\title{
Injury Experience in Coal Mining, 1989
}

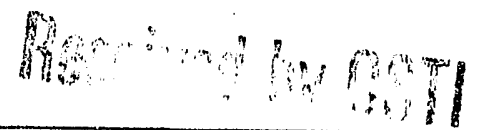

U.S. Department of Labor

Elizabeth H. Dole, Secretary

Mine Safety and Health Administration

UTEO 21990

William J. Tattersall, Assistant Secretary

Information Report

IR 1196

1990

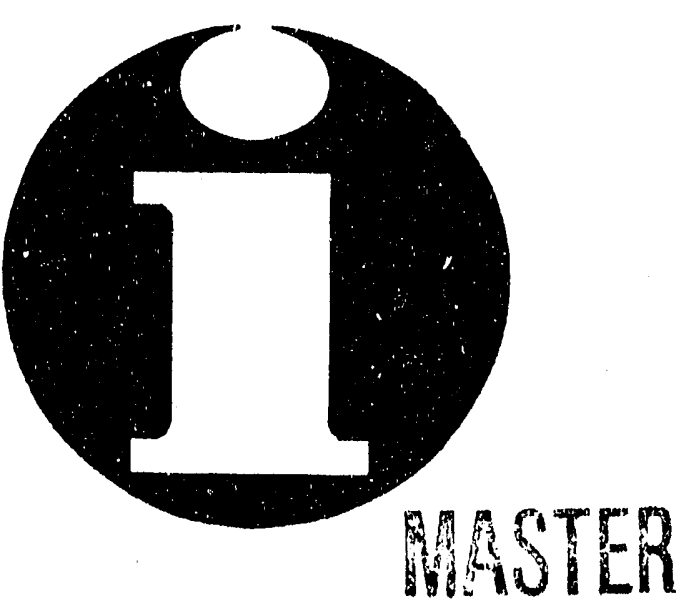


This publication has been reviewed and approved for distribution to the mining public by the Office of the Assistant Secretary for Mine Safety and Health. 
Abstract-- 1

Introduction-

Scope of statistics- 2

Definition of terms-… 3

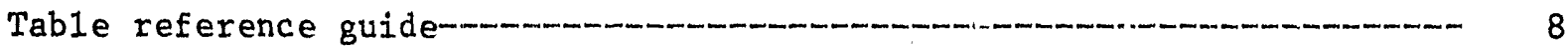

\section{ILLUSTRÁTIONS}

Figure 1. Scheduled charges in 1ost workdays for 10ss or 10 ss of use involving hand or foot- coal production report formFigure 2. Quarterly mine employment and coal production report form-
Figure 3. Mine accident, injury, and illness report form-

TABLES FOR COAL OPERATIONS IN THE UNITED STATES, 1989 (OPERATOR DATA)

1. General statistics on injury experience, worktime, production, and productivity - (a11 coal) -

1A. General statistics on injury experience, worktine, production,

1B. Genera1 statistics on injury experience, worktime, production.

2. Injury experience by degree, worktime, and producrion for work location - (al1 coal)-

2A. Injury experience by degree, worktime, and production for work
location - (anthracite)

$2 B$. Injury experience by degree, worktime, and production for work location - (bituminous)

3. Number of injuries, 1ost workdays, and average severity by degree for work location - (a11 coal)-

3A. Number of injuries, 1ost workdays, and average severity by degree for work location - (anthracite)- -

3B. Number of injuries, 10st workdays, and average severity by degree for work location - (bituminous)-

4. Injury experience by degree, worktime, and production for work location and employment size group - (al1 coal)- - -

4A. Injury experience by degree, worktime, and production for work location asd employment size group - (anthracite)-- - - - - -

4B. Injury experience by degree, worktime, and production for work location and exployment size group - (bituminous)--.--

5. Number of injuries, Iost workdays, and average severity by degree for work location and employment size group - (al1 coal)--.-.--

5A. Number of injuries, 10st workdays, anc average severity by degree for work location a:d employment size group - (anthracite)----

5B. Number of injuries, 1ost workdays, and average severity by degree for work location and employment size group - (bituminous)-----

6. Injury experience by degree, worktime, and production for work location and State - (a11 coal) -

7. Number of injuries, 1ost workdays, and average severity by degree for work location and State - (al1 coal)-

8. Number of injuries, incidence rates, and severity measures by dsgree for work location anci part of body injured - (a11 coal) 
TABLES FOR C.OAL OPERATIONS IN THE UNITED STATES, 1989 (OPERATOR DATA)

Continued

$\underline{\text { Page }}$

8A. Number of injuries, incidence rates, and severity measures by degree for work location and part of body injured (anthracite)

8B. Number of injuries, incidence rates, and severity measures by degree for work location and part of body injured (bituminous)

9. Number of injuries, 1ost workdays, and average severity by degree for work location and part of body injured - (a11 coa1)--..--

9A. Number of injuries, 10st workdays, and average severity by degree for work location and part of body injured - (anthracite)-.-.-.-

9B. Number of injuries, 10st workdays, and average severity by degree for work location and part of body injured - (bituminous)--.--

10. Number of injuries, incidence rates, and severity measures by degree for work location and nature of injury - (a11. coal)-...-

10A. Number of injuries, incidence rates, and severity measures by degree for work location and nature of injury - (anthracite)---

10B. Number of injuries, incidence rates, and severity measures by degree for work location and nature of injury - (bituminous)---

11. Number of injuries, 10st workdays, and average severity by degree for work location and nature of injury - (a11 coa1)--..--

11A. Number of injuries, lost workdays, and average severity by degree for work location and nature of injury - (anthracite)---

11B. Number of injuries, 10st workdays, and average severity by degree for work location and nature of injury - (bituminous)---

12. Number of injuries, 1ost workdays, and average severity by degree for part of body injured and nature of injury - (al1 coal) -...-

12A. Number of injuries, lost workdays, and average severity by degree for part of body injured and nature of injury - (anthracite)---

12B. Number of injuries, 10st workdays, and average severity by degree for part of body injured and nature of injury - (bituminous)---

13. Number of injuries by accident classification and degree for work location and State - (a11 coa1)- -

14. Number of lost workdays by accident classification and degree of injury for work location and State - (a11 coal) - - - - -

15. Injury experience by degree, worktime, and production for work location and production size group - (a11 coal)--

15A. Injury experience by degree, worktime, and pruduction for work location and production size group - (anthracite)- - - -

15B. Tnjury experience by degree, worktime, and production for work location and production size group - (bituminous)-- - -

16. Number of injuries, 1ost workdays, and average severity by degree for work location and production size group - (a11 coal)----

16A. Number of injuries, lost workdays, and average severity by degree for work location and production size group - (anthracite)-----

16B. Number of injuries, 10st workdays, and average severity by degree for work location and production size group - (bituminous)----

17. Number of injuries, 10st workdays, and average severity by degree for work location and occupation at time of injury - (a11 coa1) 
TABLES FOR COAL OPERATIONS IN THE UNITED STATES, 1989 (OPERATOR DATA)--Continued

Page

17A. Number of injuries, 10st workdays, and average severity by degree for work location and occupation at time of injury (anthracite)

17B. Number of injuries, 10st workdays, and average severity by (bituminous) 18. Number of injuries, lost workdays, and average severity by degree
for work location and accident classification - (all coal)18. Number of injuries, lost workdays, and average severity by degree
for work location and accident classification - (all coal)-are-
18A. Number of injuries, lost workdays, and average severity by degree

\section{degree for work location and occupation at time of injury -} for work location and accident classification - (anthracite)--..-

18B. Number of injuries, lost workdays, and average severity by degree
for work location and accident classification - (bituminous)--...

19. Number of injuries, lost workdays, and average severity by degree for accident classification and part of body - (all coal)-- - - -

19A. Number of injuries, 10st workdays, and average severity by degree for accident classification and part of body - (anthracite)-----

19B. Number of injuries, 10st workdays, and average severity by degree for accident classification and part of body - (bituminous)---.--

20. Number of injuries, 1ost workdays, and average severity by degree for accident classification and nature of injury - (a11 coal)----

20A. Number of injuries, lost workdays, and average severity by degree for accident classification and nature of injury - (anthracite)--

20B. Number of injuries, 1ost workdays, and average severity by degree for accident classification and nature of injury - (bituminous)--

21. Occurrences reported to MSHA as occupational illnesses (a11 coal)

21A. Occurrences reported to MSHA as occupational ilinesses (anthracite) -

21B. Occurrences reported to MSHA as occupational ilinesses (bituminous)

22. Related coal mining summary reference tabulation, number of injuries, incidence rates, average number of workexs, employeehours and production by type of coal mined and work location----

23. Related noncoal mining summary reference tabulation, number of injuries, incidence rates, average number of workers, and employee-hours by work location and mineral industry-............... 
Table reference guide (contractor data)

TABLES FOR COAL OPERATIONS IN THE UNITED STATES, 1989 (CONTRACTOR DATA)

1. General statistics on injury experience, worktime, and production--

2. Injury experience by degree, worktime, and production for work location-

3. Number of injuries, 1ost workdays, and average severity by

4. Injury experience by degree, worktime, and production for work location and employment size group

5. Number of injuries, lost workdays, and average severity by

degree for work location and employment size group---
7. Number of injuries, lost workdays, and average severity by

8. Number of injuries, incidence rates, and severity measures by

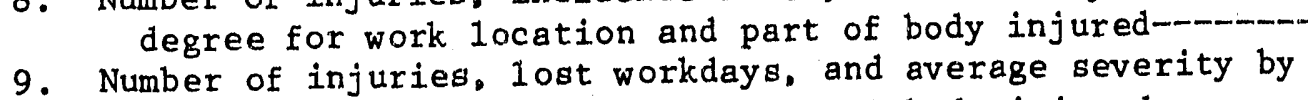
degree for work location and part of body injured-----m

10. Number of injuries, incidence rates, and severity moisures by degree for work Iocation and hature of injury--..-

11. Number of injuries, 10st workdays, and average severity by

degree for work 1ocation and nature of injury---
12. Number of injuries, 10st workdays, and average severity by degree
for part of body and nature of injury-

13. Number of injuries by accident classification and degree ror

14. Number of 10 st workdays by accident classification and degree of injury for work location and State

17. Number of injuries, 10st workdays, and average severity by degree for work location and occupation at time of j.njury

18. Number of injuries, 10st workdays, and average severity by

degree for work location and accident classification---
19. Number of injuries, 10 st workdays, and average severity by degree for accident classirication and part of body injured-- - -

20. Number of injuries, 10st workdays, and average severity by degree for accident classification and nature of injury

degree for accident 398

22. Related coal mining summary reference tabulation, number of injuries, incidence rates, average number of workers, employee-hours and production hy type of coal mined and work location- noncos1 mining summary reference tabulation, number

23. Re1ated noncosl mining summary reference tabulation, number
of injuries, incidence rates, average number of workers, and employee-hours by mineral industry and work location 
INJURY EXPERIENCE IN COAL MINING, 1989

by

Staff, Division of Mining Information Systems

Safety and Health Technology Center $1 /$

\section{AB STRACT}

This Mine Safety and Health Administration (MSHA) informational report reviews in detail the occupational injury and illness experience of coal mining in the United States for 1989. Data reported by operators of mining establishments concerning work injuries are summerized by work location, accident classification, part of body injured, nature of injury, occupation, and anthracite or bituminous coal. Related information on employment. worktime, and operating activity also is presented. Data reported by independent contractors performing certain work at mining locations are depicted separately in this report. For ease of comparison between coal mining and the metal and nonmetal mineral mining industries, summary reference tabulations are included at the end of both the operator and the contractor sections of this report.

\section{INTRODUCTION}

Data used in compiling this report were reported by operators of coal mines and preparation plants on a mandatory basis as required under the Federal Mine Safety and Health Act of 1977, Public Law 91-173 as amended by Public Law 95-164. Since January 1, 1978, Ejerators of mines or preparation plants or both which are subject to the act have been required under $30 \mathrm{CFR}$, Part 50 to submit reports of injuries, occupational illnesses, and related data.

On1y data reported by the operators and contractors themselves are utilized in this report. No estimates are made of employment, employeehours, or injuries for nonrespondent operations except as necessary to meet statistical processing requirements by providing minimum employment data for reported unmatched injury data.

Beginning with calendar year 1978, statistical measures for injury experience in mining were changed from the measures used from 1930 through 1977. The statistical measures for injury experience adopted by MSHA in 1978 compare closely with the measures used in the Office of Occupational Safety and Health Statistics, Bureau of Labor Statistics, U. S. Department of Labor. Therefore, beginning with 1978 data the mining industry can be compared on a standard basis with other United States industries reporting under the Occupational Safety and Health Act of 1970.

1/ Technical Support, Denver, Colorado. 
Occurrences identified as occupational illnesses by mine operators were reported to MSHA and processed in accordance with 30 CFR Part 50.20-6. The reported illnesses are summarized in seven categories for each work location, kind of coal, and State. Incidence rates and severity measures are not computed for occupational ilinesses.

Because of the modifications in reporting and processing procedures that became effective January 1, 1978, injury rates as current1y computed are not precisely comparable to those of previous years, except for fatality rates in which the "incidence rate" (the term used after 1977) is one-fifth of the "frequency rate" (the term used prior to 1978) for otherwise similar groupings of fatal injuries and worktimes. Because of this disparity, MSHA annual publications concerning injuries, illnesses, and operating acativity will not contain data for years prior to 1978 .

\section{SCOPE OF STATISTICS}

The statistical data in this report cover the work experience of all personnel engaged in mine exploration, development, production, maintenance, repair, and construction work, including supervisory and technical personne1, working partners, and onsite of ficeworkers.

It should be noted that amendments to legislation that funded MSHA and other government programs from December 16, 1981, through July 18, 1982 , prohibited MSHA from administering or enforcing the Federal Mine Safety and Health Act of 1977 (Mine Act) or MSHA standards and regulations with respect to any person engaged in the surface mining or milling of stone, clay, feldspar, colloidal phosphate, or sand and gravel. They also provided that MStiA could not administer or enforce the Mine Act or MSHA standards and regulations on any State or political subdivision thereof. Further, MSHA could not administer or enforce the Mine Act or MSHA standards or regulations with respect to any independent construction contractor who was engaged by an operator for the construction, repair or alteration of struccures, facilities, utilities or private ways or roads located on (or appurtenant to) the surface areas of any coal or other mine, and whose employees worked in a specifically demarcated area, separate from actual mining or extraction activities. No data on exempted activities are included for that period of time in Table 1 of this report.

MSHA issued guidelines which additionally reduced the reporting requirements of independent contractors. Reporting of employment, hours worked, and injury/iliness data by independent contractors is 1 imited to the following types of work performed: mine development, including shaft and slope sinking; construction or reconstruction of mine facilities, including building or rebuilding preparation plants and mining equipment, and building additions to existing facilities; demolition of mine facilities; construction of dams; excavation or earthmoving activities involving mobile equipment; equipment installation, such as crushers and mills; equipment service or repair done underground or in the pit for a period exceeding five consecutive days at a particular mine; material handling within mine property, including haulage of coal, ore, refuse, etc.. unless for the sole purpose of direct removal from or delivery to mine property; drilling and blasting. Also, data collected for employment and hours worked in those activities reflect the contractor's total metallic/nonmetallic work locations and total coal work locations and not the individual mine sites. For more detailed information concerning these changes, see the guidelines accompanying MSHA Policy Memoranda Nos. 82-6A MM and 81-35A C. 


\section{DEFINITION OF TERMS}

Occupational Injury.--An occupational injury is any injury to a mine worker which occurs at a mine and for which medical treatment is administered, or which results in death or loss of consciousness, restriction of work or motion, inability to perform all job duties on any day after an injury, 1ost workdays, temporary assignment to other duties, transfer to another job, or termination. The injury must result from a recognizable single ncident. For example, an explosion of a battery which splattered a worker with sulfuric acid would be a single incident and the worker would be considered to have suffered an injury. Another example would be an injury where a worker was overcome by hydrogen sulfide gas released from an exploding vesse1.

Occupational Illness.--An occupational illness is an illness or disease of a mine worker which may have resulted from work at a mine or for which an award of compensation is made. To be classified as an occupational illness, the disability must result from repeated exposure to the condition or substance which caused the disability. A classic illness example is a pneumoconiosis disability which may have been induced by repeated exposure to the causative dusty condition. Dermatitis resulting from repeated exposure to lime dust or other material in the work environment also should be reported as an illness. In cases where the time of onset of iliness is in doubt, the day of diagnosis of illness can be considered as the first day of illness.

Number of Operations and Associated Work Locations.--Number of operations is the number of individual respondents with a unique Federal identification number that have reported worktiwe data. The count and kind of operation are based on the lowest numbered (primary) subunit on employment report Form 7000-2 (see figure 2, p. 10) that contains worktime data. An associated work $10 c a t i o n$ is a subunit reported with a primary subunit; e.g., an "office" is almost always an associated work location. Independent contractors working on mine property performing the types of work described under "Scope of Statistics" are assigned an identification number, report to MSHA, but are not included in the count of operations; 2,036 contractors in the coal mining industry reported to MSHA in 1989 . Contractor data are depicted separately in this report.

Worktime.--Worktime includes number of workers and number of employeehours worked. "Average number of workers" is a summary of the average number of persons working at individual establishments during calendar quarters of active operations. "Employee-hours" is a sumbily of employeehours reported.

Employment Size Group.--Reporting operations are classified into size groups according to the average number of workers in specifled work locations.

Production Size Group.--Reporting operations are classified into production size groups according to the number of short tons of coal produced. 
Degree of Injury.--Degree of injury indicates the seriousness of injuries. FATAL injuries are those occurrences resulting in death, NEDL, (NonFatal with Days Lost) injuries are nonfatal occurrences that result in days away from work, statutory days charged, or days of restricted work activity. NDL (No Days Lost) injuries are occurrences having no $10 \mathrm{st}$ workdays; that is, nonfatal injury occurrences resulting only in temporary 10ss of consciousness or medical treatment other than first aid.

A permanent total disability is caused by any work injury or illness other than death that permanently and totally incapacitates a worker from following any gainful occupation, or that results in the loss, or the complete loss of use, of both or any combination of: hands, arms, legs, feet, or eyes.

A permanent partial disability is caused by any work injury or illness other than death or permanent total disability that results in the loss, or the complete loss of use, of any member or part of a member of the body, or any permanent impairment of functions of the body or part thereof, regardless of any preexisting disability of the injured member or impaired body function.

Incidence Rate.--Incidence rate is defined as the number of injuries per 200,000 employee-hours, rounded to two decimal places. The highest rate given is 99.99. If an incidence rate is greater than 99.99, then 99.99 is printed. If no data were reported, then a hyphen is printed. If a computation is 1 ess than 0.01 after rounding, then an asterisk inside parentheses $(*)$ is printed. The following is the standard incidence rate formula:

$$
I R=\frac{\text { number of injuries } \times 200,000}{\text { number of employee-hours }}
$$

Lost Workdays (LWD).--Lost workdays consist of days away from work (DAW) and days of restricted work activity (DRA), or statutory days charged as prescribed from a table of standard charges (see p. 7), e.g., 6,000 days for a fatality or permanent total disability.

Severity Measure.--Severity measure is the number of 1 ost workdays per 200,000 employee-hours, rounded to whole numbers. The highest rate given is 99,999. If the computed rate is greater than 99,999, then 99,999 is printed. If no data were reported, then a hyphen is printed. If the computation results in a zero after rounding, then an asterisk inside parentheses (*) i.s printed. The following is the standard severity measure formula:

$$
S M=\frac{\text { number of } 1 \text { ost workdays } \times 200,000}{\text { nimber of employ ee- hours }}
$$

Average Severity.--Average severity is the average number of $108 \mathrm{t}$ workdays per injury and is computed only for injuries classified as FATAL or NEDL. Lost workdays are selected on the same basis as that for computing the severity measure. The following is the standard average severity formula:

$$
\text { AS }=\frac{\text { number of lost workdays }}{\text { number of injuries contributing the lost workdays }}
$$


Classification of Accident.--Accident classification identifies circumstances which contributed most directly to the resulting accident. The 21 classifications in the following 1 ist are designed to categorize all accidents, whether injury-producing or not.

Electrical.--Accidents in which the electric current is most. directly responsible for the resulting accident.

Entrapment.--Accidents involving entrapment of persons.

Exploding Vesse1s Under Pressure.--Accidents involved with bursting of air hoses, air tanks, hydraulic 1 ines, hydraulic hoses, stand pipes, etc., due to internal pressure.

Explosives and Breaking Agents.--Accidents involving the detonation of manufactured explosives, includes Airdox and Cardox.

Falling, Rolling, or Sliding Rock or Material of Any Kind.-Accidents caused directly by falling material other than material from the roof or face. If material was set in motion by machinery, by haulage, by handtools, or while being handled or disturbed, etc., the force that set the material in motion determines the classification. For example, where a rock was pushed over a highwall by a bulldozer and the rock hit another rock which hit and injured a worker--the accidene is classified as machinery. Machinery (a bulldozer) most directly caused the resulting accident.

Fal1 of Face, Rib, Pillar, Side, or Highwal1 (from in place).-Accidents in this claseification inc1ude falls of material while barring down or placing props, also pressure bumps and bursts. Not included are accidents in which the motion of machinery or haulage equipment caused the fall either directly or by knocking out support.

Fal1 of Roof, Back, or Brow (from in place).--Underground only Accidents which include falls while barring down or placing props, also pressure bumps and bursta. Not included are accidents in which the motion of machinery or haulage equipment caused the fall either directly or by knocking out support.

Fire.-Accidents related to uncontrolled burning of material or mineral in the mine environment. Not included are fires initiated by electricity or by ignition or explosion of gas or dust.

Handling Material.--Accidents related to handling packaged or loose material while lifting, pulling, pushing, or shoveling.

Nonpowered Haulage.--Accidents re ated to the motion of nonpowered haulage equipment. Included are accicients involving wheelbarrows. manually pushed mine cars, timber trucks, etc. 
Fire.-Accidents related to uncontrolled burning of material or mineral in the mine environment. Not included are fires initiated by electricity or by ignition or explosion of gas or duet.

Handling Material.--Accidents related to handling packaged or loose material while jifting, puiling, pushing, or shoveling.

Handtuo1s.--Accidents related to nonpowered tools.

Nonpowered Hatlage.--Accidents related to the motion of nonpowered haulage equipment. Included are accidents involving wheelbarrows, manually pushed mine cars, timber trucks, etc.

Powered Haulage.--Accidents related to the motion of powered haulage equipment. Included are accidents involving conveyors, front-end loaders, forklifts, shuttle cars, load-haul-dump units, locomotives, railroad cars, haulage trucks, pickups, automobiles, and personnel carriers.

Hoisting.--Accidents involving cages, skips, ore buckets, and elevators. The accident results from the action, motion, or failuce of the hoisting equipment or mechanism. Included are equipment such as cranes and derricks only when used in shaft sinking; also suspended work platforms in shafts. Not included is equipment such as chain hoists, come-alongs, and winches.

Ignition or Explosion of Gas or Dust.--Accidents resulting as a consequence of the ignition or explosion of gas or dust.

Impoundment.--Accidents caused by an unstable condition or failure of an impoundment, refuse pile, or culm bank requiring cmergency preventative action or evacuation of an area.

Inundation.--Accidents caused by inundation of a surface or underground mine by a liquid (or semi-solid) or a gas.

Machinery.--Accidents related to the motion of machinery. Included are all electric and air-powered tools and mining machinery such as drills, tuggers, winches, slushers, draglines, power shovels, loaders, and compressors.

S1ip or Fal1 of Person (from an elevation or on the same leve1).-Accidents include slips or falls while getting on or of machinary and haulage equipment which is not moving, and slips or falls while servicing or repairing equipment or machinery.

Stepping or Kneeling on Object.--Accidenta are classified in this category only where the object stepped or kneeled on contributed most directly to the accident.

Striking or Bumping.--This classification is restricted to those accidents in which an individual, while moving about, strikes or bumps an object but is not handling material, using handtools, or operating equipment. 
Statutory Days Charged.--Same injuries or illnesses are of such a degree of severity that a standard time charge of lost workdays has been adopted by MSHA as follows:

Disability

Lost Workdays Charged

D zath . . . . . . . . . . . . . . 6,000

Permanent total cisability . . . . . . . . . 6,000

Dismemberment or total loss of use

Arm above elbow ........... 4,500

Arm at or below elbow and above wrist . . . 3,600

Hand at wrist . . . . . . . . . . 3,000

Leg above kt.ee.............. 4,500

Leg at or jelow knee and above ankle. . . . 3,000

Foot at ankle........... 2. 2,400

Loss of sight:

One eye (whether or not ther is sight

in the other eye)............ 1,800

Both eyes (in one accident) ....... 6,000

Complete industria1 loss of hearing:

One ear (whether or not there is hearing

in the other ear)........... 600

Both ears (in one accident) ....... 3,000

Unrepaired hernia........... 50

2/ Not more than 6,000 lost workdays will be charged in a single accident that results in multiple injuries to a mine worker. (See definition of permanent total disability on page 4.)

\section{TABLE REFERENCE GUIDE (OPERATOR DATA)}

The following reference guide facilitates locating particular data elements contained in the annual statistical tables for operators.

A comparable guide precedes the contractor section of this report. The number appearing in each cell indicates the table number in which that particular element may be found. All elements listed under the stub headings appear as vertical headings in the tables. Elements listed under column headings appear as horizontal headings. The only exception ro this is table 1, in which the headings have been reversed.

To use this guide, read down or across the elements 1 isted in both the stub and column hearings to determine which combinations are of interest. For example, incidence rates by work location appear in seven different tables, numbers $2,4,6,8,10,22$, and 23 . However, only one, table 10 , provides a further division by nature of injury as well. The occupation of the injured is found in table 17 and, as seen in the column headings, provides the number of injuries, lost workdays, and average severity. With the exception of tables $6,7,13,14,22$, and 23 , a11 remaining tables are followed by additional tables ( $A$ and $B$ ) which divide the information into anthracite (e.g., table 1A) and bituminous (e.g., table 1B). 
BEFERENCE GUIDE TO COAL STATISTICAL TABLES (OPERATOR DATA)

\begin{tabular}{|c|c|c|c|c|c|c|c|c|c|c|c|c|c|c|c|}
\hline STUB HEADINGS & 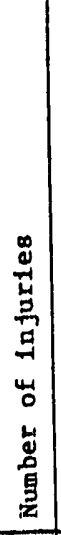 & 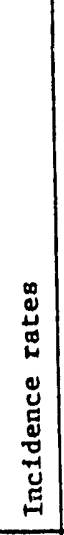 & 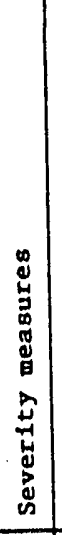 & 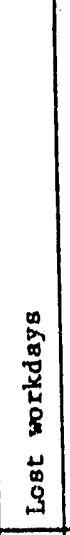 & 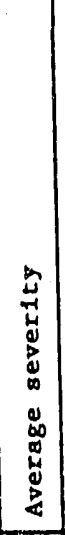 & 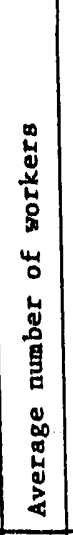 & 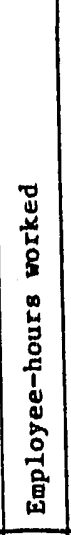 & 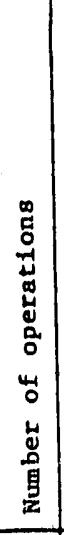 & 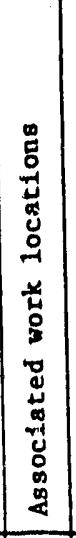 & 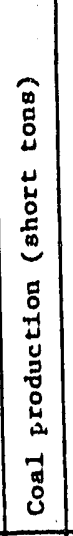 & 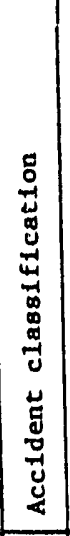 & 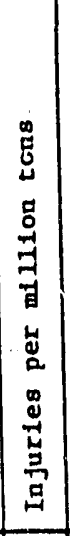 & 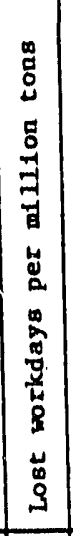 & 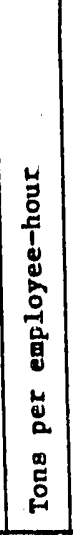 & 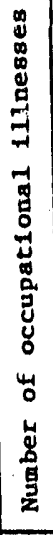 \\
\hline $\begin{array}{l}\text { Pritor year data } \\
\text { wlth and without office I/ }\end{array}$ & 1 & 1 & 1 & & 1 & 1 & 1 & 1 & 1 & 1 & & 1 & 1 & 1 & \\
\hline \multirow{17}{*}{ Work location } & 2 & 2 & 2 & & & 2 & 2 & 2 & 2 & 2 & & & & & \\
\hline & 3 & & & 3 & 3 & & & & & & & & & & \\
\hline & 4 & 4 & 4 & & & 4 & 4 & 4 & 4 & 4 & & & & & \\
\hline & 5 & & & 5 & 5 & & & & & & & & & & \\
\hline & 6 & 6 & 6 & $T$ & $\pi$ & 6 & 6 & 6 & 6 & 6. & & & & & \\
\hline & $\frac{7}{8}$ & 8 & 8 & - & 7 & & & & & & & & & & \\
\hline & 9 & & & 9 & 9 & & & & & & & & & & \\
\hline & 10 & 10 & 10 & & & & & & & & & & & & \\
\hline & II & & & 11 & II & & & & & & & & & & \\
\hline & 13 & & & & & & & & & & 13 & & & & \\
\hline & & & & 14 & & & & & & & 14 & & & & \\
\hline & 15 & & & & & 15 & 15 & 15 & 15 & 15 & & 15 & 15 & & \\
\hline & 16 & & & 16 & 16 & & & & & & & & & & \\
\hline & 17 & & & 17 & 17 & & & & & & & & & & \\
\hline & 18 & & & 18 & 18 & & & & & & & & & & \\
\hline & 22 & 22 & & & & 22 & 22 & & & 22 & & & & & 21 \\
\hline & 23 & 23 & & & & $\frac{26}{23}$ & $\frac{22}{23}$ & & & 10.2 & & & & & \\
\hline \multirow{2}{*}{ Employment size group } & 4 & 4 & 4 & & & 4 & 4 & 4 & 4 & 4 & & & & & \\
\hline & 5 & & & 5 & 5 & & & & & & & & & & \\
\hline \multirow{4}{*}{ State } & 6 & 6 & 6 & & & 6 & 6 & 6 & 6 & 6 & & & & & \\
\hline & 7 & & & 7 & 7 & & & & & & & & & & \\
\hline & 13 & & & & & & & & & & $\frac{13}{14}$ & & & & \\
\hline & & & & 14 & & & & & & & 14 & & & & \\
\hline \multirow{3}{*}{ Part of body infured } & 87 & 8 & 8 & & & & & & & & & & & & 21 \\
\hline & 9 & & & 91 & 9 & & & & & & & & & & \\
\hline & 12 & & & 12 & 12 & & & & & L & & & & & \\
\hline \multirow{4}{*}{ Nature of 1nfury } & $\frac{19}{10}$ & & & 19 & 19 & & & & & & & & & & \\
\hline & $\frac{10}{11}$ & 10 & 10 & & & & & & & & & & & & \\
\hline & $\frac{11}{12}$ & & & 11 & 11 & & & & & & & & & & \\
\hline & $\frac{12}{20}$ & & & 12 & 12 & & & & & & & & & & \\
\hline \multirow{2}{*}{ Production size group } & $\frac{20}{15}$ & & & 20 & 20 & 15 & 15 & 15 & 15 & 15 & & 15 & 15 & & \\
\hline & 16 & & & 16 & 16 & & & & & & & & & & \\
\hline $\begin{array}{l}\text { Occupation af time of } \\
\text { Injury }\end{array}$ & 17. & & & 17 & 27 & & & & & & & & & & \\
\hline \multirow{2}{*}{ Accident classification } & 18 & & & 18 & 18 & & & & & & & & & & \\
\hline & 19 & & & 19 & +9 & & & & & & & & & & \\
\hline Type of coal & 20 & & & 20 & 20 & & & & & & & & & & \\
\hline $\begin{array}{l}\text { Type of coal } \\
\text { Mineral Industry }\end{array}$ & $\frac{22}{23}$ & $\frac{22}{23}$ & & & & \begin{tabular}{|l|}
22 \\
23 \\
\end{tabular} & $\frac{22}{23}$ & & & 22 & & & & & \\
\hline
\end{tabular}

1/ For table 1 only, the headings have been reversed to simplify this gulde; that 18, the data elements indicated in the column headings for this table actually appear as vertical headings. 

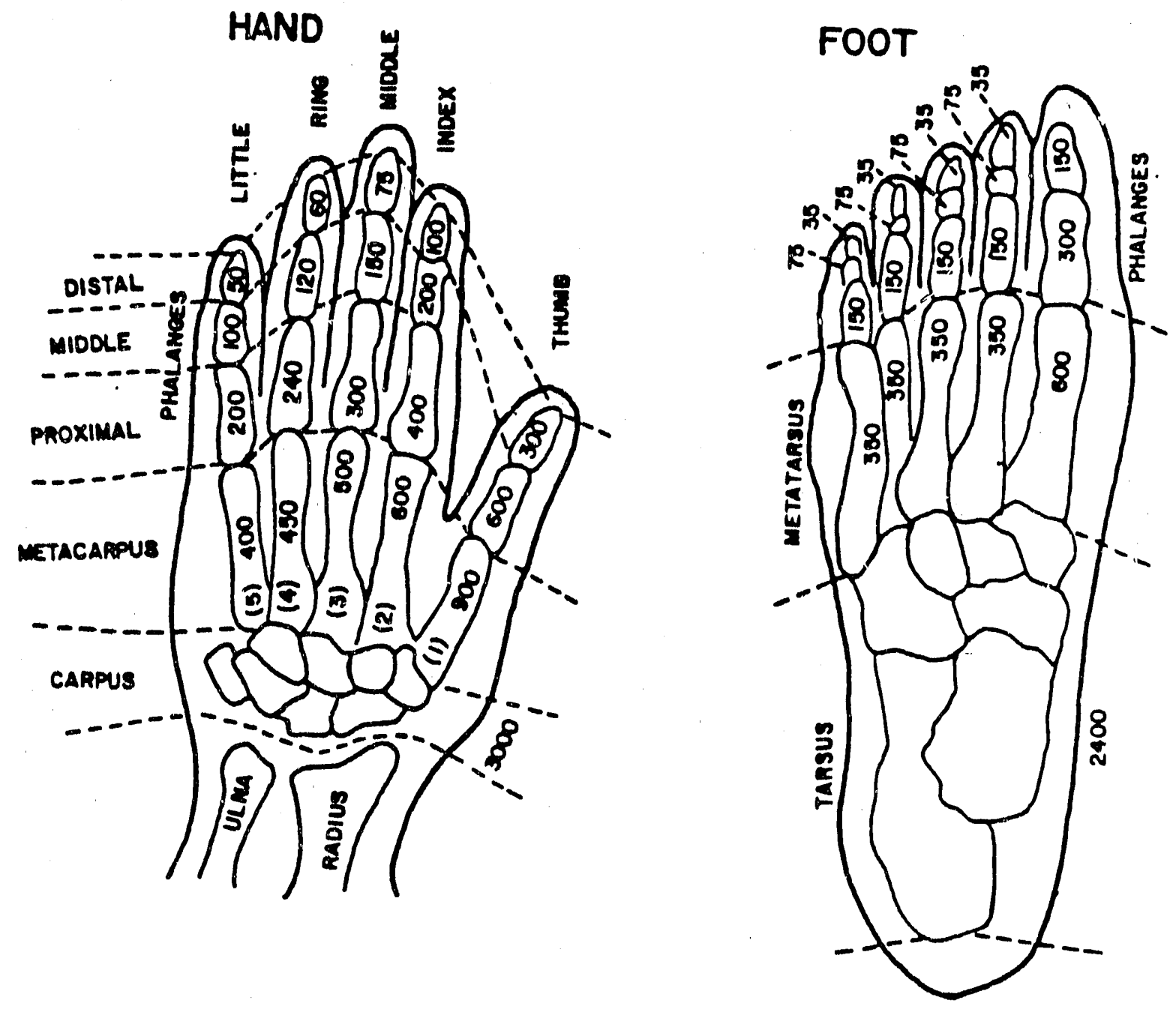

Note:

Numbers shown on diagrams are statutory charges of lost workdays for loss of all or part of bone.

Proportional charges may be computed based on medical determination of percentage loss of use.

FIGURE I. Scheduled charges in lost workdays for loss or loss of use involving hand or foot. 


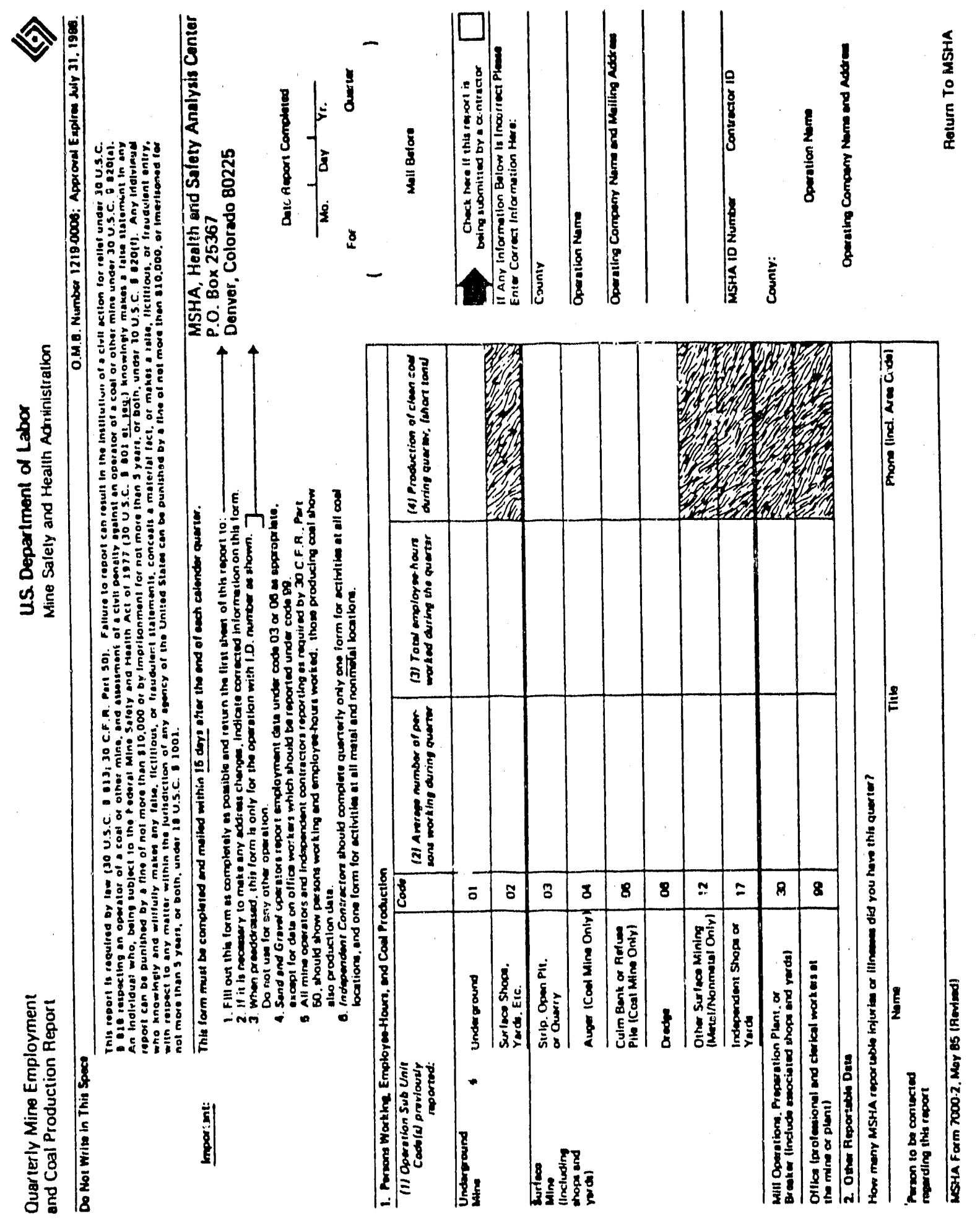

FIGURE 2. Quarterly mine employment and coal production report form. 
Mine Accident, Injury and IIIness Report

US. Department of Labor

Mine Safety and Heaith Adrinistration

Sectoon A-tamnfiemon Dew

\begin{tabular}{|c|c|c|c|c|}
\hline \multicolumn{5}{|c|}{ 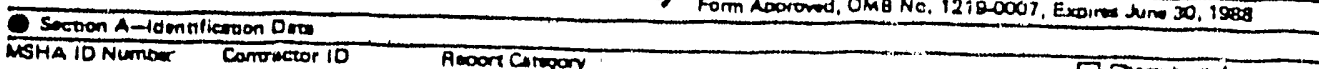 } \\
\hline MSHA IO NumOar & Cantuctor iD & 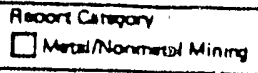 & 口 Cool Mining & 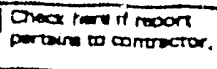 \\
\hline
\end{tabular}

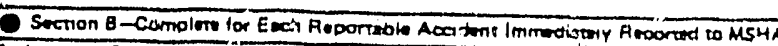

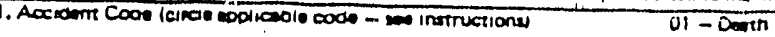

or - Imunarion

05 - Ges or Oun Ionition

$\infty$ - Mino Fir

10 - Imvewnding Dain 11 - Hointing

$02-5$ iniou Injury

07 - Explosine

12 - Ottrit Injury
C) - Entrapremt

$\infty$ - Aool Fall

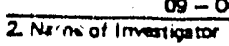
3 Dare Imertiontro, Startad

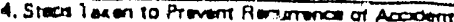
Mor". Der Tlas

Section C-Complerv tor Emch Remortoole Aceroent, Iniurv or llingy

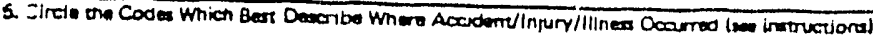

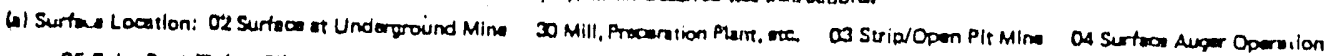

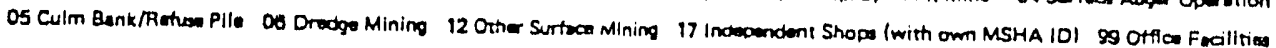

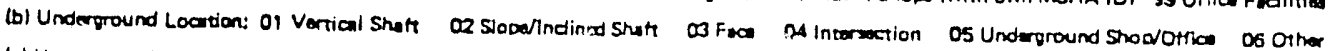

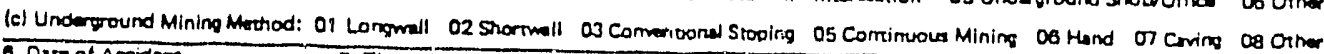
C. Dirn of Axridert

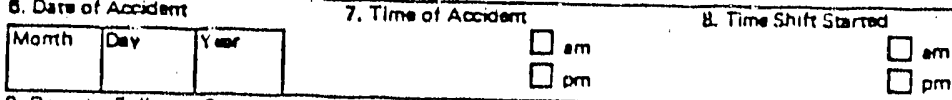

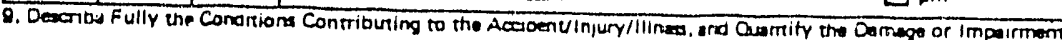

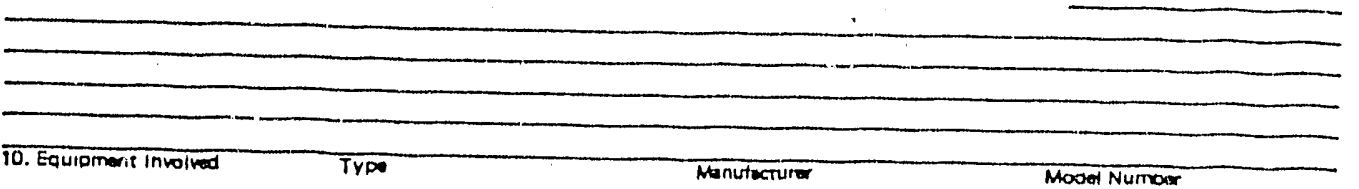

11. Name of Witnes to Acordent/injuryfliliness

12 Number of Reporable Injurite or Mosel NurTour

13. Name of injurad/III Employee IIIneases Resulting from This Oecurn ma

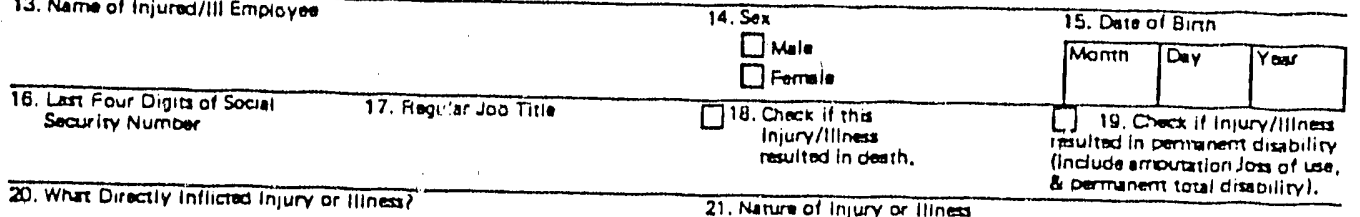

20. Wha Direaly inflictod Injury or llines?

21. Nature of Injury or llines

\begin{tabular}{|c|c|c|c|}
\hline \multirow[t]{2}{*}{$\begin{array}{l}\text { 22. Part of boor injured } \\
\text { or Altected }\end{array}$} & \multirow{2}{*}{\multicolumn{3}{|c|}{ 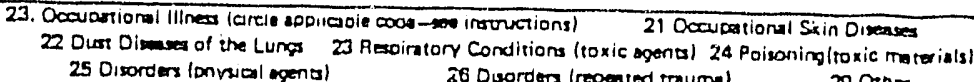 }} \\
\hline & & 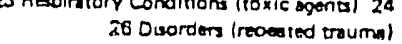 & $\begin{array}{l}\text { altoxic materials) } \\
29 \text { Giner }\end{array}$ \\
\hline
\end{tabular}

\begin{tabular}{|c|c|c|}
\hline Experience & Yoan & Wenks \\
\hline IE. Exovriencs in Thus 200 Title & & \\
\hline 25. Experience st inga Mine & & \\
\hline 27. ToDl Mining Ex oerience & & \\
\hline
\end{tabular}

Secrion O-Rerum io Dut Iniommion

28. Pormanantly Transerred or

Teminated lit enecked.

complete items 29,30 . \& 31 Answ 30 \& 31 men case is clased

29. Oale Aerumed to Facular joo at 30 . Tumoer of Davi31. Mumber of Dary 30 inumber of Davi 31 . Number of Dary

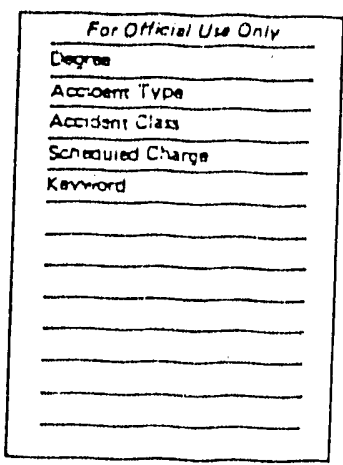

Porson Eumpleting Form (nama) Aestivied Work enter ol

Dare This Reoort Prexured imonin, day, year Mionit ray

\section{MSHA Form 7000-1, Feo 60 Irevised)}

MAIL THIS PAGE TO THE HEALTH AND SAFETT ANALYSIS CENTER, MINE SAFETY ANO HEALTH ADMINISTAATION, P.O. BOX $\$ 367$, DENVEA, COLORADO 8OZ25

FIGURE 3. Mine accident, injury, and illness report form. 


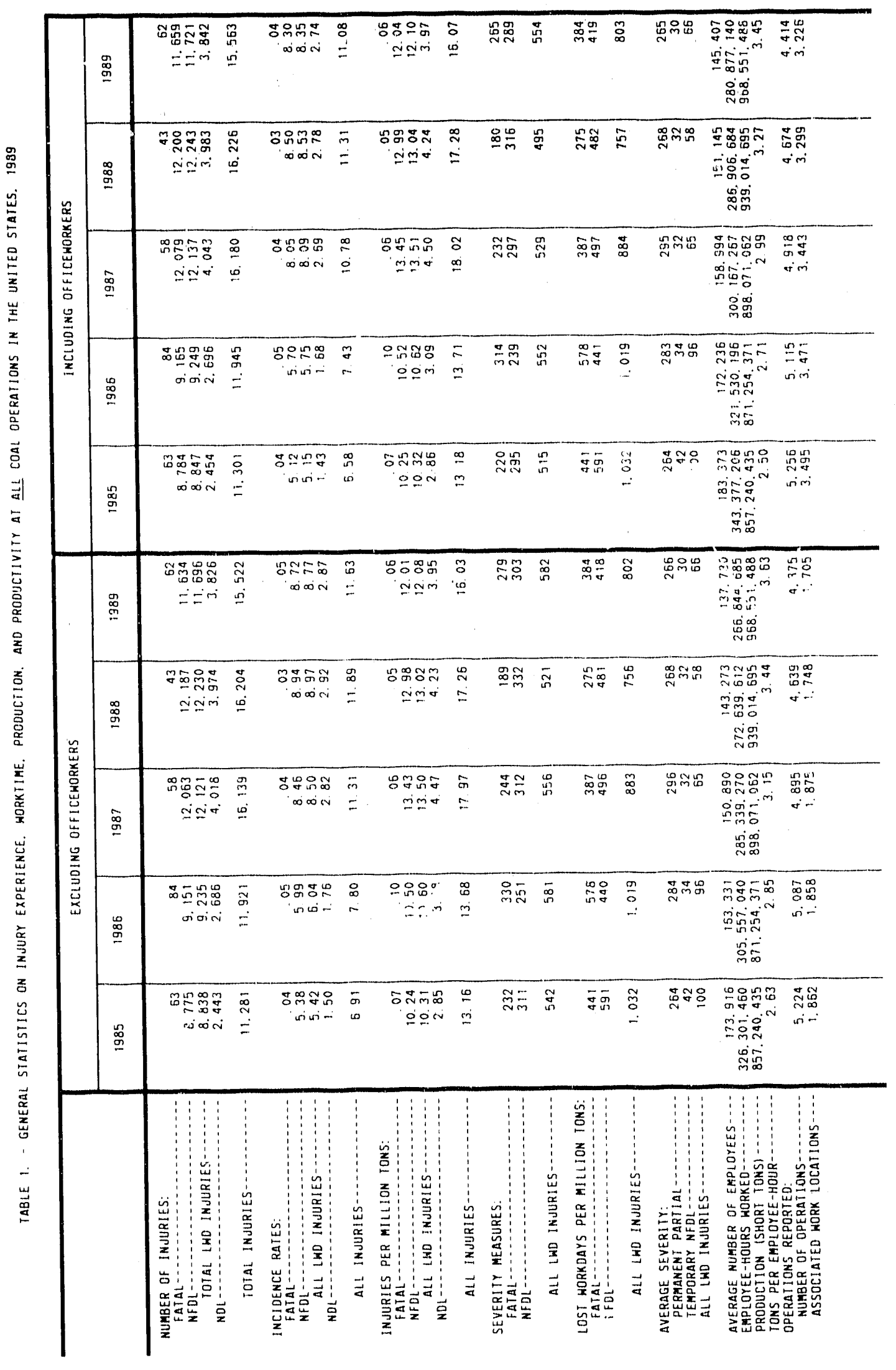




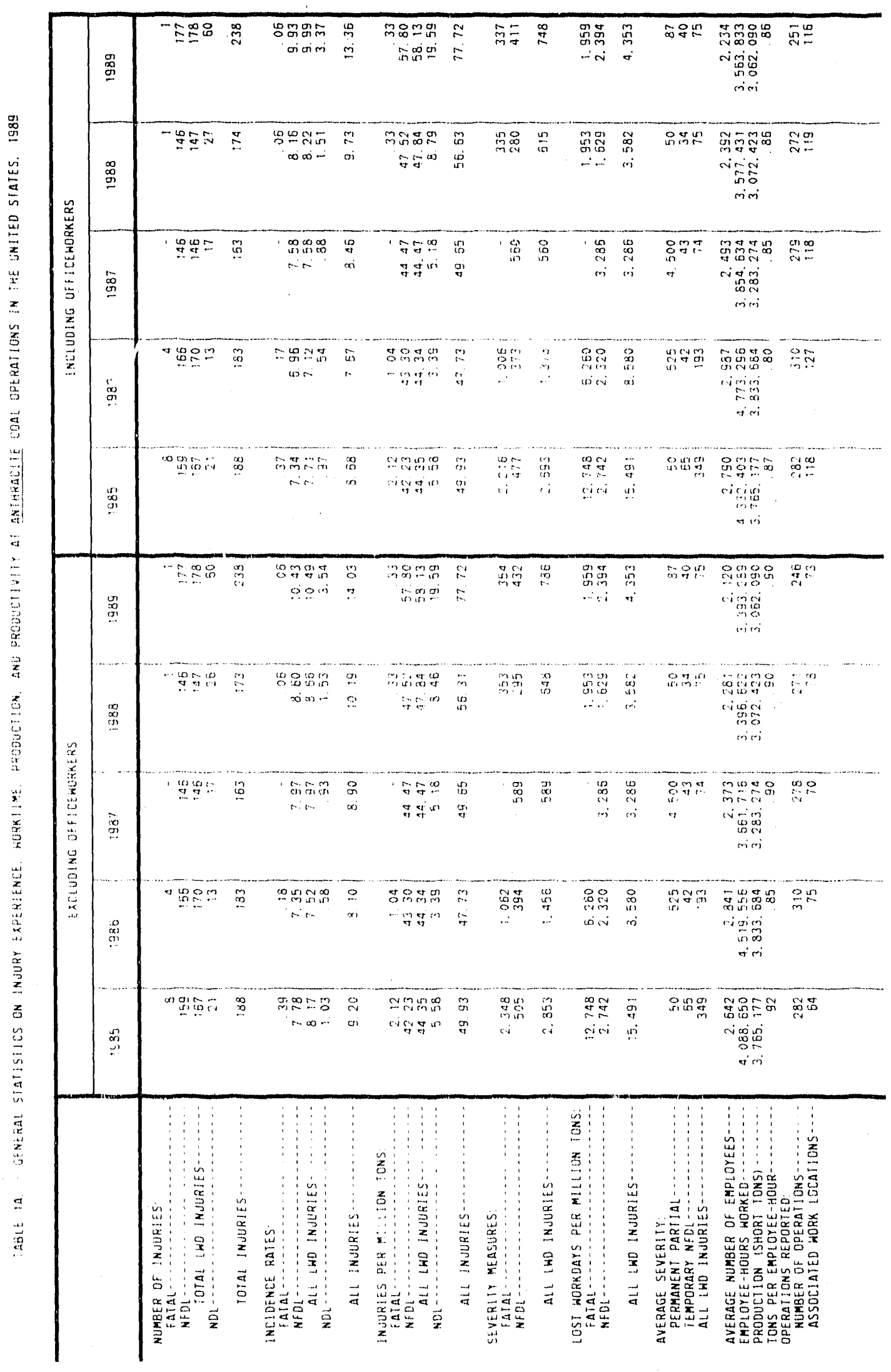




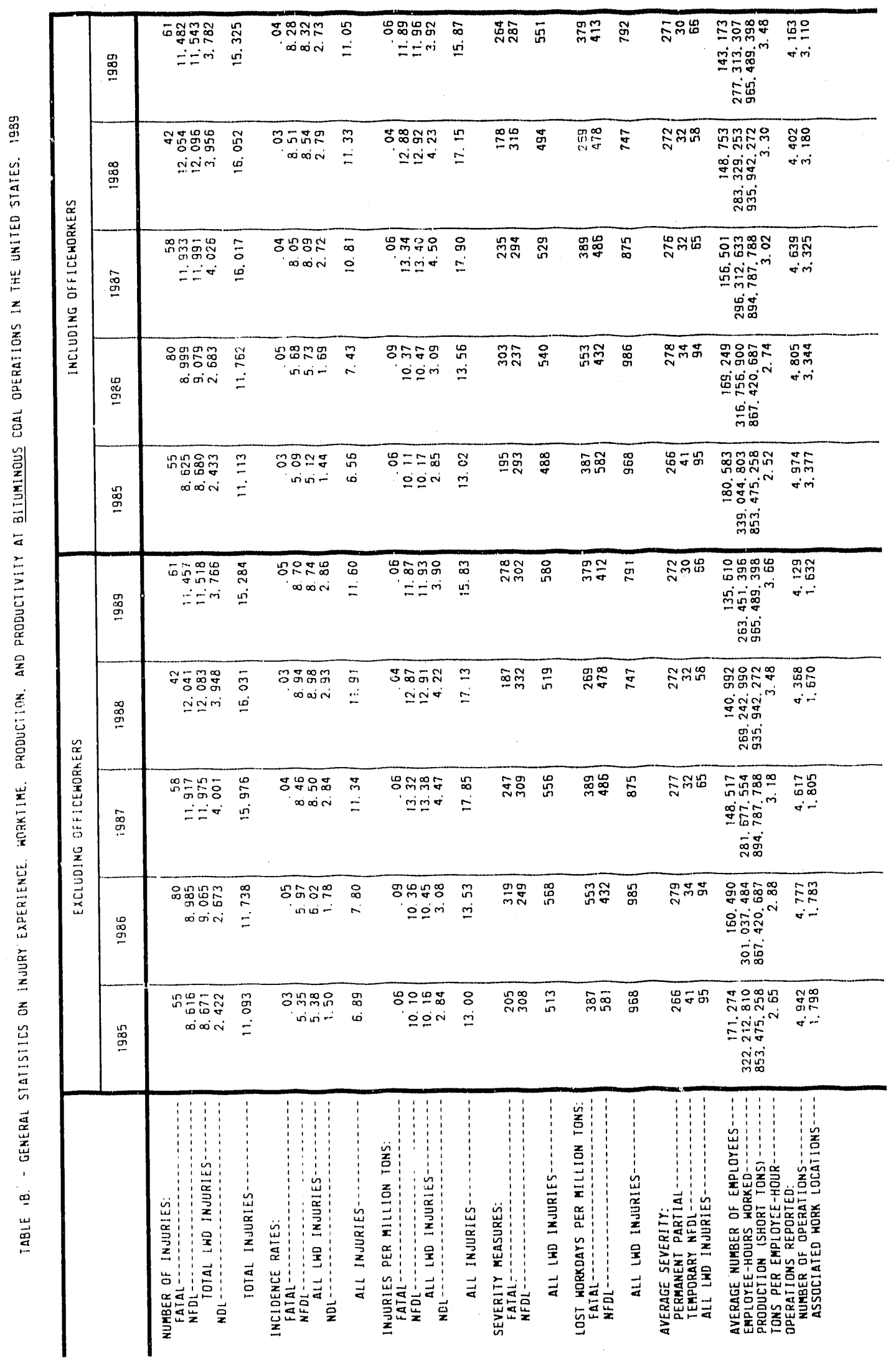




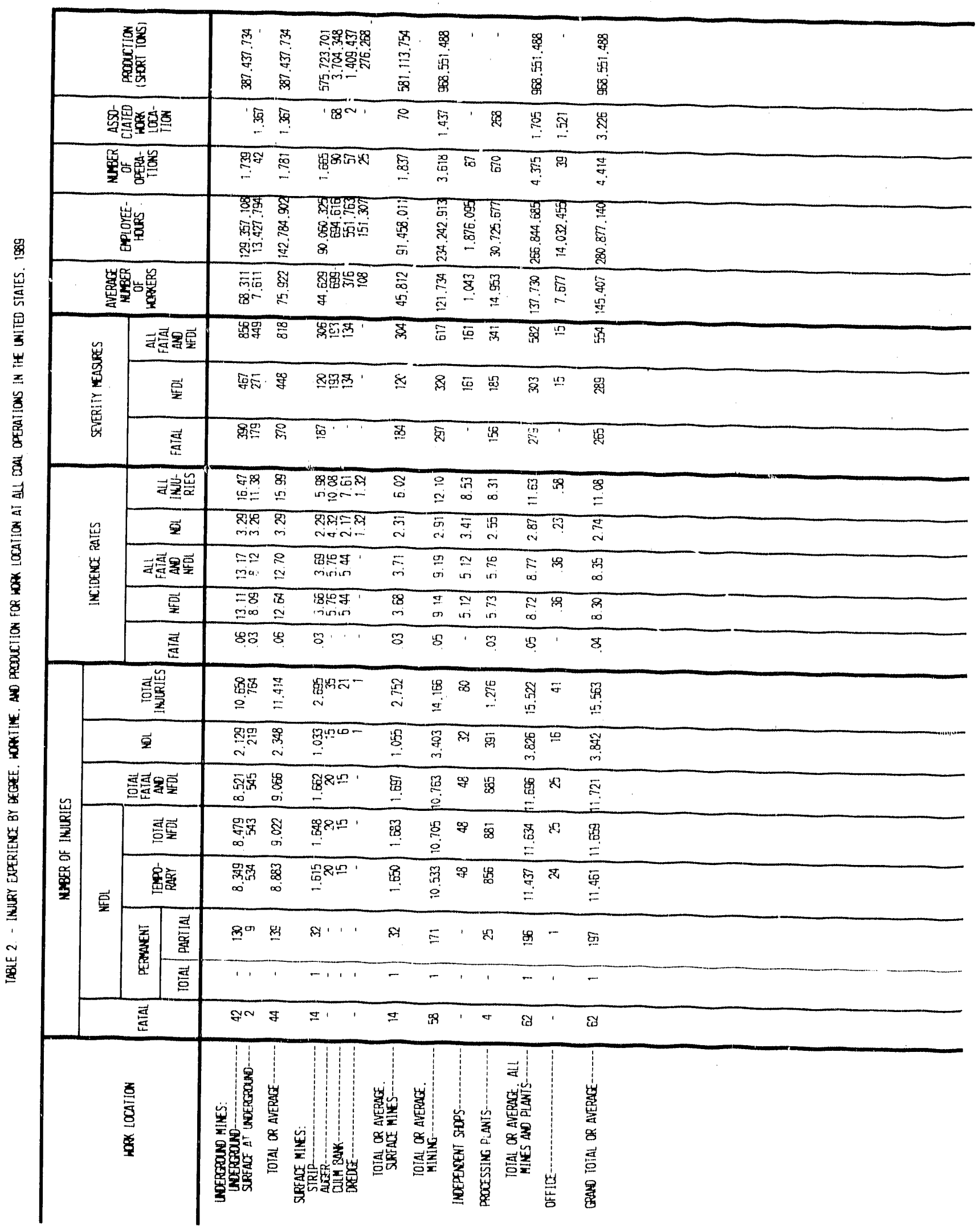




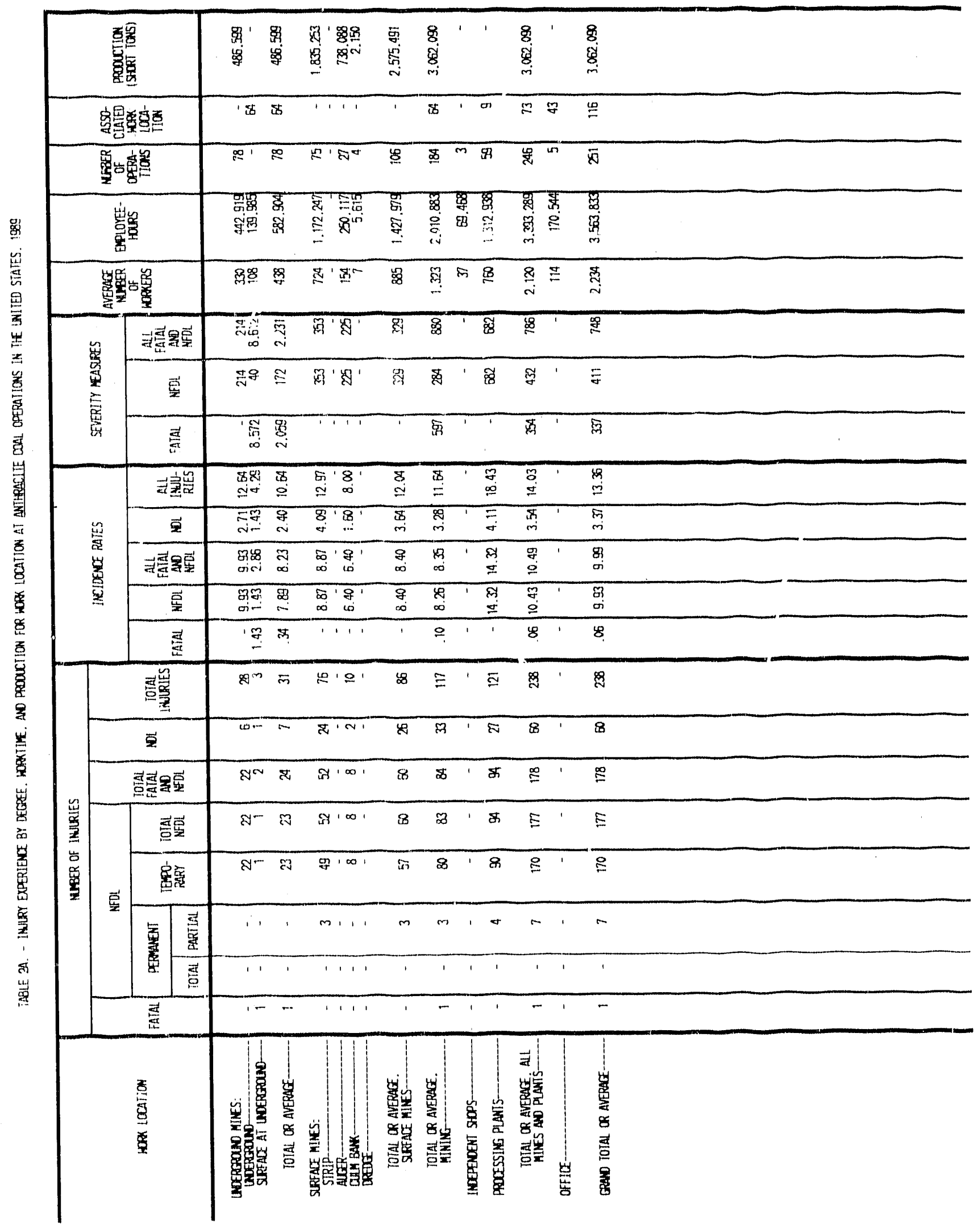




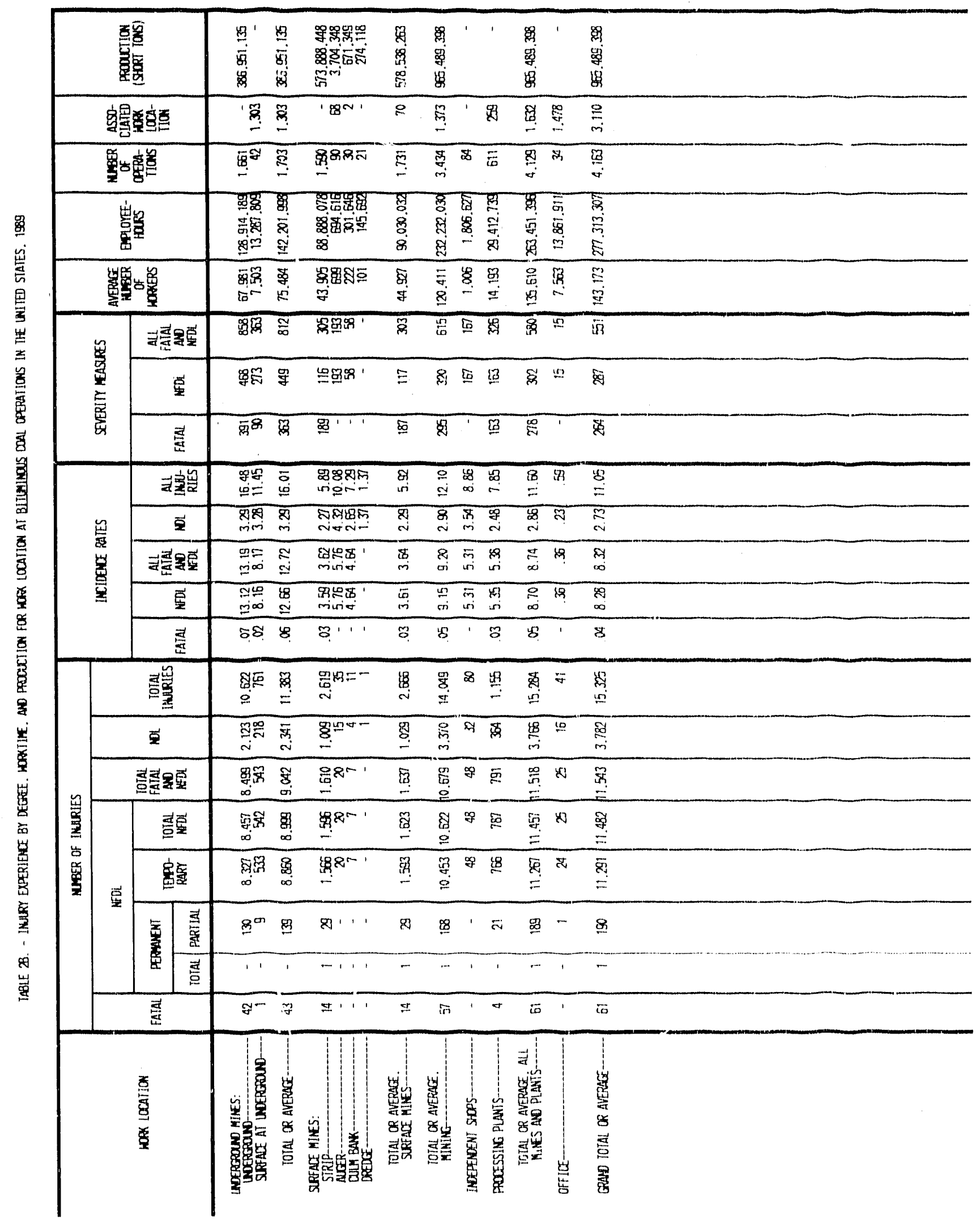




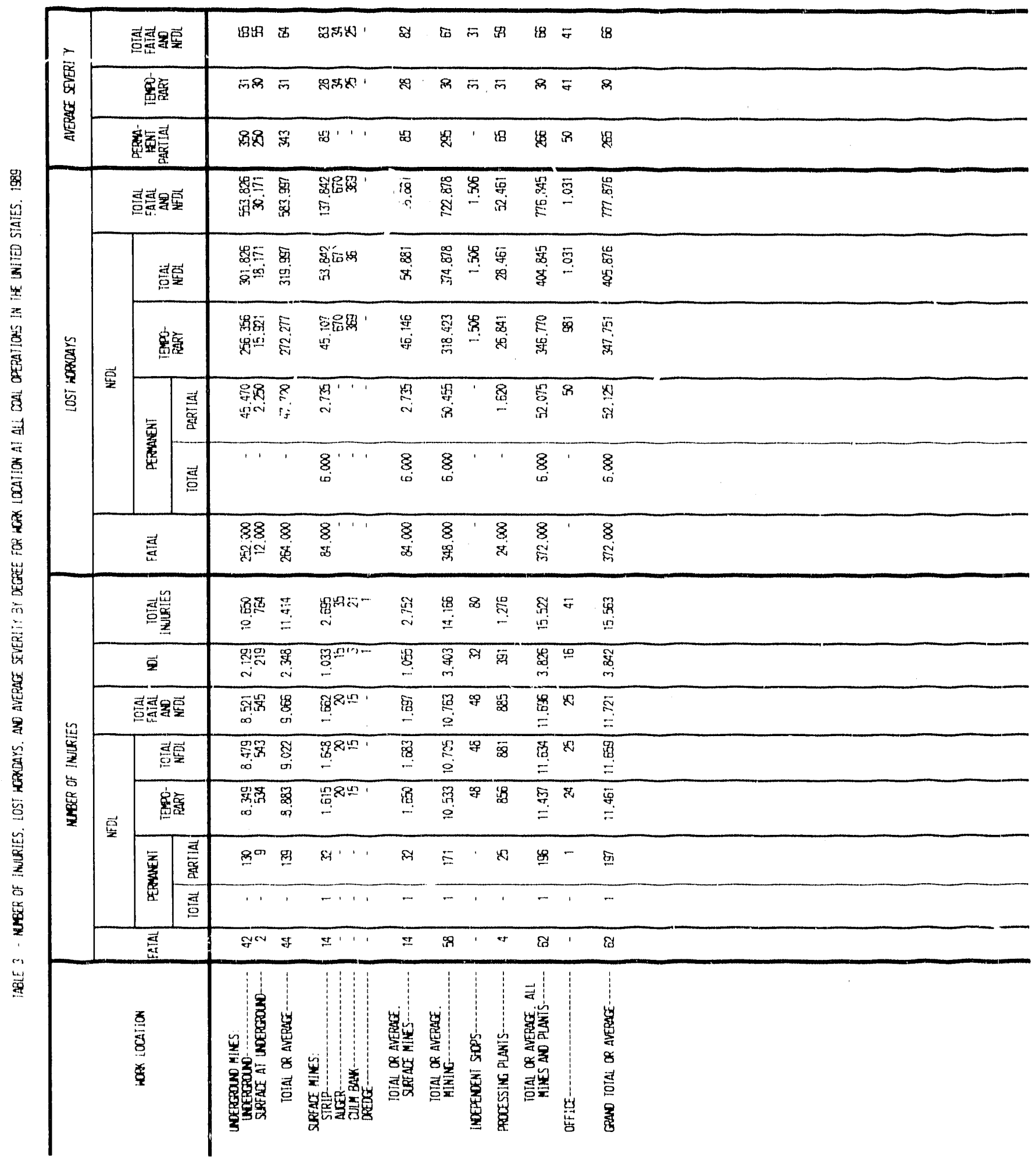




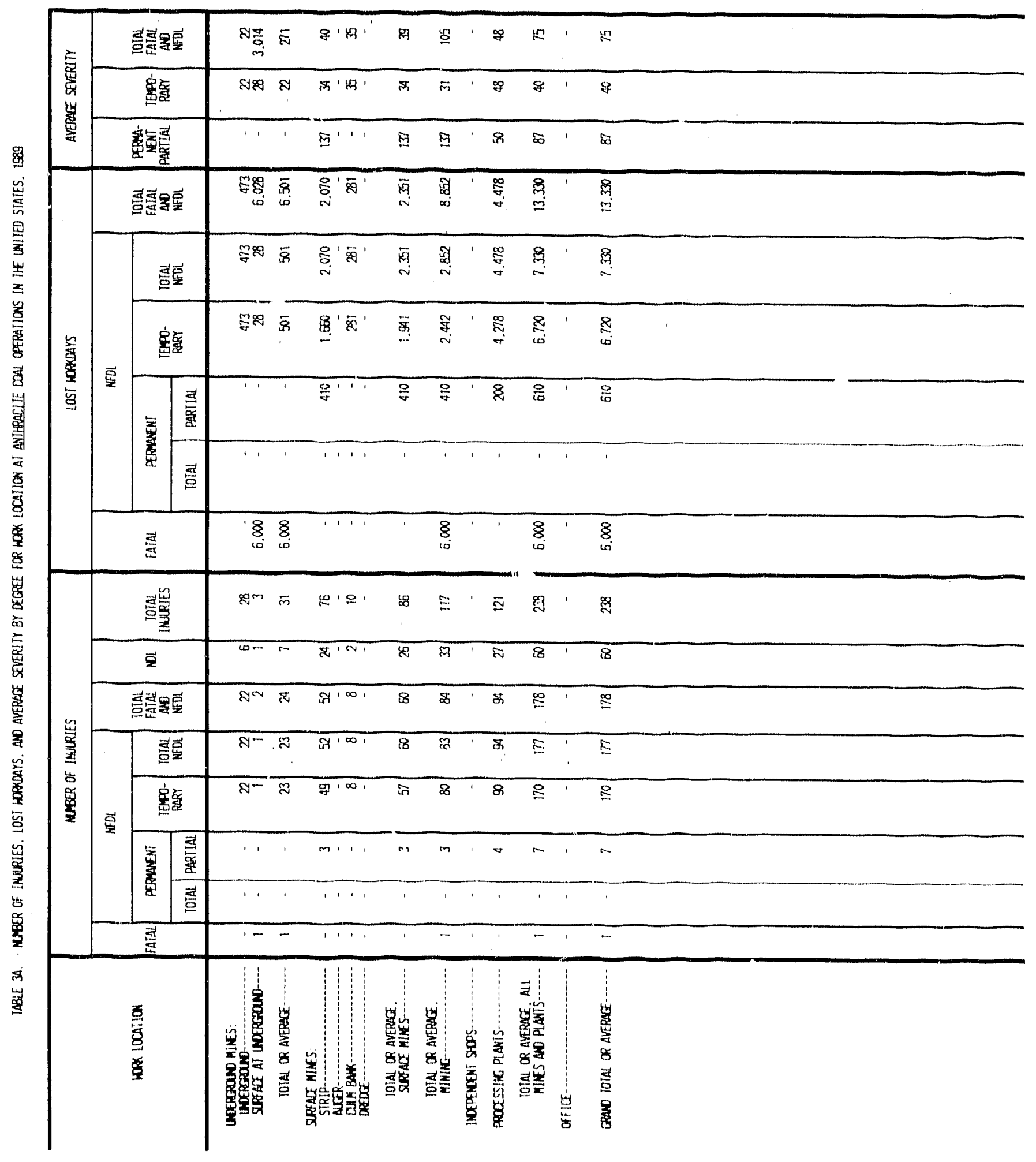




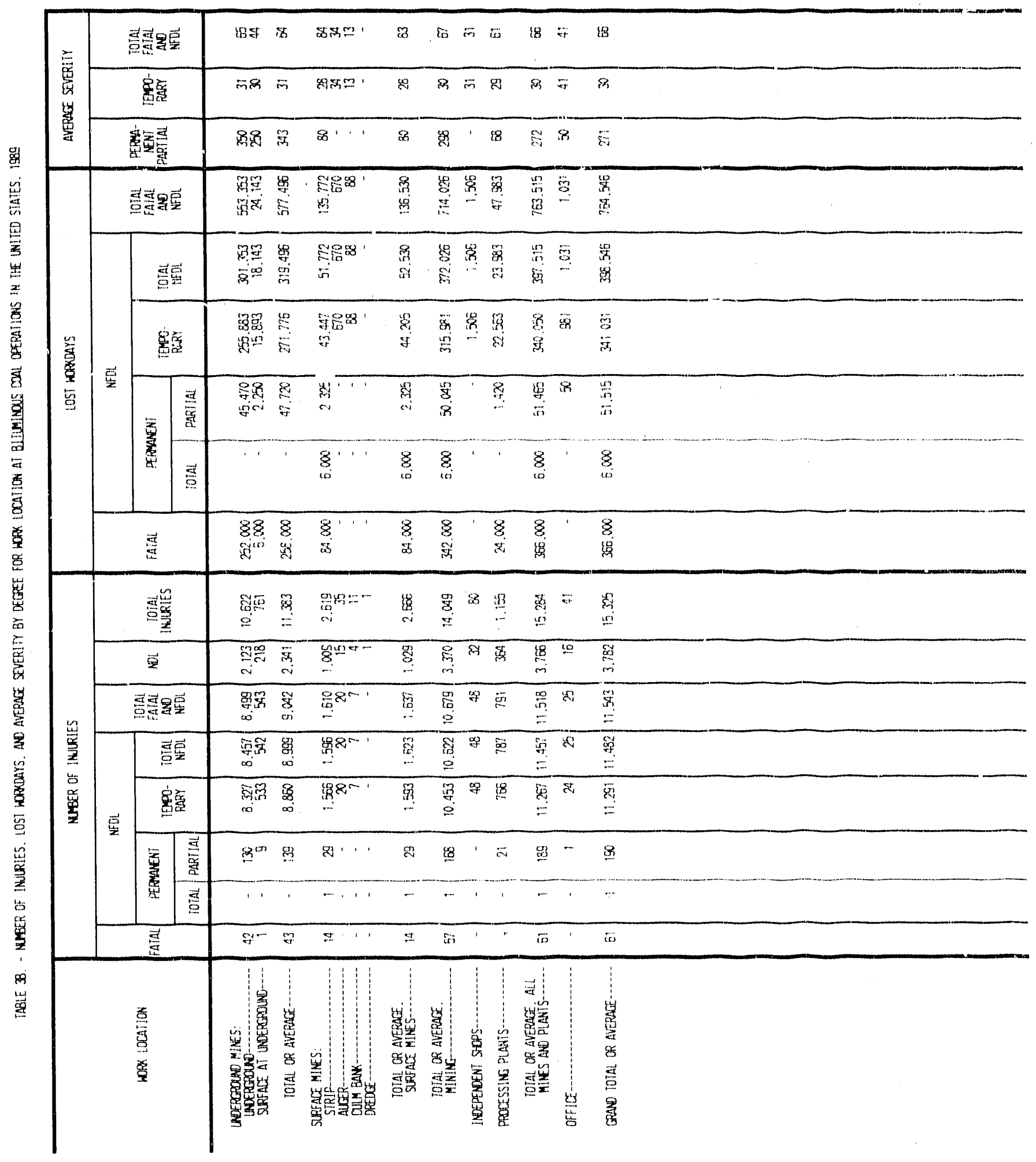




\begin{tabular}{|c|c|c|c|c|c|c|c|c|c|c|c|c|}
\hline & \multicolumn{2}{|l|}{ 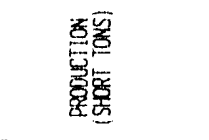 } & 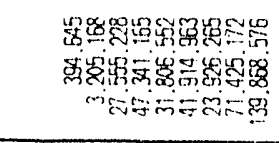 & & 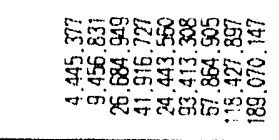 & & 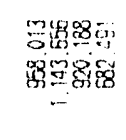 & 然 & 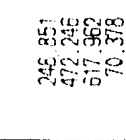 & $\begin{array}{l}\text { 筞 } \\
\text { 察 }\end{array}$ & 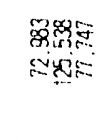 & 总 \\
\hline & \multicolumn{2}{|c|}{ 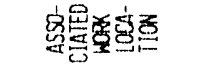 } & 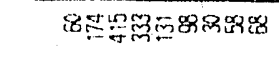 & 奥 & ' ' ' ' ' ' ' & ' & 890 & 8 & $\ldots$ & "is & $\cdots$ & 1 \\
\hline & \multicolumn{2}{|l|}{ 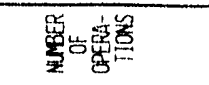 } & 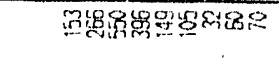 & $\bar{\alpha}$ & 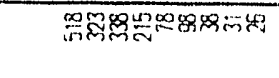 & 8 & 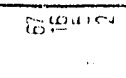 & 8 & $x:=\infty$ & is & tomen & प्: \\
\hline & \multicolumn{2}{|l|}{ 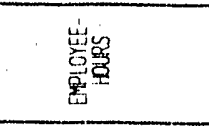 } & 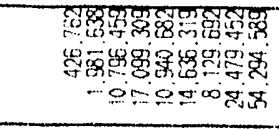 & 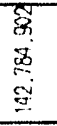 & 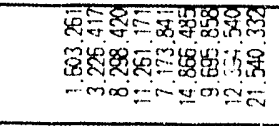 & $\begin{array}{l}\text { हू: } \\
8 \\
8 \\
8\end{array}$ & 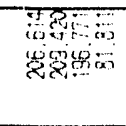 & 位 & 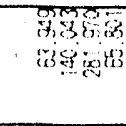 & 高 & 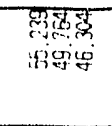 & 策 \\
\hline \multicolumn{3}{|c|}{ 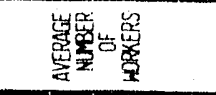 } & 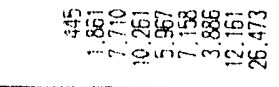 & 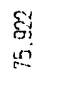 & 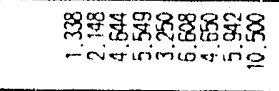 & 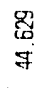 & 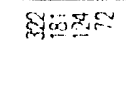 & 照 & 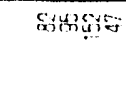 & 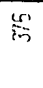 & Fy & 骂 \\
\hline \multirow{3}{*}{ 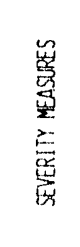 } & $\Leftrightarrow \quad \vec{z}$ & 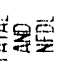 & 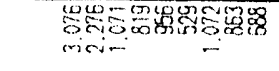 & 要 & 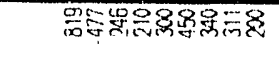 & 妴 & gitiong & 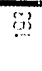 & 法的 & 3 & & \\
\hline & 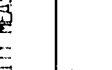 & 可 & 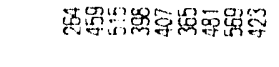 & 等 & 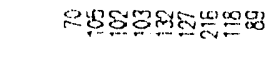 & $\varepsilon$ & cys & 舀 & 赵绍证 & $3_{3}$ & & \\
\hline & & 亲 & 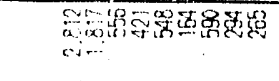 & i & 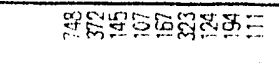 & $\underline{\square}$ & & & & & & \\
\hline & & 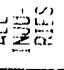 & 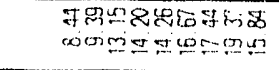 & $\begin{array}{l}83 \\
6 \\
?\end{array}$ & 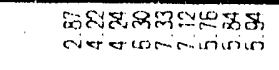 & 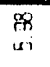 & 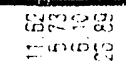 & 8 & 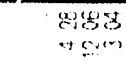 & is & 8 & $\bar{N}$ \\
\hline \multirow{3}{*}{ 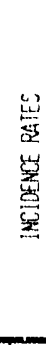 } & & $\bar{z}$ & 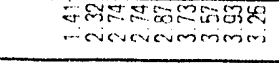 & 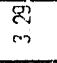 & 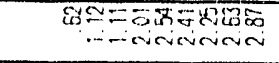 & $\stackrel{\text { gu }}{i}$ & 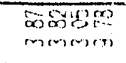 & $\Leftrightarrow$ & 4 & 5 & $\begin{array}{l}8 \\
5\end{array}$ & $\overline{i n}$ \\
\hline & $\vec{z}$ & 夥自 & 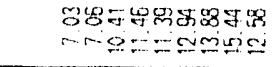 & $\stackrel{?}{\varrho}$ & 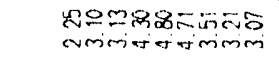 & 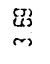 & 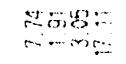 & 些: & 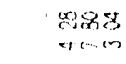 & 落 & ' & I \\
\hline & & 苞 & 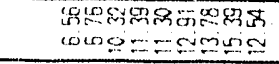 & 8 & 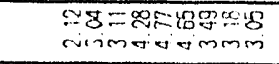 & 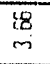 & 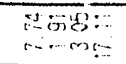 & (5) & 988 & a & ' & \\
\hline & & 恶 & 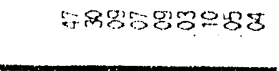 & 8 & 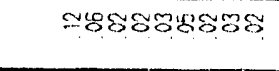 & 8 & $\cdots$ & ' & & . & . & ' \\
\hline \multirow{8}{*}{ 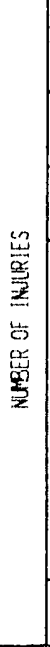 } & 跣: & & 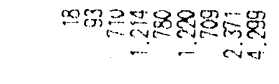 & 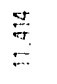 & 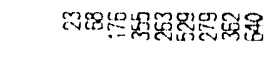 & 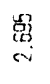 & $\cos (x)=$ & $y$ & $m \ldots$ & $\therefore$ & - & $\cdots$ \\
\hline & 家 & & 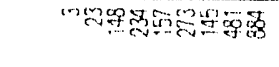 & 器 & 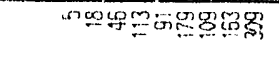 & $\stackrel{9}{g}$ & $\cos (\cos )$ & 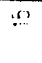 & 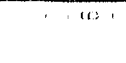 & $\omega$ & 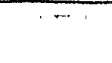 & $\cdots$ \\
\hline & 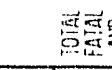 & & 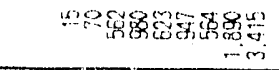 & $\begin{array}{l}\text { 呰 } \\
\text { cij }\end{array}$ & 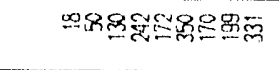 & 兽 & nocumaty & 2 & $m \ldots$ & 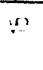 & & ' \\
\hline & \multirow{4}{*}{ 可 } & 参要 & 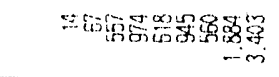 & छे & $=$ g్g & 器 & $\cos x \cos x$ & $\&$ & $\cdots \cdots$ & $\underline{2}$ & & ' \\
\hline & & 密蛋 & 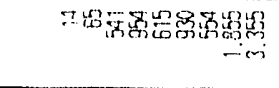 & $\begin{array}{c}\mathscr{\mathscr { O }} \\
\infty \\
\infty\end{array}$ & 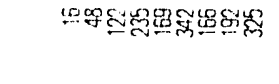 & 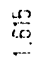 & a) & 2 & $m$ & $\because$ & & ' \\
\hline & & 苞 & - Nes & 出 & 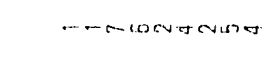 & $\Leftrightarrow$ & & & & I & & . \\
\hline & & 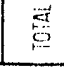 & $\cdots$ & & $\cdots$ & $\cdots$ & & & & 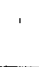 & & \\
\hline & \multicolumn{2}{|c|}{ 竞 } & 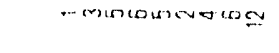 & 和 & dat & $s$ & & & & . & & ' \\
\hline & \multicolumn{2}{|l|}{ 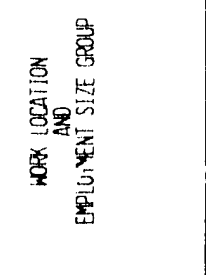 } & 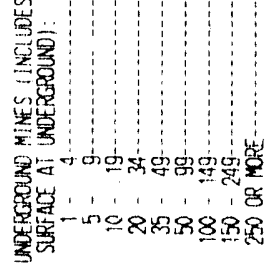 & 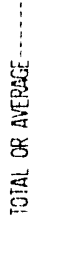 & 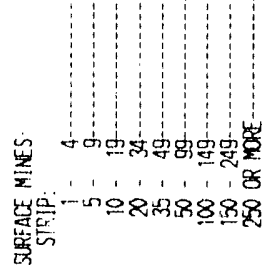 & 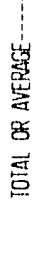 & 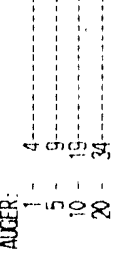 & 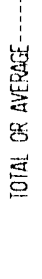 & 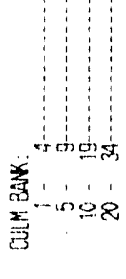 & 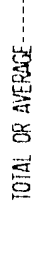 & 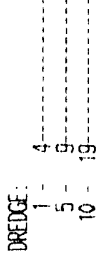 & 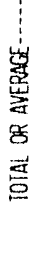 \\
\hline
\end{tabular}




\begin{tabular}{|c|c|c|c|c|c|c|c|c|c|c|}
\hline \multicolumn{3}{|c|}{ 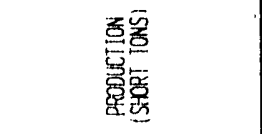 } & 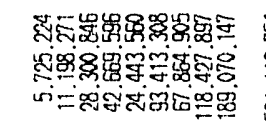 & 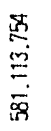 & 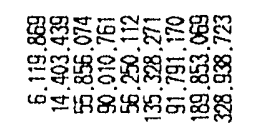 & 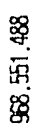 & 111111 & & 111,1111 & \\
\hline \multicolumn{3}{|c|}{ 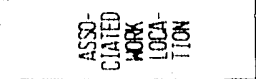 } & พx=0.1111 & $?$ & 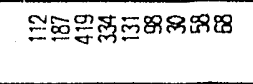 & g. & 111111 & 1 & $8948 \sin =-$ & 究 \\
\hline \multicolumn{3}{|c|}{ 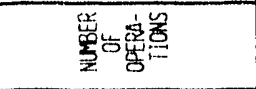 } & 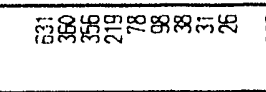 & $\underbrace{\infty}_{\infty}$ & 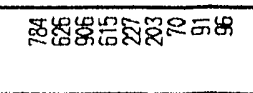 & 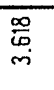 & 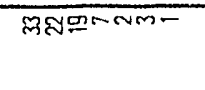 & $\bar{\infty}$ & 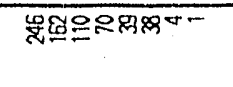 & \& \\
\hline \multicolumn{3}{|c|}{ 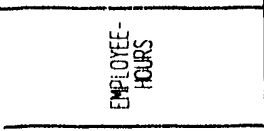 } & 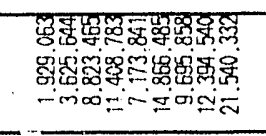 & 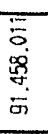 & 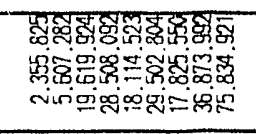 & 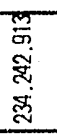 & 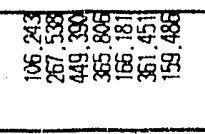 & 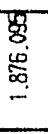 & 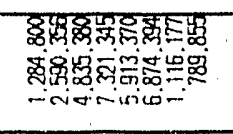 & हैं \\
\hline \multicolumn{3}{|c|}{ 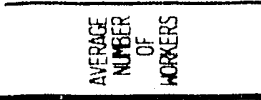 } & 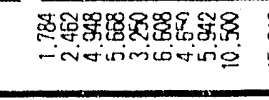 & 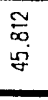 & 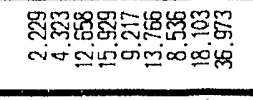 & 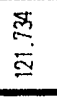 & 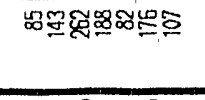 & $\underline{9}$ & 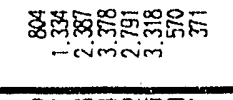 & 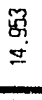 \\
\hline \multirow{3}{*}{ 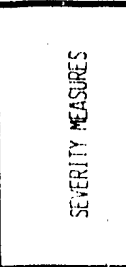 } & \multicolumn{2}{|c|}{ 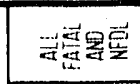 } & 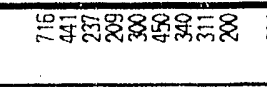 & $\bar{d}$ & 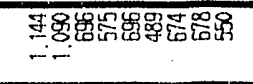 & 5 & 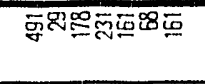 & $\overline{0}$ & 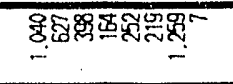 & $\bar{\nabla}$ \\
\hline & \multicolumn{2}{|c|}{ 总 } & 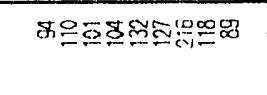 & 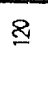 & 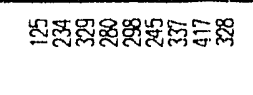 & \&্ল & 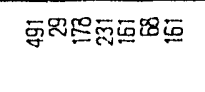 & $\bar{E}$ & 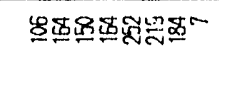 & $\stackrel{\infty}{\infty}$ \\
\hline & 点 & 蔗 & త్ల్ల & $\Phi$ & 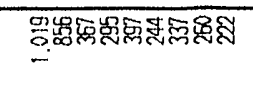 & छ & 1111111 & T & 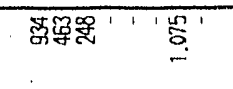 & $\underline{R}$ \\
\hline \multirow{4}{*}{ 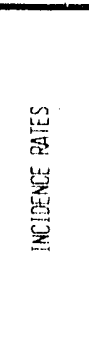 } & \multicolumn{2}{|c|}{ 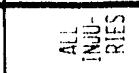 } & 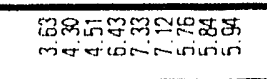 & $\begin{array}{c}8 \\
0 \\
60\end{array}$ & 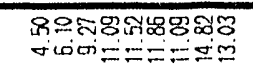 & $\stackrel{ }{\dddot{2}}$ & 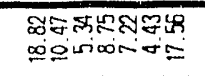 & 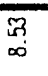 & 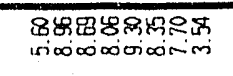 & $\bar{m}$ \\
\hline & \multicolumn{2}{|c|}{$\vec{z}$} & 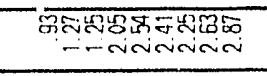 & $\bar{m}$ & 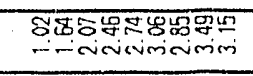 & $\stackrel{\bar{\sigma}}{i}$ & 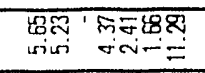 & $\overline{\bar{F}}$ & 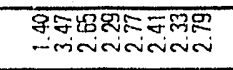 & $\stackrel{\text { 㞻 }}{\sim}$ \\
\hline & \multicolumn{2}{|c|}{ 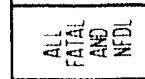 } & 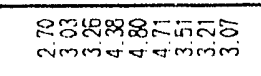 & $\bar{m}$ & 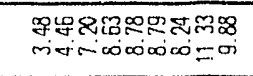 & $\frac{g}{\sigma i}$ & 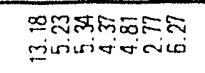 & $\underset{\text { si }}{\cong}$ & 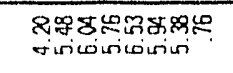 & $\begin{array}{l}\text { gin } \\
\text { in }\end{array}$ \\
\hline & \multicolumn{2}{|c|}{ 官 } & 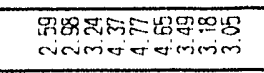 & $\begin{array}{l}98 \\
i n \\
\end{array}$ & 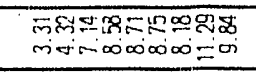 & $\begin{array}{l}\square \\
\sigma\end{array}$ & 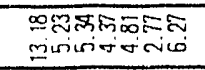 & $\frac{\pi}{\sin }$ & 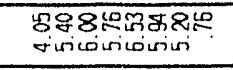 & mi \\
\hline & \multicolumn{2}{|c|}{ 焉 } & 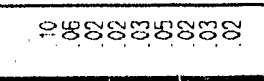 & $\%$ & $= \pm 985554858$ & 骂 & 11111 & ' & פg8 ' ' ' & $\mathscr{O}$ \\
\hline \multirow{8}{*}{$\begin{array}{l}\text { 总 } \\
\text { 焉 } \\
\text { 岩 } \\
\text { 品 } \\
\text { 至 }\end{array}$} & \multicolumn{2}{|c|}{ 总 } & 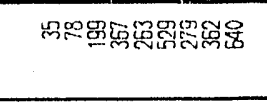 & 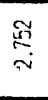 & 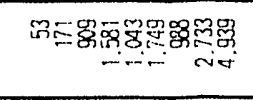 & $\begin{array}{l}\stackrel{g}{g} \\
\stackrel{\square}{\square}\end{array}$ & $0.1,100000$ & 8 & 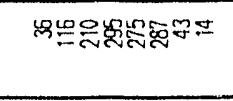 & $\stackrel{\infty}{\stackrel{\infty}{*}}$ \\
\hline & \multicolumn{2}{|l|}{ 臣 } & 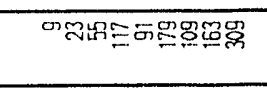 & 范 & 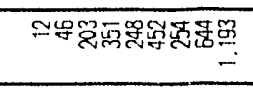 & $\begin{array}{l}\text { 总 } \\
\text { i } \\
\end{array}$ & me-100तmo & ह্ল & 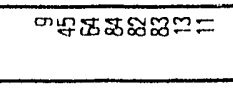 & $\overline{\text { मे }}$ \\
\hline & 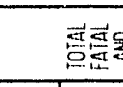 & & 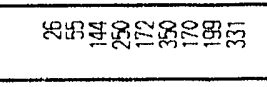 & 曷 & 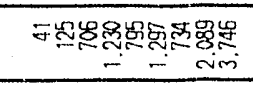 & $\begin{array}{r}0 \\
\stackrel{0}{0} \\
0 \\
\end{array}$ & $-125000(5)$ & 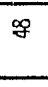 & 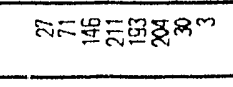 & $\ddot{8}$ \\
\hline & & 愛要 & 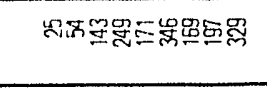 & $\stackrel{\mathscr{m}}{-}$ & 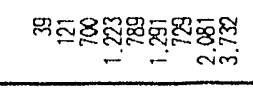 & $\begin{array}{l}\text { 号 } \\
\therefore \\
\end{array}$ & matsooginin & g & 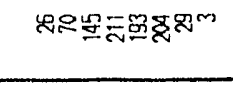 & $\overline{8}$ \\
\hline & 偓 & 薑舜 & 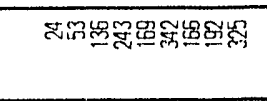 & 8 & 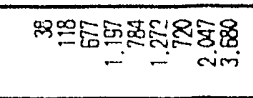 & 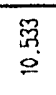 & 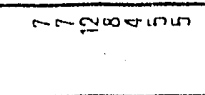 & $\overline{o g}$ & வ88: & 岁 \\
\hline & \multirow{2}{*}{$\begin{array}{l}\text { 䗎 } \\
\text { 品 }\end{array}$} & 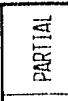 & -mingenor & $\approx$ & 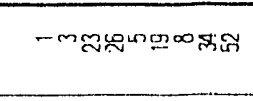 & $E$ & $1,1,1$ & ' & -N i & W \\
\hline & & 总 & $1,1,1$ & - & $11-11$ & - & $1,1,1$ & , & $1,1,1$ & , \\
\hline & \multicolumn{2}{|l|}{ 焉 } & $--m-$ d w wav & \pm & Nocon-cosingo & 禺 & $1,1,11$ & ' & $-\infty, 1,1$, & $\sigma$ \\
\hline & 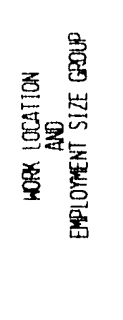 & & 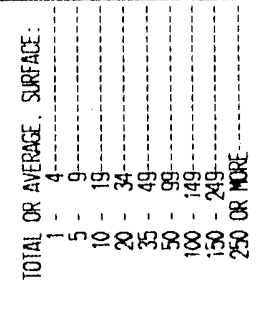 & 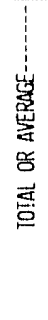 & 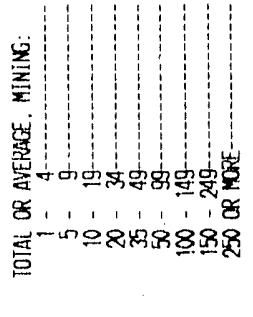 & 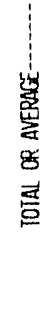 & 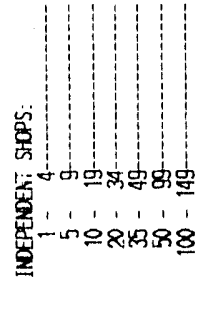 & 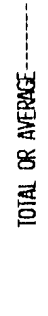 & 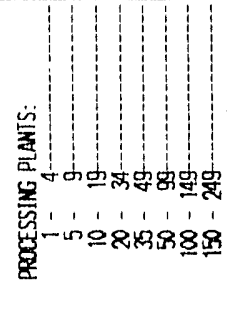 & 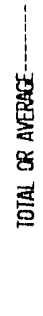 \\
\hline
\end{tabular}




\begin{tabular}{|c|c|c|c|c|c|c|c|c|}
\hline \multicolumn{3}{|c|}{ 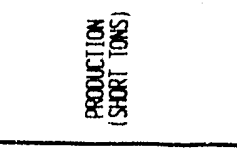 } & 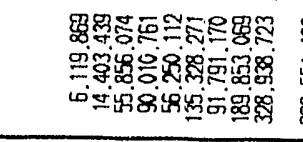 & 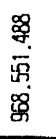 & 11111 & ' & 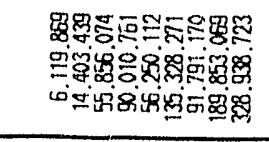 & 唯 \\
\hline \multicolumn{3}{|c|}{ 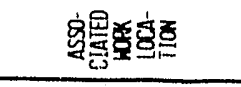 } & 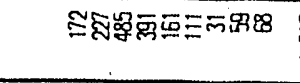 & $\stackrel{\text { Le }}{=}$ & 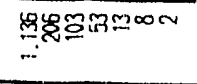 & 똑 & 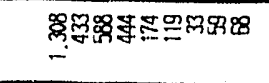 & ष্ষ \\
\hline \multicolumn{3}{|c|}{ 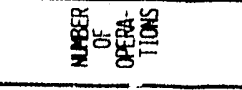 } & 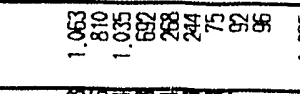 & 点 & mimm & क्ल & 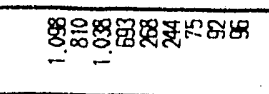 & 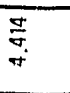 \\
\hline \multicolumn{3}{|c|}{ 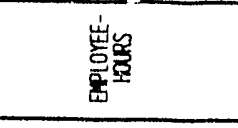 } & 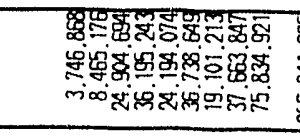 & 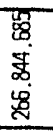 & 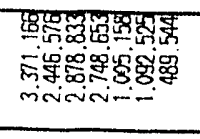 & 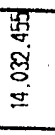 & 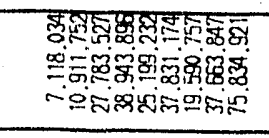 & 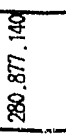 \\
\hline \multicolumn{3}{|c|}{ 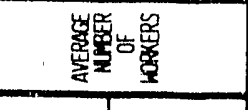 } & 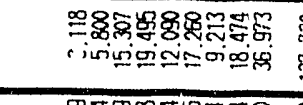 & 总 & 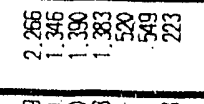 & 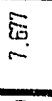 & 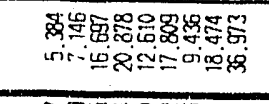 & $\begin{array}{l}\overline{9} \\
\text { 垔 } \\
\end{array}$ \\
\hline & 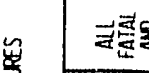 & 울울 & 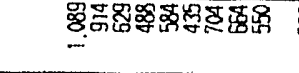 & $\widetilde{q}$ & gasmin & 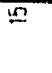 & 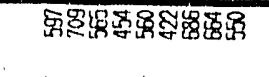 & 离 \\
\hline & 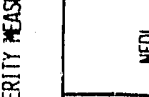 & $\overrightarrow{\underline{\underline{z}}}$ & 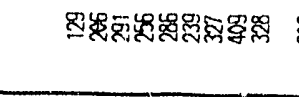 & m & gIom, & 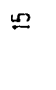 & 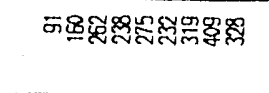 & $g$ \\
\hline & & 槖 & 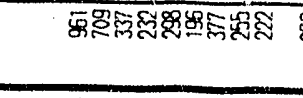 & 罚 & 111 & $T$ & 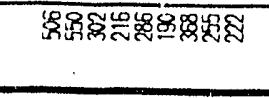 & 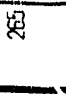 \\
\hline & & 表票 & 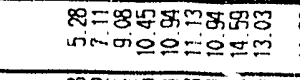 & $\stackrel{\mathscr{B}}{=}$ & 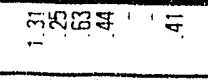 & 요 & 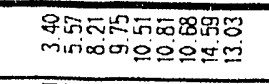 & $\stackrel{\text { gos }}{=}$ \\
\hline & & $\overrightarrow{\underline{z}}$ & 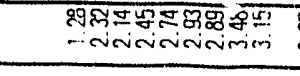 & $\stackrel{\substack{\infty \\
i}}{i}$ & 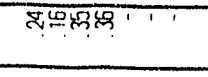 & $\bar{N}$ & 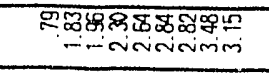 & $\stackrel{j}{i}$ \\
\hline & 喾 & 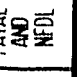 & 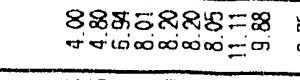 & $\begin{array}{l}:= \\
\infty \\
\infty\end{array}$ & 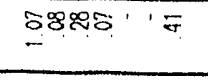 & 亗 & 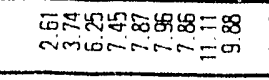 & $\begin{array}{l}4 \\
\infty\end{array}$ \\
\hline & & 亚 & 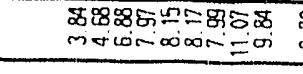 & $\begin{array}{c}\mathbb{N} \\
\infty \\
\end{array}$ & 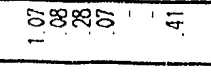 & 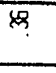 & 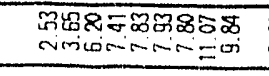 & $\begin{array}{c}8 \\
\infty \\
\infty\end{array}$ \\
\hline & & $\underline{\underline{t}}$ & 요8848\%88 & 4 & $\cdots \cdots$ & ' & 88948.89480 & 8 \\
\hline \multirow{8}{*}{ 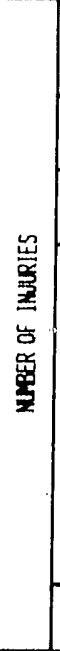 } & \multicolumn{2}{|c|}{ 总掌 } & 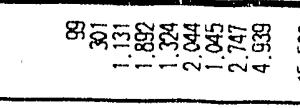 & 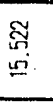 & Emone. & $\bar{\sigma}$ & 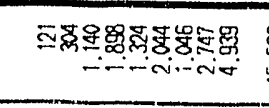 & 茴 \\
\hline & \multicolumn{2}{|l|}{ 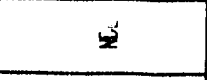 } & 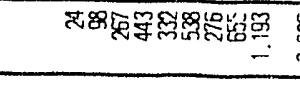 & 发 & Tnisnon 111 & $\Phi$ & 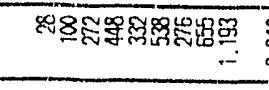 & $\bar{s}$ \\
\hline & \multicolumn{2}{|c|}{ 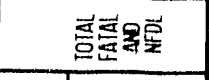 } & 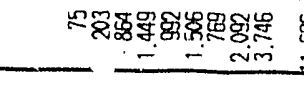 & 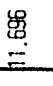 & $\infty-\sigma-11-$ & 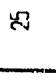 & 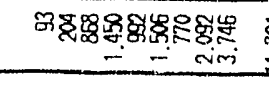 & זָ \\
\hline & \multicolumn{2}{|c|}{ 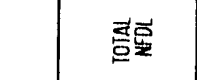 } & 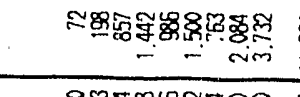 & 苔 & 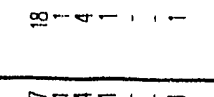 & 准 & 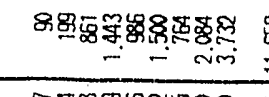 & $\stackrel{9}{\text { 照 }}$ \\
\hline & \multicolumn{2}{|c|}{ 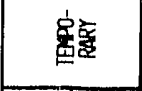 } & 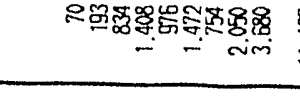 & 㞼 & $=-\pi-11-$ & Zt & 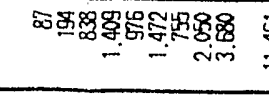 & $\stackrel{\text { 垔 }}{=}$ \\
\hline & & \begin{tabular}{|l}
$\vec{z}$ \\
妾 \\
\end{tabular} & 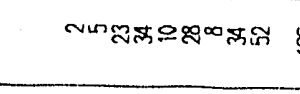 & $\mathscr{g}$ & $-1, \ldots$ & - & 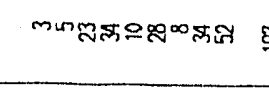 & $\Phi$ \\
\hline & & 흘 & $11,1,1-1$. & -- & 11 & ' & $\cdots 1$ & - \\
\hline & \multicolumn{2}{|l|}{ 竞 } & minntioweos & $\tilde{0}$ & 1 ' & 1 & munnarowoog & $\widetilde{0}$ \\
\hline & \multicolumn{2}{|l|}{ 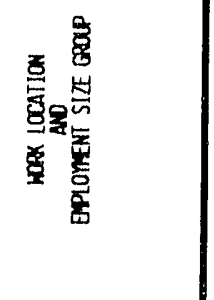 } & 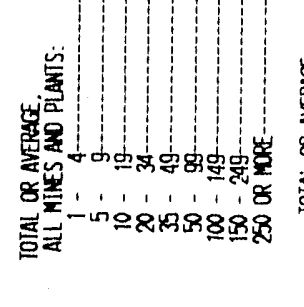 & 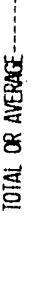 & 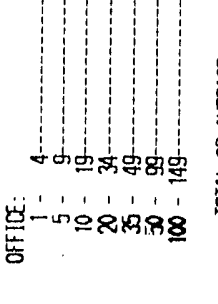 & 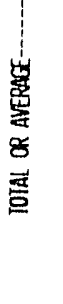 & 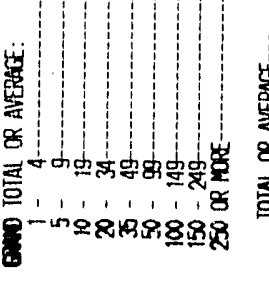 & 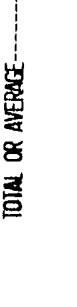 \\
\hline
\end{tabular}




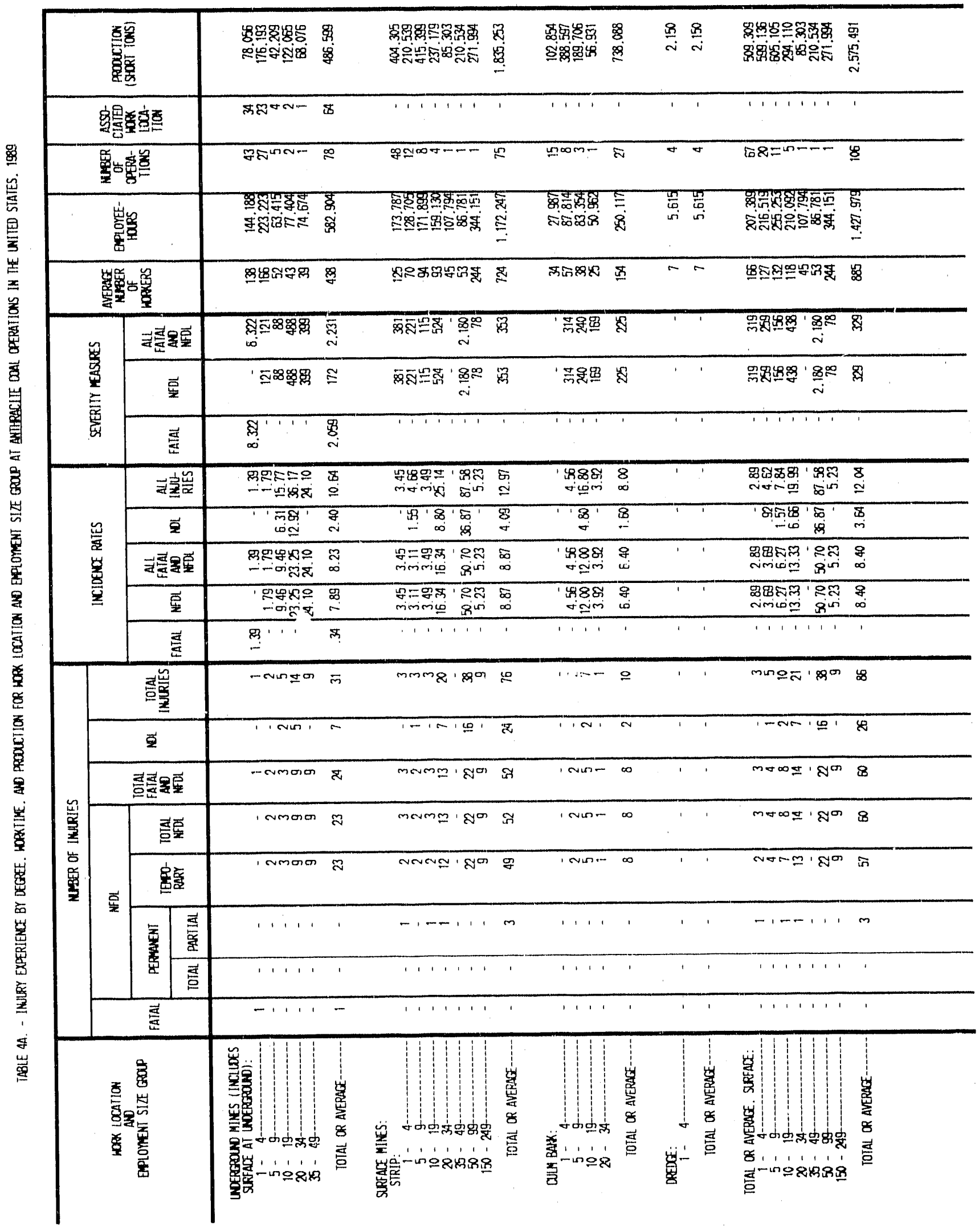




\begin{tabular}{|c|c|c|c|c|c|c|c|c|c|c|c|c|c|}
\hline \multicolumn{3}{|c|}{ 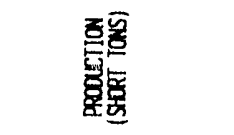 } & 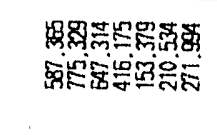 & & & 11 & & 11111 & ' & 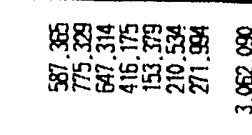 & & ' 1 & \\
\hline \multicolumn{3}{|c|}{ 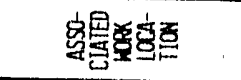 } & 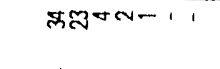 & के & & & ' & - mavm 1 & or & Hedour 17 & M & gूm-- & F \\
\hline \multicolumn{3}{|c|}{ 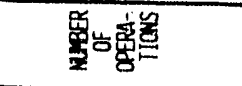 } & 워메 & $\Phi$ & & -- & $m$ & dy= & 9 & 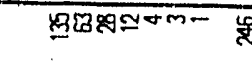 & 罚 & 51 & \\
\hline \multicolumn{3}{|c|}{ 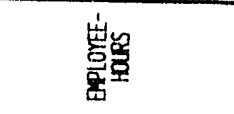 } & 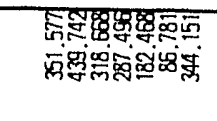 & 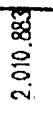 & & 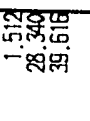 & 荨 & 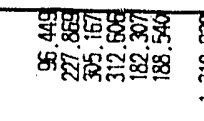 & 器 & 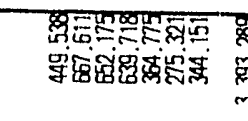 & 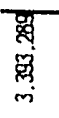 & 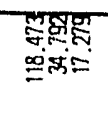 & 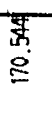 \\
\hline \multicolumn{3}{|c|}{ 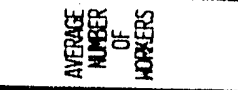 } & 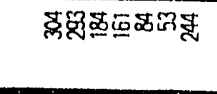 & ఇ్ల & & M됴 & m & 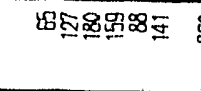 & 8 & 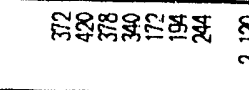 & 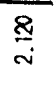 & 뼈으응 & $\Xi$ \\
\hline \multirow{3}{*}{ 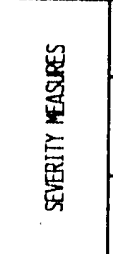 } & & 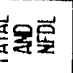 & $\underset{j}{\bar{c}}$ & 8 & & & & 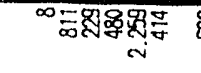 & $\widetilde{\sharp}$ & 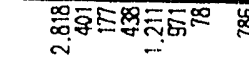 & 总 & & \\
\hline & 里 & 营 & 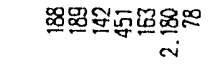 & 总 & & 1. & ' & 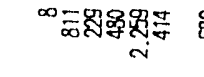 & 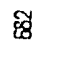 & 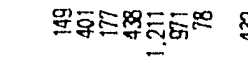 & 영 & 11 ' & ' \\
\hline & & $\frac{\vec{x}}{\underline{z}}$ & 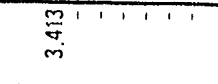 & $\overline{5}$ & & & $T$ & 1.1. & & $\underset{\sim}{g} 1+1+1 k$ & 要 & 11 & $T$ \\
\hline & & 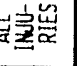 & 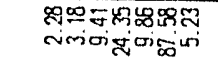 & $\stackrel{\square}{=}$ & & & ' & 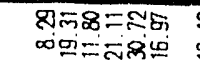 & $\underset{\substack{3 \\
0}}{2}$ & 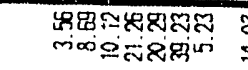 & $\begin{array}{l}\text { mis } \\
\dot{s} \\
\dot{s}\end{array}$ & & \\
\hline \multirow{3}{*}{\multicolumn{2}{|c|}{ 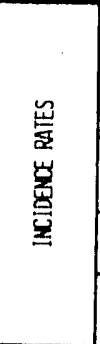 }} & $\overrightarrow{\underline{2}}$ & 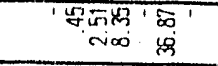 & $\underset{m}{2}$ & & & ' & 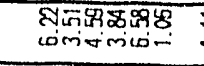 & $\begin{array}{l}\bar{\square} \\
\bar{q}\end{array}$ & 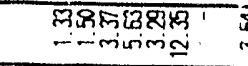 & 楤 & & \\
\hline & & 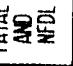 & 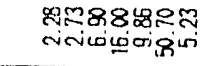 & $\underbrace{\infty}_{\infty}$ & & & $\cdot$ & 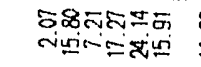 & 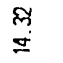 & 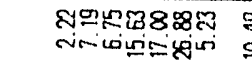 & 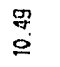 & $\cdots$ & ! \\
\hline & & $\overrightarrow{\mathrm{P}}$ & 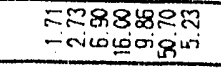 & 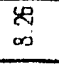 & & & ' & 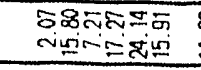 & 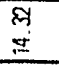 & 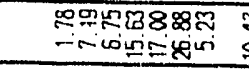 & 举 & .1. & 1 \\
\hline & & 론 & 的' $11+1$ & 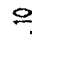 & & 11. & ' & 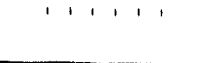 & ' & $\neq, \ldots, \ldots, \varepsilon$ & 8 & ' ' & ' \\
\hline \multirow{8}{*}{ 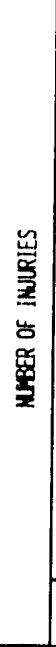 } & \multicolumn{2}{|c|}{ 砉营 } & 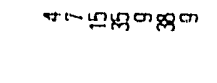 & $\cong$ & & ' & ' & 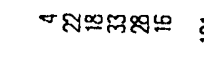 & $\Xi$ & 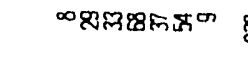 & $\stackrel{8 g}{9}$ & 11 ' & \\
\hline & \multicolumn{2}{|l|}{$\overrightarrow{\underline{x}}$} & 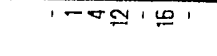 & $\mathscr{m}$ & & & ' & mancoor & $\varepsilon$ & $\min =000=1$ & 8 & 111 & \\
\hline & \multicolumn{2}{|c|}{ 총켠운물 } & $\sigma^{\infty}=\approx \infty \mathbb{N}^{\infty}$ & $\$$ & & ' & ' & $-\infty=\Sigma \mathbb{E}$ & क & 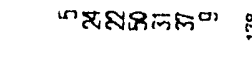 & $\cong$ & ' ' & ' \\
\hline & \multirow{4}{*}{ 官 } & 悹畐 & 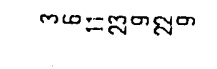 & $\mathscr{\infty}$ & & ' & ' & - & क & 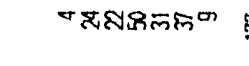 & $E$ & ' ' & \\
\hline & & 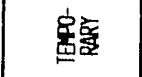 & N's? & 8 & & ' & ' & - $=4 \approx m$ & 8 & m资 & $?$ & 1 . ' & ' \\
\hline & & 亲 & $-1--1$, & $m$ & & & ' & $1,1,-\infty$ & $\sigma$ & $-1-n-n 1$ & - & , & , \\
\hline & & 홍 & $1 \ldots 1,1$ & ' & & 1 & ' & . & ' & $\cdots \cdots$ & I & ' & ' \\
\hline & \multicolumn{2}{|c|}{ 롤 } & $-1,111$, & - & & & & 1 & . & 111 & - & & \\
\hline \multicolumn{3}{|c|}{ 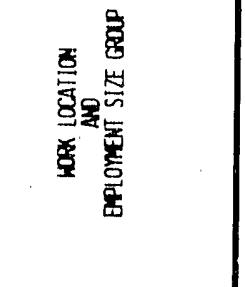 } & 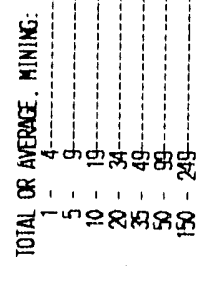 & 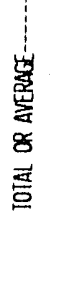 & 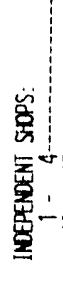 & 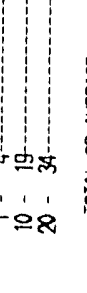 & 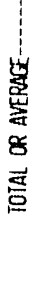 & 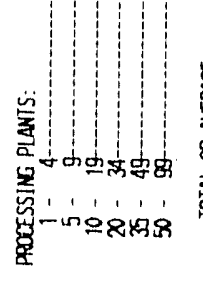 & 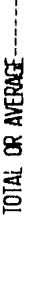 & 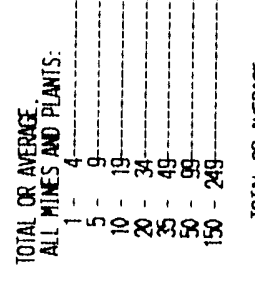 & 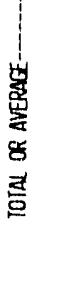 & 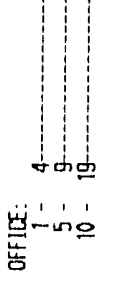 & 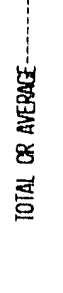 \\
\hline
\end{tabular}




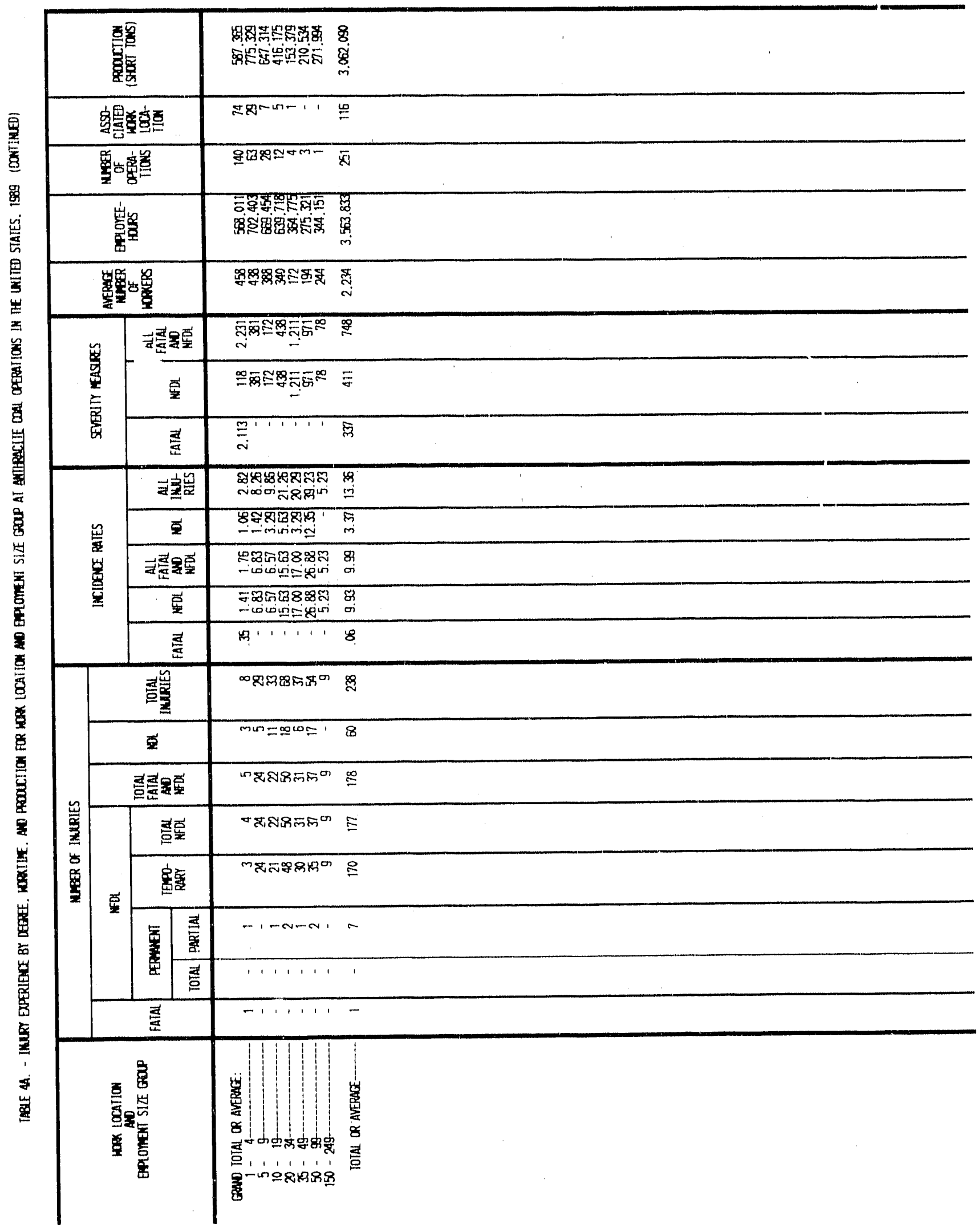




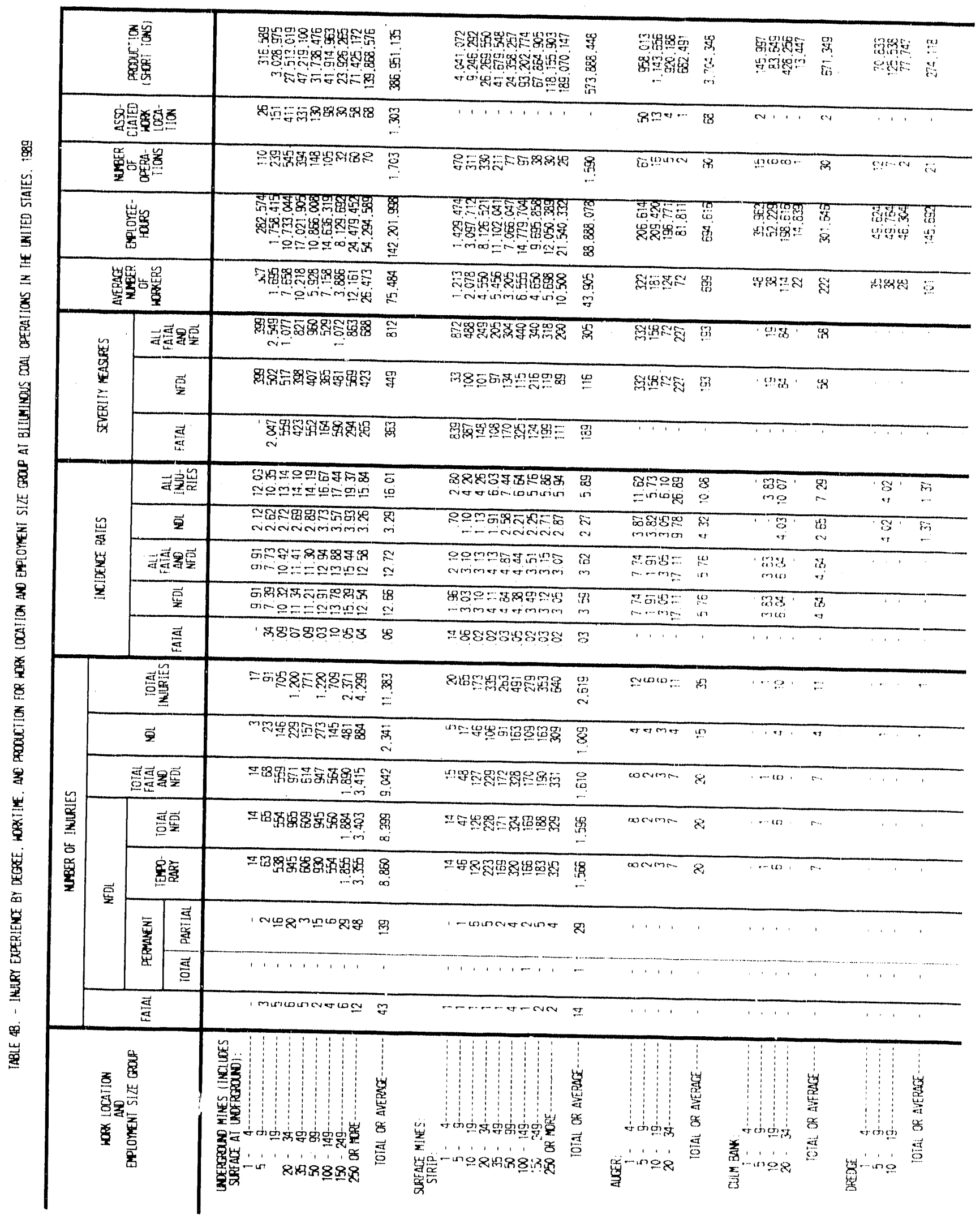




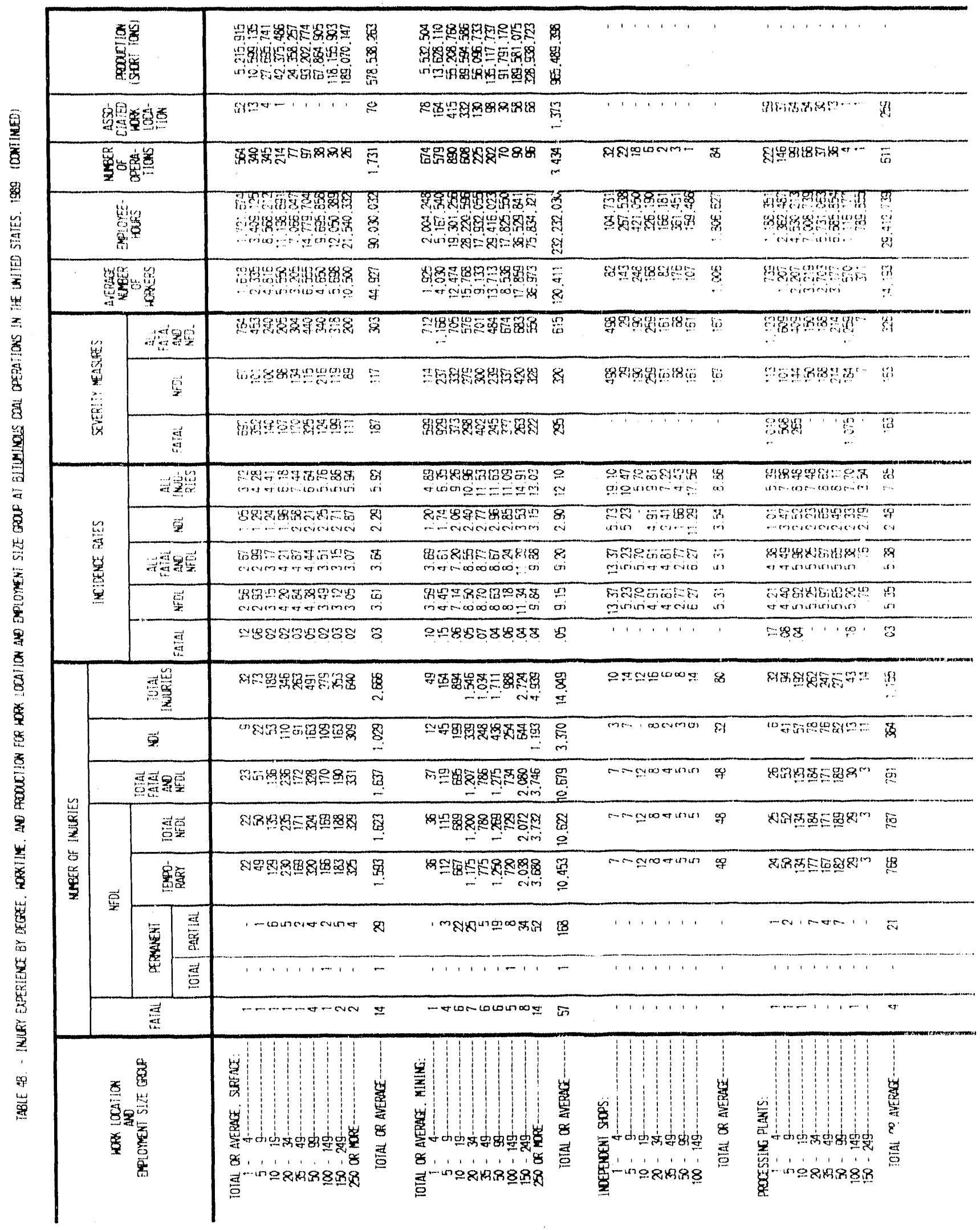




\begin{tabular}{|c|c|c|c|c|c|c|c|c|c|}
\hline \multicolumn{3}{|c|}{ 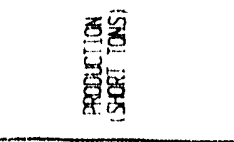 } & 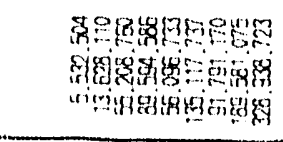 & & . ' & & 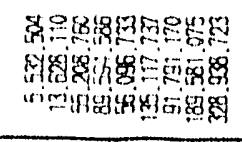 & 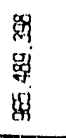 & \\
\hline \multicolumn{3}{|c|}{ 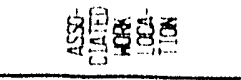 } & 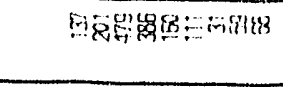 & y & 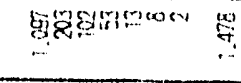 & 粱 & 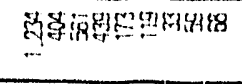 & $\stackrel{9}{i}$ & \\
\hline \multicolumn{3}{|c|}{ 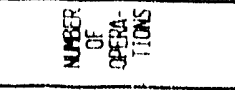 } & 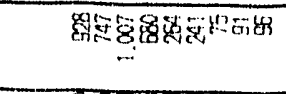 & $\frac{d}{\pi}$ & $81 m-11 \%$ & 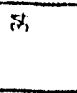 & 498968 & 黑 & \\
\hline \multicolumn{3}{|c|}{ 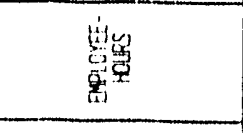 } & 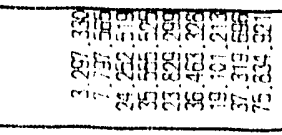 & 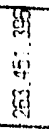 & 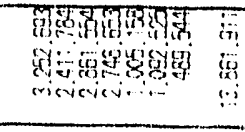 & 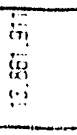 & 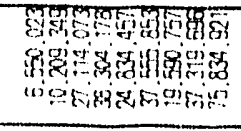 & 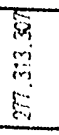 & \\
\hline \multicolumn{3}{|c|}{ 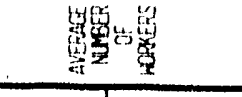 } & 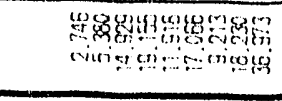 & 空 & 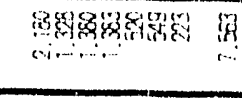 & 解 & 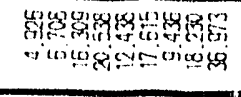 & 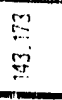 & \\
\hline \multirow{3}{*}{ 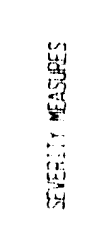 } & 邹 & 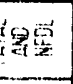 & 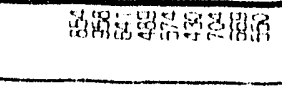 & 僢 & 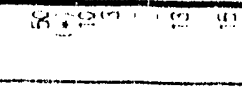 & $!$ & 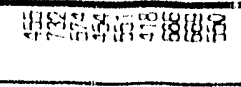 & R & \\
\hline & 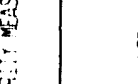 & $\underline{\underline{s}}$ & 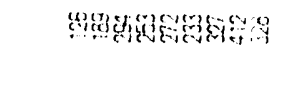 & $\xi$ & 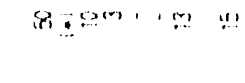 & $\because !$ & 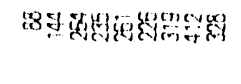 & 8 & \\
\hline & Fy & it & 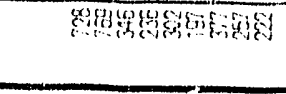 & 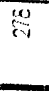 & $T$ & $T$ & 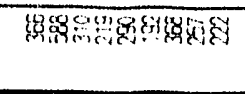 & sis & \\
\hline & & 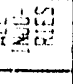 & 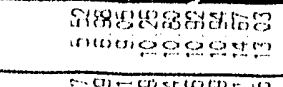 & $\begin{array}{l}88 \\
\cdots \\
\end{array}$ & 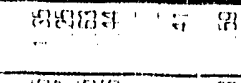 & : & 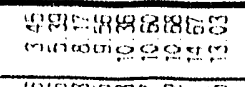 & 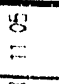 & \\
\hline & 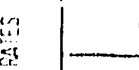 & 寊 & 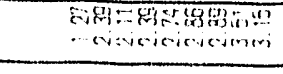 & 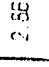 & 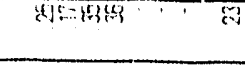 & 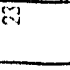 & 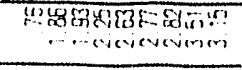 & 2 & \\
\hline & tit & 鼠要 & 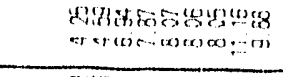 & $\begin{array}{l}\because \\
i \\
i n\end{array}$ & 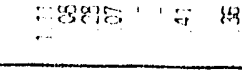 & 4 & 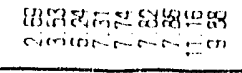 & $\begin{array}{l}\text { \&i } \\
\infty\end{array}$ & 1 \\
\hline & & $\ddot{z}$ & 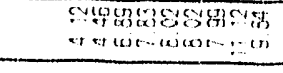 & (i) & ascex & 4 & 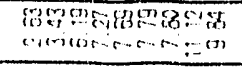 & $\begin{array}{l}\infty y \\
\infty \\
\infty\end{array}$ & 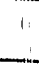 \\
\hline & & it & 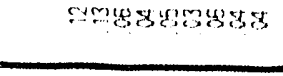 & 4 & $\cdots 1$. & ' & 4о4849858 & is & \\
\hline \multirow{8}{*}{ 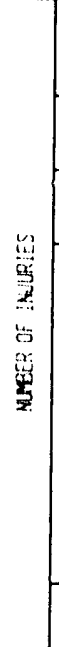 } & 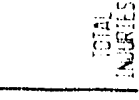 & & 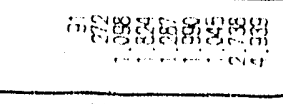 & in & symerna, , , - & $\because r$ & 945868089 & 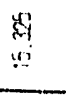 & \\
\hline & $\ddot{\oplus}$ & & न & 黙 & 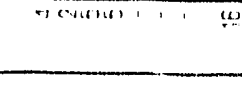 & بيt & WG & $\begin{array}{c}\text { sy } \\
\text { s. } \\
i n \\
\end{array}$ & \\
\hline & 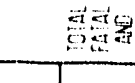 & & 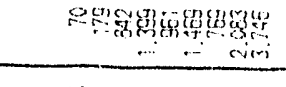 & $\dddot{1}$ & $(x) \cdots+\cdots+\cdots$ & 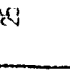 & 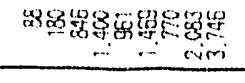 & 8 & \\
\hline & \multirow{3}{*}{$\overrightarrow{\mathrm{s}}$} & 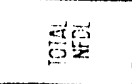 & 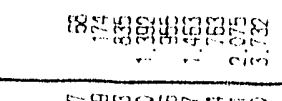 & $\stackrel{i}{i=1}$ & $m, \cdots+\cdots, \cdots$ & 0 & 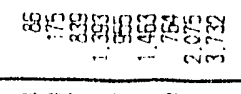 & $\stackrel{q u}{\underline{E}}$ & \\
\hline & & 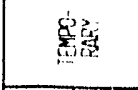 & 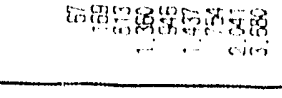 & 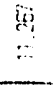 & $\cdots+\cdots+z_{t}$ & 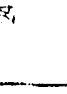 & 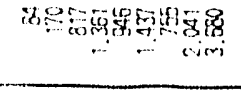 & 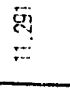 & \\
\hline & & 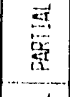 & “" & 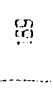 & $\cdots$ & 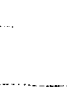 & 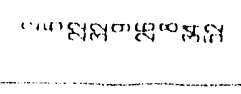 & 8 & \\
\hline & & 8 & '. & $\cdots$ & & & & $\cdots$ & \\
\hline & \multicolumn{2}{|l|}{ it } & 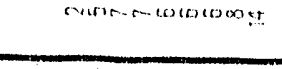 & $i$ ii & & & 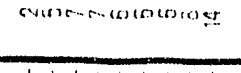 & is & \\
\hline \multicolumn{3}{|c|}{ 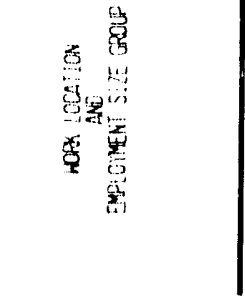 } & 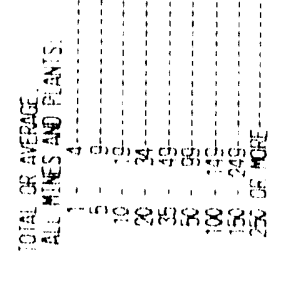 & 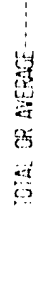 & 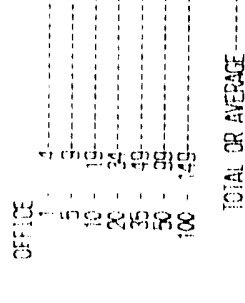 & 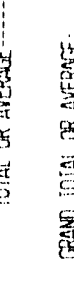 & 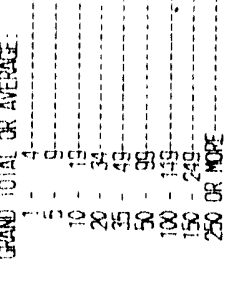 & 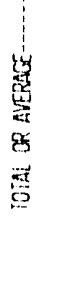 & \\
\hline
\end{tabular}




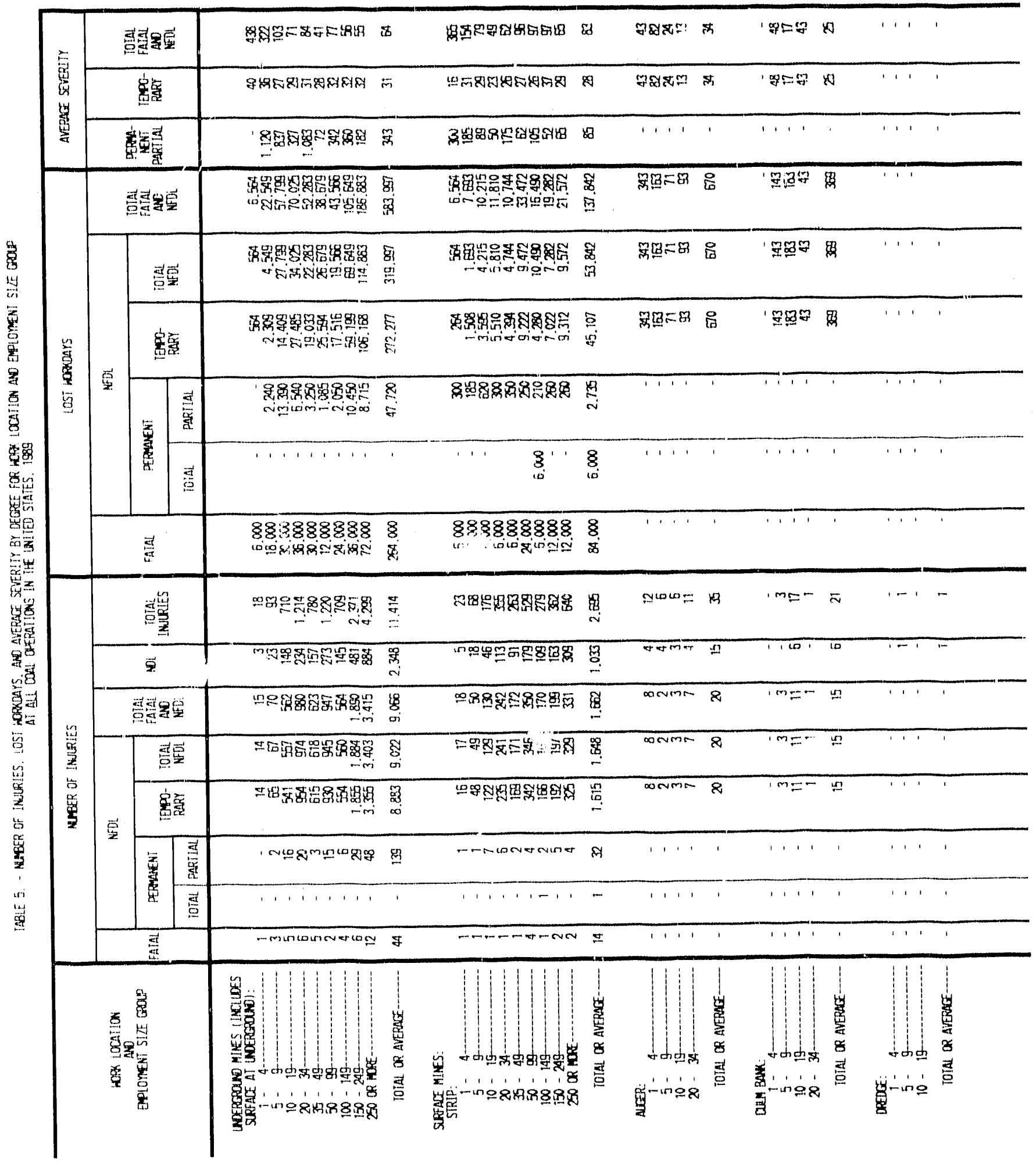




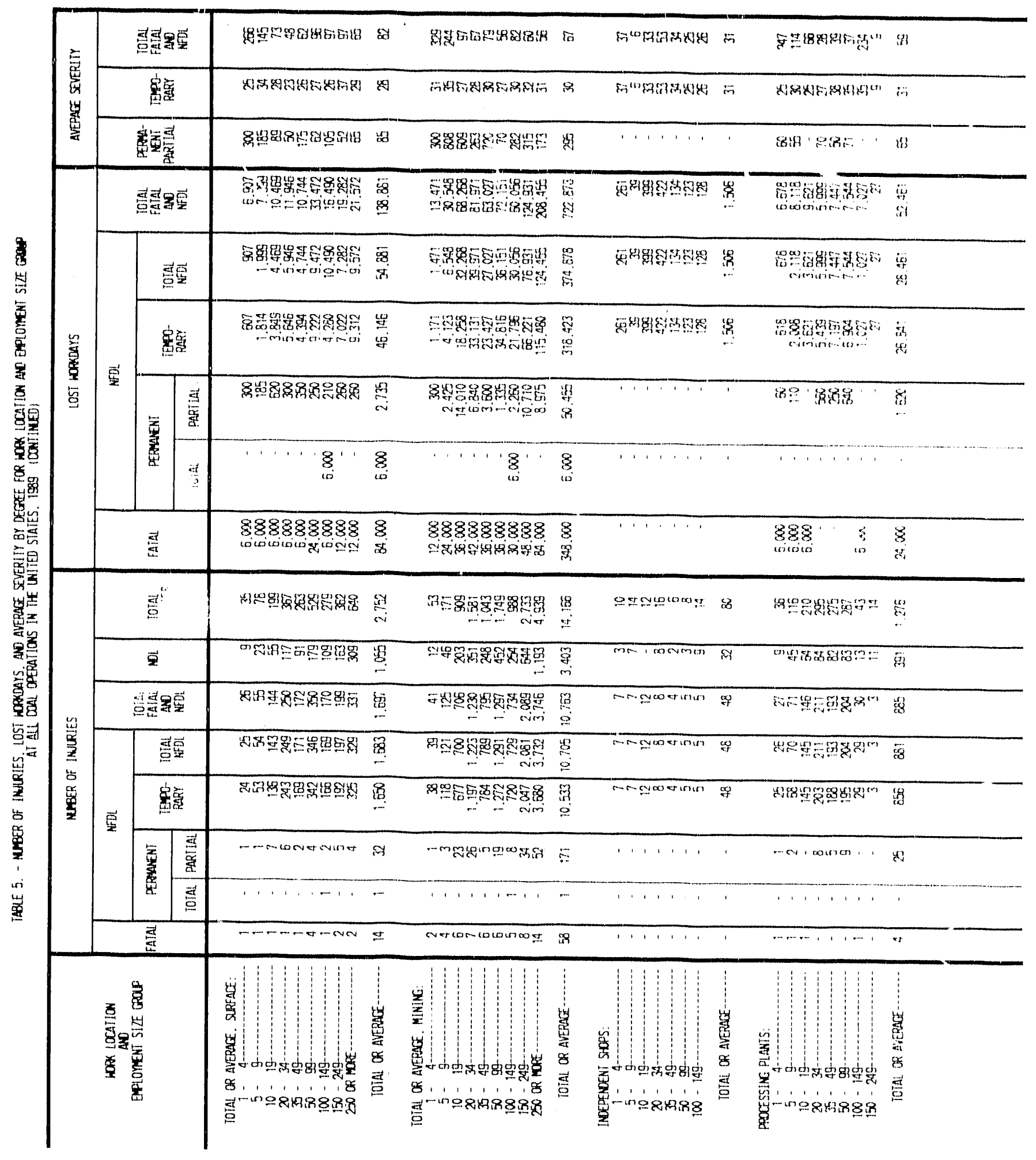




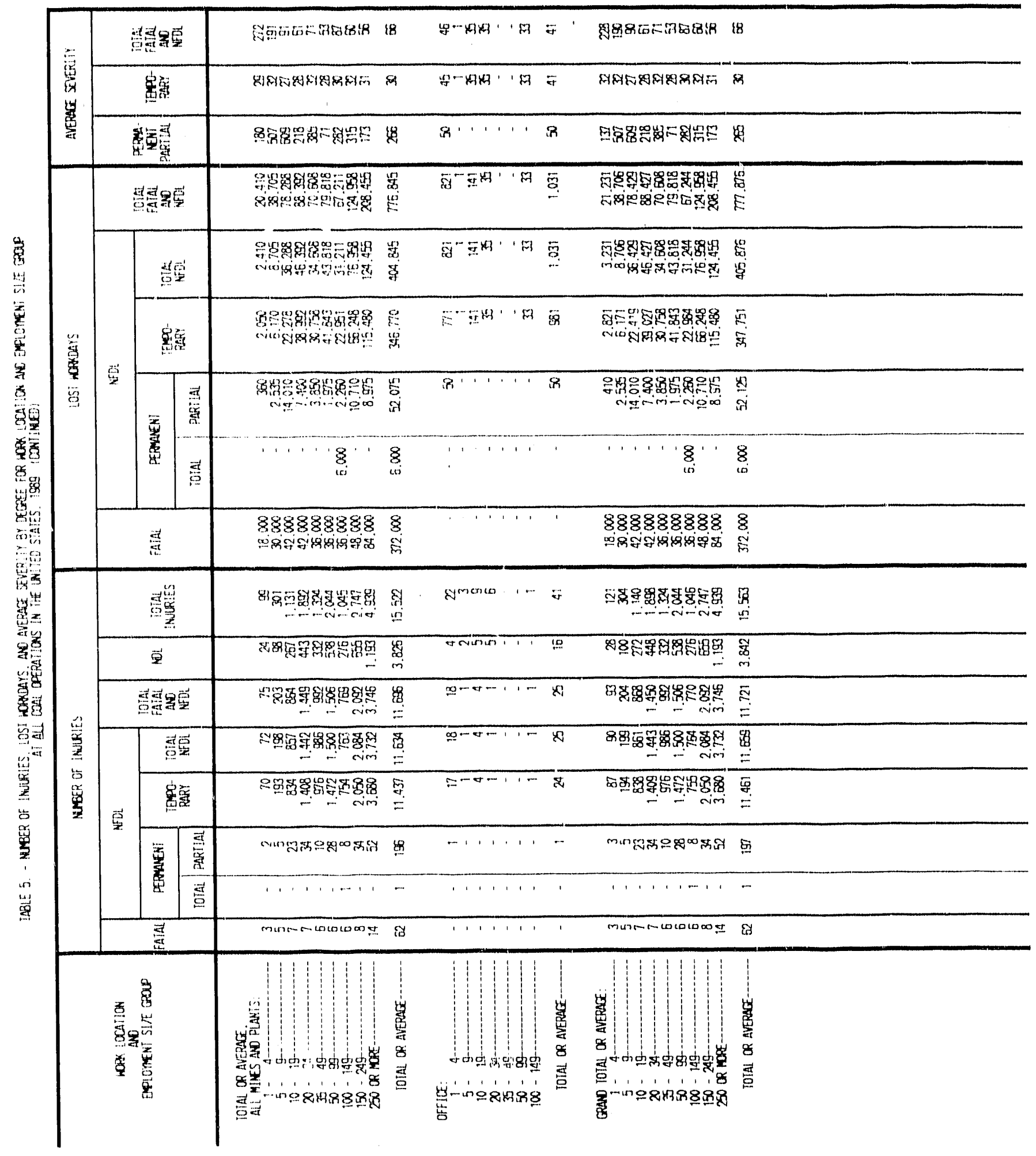




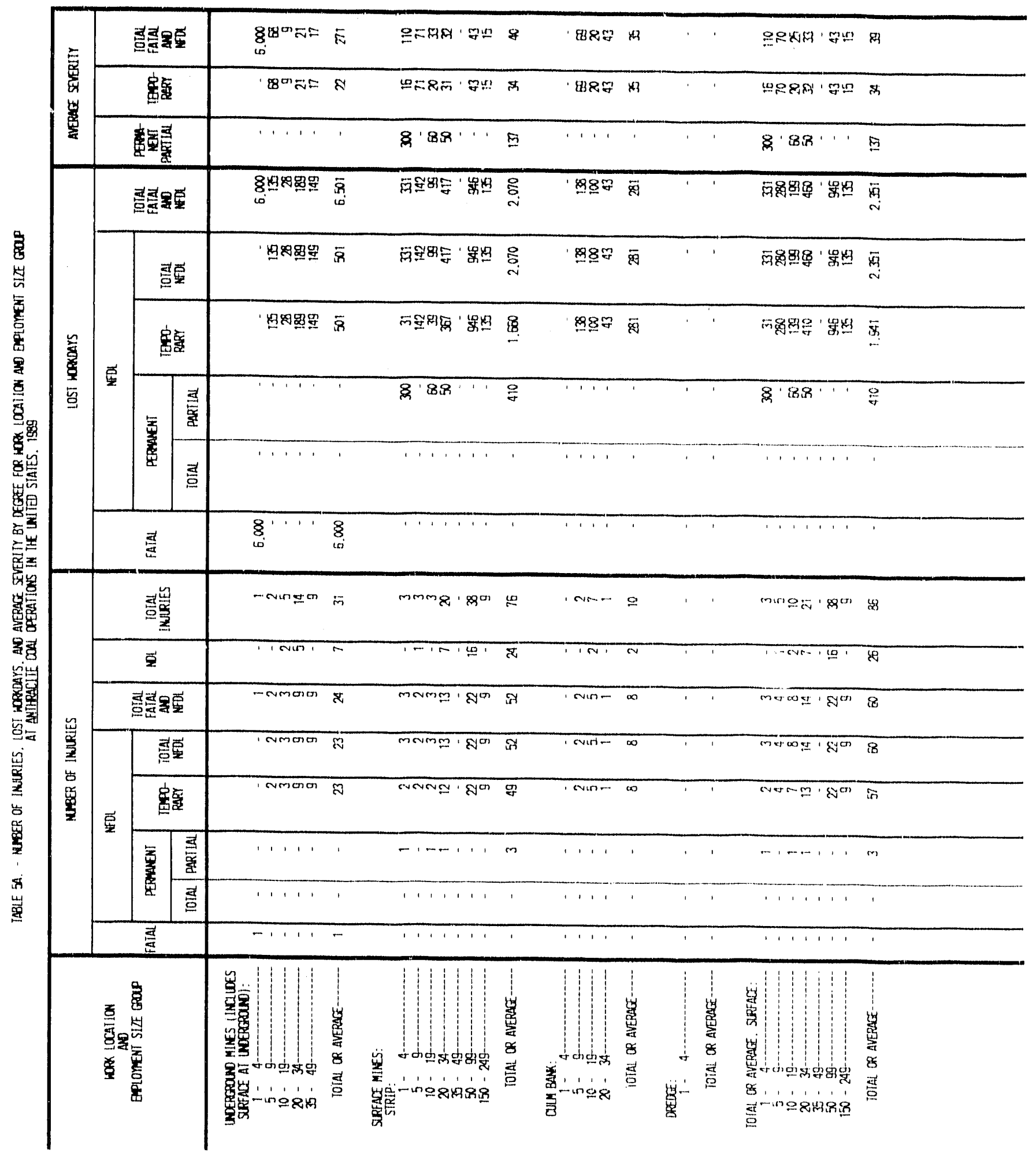




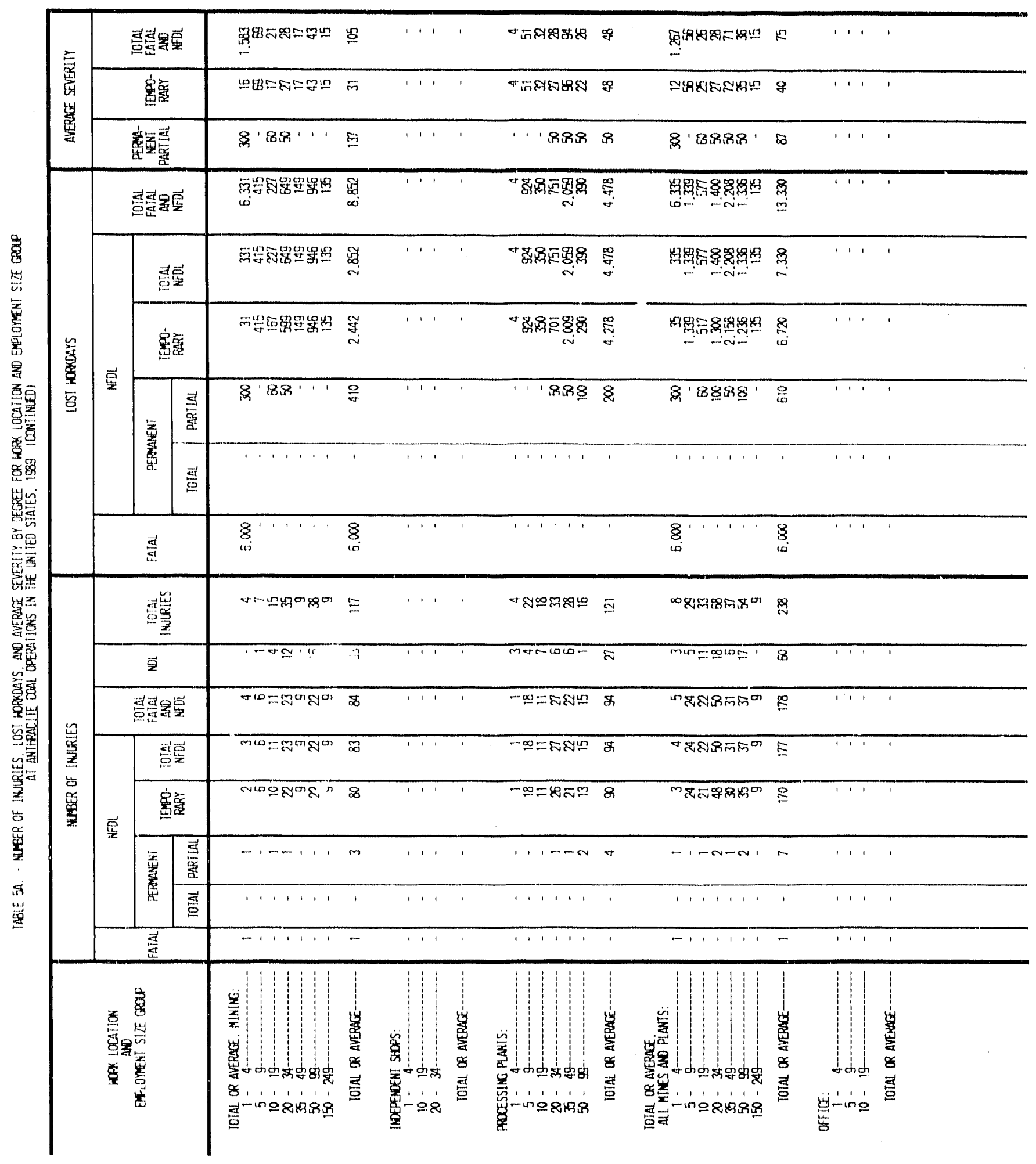




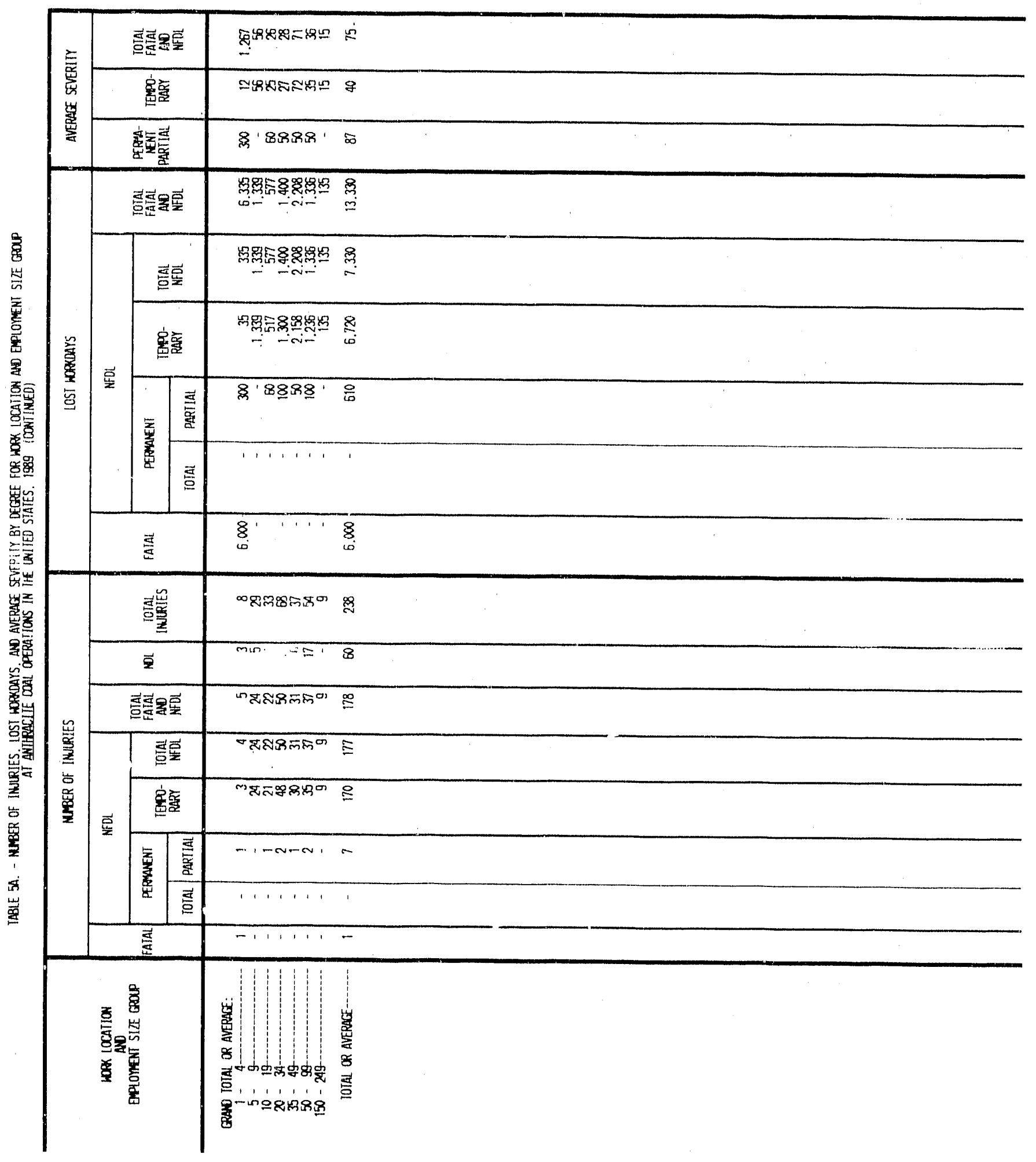




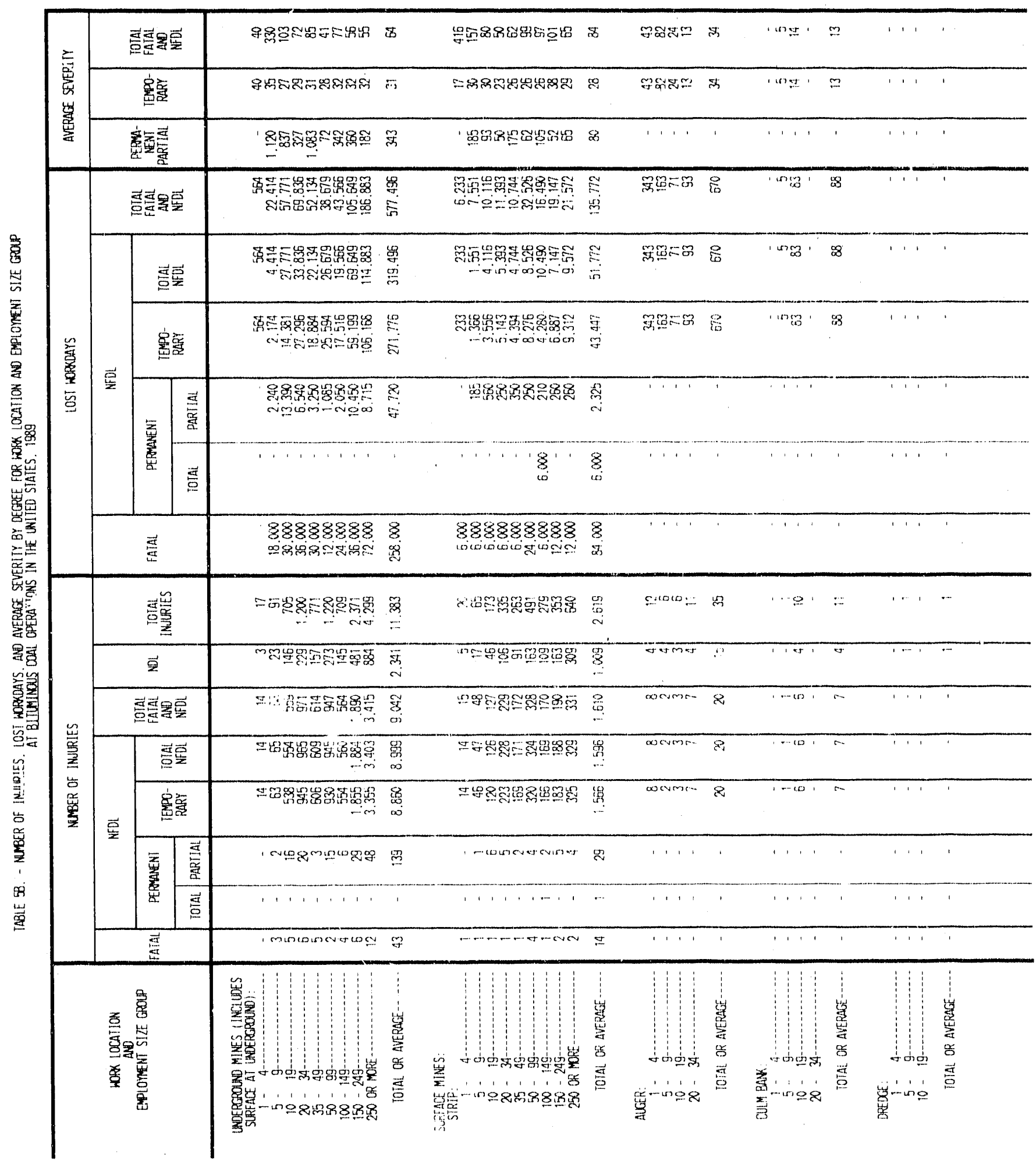




\begin{tabular}{|c|c|c|c|c|c|c|c|c|c|c|}
\hline \multirow{3}{*}{ 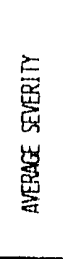 } & \multicolumn{2}{|c|}{ 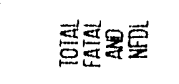 } & 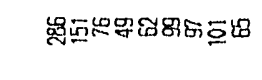 & & 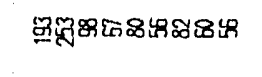 & 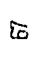 & 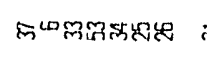 & $\bar{m}$ & 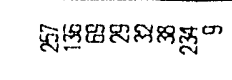 & $\pi$ \\
\hline & \multicolumn{2}{|c|}{ 寡旁 } & 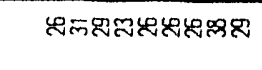 & $\mathscr{q}$ & 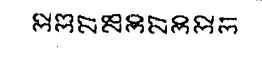 & 8 & 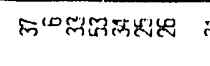 & $\bar{m}$ & 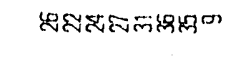 & 8 \\
\hline & \multicolumn{2}{|c|}{ 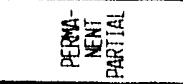 } & 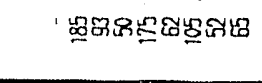 & 8 & 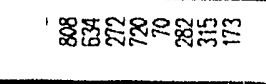 & 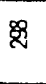 & 11,1 & , & BHA ' MRE' ' & 18 \\
\hline \multirow{6}{*}{ 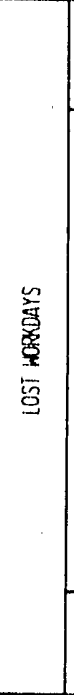 } & \multicolumn{2}{|c|}{ 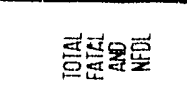 } & 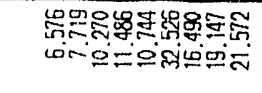 & 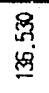 & 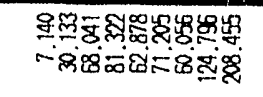 & 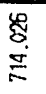 & 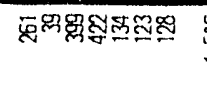 & 罗 & 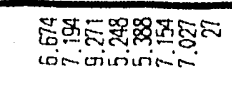 & $\stackrel{5}{8}$ \\
\hline & \multirow{2}{*}{\multicolumn{2}{|c|}{ 害学 }} & 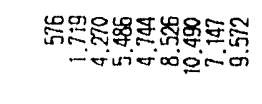 & $\begin{array}{l}\text { 急 } \\
\text { 品 }\end{array}$ & 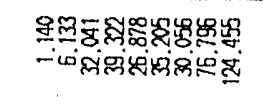 & 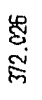 & 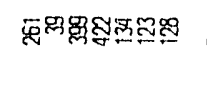 & 䍐 & [ా. & $\underset{\sim}{\mathscr{\sim}}$ \\
\hline & & & 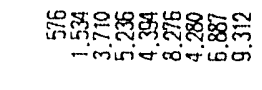 & $\begin{array}{l}\text { 梠 } \\
\text { g }\end{array}$ & 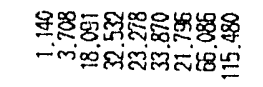 & 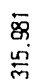 & 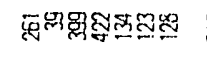 & 罩 & 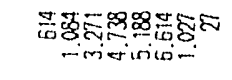 & $\underset{\mathscr{R}}{\mathbb{N}}$ \\
\hline & \multirow{2}{*}{ 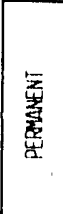 } & $\begin{array}{l}\vec{z} \\
\text { 亲 } \\
\text { 至 }\end{array}$ & 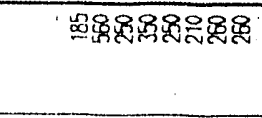 & 䒽 & 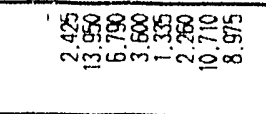 & $\begin{array}{l}\text { 桨 } \\
8 \\
8\end{array}$ & & 1 & 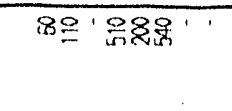 & 8 \\
\hline & & 롱 & ' & 8 & ' ' ' ' & 8 & $\cdots \cdots$ & ' & 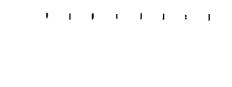 & \\
\hline & \multicolumn{2}{|l|}{ 峦 } & 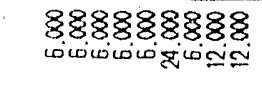 & $\begin{array}{l}8 \\
\text { \$ }\end{array}$ & 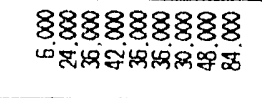 & 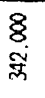 & ' & ' & $\begin{array}{l}888 \\
\text { फisios }\end{array}$ & $\begin{array}{l}\delta \\
\dot{x}\end{array}$ \\
\hline \multirow{8}{*}{ 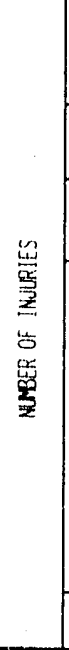 } & \multicolumn{2}{|c|}{ 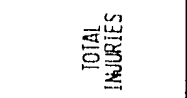 } & 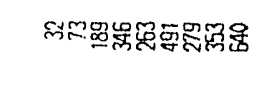 & 奪 & 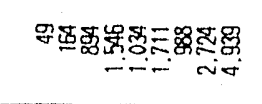 & 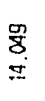 & 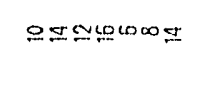 & 8 & 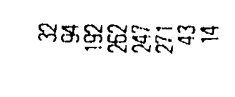 & $\stackrel{\text { 照 }}{-}$ \\
\hline & \multicolumn{2}{|l|}{$\ddot{z}$} & 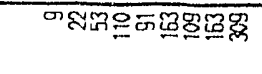 & 啇 & 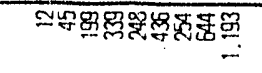 & 옹 & ma. & ब্ল & 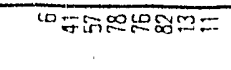 & 离 \\
\hline & \multicolumn{2}{|c|}{ 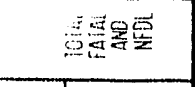 } & 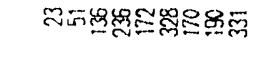 & $\tilde{\varepsilon}$ & m.9.8 & 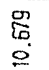 & ans & $\ddot{q}$ & 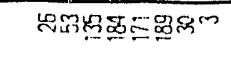 & $\overline{\underline{9}}$ \\
\hline & \multicolumn{2}{|c|}{ 홍롤 } & 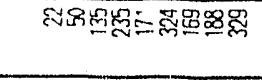 & $\widetilde{\widetilde{\sigma}}$ & 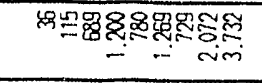 & 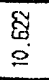 & 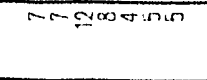 & og & प्रतिक्तु & 鱼 \\
\hline & \multicolumn{2}{|c|}{ 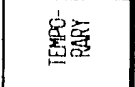 } & 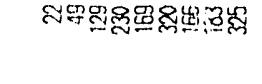 & 思 & 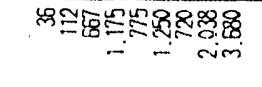 & $\begin{array}{l}\stackrel{9}{9} \\
\stackrel{9}{9}\end{array}$ & 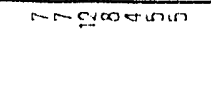 & 受 & 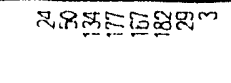 & 18 \\
\hline & 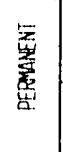 & 矛 & 1 - minnervinet & $\mathscr{D}$ & 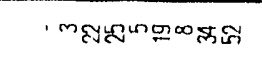 & $\ddot{8}$ & 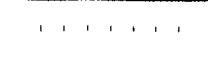 & & matman & $\approx$ \\
\hline & & 总 & $11,1,-$ & - & $\cdots$ &.- & ' & & I & \\
\hline & \multicolumn{2}{|l|}{ 高 } & $\rightarrow-\infty n$ & \pm & 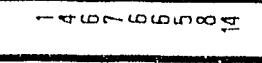 & in & ' & & $-\ldots+1,1$ & $\pi$ \\
\hline & \multicolumn{2}{|l|}{ 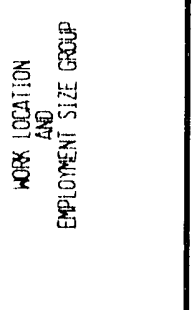 } & 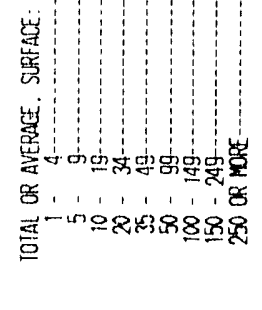 & 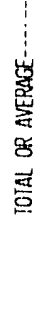 & 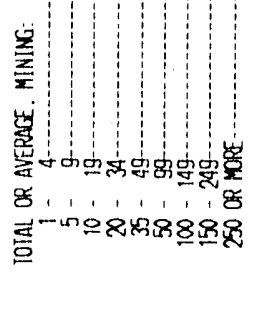 & 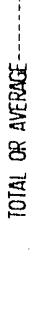 & 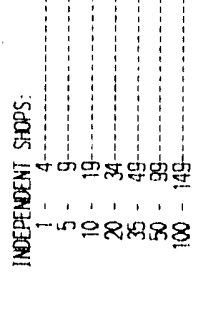 & 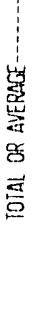 & 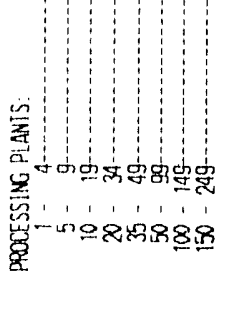 & 岁 \\
\hline
\end{tabular}




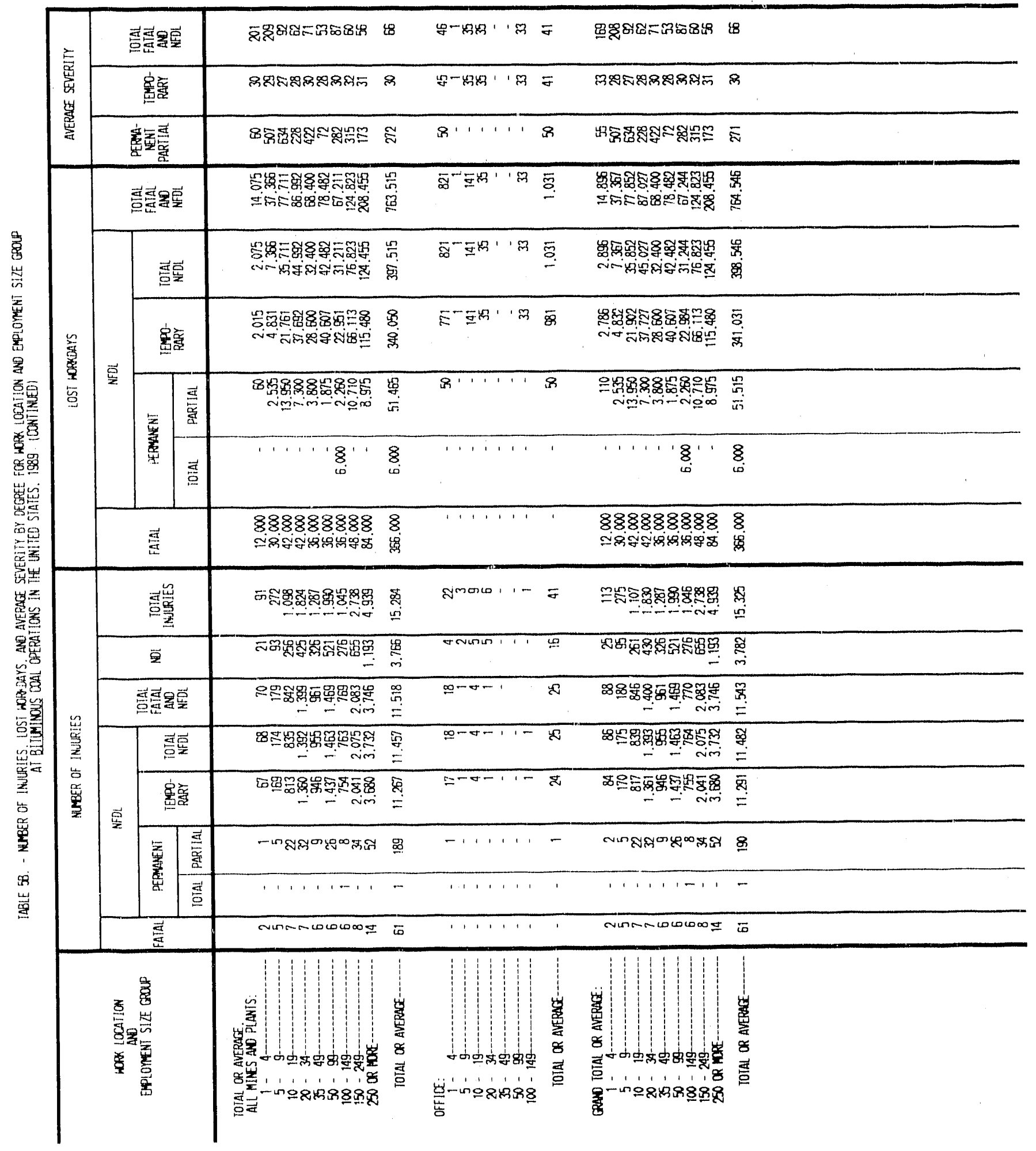




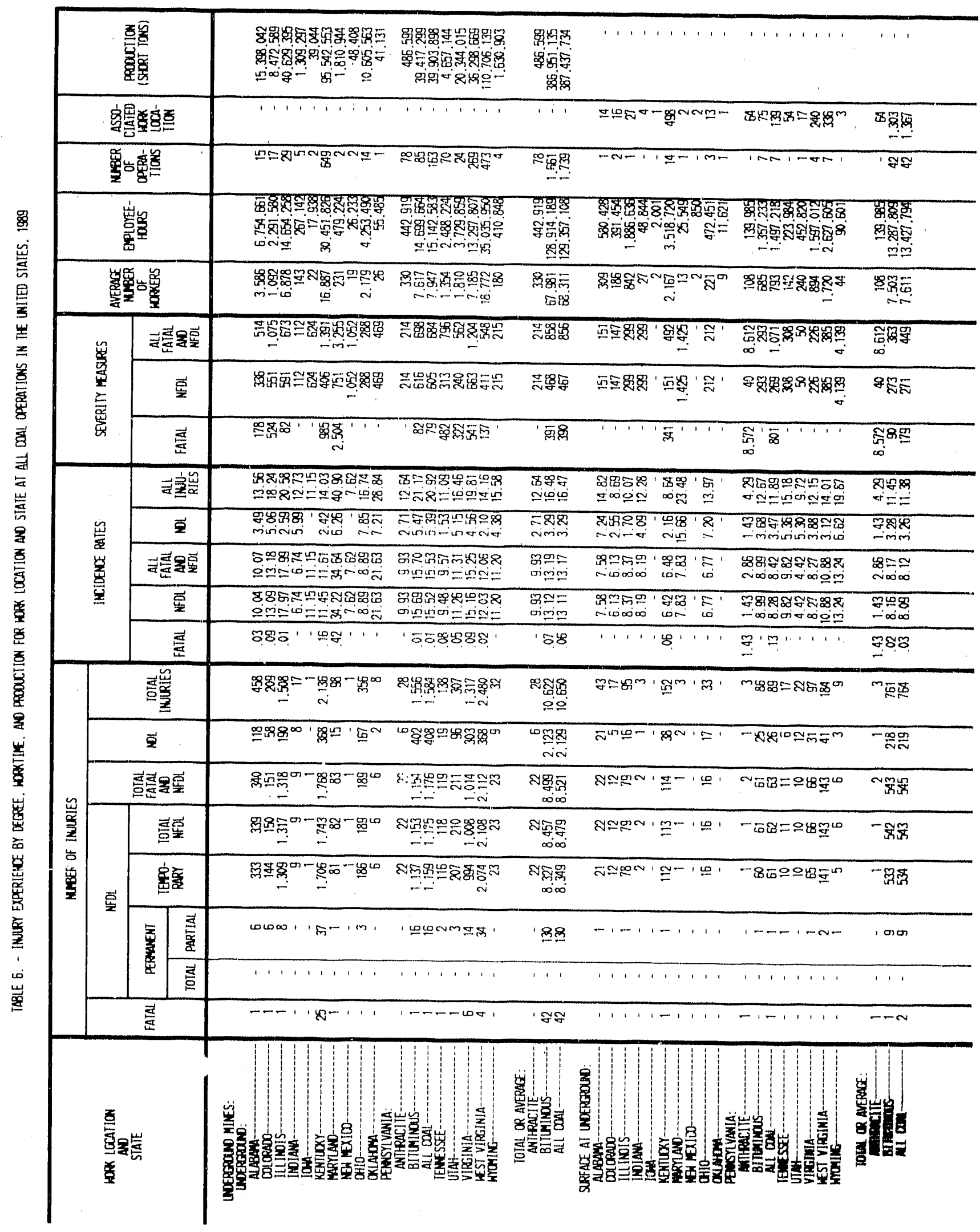




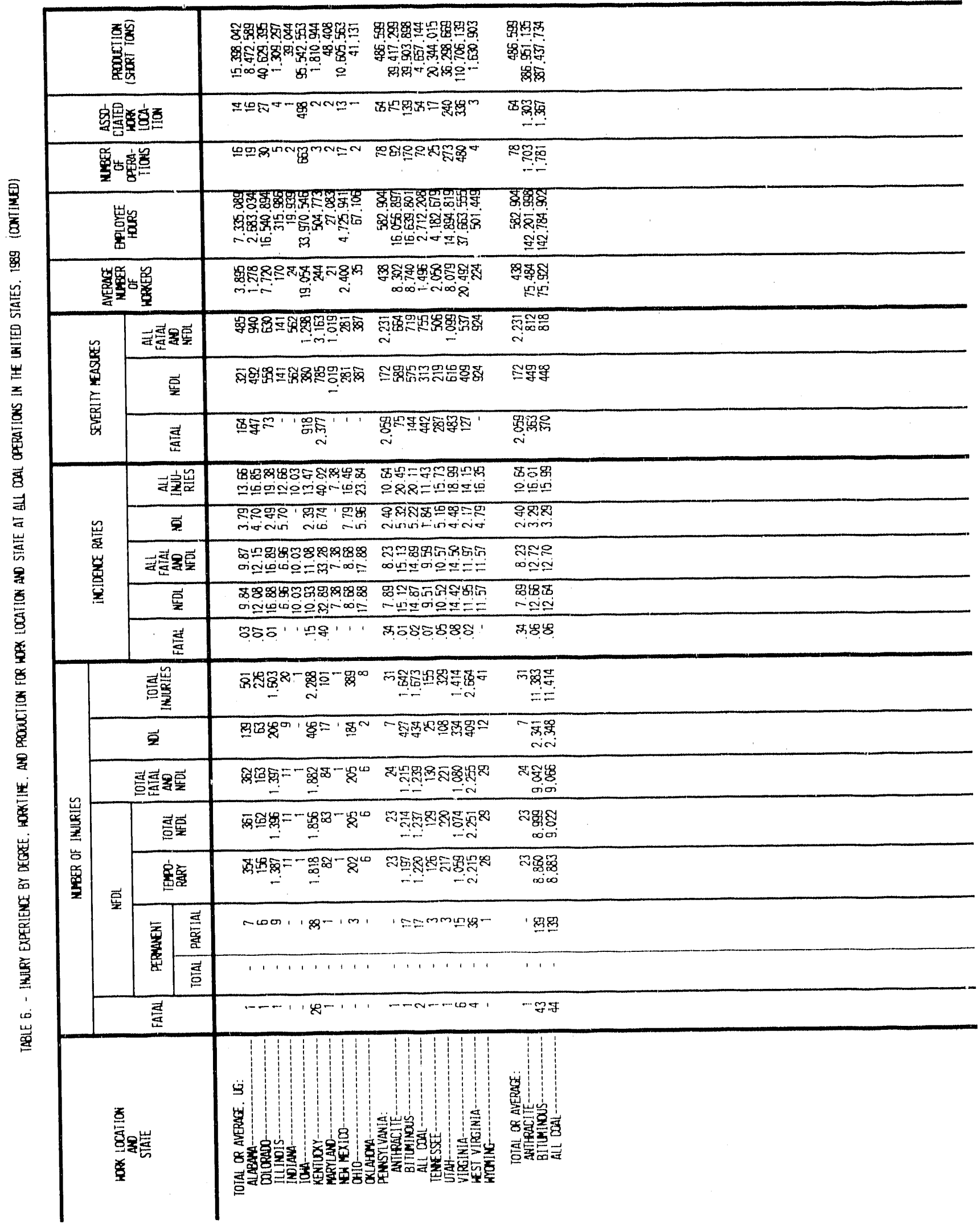




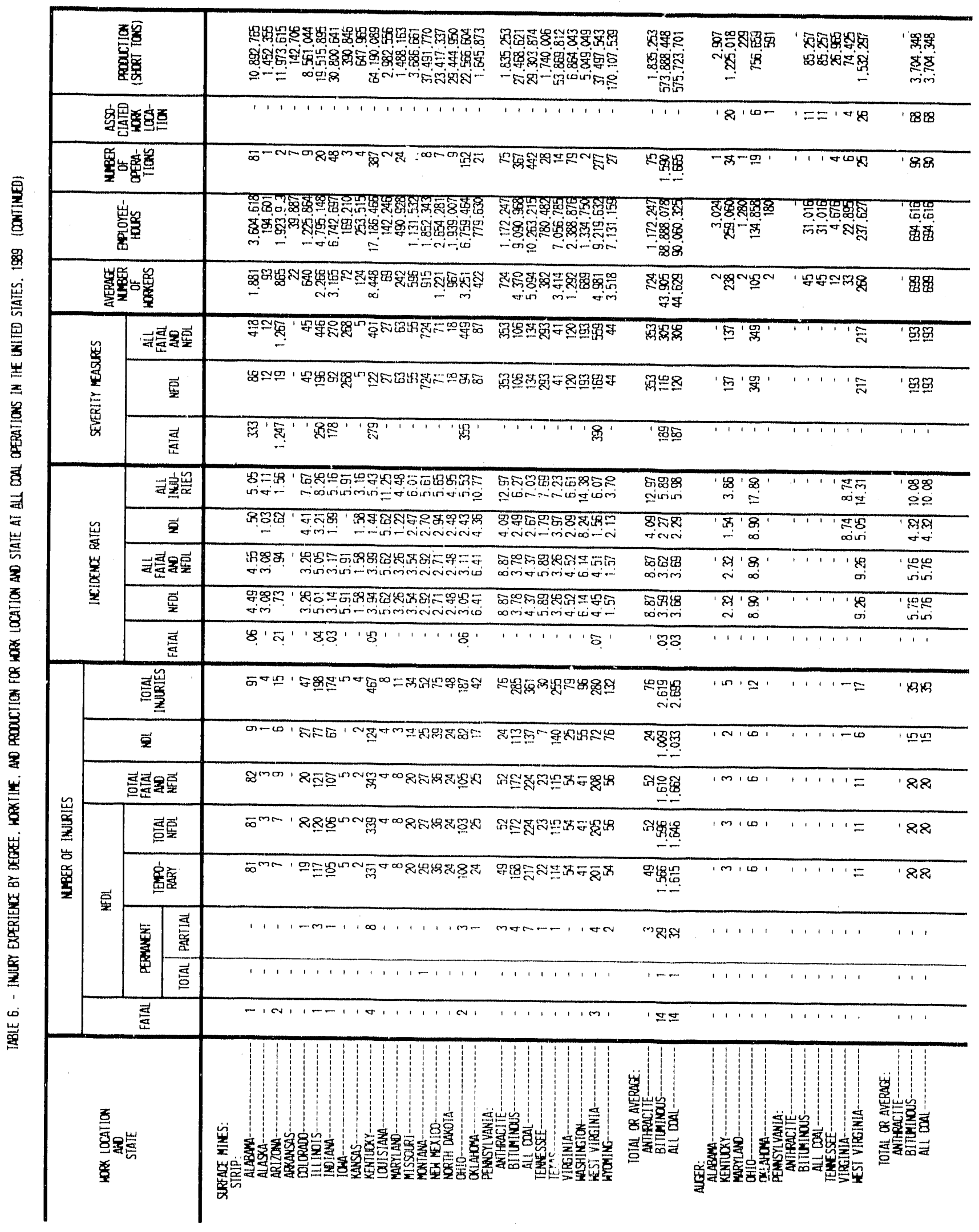




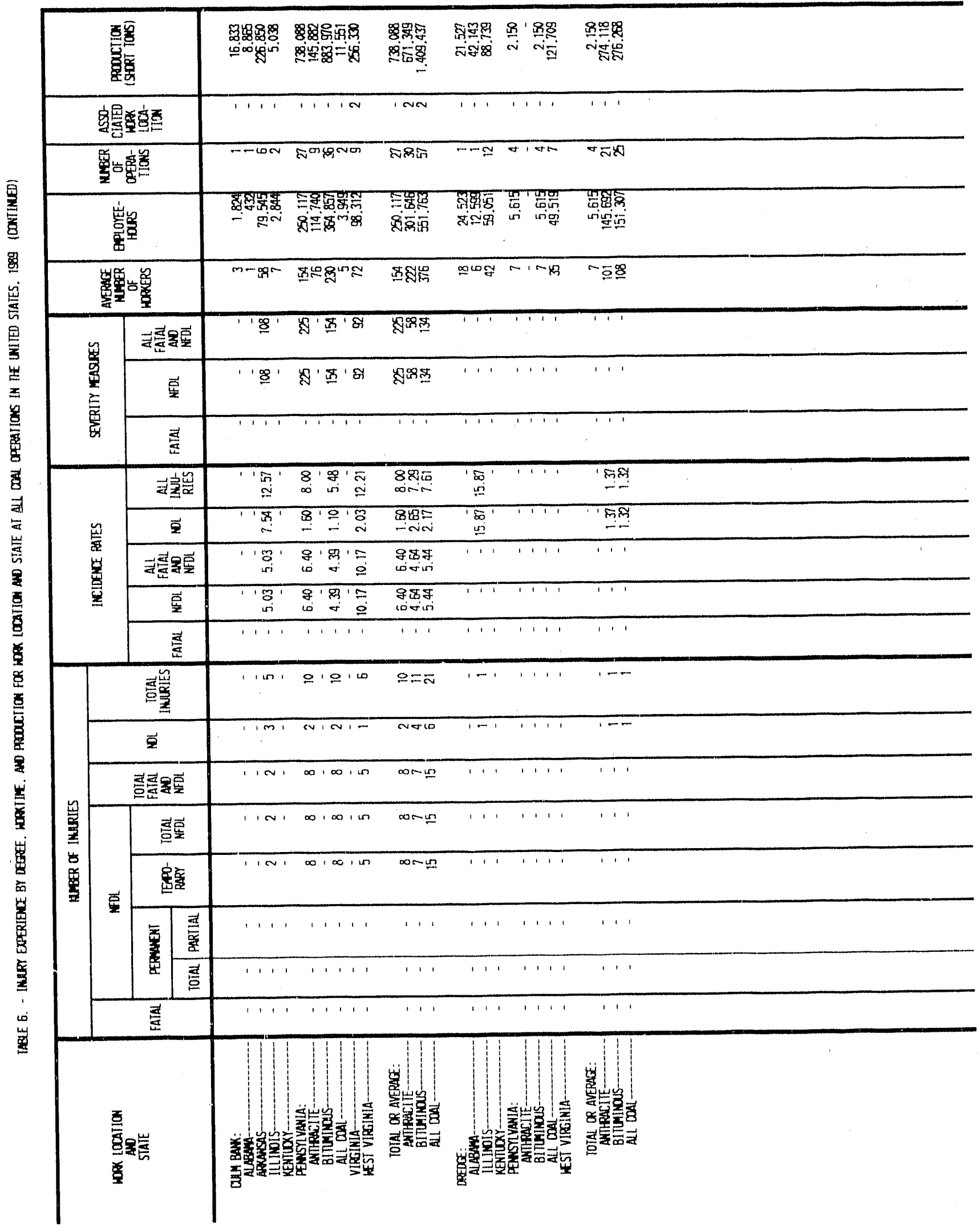




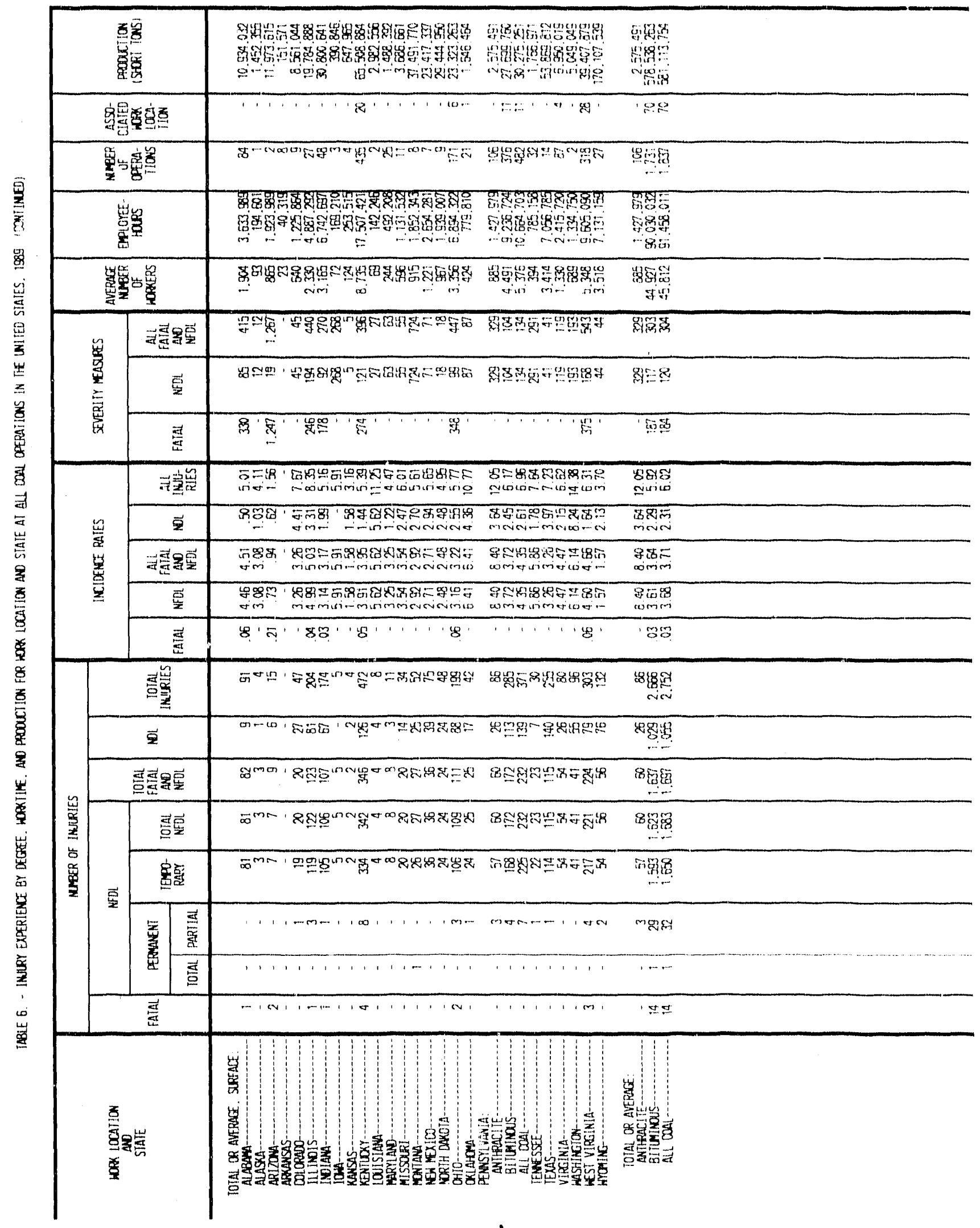




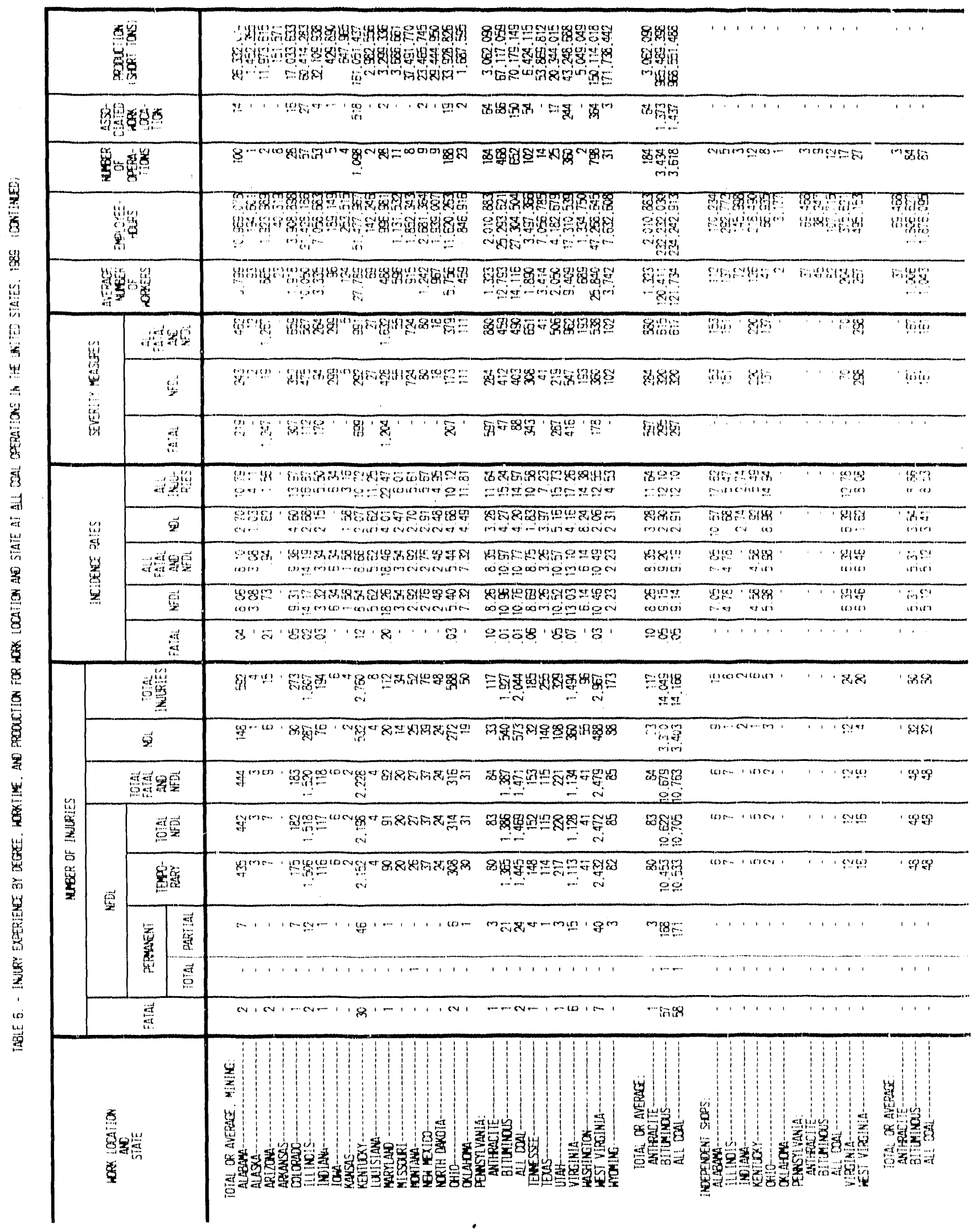




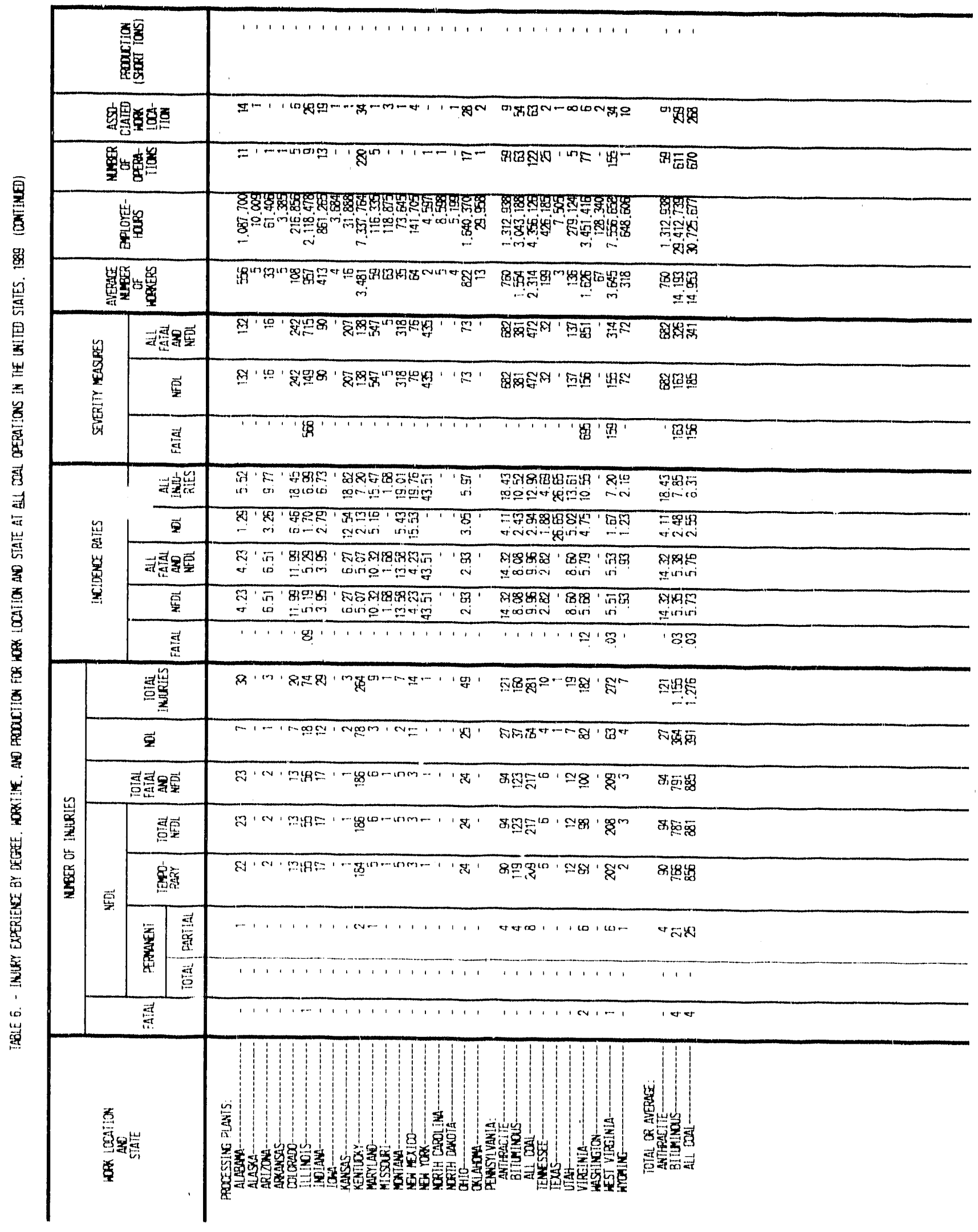




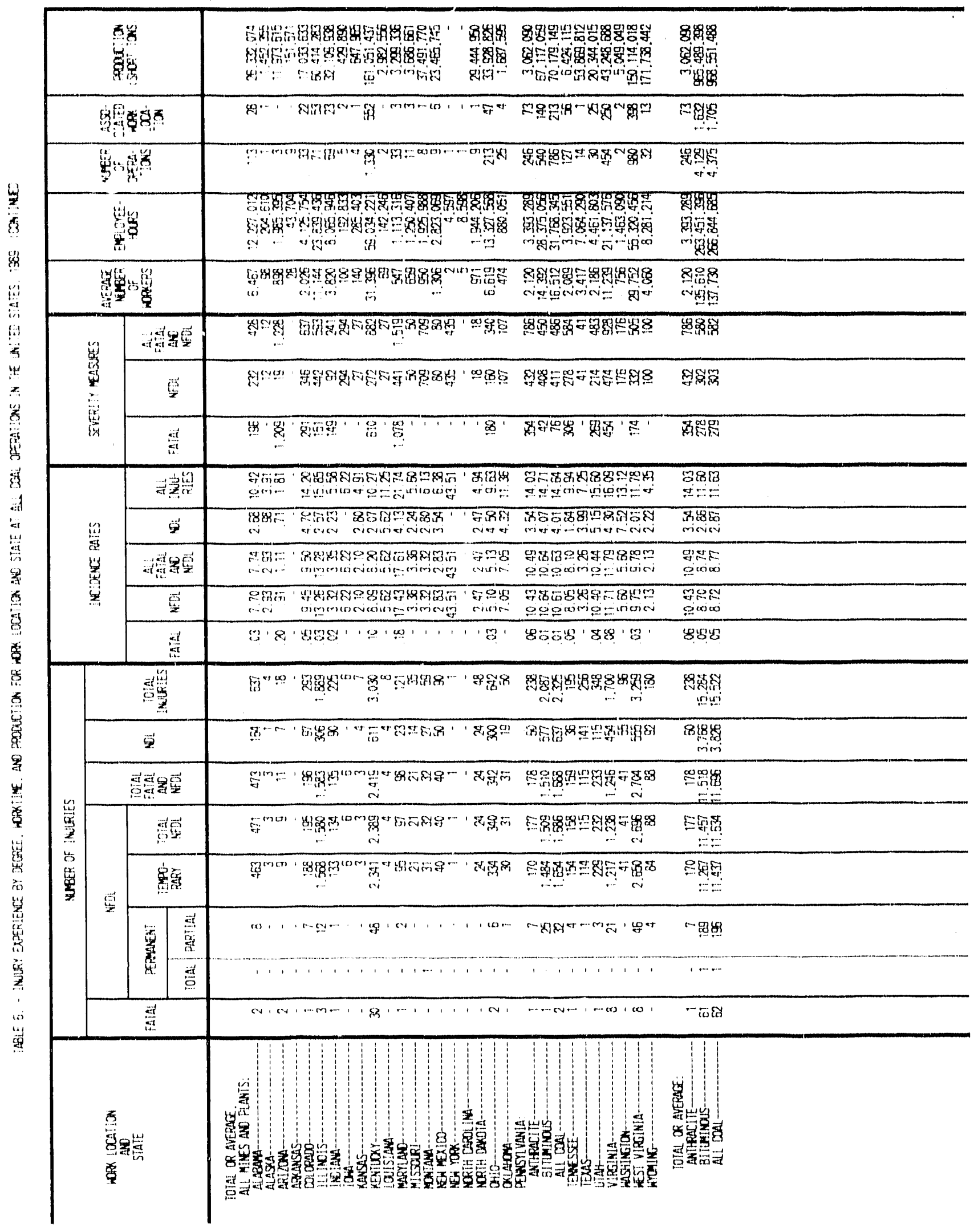




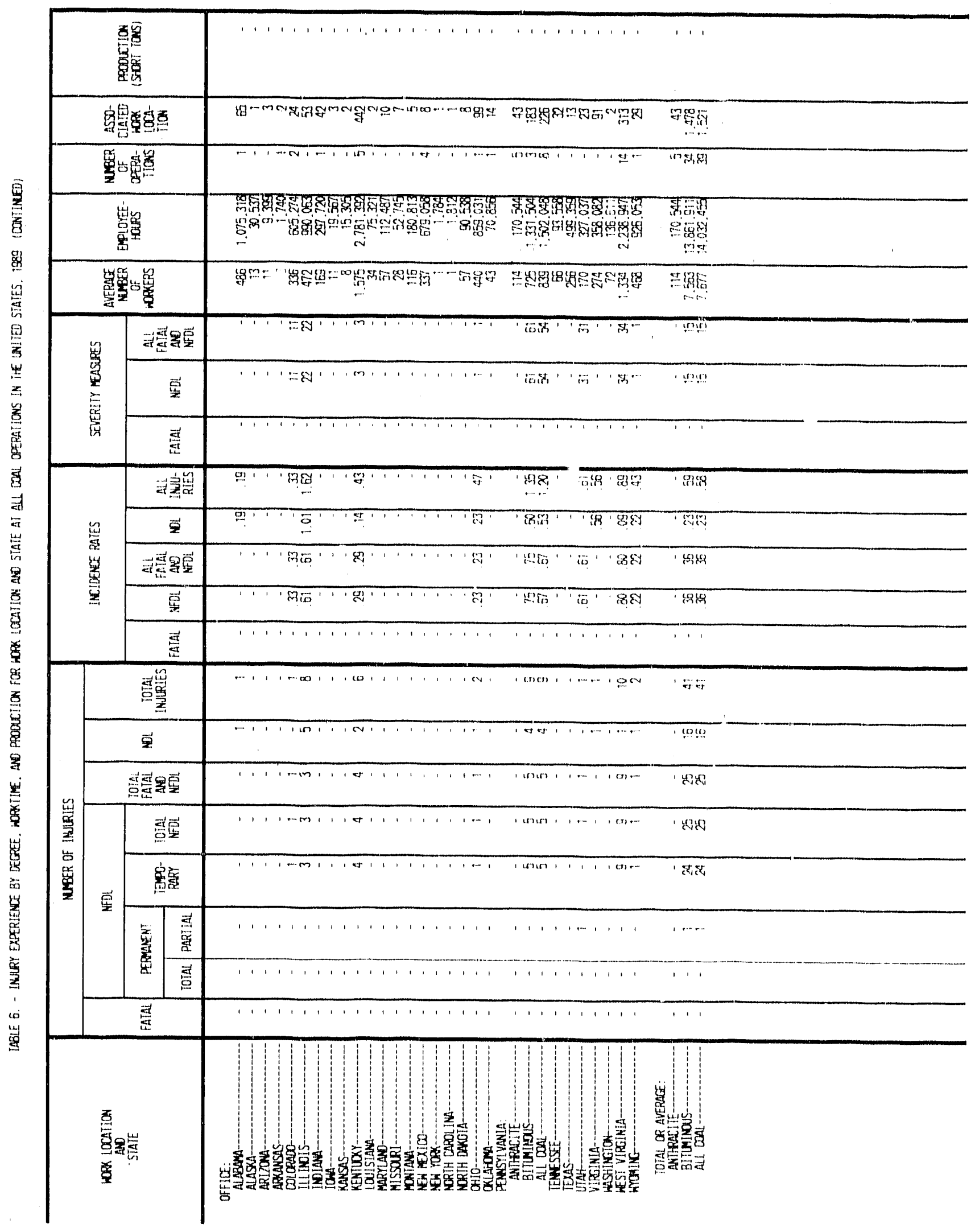




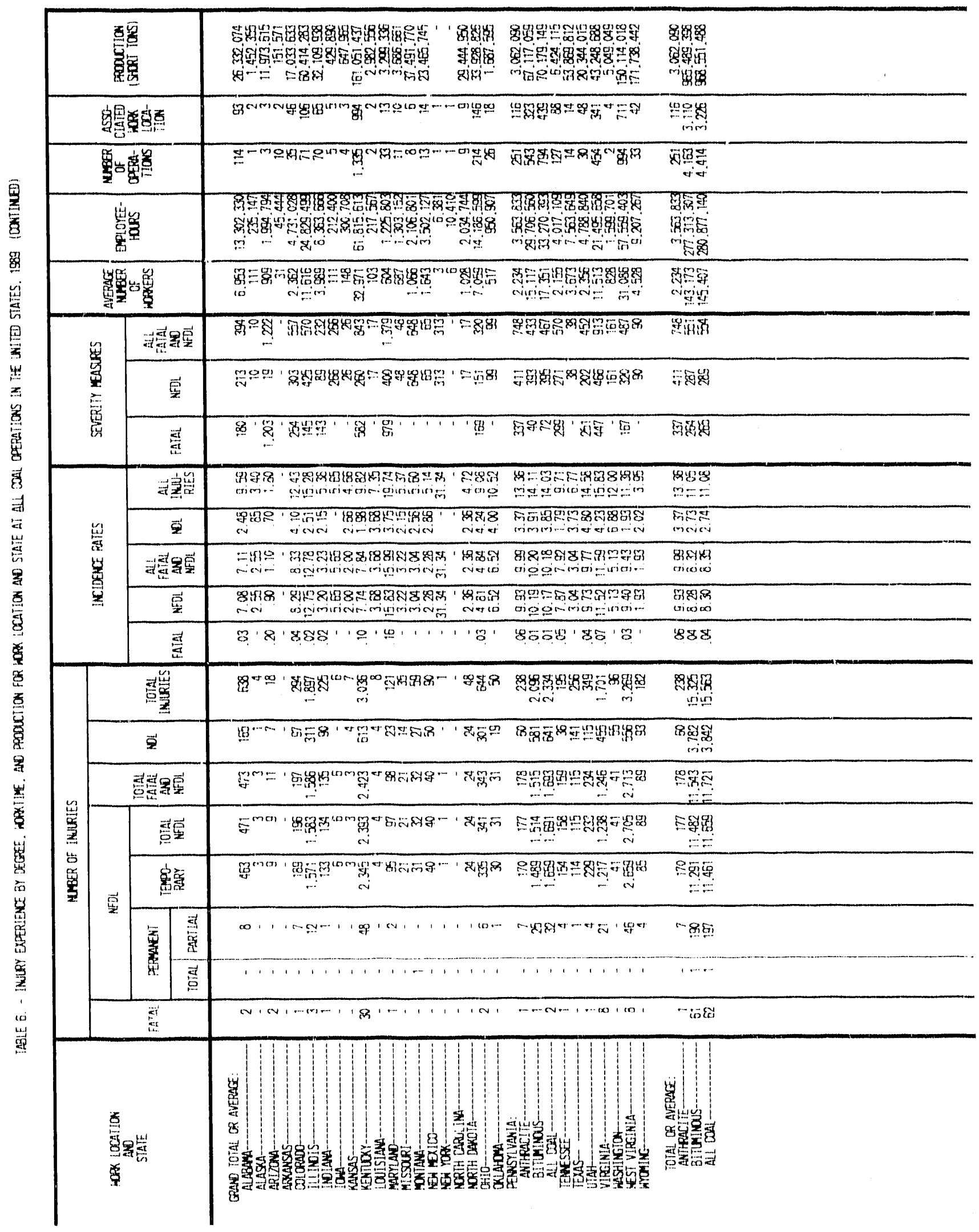




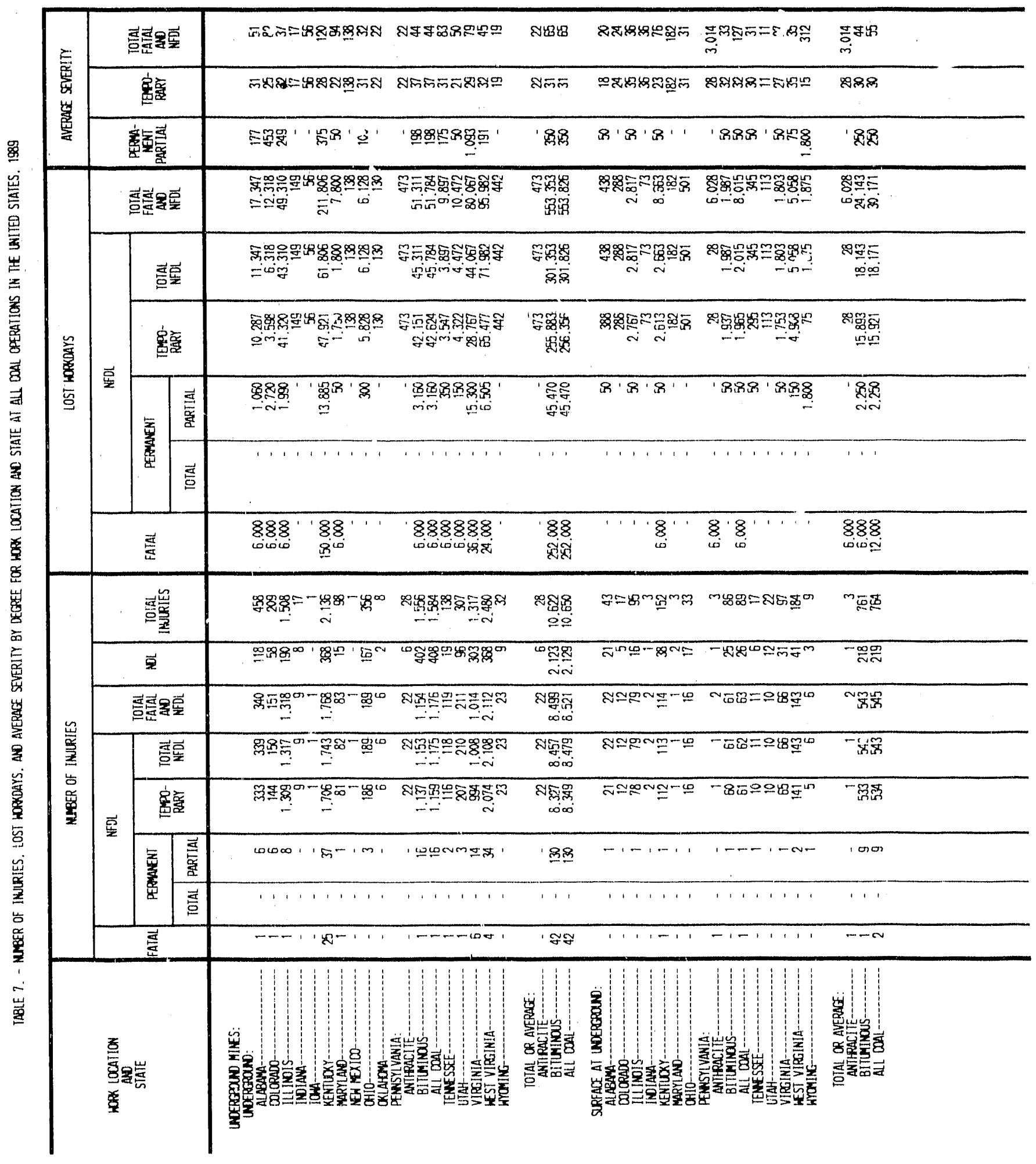




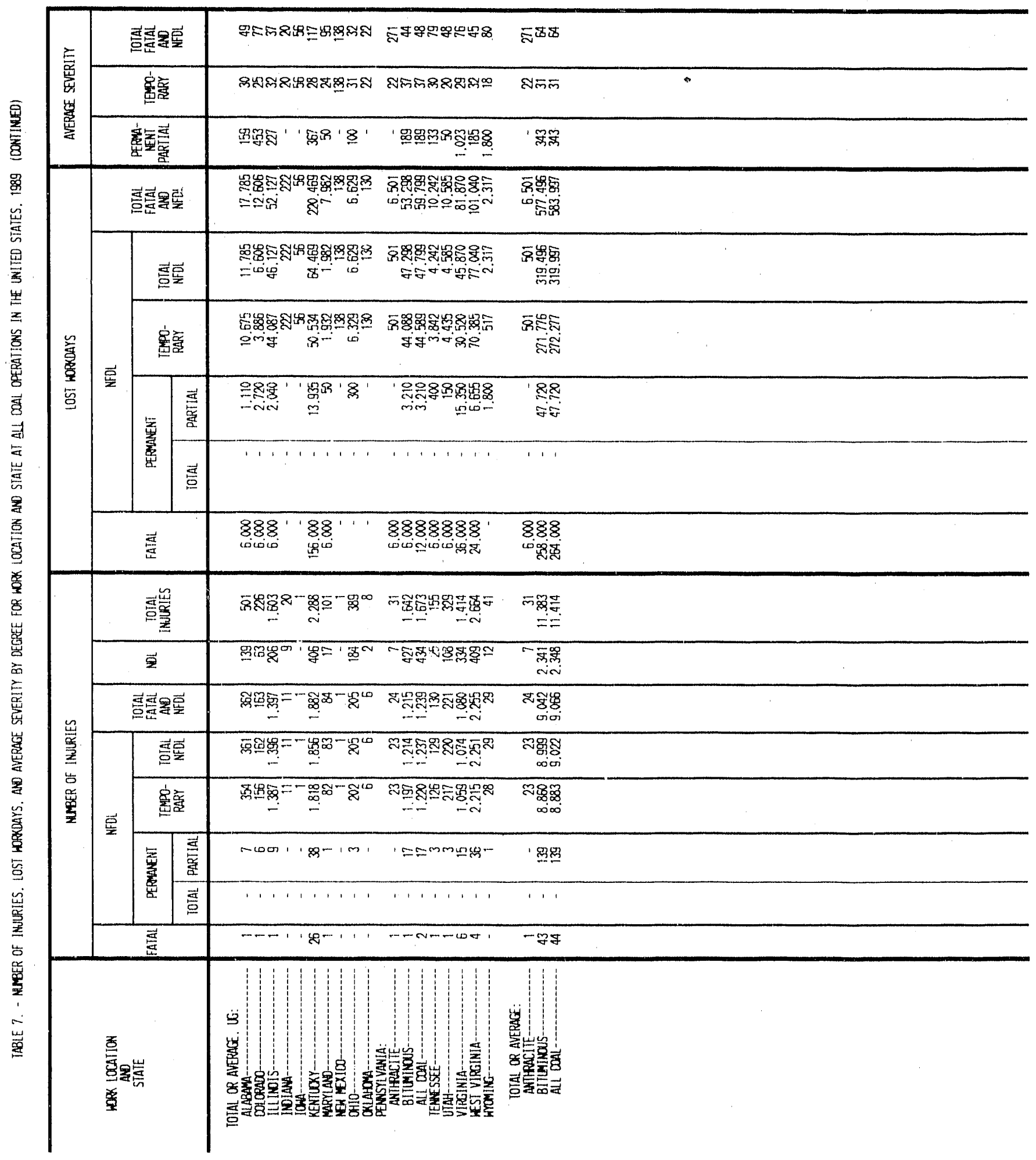




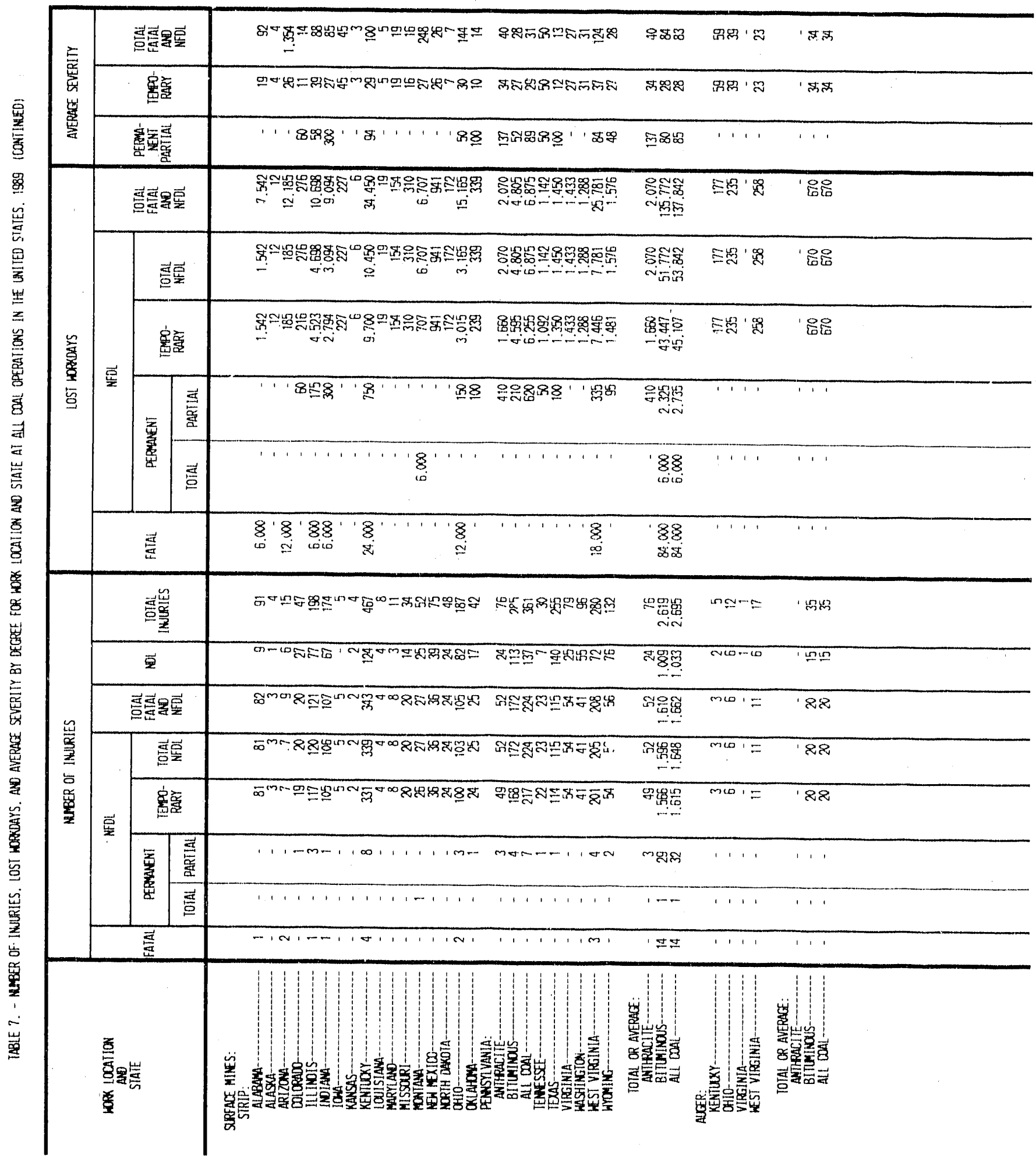




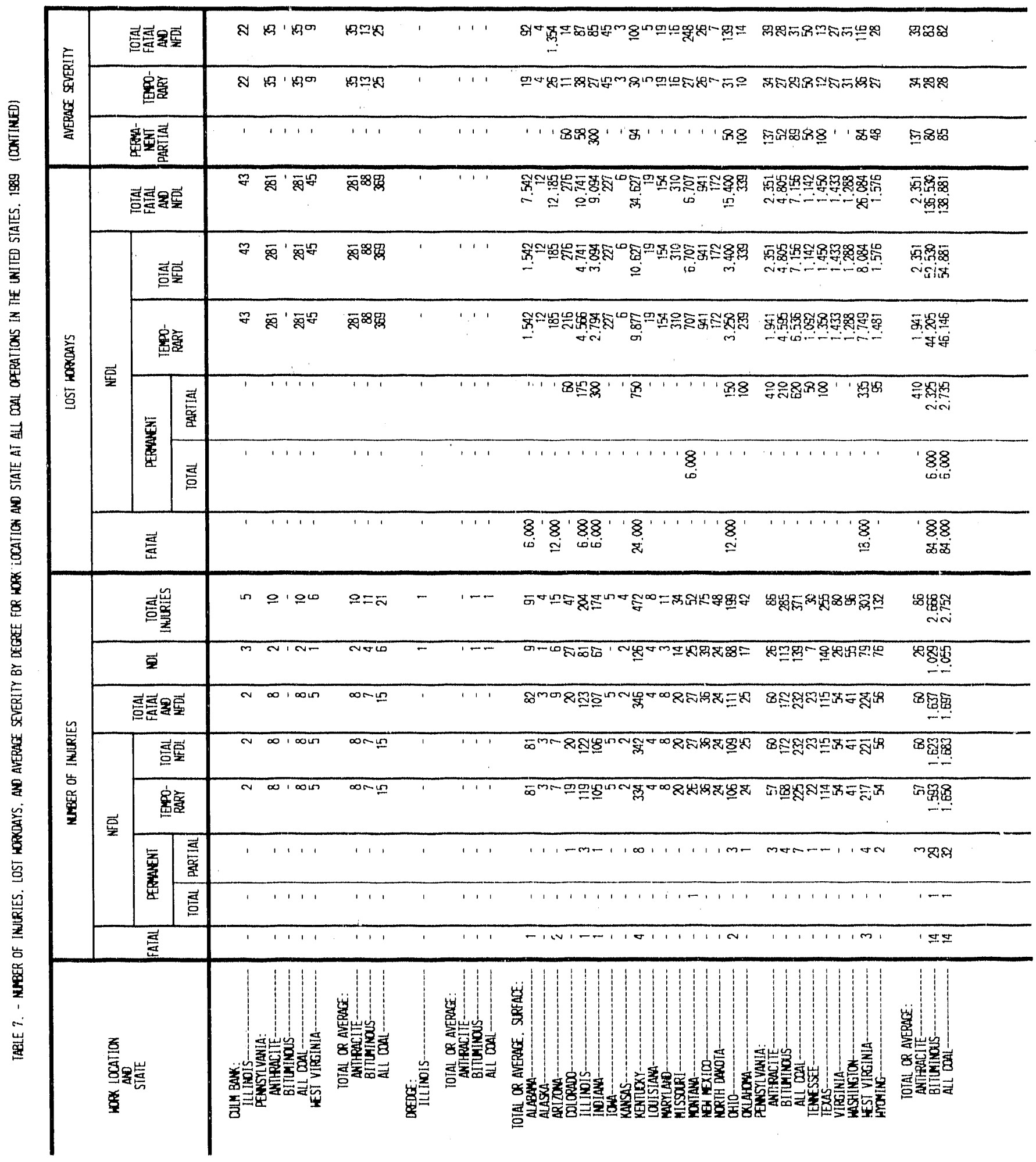




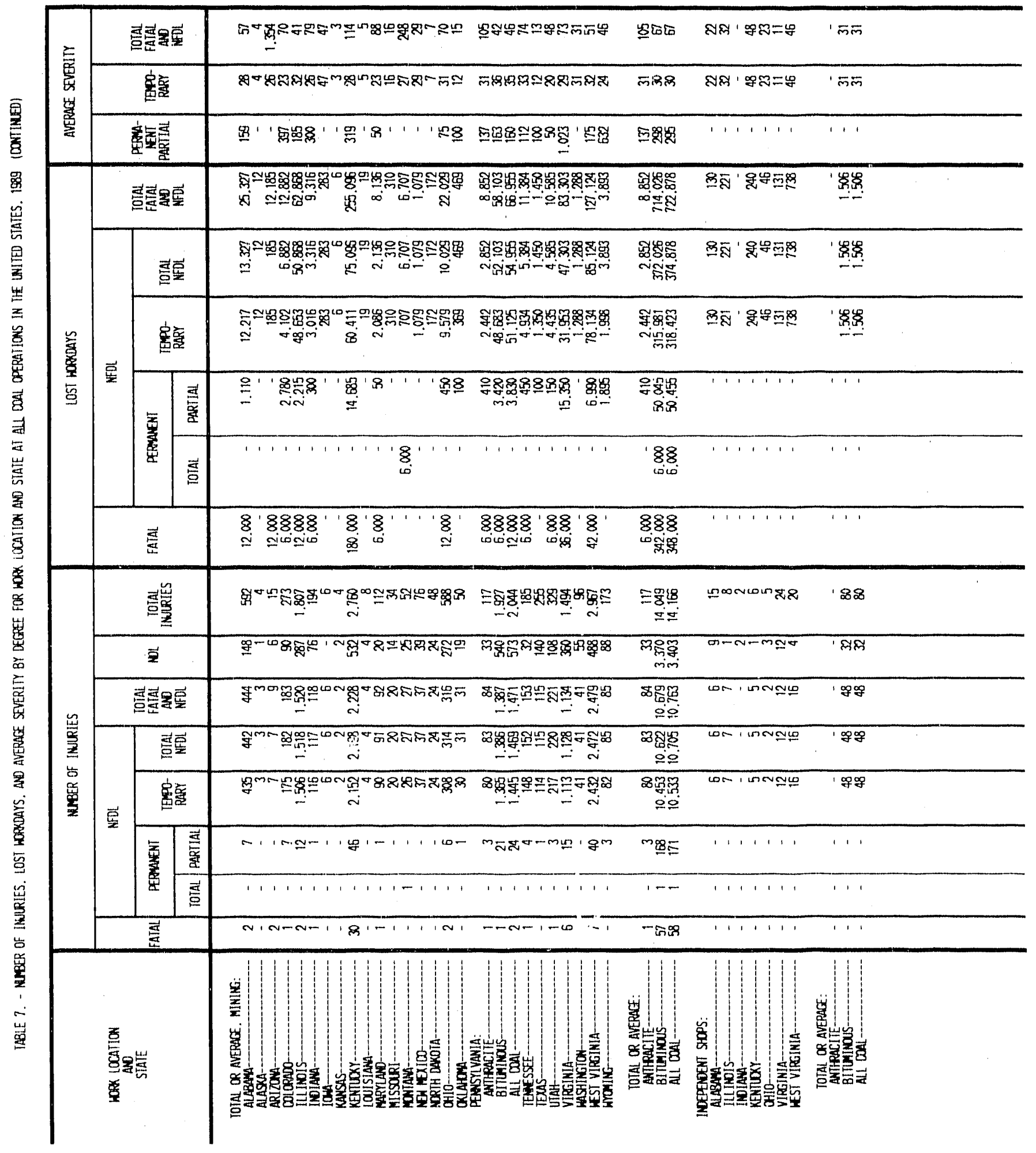




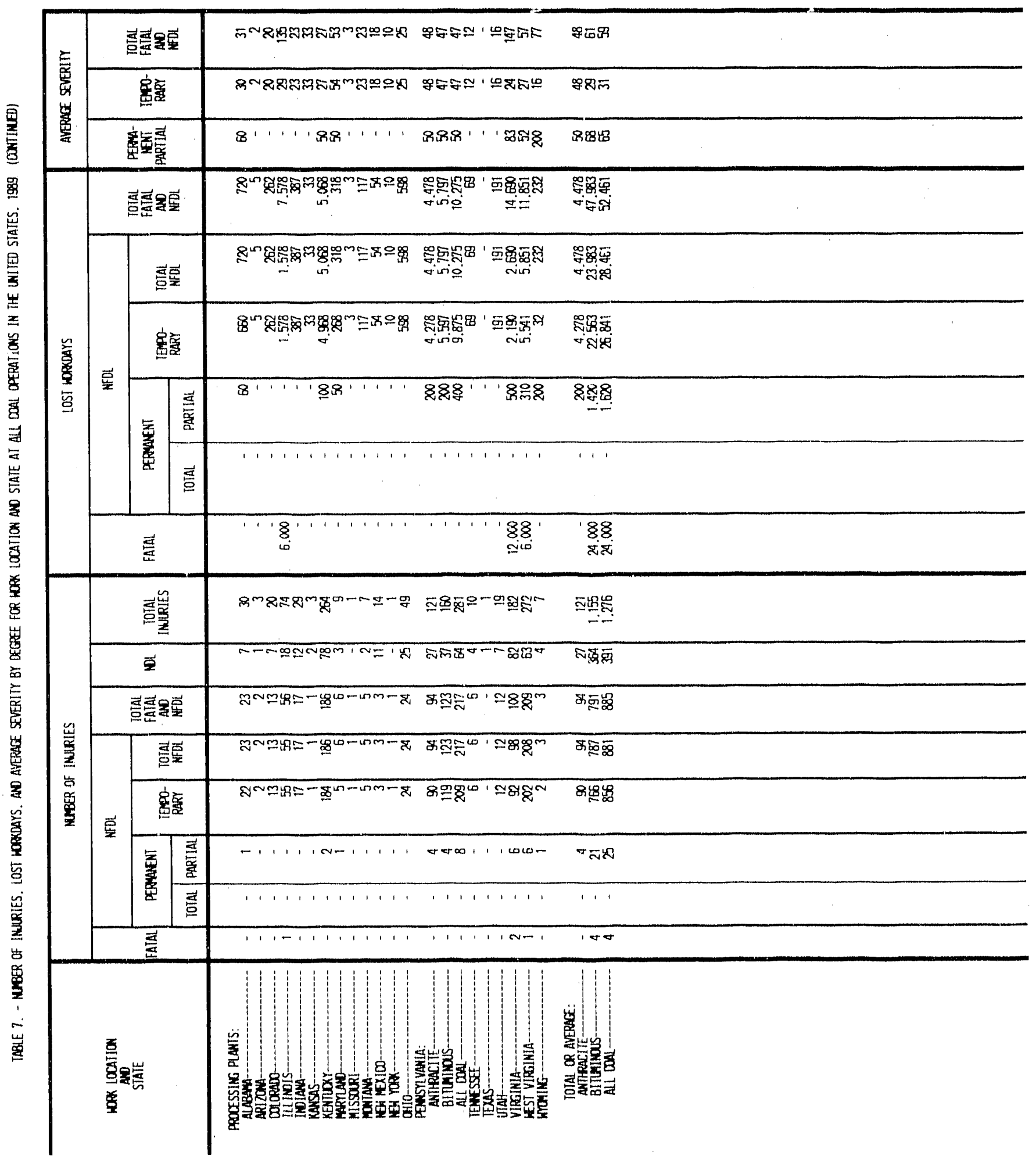




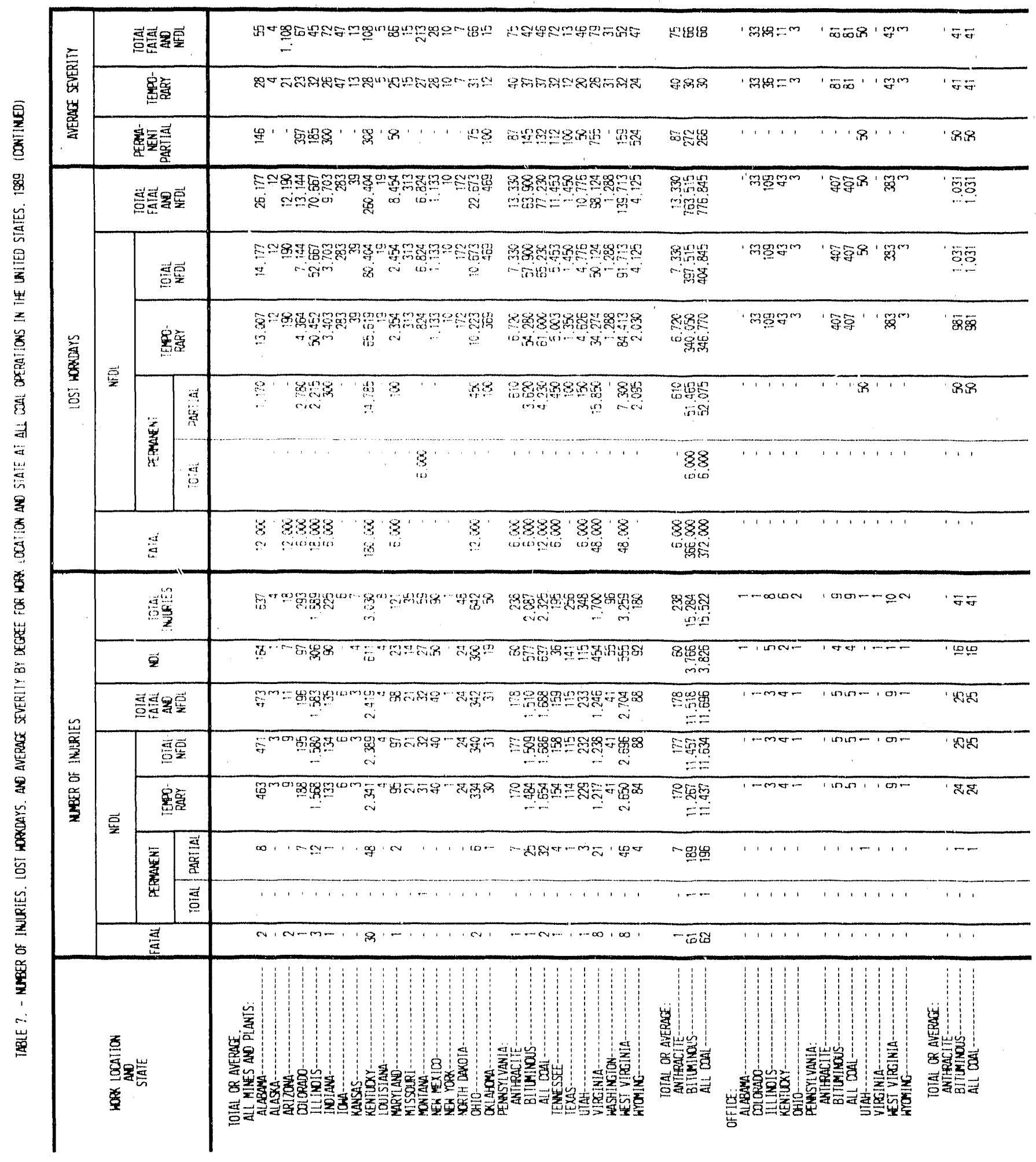




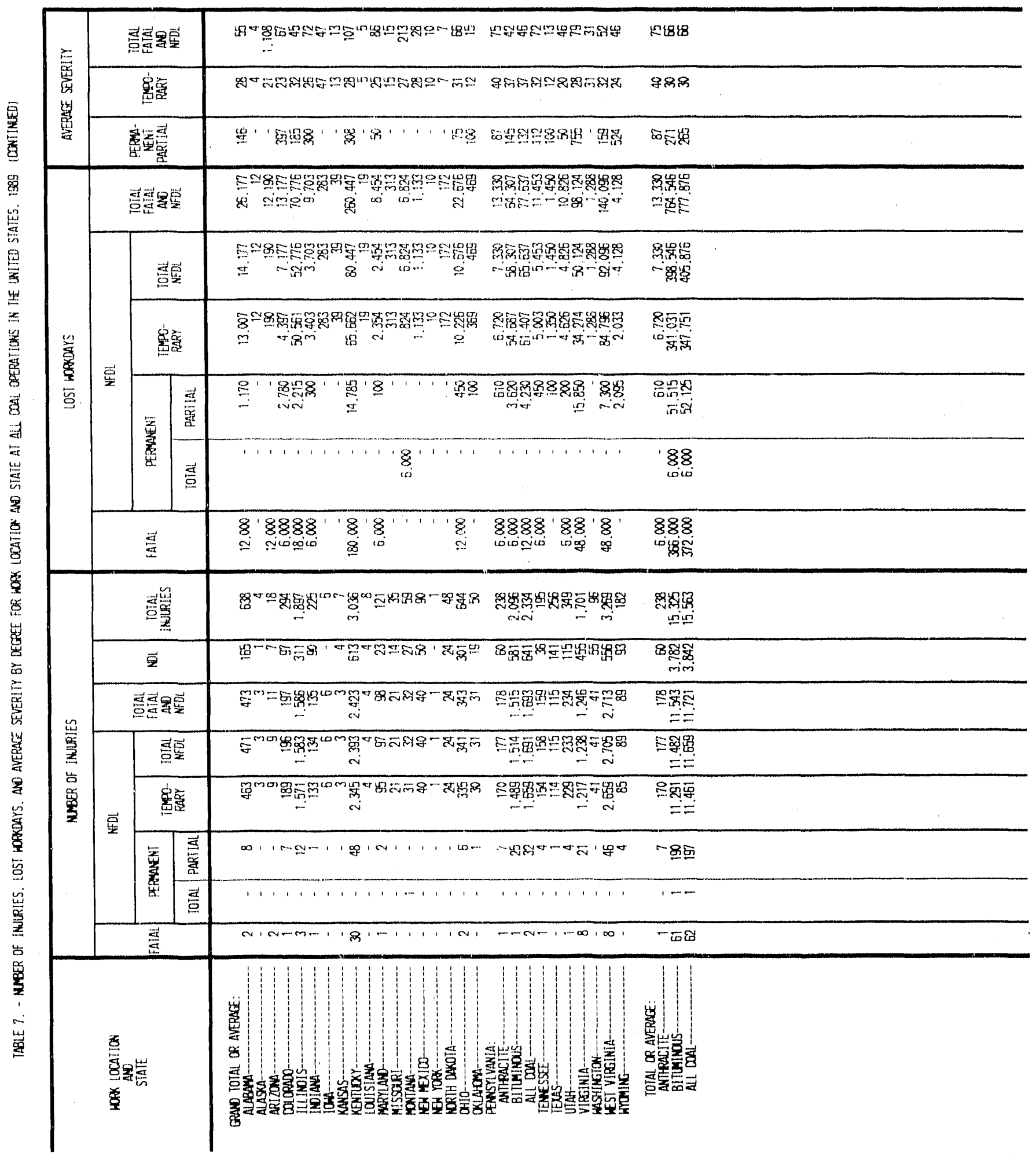




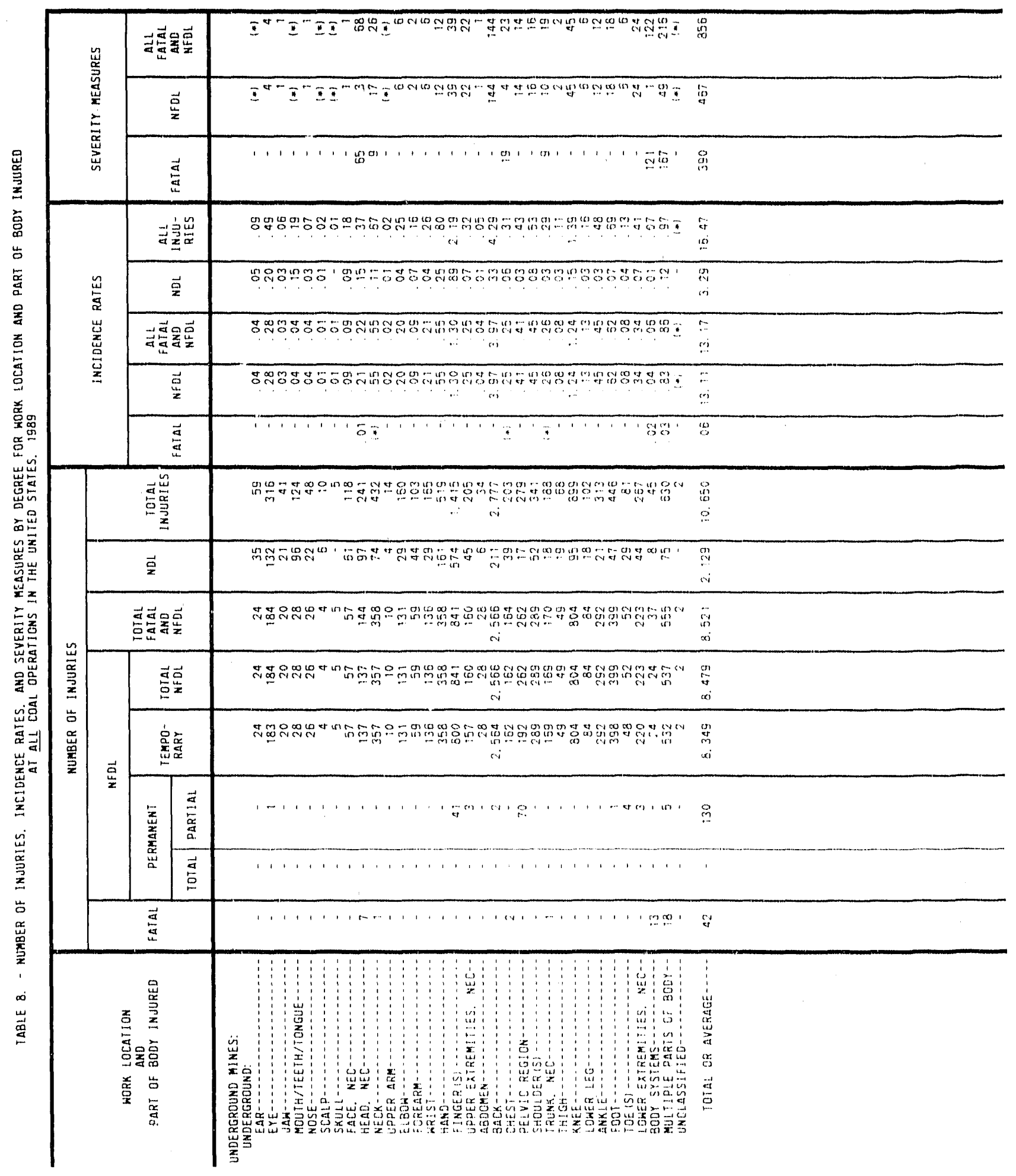




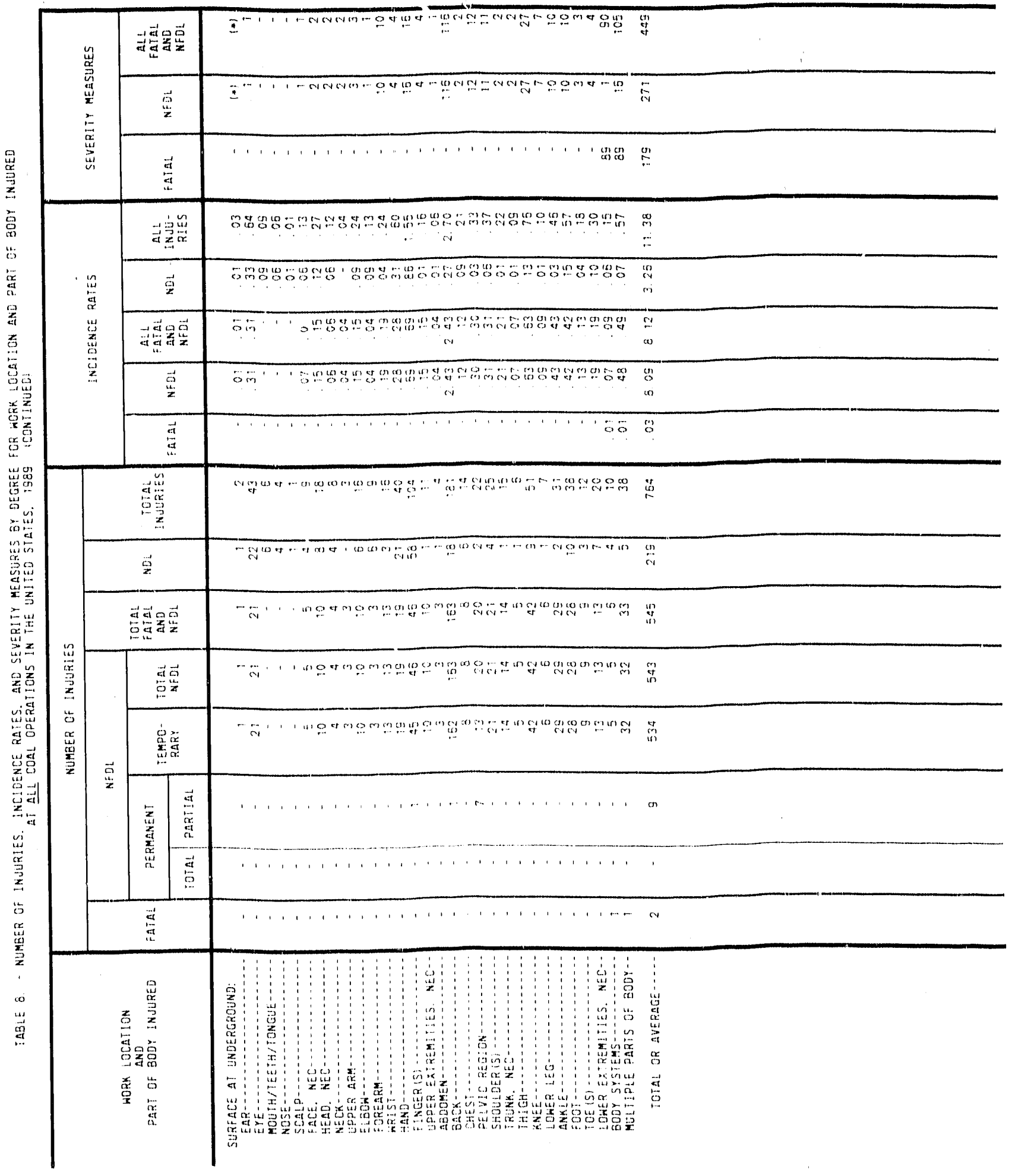




\begin{tabular}{|c|c|c|c|}
\hline \multirow{3}{*}{\multicolumn{2}{|c|}{ 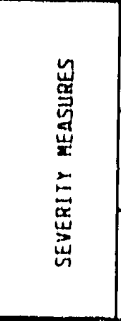 }} & 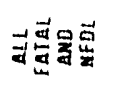 & 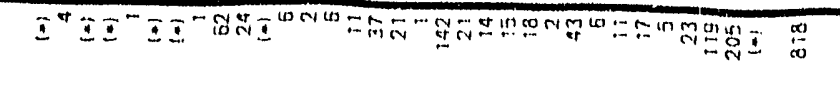 \\
\hline & & 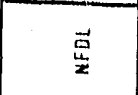 & 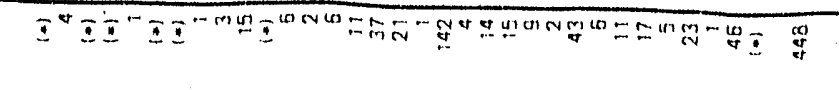 \\
\hline & \multicolumn{2}{|r|}{ 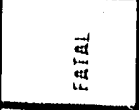 } & 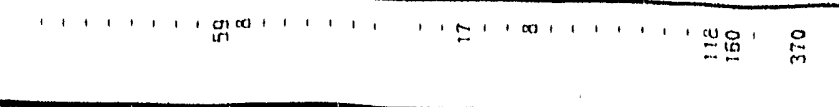 \\
\hline & & 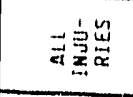 & 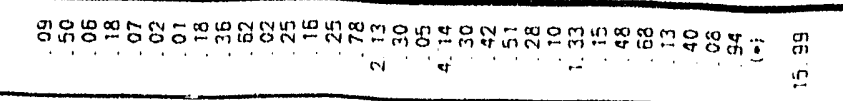 \\
\hline \multirow{4}{*}{\multicolumn{2}{|c|}{ 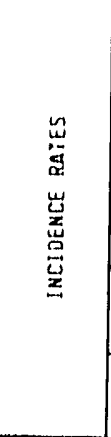 }} & $\overrightarrow{\underline{z}}$ & 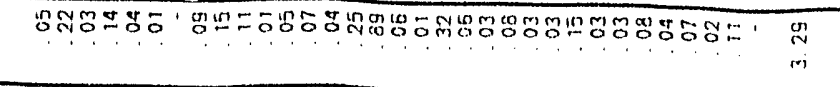 \\
\hline & & 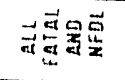 & 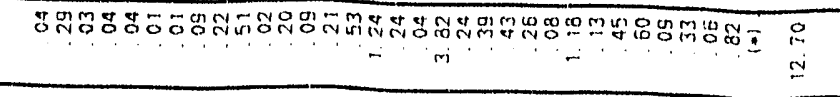 \\
\hline & \multicolumn{2}{|r|}{ 豆 } & tong \\
\hline & & $\frac{\vec{a}}{4}$ & $I^{\prime} I^{\prime}$ \\
\hline \multirow{7}{*}{\multicolumn{2}{|c|}{ 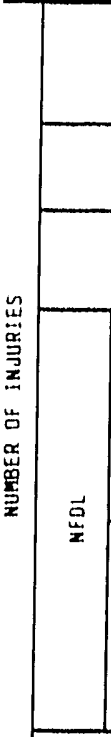 }} & & 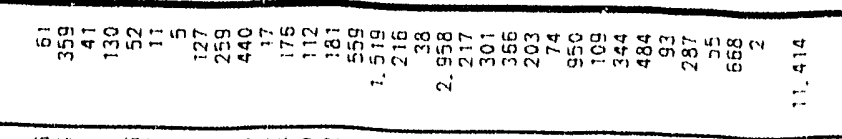 \\
\hline & & $\overrightarrow{\underline{\alpha}}$ & 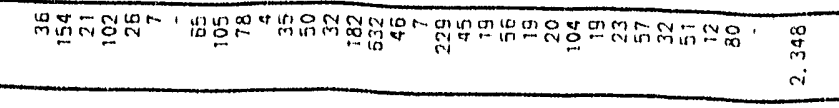 \\
\hline & \multicolumn{2}{|c|}{ 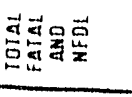 } & 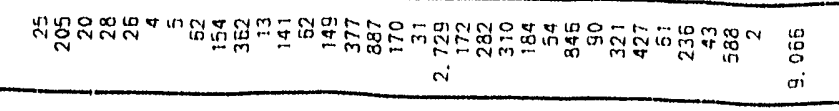 \\
\hline & & 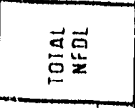 & 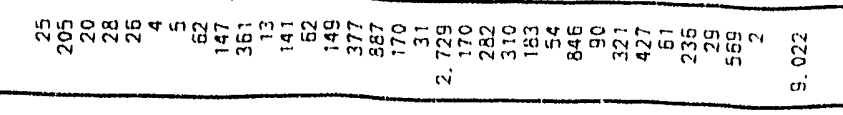 \\
\hline & & 它事 & 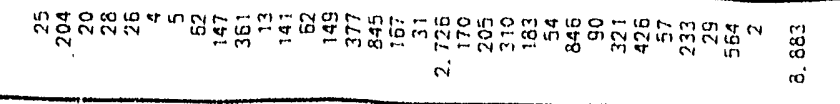 \\
\hline & & 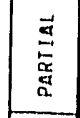 & m，惫 \\
\hline & & 可 & 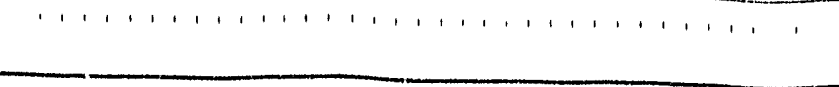 \\
\hline & & 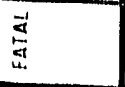 & $\pi$ \\
\hline & 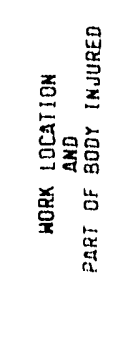 & & 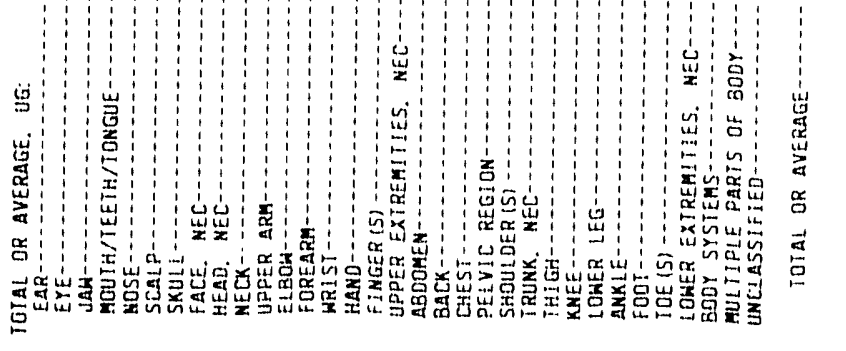 \\
\hline
\end{tabular}




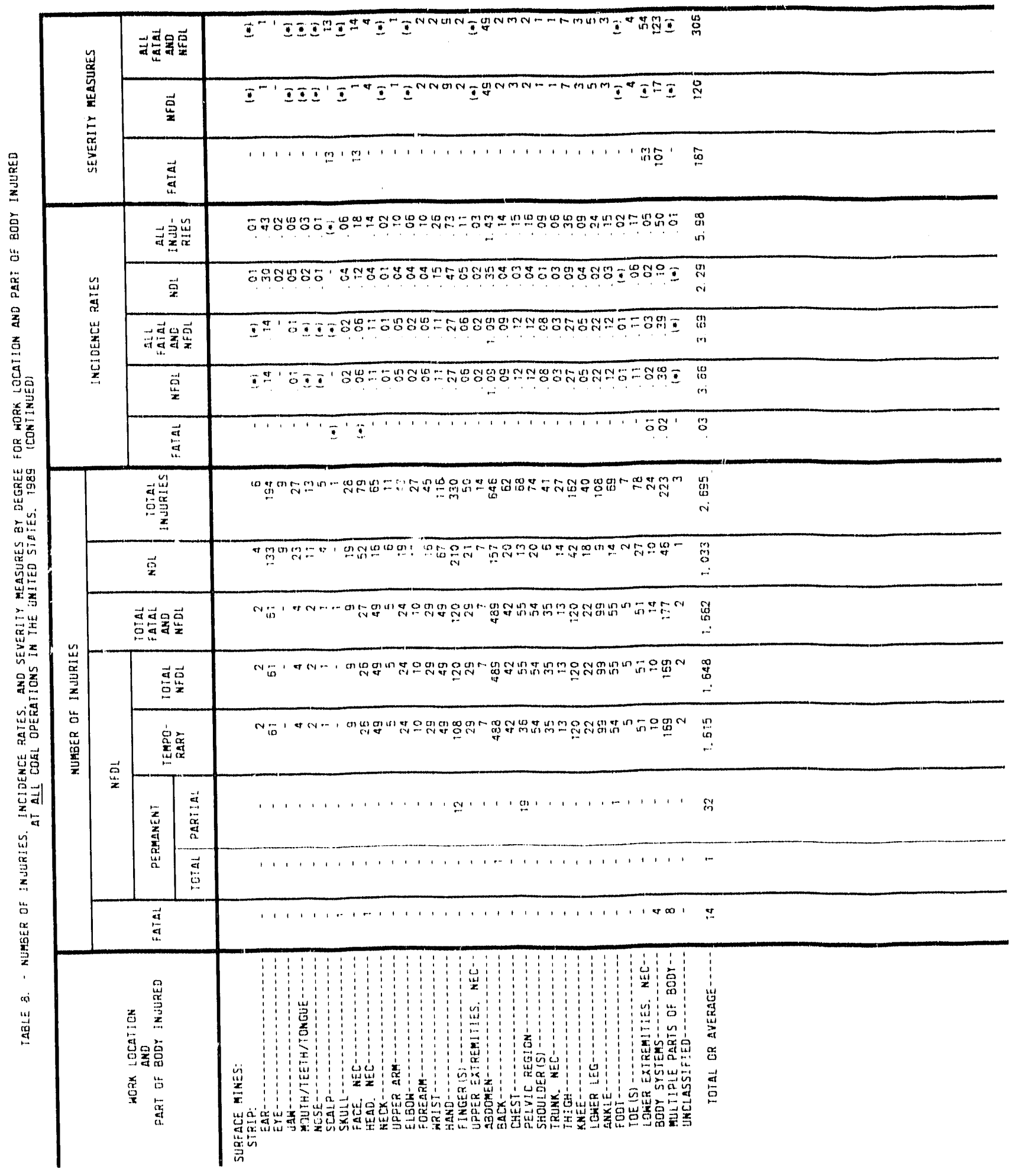




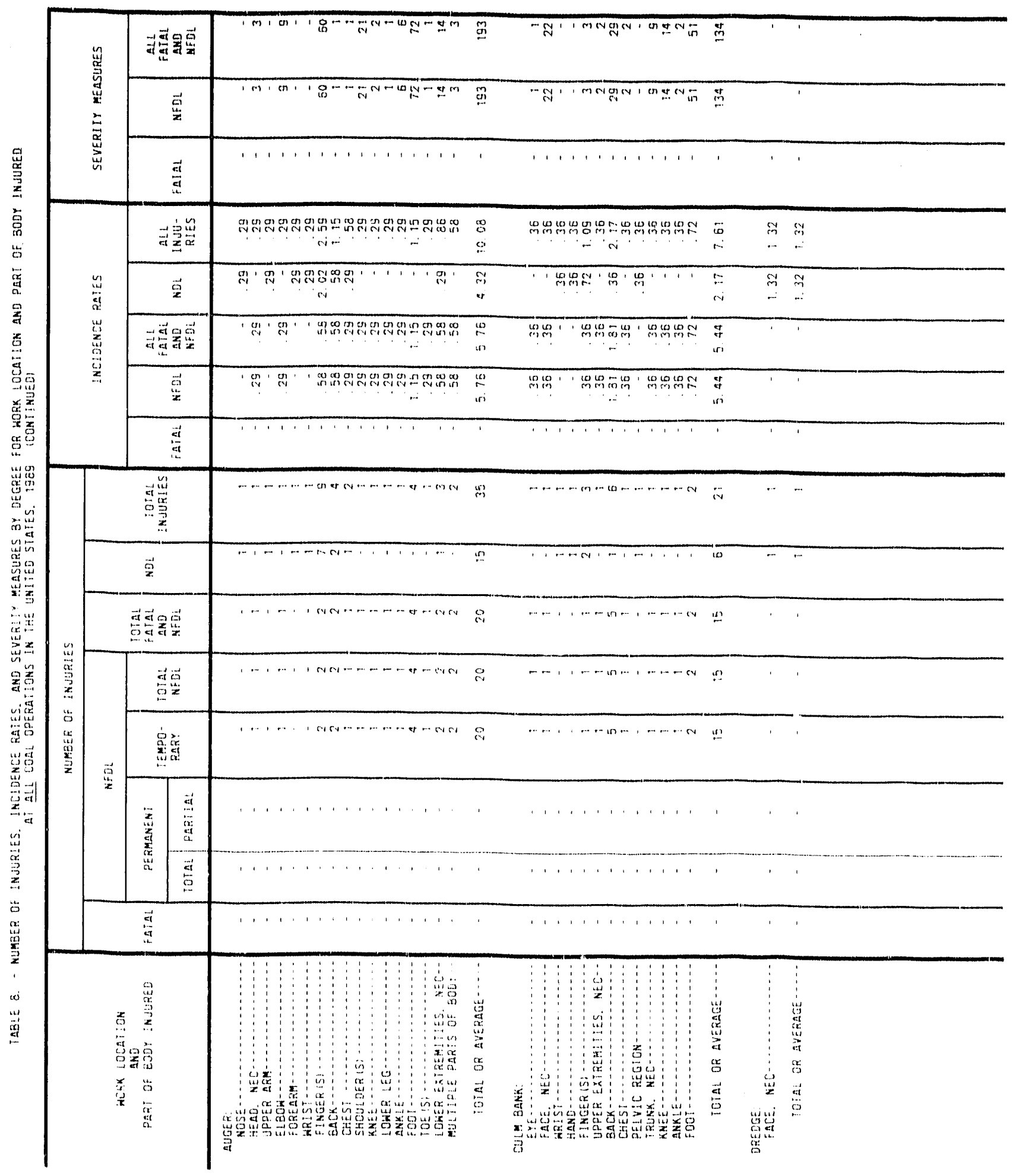




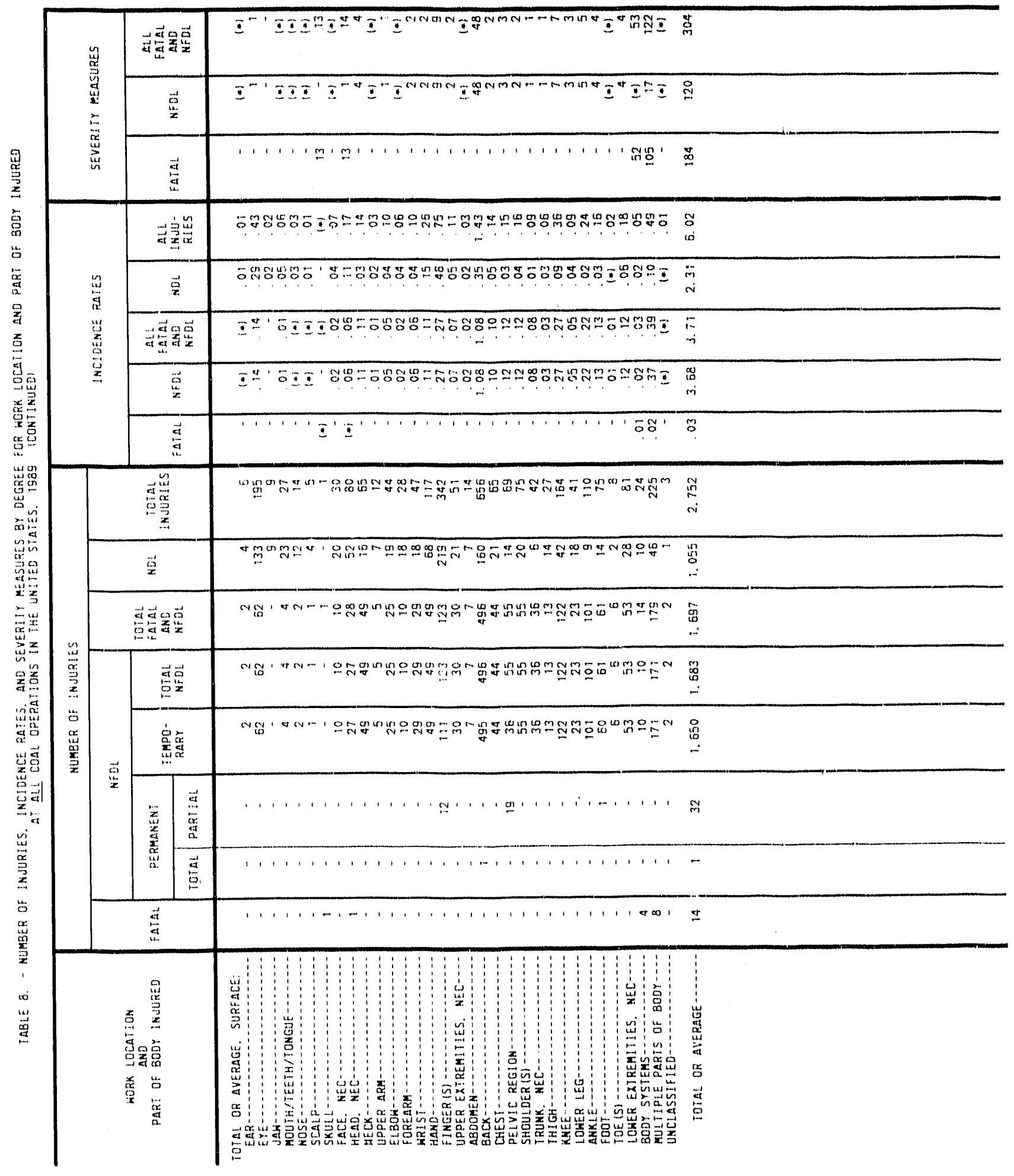




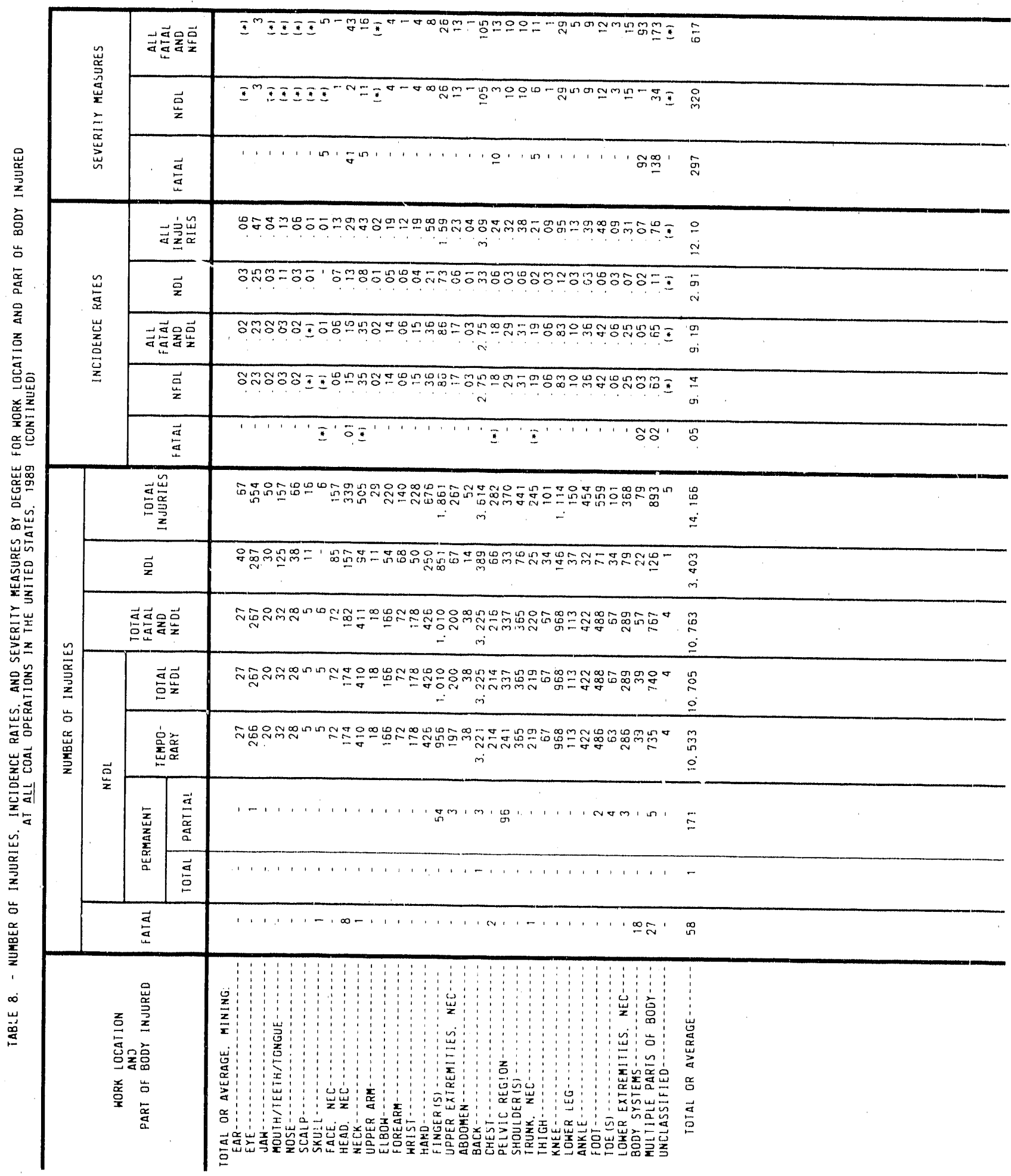




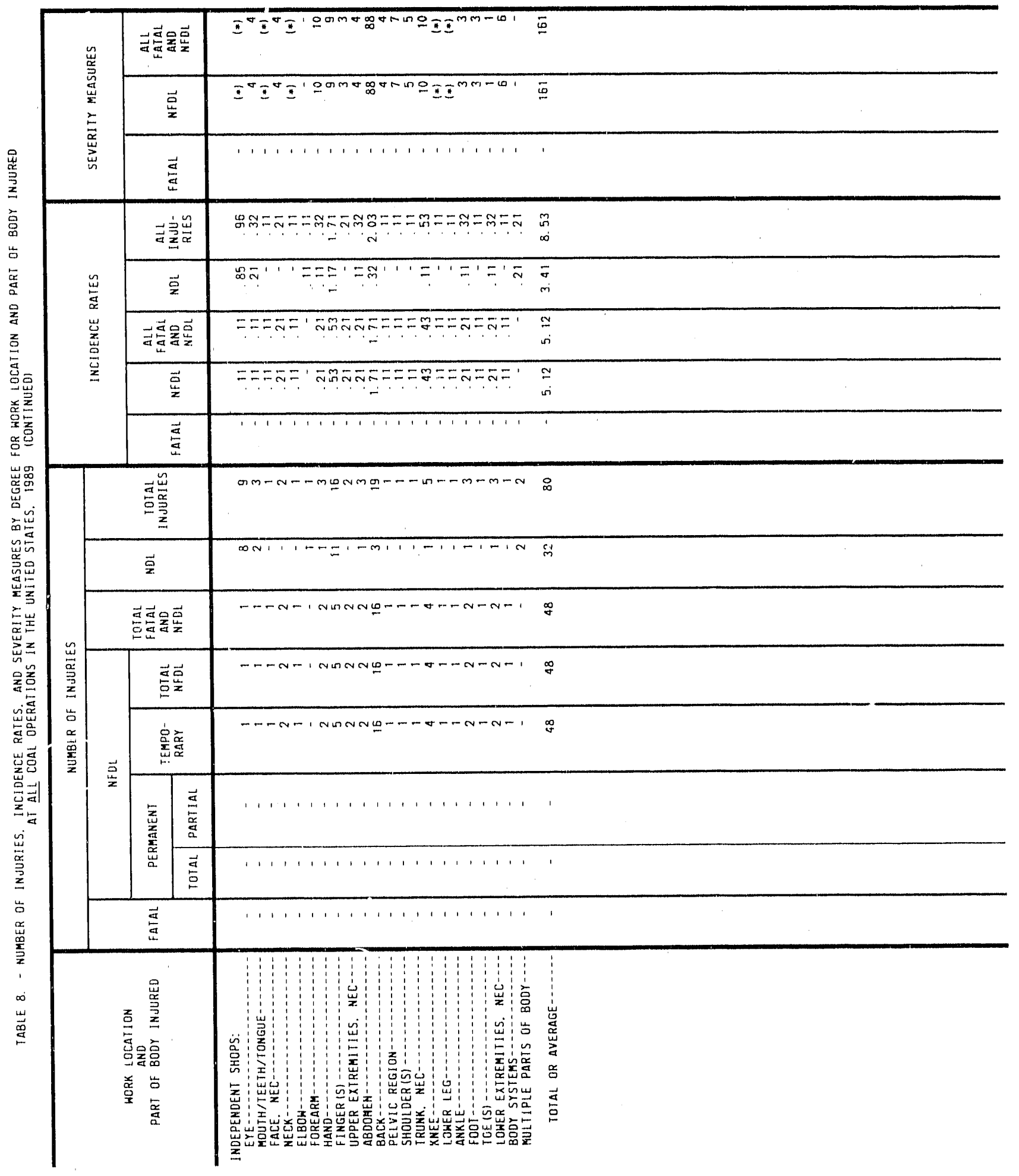




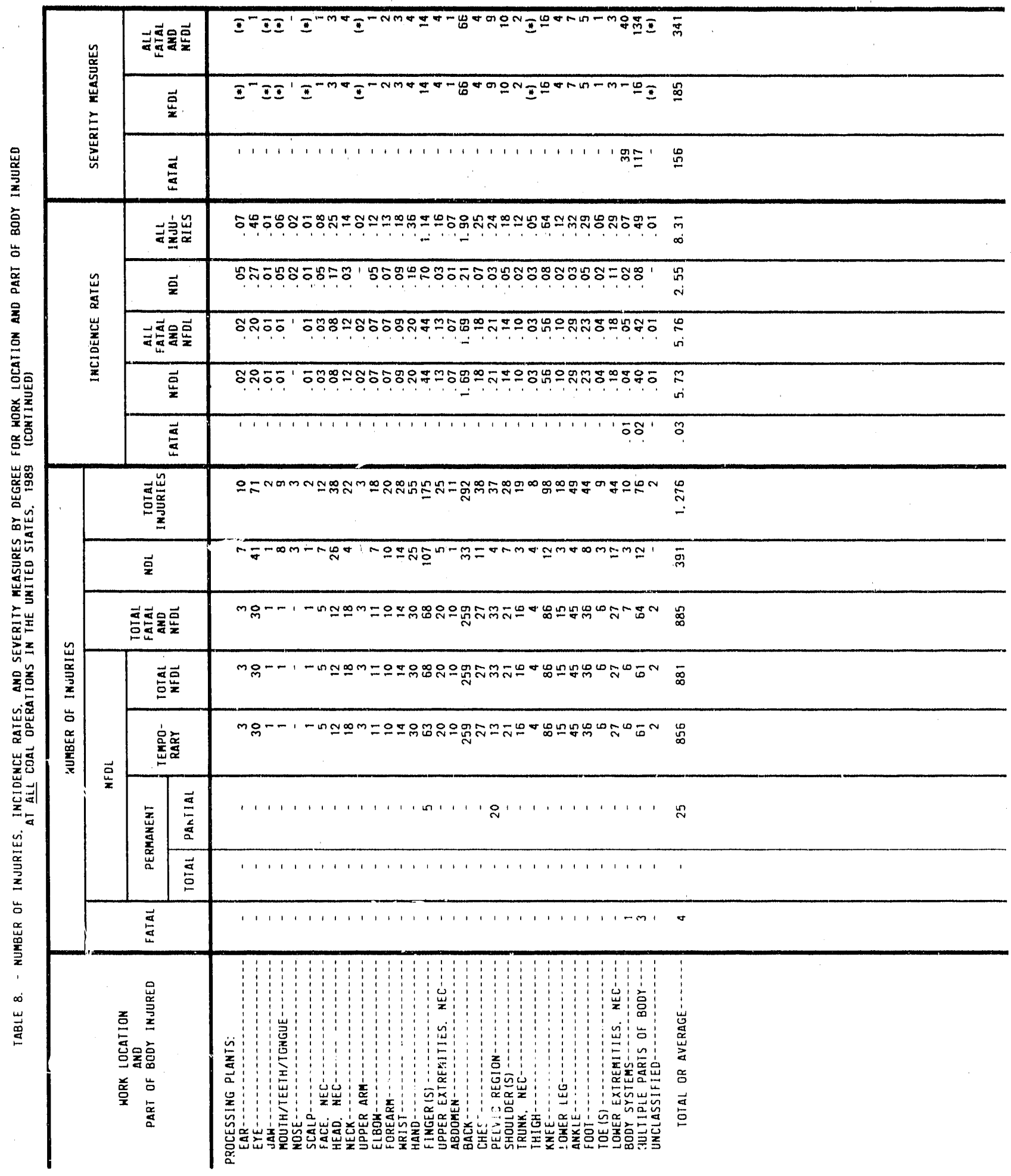




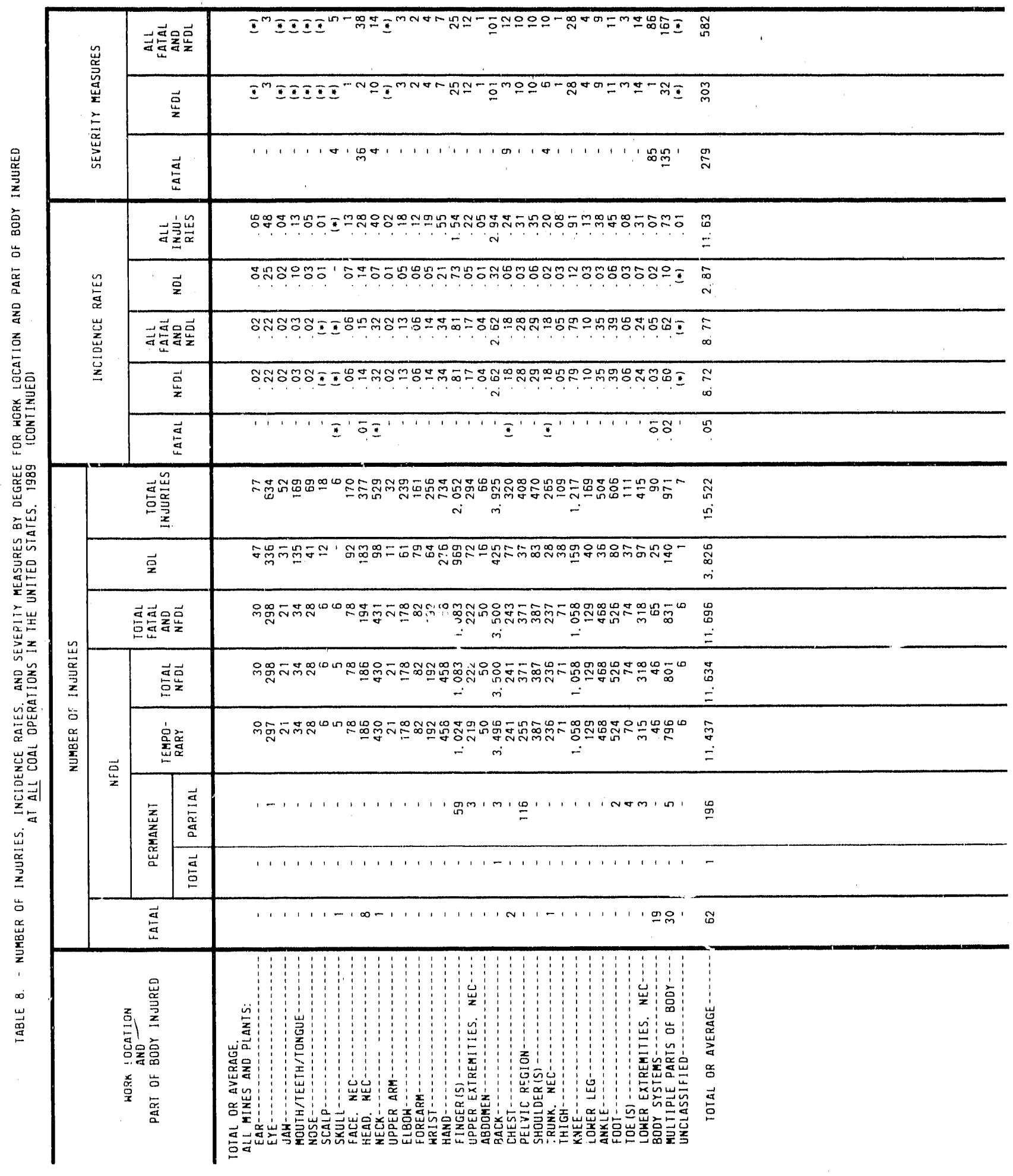




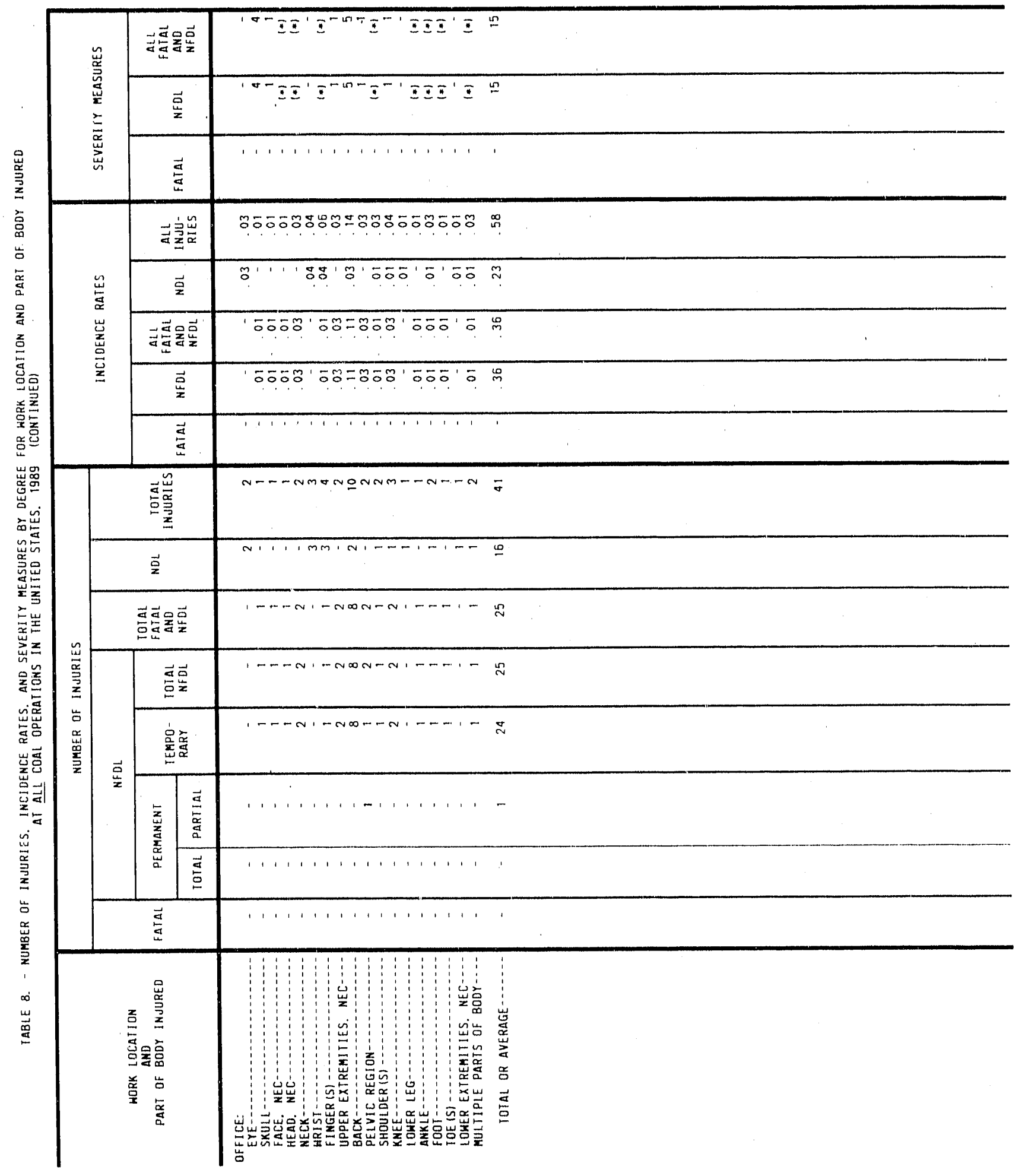




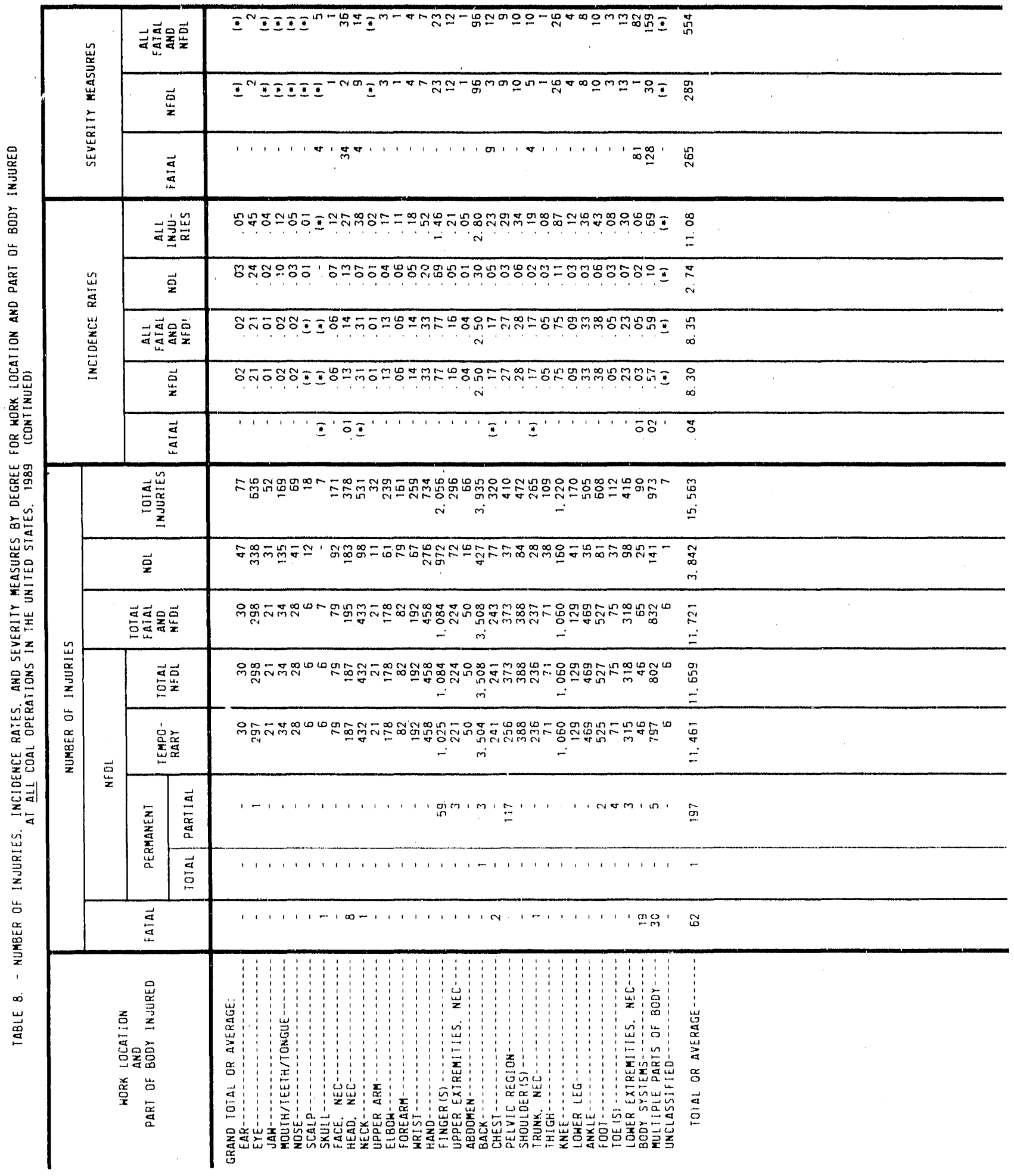




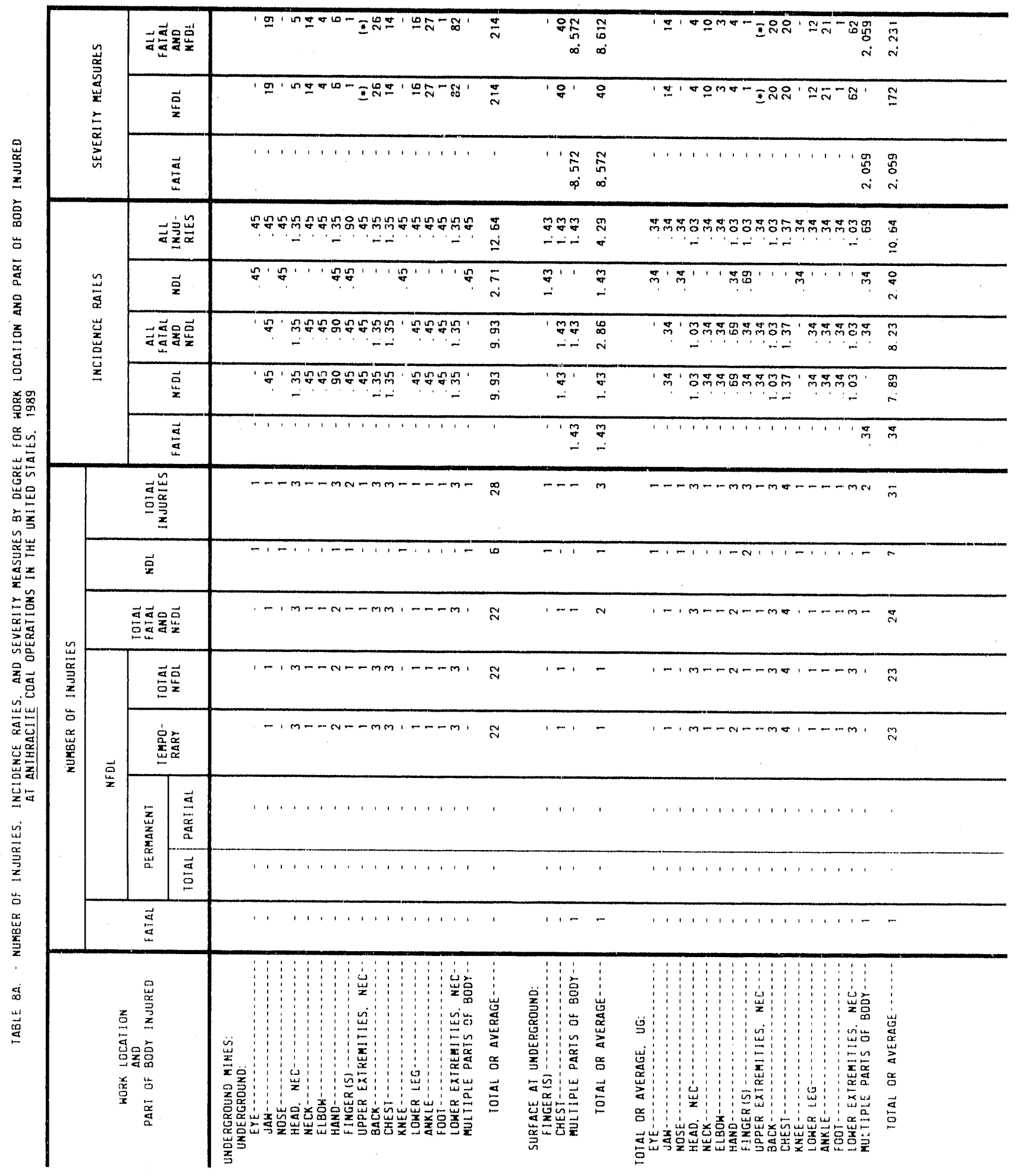




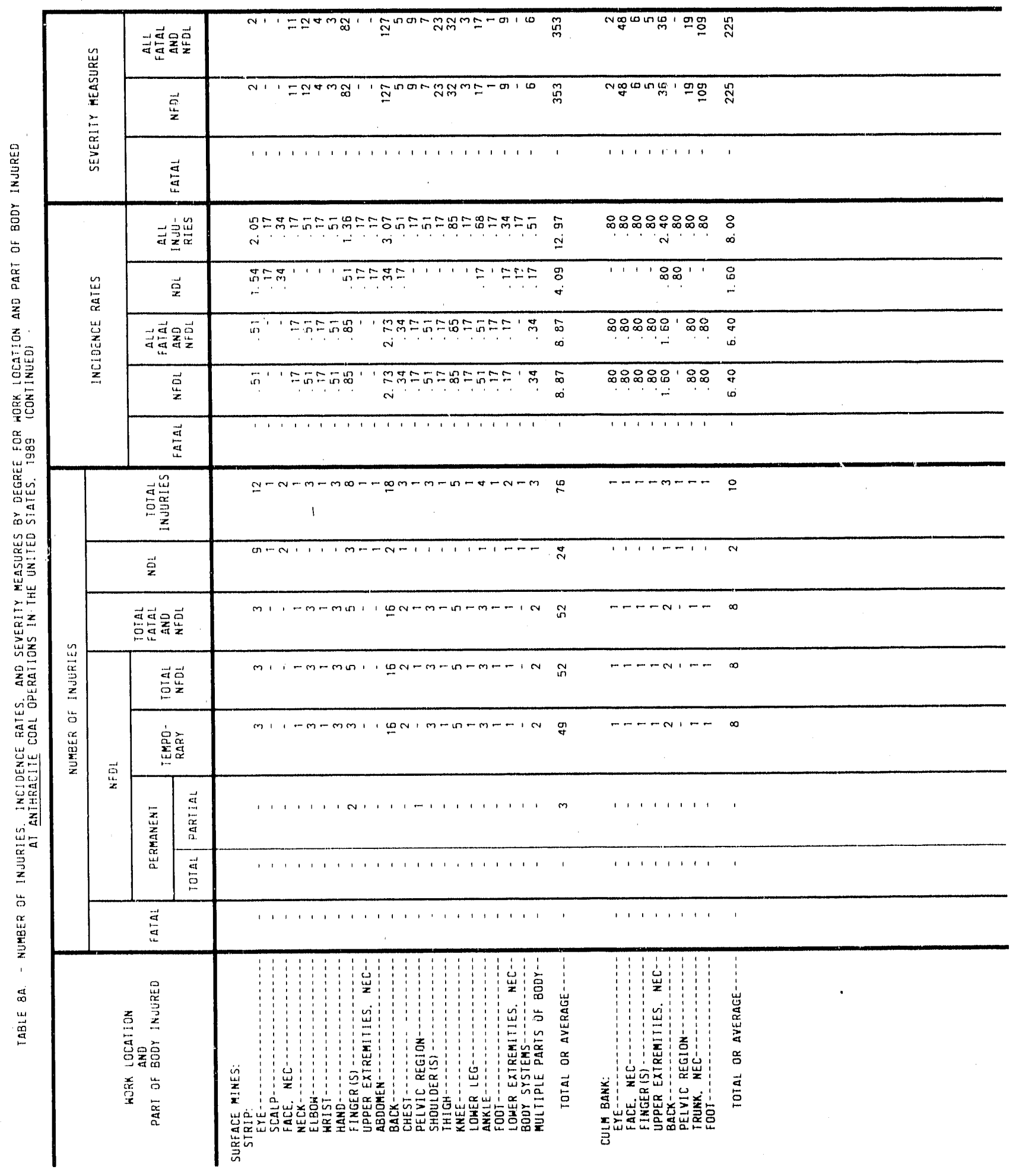




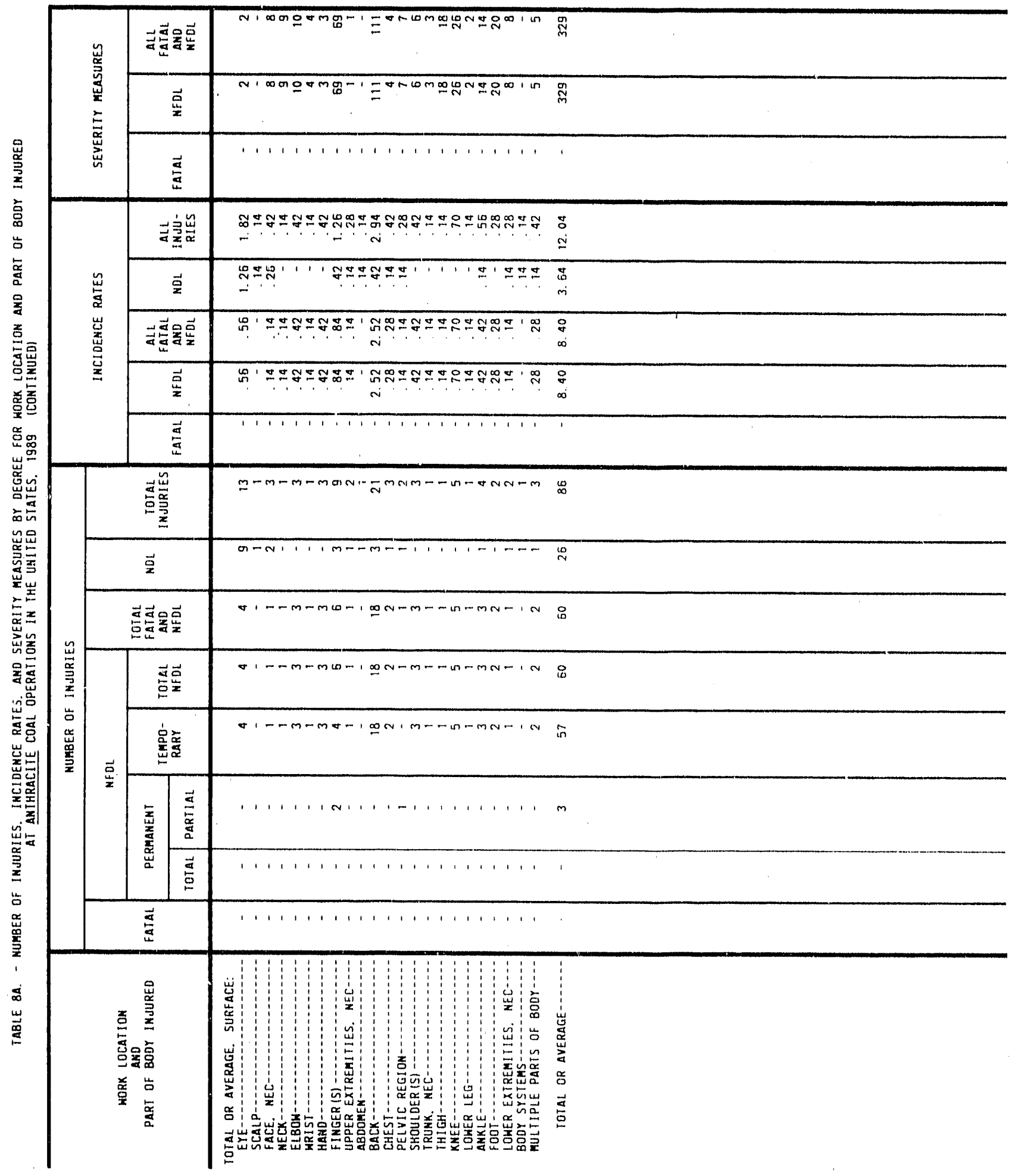




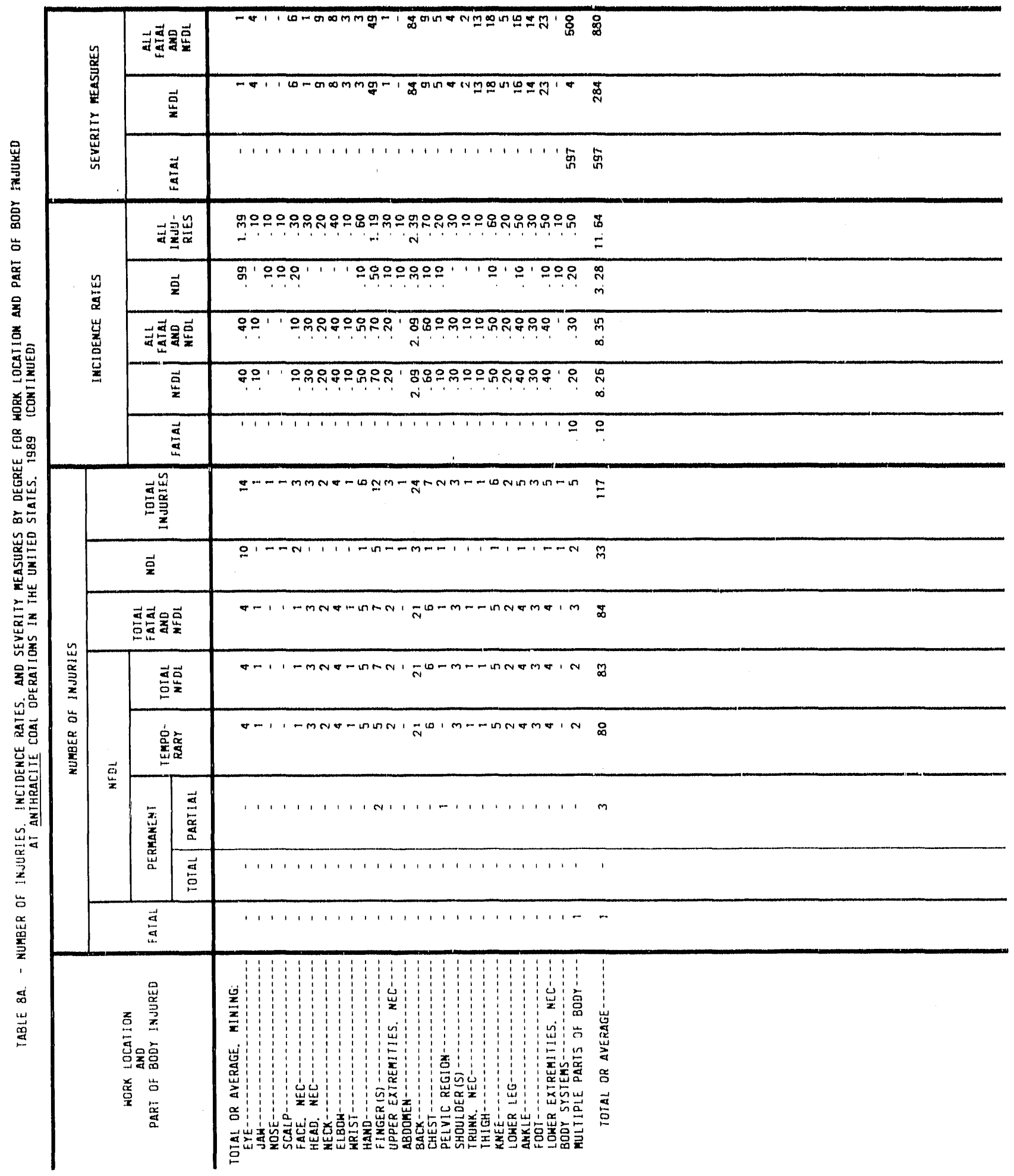




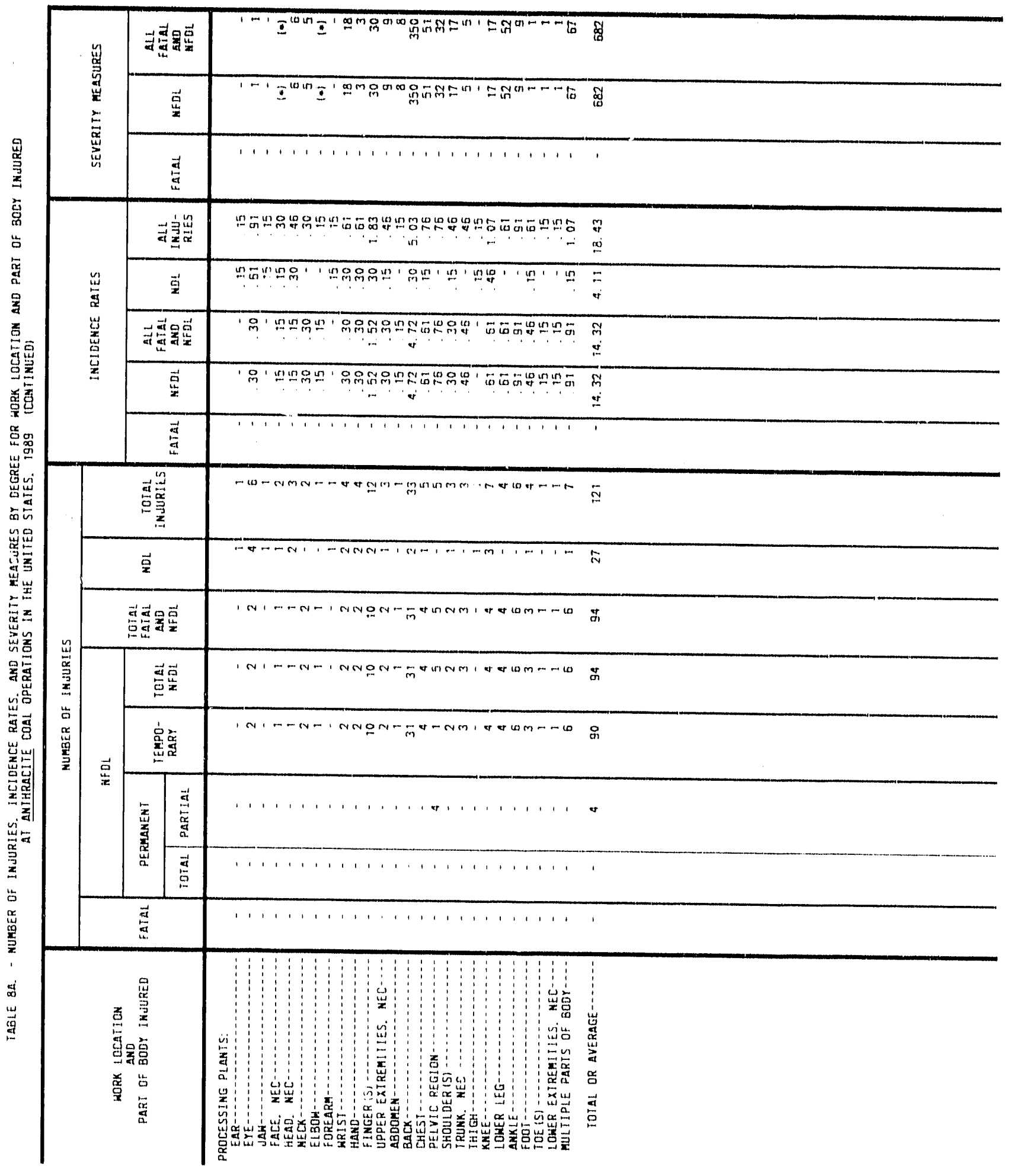




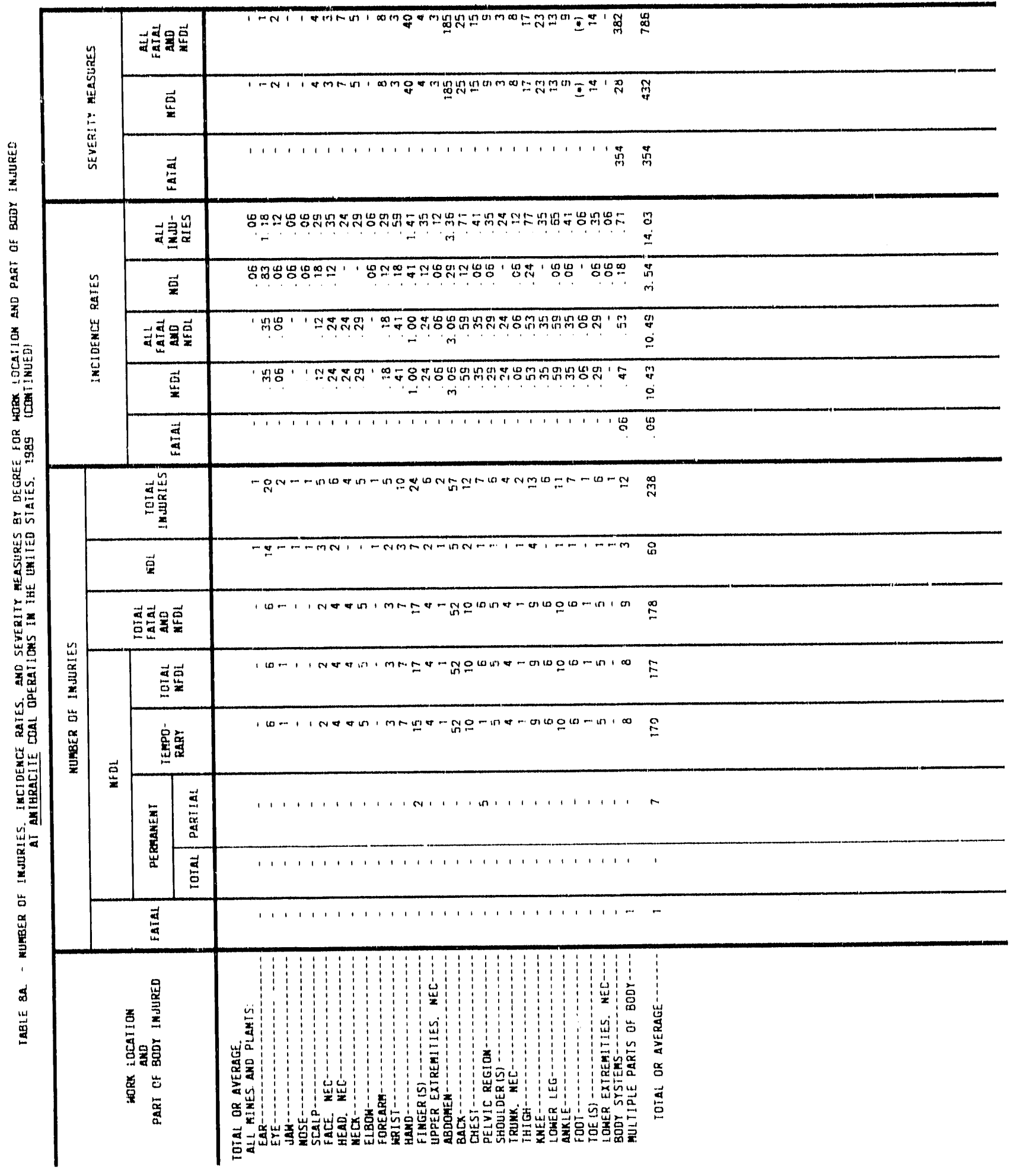




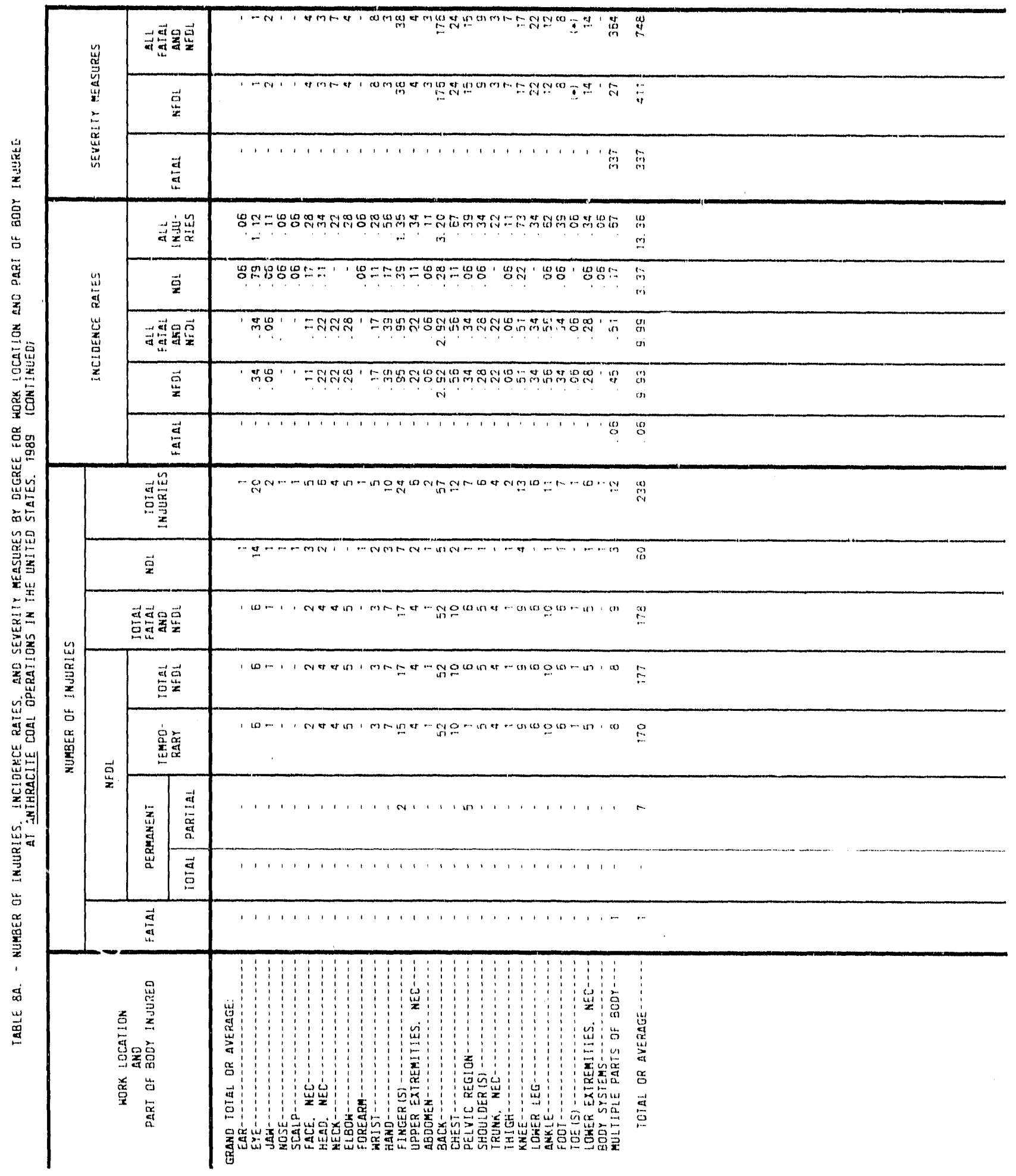




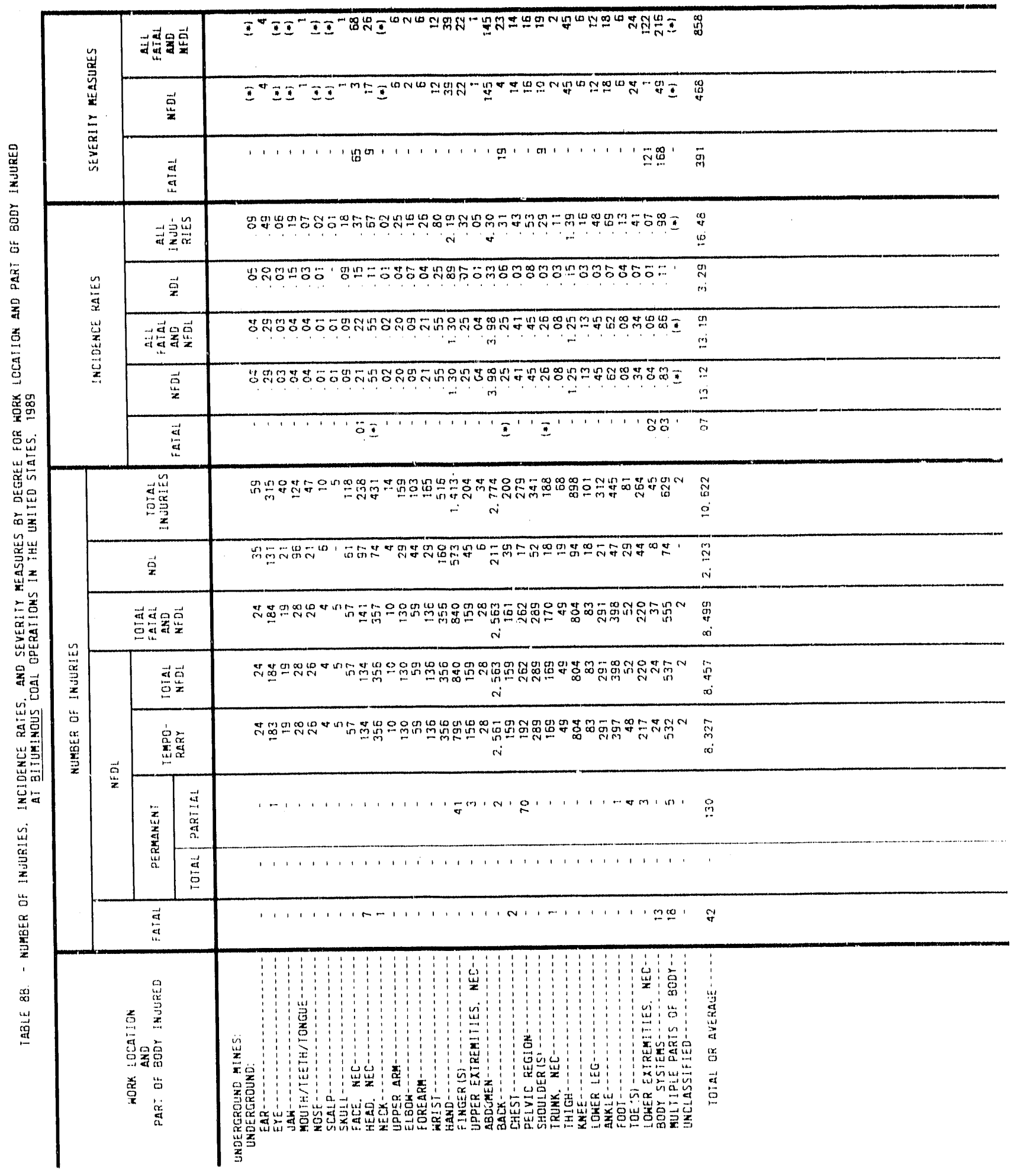




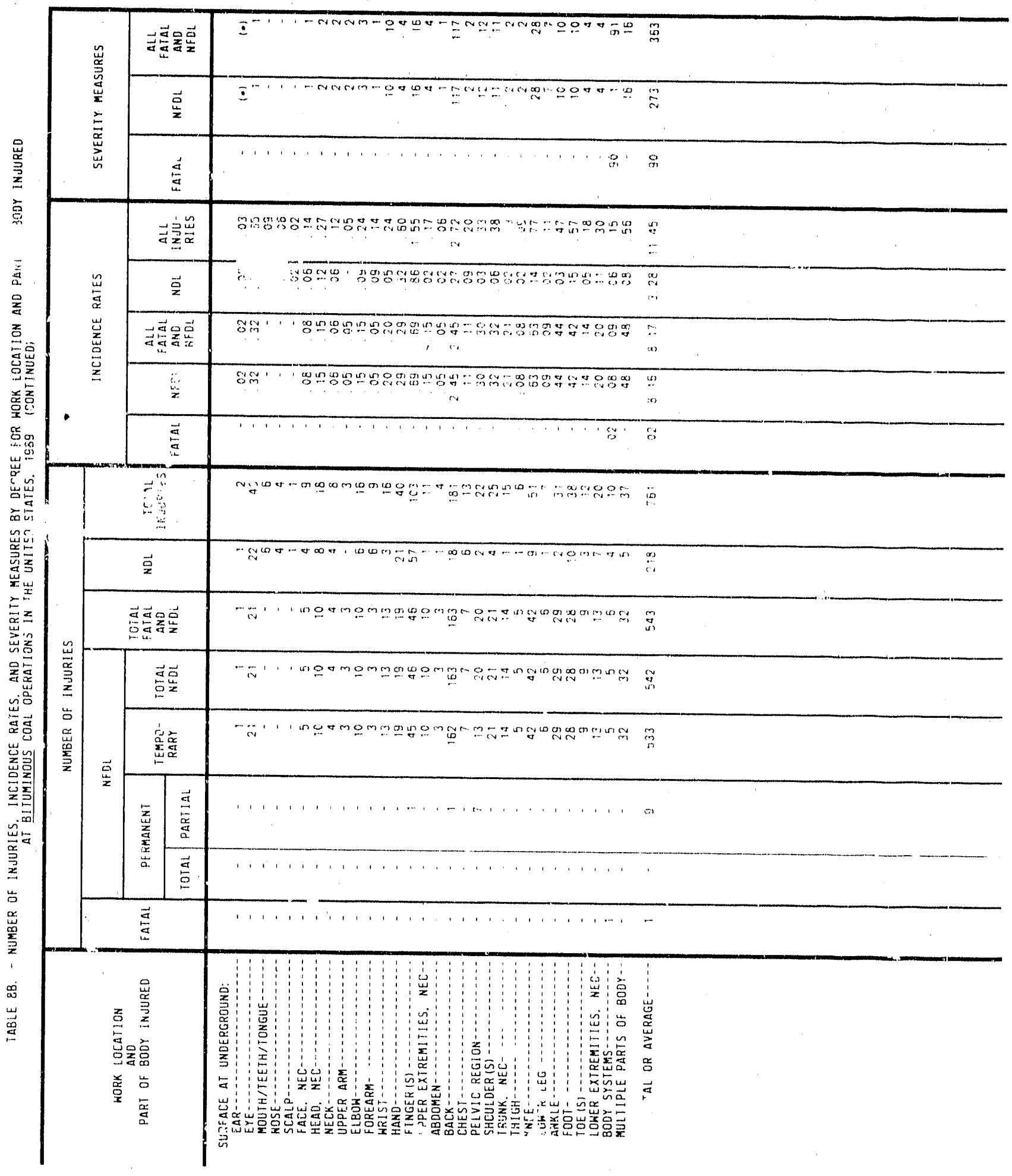




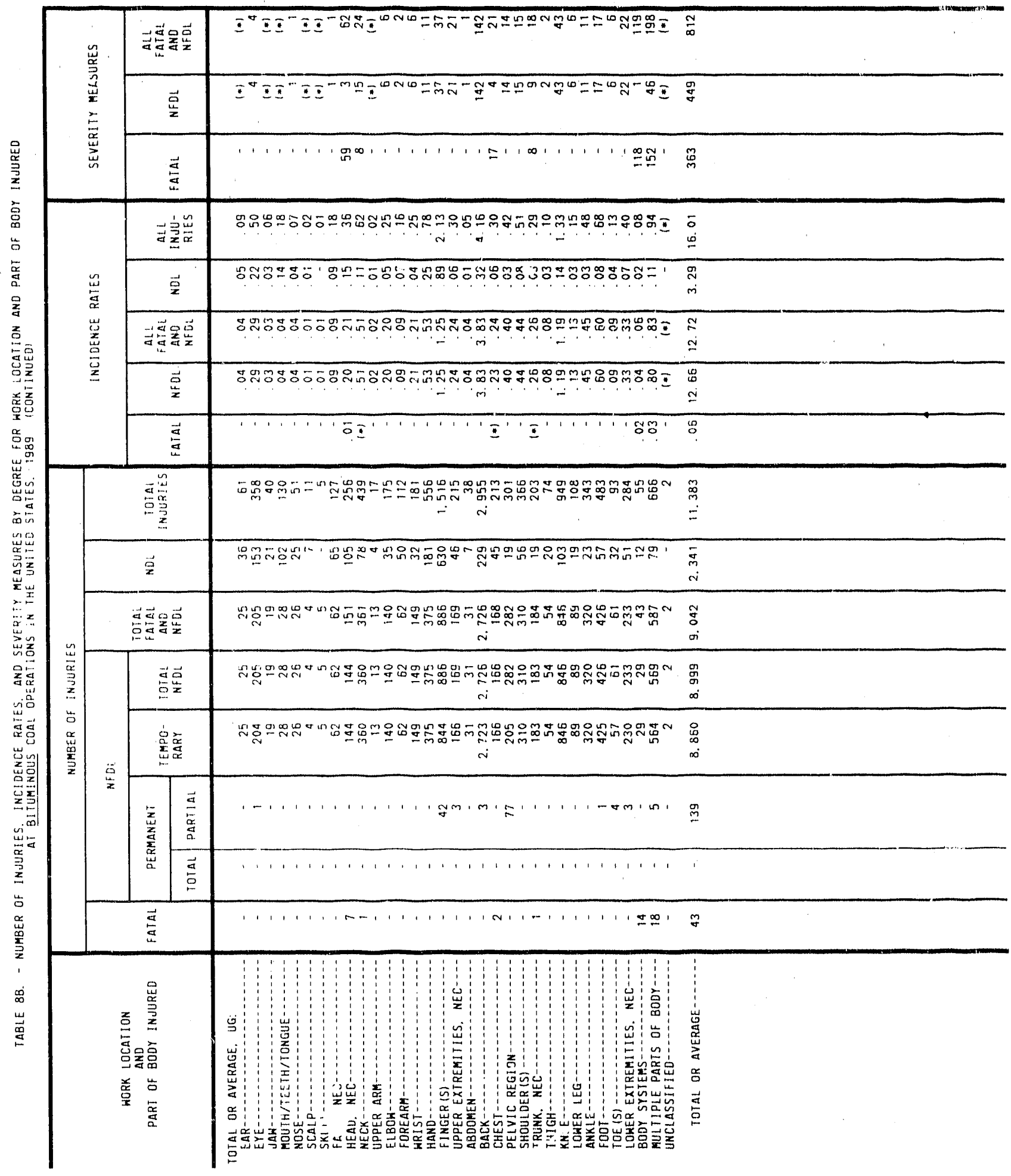




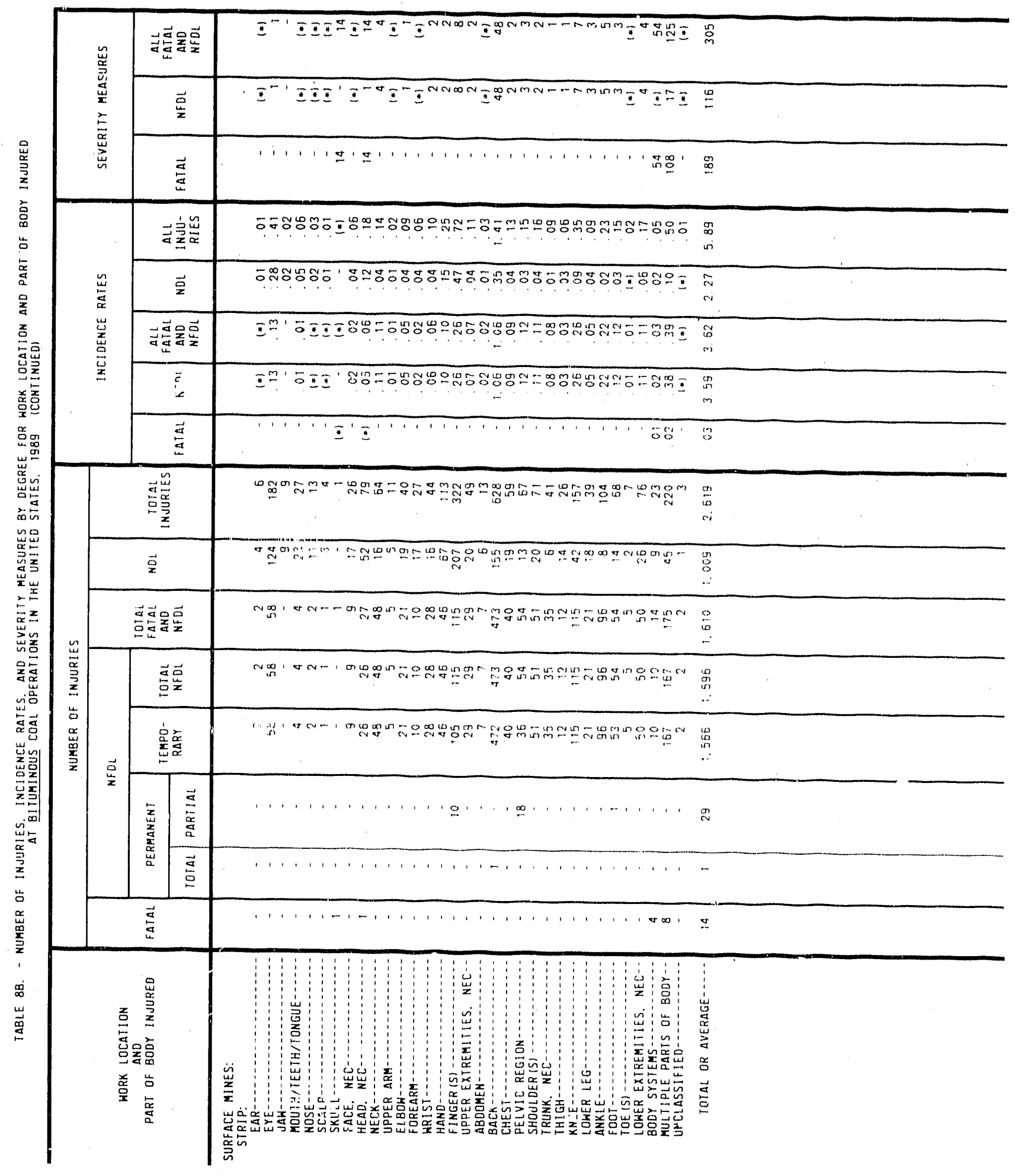




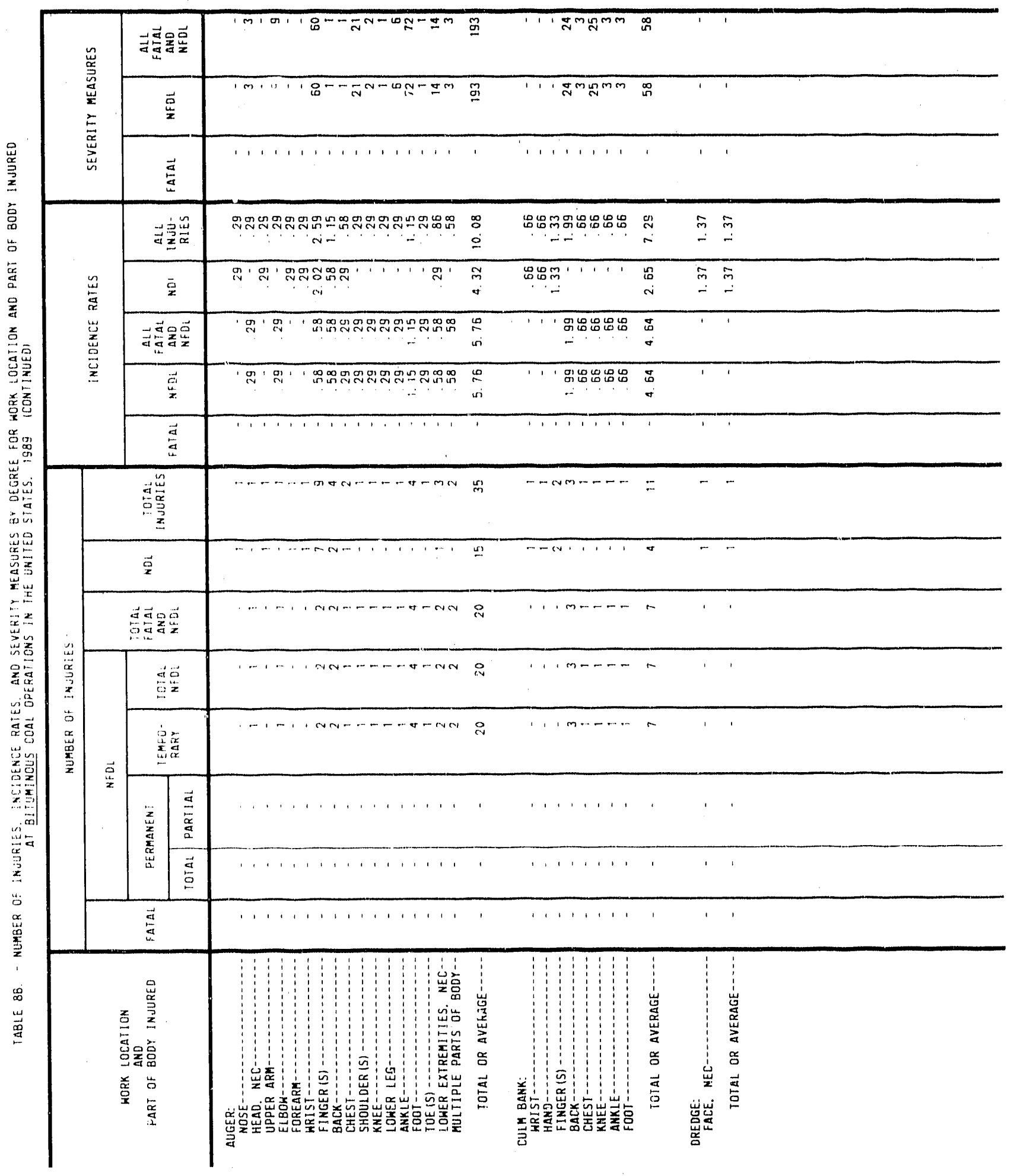




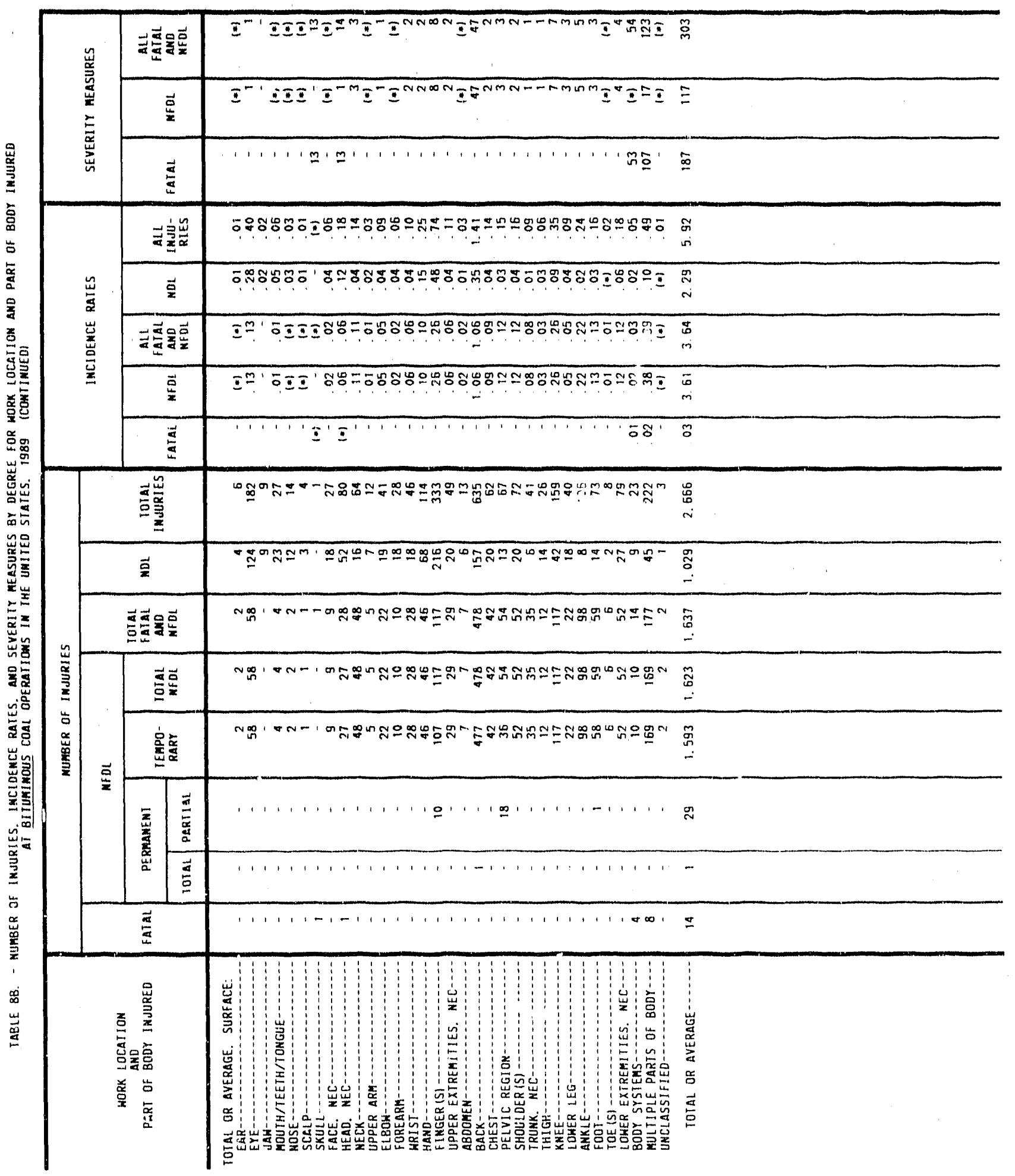




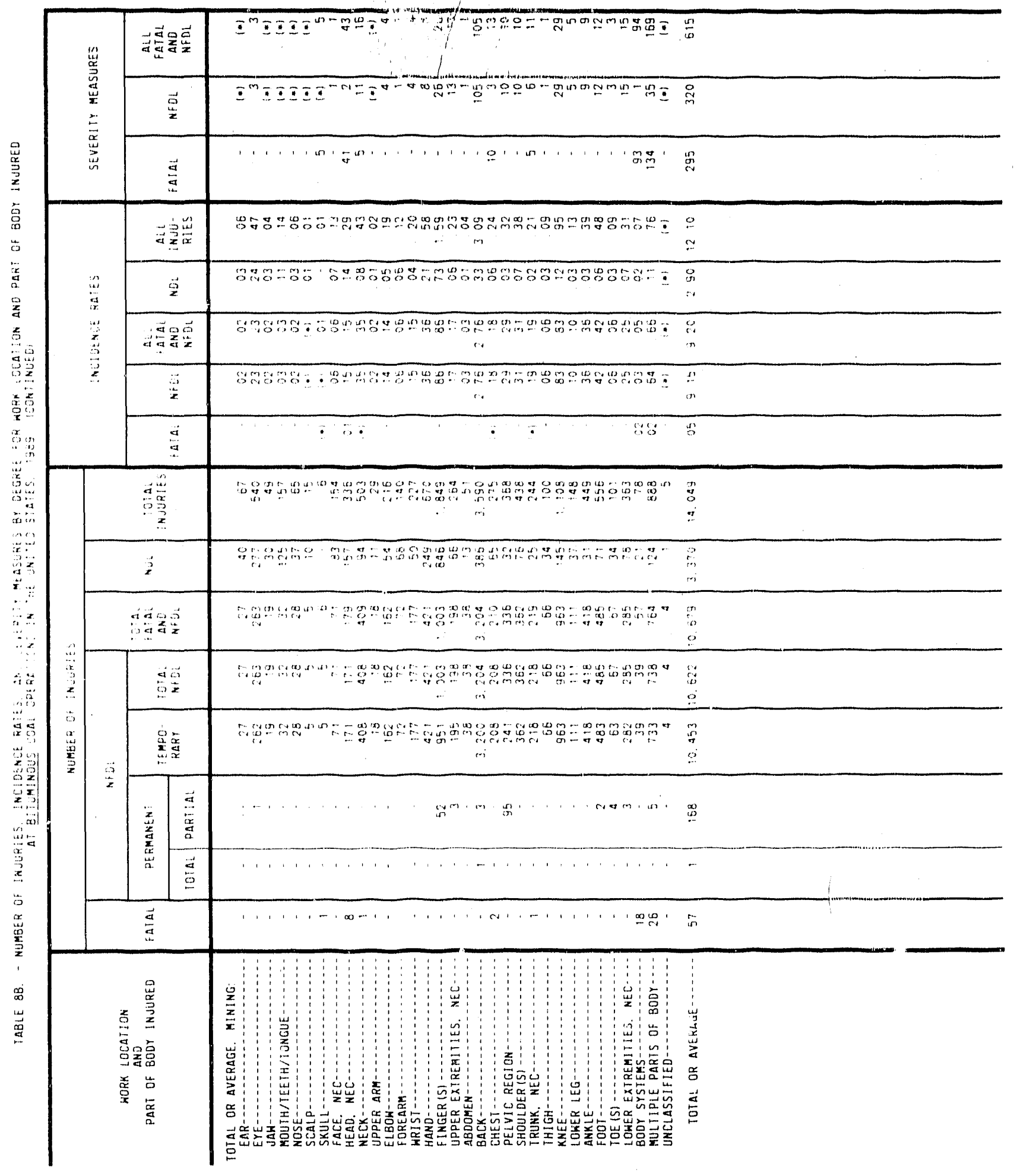




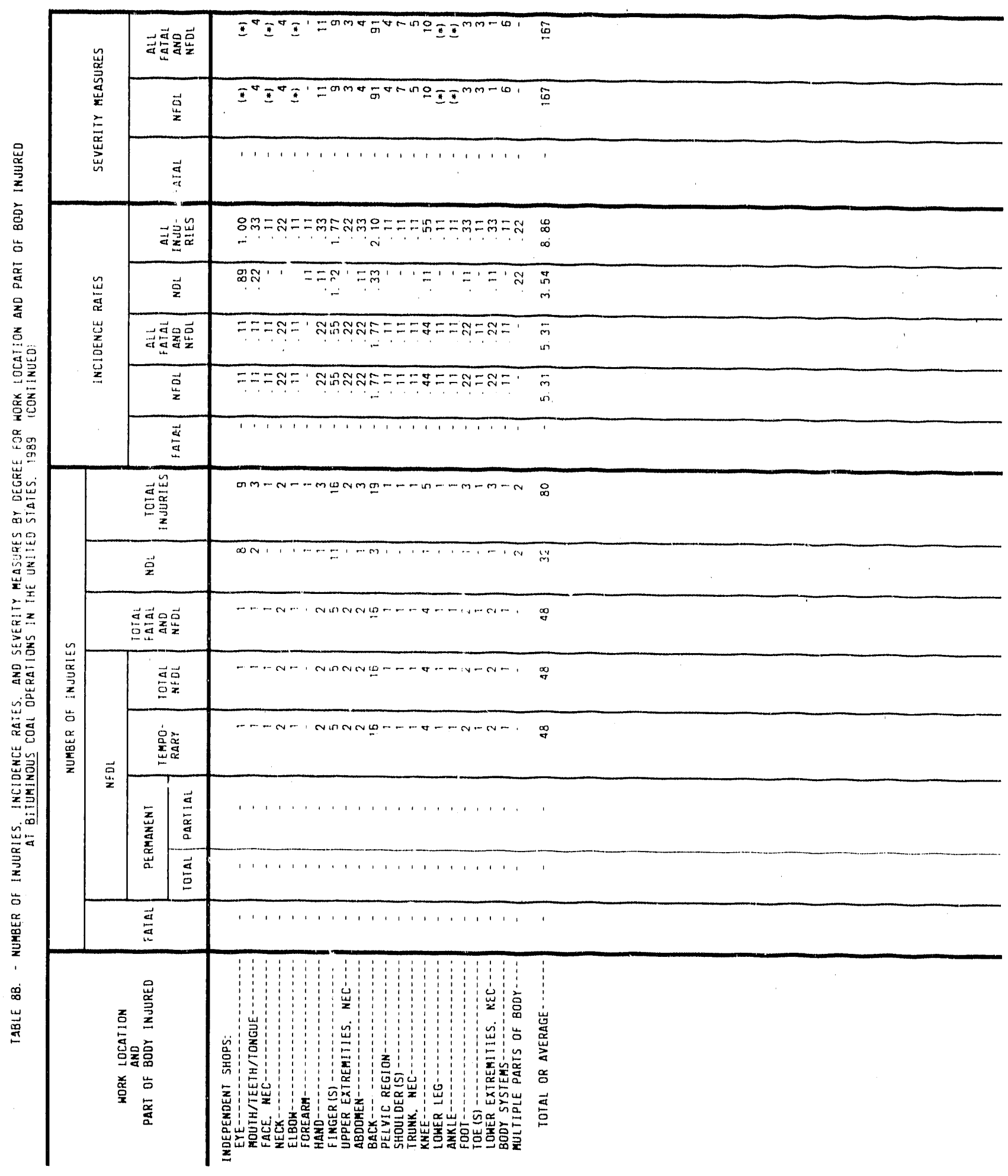




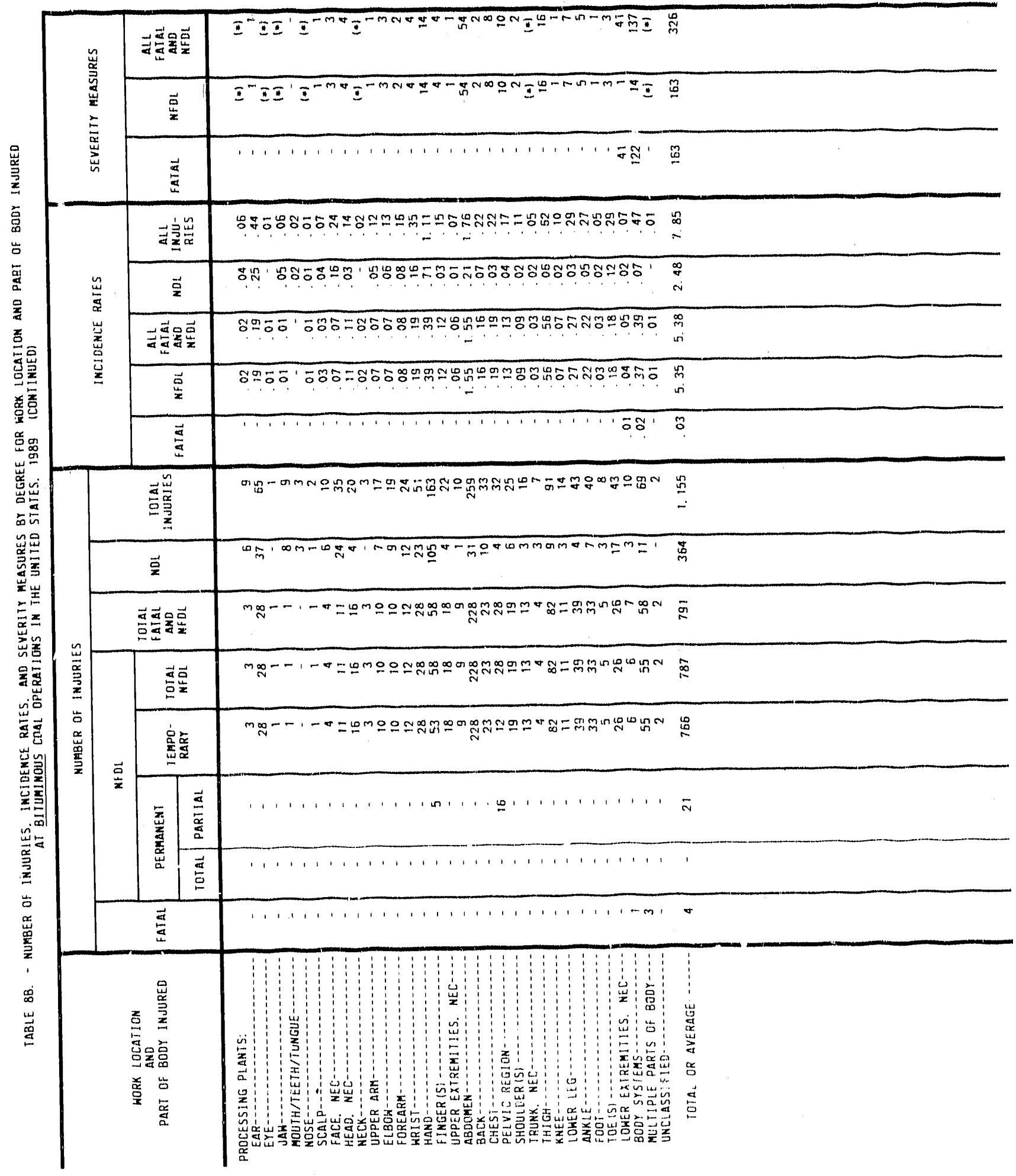




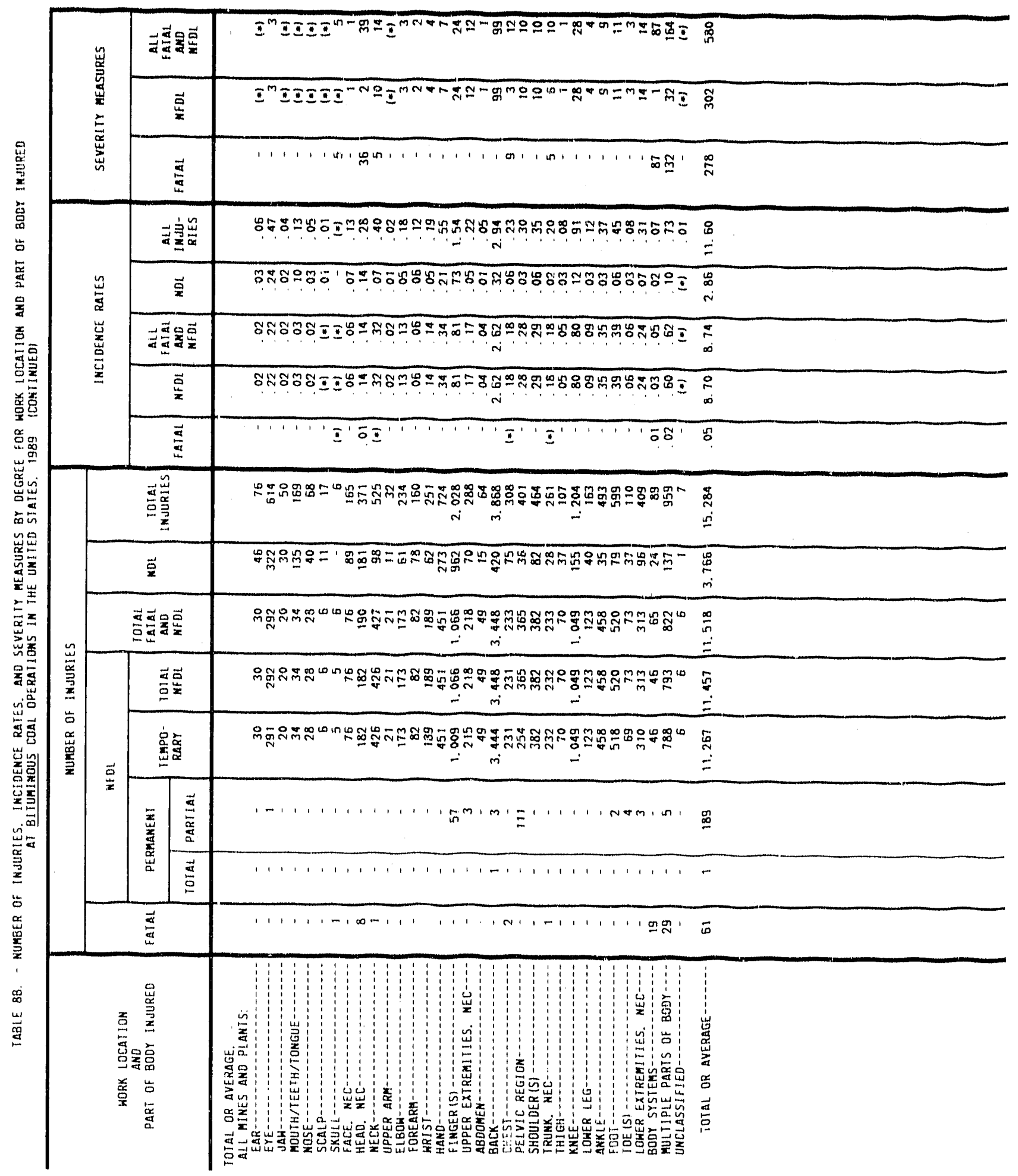




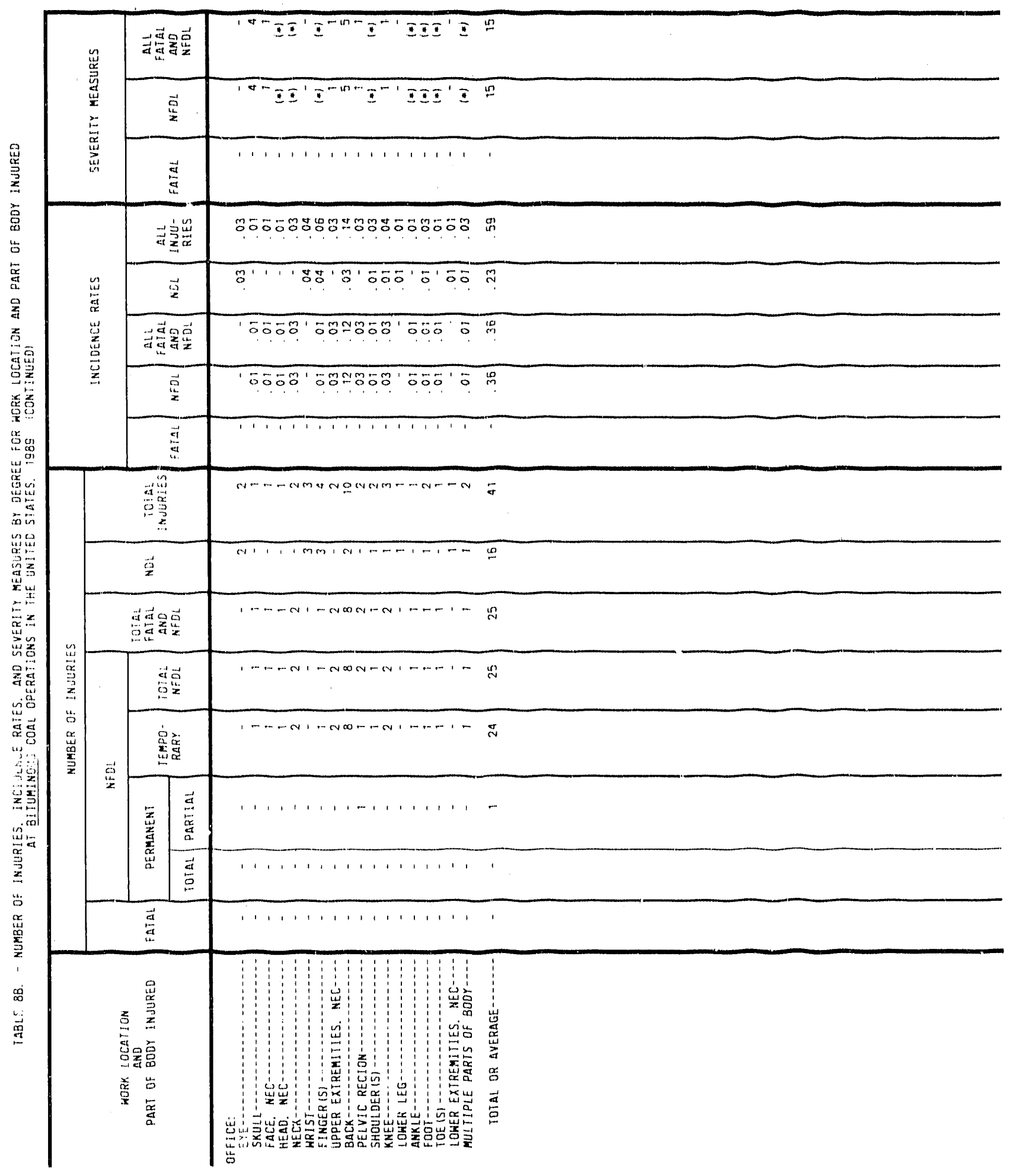




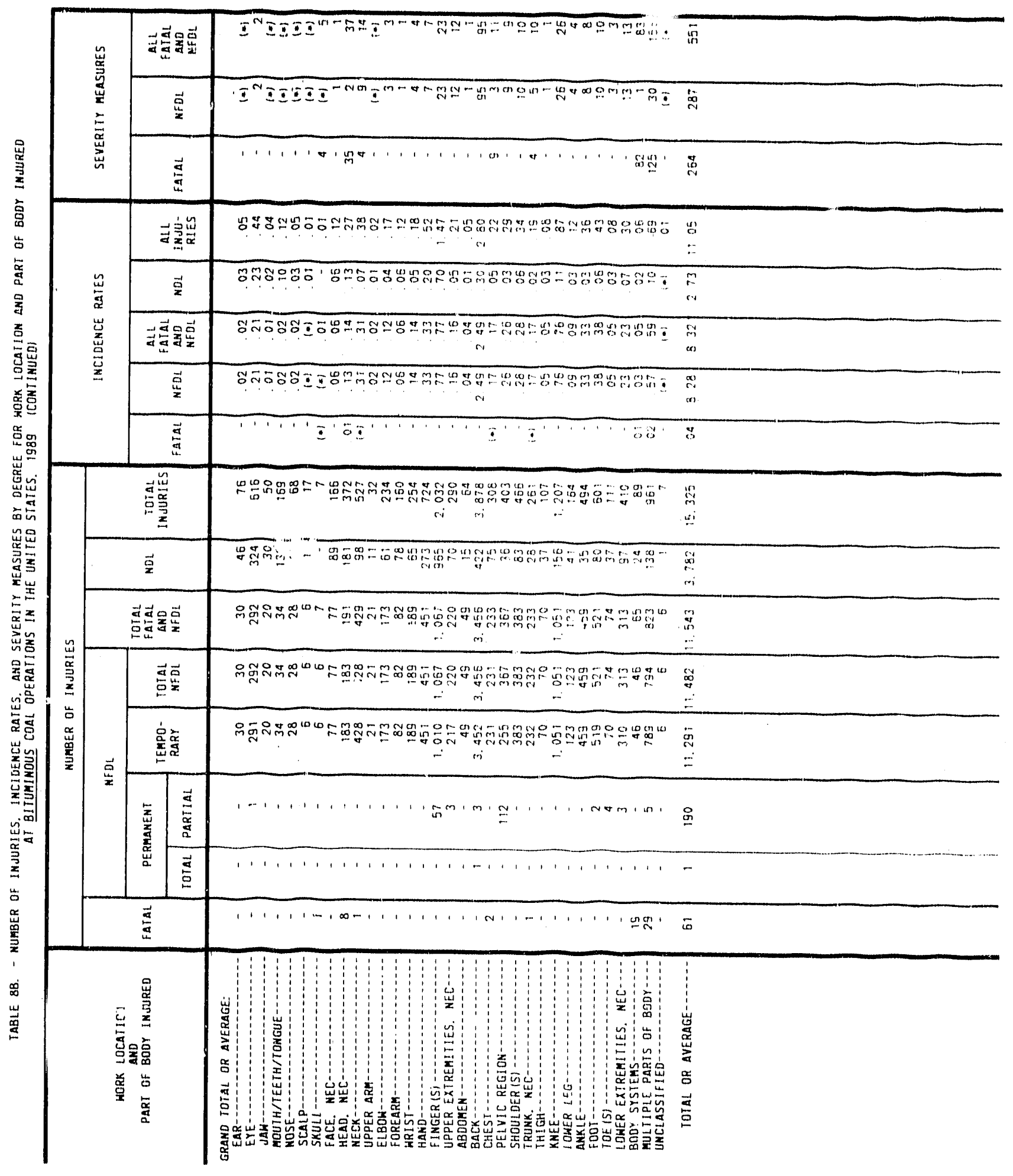




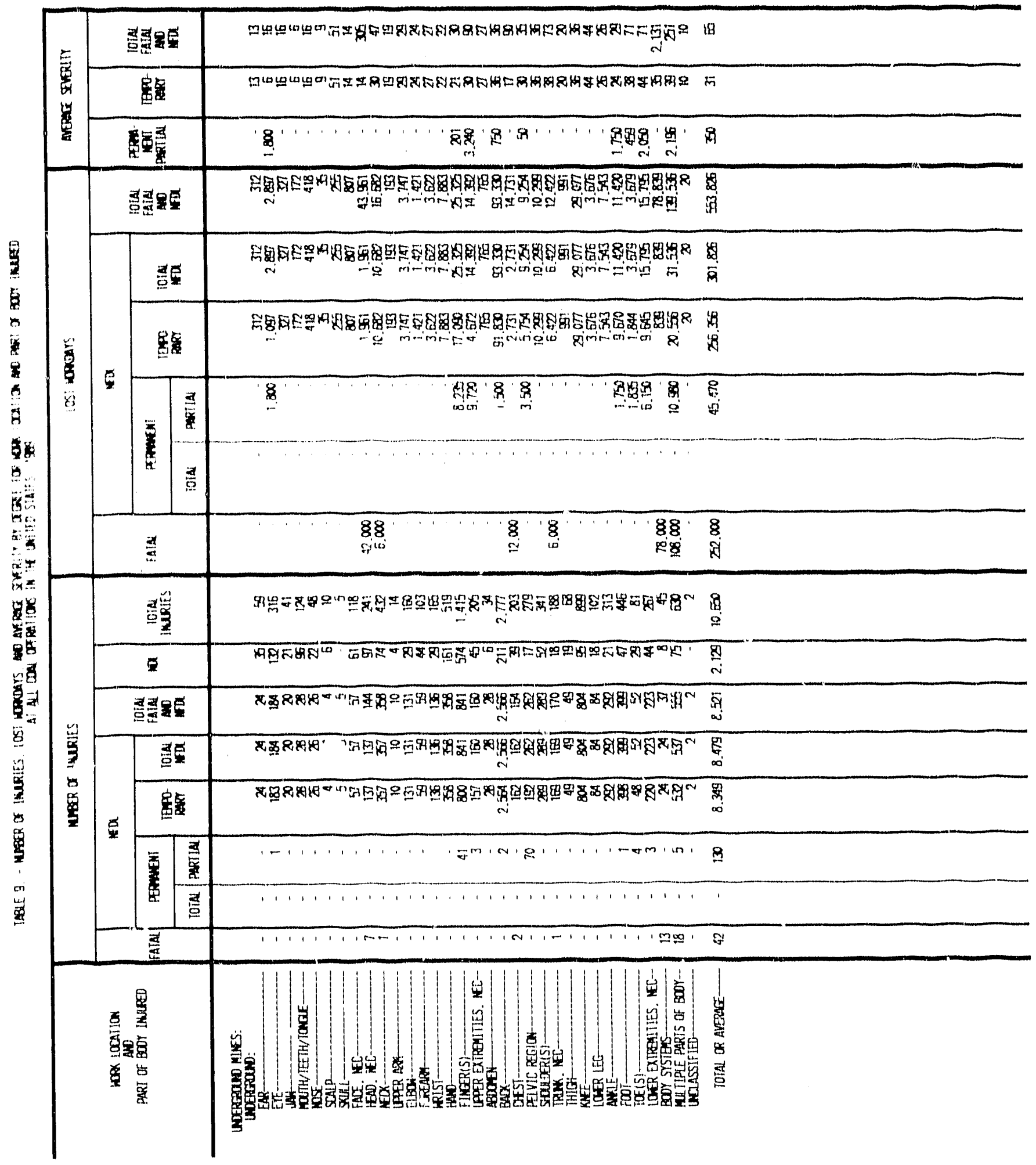




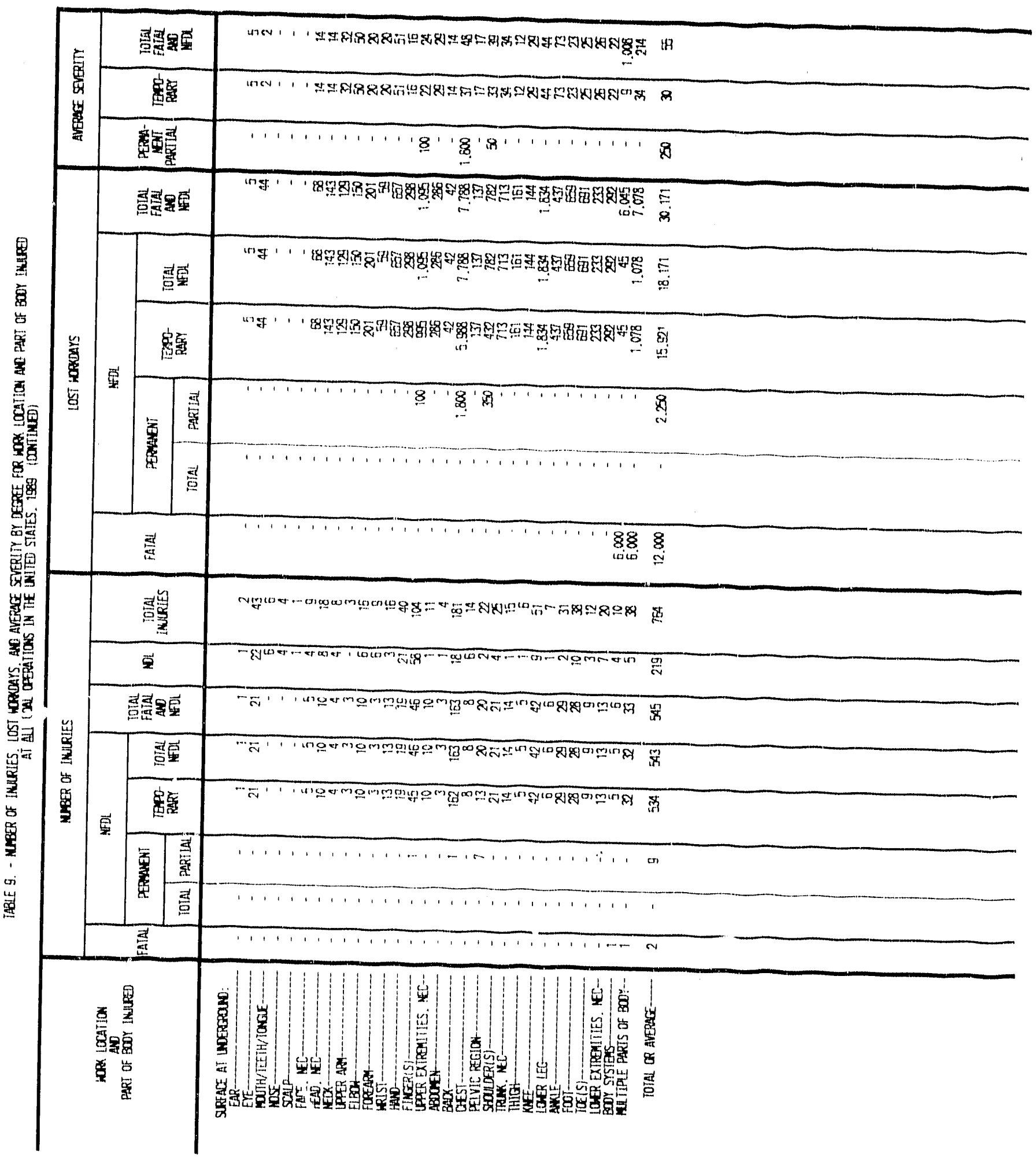




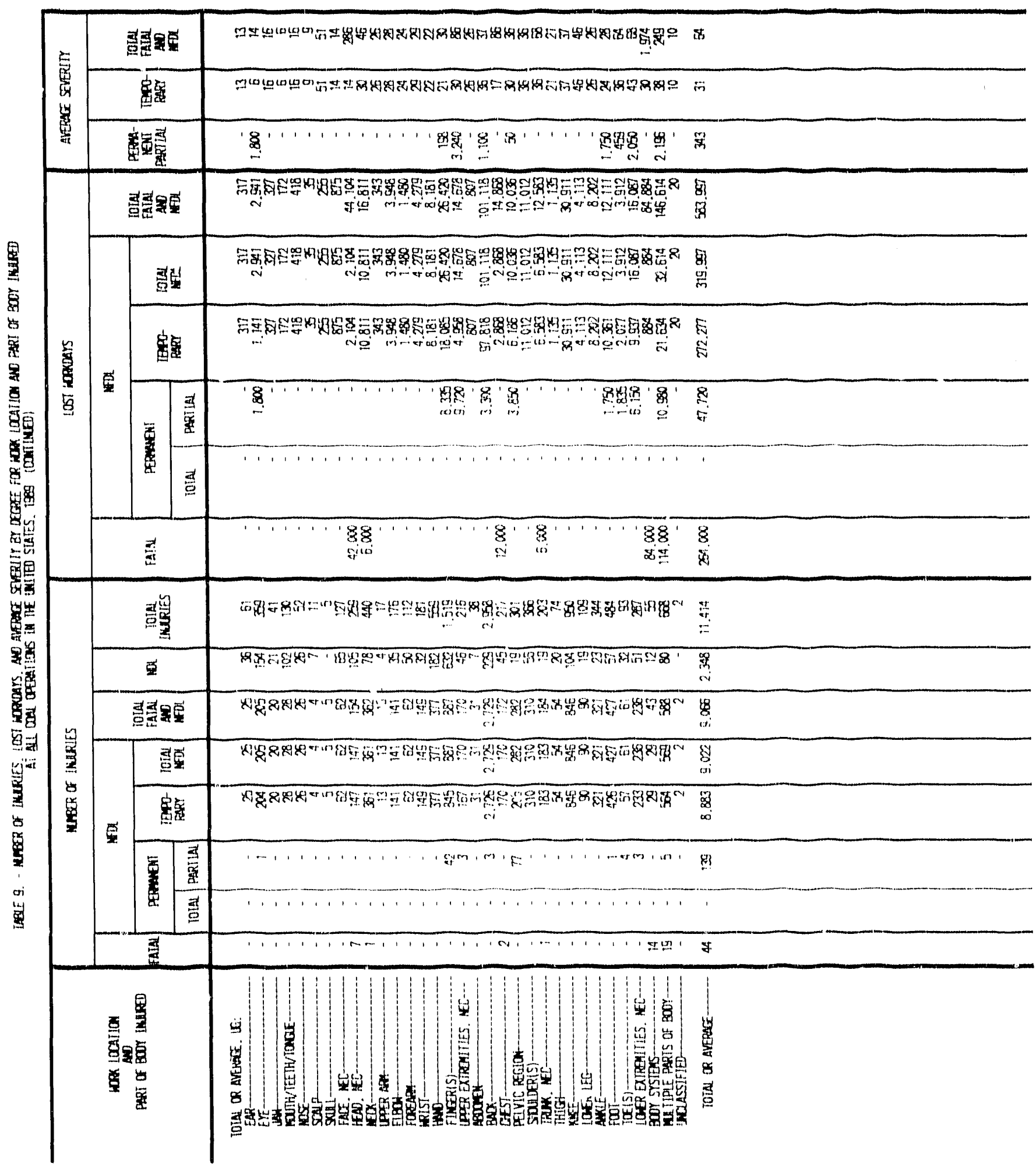




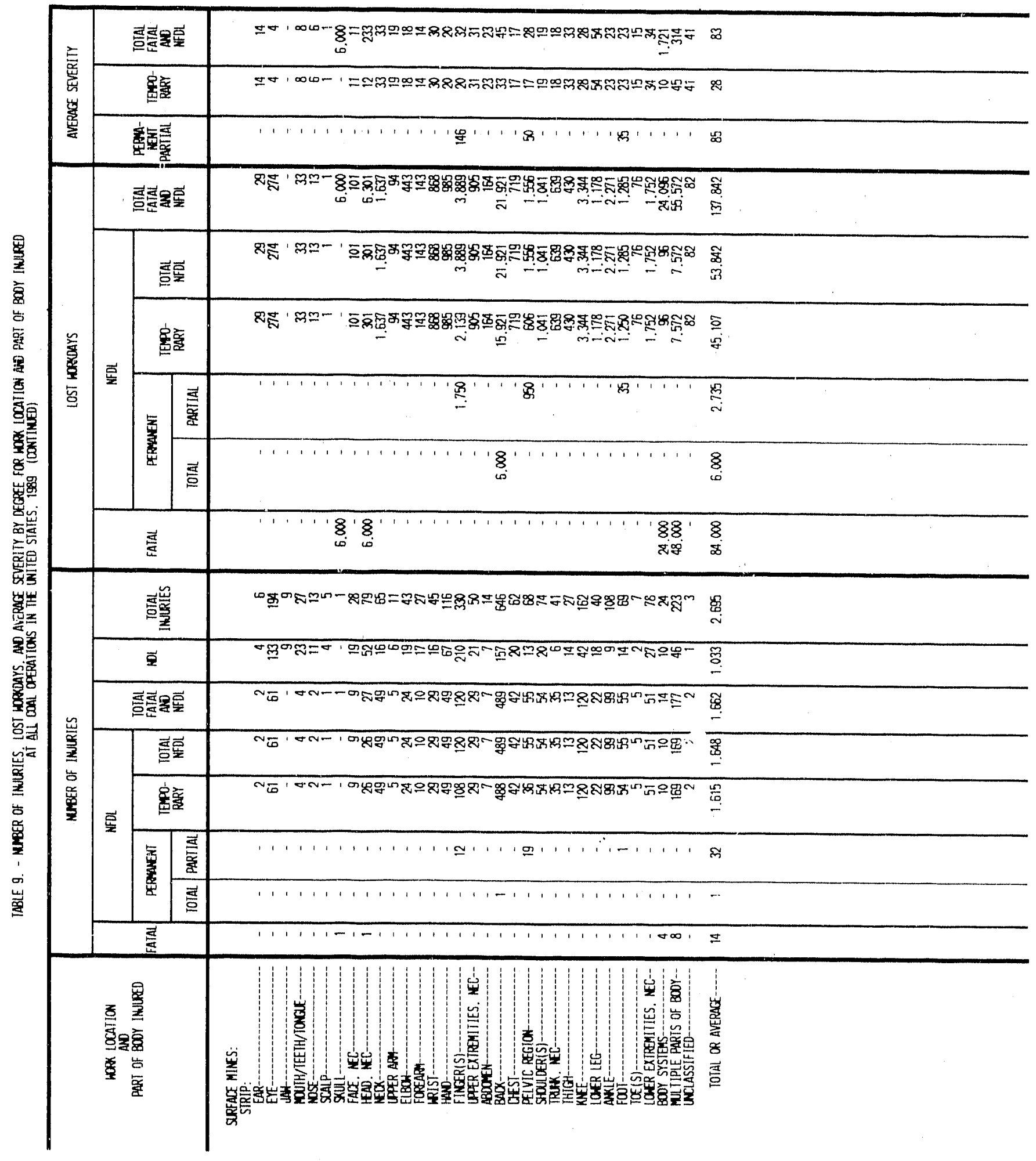




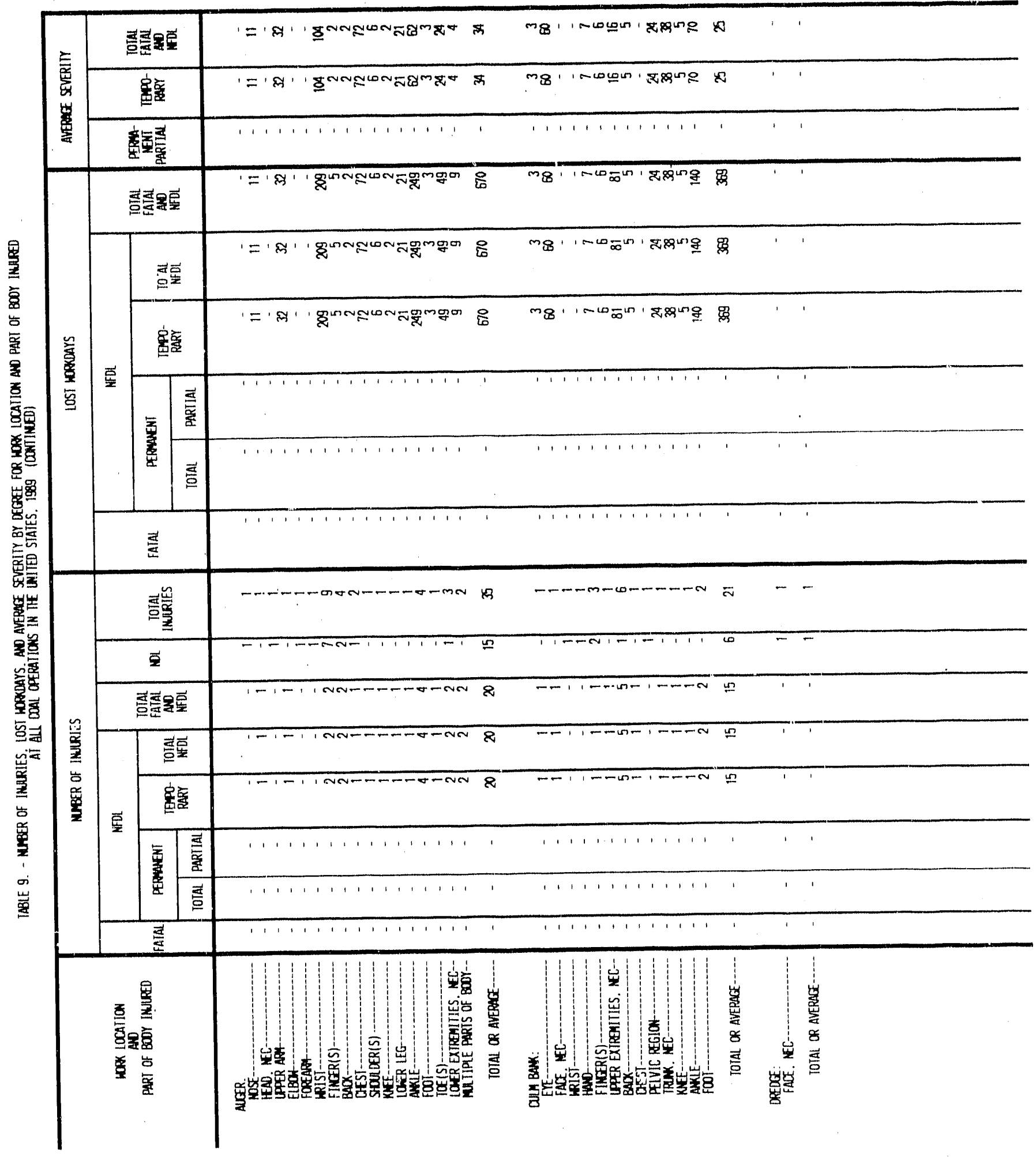




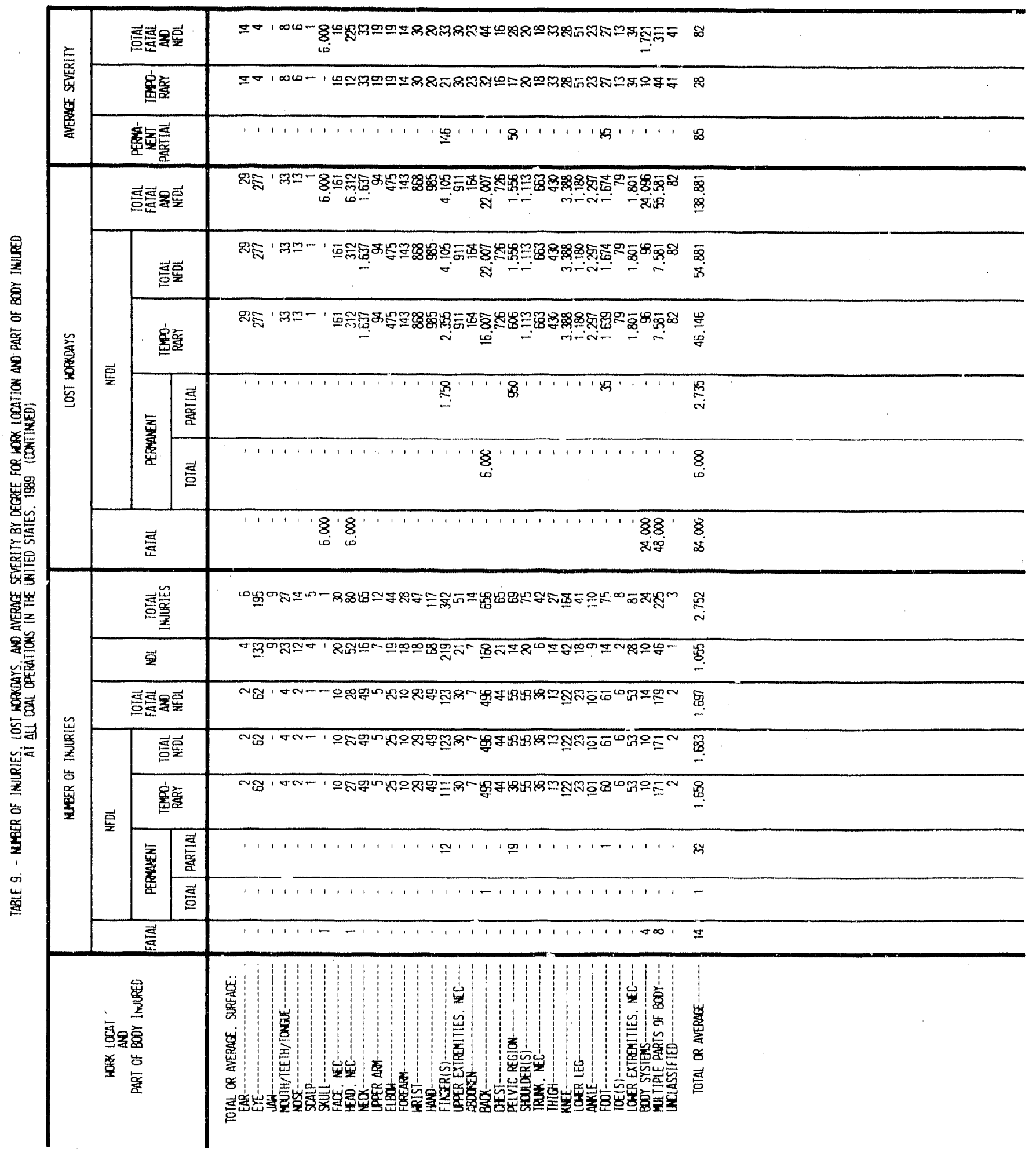




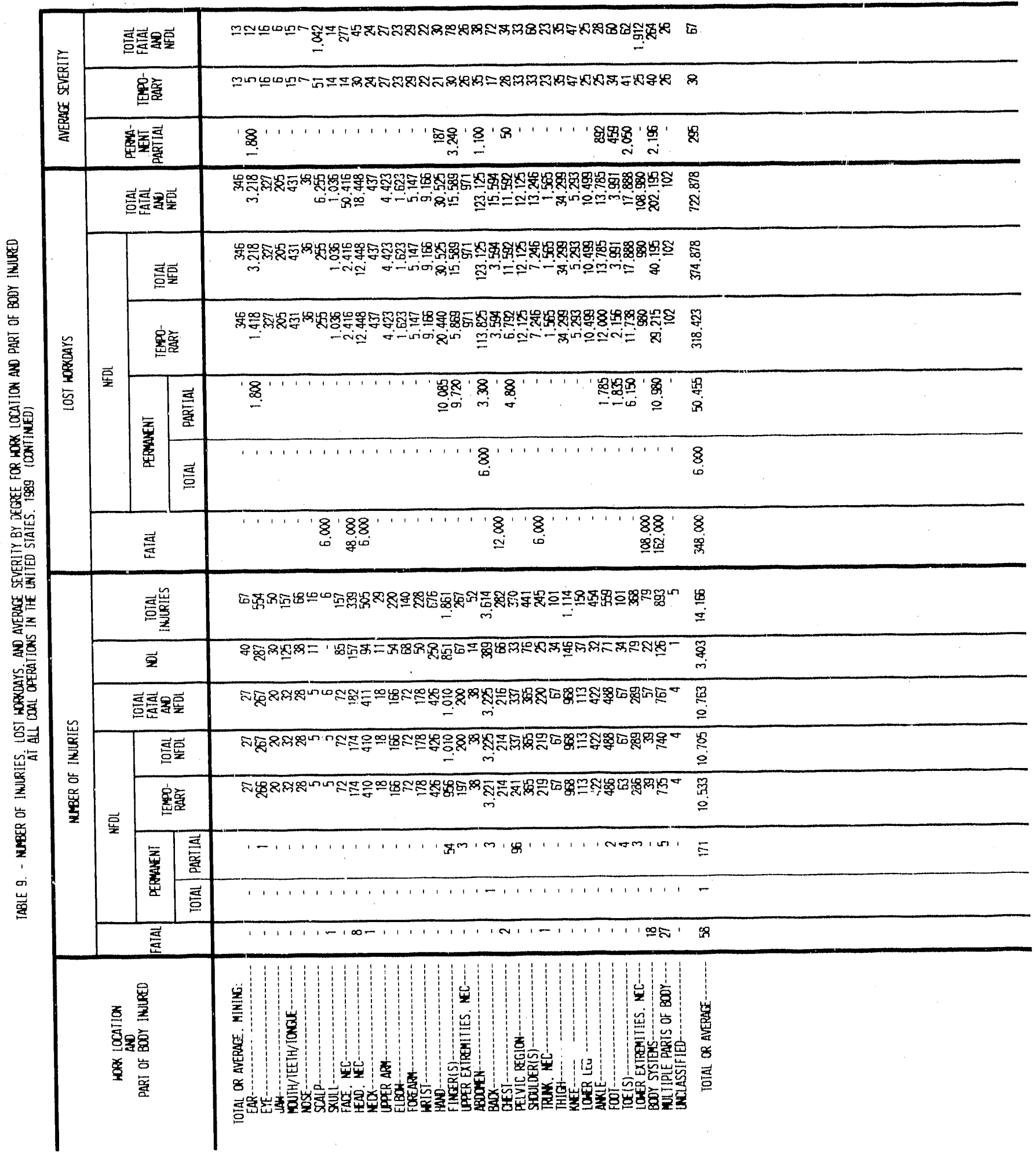




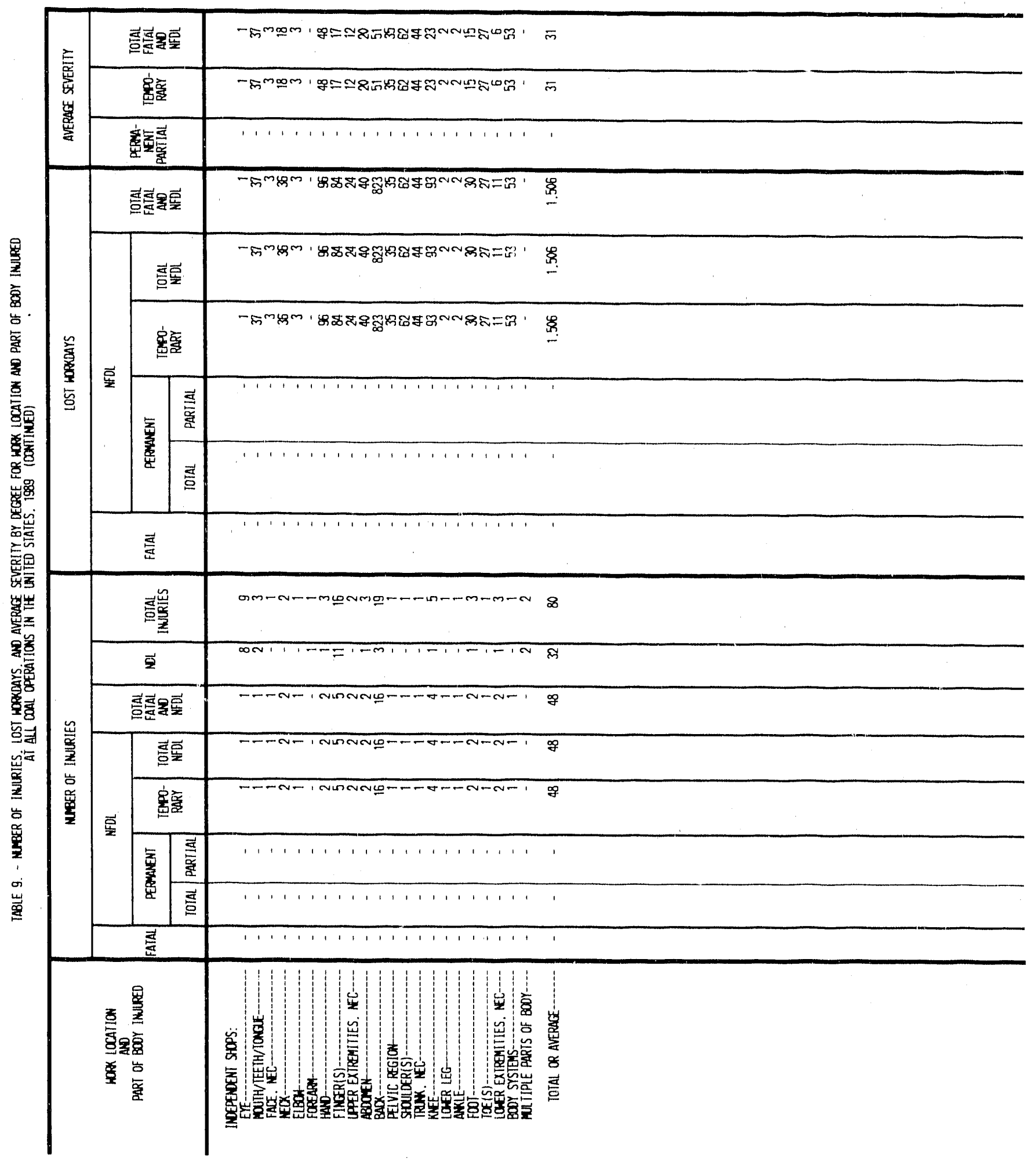




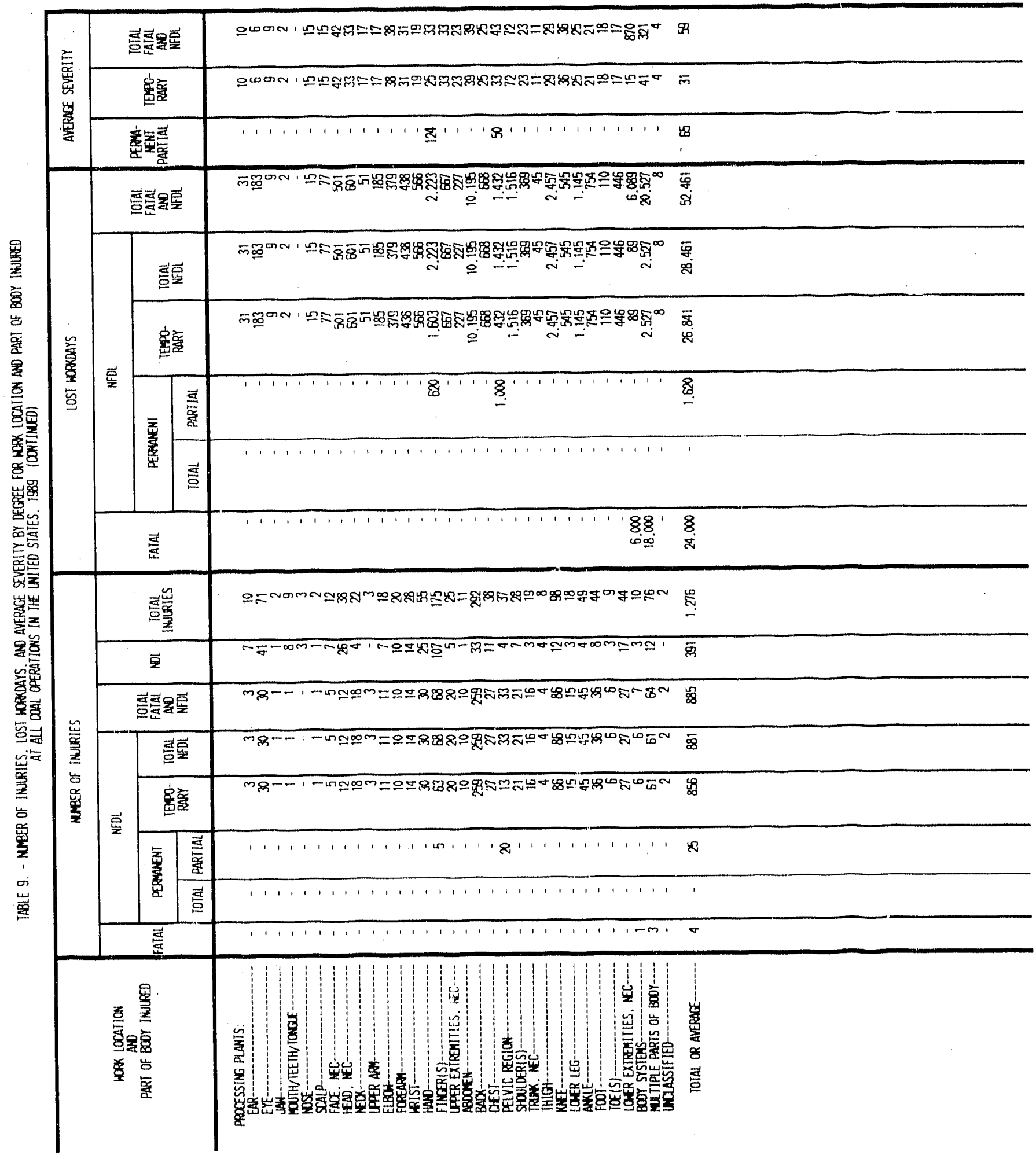




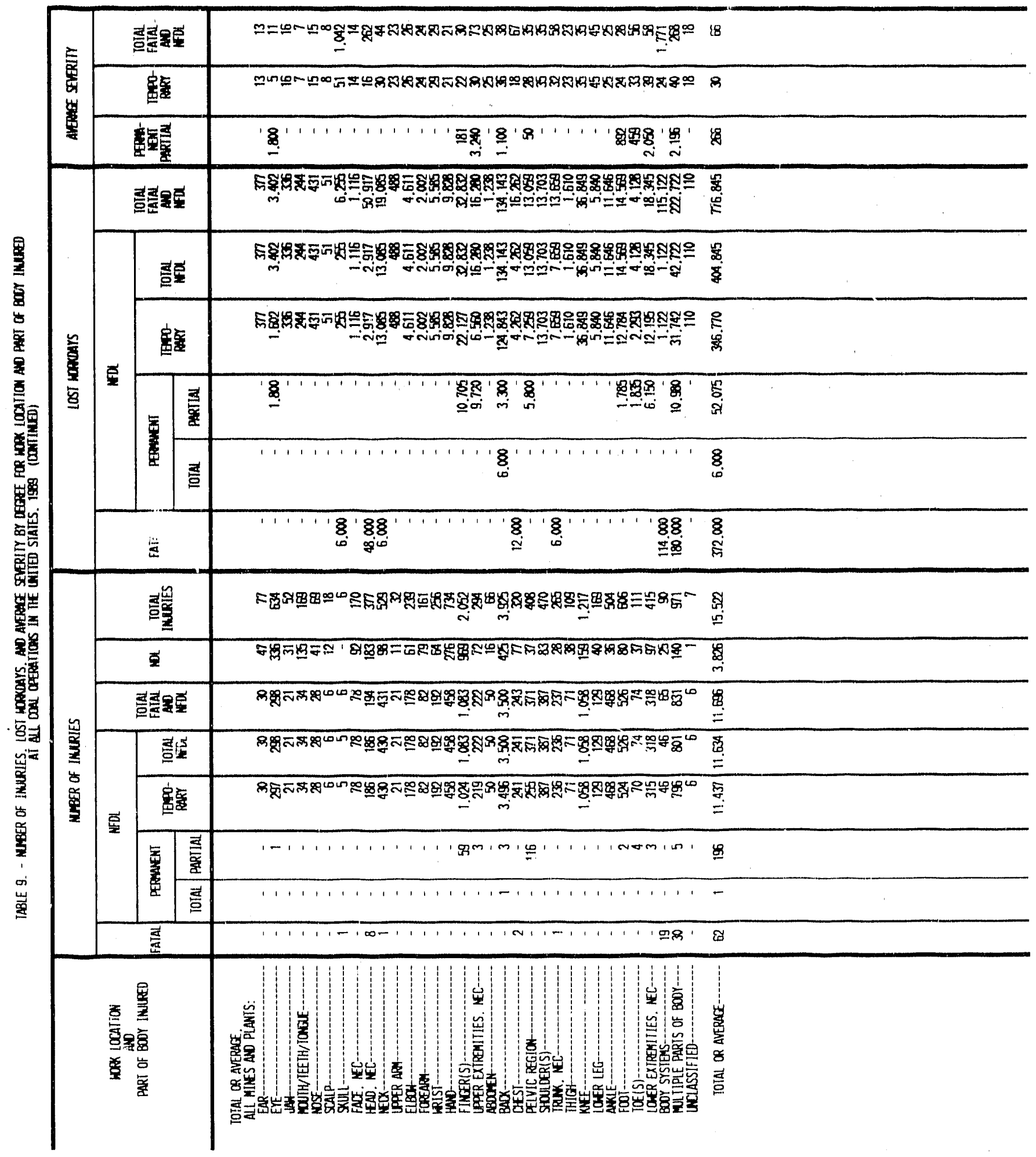




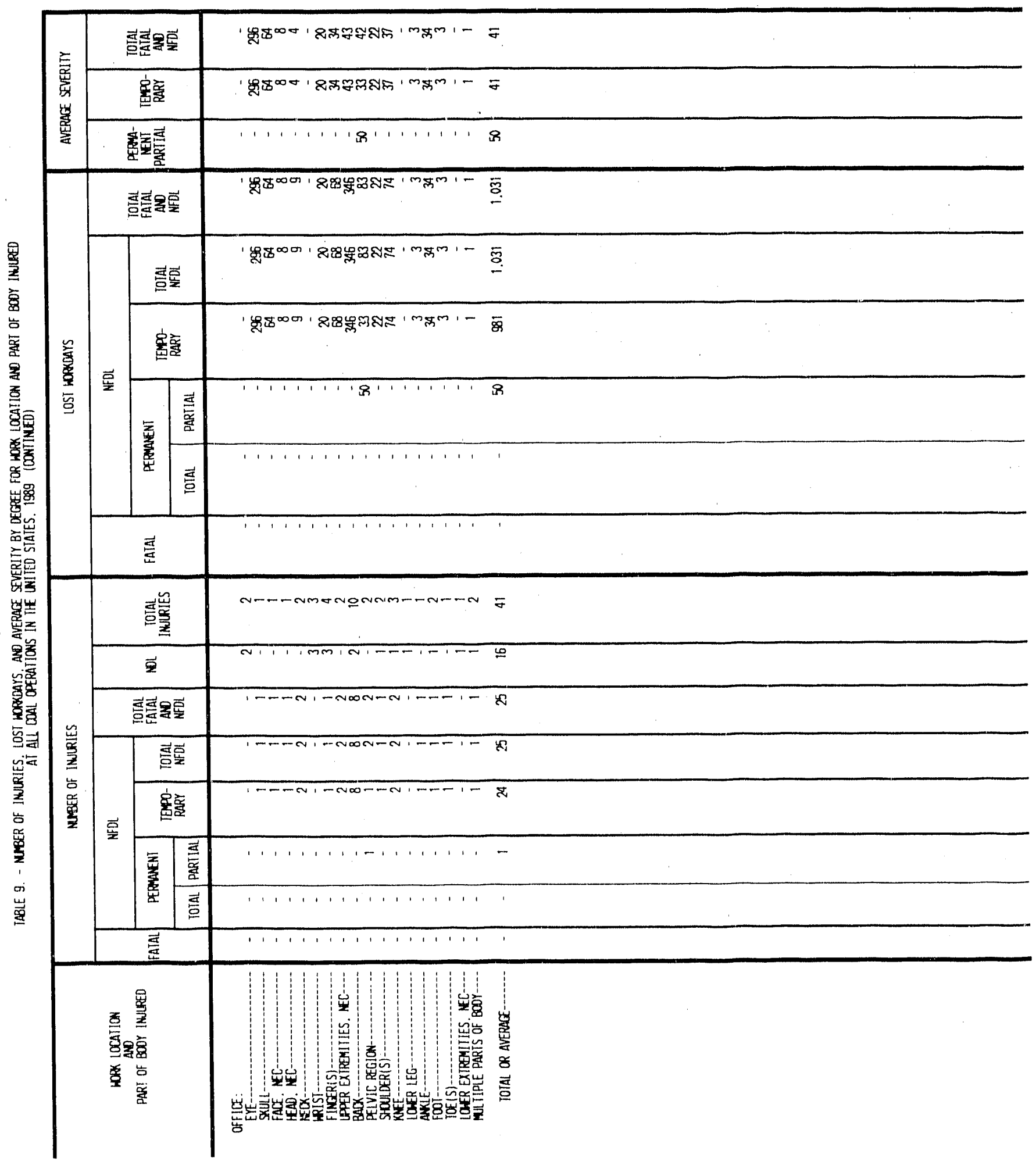




\begin{tabular}{|c|c|c|c|c|}
\hline \multirow{3}{*}{ 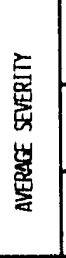 } & \multirow{2}{*}{\multicolumn{2}{|c|}{ 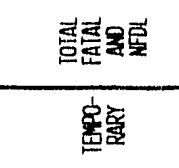 }} & 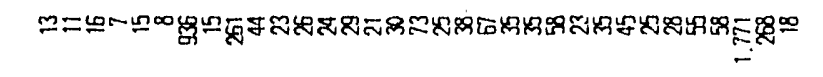 & 8 \\
\hline & & & 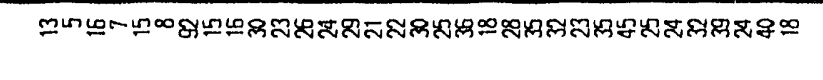 & s \\
\hline & \multicolumn{2}{|l|}{ 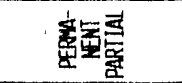 } & 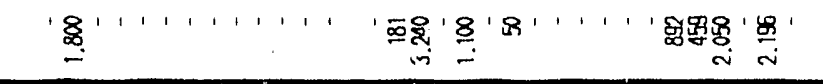 & 逢 \\
\hline \multirow{6}{*}{ 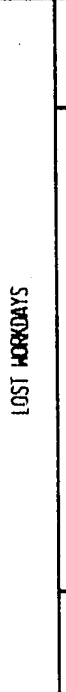 } & \multicolumn{2}{|c|}{ 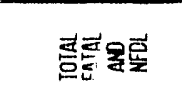 } & 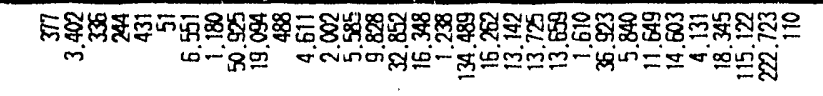 & 崖 \\
\hline & \multirow{4}{*}{ 茞 } & 홍릴 & 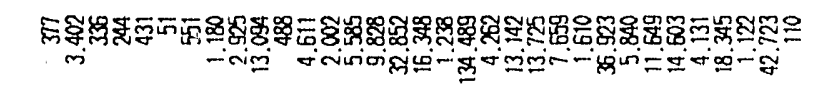 & 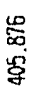 \\
\hline & & 衰衰 & 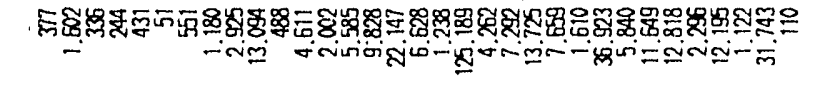 & ד. \\
\hline & & 章 & 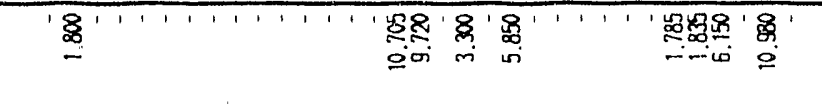 & 芠 \\
\hline & & 흠 & 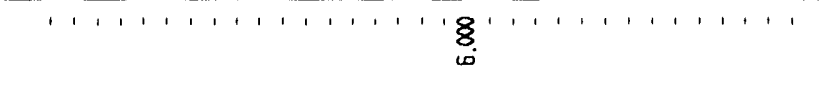 & 8 \\
\hline & \multicolumn{2}{|l|}{$\frac{\vec{z}}{4}$} & 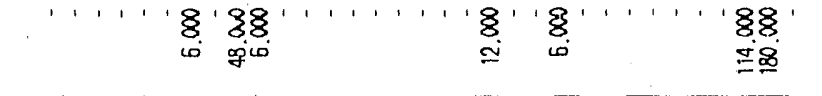 & $\begin{array}{l}8 \\
\overparen{m}\end{array}$ \\
\hline \multirow{8}{*}{ 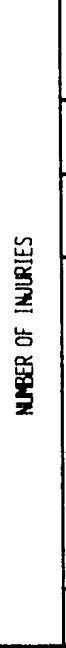 } & \multicolumn{2}{|c|}{ 趏容 } & 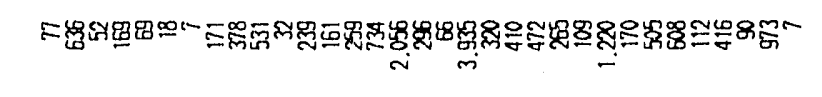 & 莡 \\
\hline & \multicolumn{2}{|l|}{$\overline{\mathbf{z}}$} & 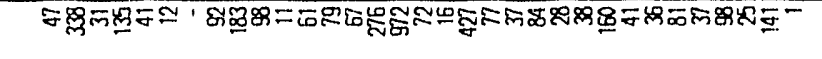 & $\tilde{\widetilde{z}}$ \\
\hline & \multicolumn{2}{|c|}{ 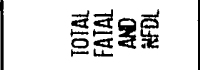 } & 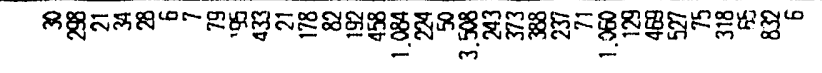 & $\underset{\Sigma}{\Sigma}$ \\
\hline & \multicolumn{2}{|c|}{ 형홀 } & 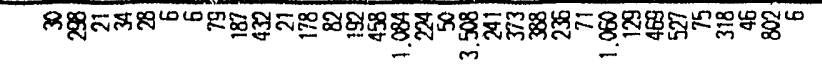 & $\stackrel{g}{g}$ \\
\hline & \multicolumn{2}{|c|}{ 害童 } & 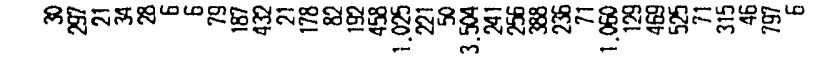 & $\stackrel{\text { 㶾 }}{=}$ \\
\hline & 畐 & 产 & 'gmimin' & 巨్ \\
\hline & & 总 & $1,1,1,1,1,1,1$ & - \\
\hline & \multicolumn{2}{|l|}{$\frac{\vec{b}}{\underline{\underline{E}}}$} & ' ' ' ' 모요 & $\tilde{\varpi}$ \\
\hline & \multicolumn{2}{|l|}{ 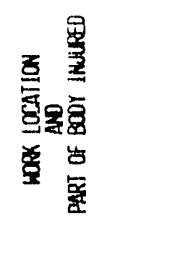 } & 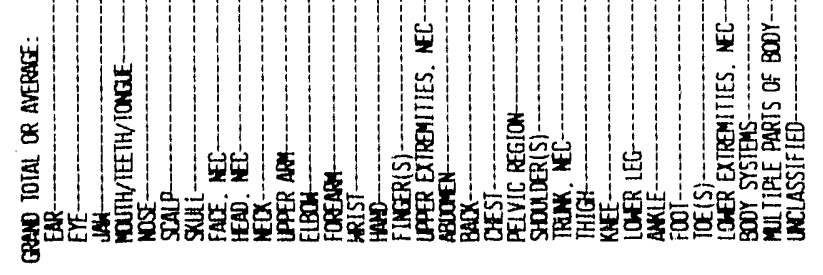 & 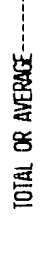 \\
\hline
\end{tabular}




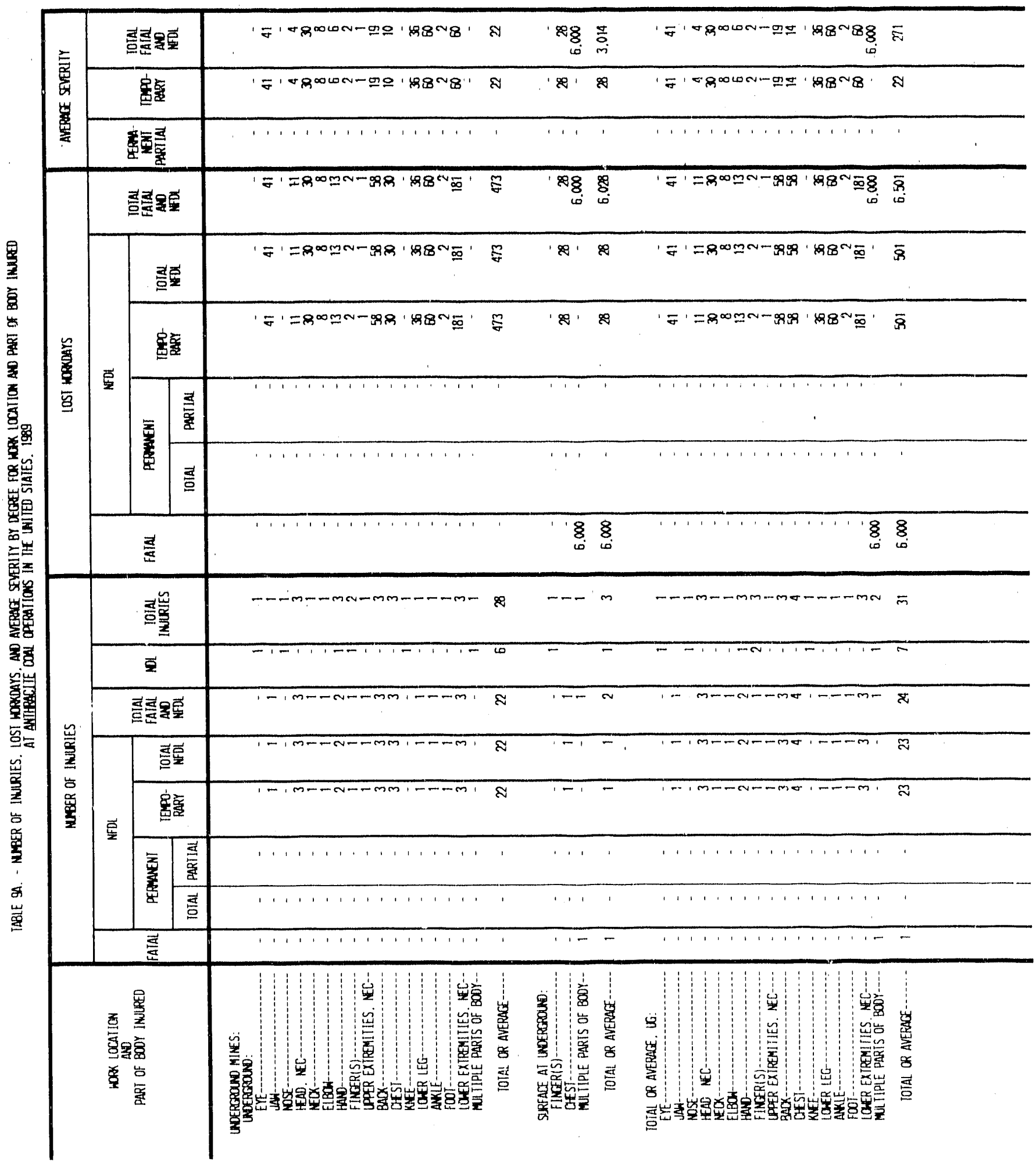




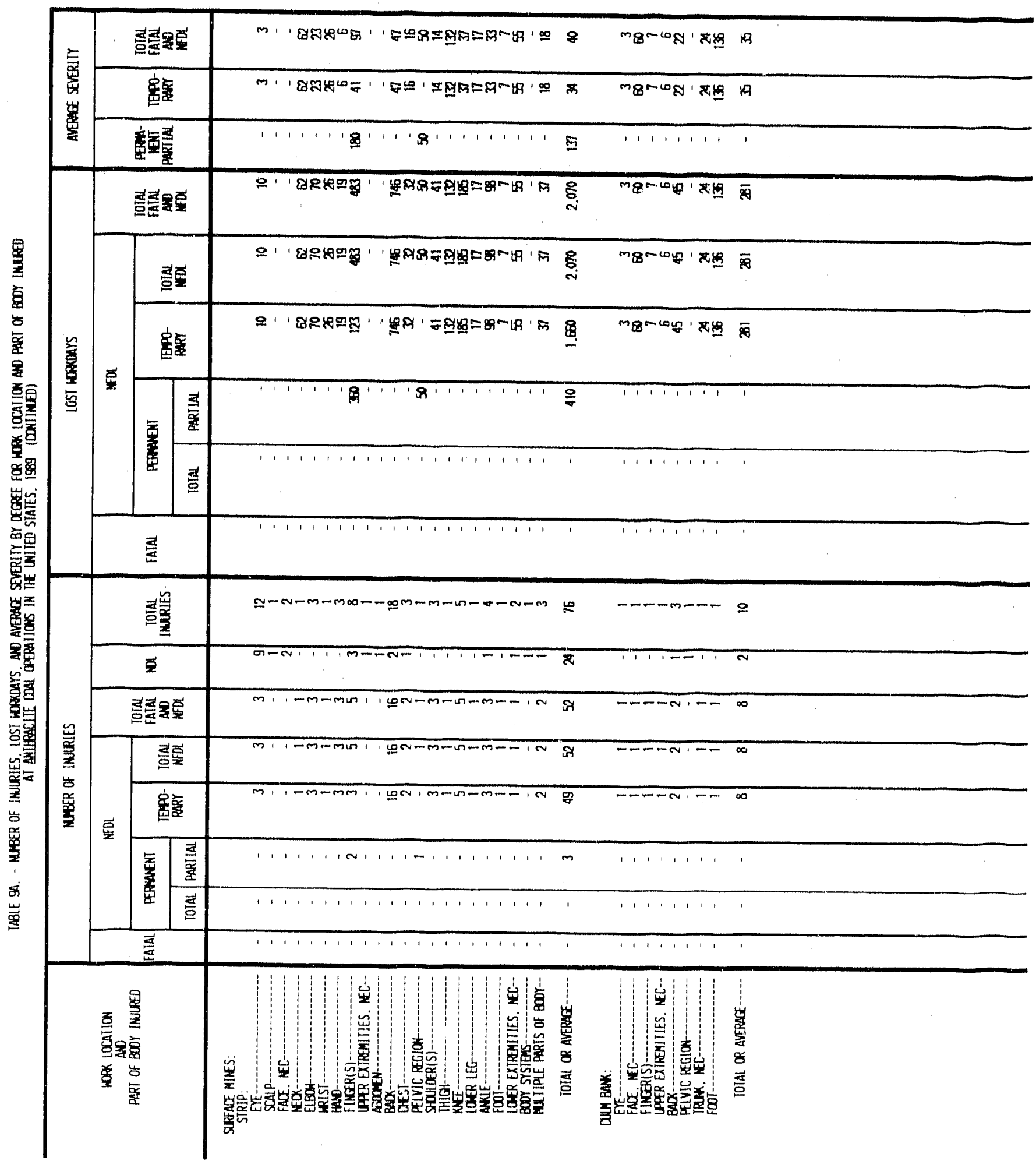




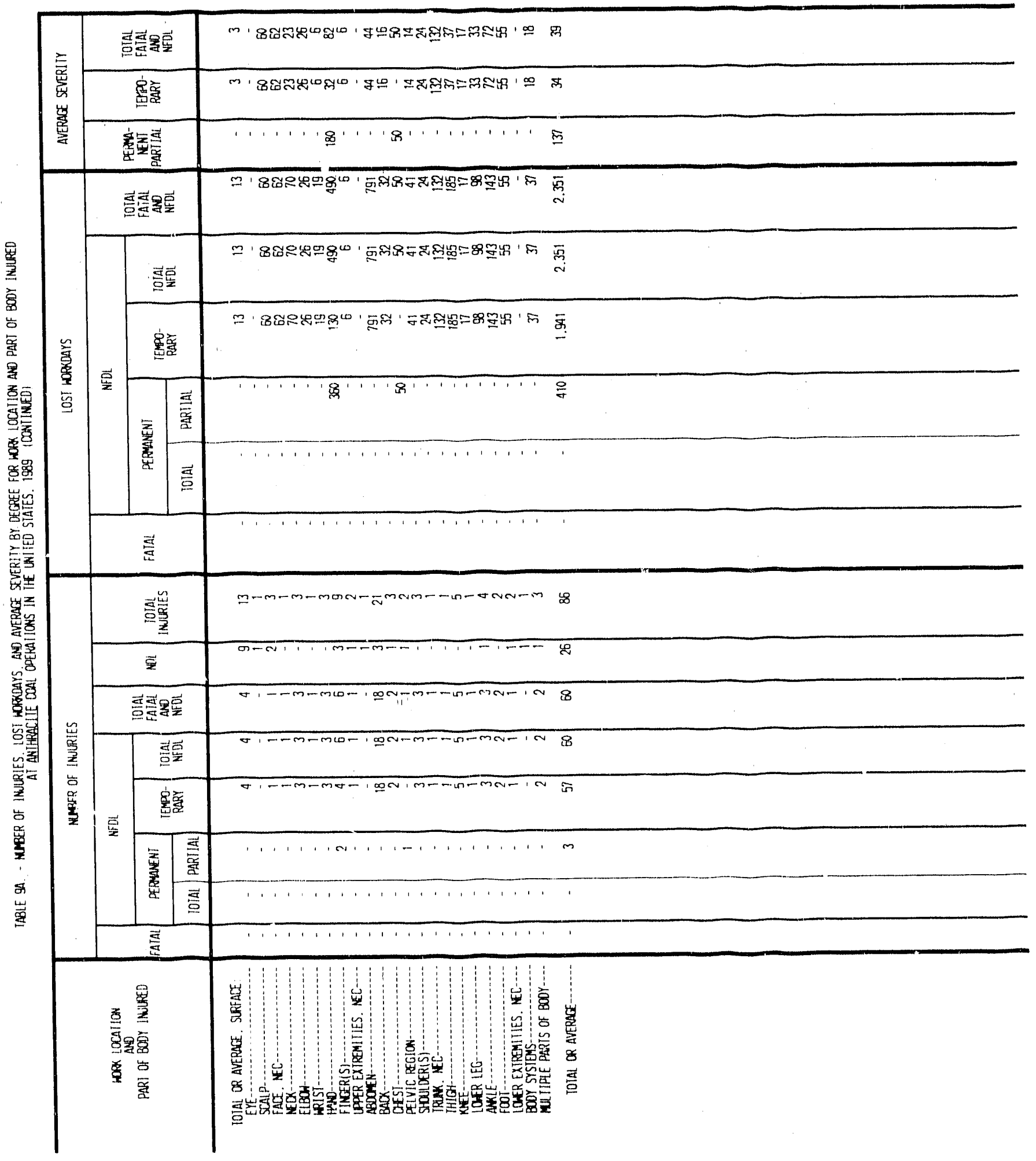




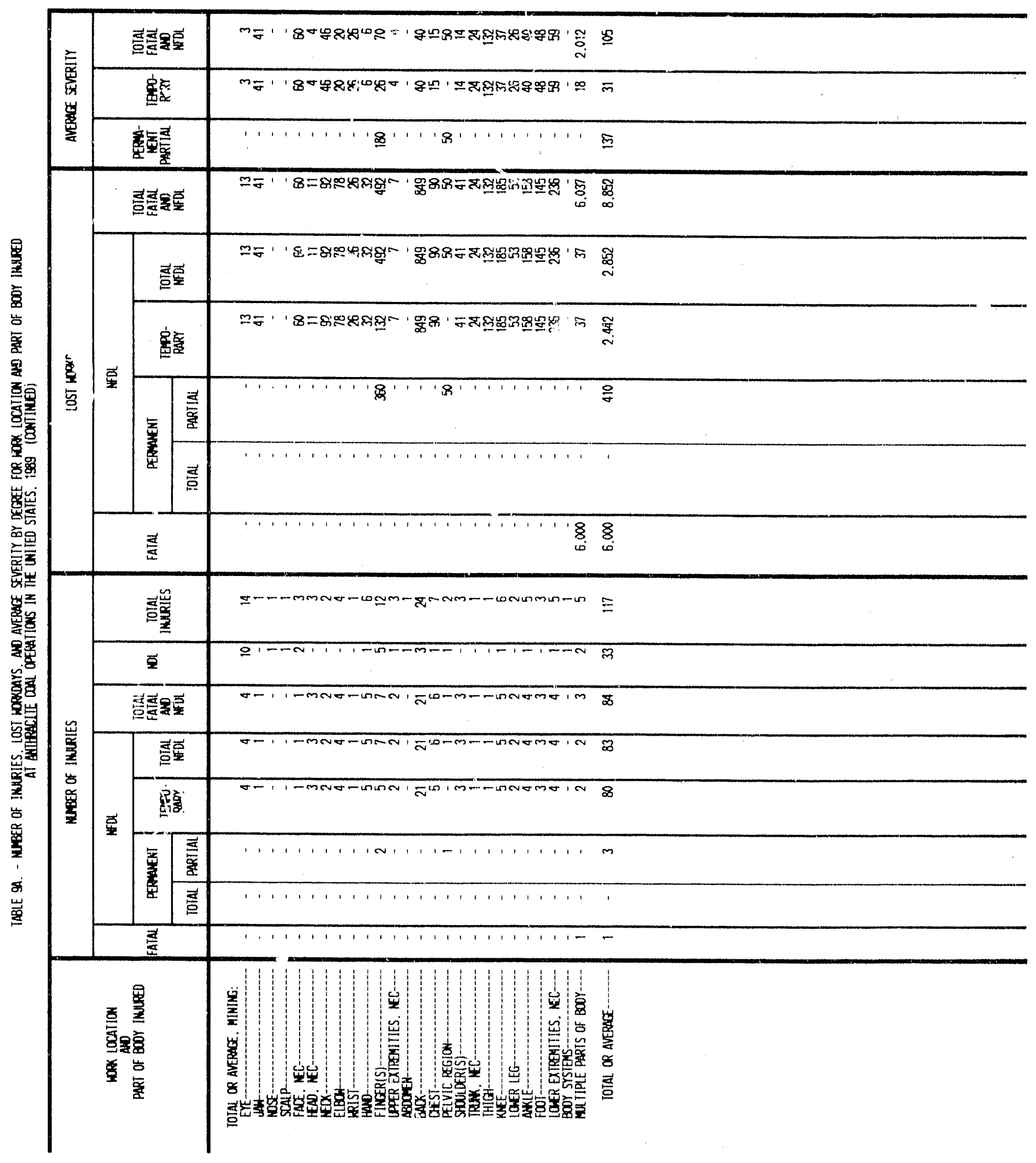




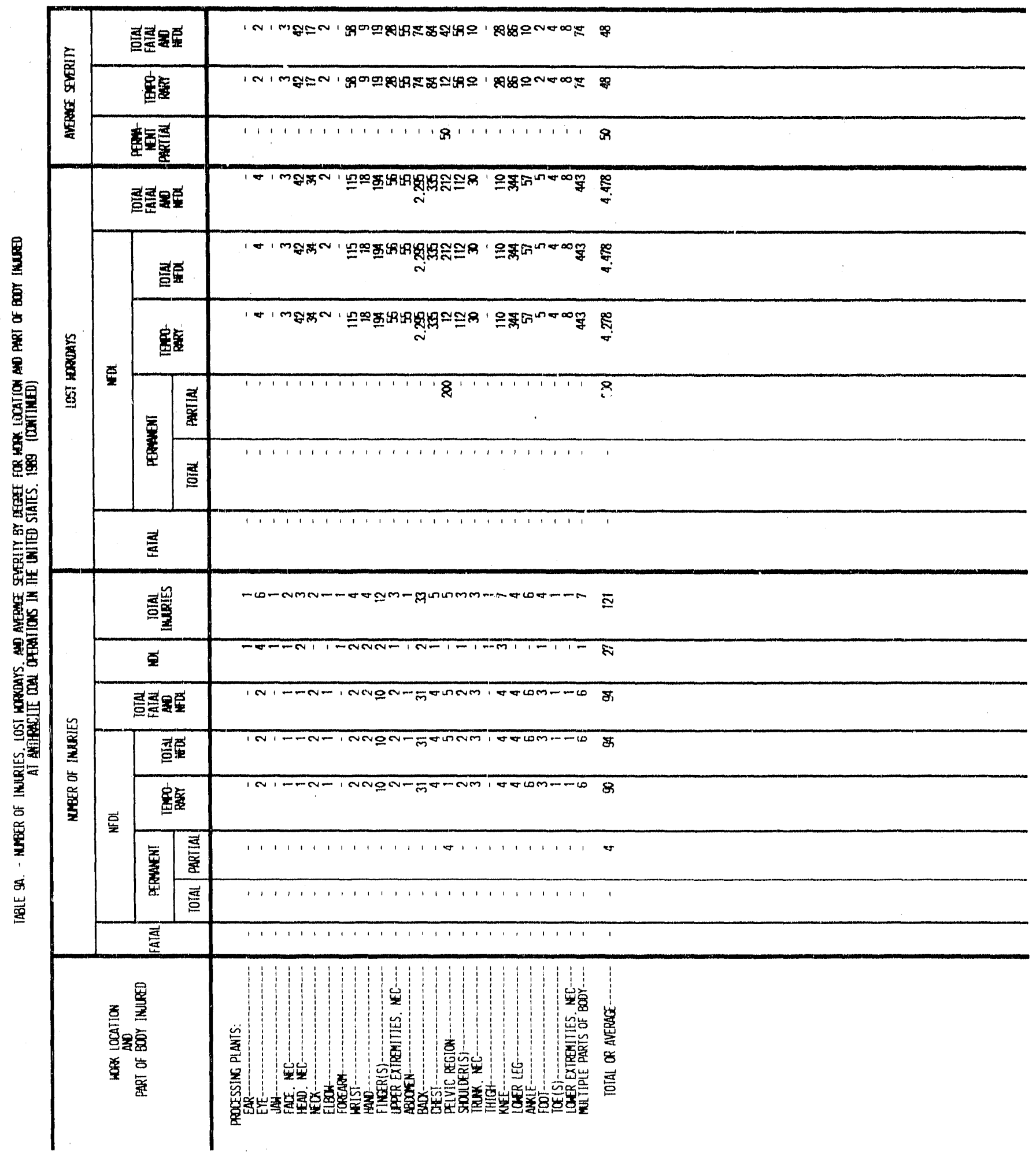




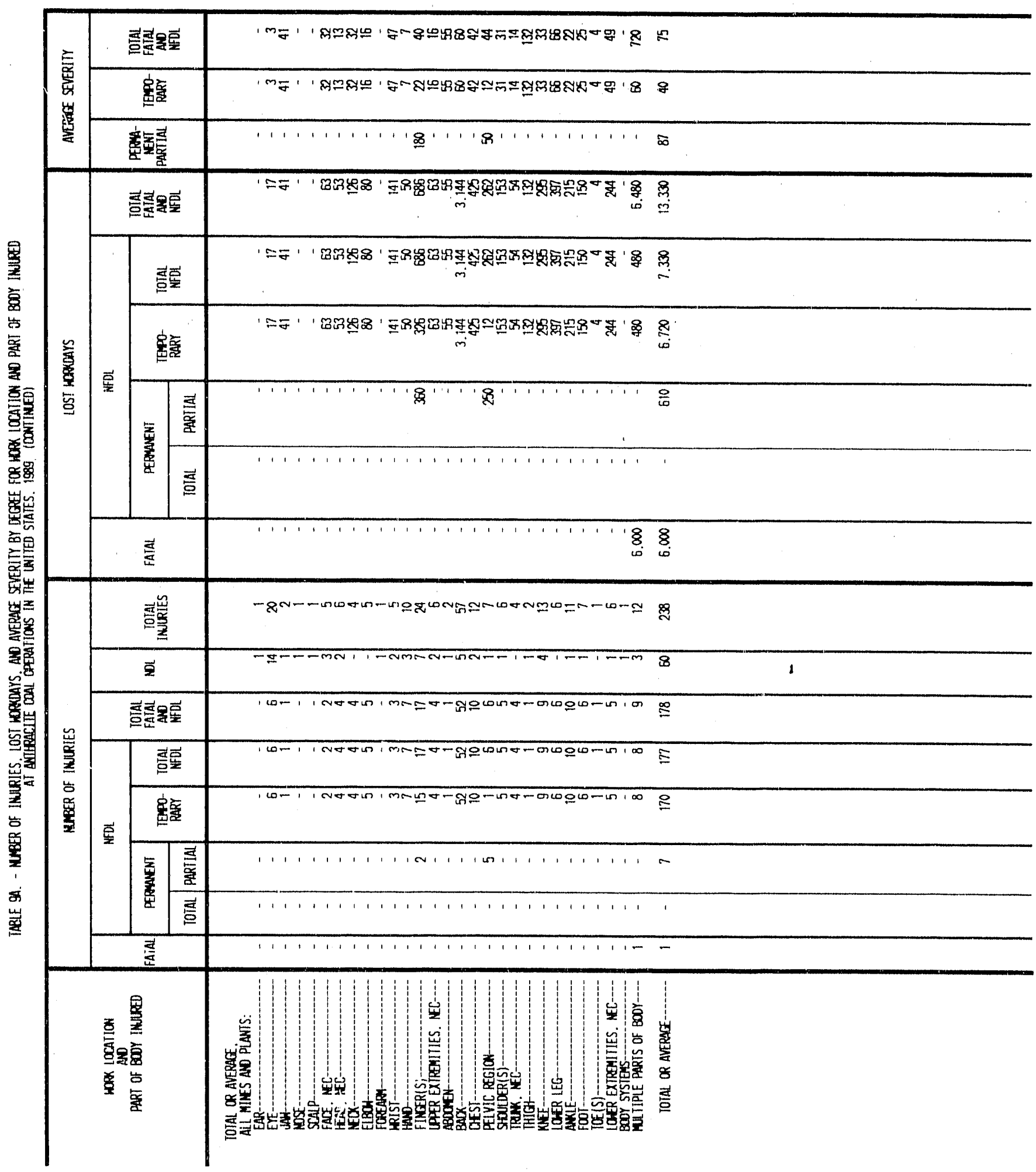




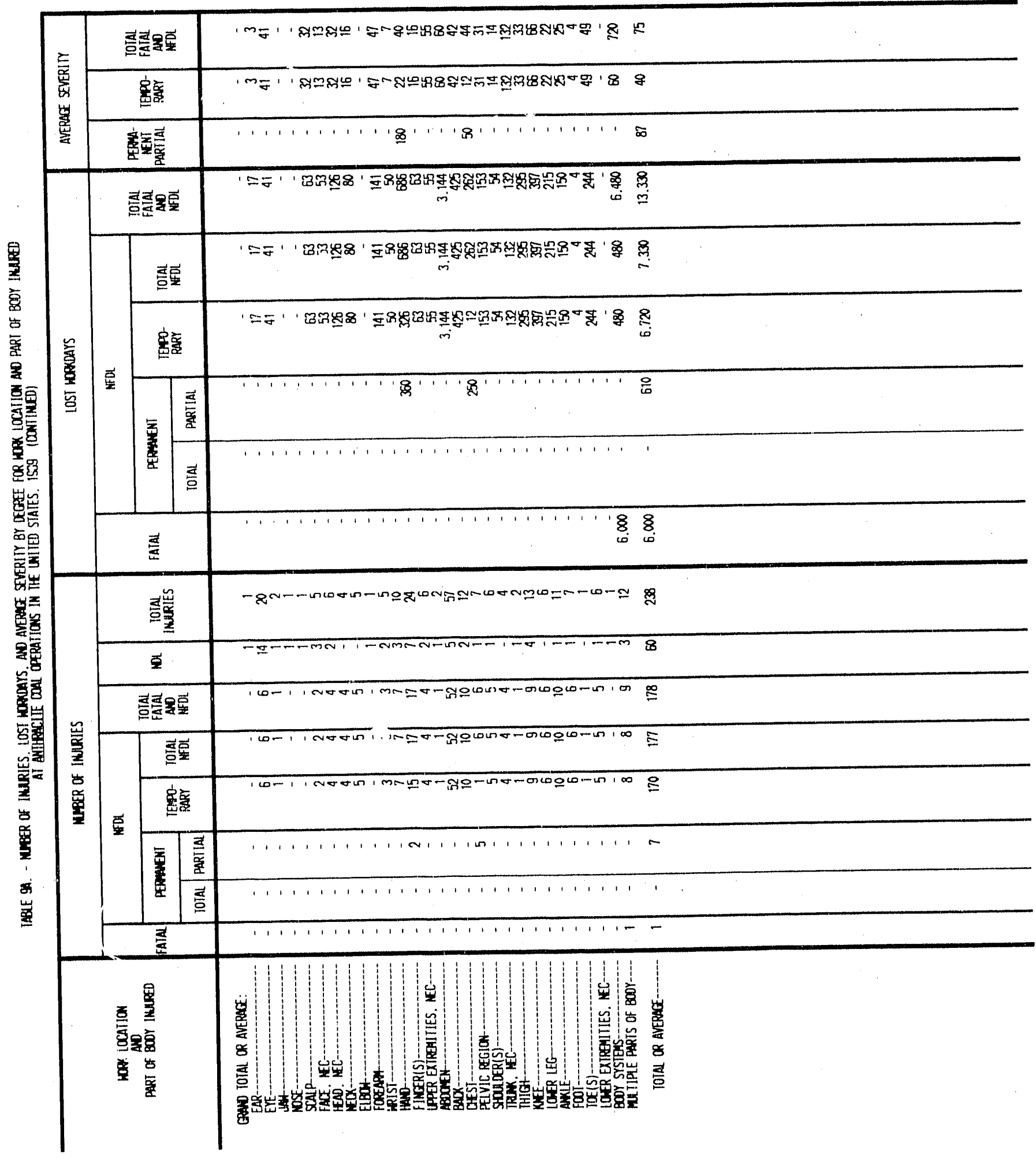




\begin{tabular}{|c|c|c|c|c|}
\hline \multirow{3}{*}{ 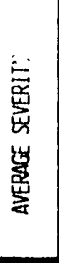 } & \multicolumn{2}{|c|}{ 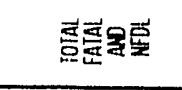 } & 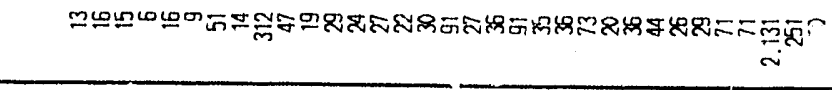 & 岕 \\
\hline & \multicolumn{2}{|c|}{ 鱼害 } & 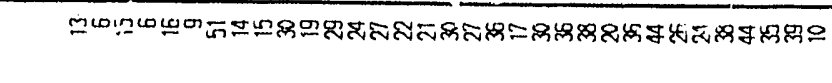 & $\bar{m}$ \\
\hline & \multicolumn{2}{|c|}{ 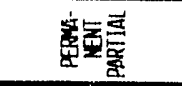 } & 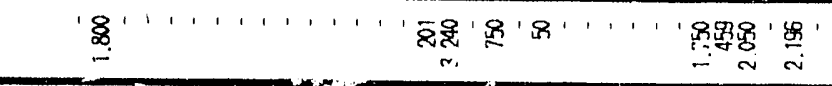 & 8 \\
\hline \multirow{6}{*}{ 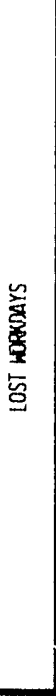 } & \multicolumn{2}{|c|}{ 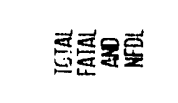 } & 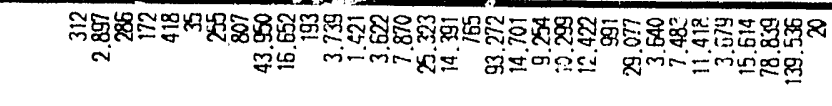 & 总 \\
\hline & \multicolumn{2}{|c|}{ 흥물 } & 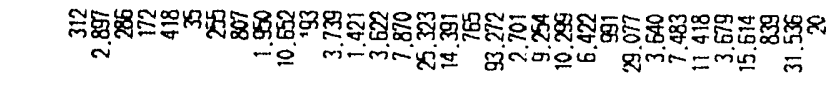 & $\frac{m_{n}}{m_{m}}$ \\
\hline & \multicolumn{2}{|c|}{ 旁蜜 } & 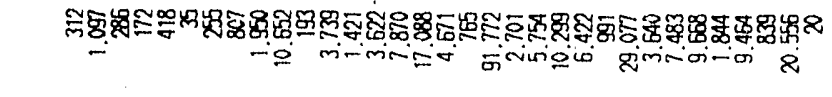 & 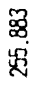 \\
\hline & \multirow{2}{*}{ 兽 } & 妾 & 兽 & 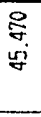 \\
\hline & & 趌 & 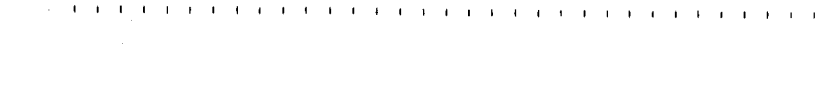 & . \\
\hline & \multicolumn{2}{|l|}{ 运 } & 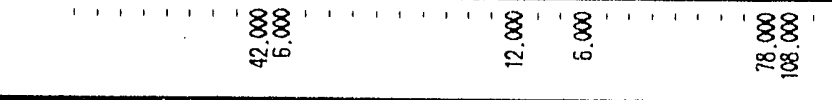 & $\begin{array}{l}8.8 \\
\tilde{~}\end{array}$ \\
\hline \multirow{8}{*}{ 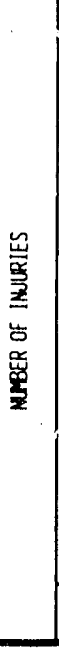 } & \multicolumn{2}{|c|}{ 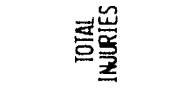 } & 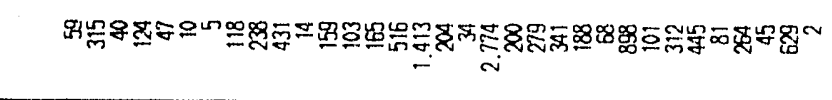 & $\underset{\dddot{g}}{\mathbb{Q}}$ \\
\hline & \multicolumn{2}{|l|}{$\overrightarrow{\underline{2}}$} & 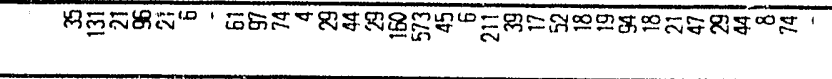 & 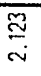 \\
\hline & \multicolumn{2}{|c|}{ 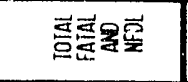 } & 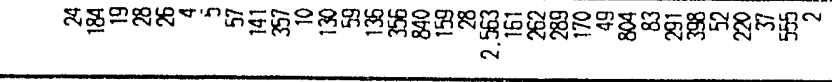 & 畩 \\
\hline & \multicolumn{2}{|c|}{ 홍휴 } & 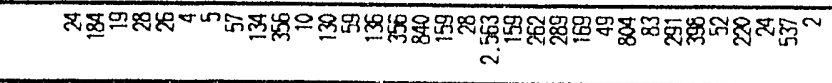 & 密 \\
\hline & \multicolumn{2}{|c|}{ 窟害 } & 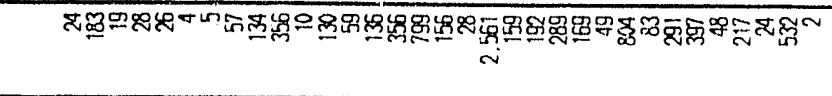 & స్తj \\
\hline & & \begin{tabular}{|l|l|}
$\vec{E}$ \\
\multirow{2}{*}{} \\
\end{tabular} & 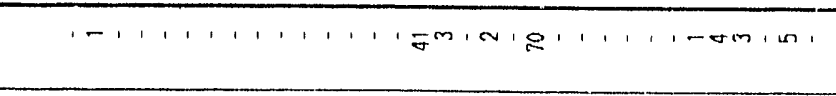 & 8 \\
\hline & & 릉 & $\cdots, \ldots$ & . \\
\hline & \multicolumn{2}{|l|}{ 迹 } & '1' ' & $\bar{F}$ \\
\hline & \multicolumn{2}{|l|}{ 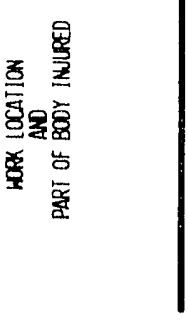 } & 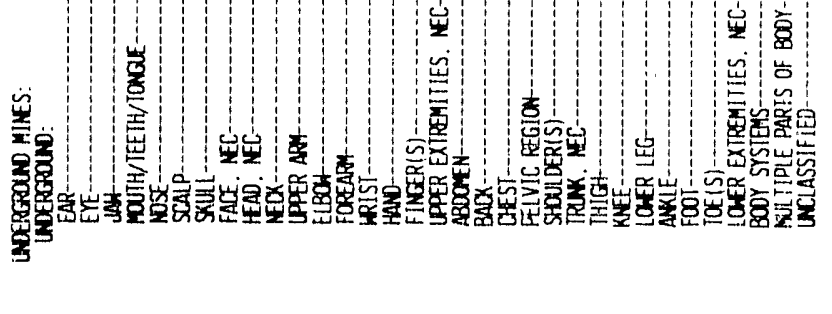 & 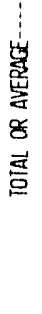 \\
\hline
\end{tabular}




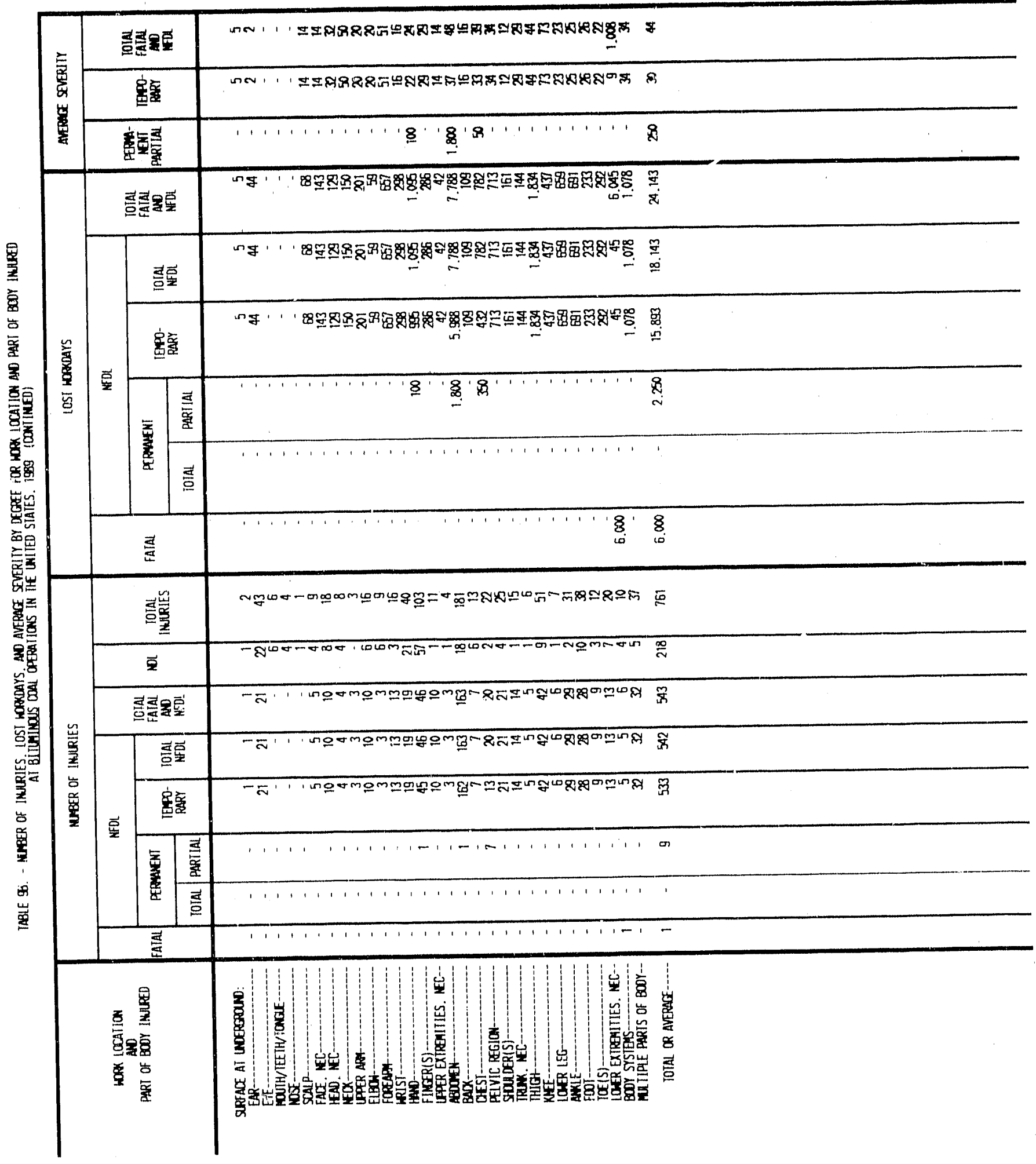




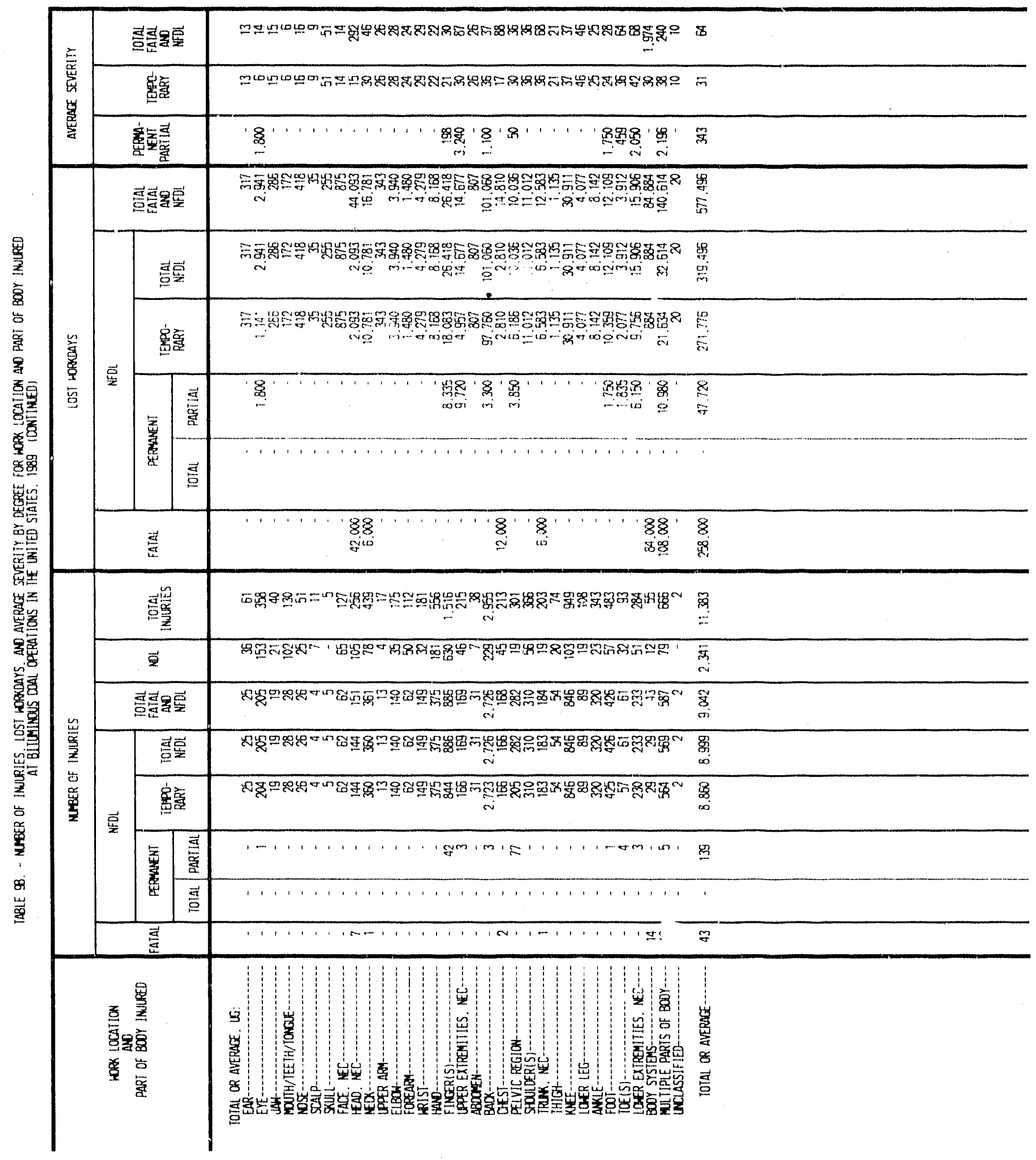




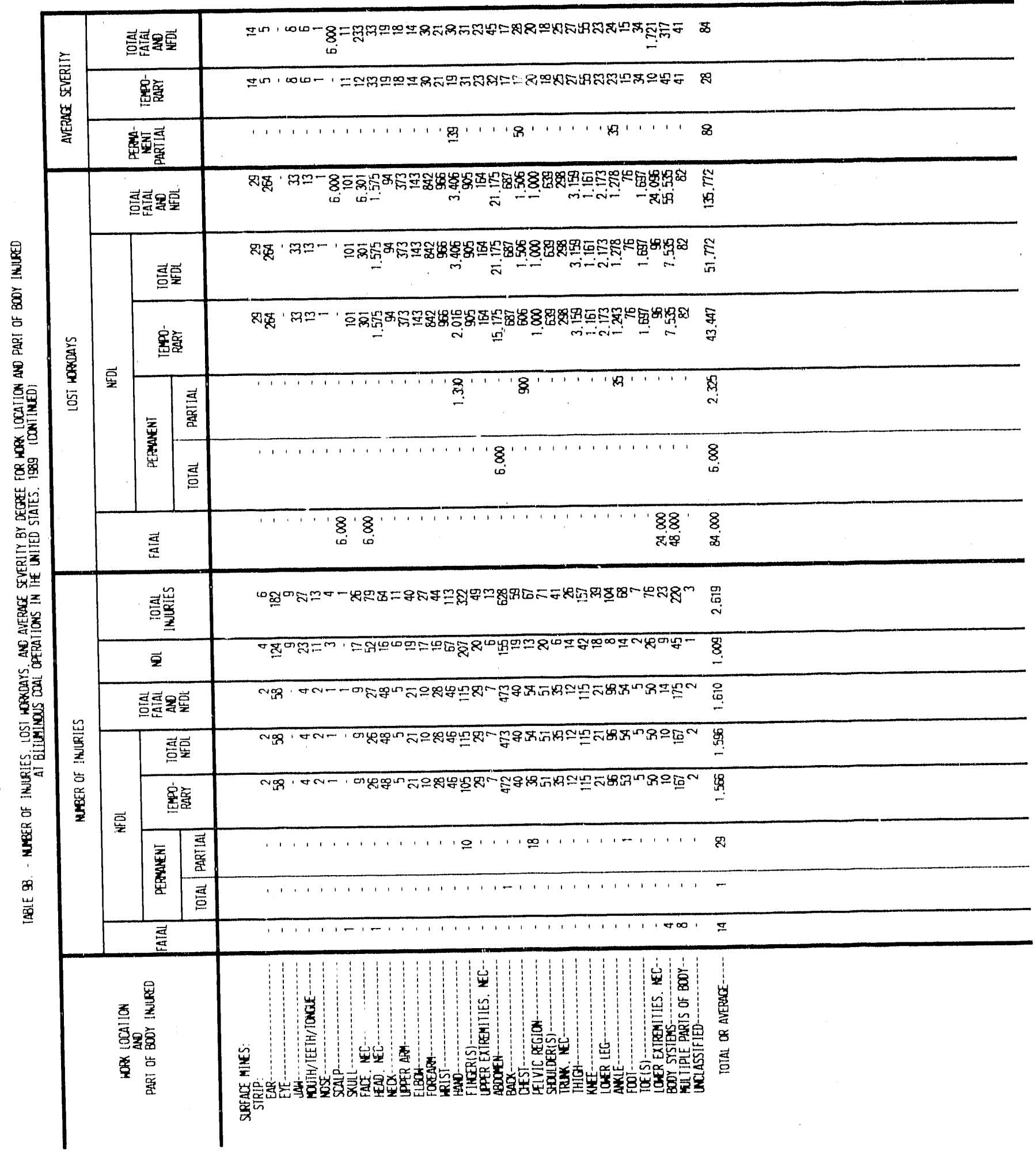




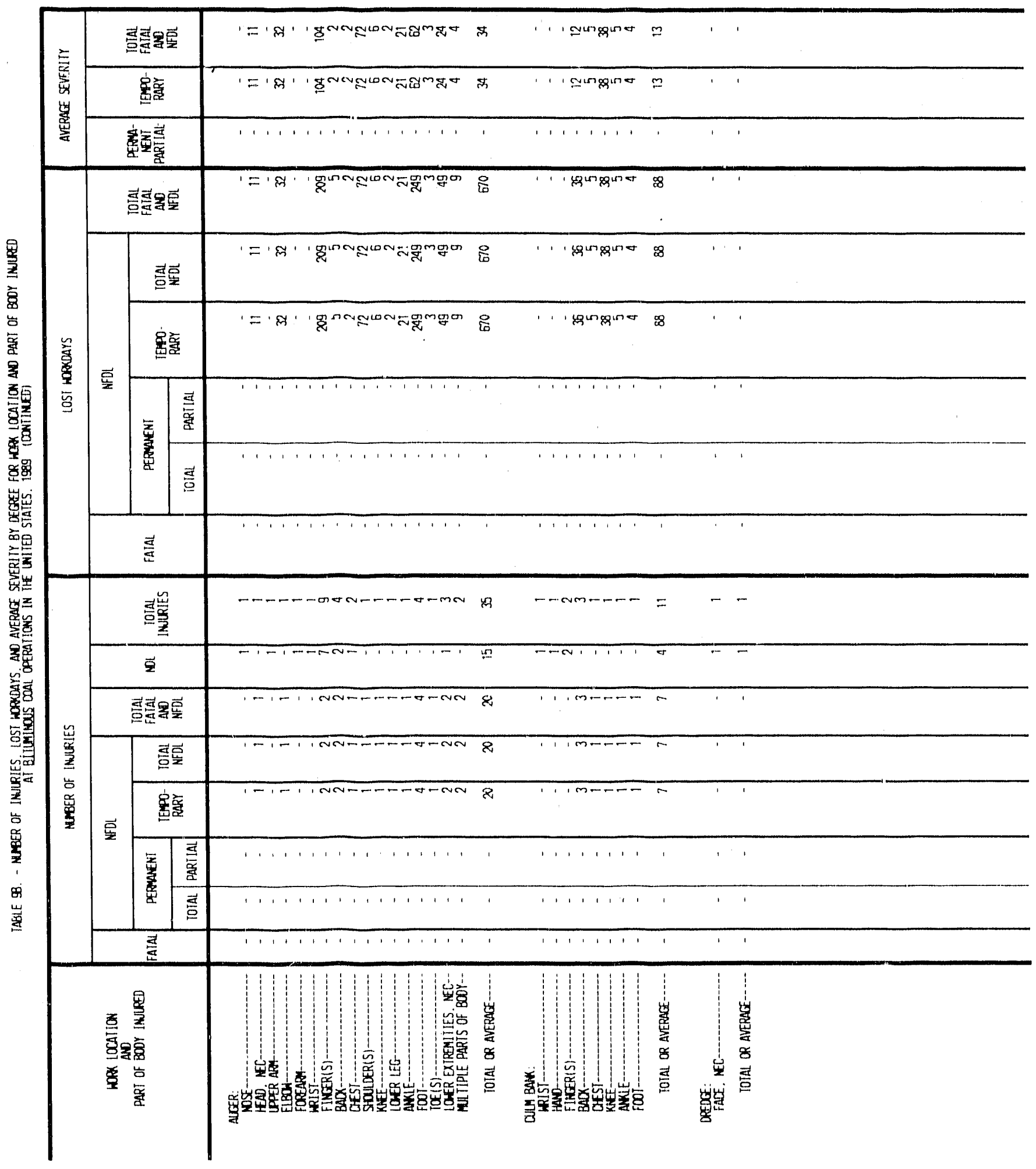




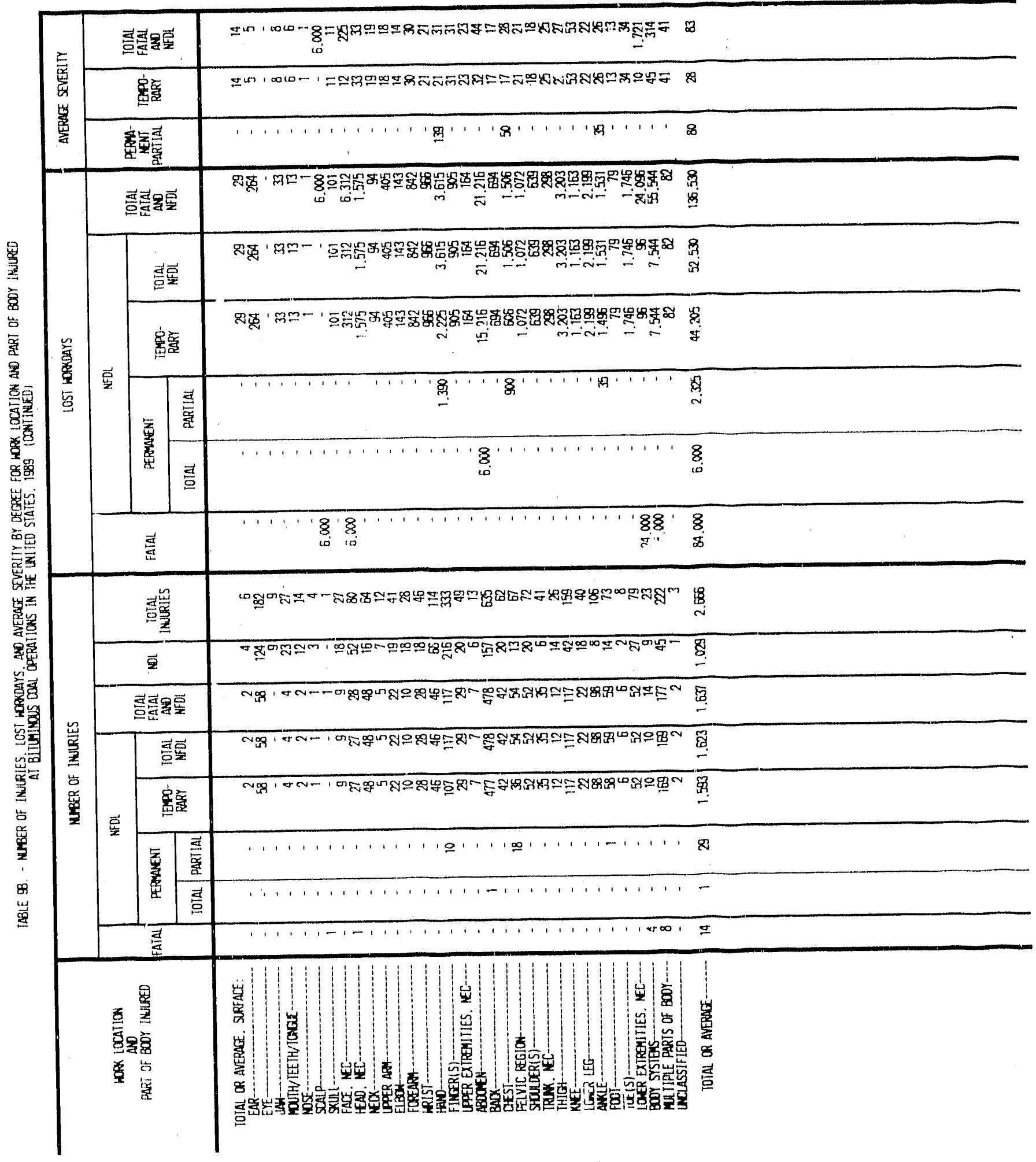




\begin{tabular}{|c|c|c|c|}
\hline \multirow{3}{*}{ 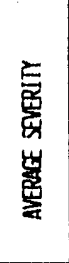 } & 형현연룰 & 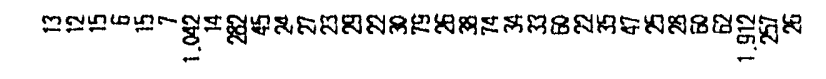 & \\
\hline & 重害 & 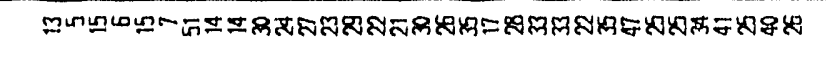 & 8 \\
\hline & 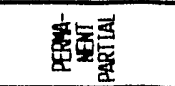 & 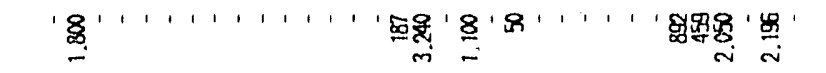 & \\
\hline \multirow{6}{*}{ 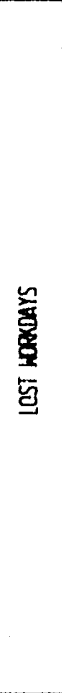 } & 홍련율 & 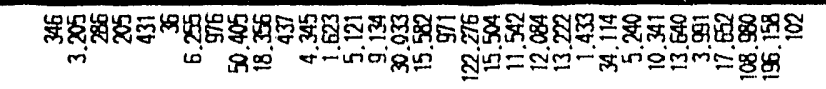 & 客 \\
\hline & 害重 & 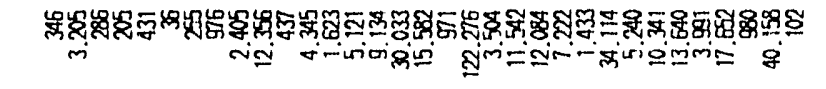 & 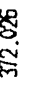 \\
\hline & 宦露 & 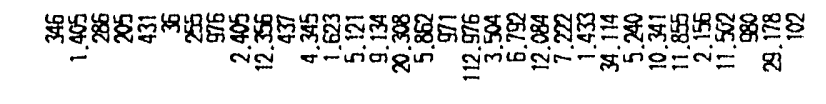 & $\frac{\overline{s i j}}{m}$ \\
\hline & \multirow{2}{*}{ 商 } & 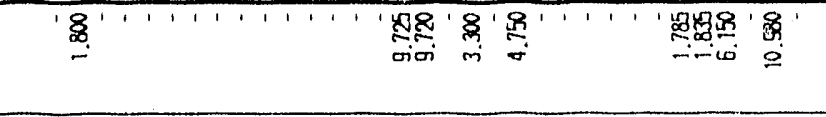 & $\begin{array}{l}\frac{7}{8} \\
8 \\
8\end{array}$ \\
\hline & & 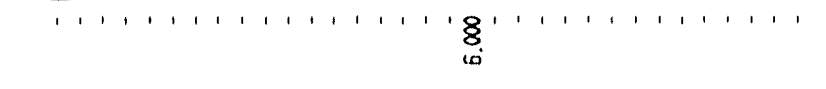 & $8_{\omega}^{8}$ \\
\hline & $\frac{7}{4}$ & 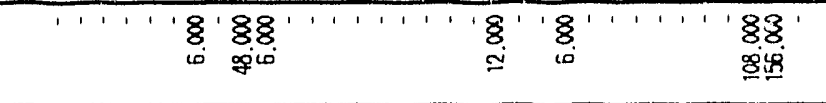 & 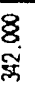 \\
\hline \multirow{8}{*}{ 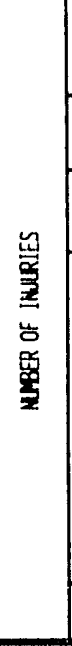 } & 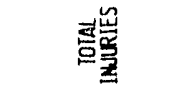 & 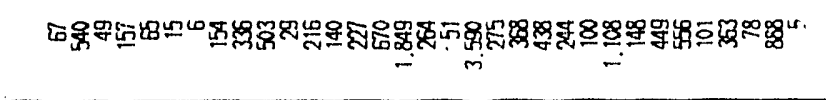 & gig \\
\hline & $\ddot{z}$ & QE্র & ह্户 \\
\hline & 흥현원물 & 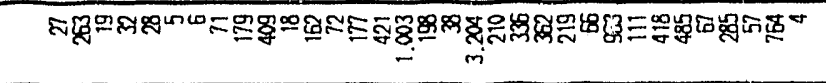 & 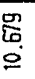 \\
\hline & 홍몽 & 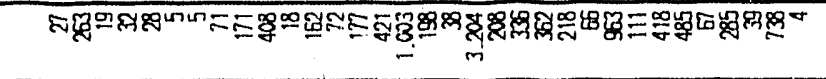 & đֶֶ̆ \\
\hline & 䔡害 & 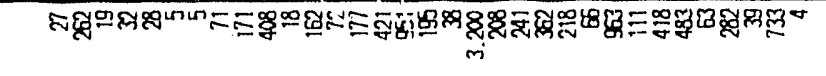 & 孚 \\
\hline & 资震 & gm! & 18 \\
\hline & 穿 & $1 \ldots 1$ & - \\
\hline & 至 & ' ' 1 此 ' & in \\
\hline & 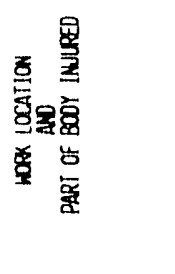 & 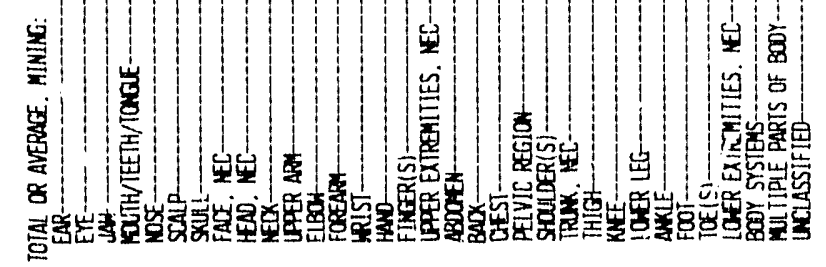 & 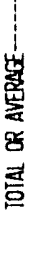 \\
\hline
\end{tabular}




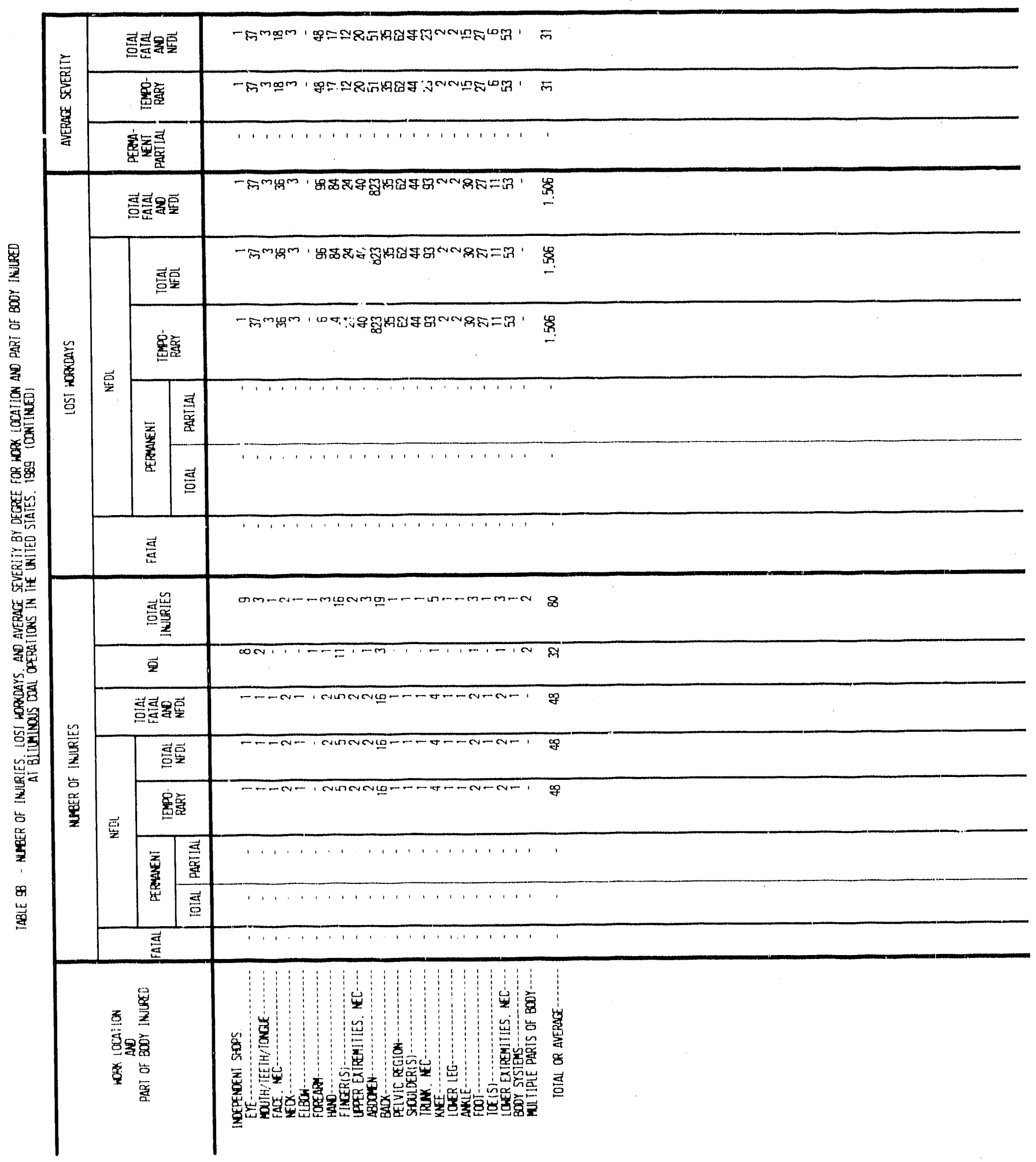




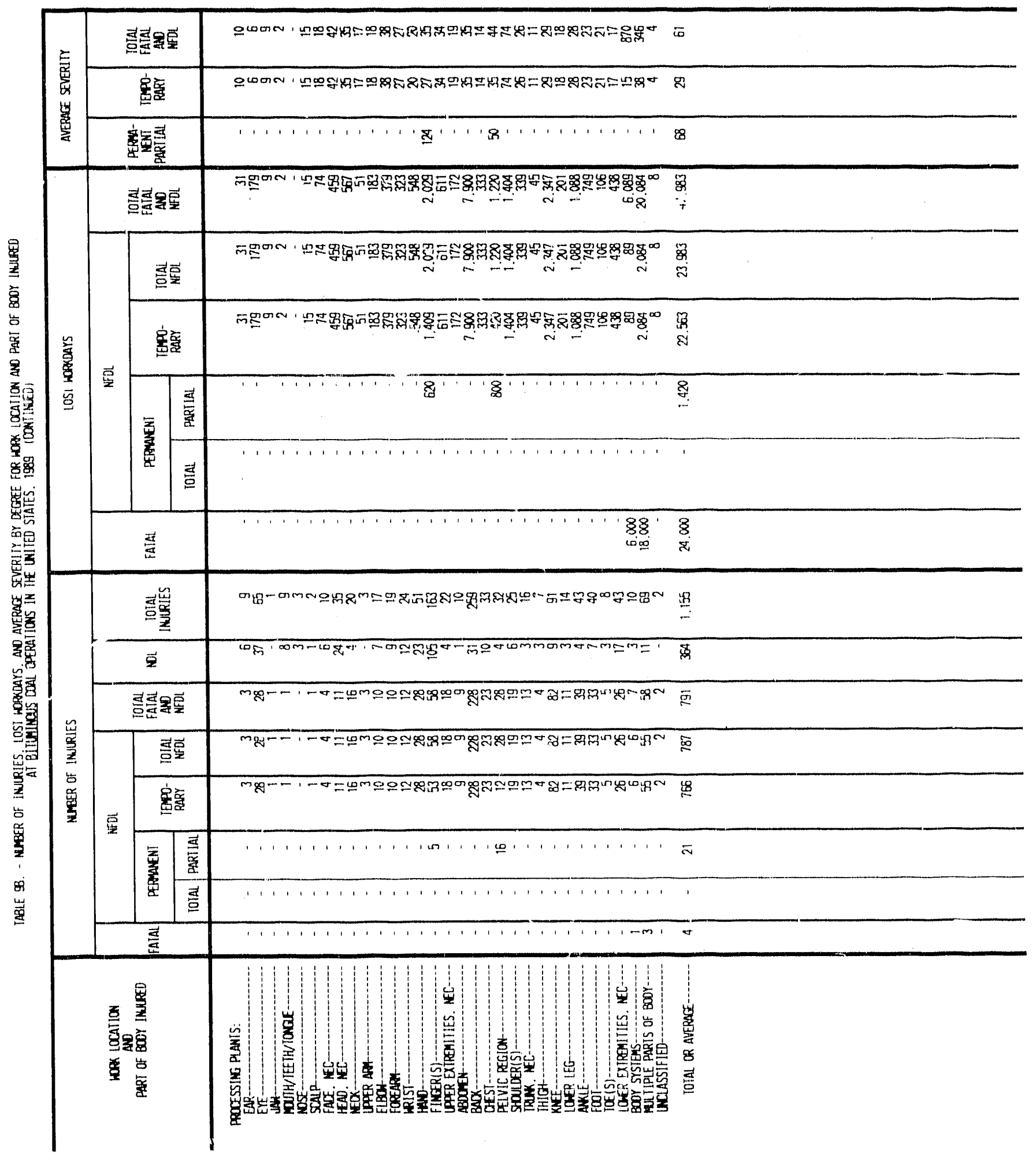




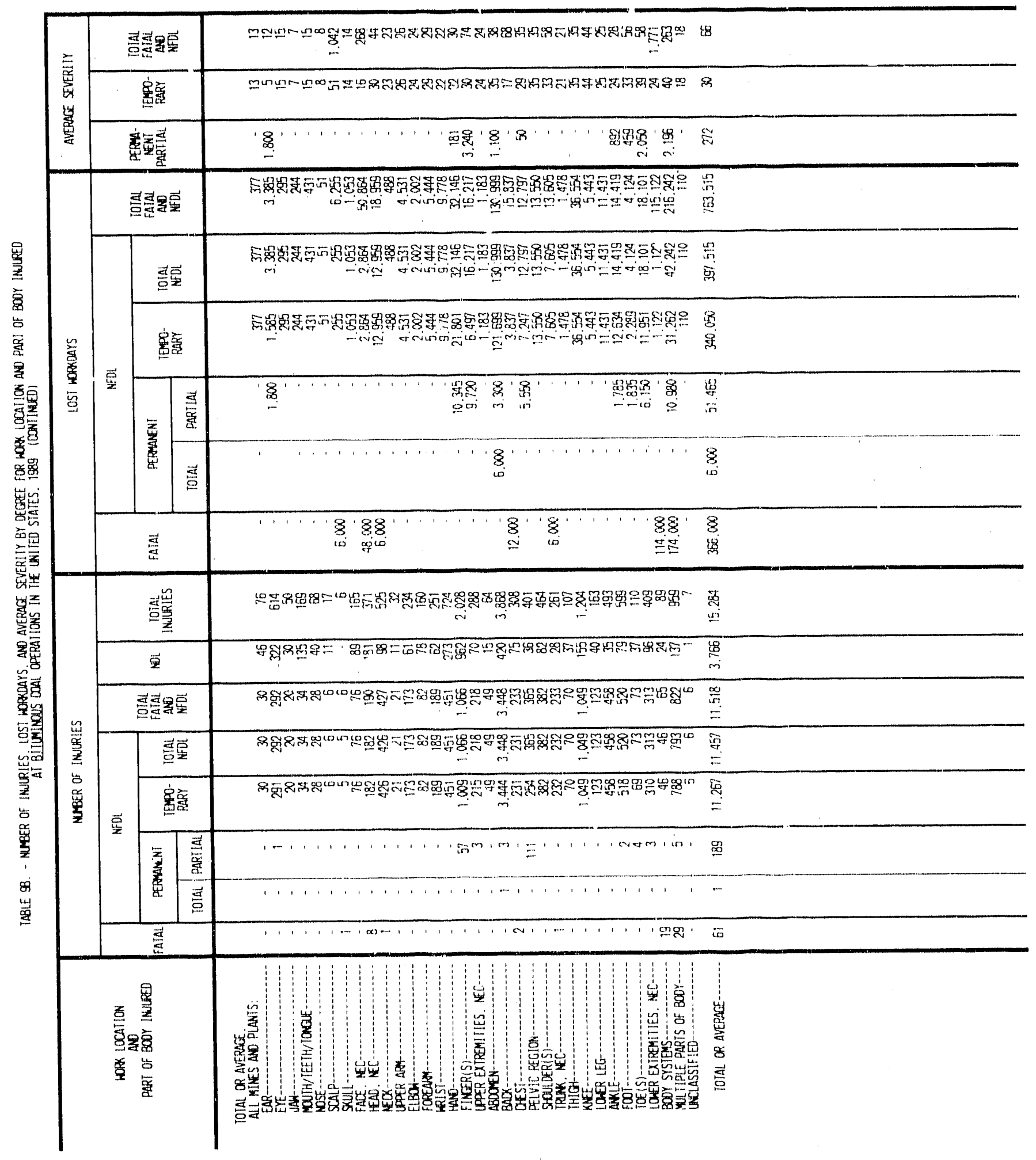




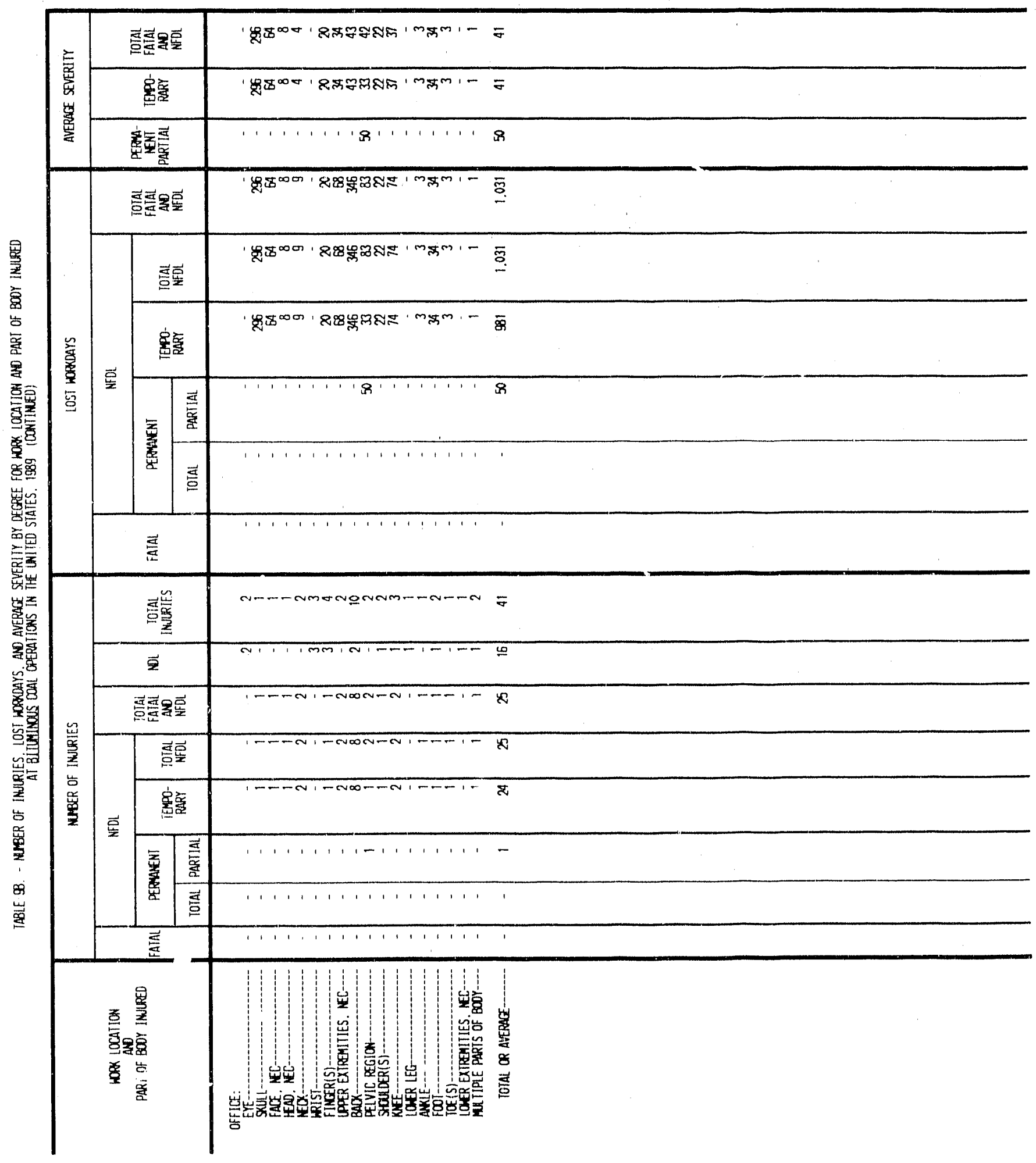




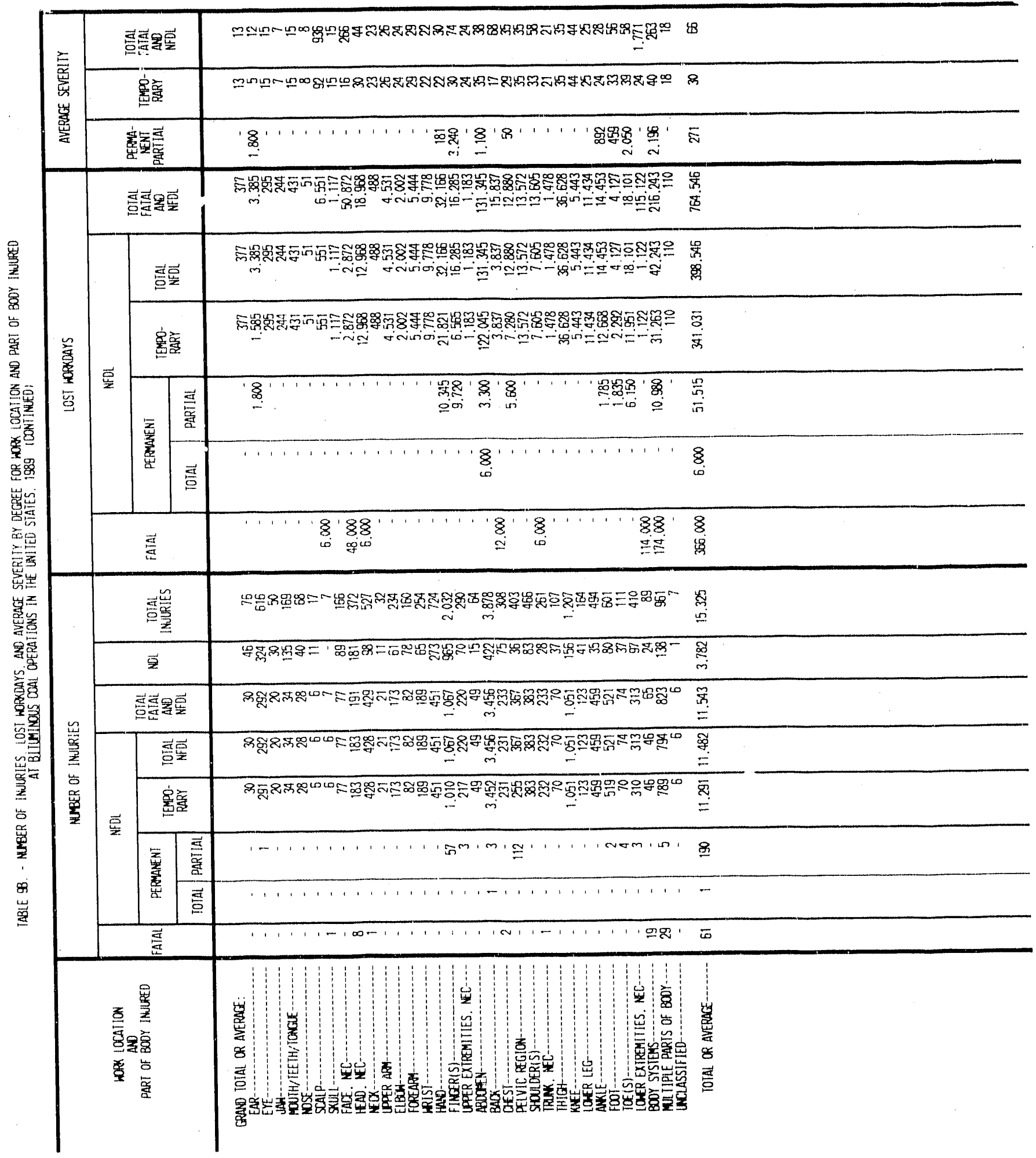




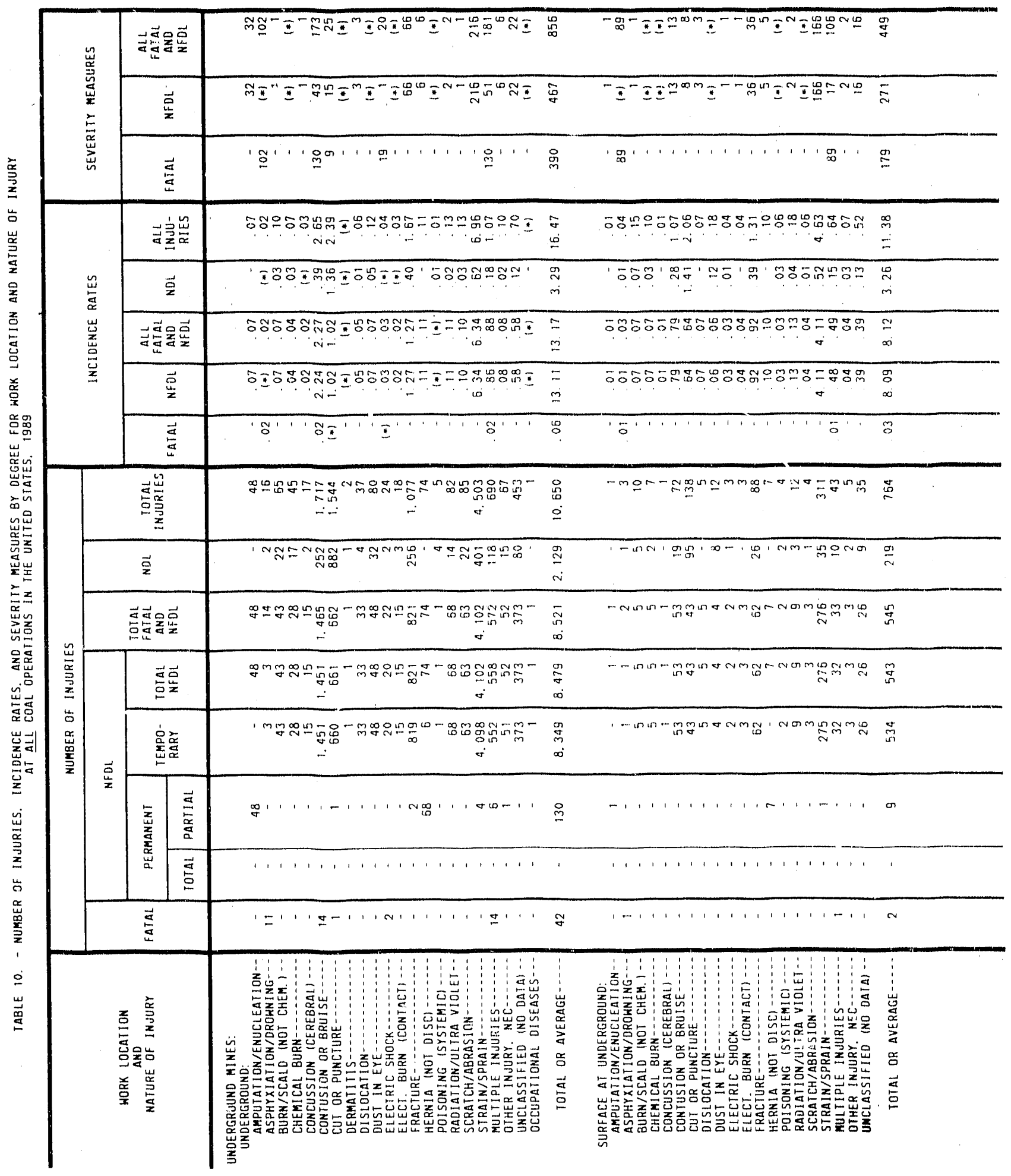




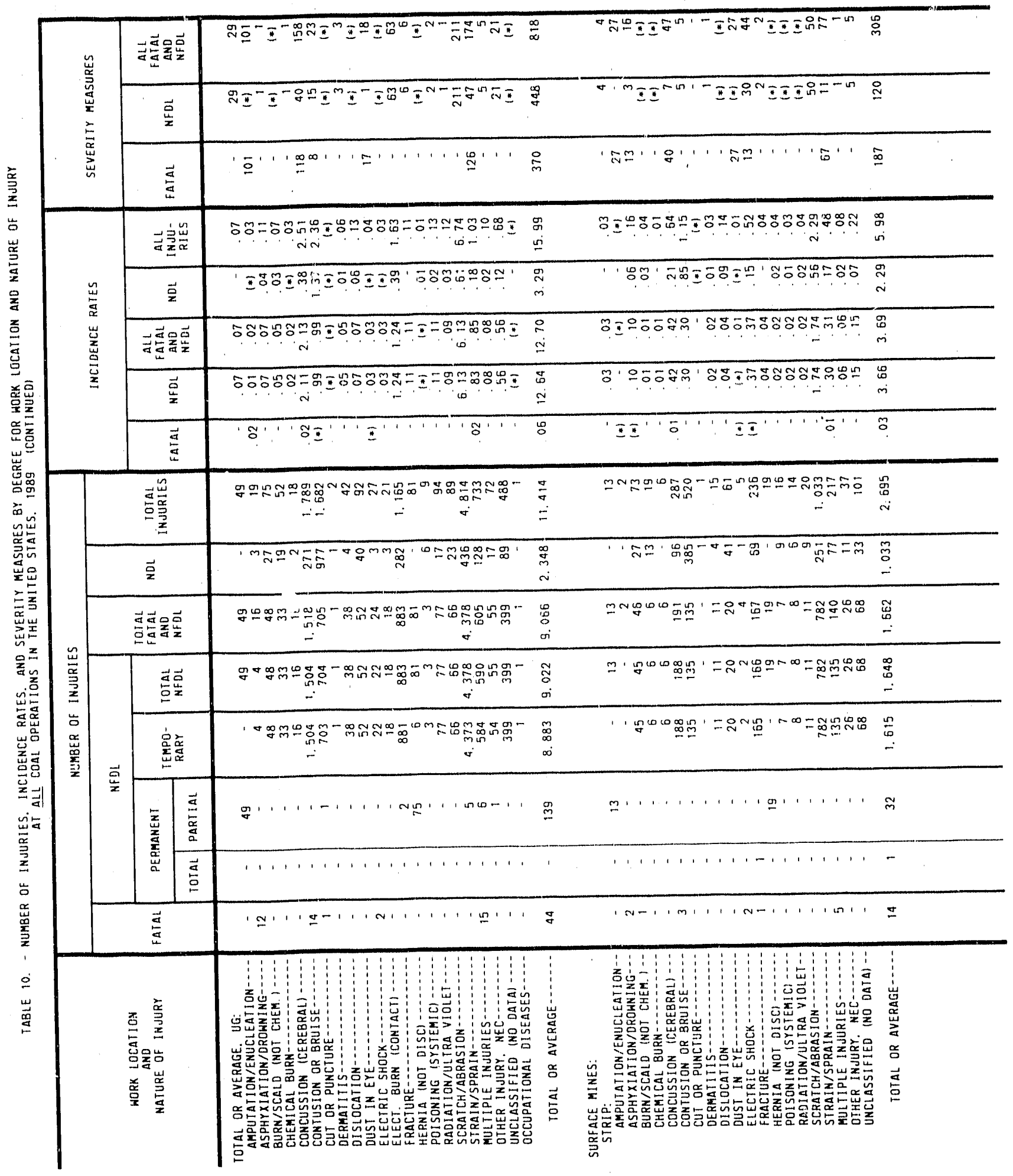




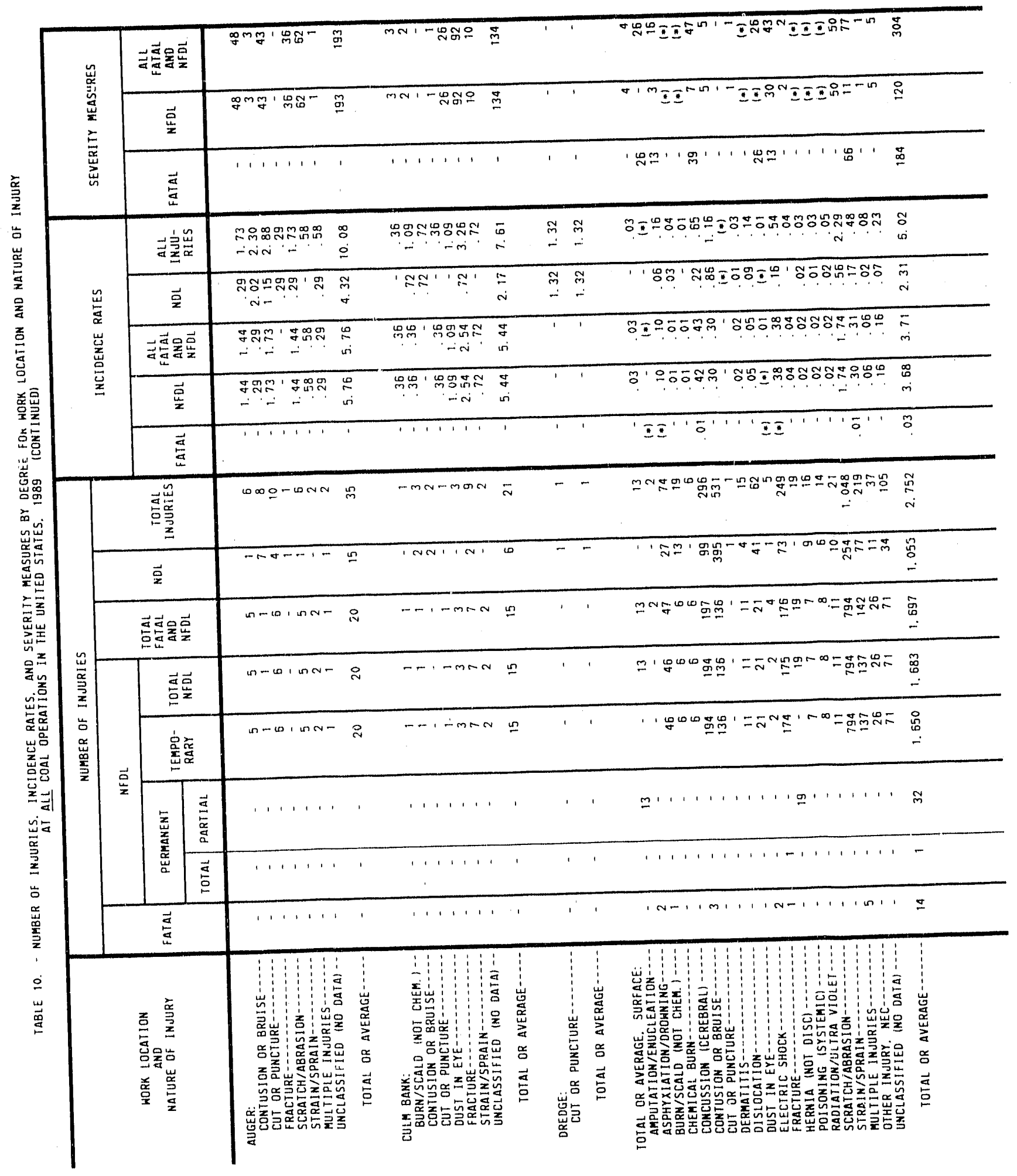




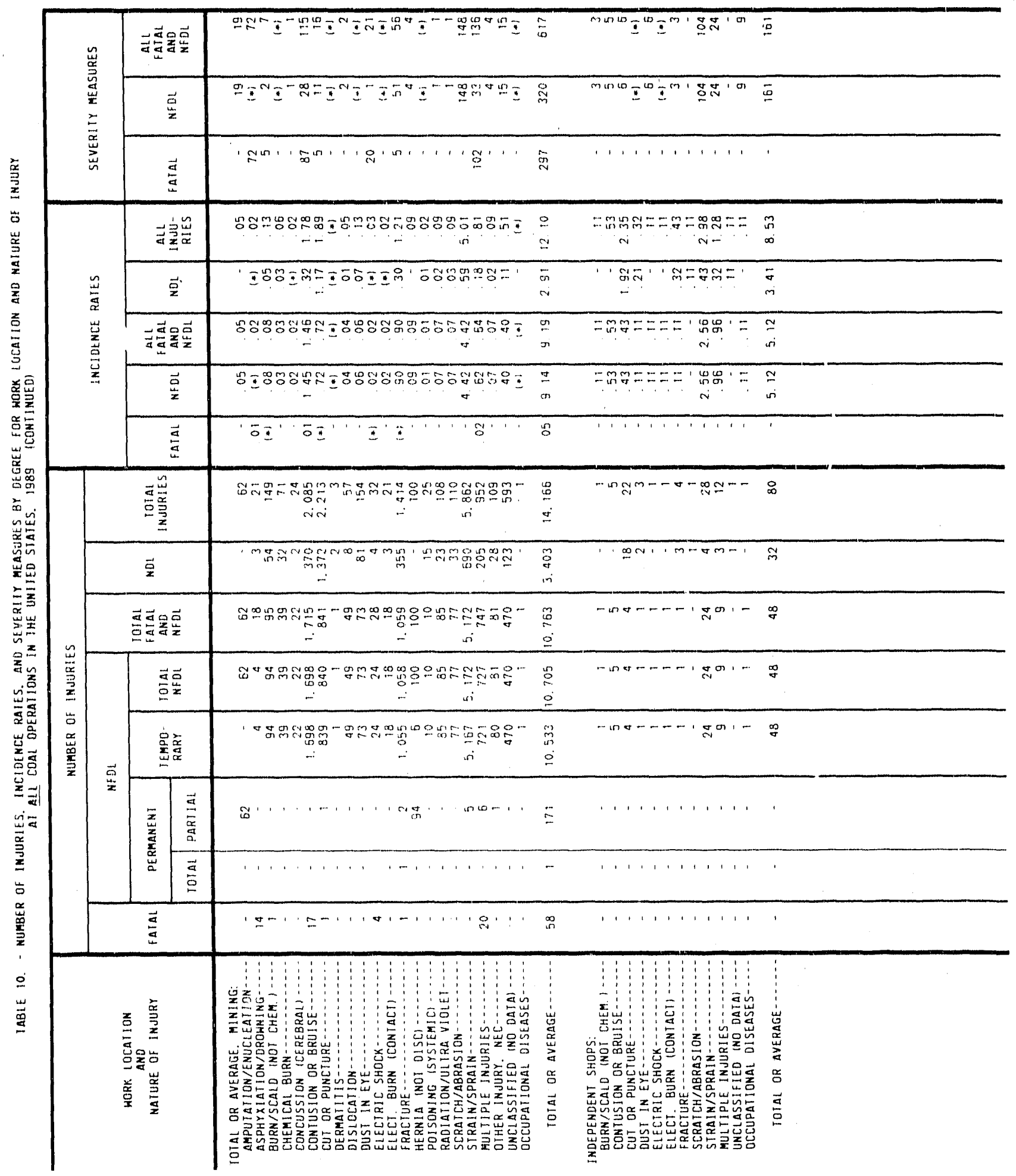




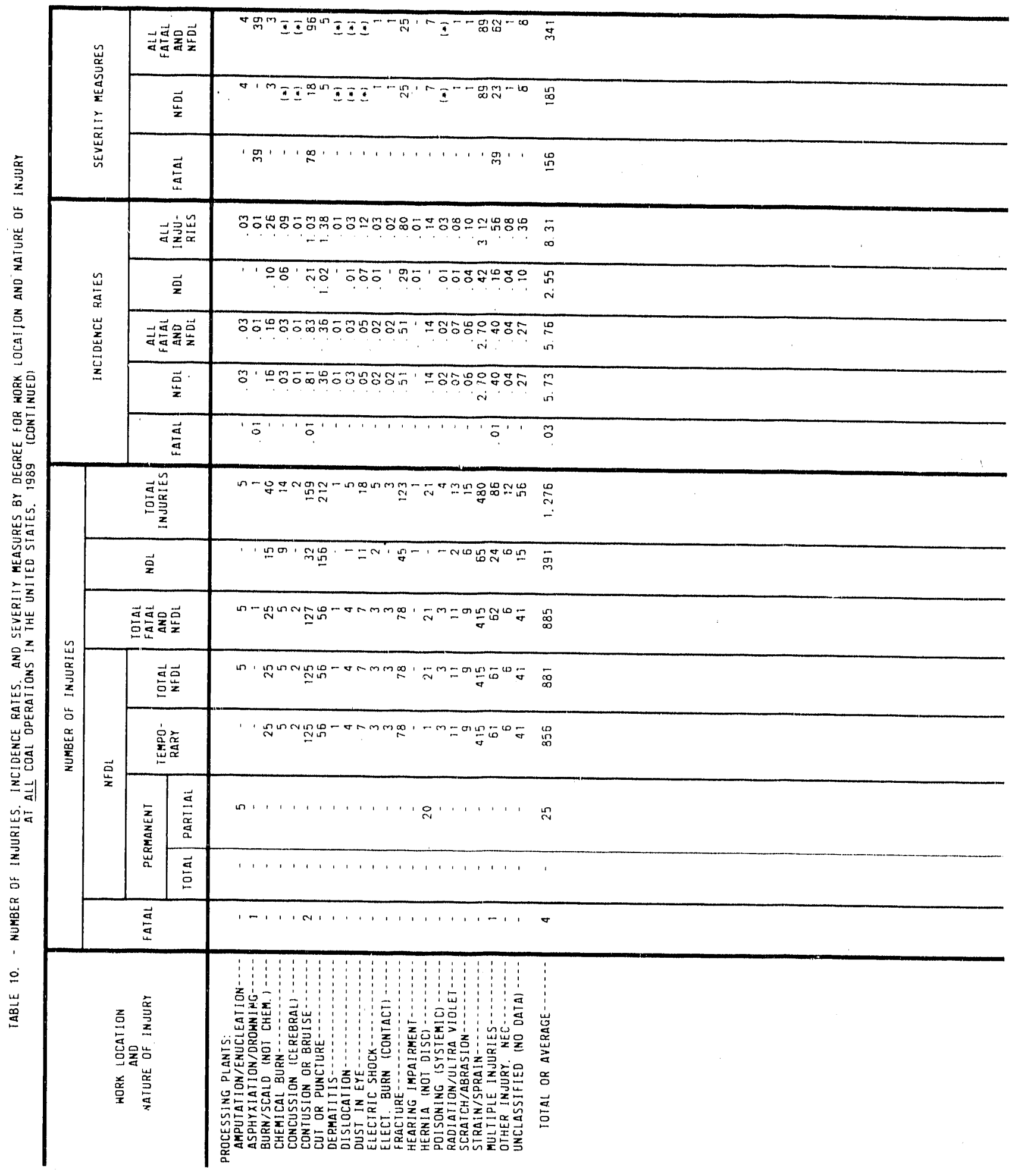




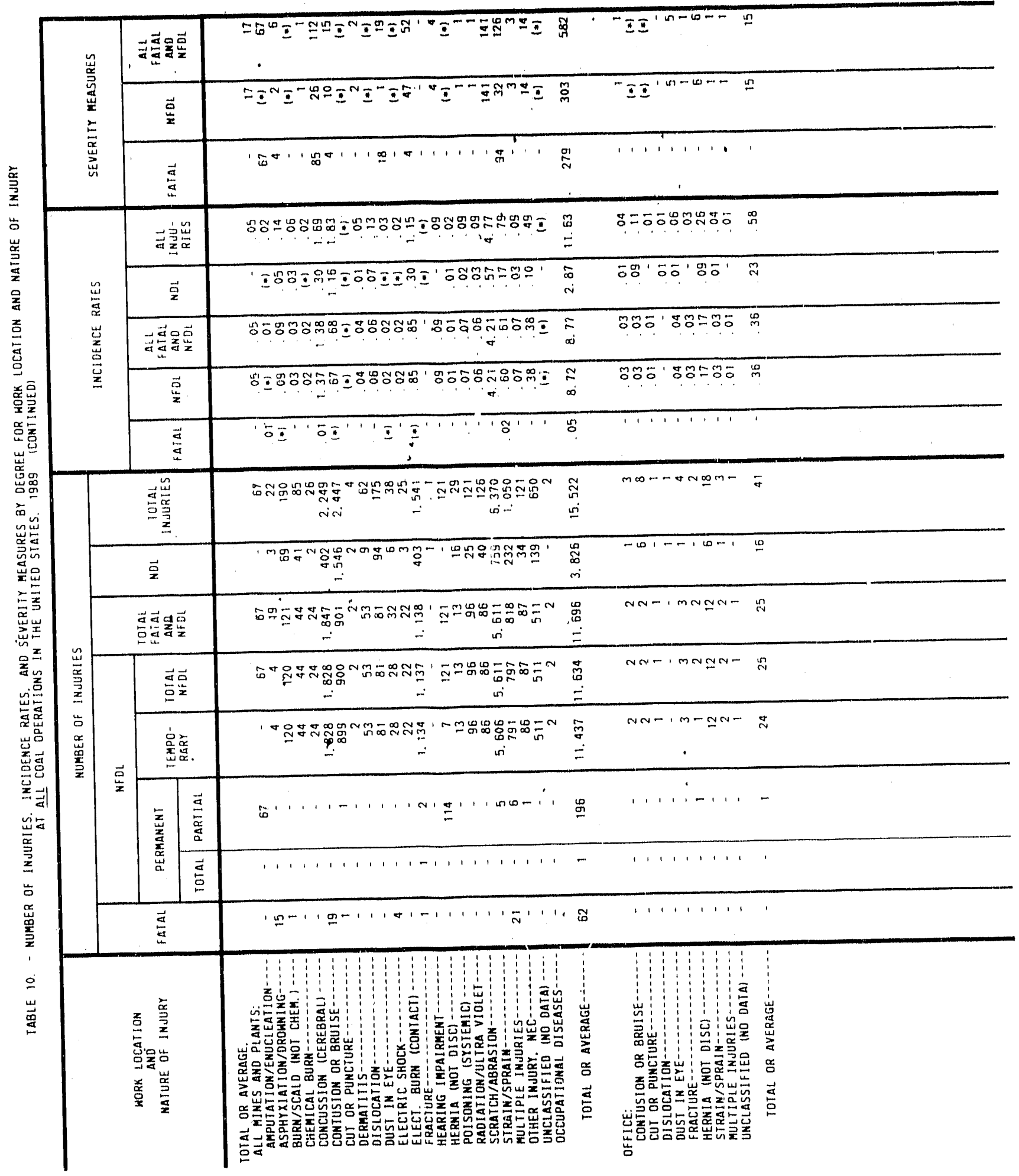




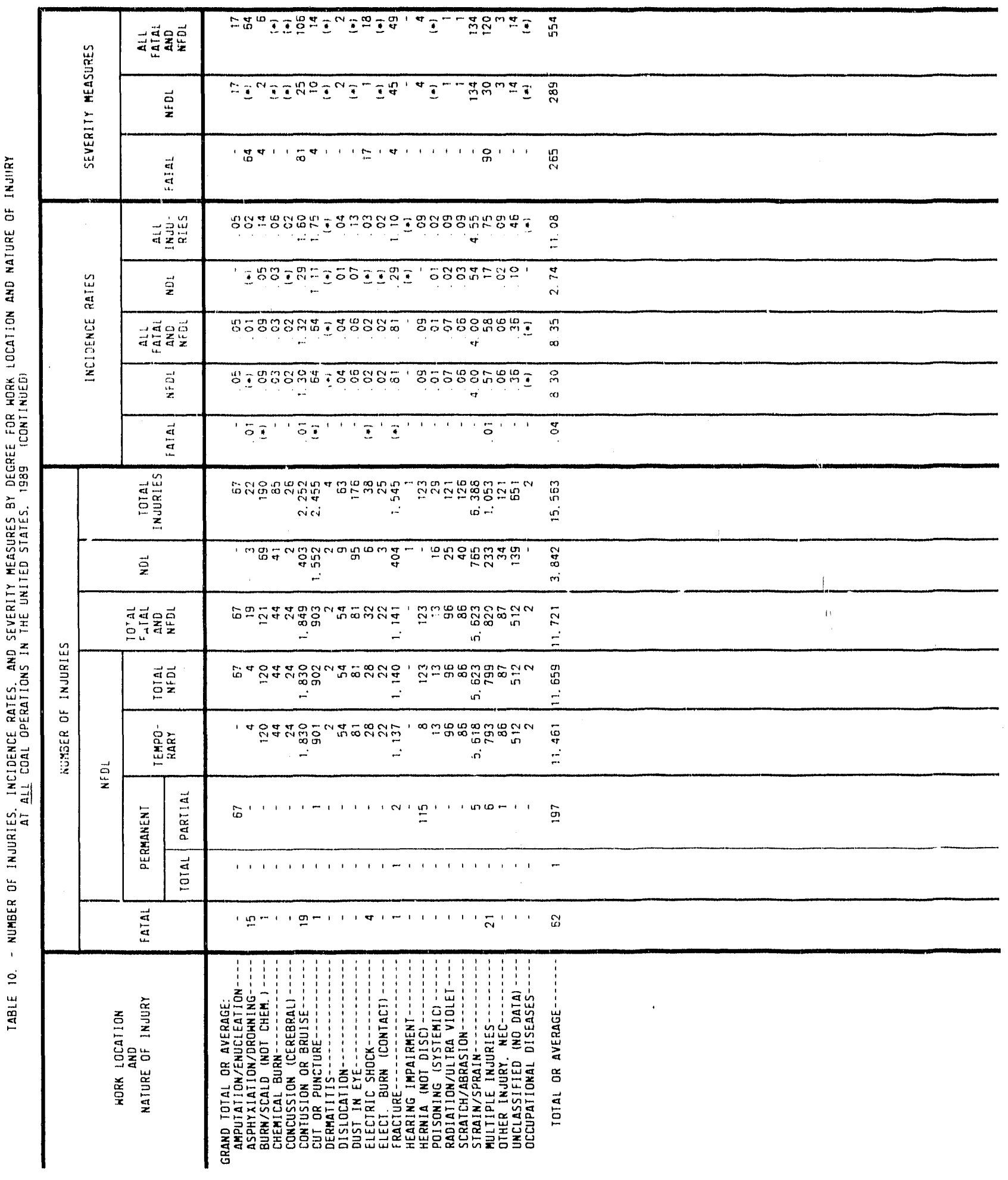




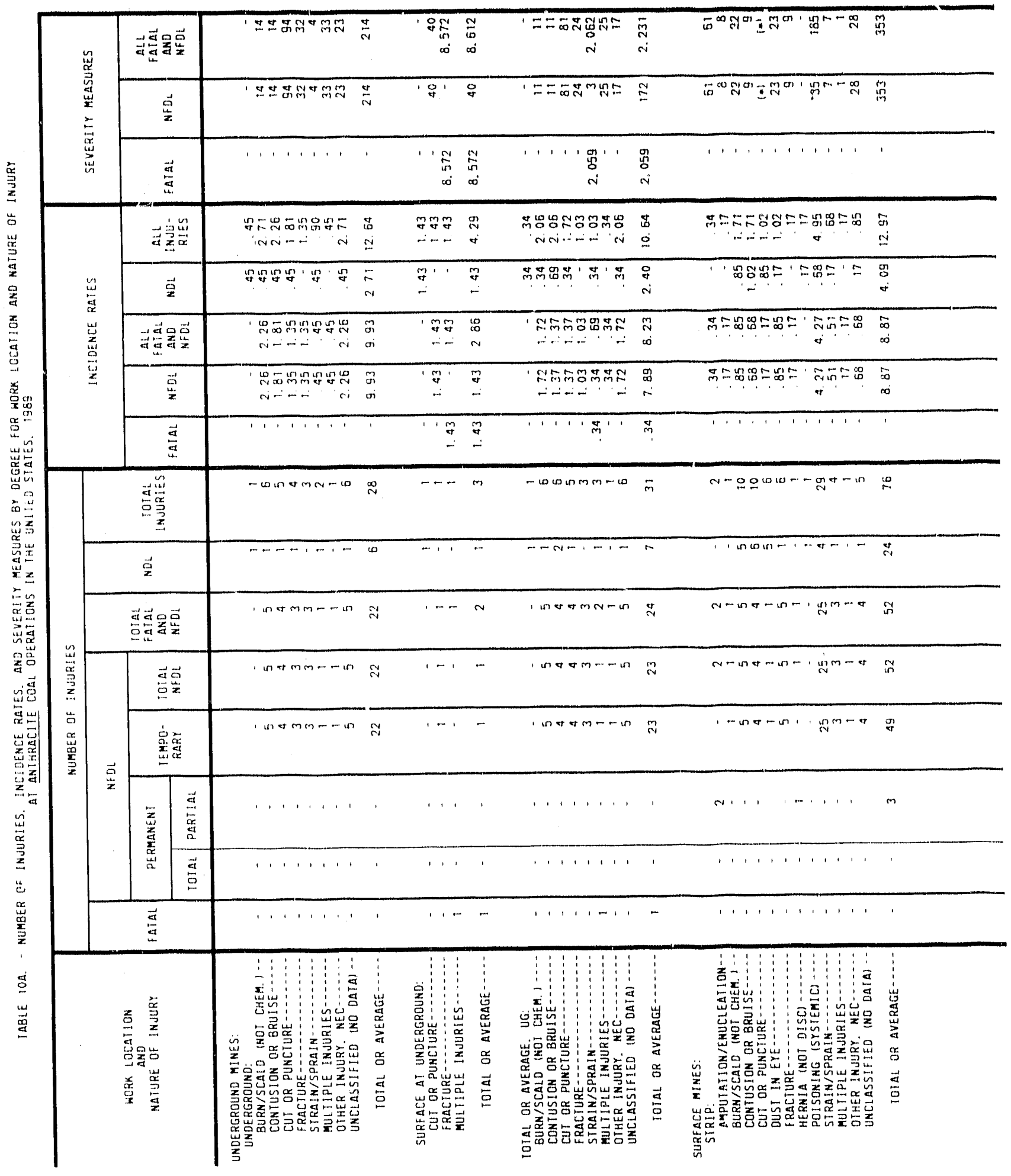




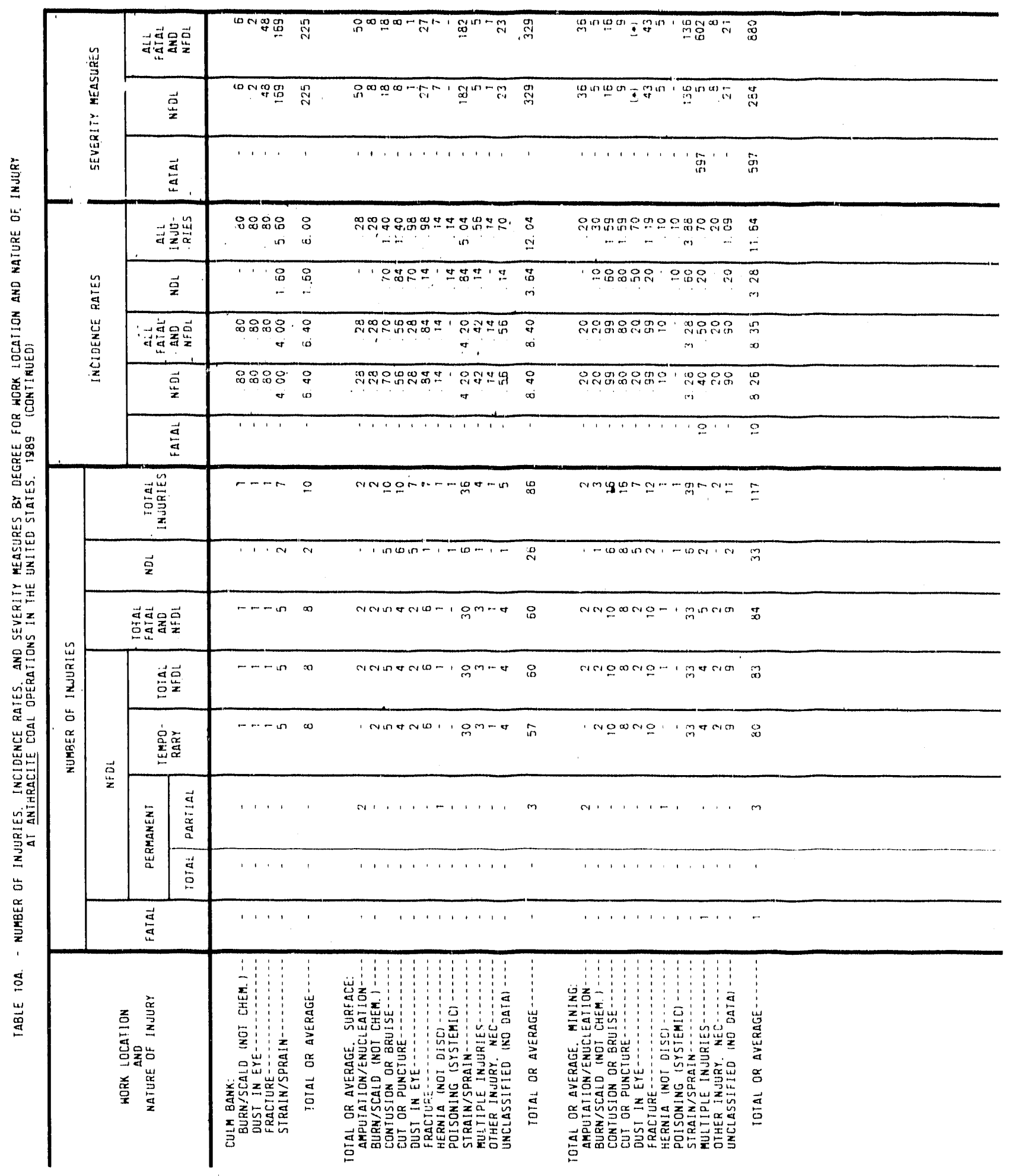




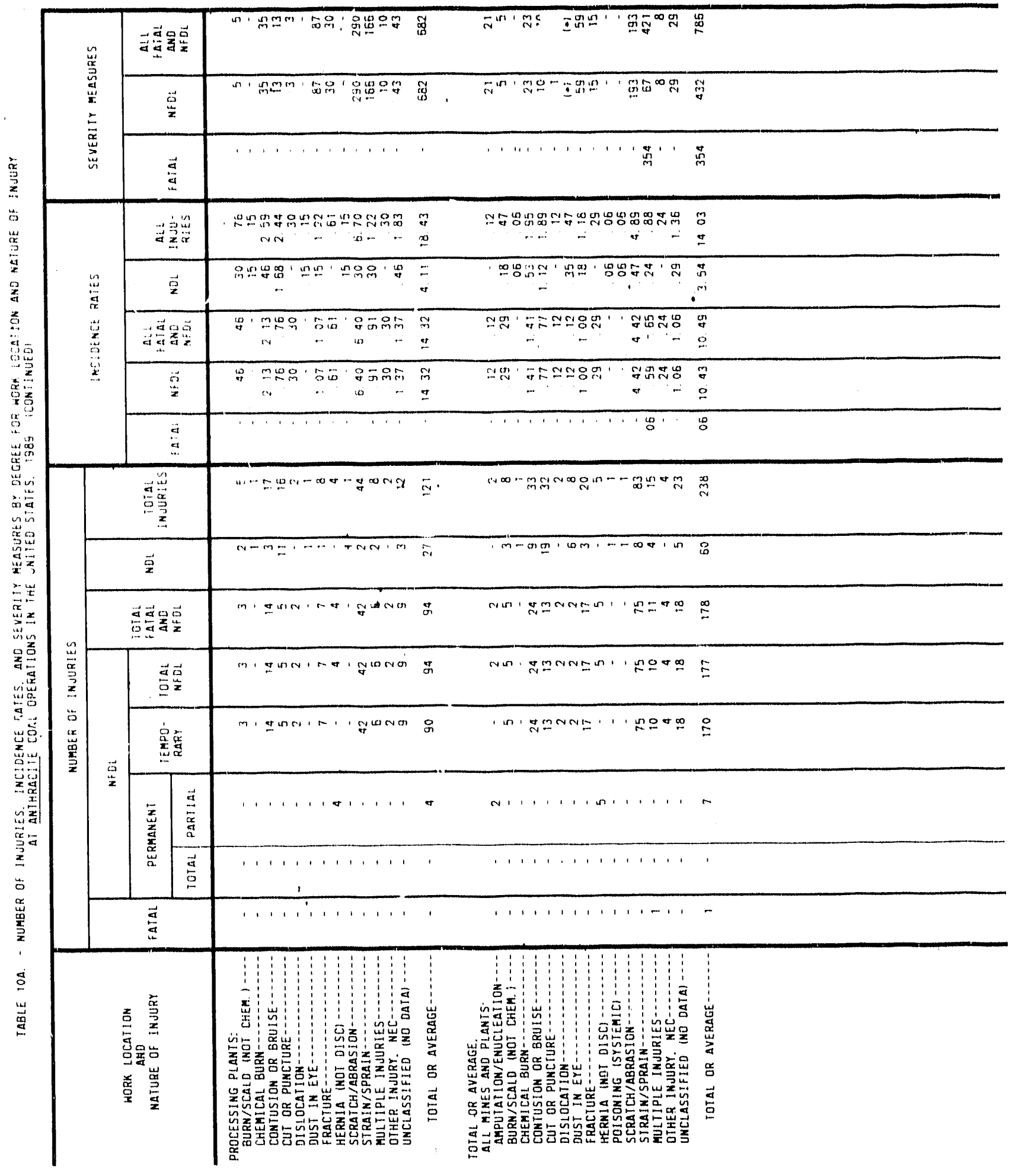




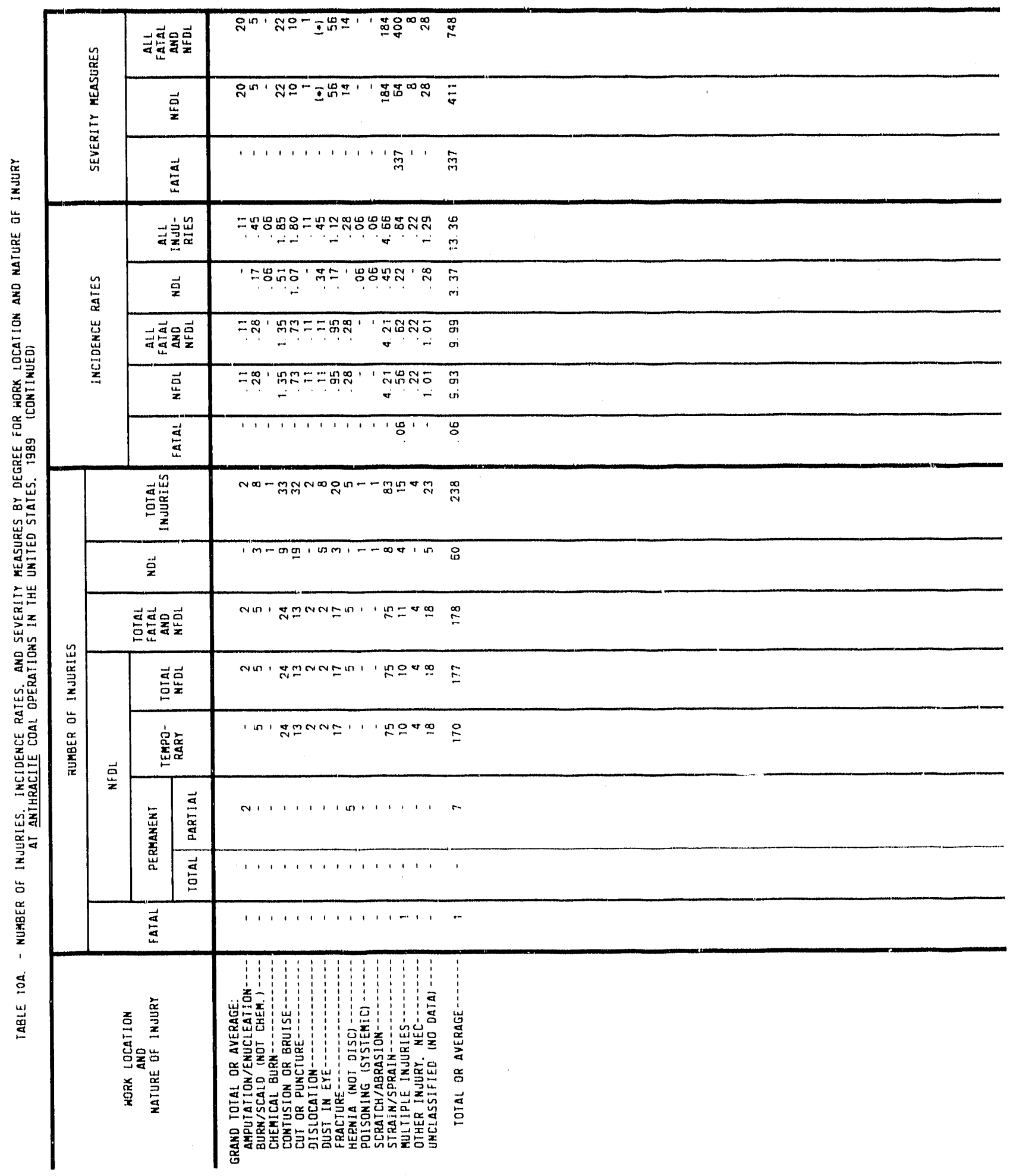




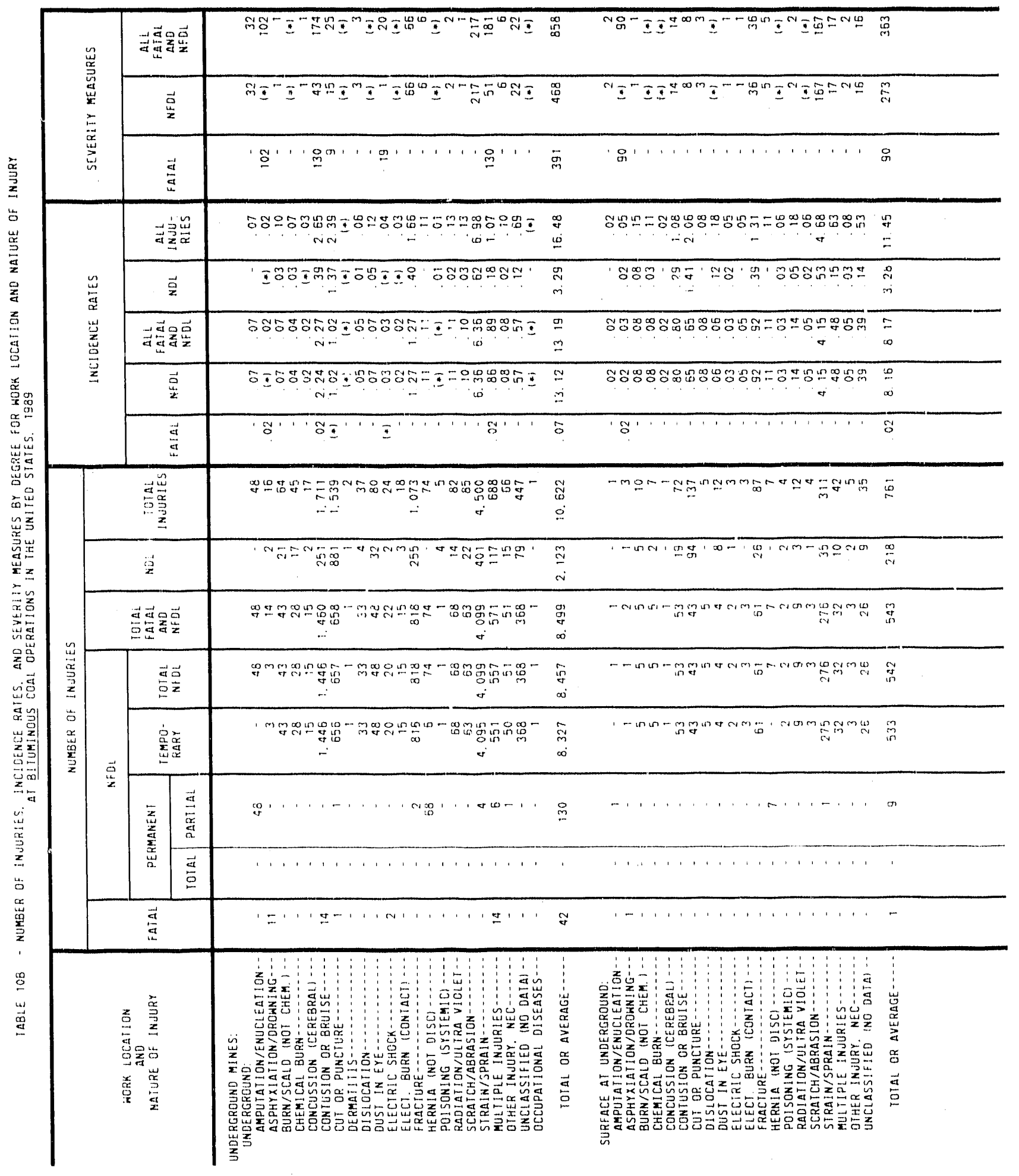




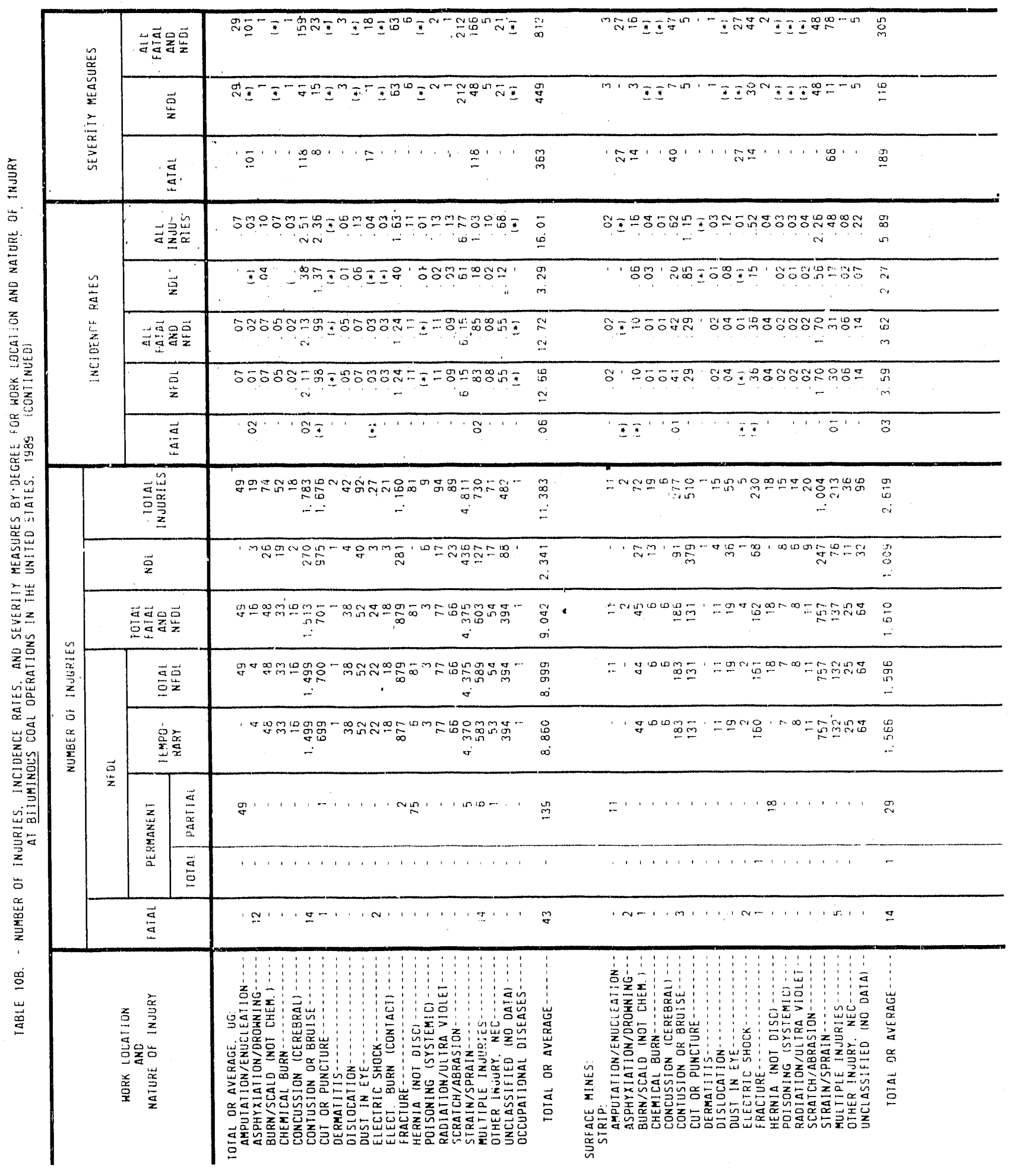




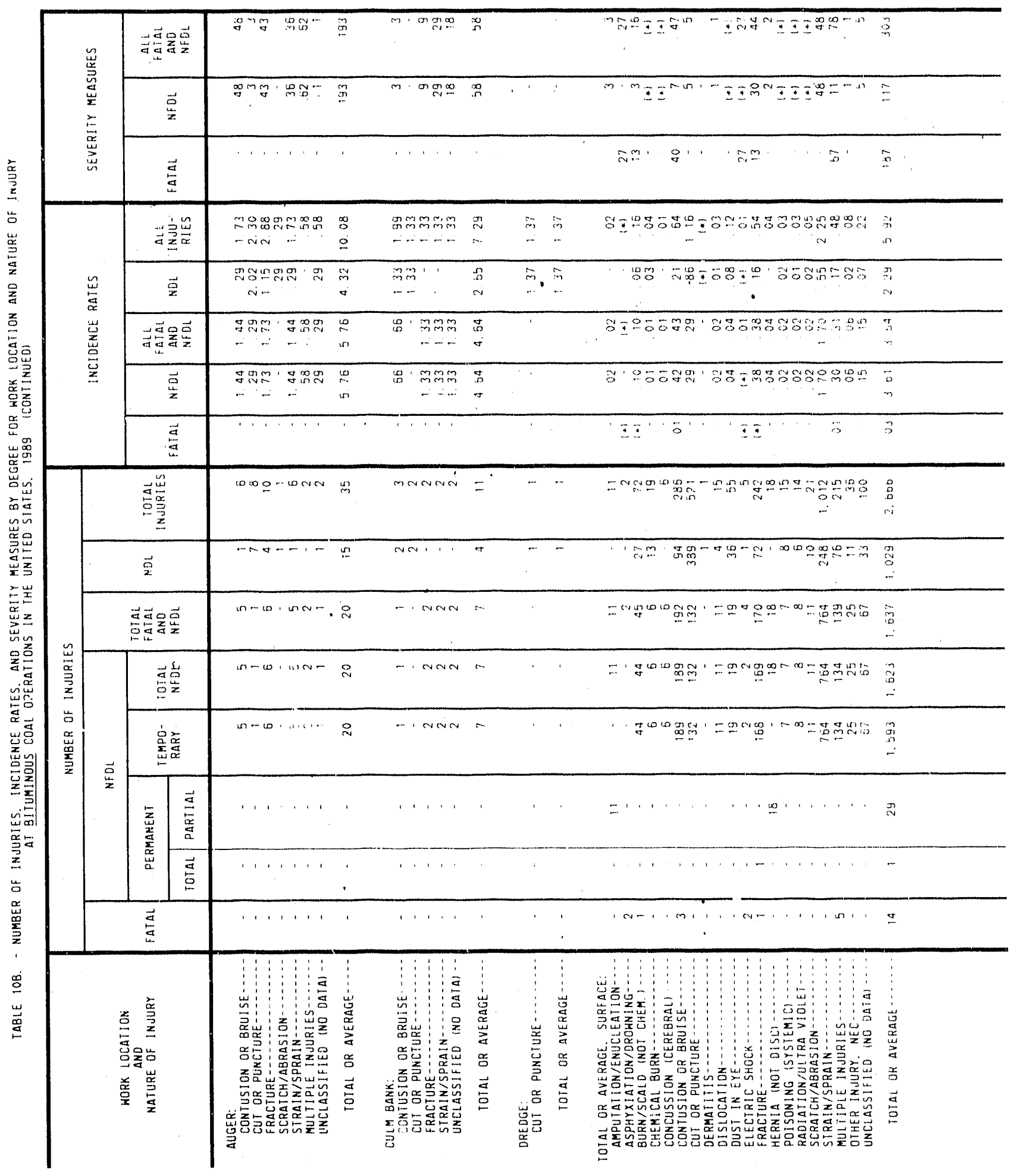




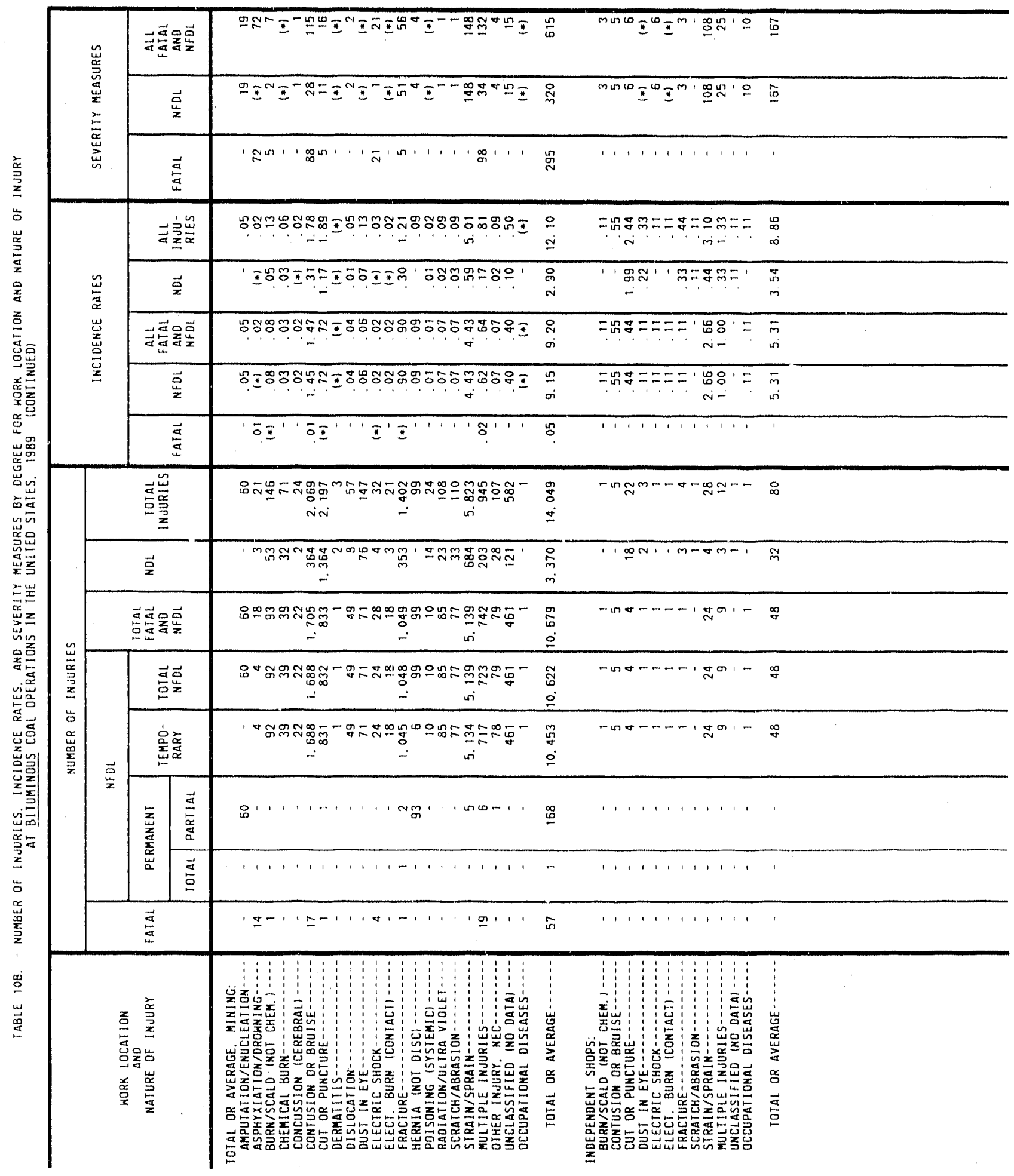




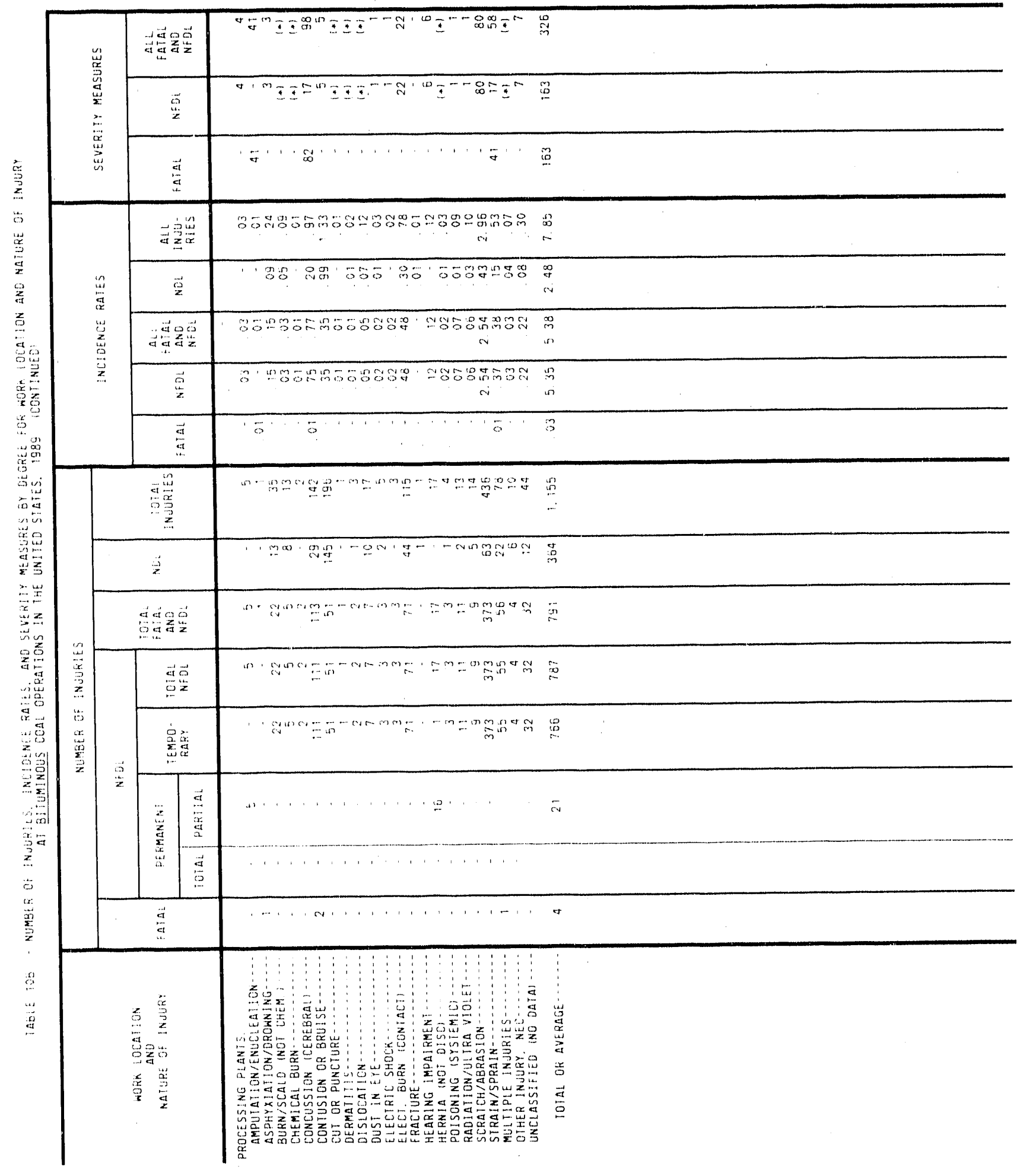




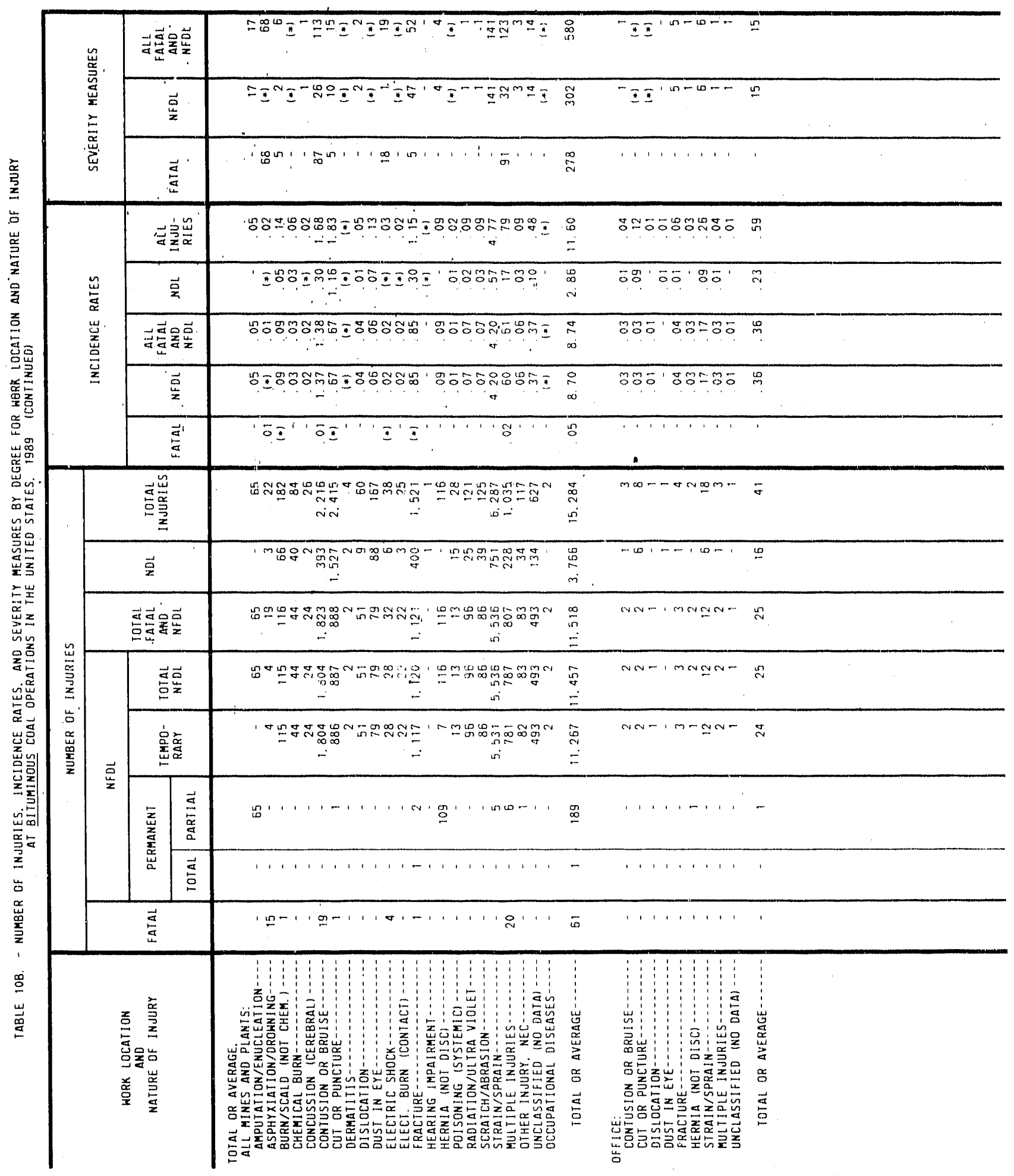




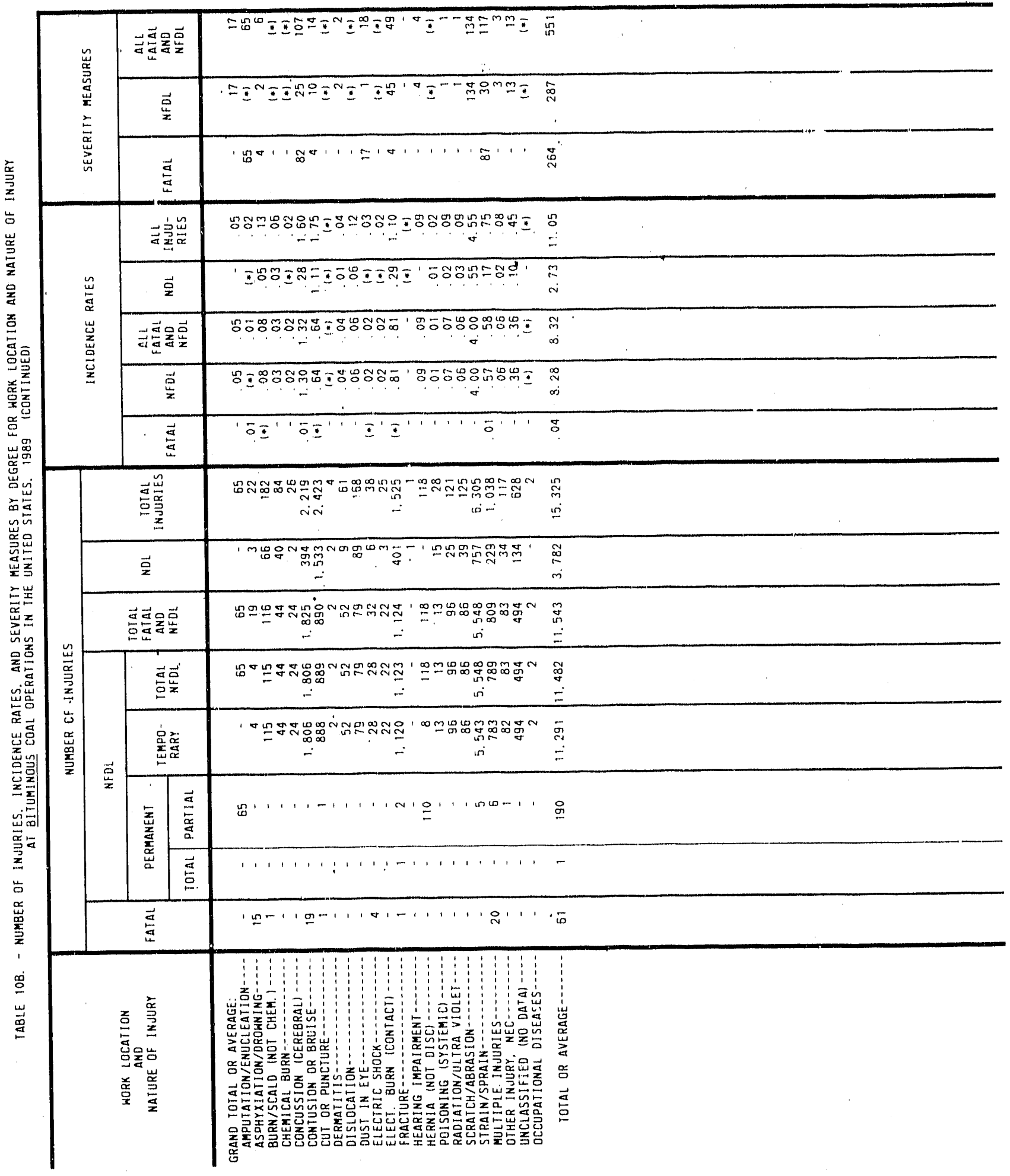




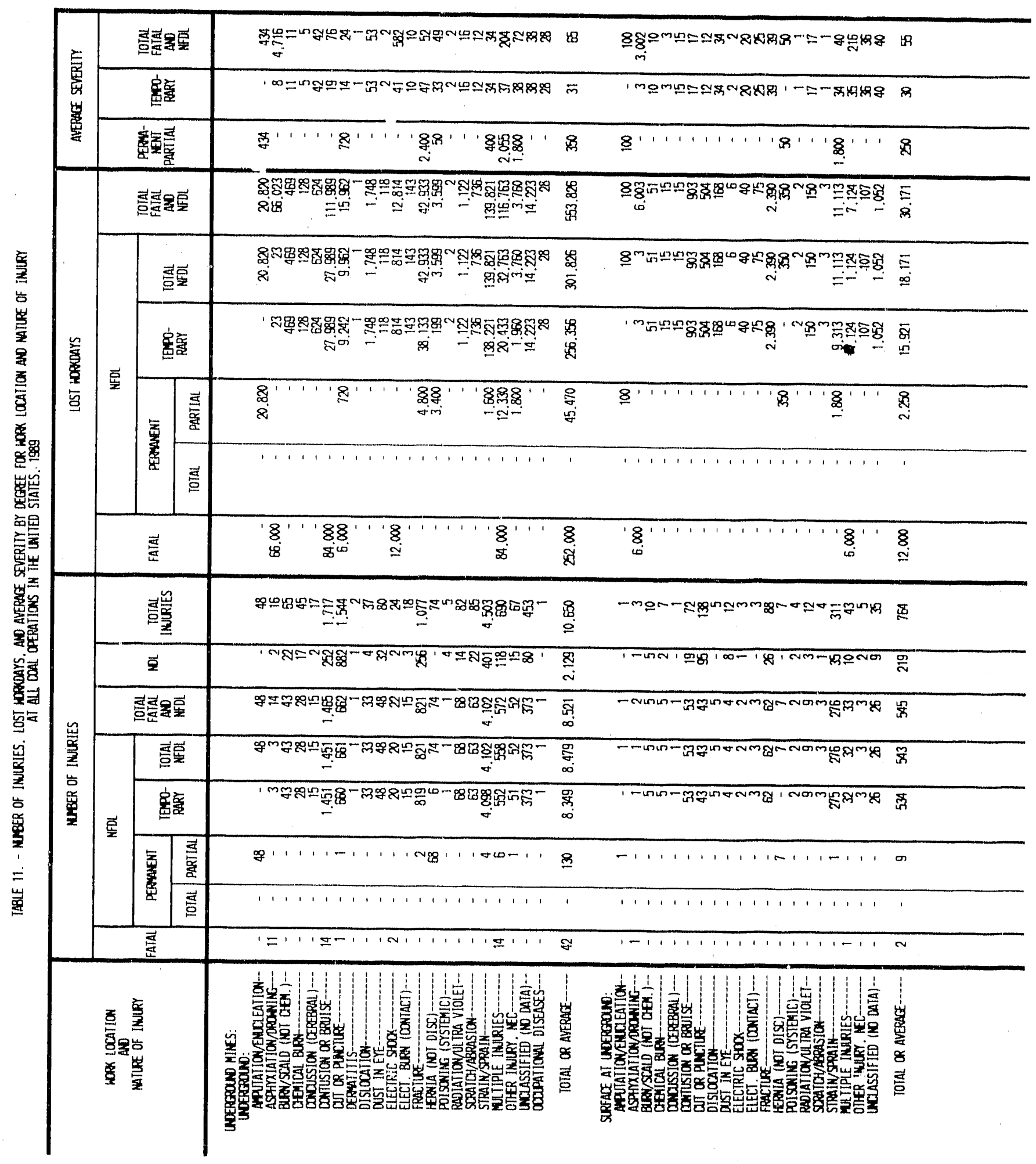




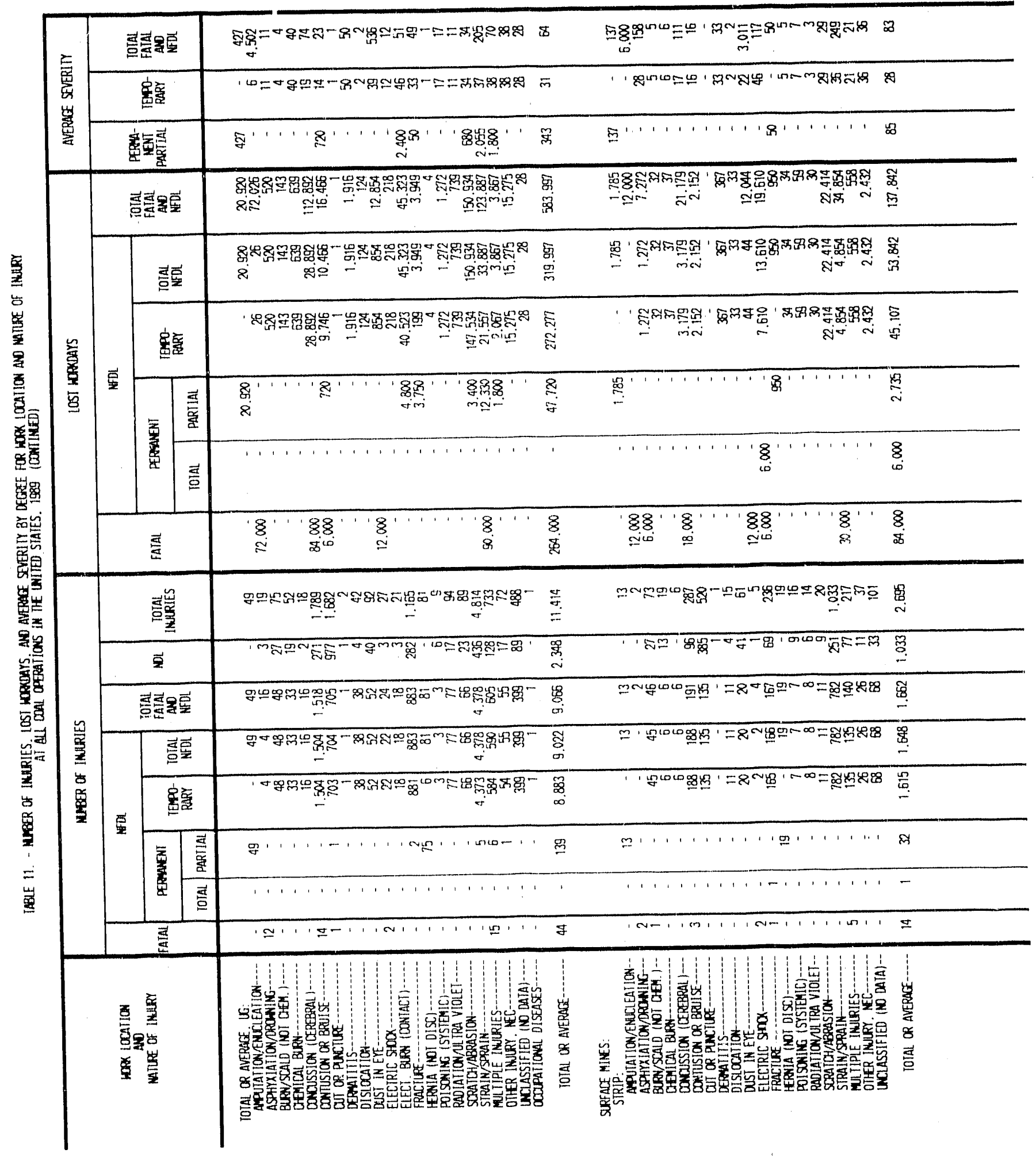




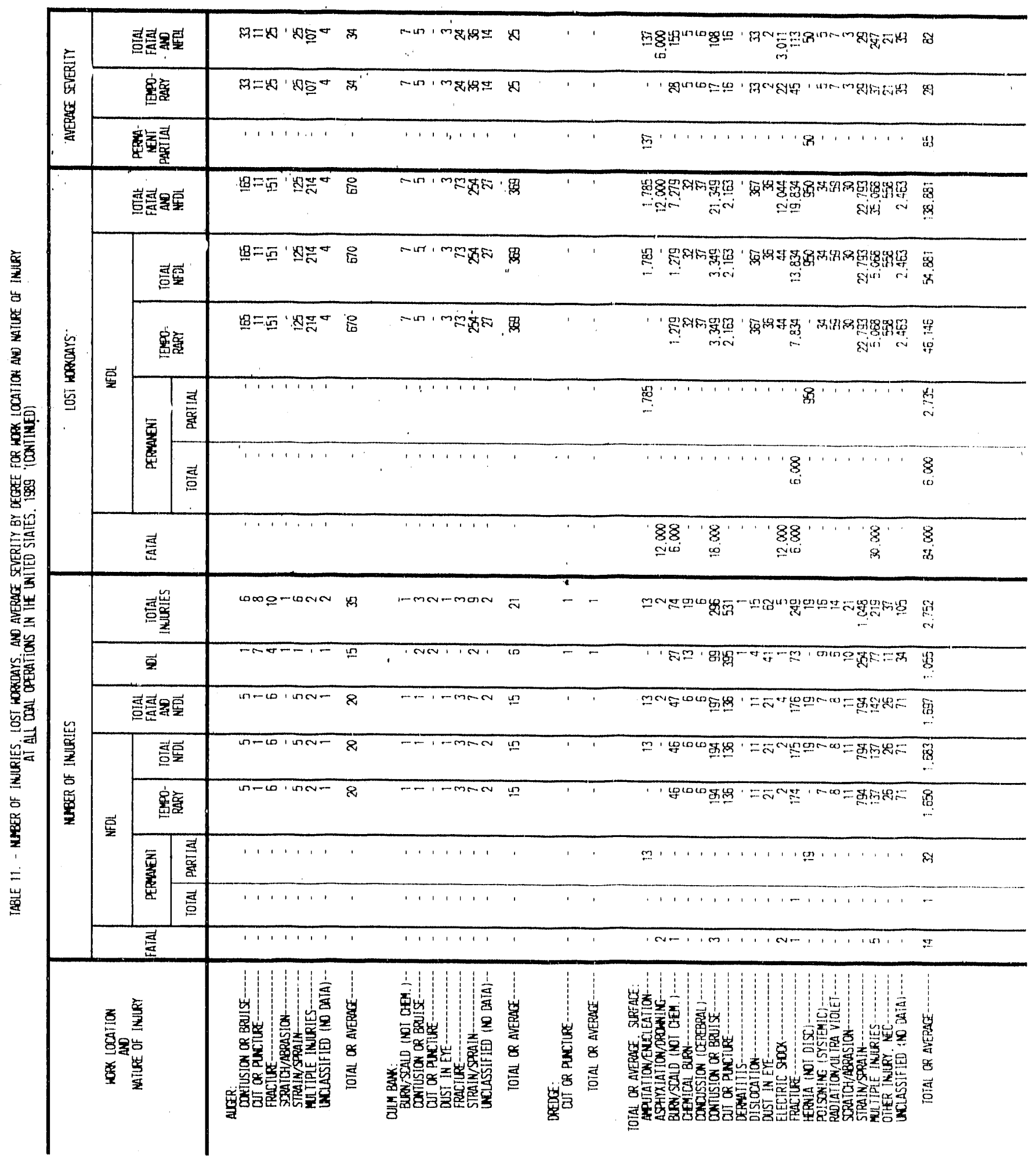




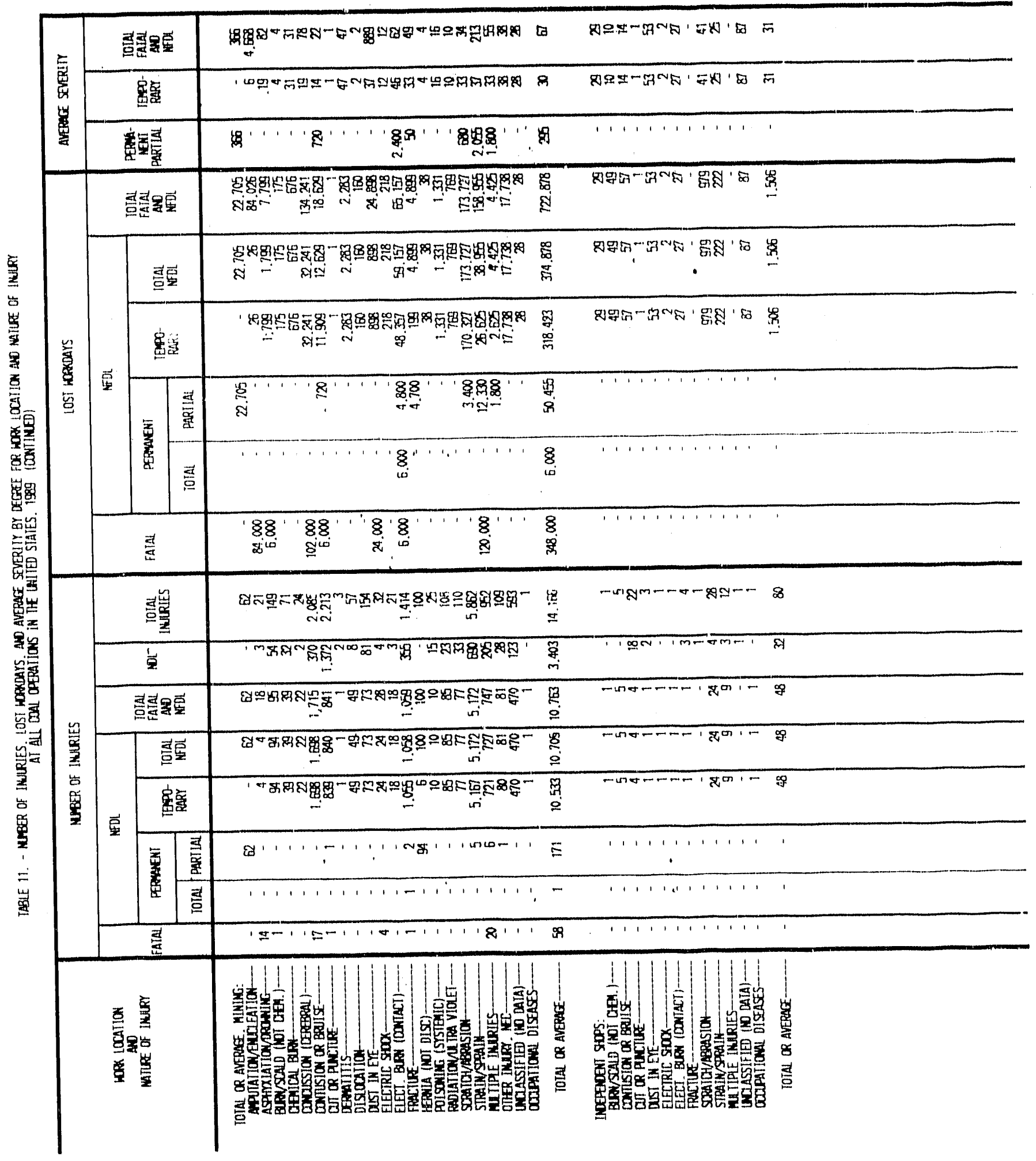




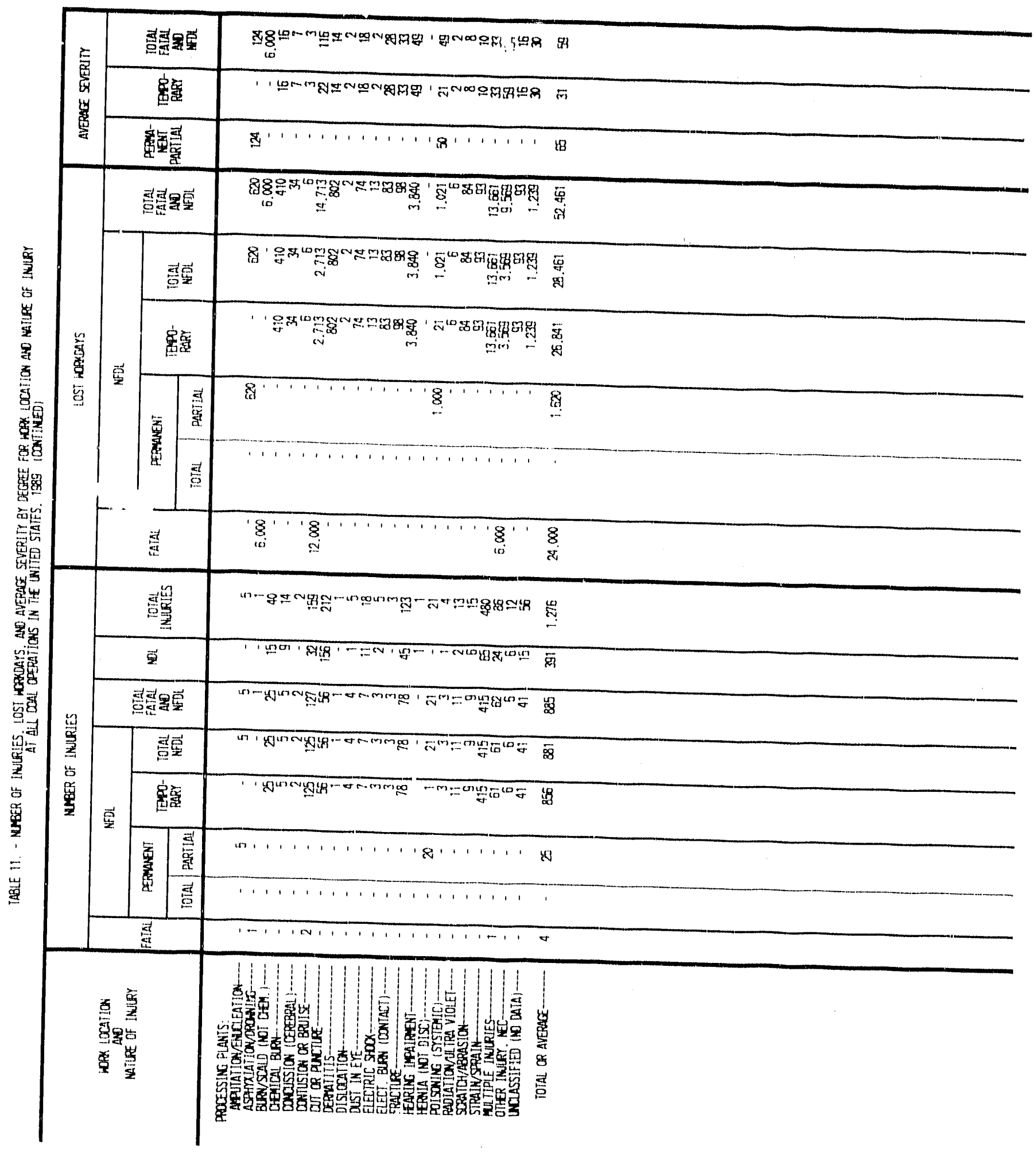




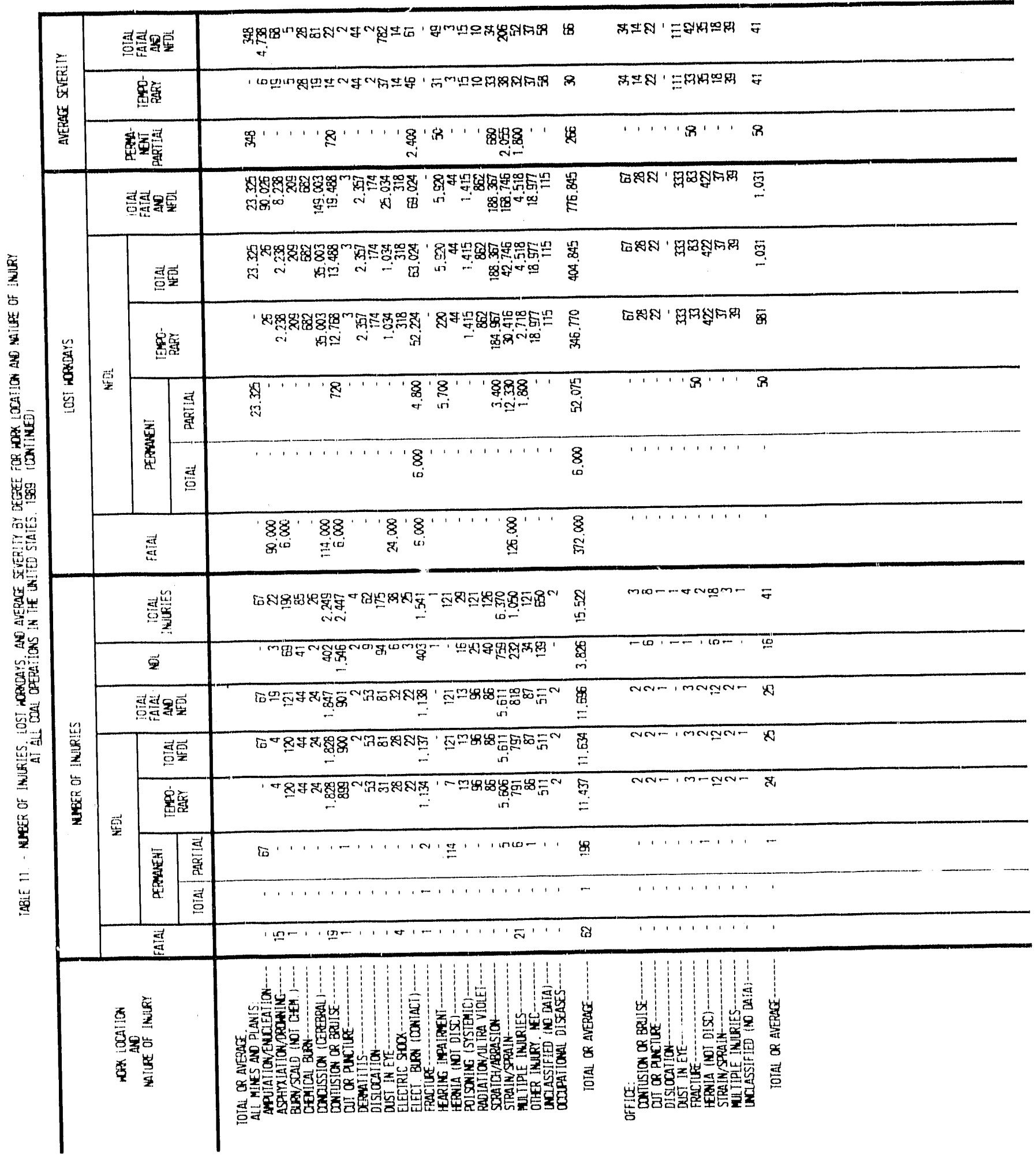




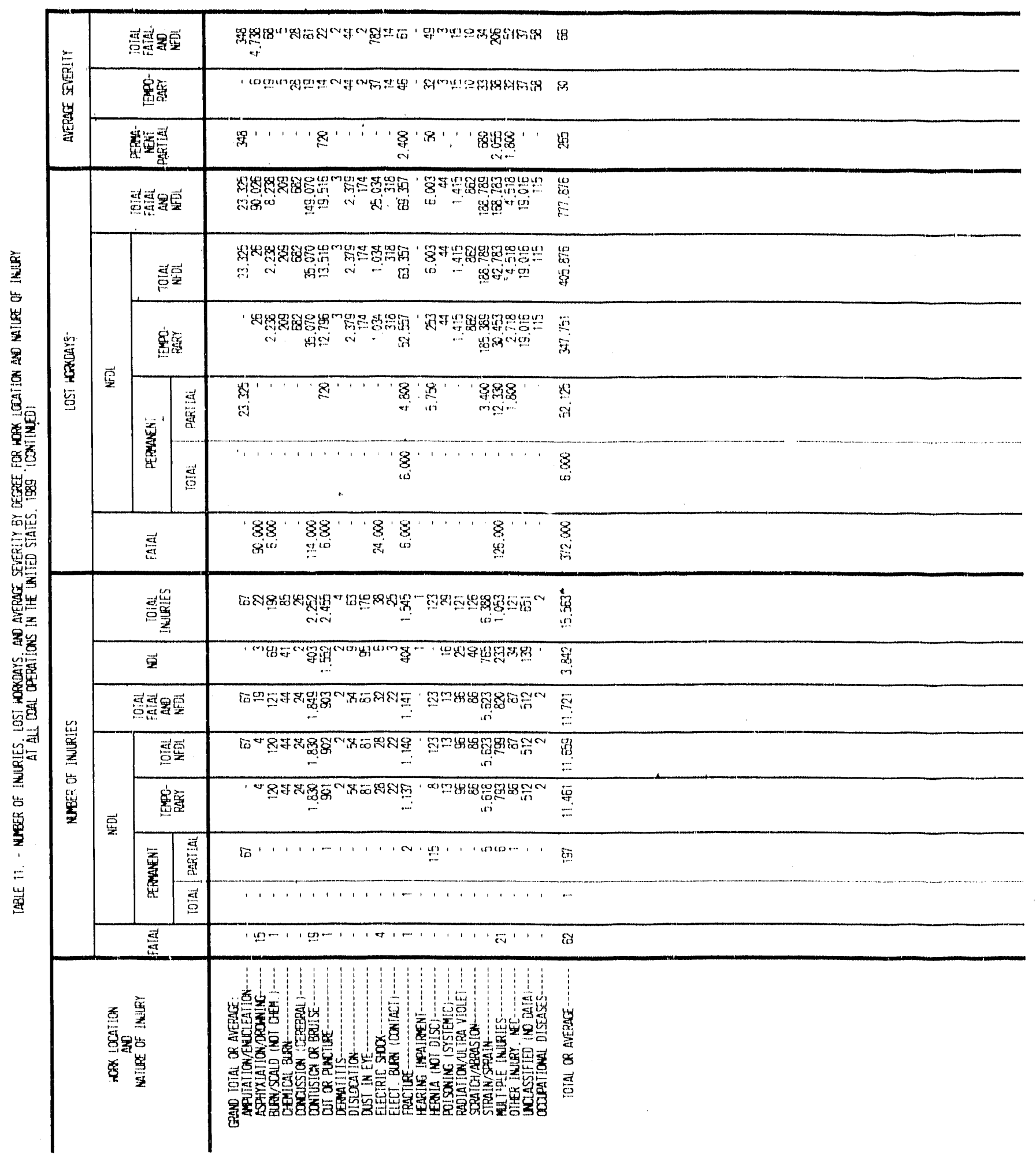




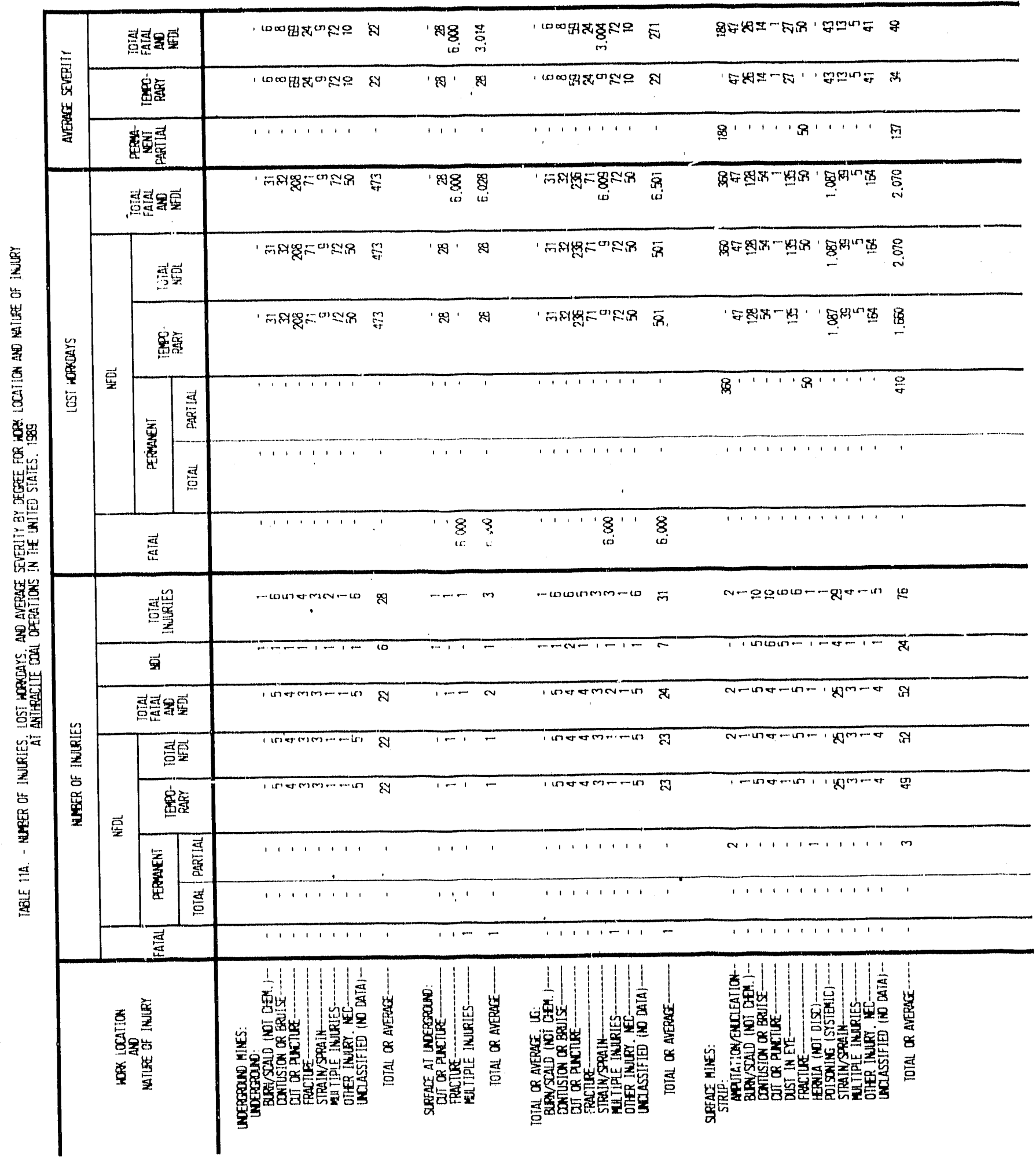




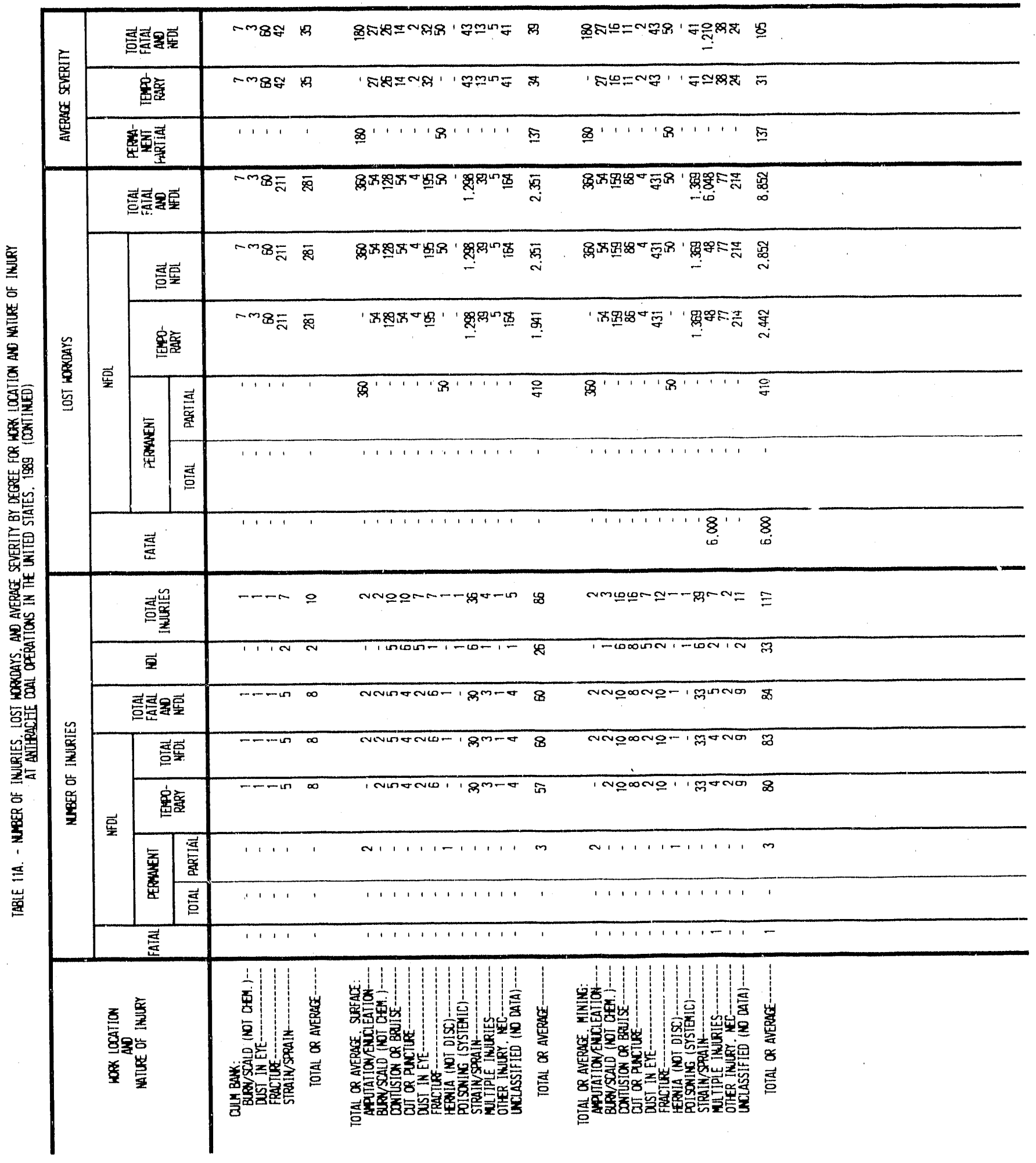




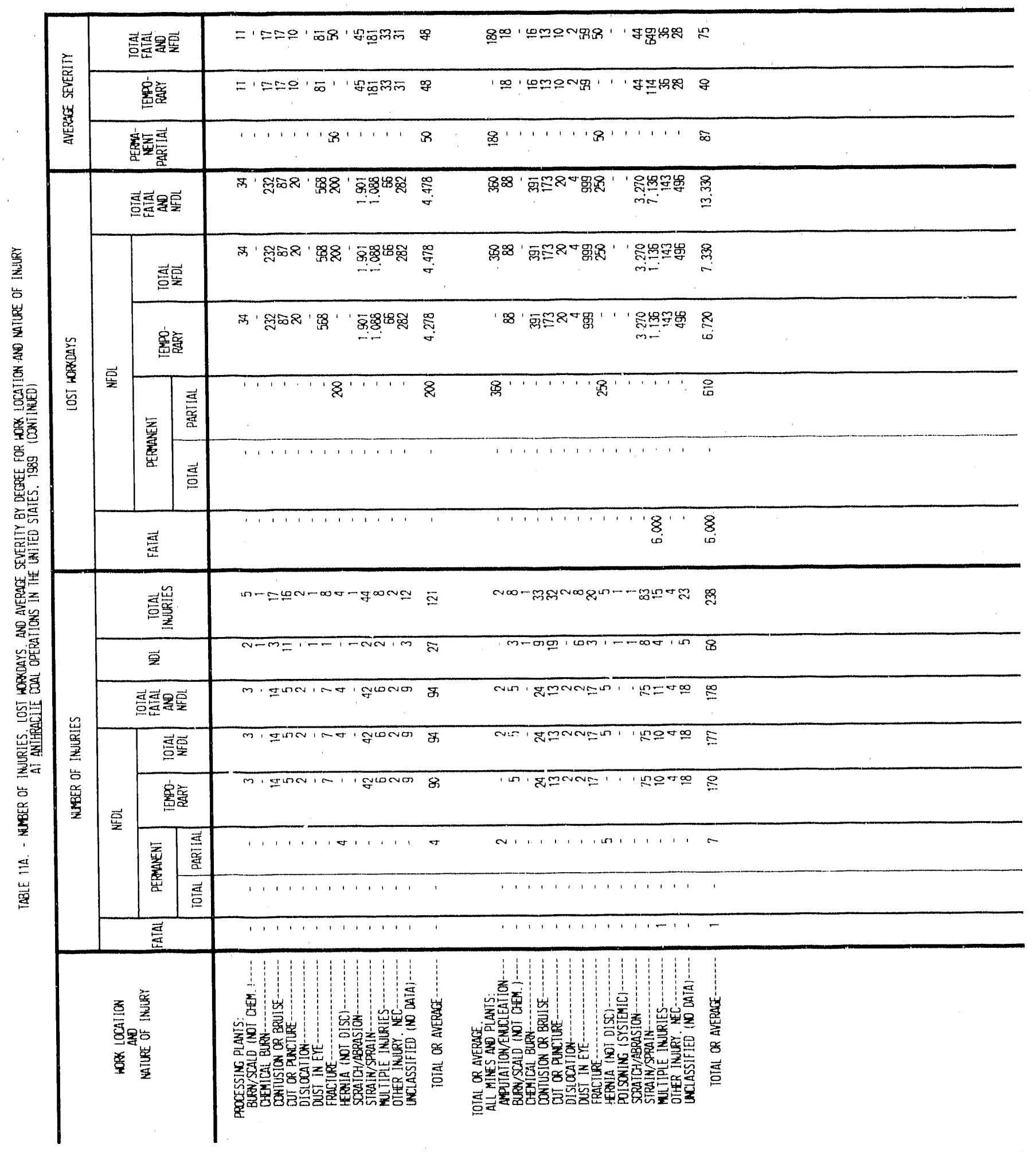




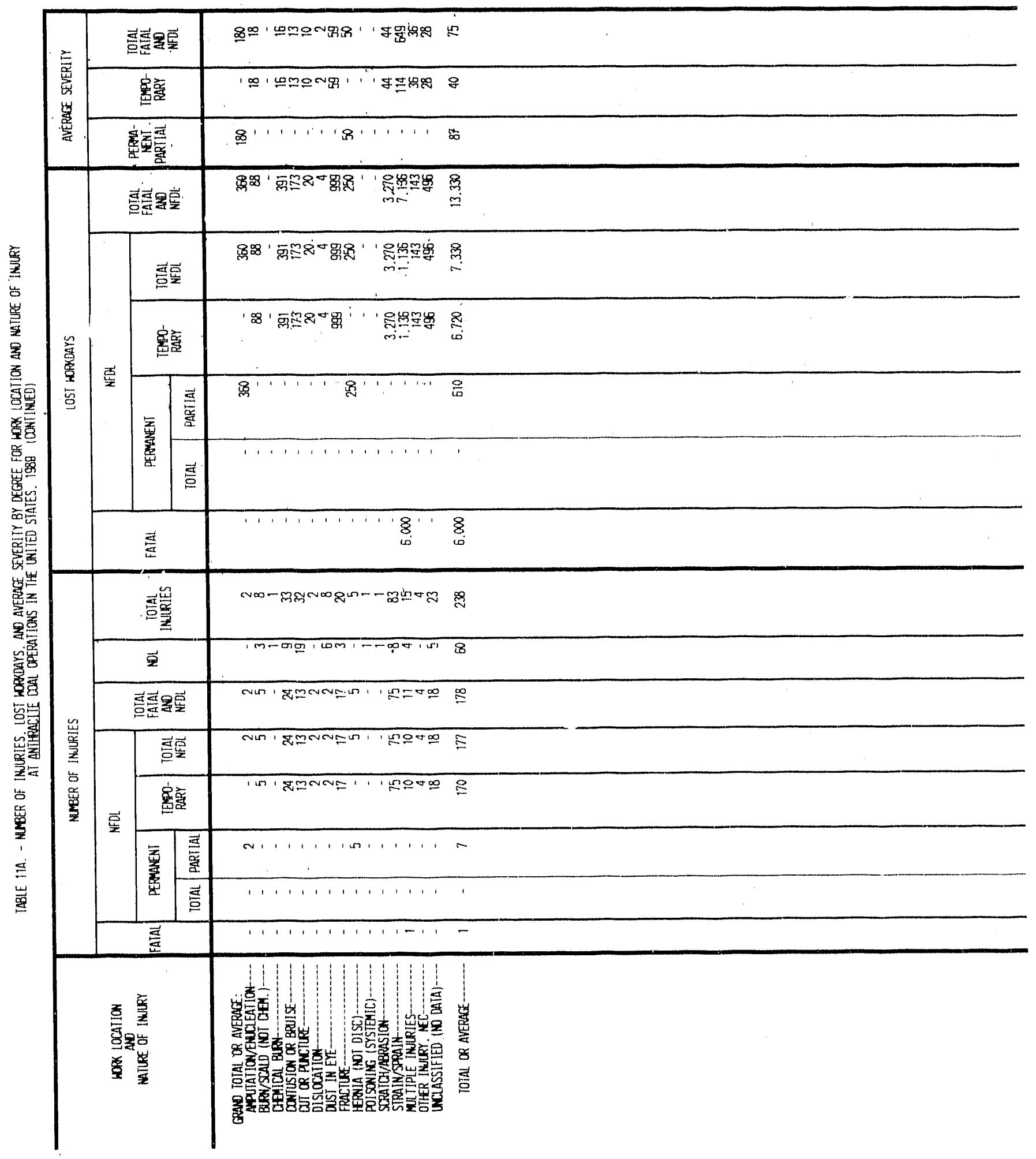




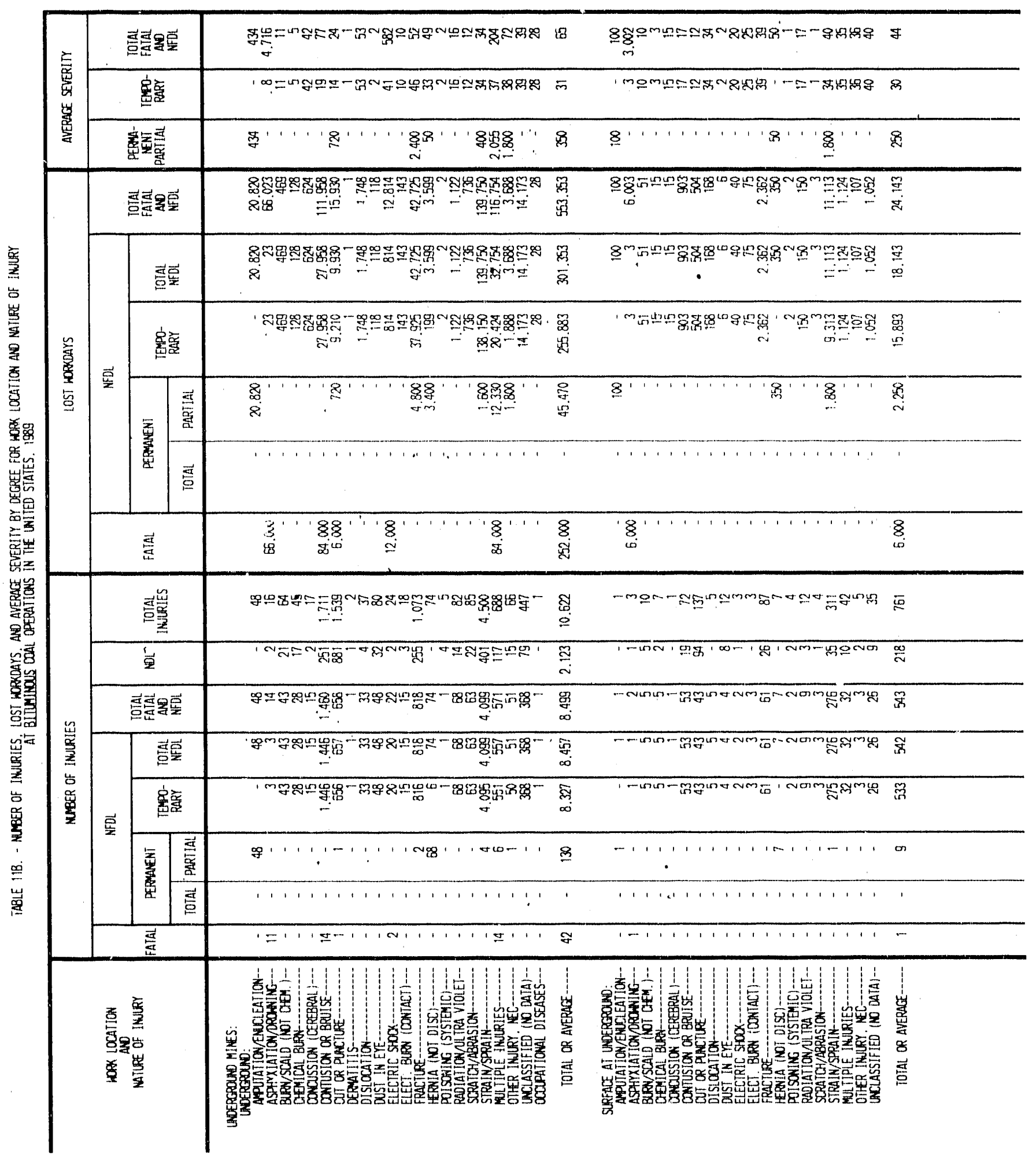




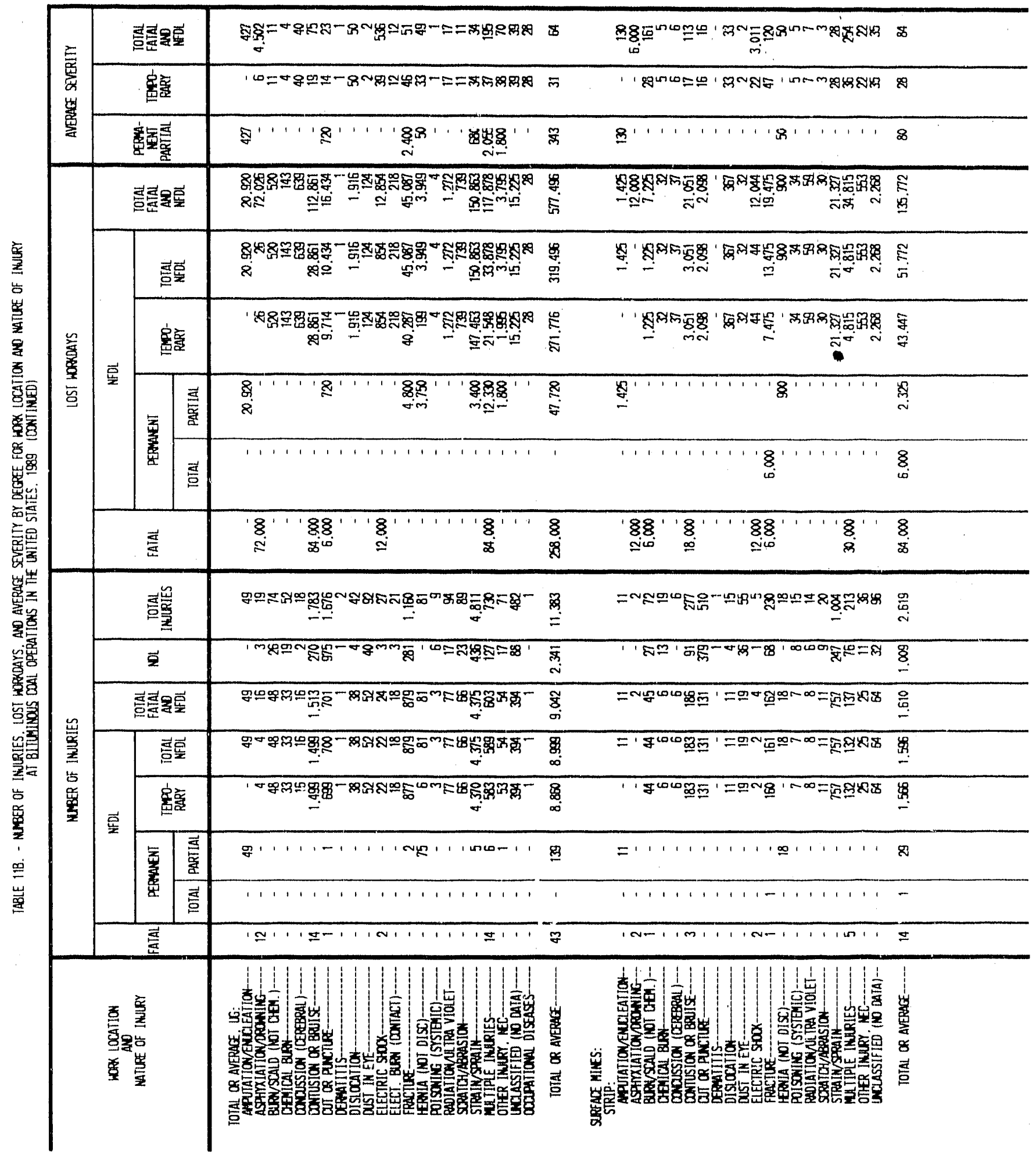




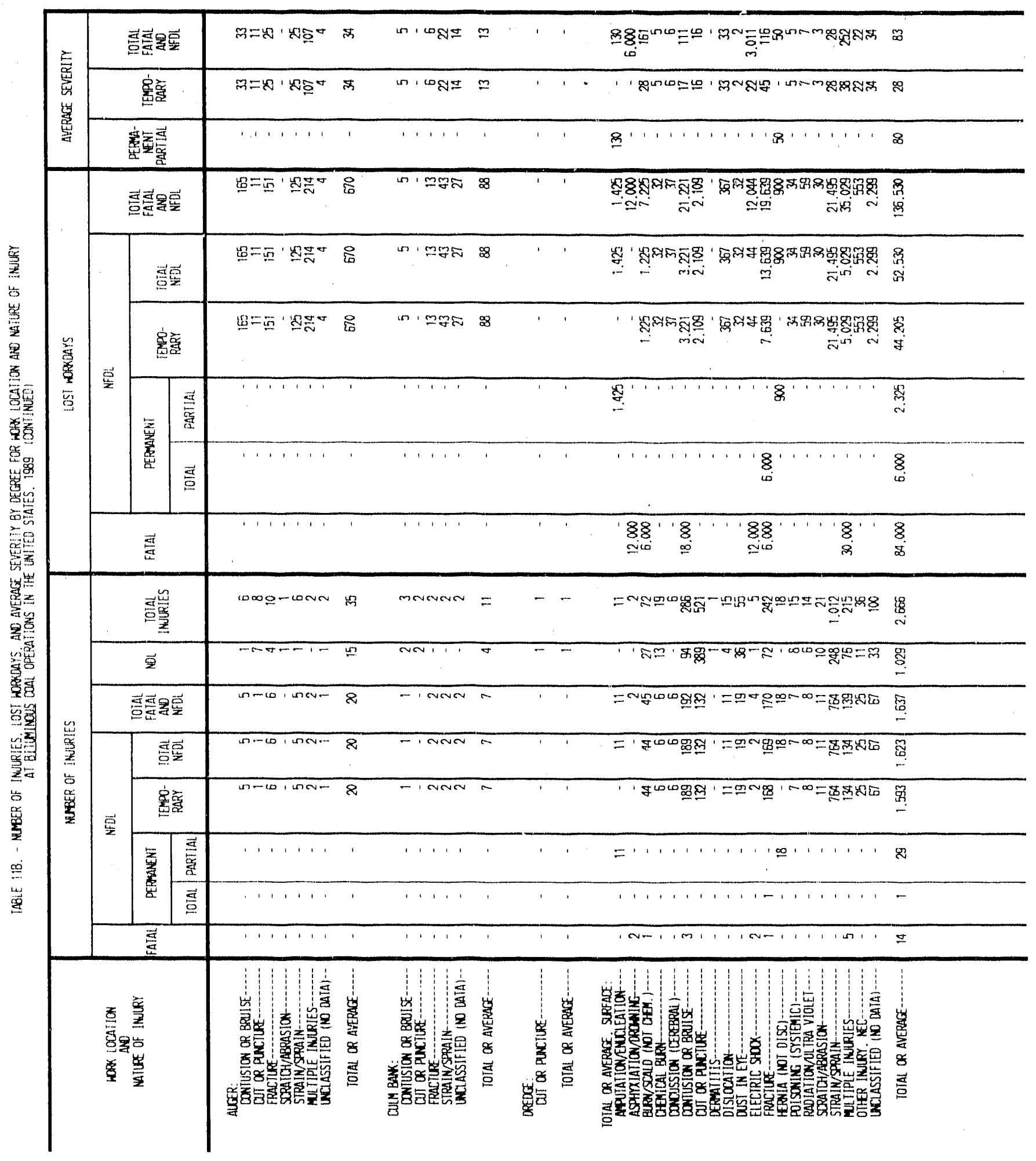




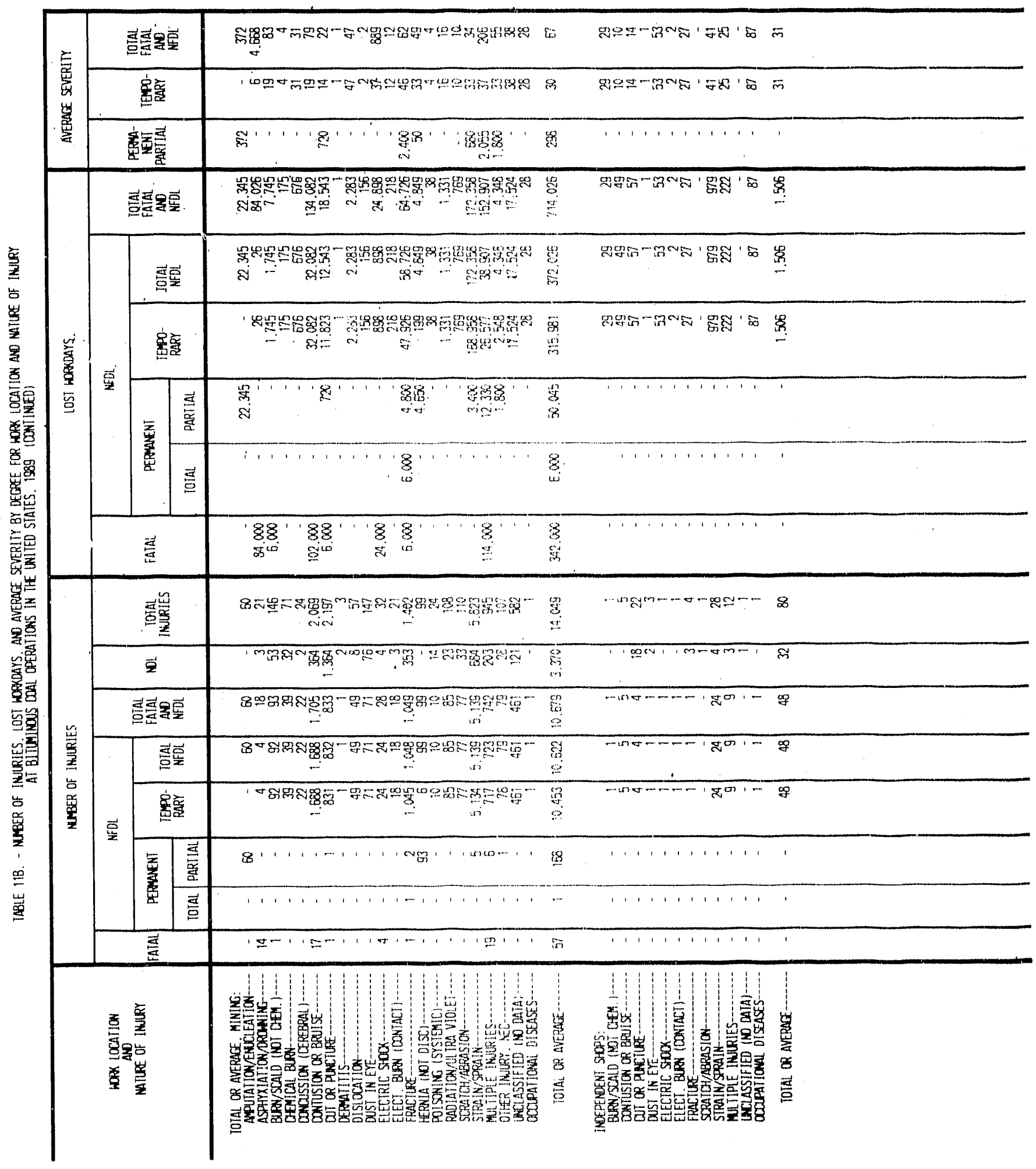




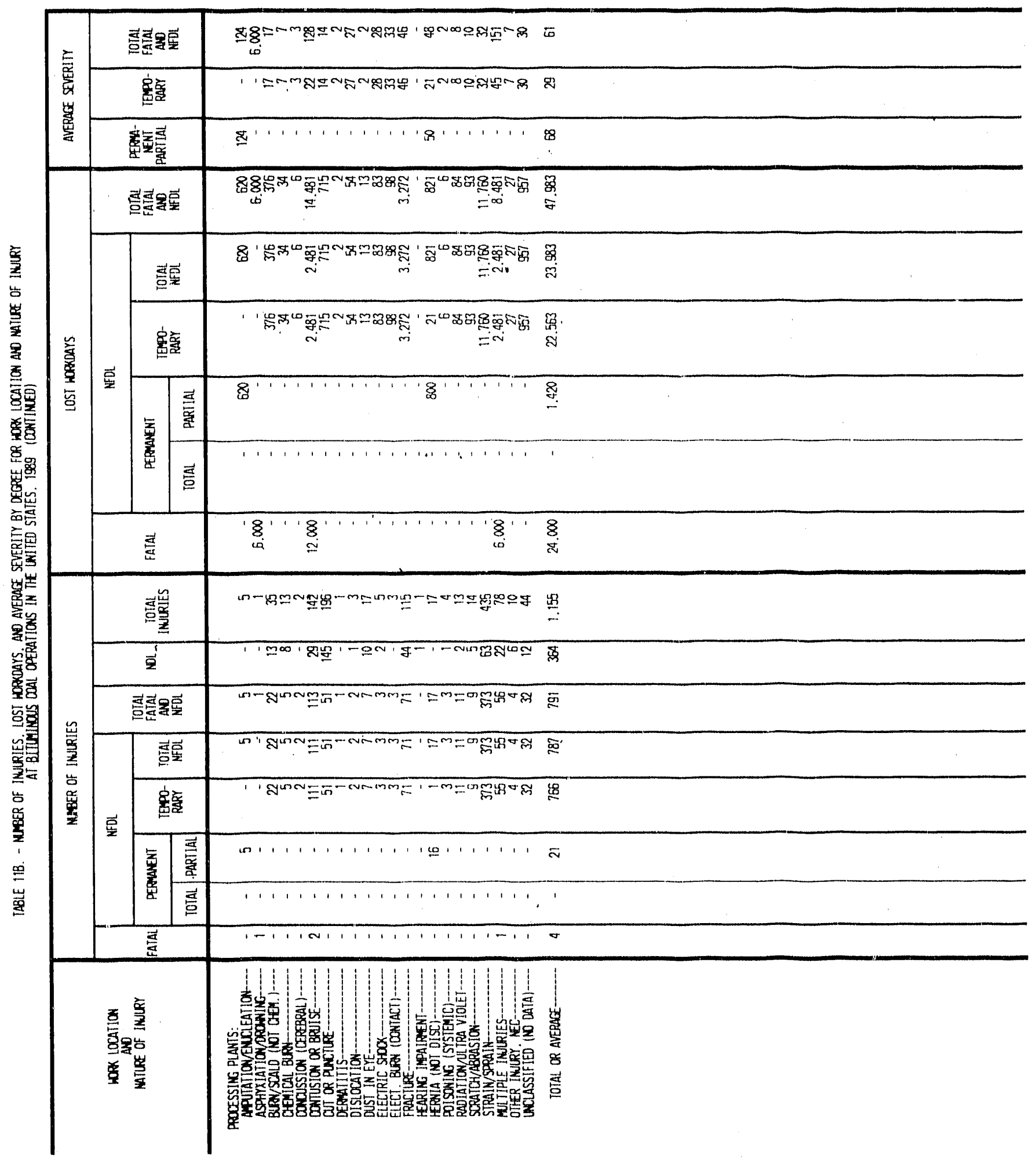




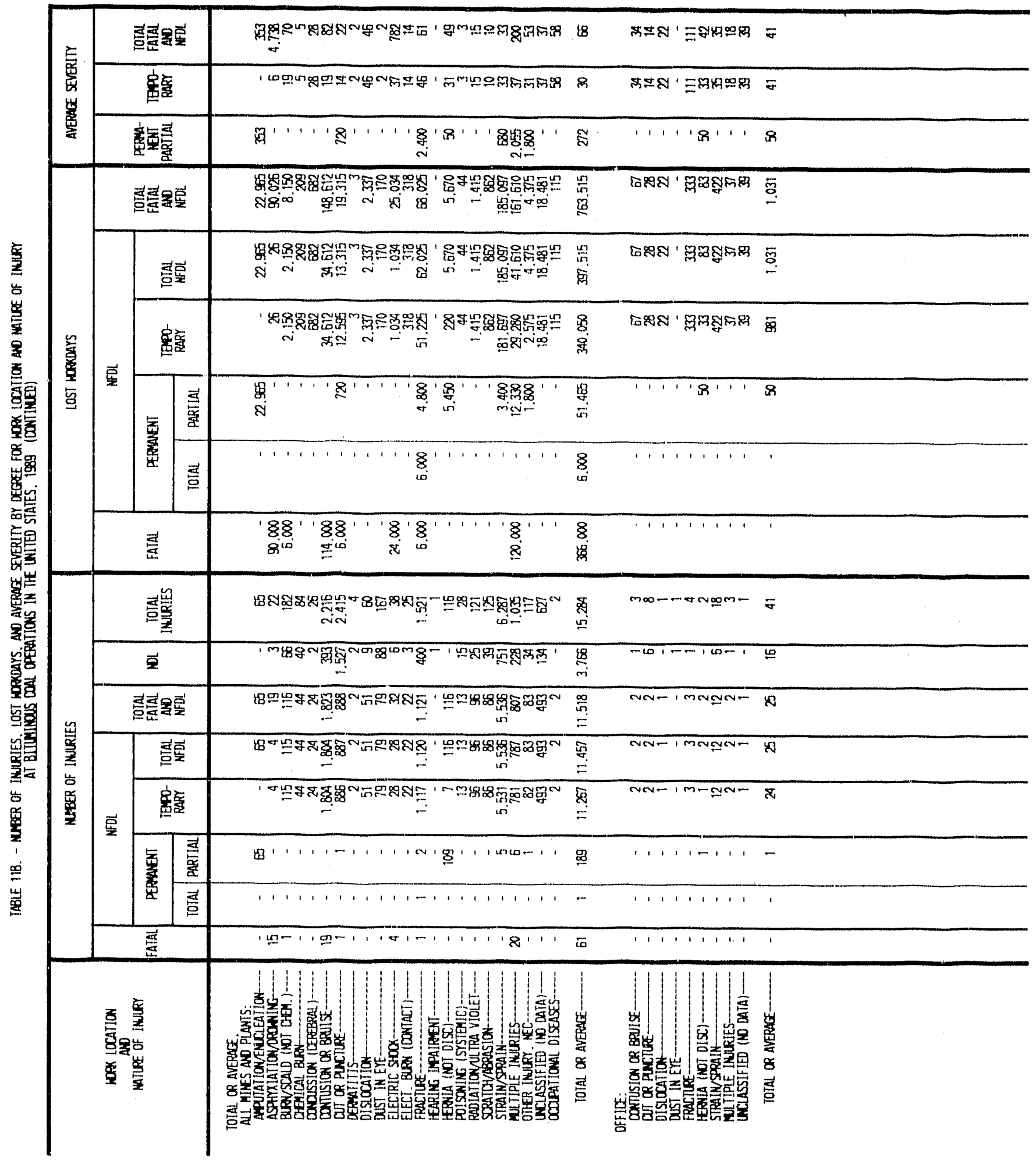




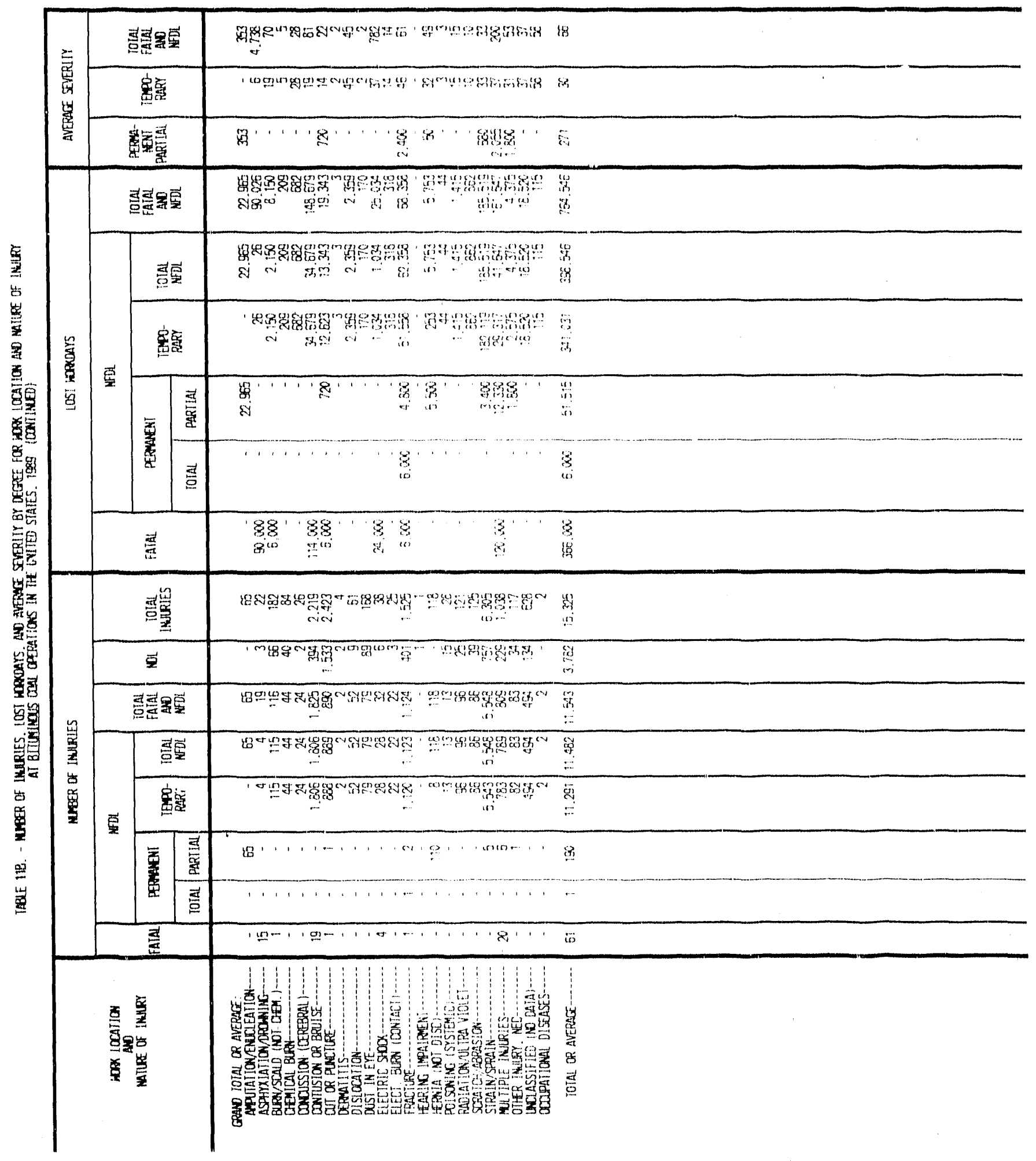




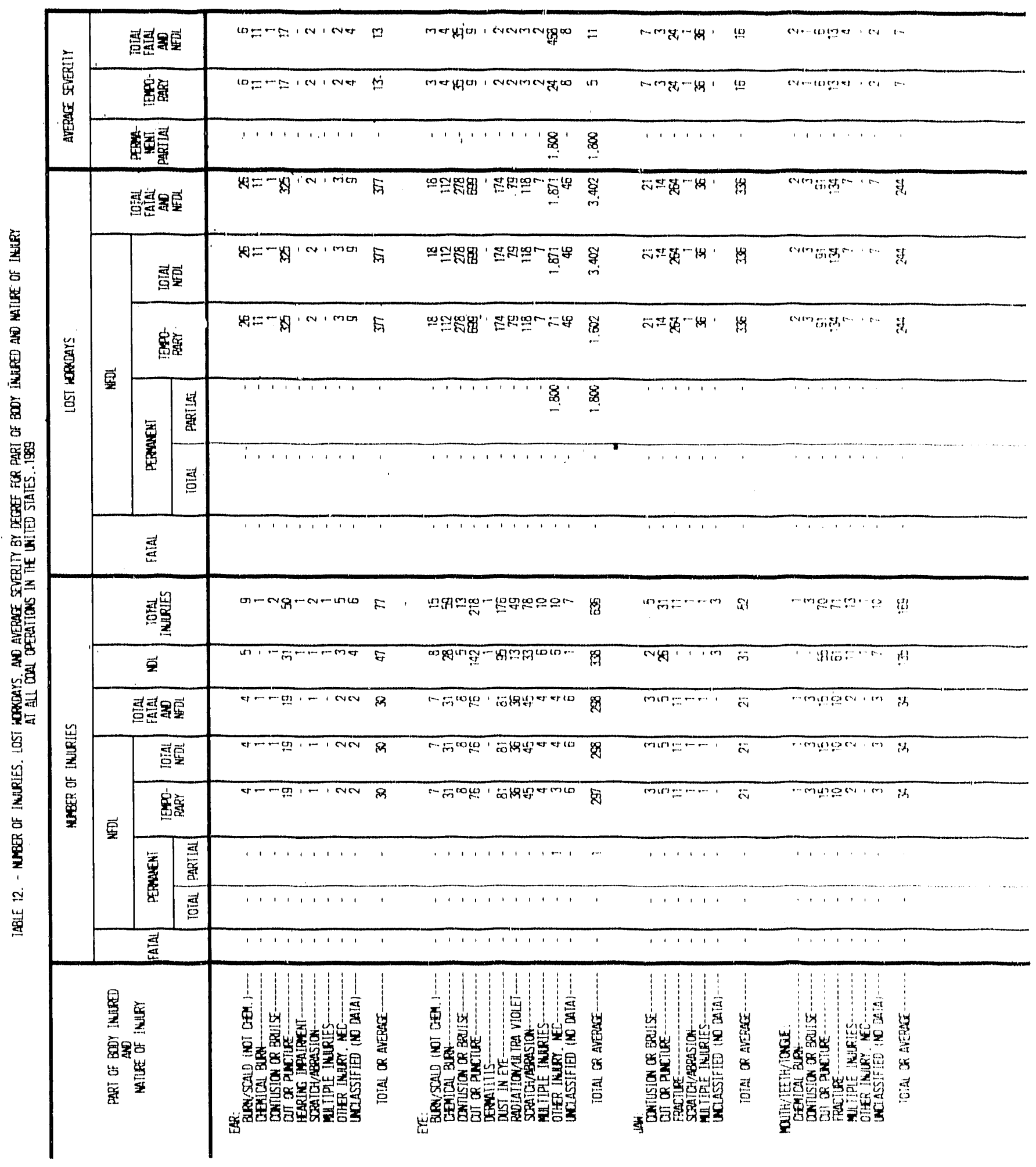




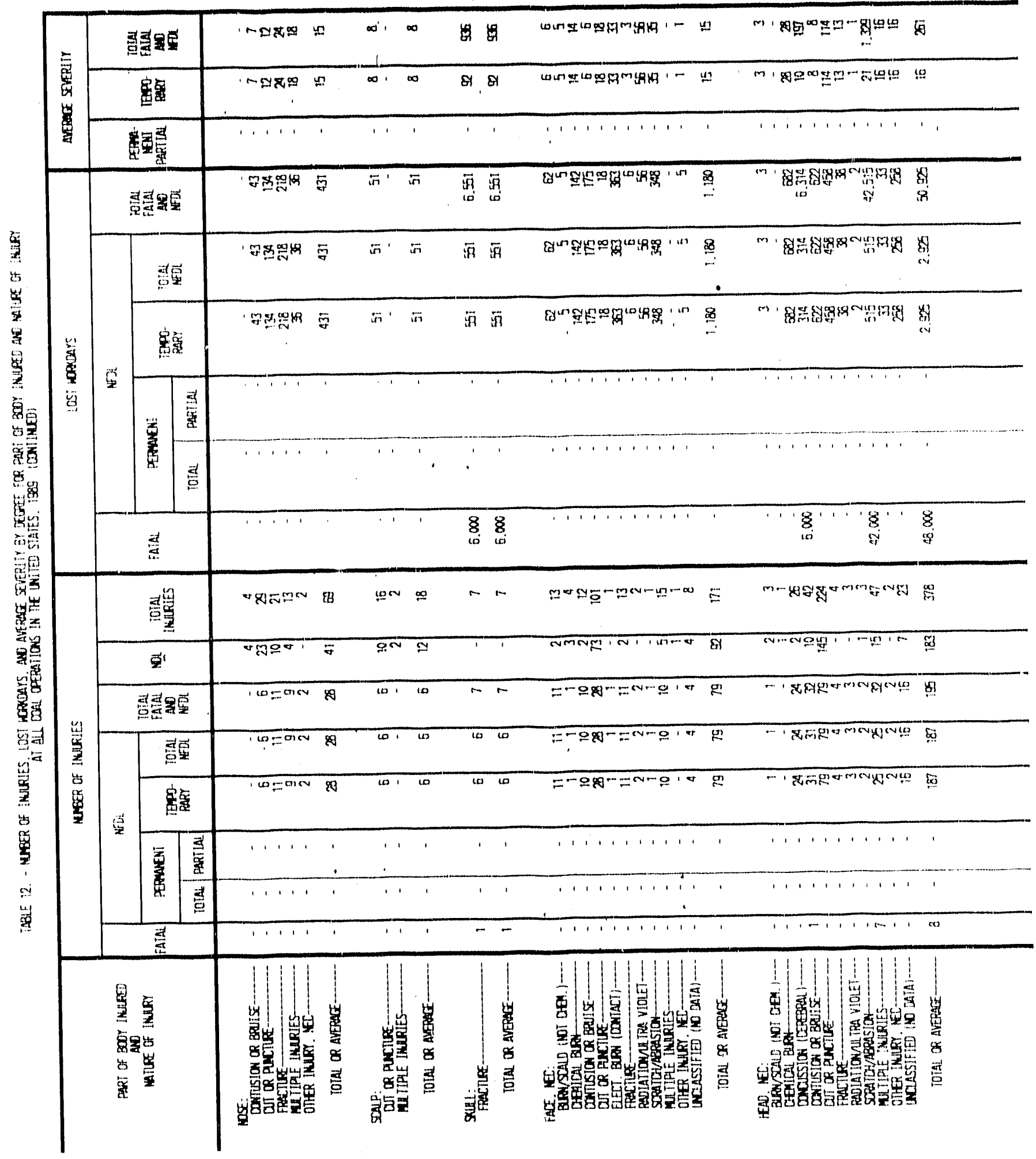




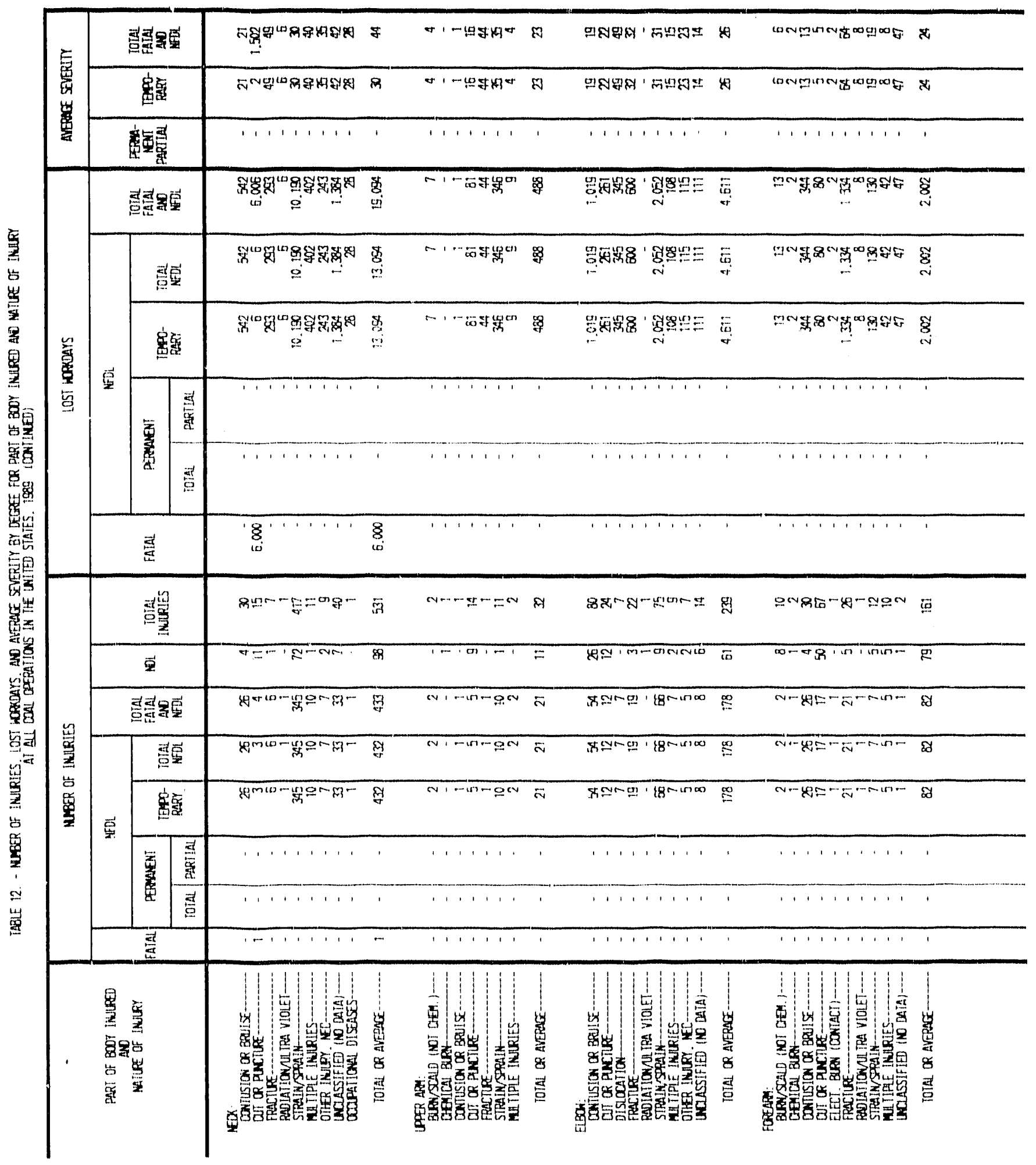




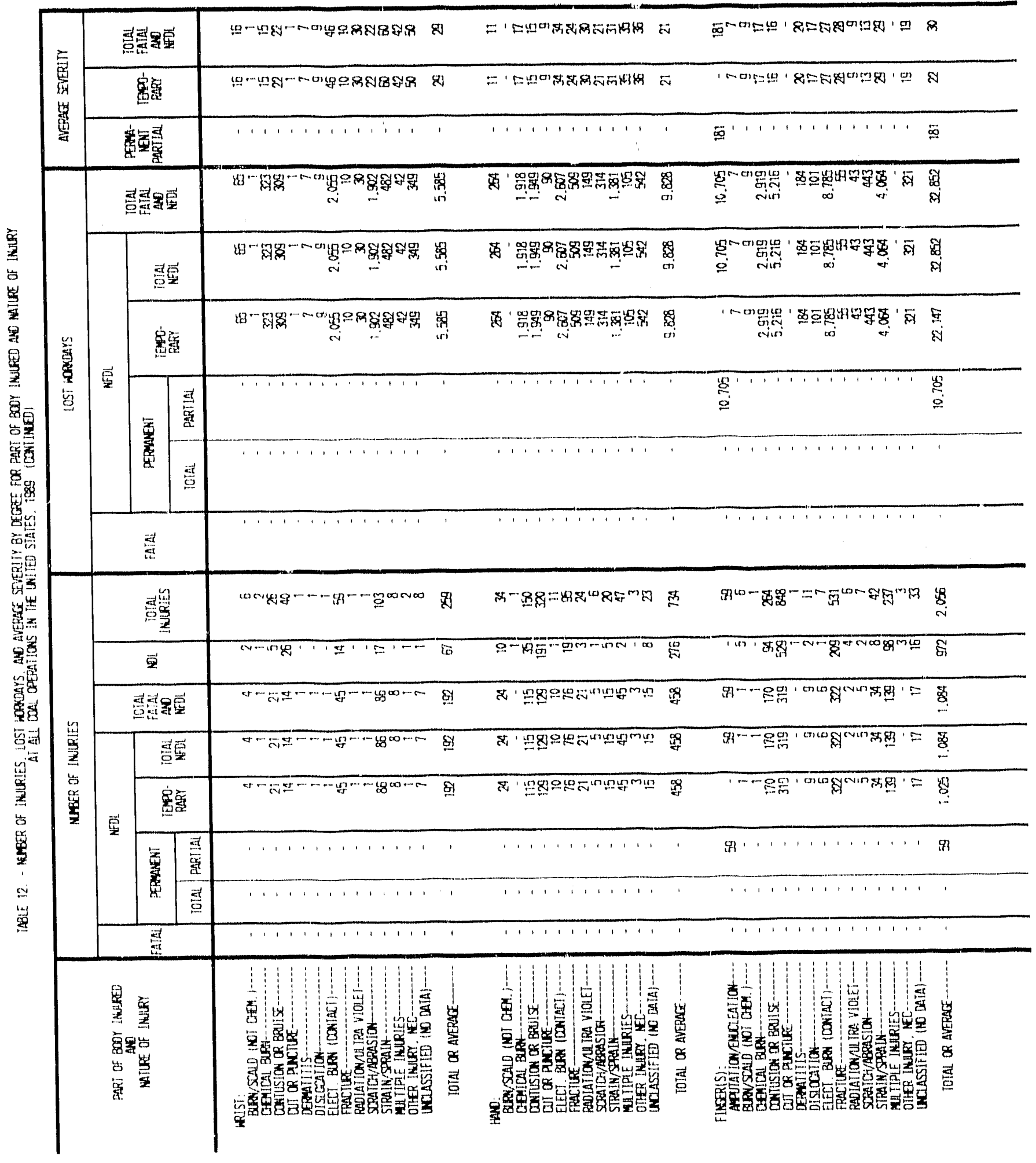




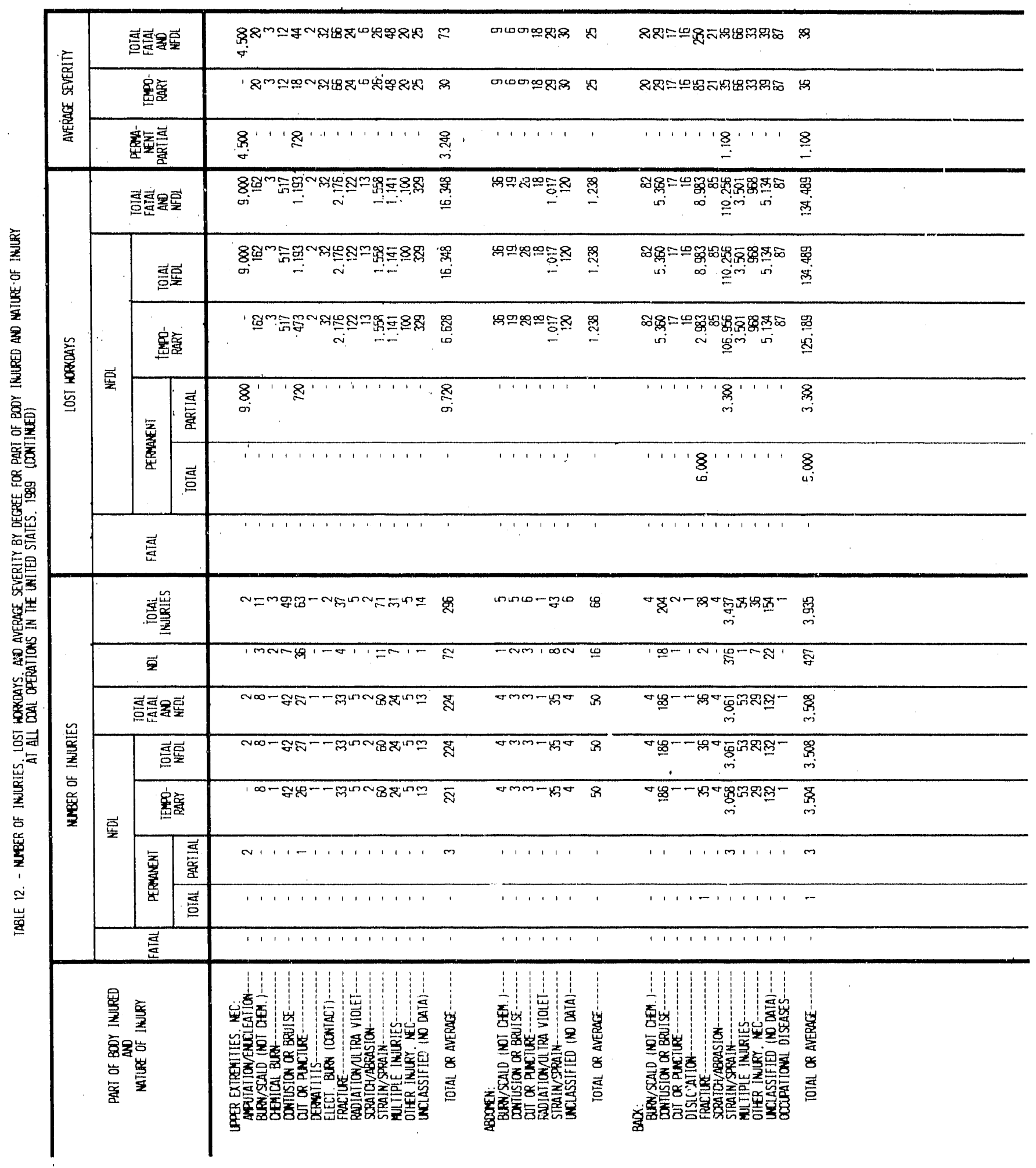




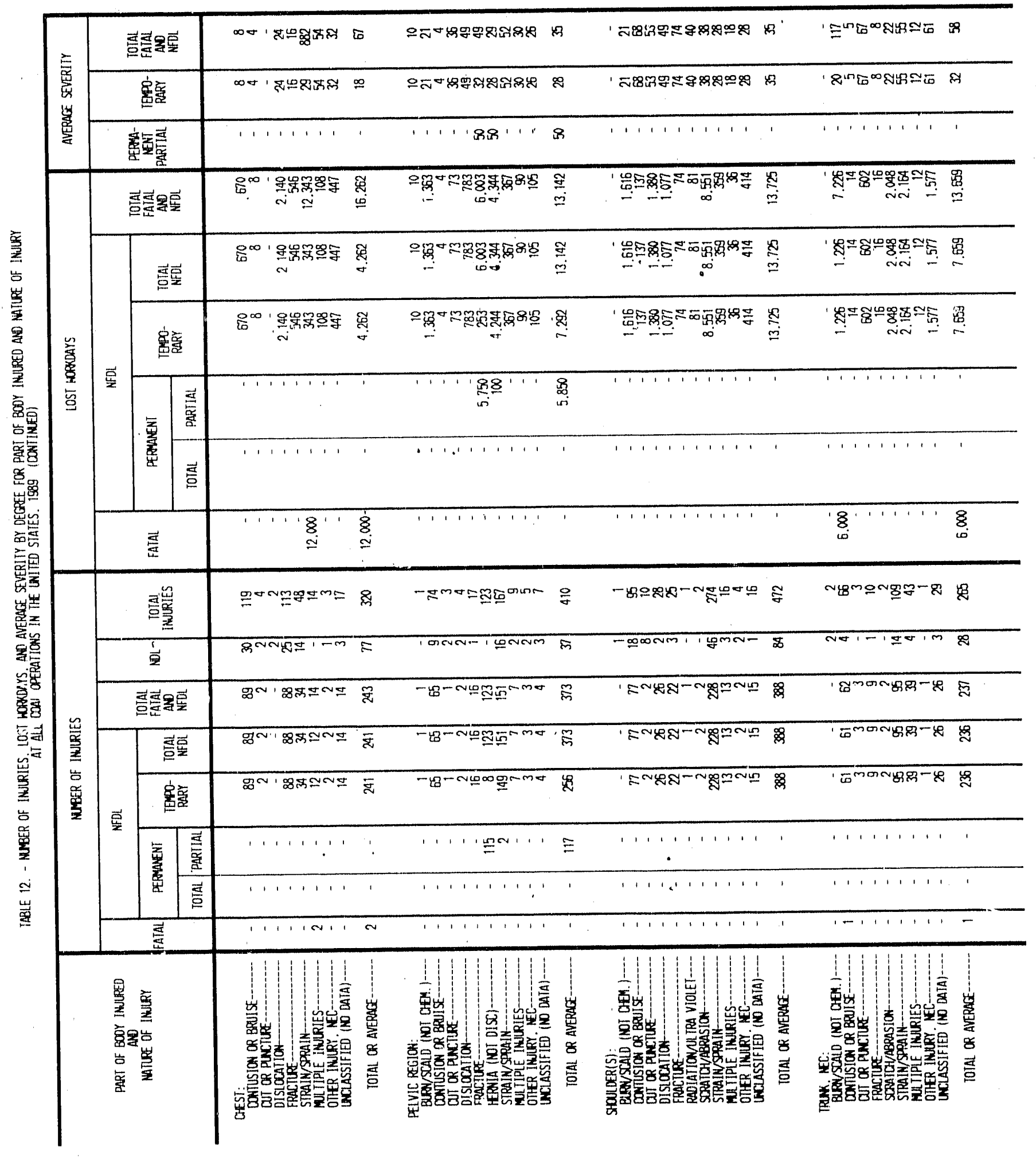




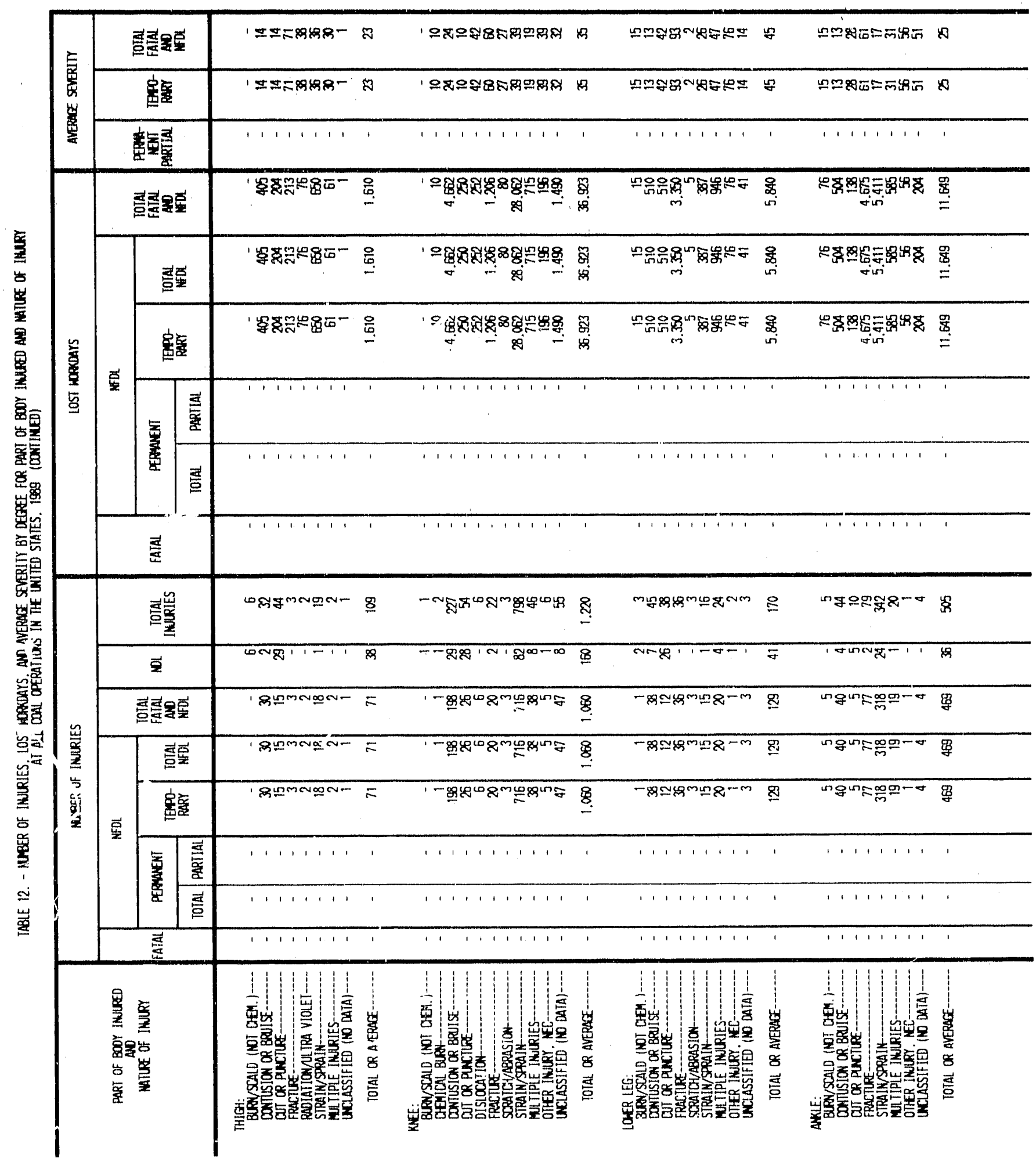




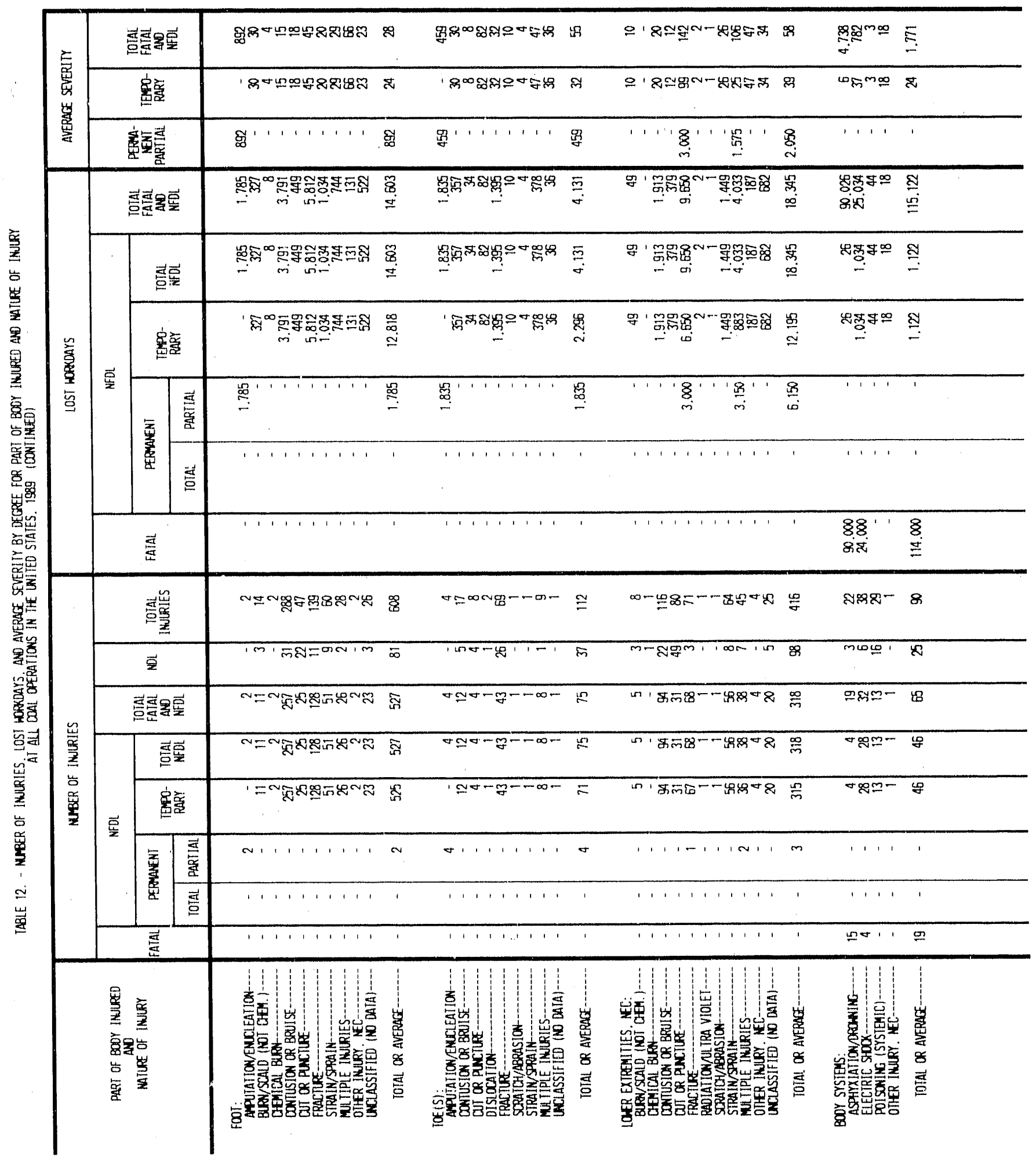




\begin{tabular}{|c|c|c|c|c|c|c|}
\hline \multirow{3}{*}{ 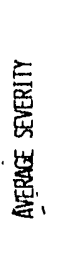 } & \multicolumn{2}{|c|}{ 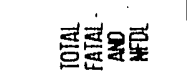 } & 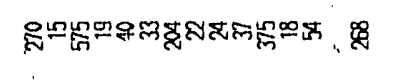 & 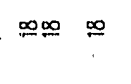 & 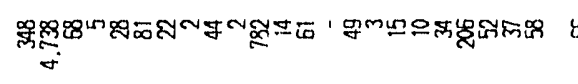 & 8 \\
\hline & \multicolumn{2}{|l|}{ 害产 } & 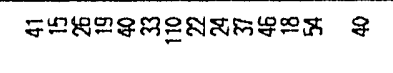 & $\propto ⿻ 上$ & 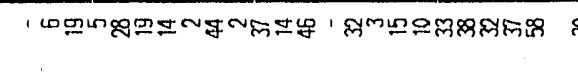 & 8 \\
\hline & \multicolumn{2}{|l|}{ 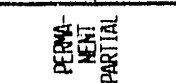 } & 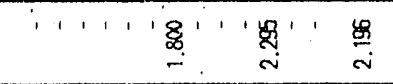 & ' & 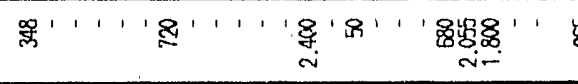 & 实 \\
\hline \multirow{6}{*}{ 謇 } & \multicolumn{2}{|c|}{ 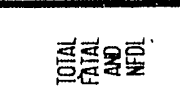 } & 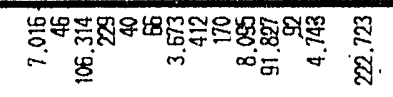 & $\infty q^{\prime} \cdot$ 음 & 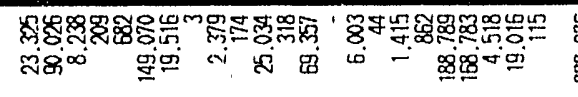 & 离 \\
\hline & \multicolumn{2}{|c|}{ 忽畐 } & 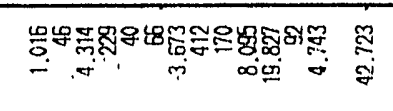 & 요 «음 & 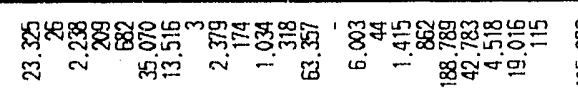 & 类 \\
\hline & \multicolumn{2}{|c|}{ 富 } & 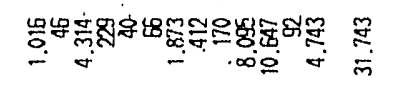 & 빼. $\cong$ & 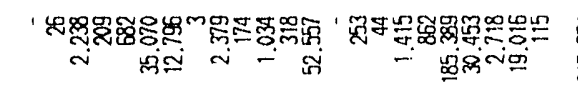 & 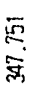 \\
\hline & \multirow{2}{*}{ 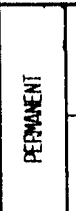 } & 离 & 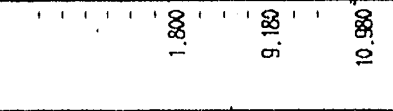 & 1.1 & 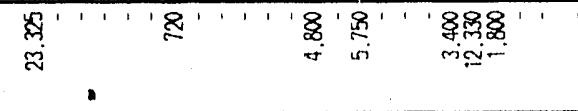 & 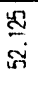 \\
\hline & & 츰 & $11,1,1,1,11,11 \ldots 1$ & $\cdots 1$ & 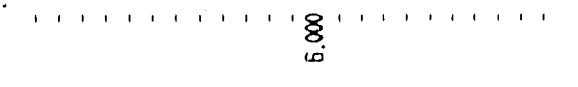 & $\underbrace{8}_{i \infty}$ \\
\hline & \multicolumn{2}{|l|}{$\frac{\vec{t}}{\underline{s}}$} & 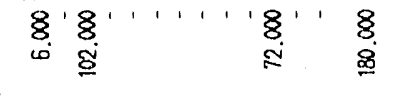 & 1 & 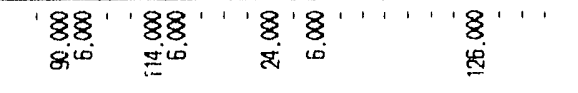 & 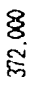 \\
\hline \multirow{8}{*}{ 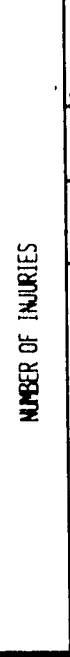 } & \multicolumn{2}{|c|}{ 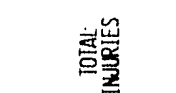 } & "ு & $-\infty-$ & 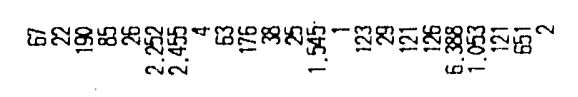 & 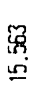 \\
\hline & \multicolumn{2}{|l|}{$\overline{\mathbf{s}}$} & F्थशून & $1--$ & 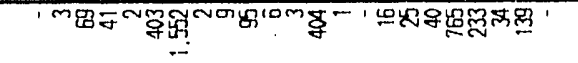 & $\underset{\tilde{m}}{\tilde{m}}$ \\
\hline & \multicolumn{2}{|c|}{ 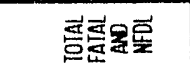 } & 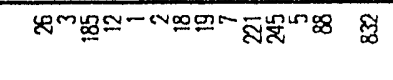 & $-\infty 10$ & 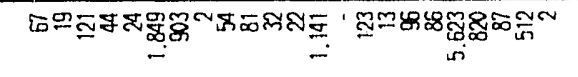 & $\stackrel{\Xi}{=}$ \\
\hline & \multicolumn{2}{|c|}{ 형룰 } & 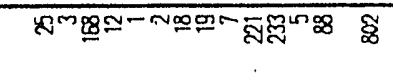 & $-\infty \omega$ & 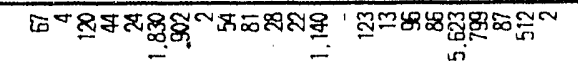 & 莒 \\
\hline & \multicolumn{2}{|c|}{ 害蜜 } & 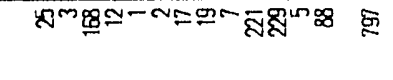 & $-\ln 6$ & 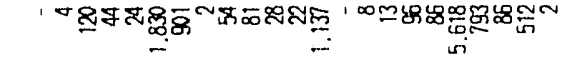 & $\underset{\text { 要 }}{\stackrel{F}{F}}$ \\
\hline & \multirow{2}{*}{ 要 } & 章 & in & ' & 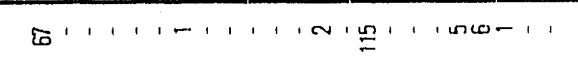 & छ \\
\hline & & 志 & $1,1,1,1$ & ' ' & ' ' ' & - \\
\hline & \multicolumn{2}{|l|}{ 层 } & $-1=$ & ' & 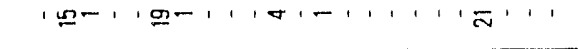 & $\widetilde{0}$ \\
\hline & \multicolumn{2}{|l|}{ 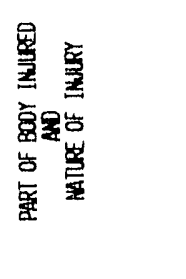 } & 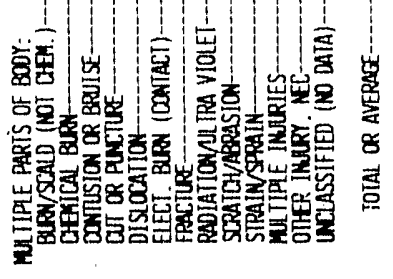 & 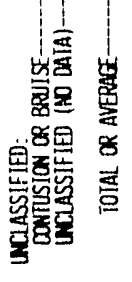 & 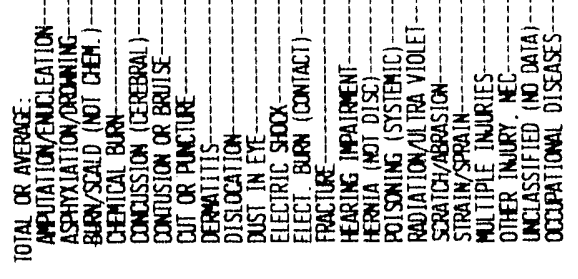 & 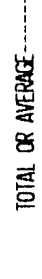 \\
\hline
\end{tabular}




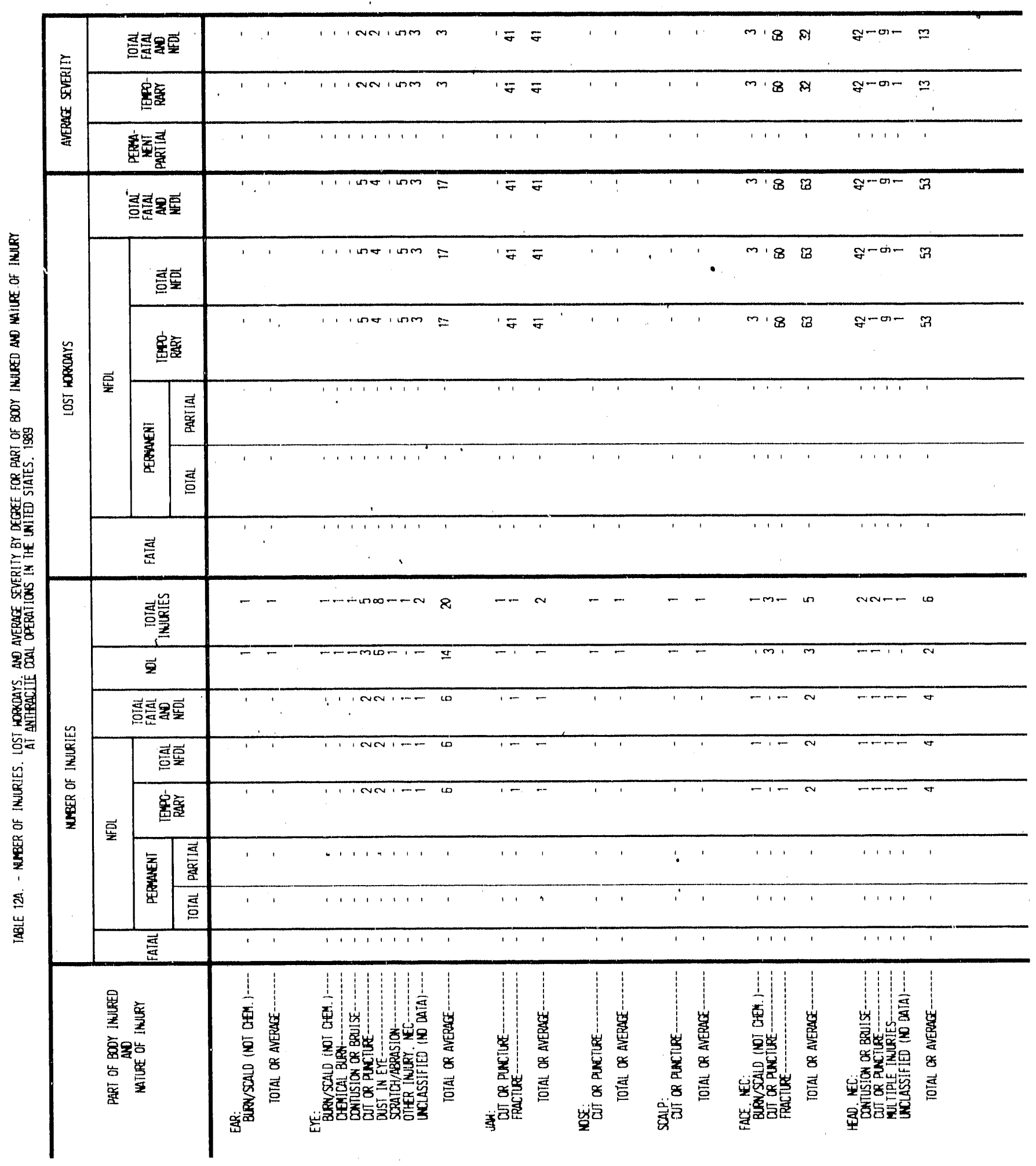




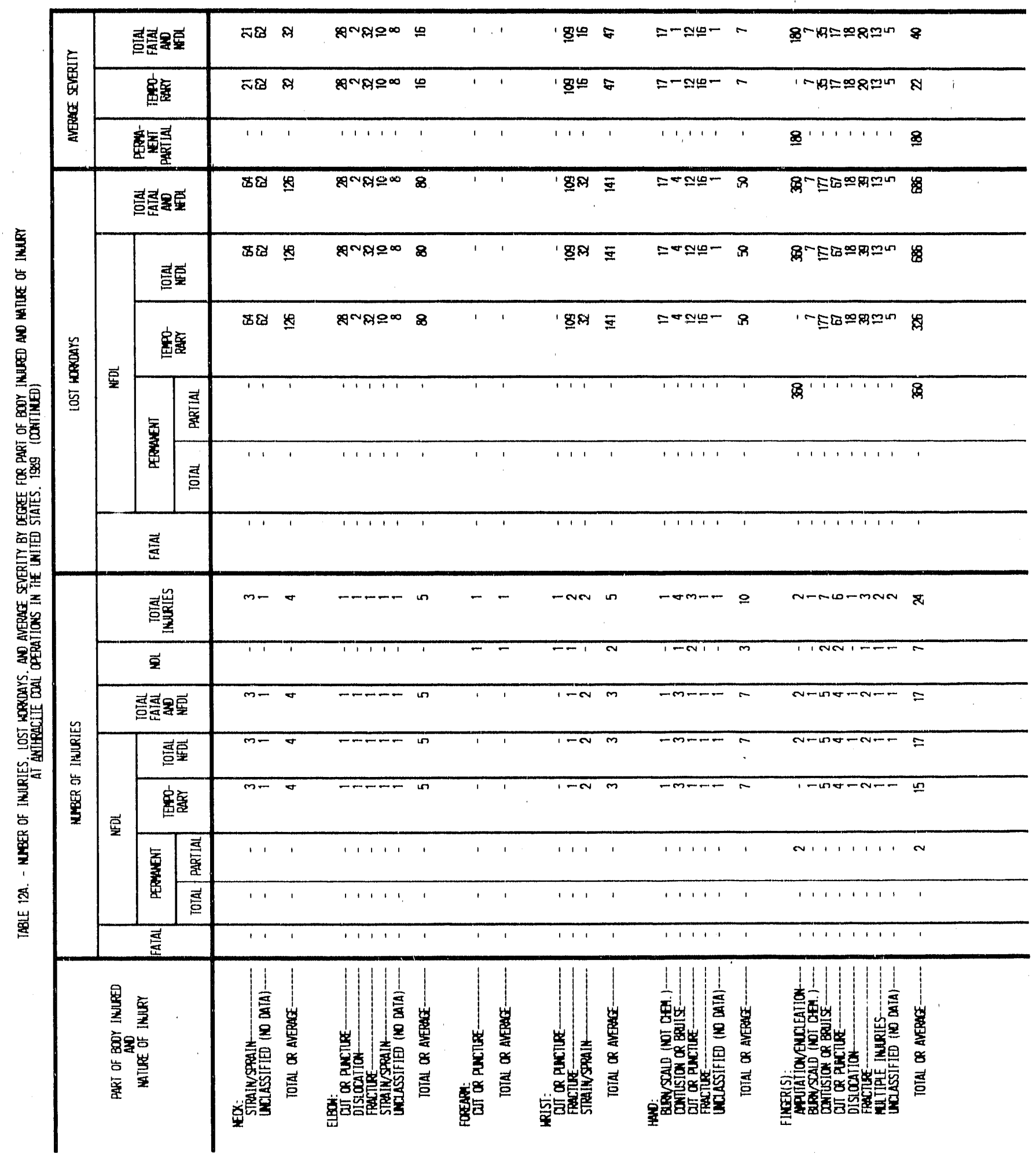




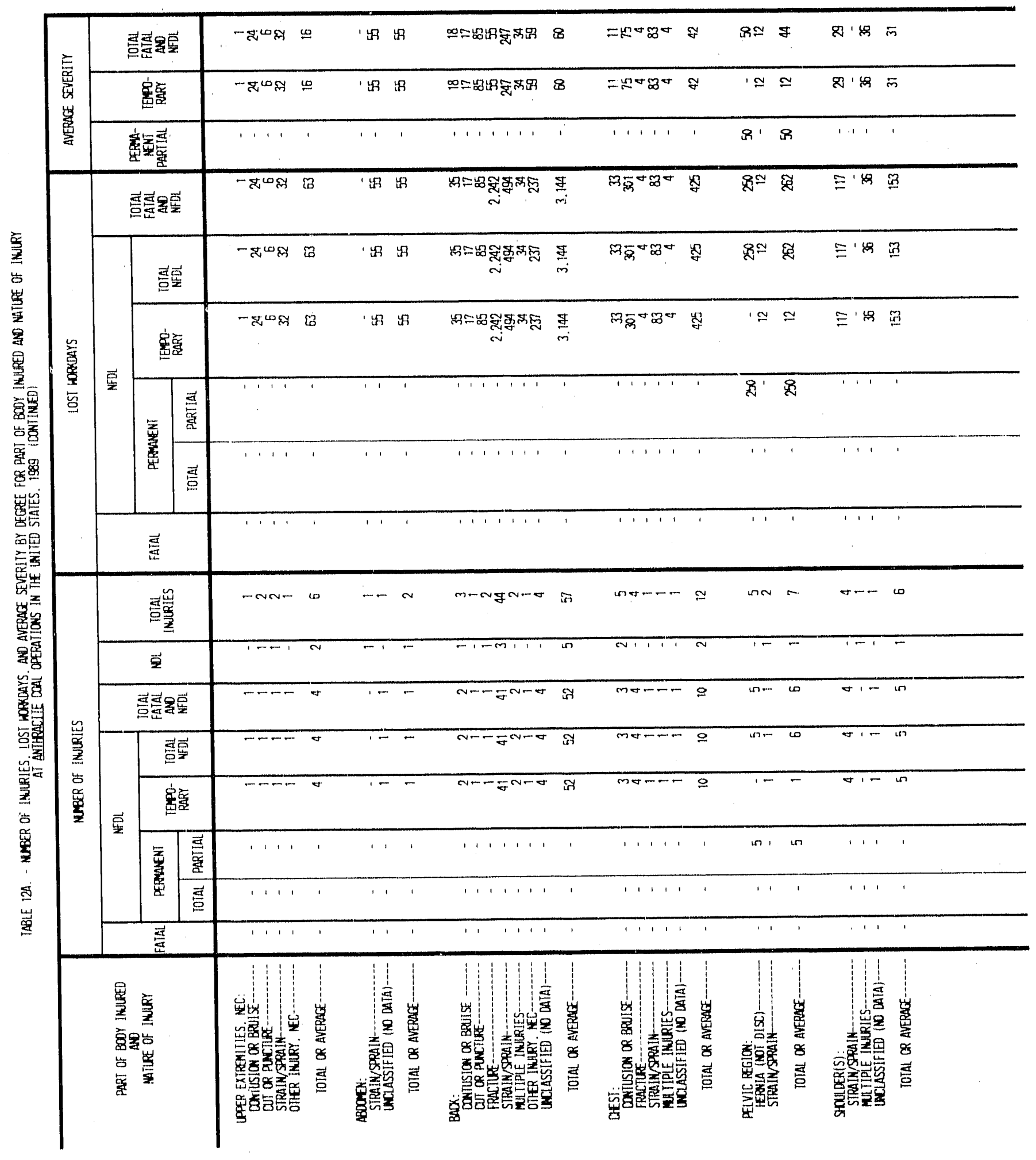




\begin{tabular}{|c|c|c|c|c|c|c|c|c|c|c|c|c|c|c|c|c|}
\hline \multirow{3}{*}{ 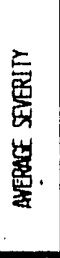 } & \multicolumn{2}{|c|}{ 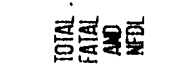 } & $=N:$ & & '믐 & 쯤 & 1 हn & $\mathscr{B}$ & =品요 & 18 & $F-D=$ & $\mathfrak{A}$ & m & $\mathbb{Q}$ & $\forall$ & $\sigma$ \\
\hline & \multicolumn{2}{|c|}{ 裹变 } & $=N$ : & $\Xi$ & '묨 & $\not ్$ & ' क्या & 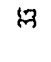 & 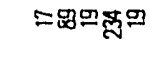 & $\mathscr{B}$ & $F-9=$ & જ્ & $m$ & $\infty$ & 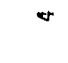 & $\theta$ \\
\hline & \multicolumn{2}{|c|}{ 密鰝 } & $\cdot$ & 1 & 11 & $i$ & 11 & 1 & 1111 & , & 1111 & 1 & 11 & 1 & ' & ' \\
\hline \multirow{6}{*}{$\begin{array}{l}\text { 号 } \\
\text { 兽 } \\
\text { 旨 }\end{array}$} & \multicolumn{2}{|c|}{ 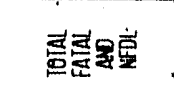 } & ঞ্ণে & 5 & 1 염 & 욤 & $1 \widehat{y}^{N}$ & '大 & =品严总 & 然 & $8-49$ & $\stackrel{n}{N}$ & $\pm \underline{8}$ & 9 & $\sigma$ & $\nabla$ \\
\hline & \multirow{3}{*}{ 要 } & 莡营 & $\tilde{w n}^{2}$ & $\pi$ & '멈 & $\not$ & $1 \hat{D}^{N}$ & 这. & 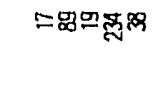 & 鸟 & $F-48$ & $\stackrel{n}{\mathrm{~N}}$ & $\pm \underline{\underline{B}}$ & 9 & $\sigma$ & $\nabla$ \\
\hline & & 容察 & त्ञ⿰氵 & क & ' ్ָల & Д્త & '苏 & प्र & 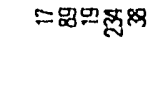 & 包 & $F-495$ & $\stackrel{n}{\sim}$ & $\pm \underline{\underline{6}}$ & 8 & $\sigma$ & $\nabla$ \\
\hline & & 烝 & 11 & 1 & 11 & 1 & 111 & 1 & 1111 & 1 & 111 & 1 & 11 & 1 & T & 1 \\
\hline & 蛙 & 롱 & 11 & 1 & 11 & $\cdot$ & 111. & 1 & $11+11$ & ' & 1. 1 & 1 & 11 & 1 & . & ' \\
\hline & \multicolumn{2}{|l|}{ 疍 } & 11 & ' & 11 & ' & 1111 & ' & 11111 & 1 & 111 & ' & 11 & 1 & ' & ' \\
\hline \multirow{8}{*}{ 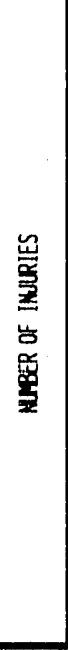 } & \multicolumn{2}{|c|}{ 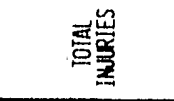 } & $m+$. & $\sigma$ & -- & $\sim$ & 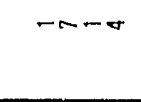 & $\mathfrak{m}$ & $-m-\infty$ & D & $--a n$ & $=$ & man & - & - & - \\
\hline & \multicolumn{2}{|l|}{$\underline{x}$} & 11 & ' & -1 & 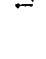 & $-1 \cdot m$ & $\sigma$ & $11+1$ & ' & $1:-$ & - & 15 & - & 1 & 1 \\
\hline & \multicolumn{2}{|c|}{ 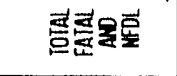 } & $m-$ & + & $1-$ & - & $1 n-5$ & or & $\cdots-\infty$ & $\omega$ & - - & 으 & nn- & $\infty$ & - & - \\
\hline & \multicolumn{2}{|c|}{ 형룰 } & m- & $\nabla$ & $1-$ & - & $10-5$ & $\sigma$ & $-\infty-\infty$ & $\omega$ & $-\cdots+N$ & 은 & (1) & $\omega$ & 5 & - \\
\hline & \multicolumn{2}{|c|}{ 衰器 } & $m+$ & $\sigma$ & 10 & - & $10-5$ & on & $----n$ & $\omega$ & $--\sqrt{-N D O}$ & 으 & in & 6 & - & - \\
\hline & \multirow{2}{*}{ 匐 } & 롤 & 11 & 1. & 11 & 1 & 1.11 & 1 & 1111 & 1 & 111, & ' & '. & ' & 1 & 1 \\
\hline & & 평 & 11 & 1 & 11 & ' & $\cdots \cdot$ & 1 & $1+11$ & 1 & 111 & ' & 1 & ' & , & ' \\
\hline & \multicolumn{2}{|l|}{ 론 } & 1 & ' & 11 & 1 & $\cdots \cdot$ & ' & $1+111$ & ' & 111 & 1 & 11 & - & 1 & ' \\
\hline & 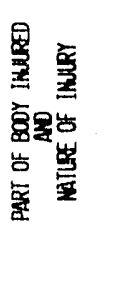 & & 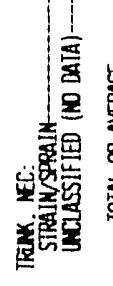 & 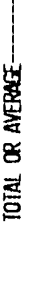 & 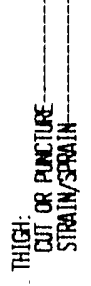 & 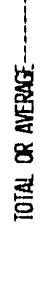 & 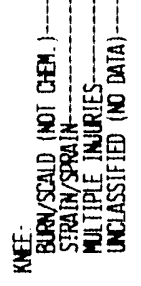 & 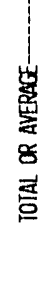 & 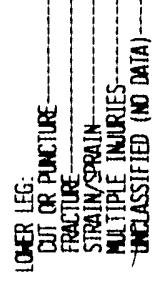 & 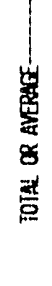 & 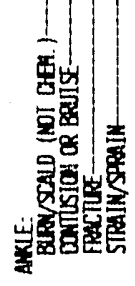 & 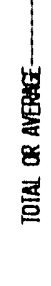 & 皆 & 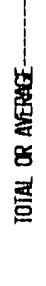 & 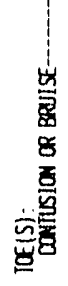 & 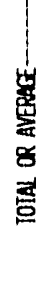 \\
\hline
\end{tabular}




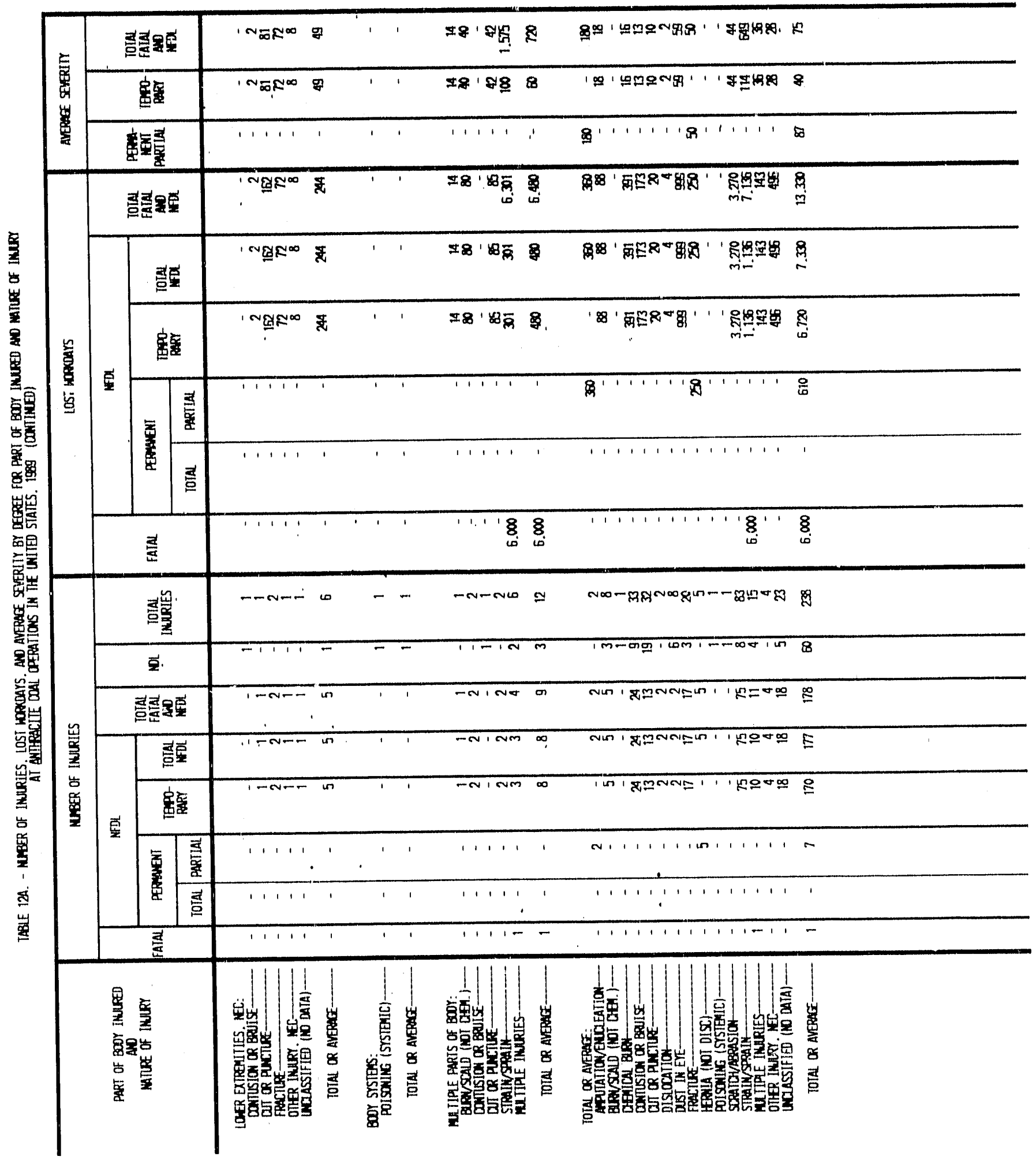




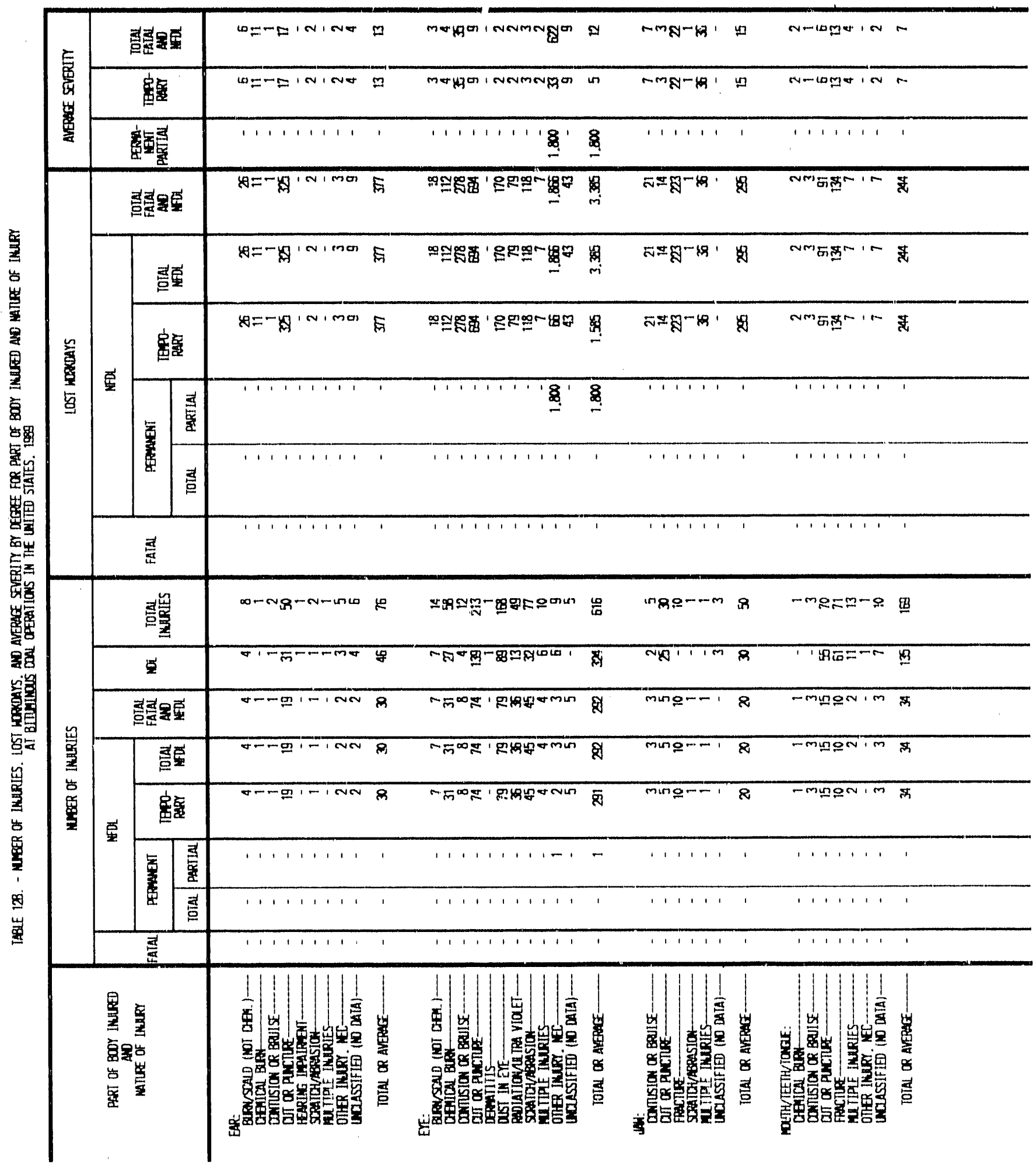




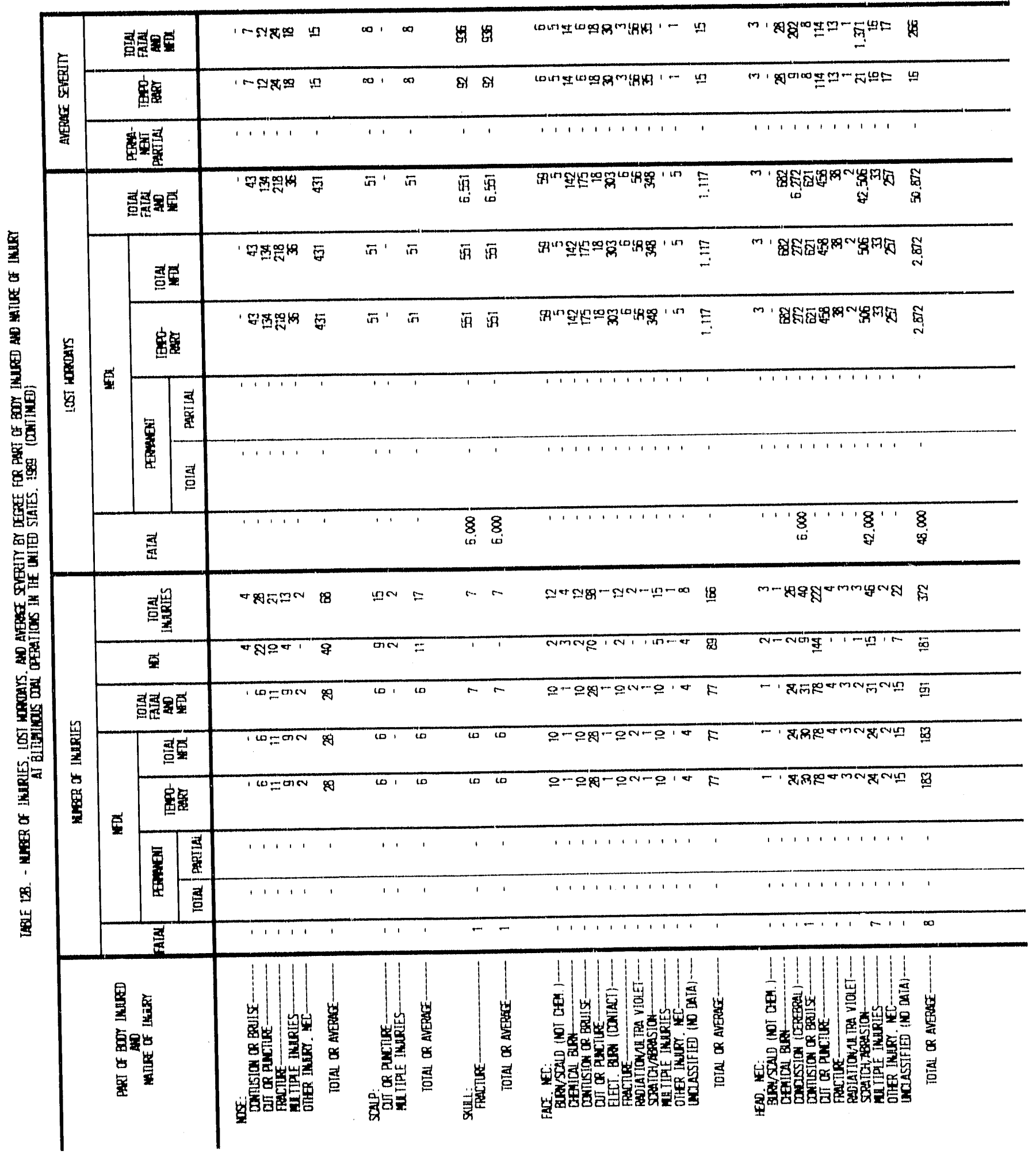




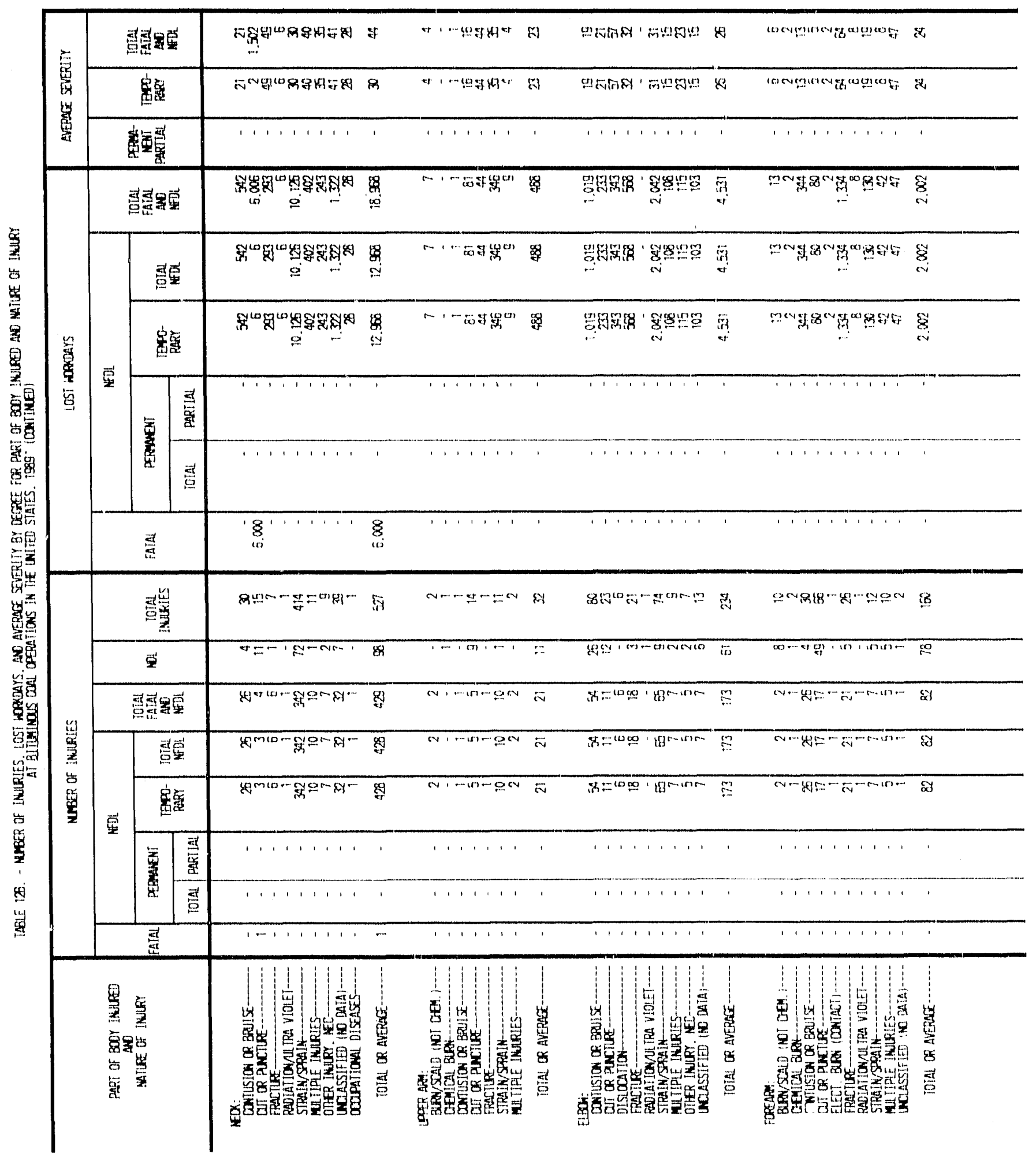




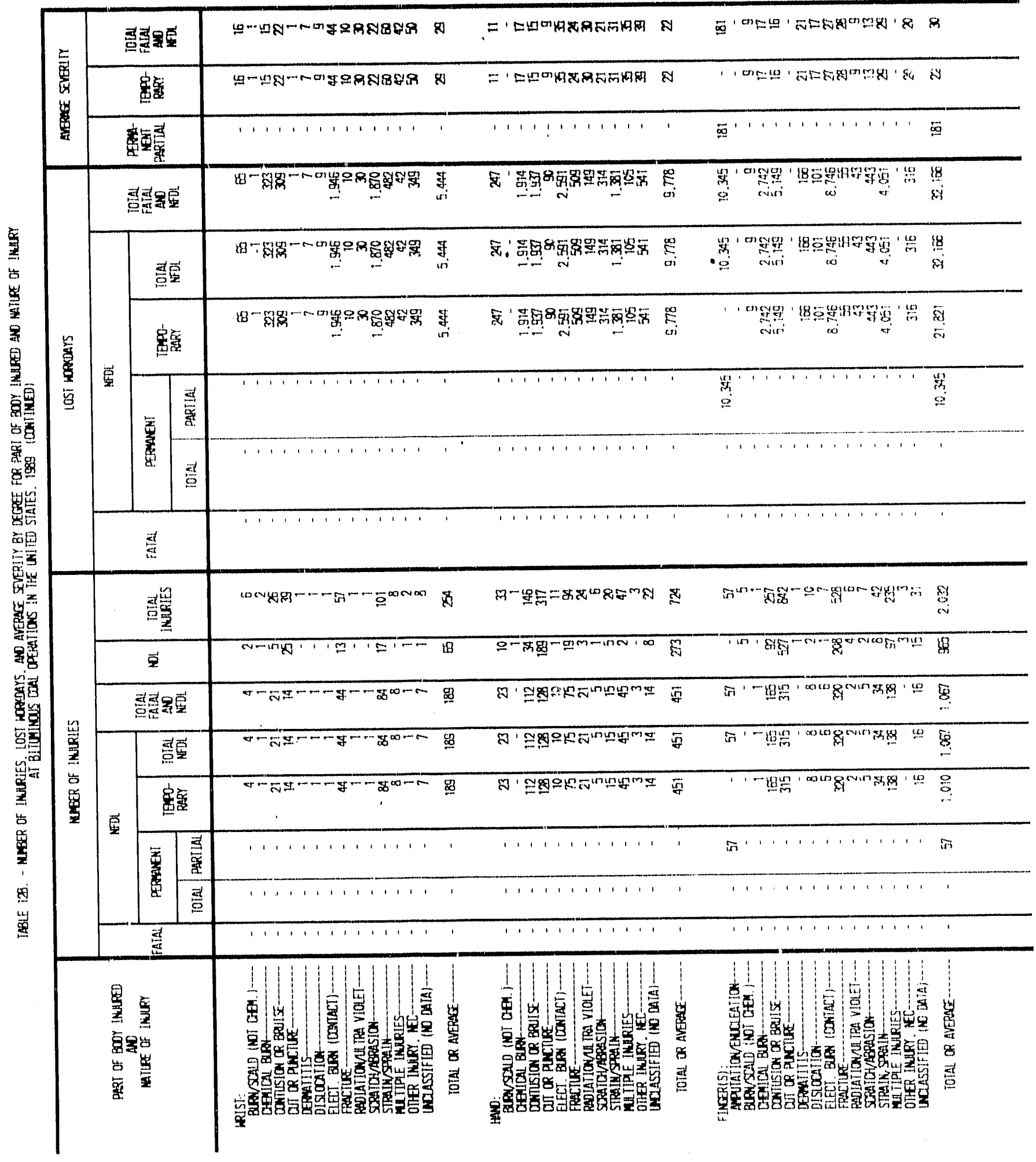




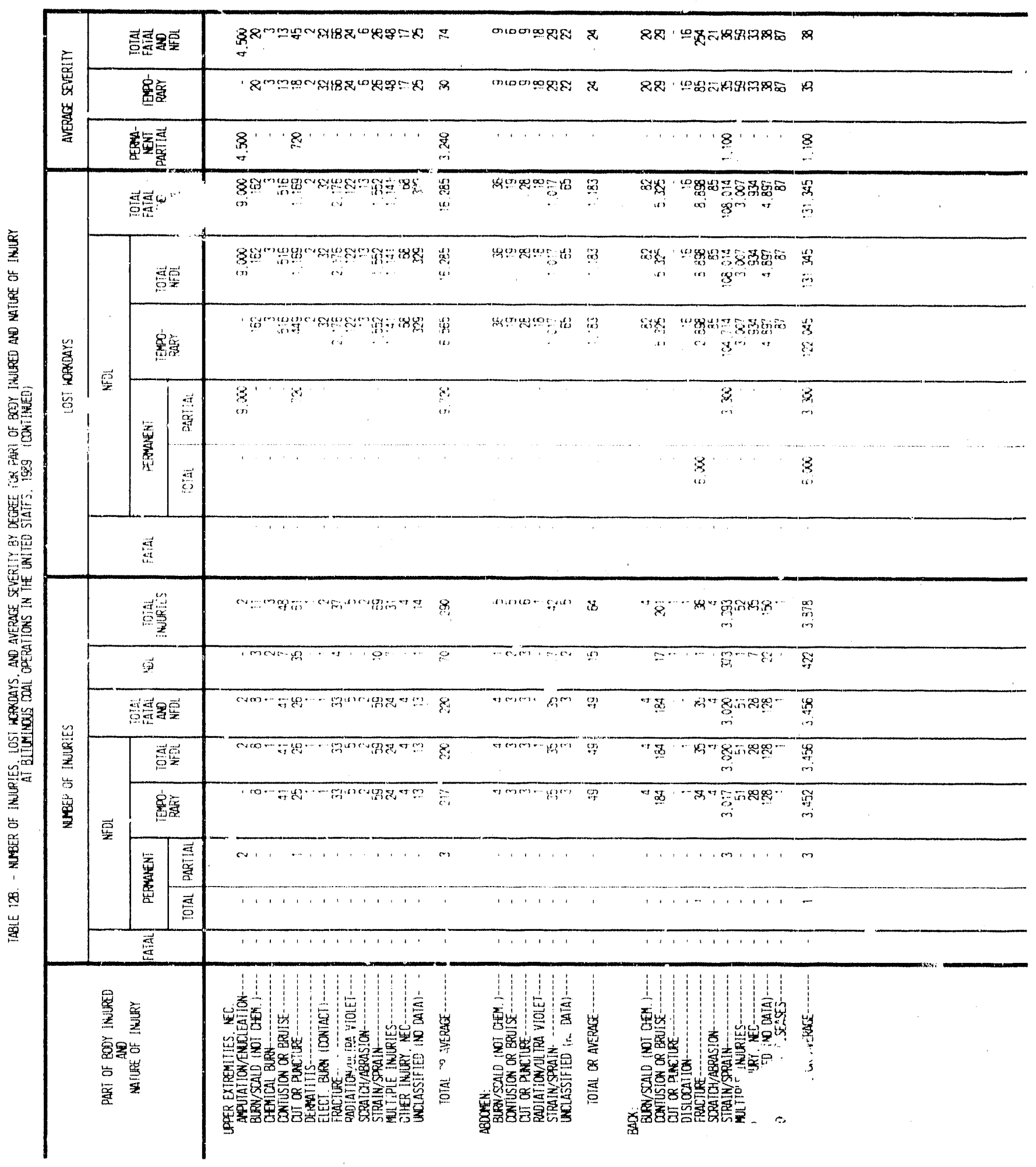




\begin{tabular}{|c|c|c|c|}
\hline 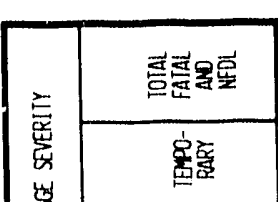 & 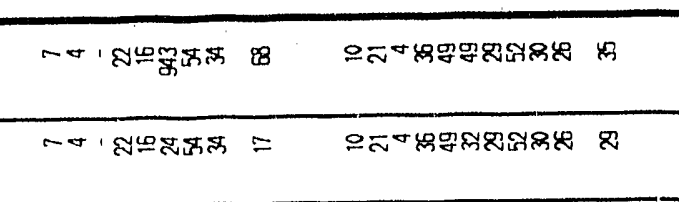 & 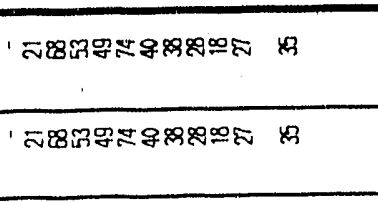 & 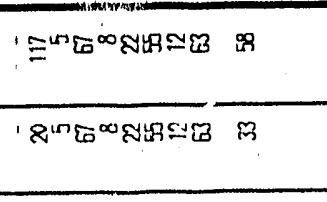 \\
\hline 部 & 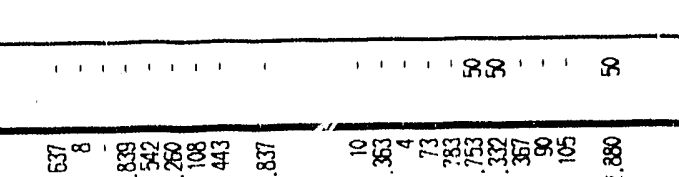 & 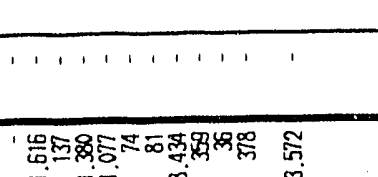 & 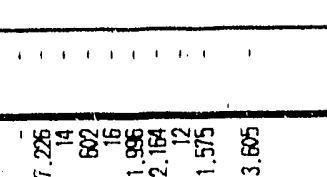 \\
\hline & 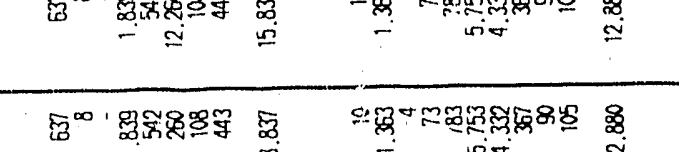 & 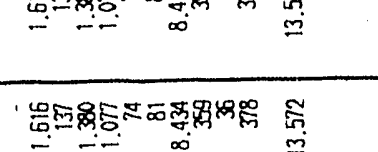 & 8 \\
\hline 籍敦 & 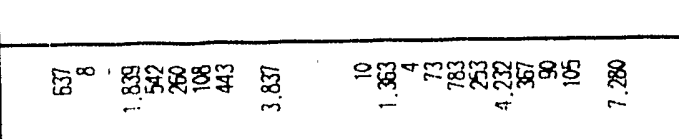 & 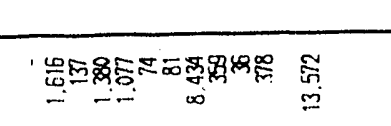 & Frepers \\
\hline & 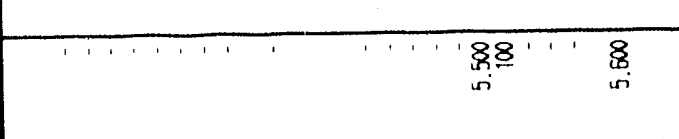 & & \\
\hline 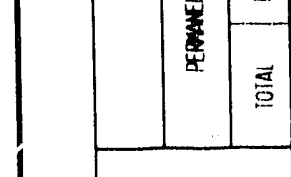 & & & \\
\hline 政 & 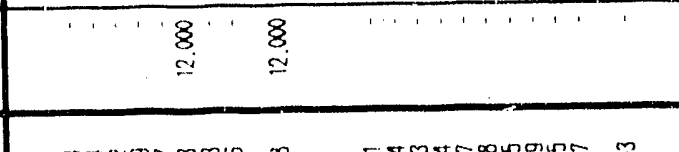 & & 3 \\
\hline & -3 & & \\
\hline 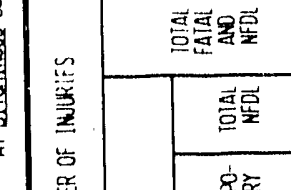 & $=2$ & & 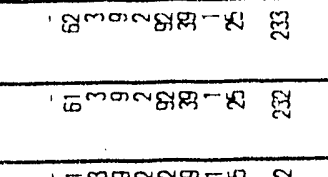 \\
\hline & DE & & \\
\hline & & & \\
\hline & & & \\
\hline
\end{tabular}




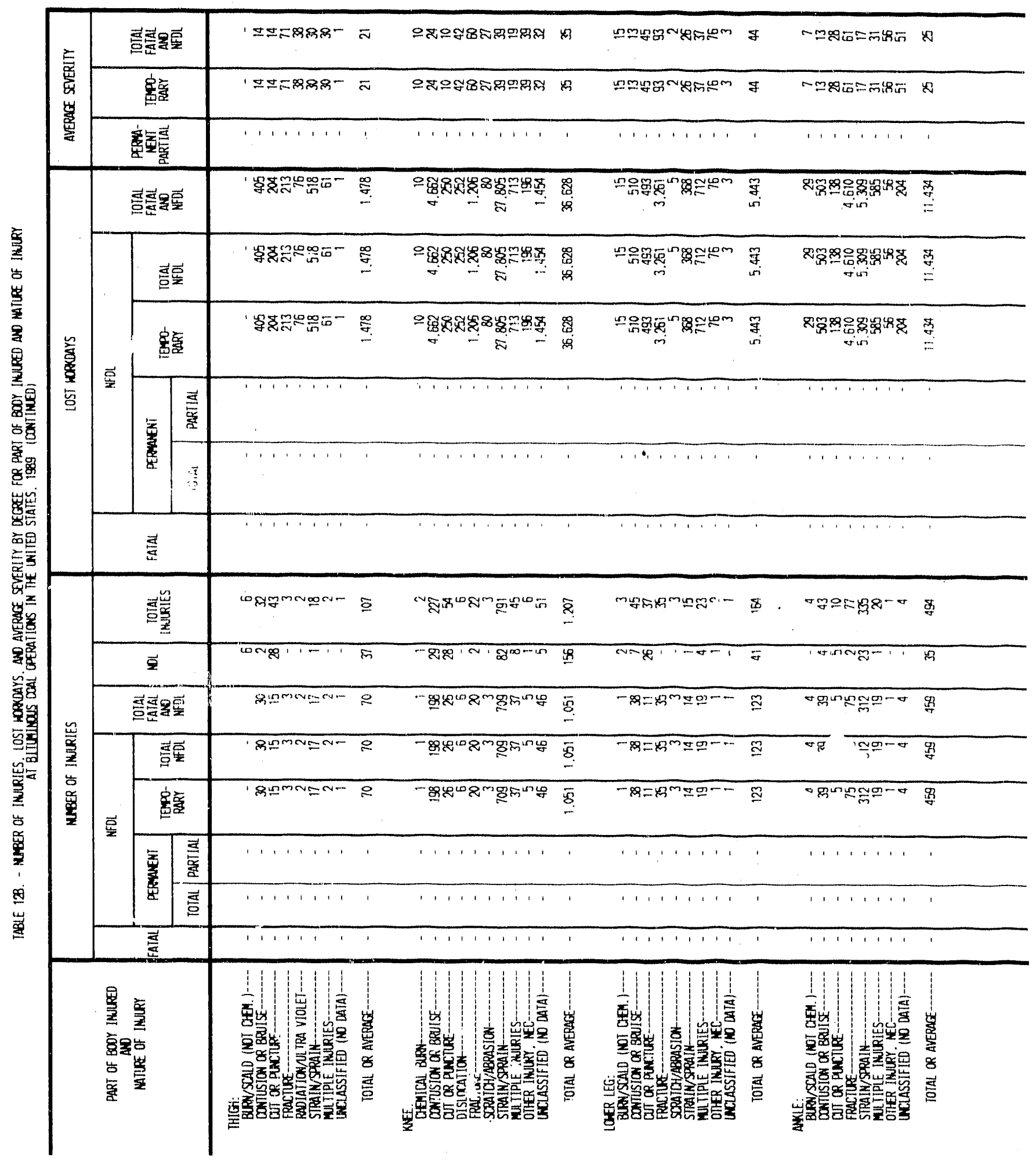




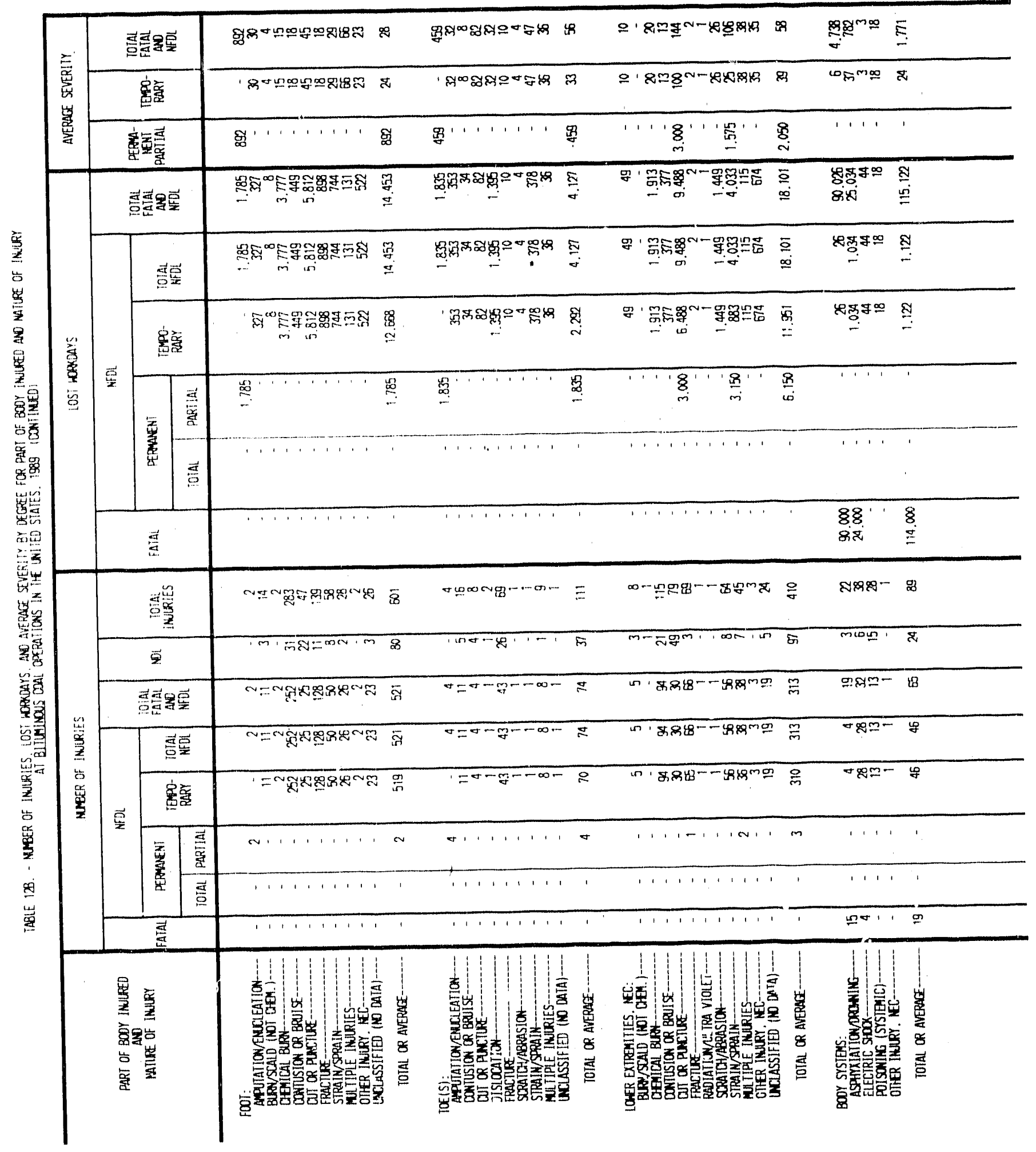




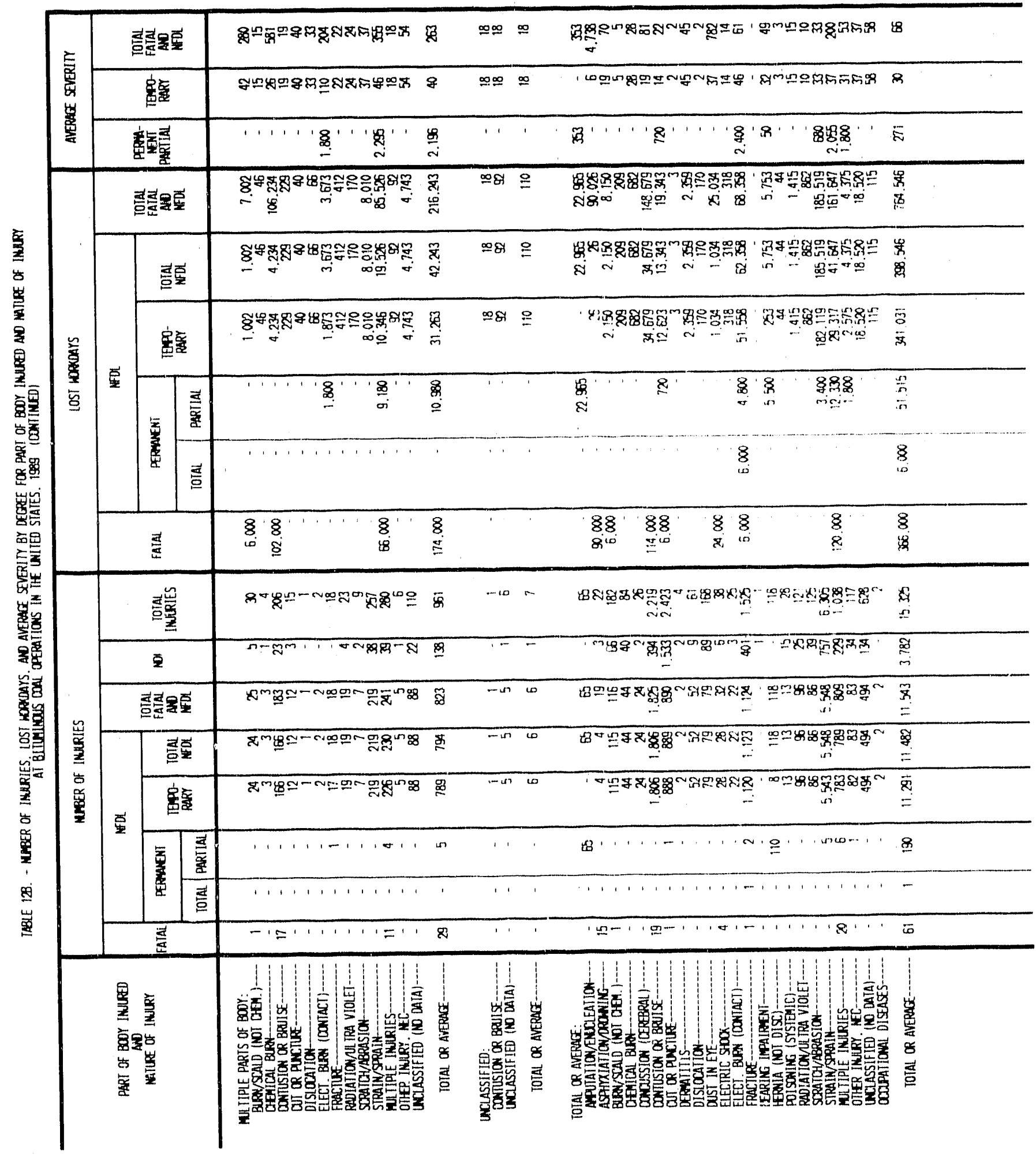




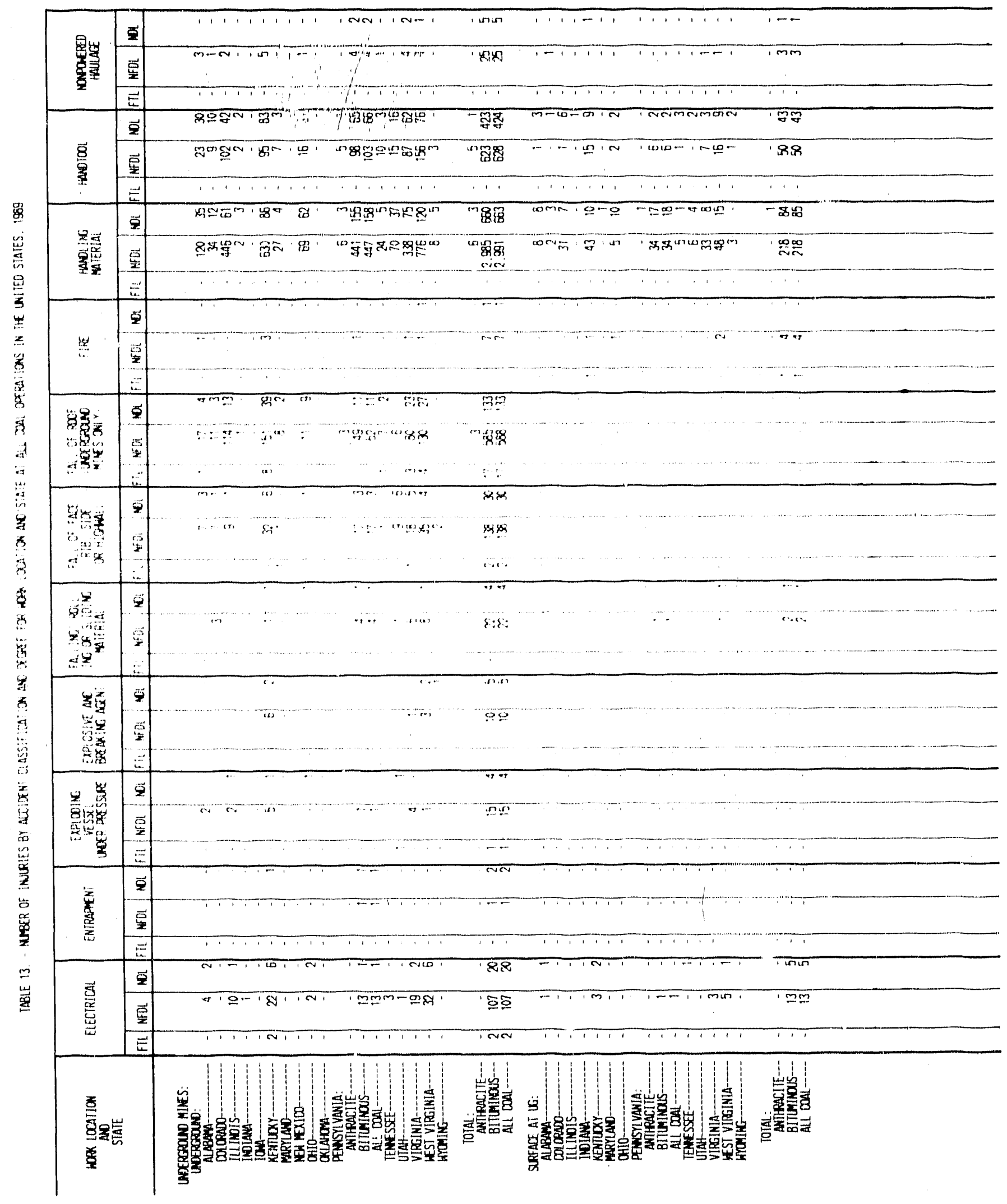




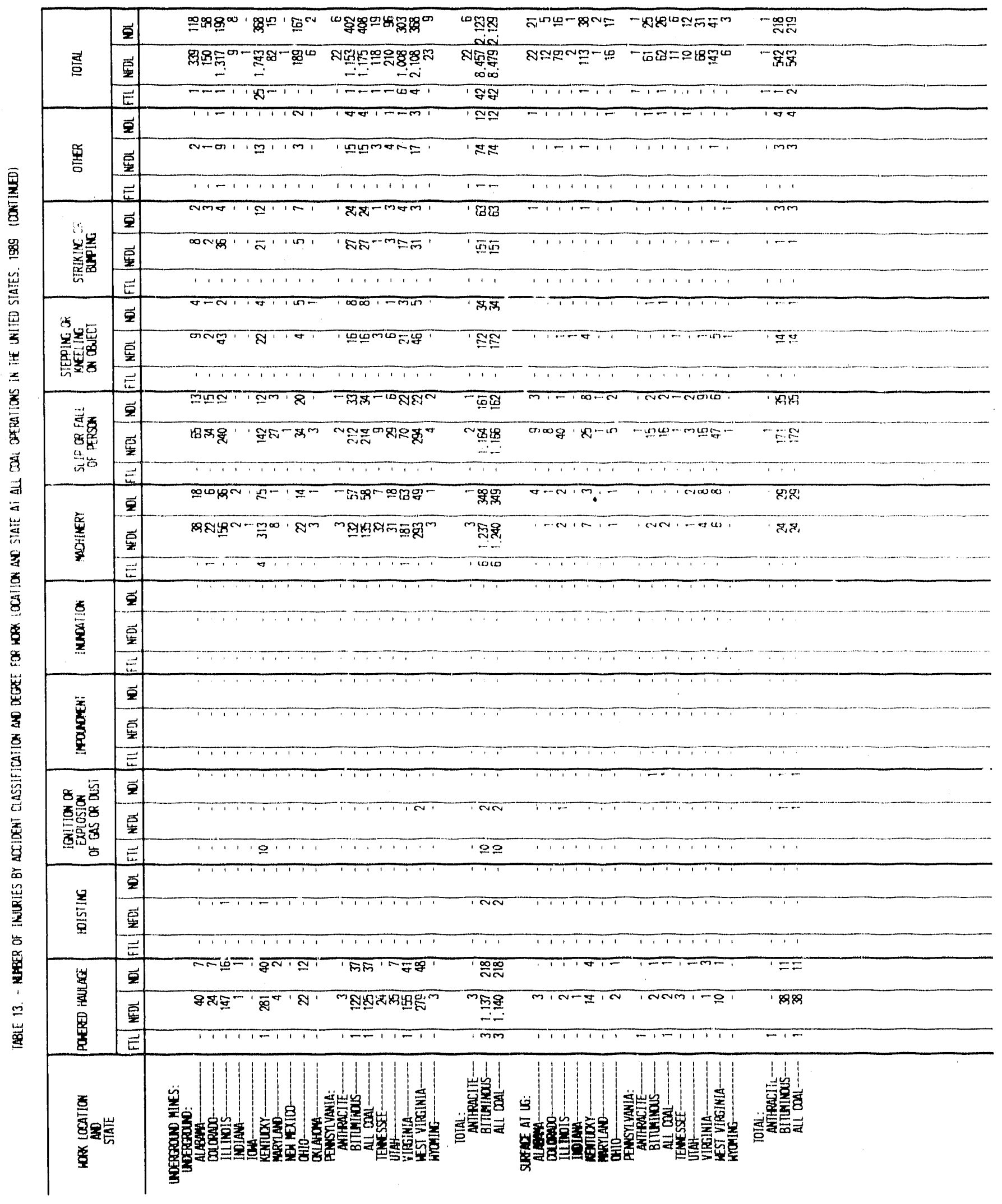




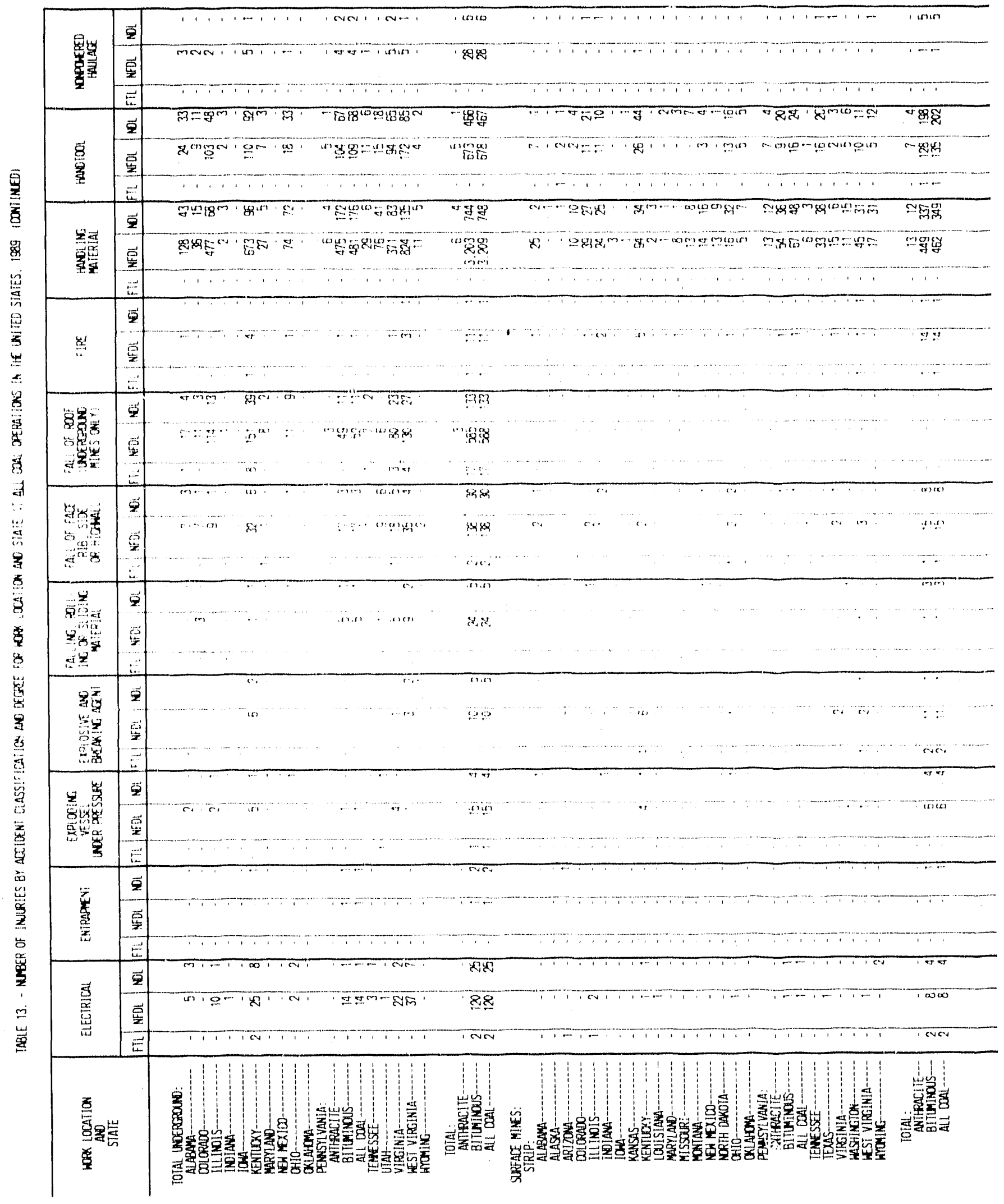




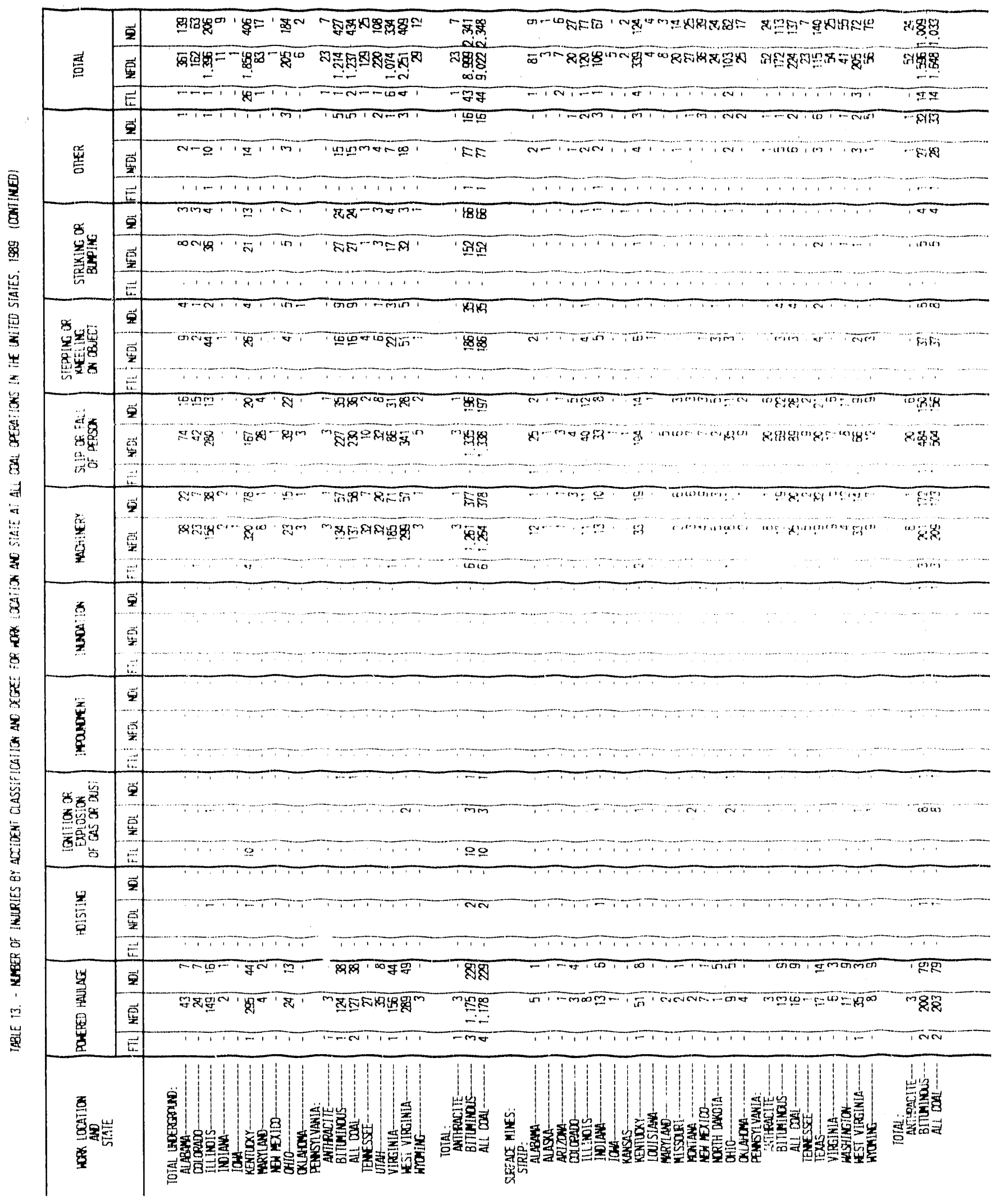




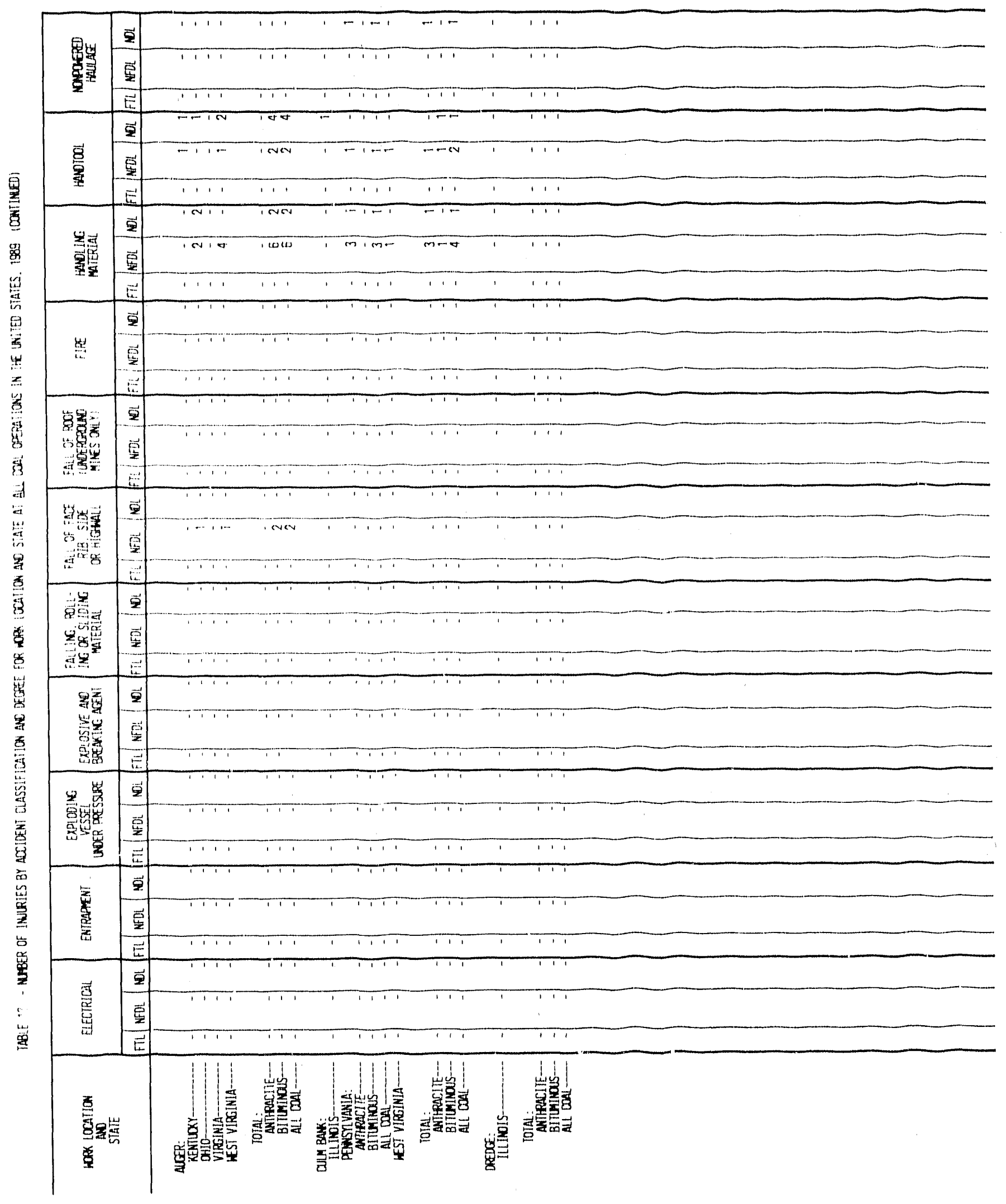




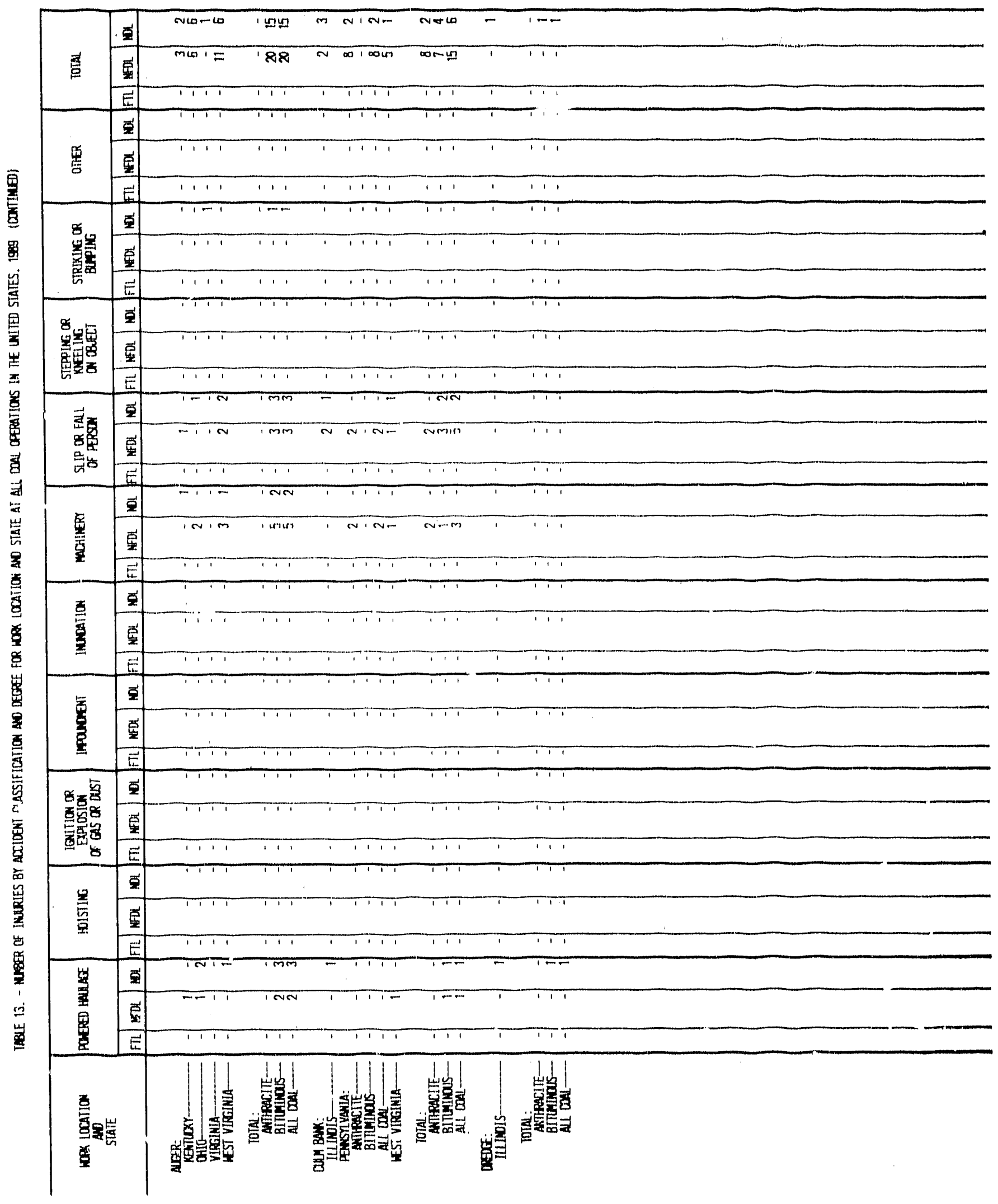




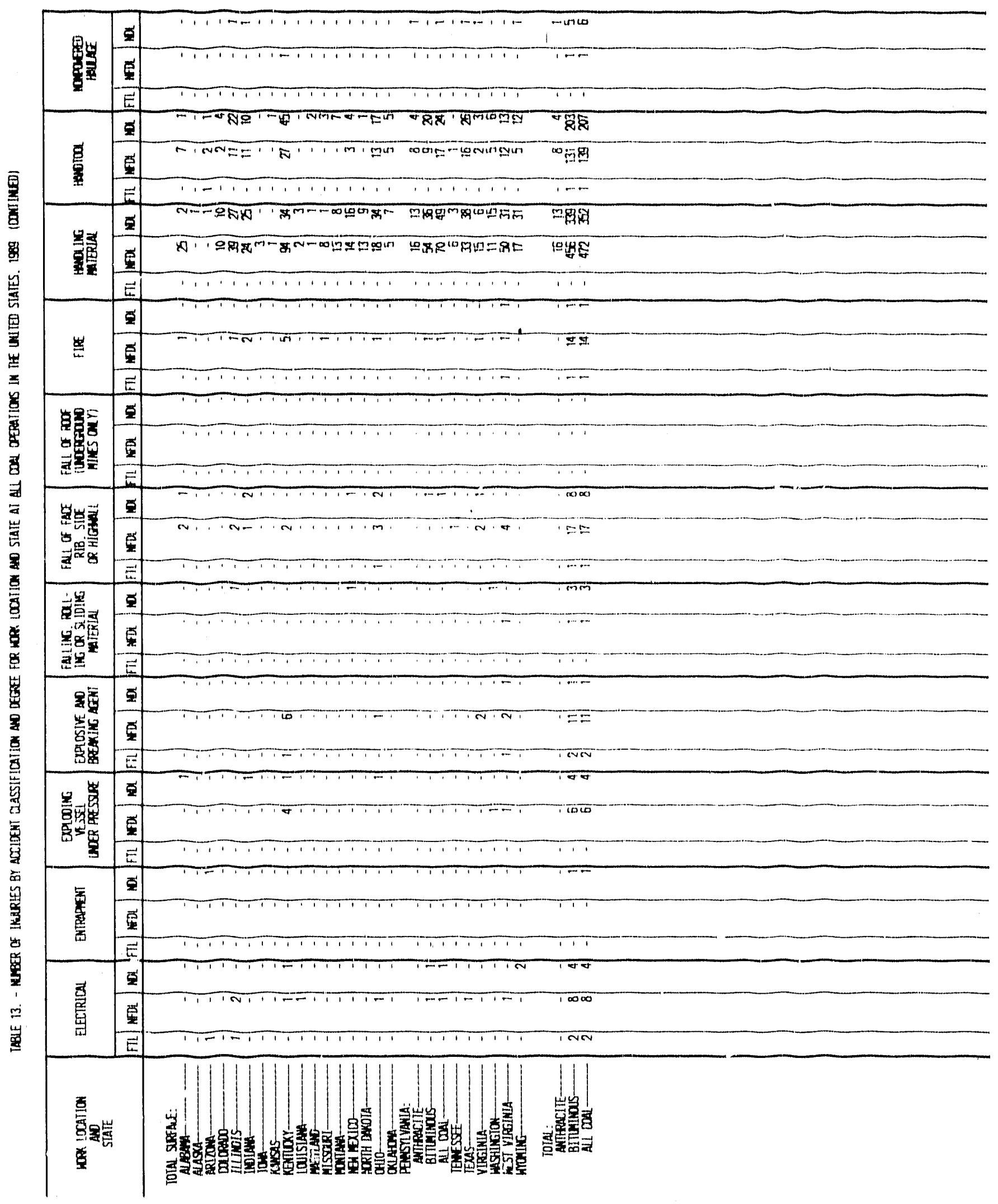




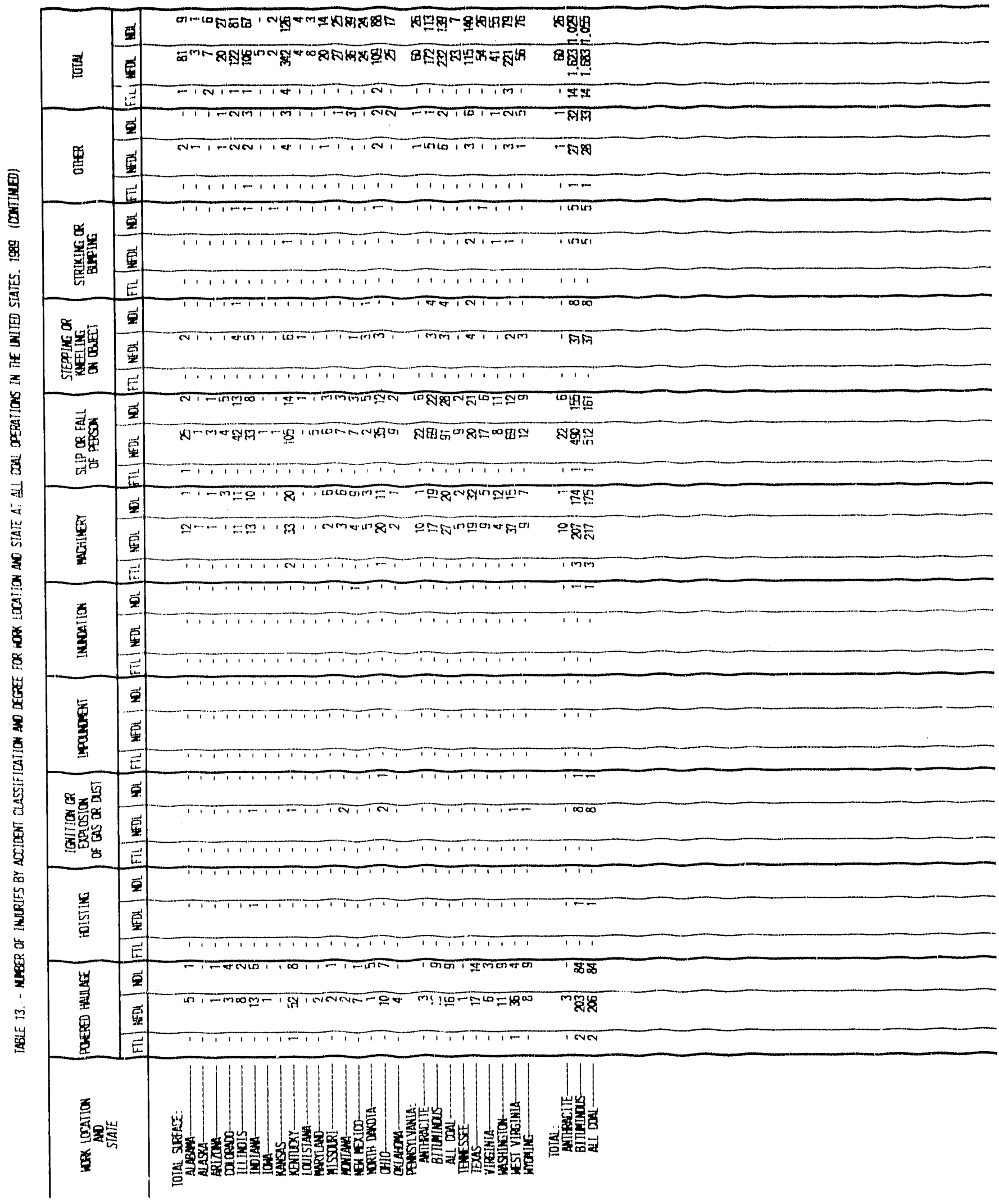




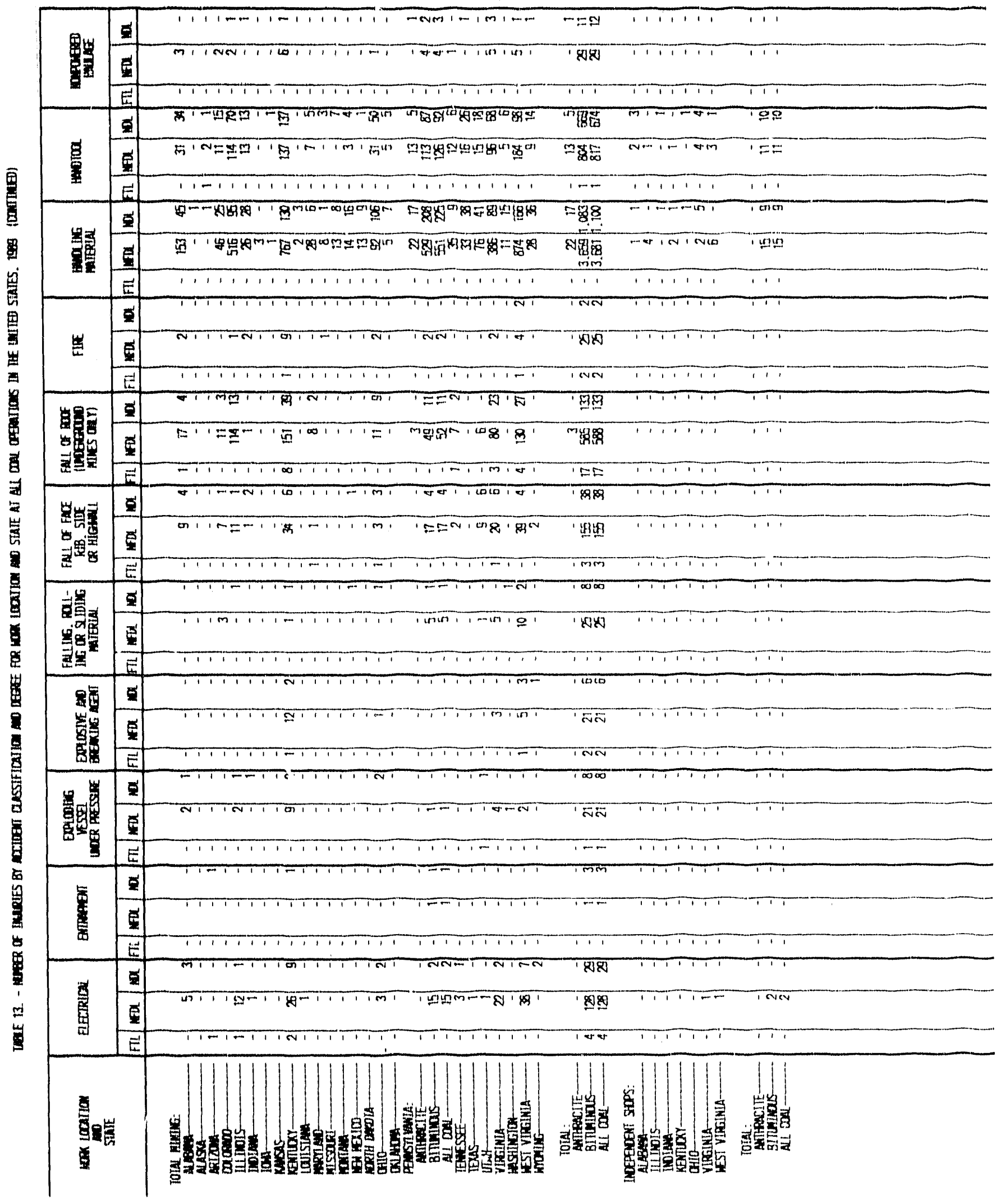




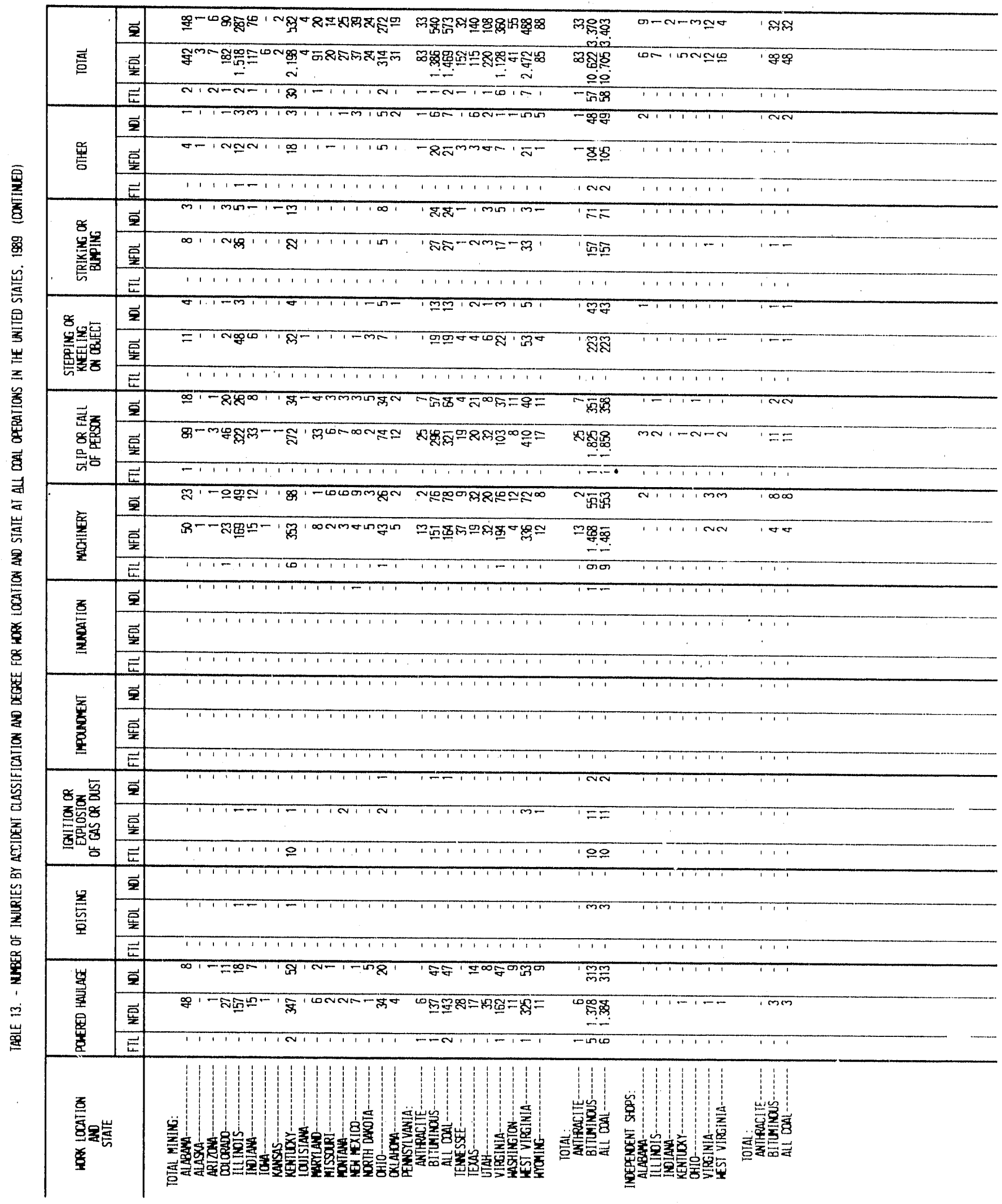




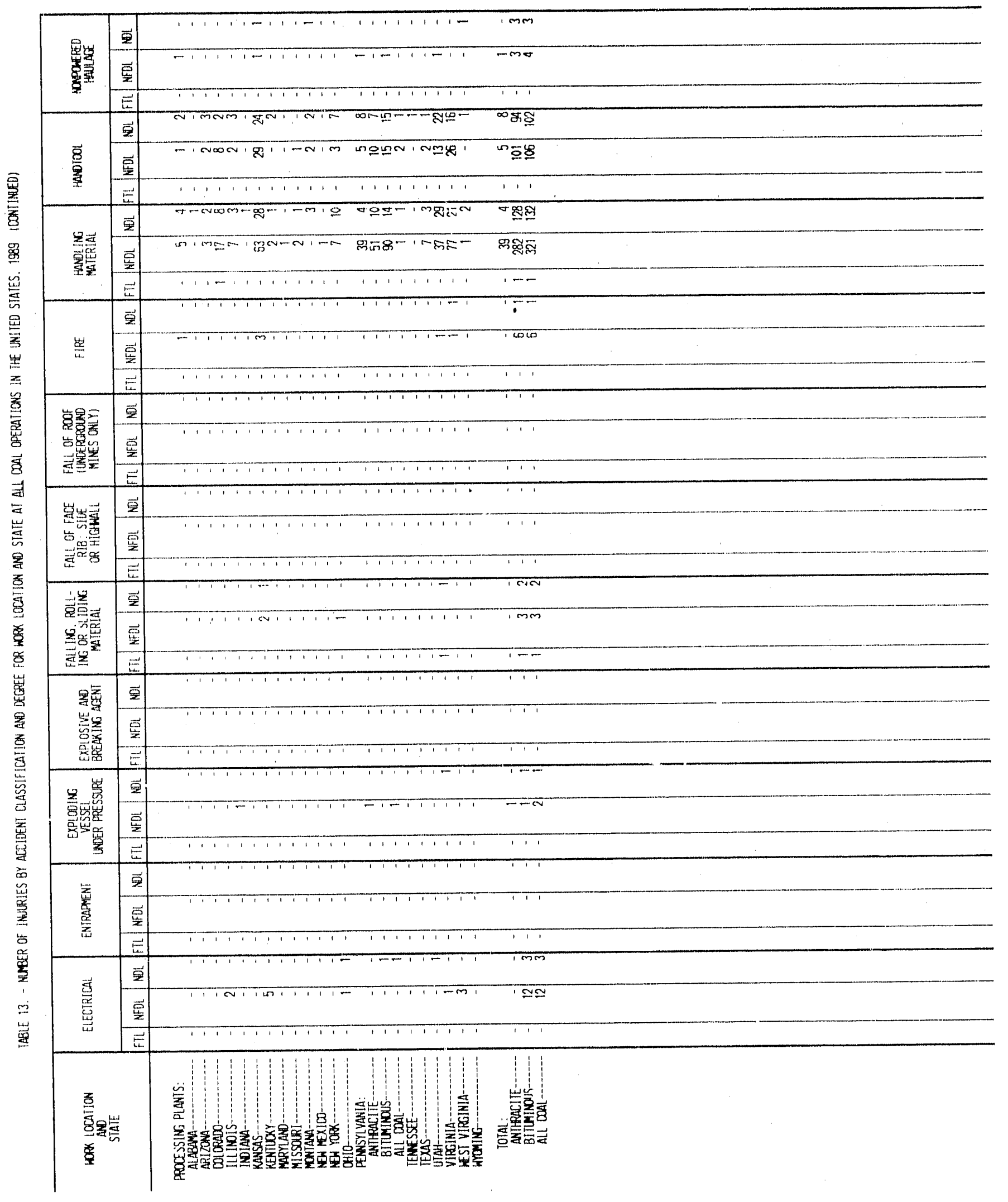




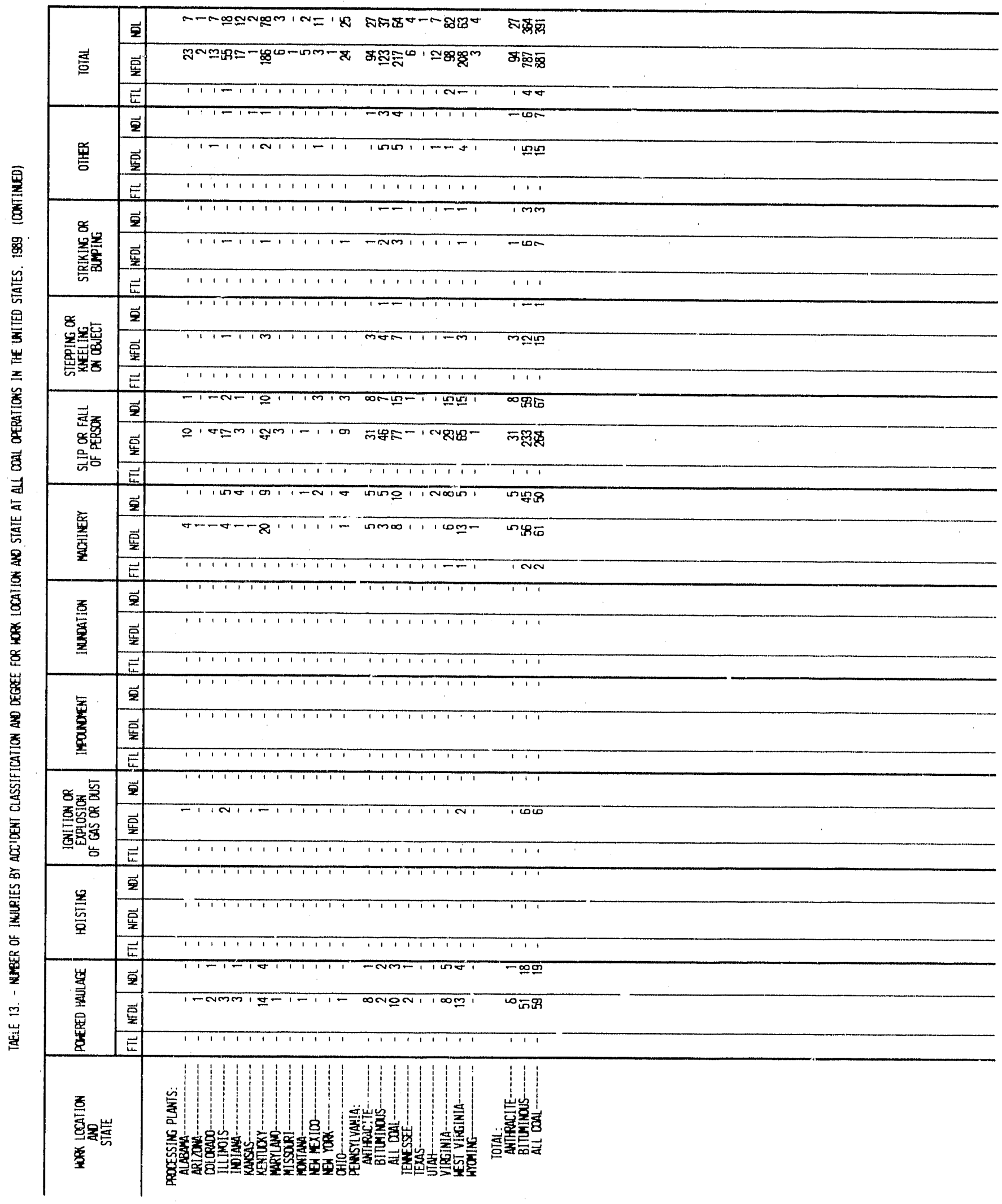




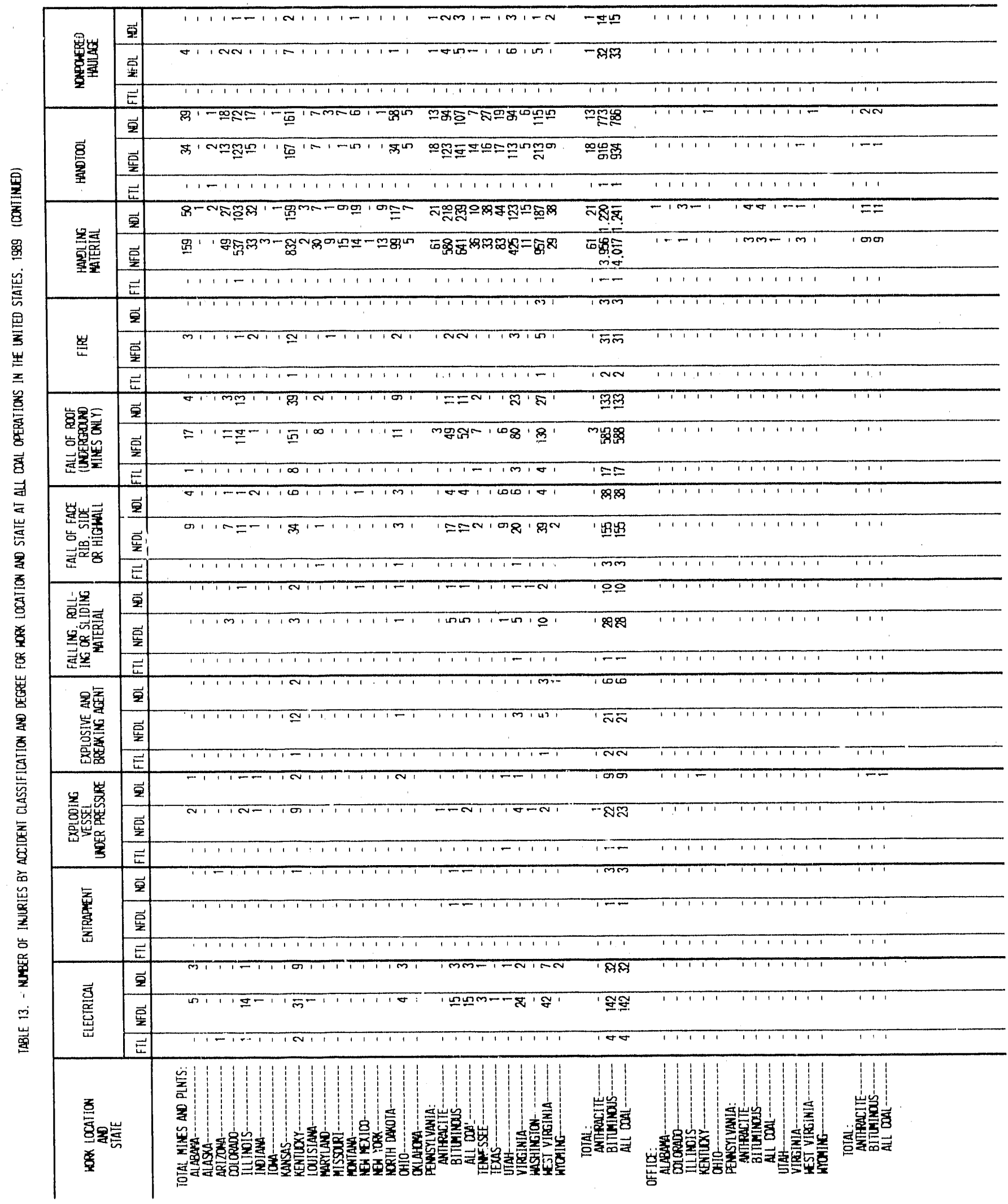




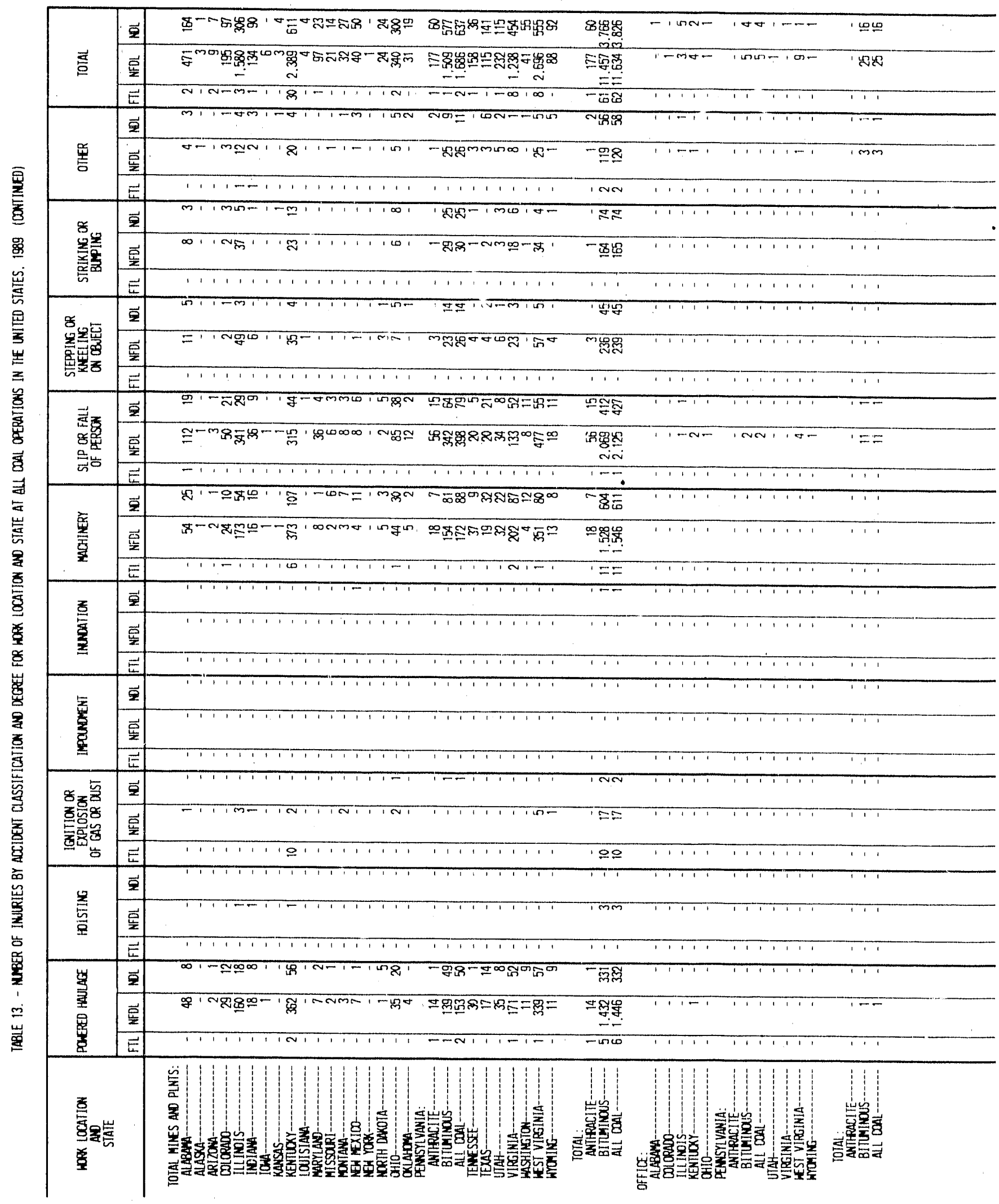




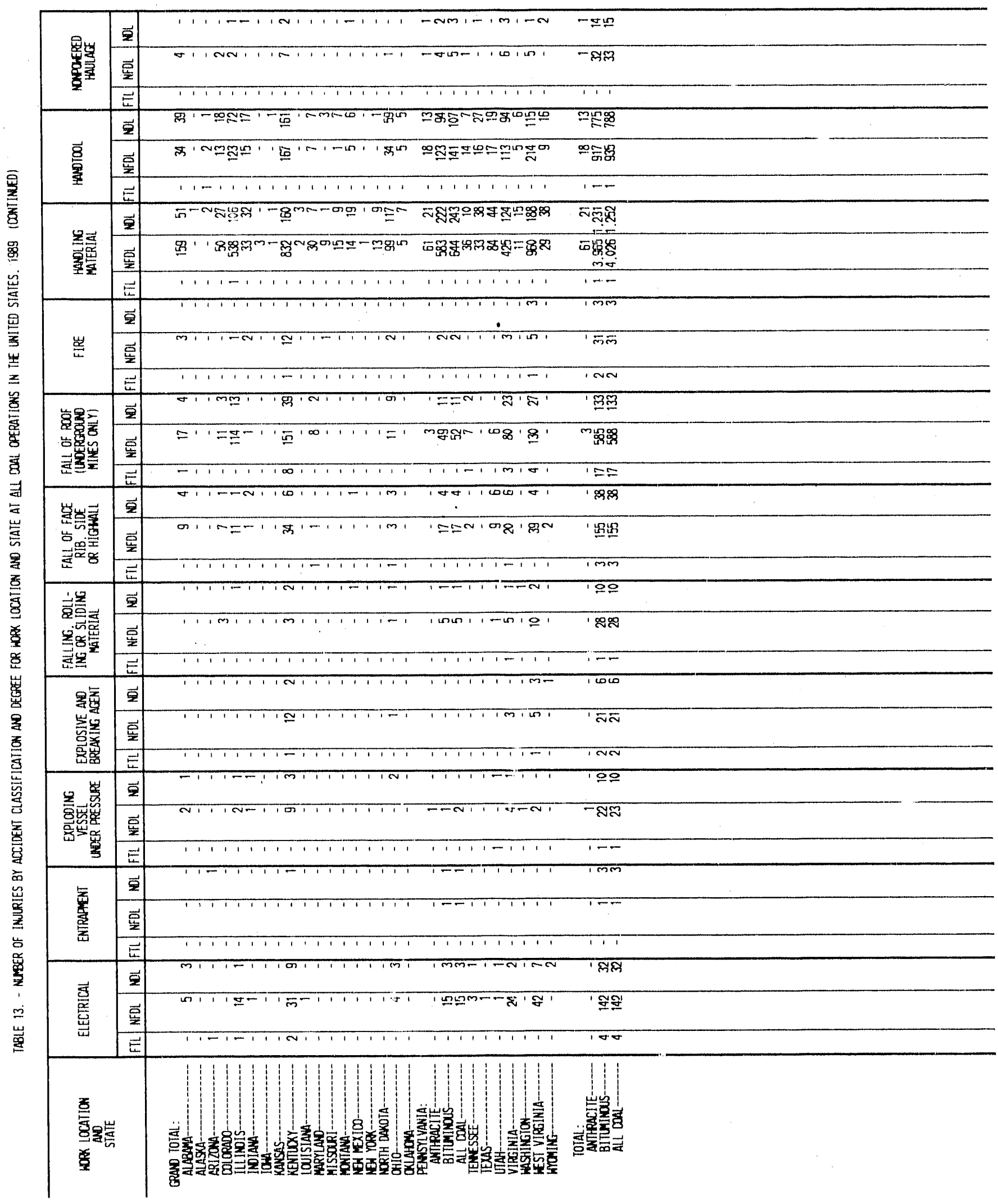




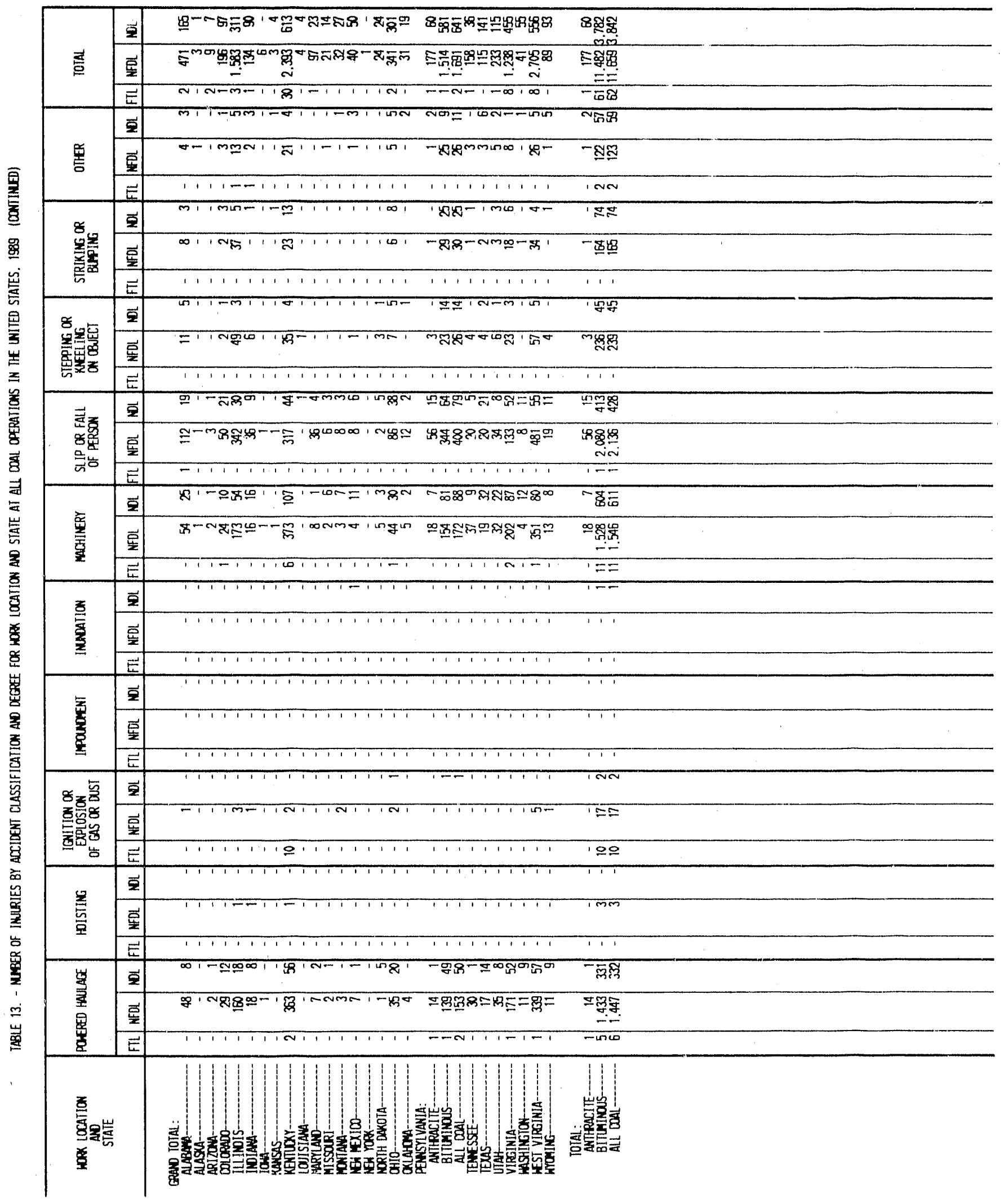




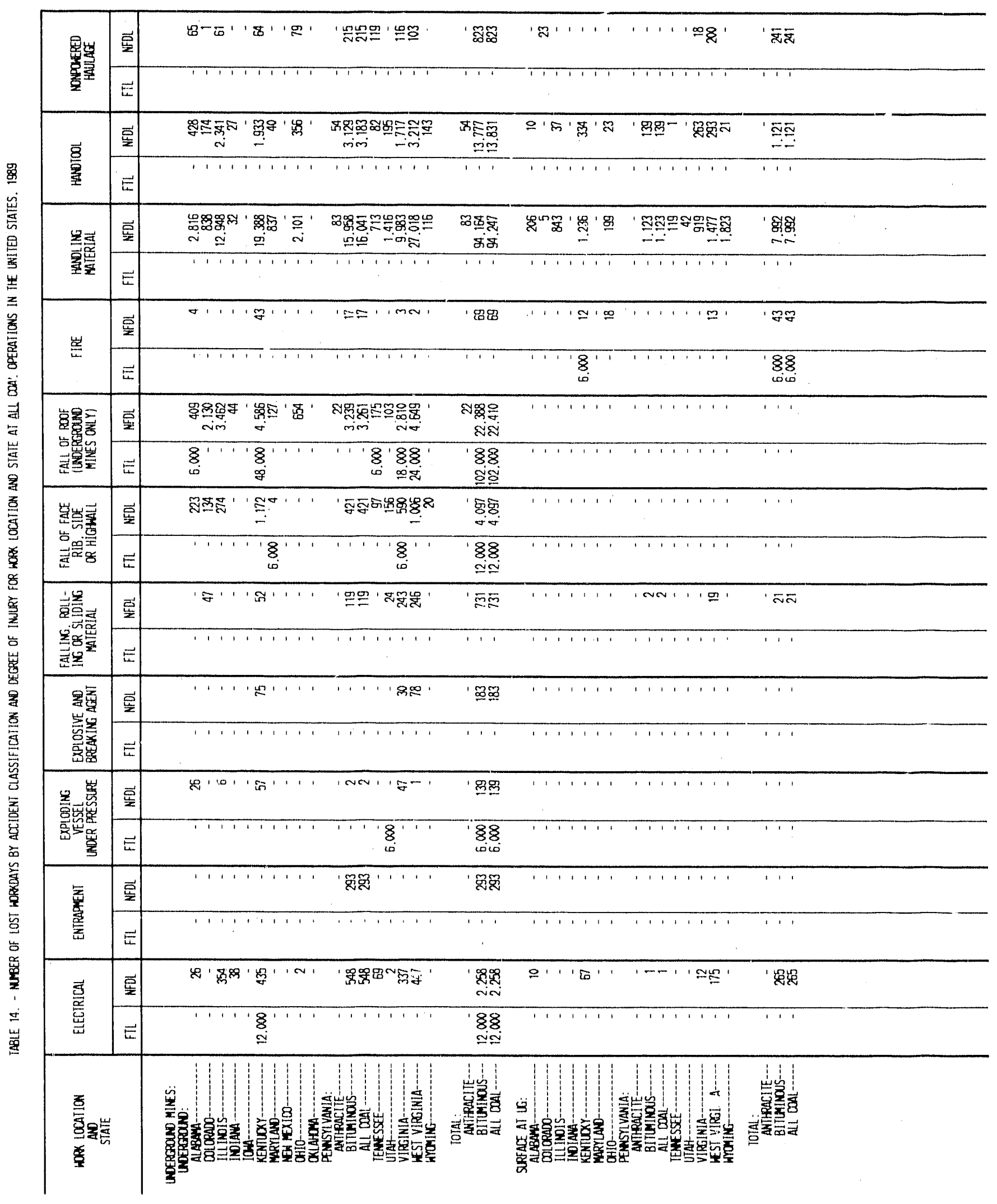




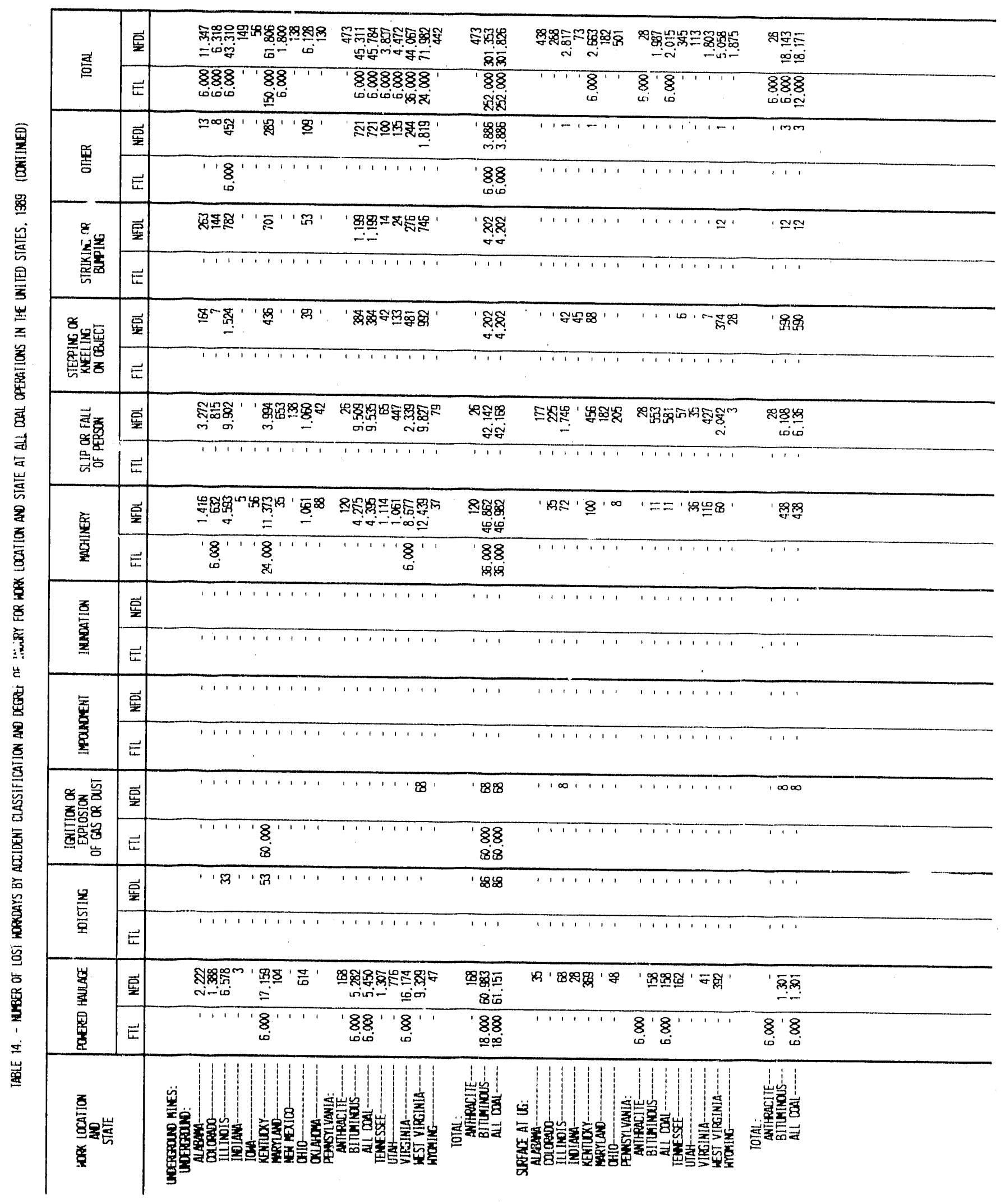




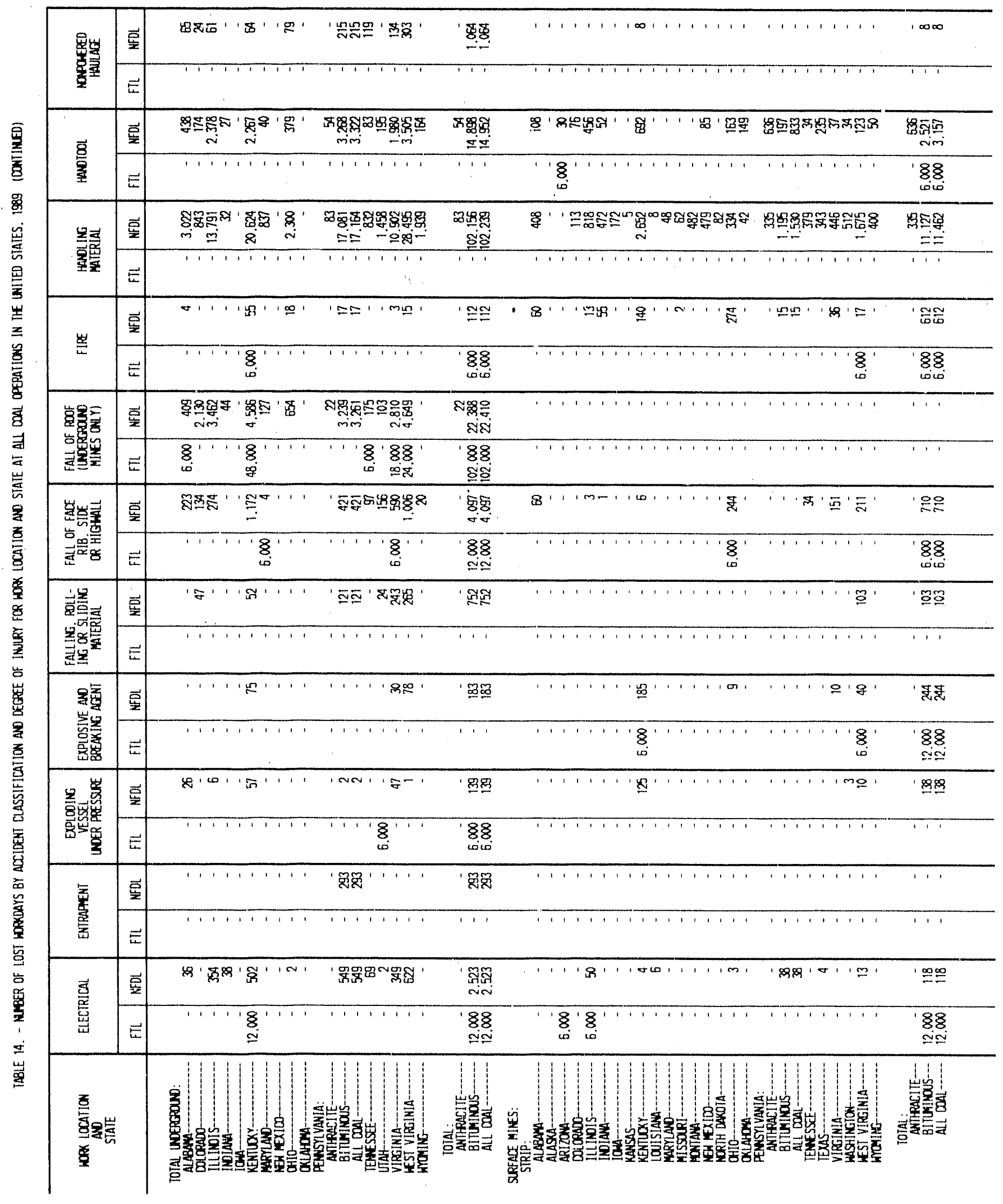




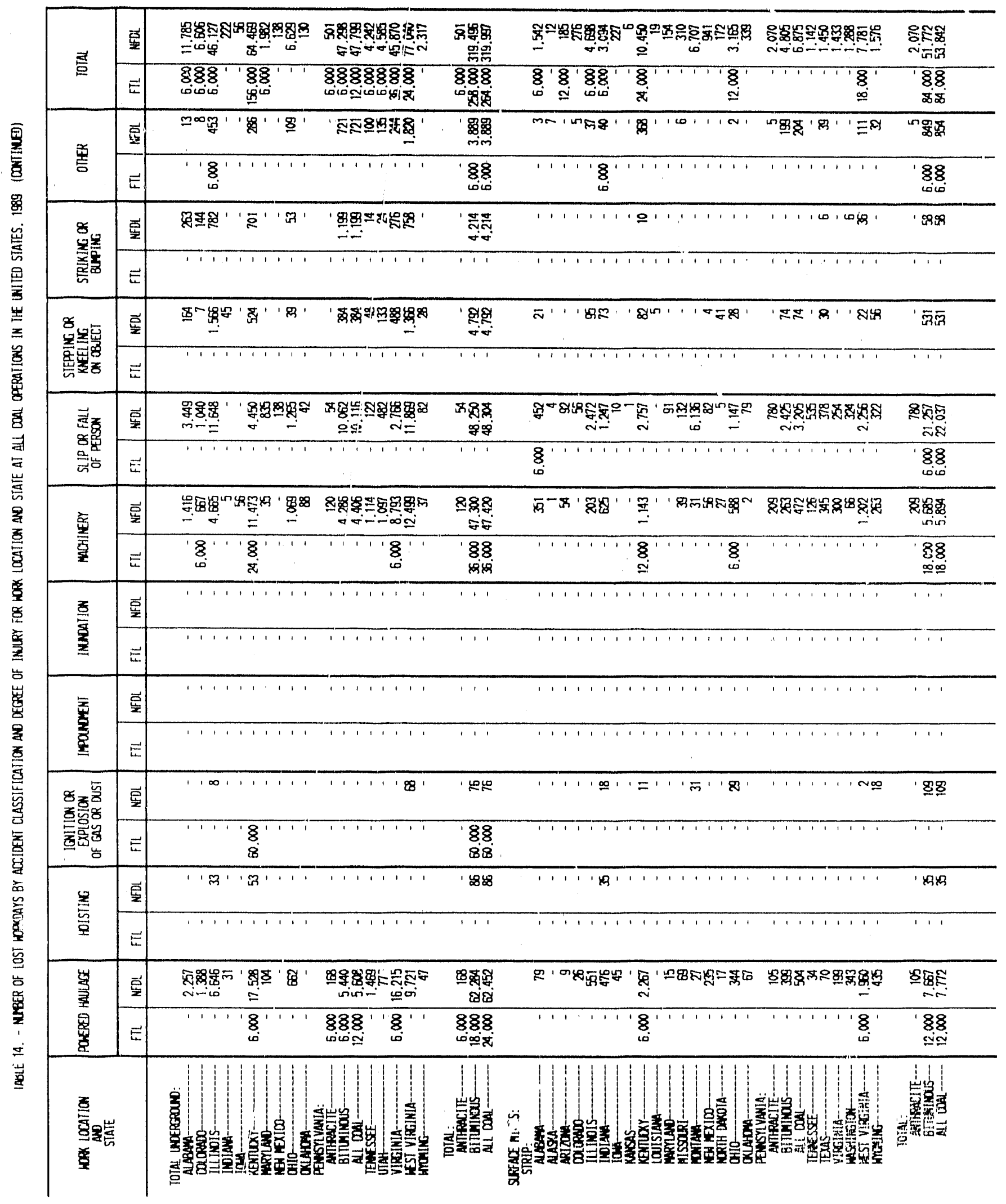




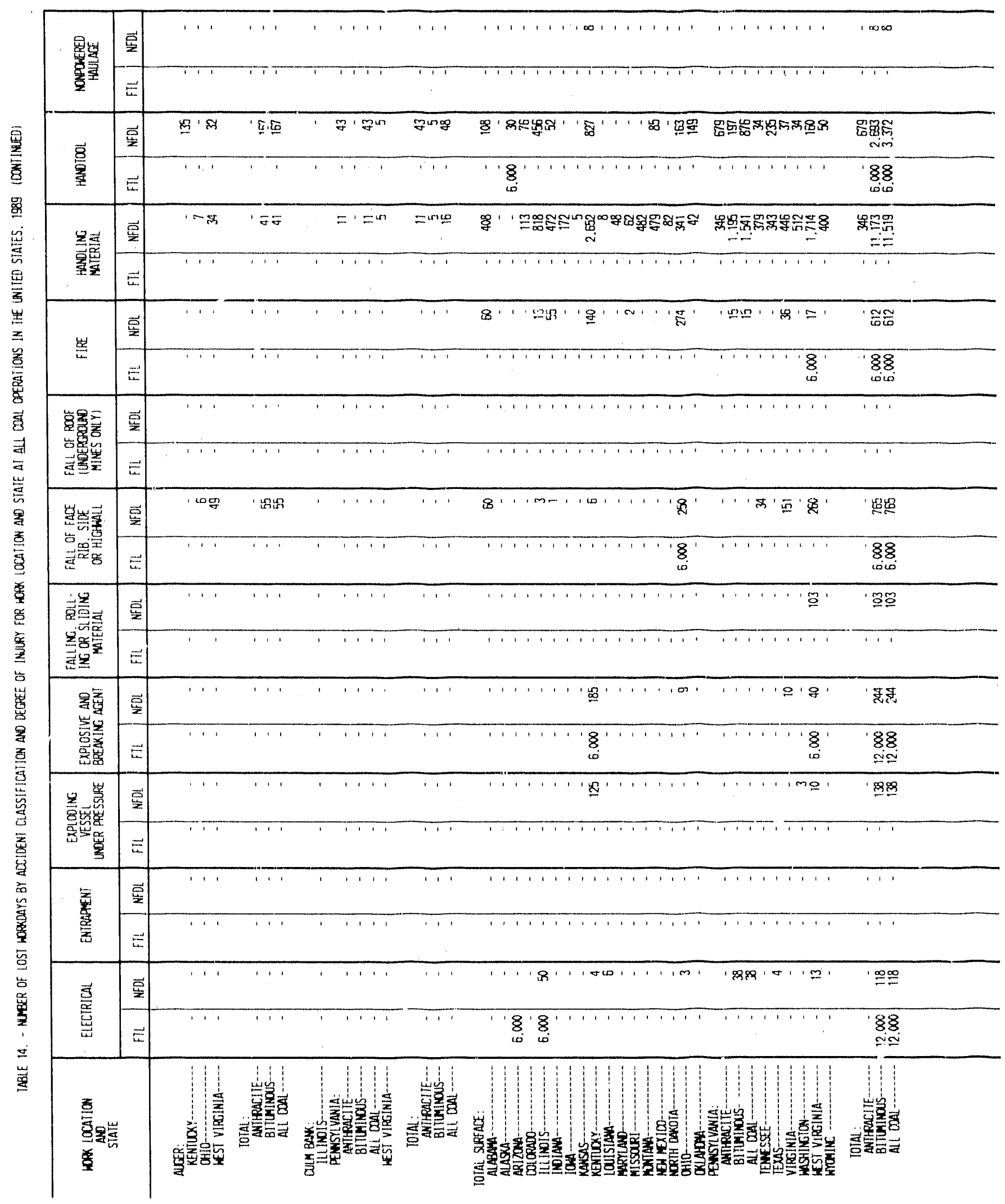




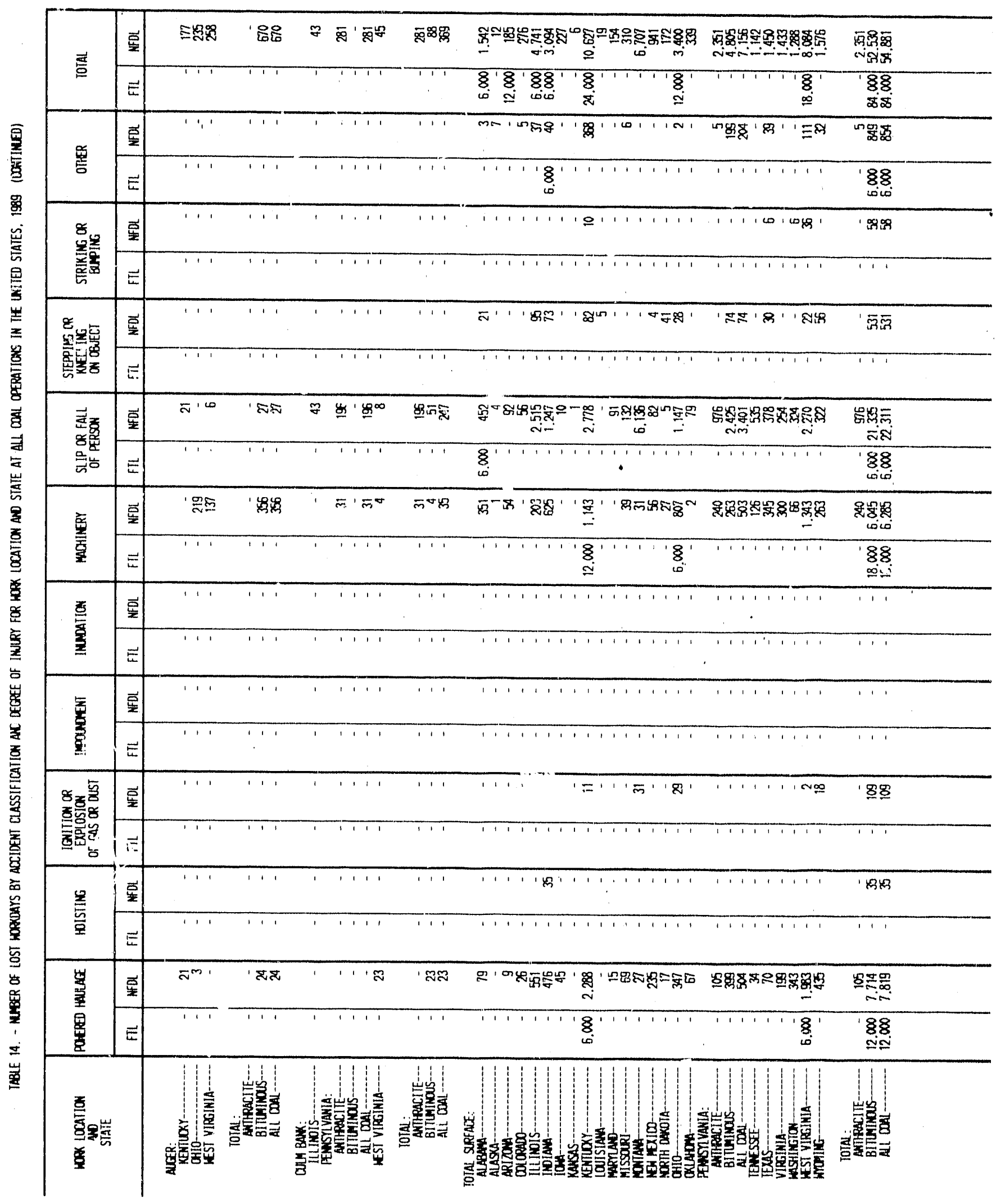




\begin{tabular}{|c|c|c|c|c|c|}
\hline \multirow{2}{*}{$\begin{array}{l}\text { 輱 } \\
\text { 至 }\end{array}$} & 稟 & 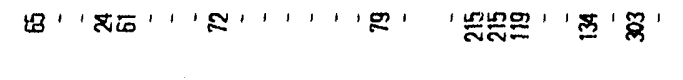 & ' & $1,1,1,1$ & ' ' \\
\hline & $E$ & & & & \\
\hline \multirow{2}{*}{$\begin{array}{l}\overline{8} \\
\text { 量 }\end{array}$} & 豆 & 罢' & 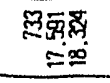 & $= \pm 0 \cdot 45$ & $\underline{\underline{\Phi}}$ \\
\hline & $E$ & 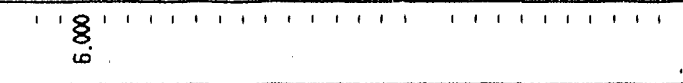 & $\underbrace{88}_{0.06}$ & 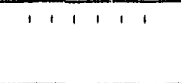 & $T$ \\
\hline \multirow{2}{*}{ 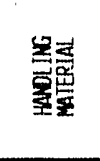 } & 要 & 勇' ' & 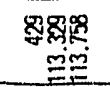 & 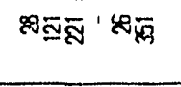 & FE \\
\hline & $\vec{E}$ & 1111111111111,1111111 & 1.11 & 11111 & 1.1 \\
\hline \multirow{2}{*}{ 嵦 } & 高 & 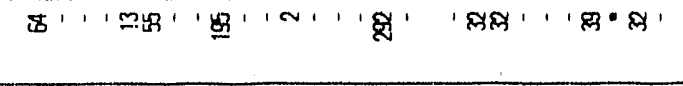 & स्रू & 11,11 & $\cdots$ \\
\hline & $E$ & 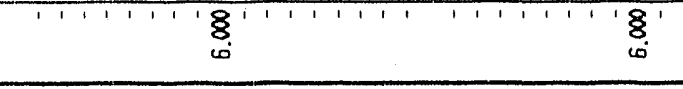 & 88 & 1111 & .11 \\
\hline \multirow{2}{*}{ 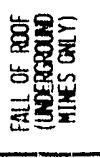 } & 氞 & 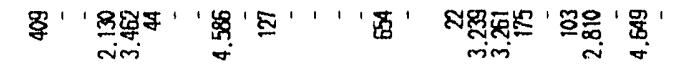 & 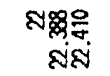 & 1111111 & 11 \\
\hline & E & 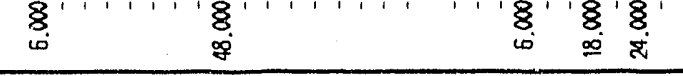 & 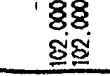 & & 11. \\
\hline \multirow{2}{*}{ 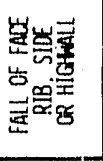 } & 苟 & 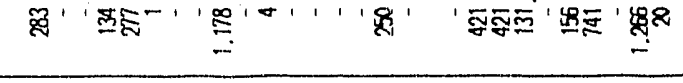 & 跴 & $1,1,1,1$, & 11 \\
\hline & 리 & 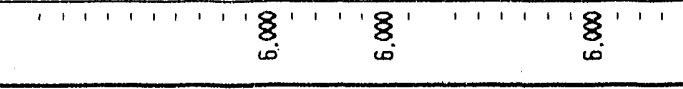 & $\begin{array}{l}88 \\
88 \\
\infty 00 \\
\end{array}$ & 11111 & 111 \\
\hline \multirow{2}{*}{ 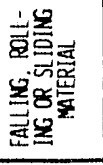 } & $\overrightarrow{\underline{\mathbf{b}}}$ & 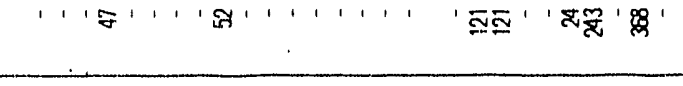 & '桨哭 & $1,1,1$ & 111 \\
\hline & $E$ & 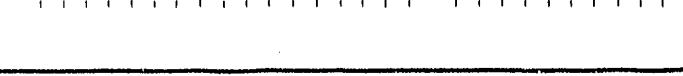 & 1' & 1 & 11. \\
\hline \multirow{2}{*}{ 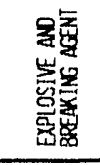 } & $\overrightarrow{\underline{a}}$ & 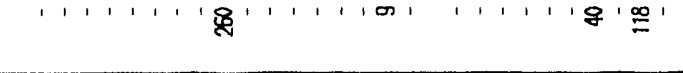 & 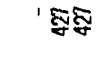 & $1+1+1$ & 11 \\
\hline & $\overrightarrow{\mathrm{E}}$ & 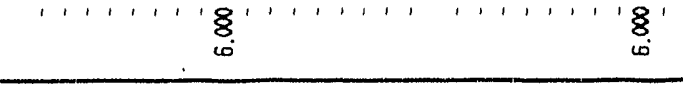 & 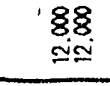 & & 111 \\
\hline \multirow{2}{*}{ 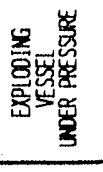 } & 豆 & 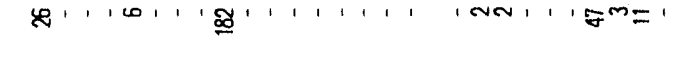 & हิ & ' 1'1'1' & ' ' \\
\hline & $\equiv$ & 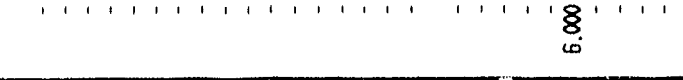 & 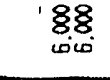 & $1+1+1$ & $1+1$ \\
\hline \multirow{2}{*}{ 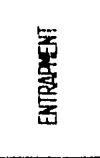 } & 可 & ' ' ' ' ' ' ' ' ' ' ' & 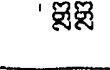 & 11.11 .1 & '. ' ' \\
\hline & 탄 & 1 & 1. & 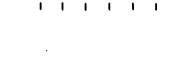 & 1.1 \\
\hline \multirow{2}{*}{ 胥 } & 豆 & 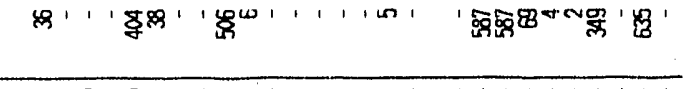 & ' & 11,10 & ' 约级 \\
\hline & $E$ & 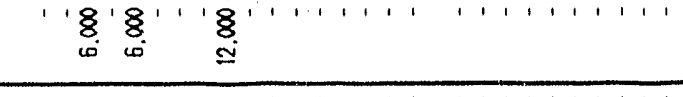 & $\begin{array}{l}188 \\
x x i \\
\end{array}$ & ' & 111 \\
\hline \multicolumn{2}{|l|}{ 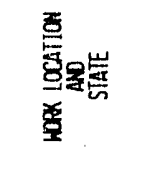 } & 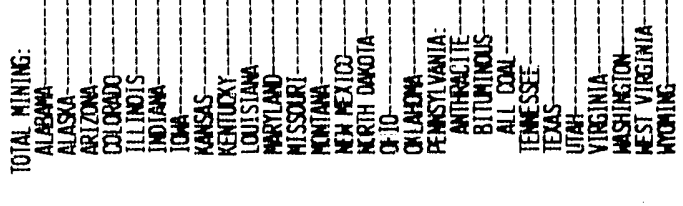 & 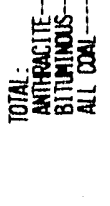 & 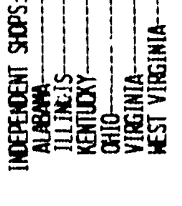 & 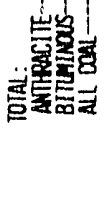 \\
\hline
\end{tabular}




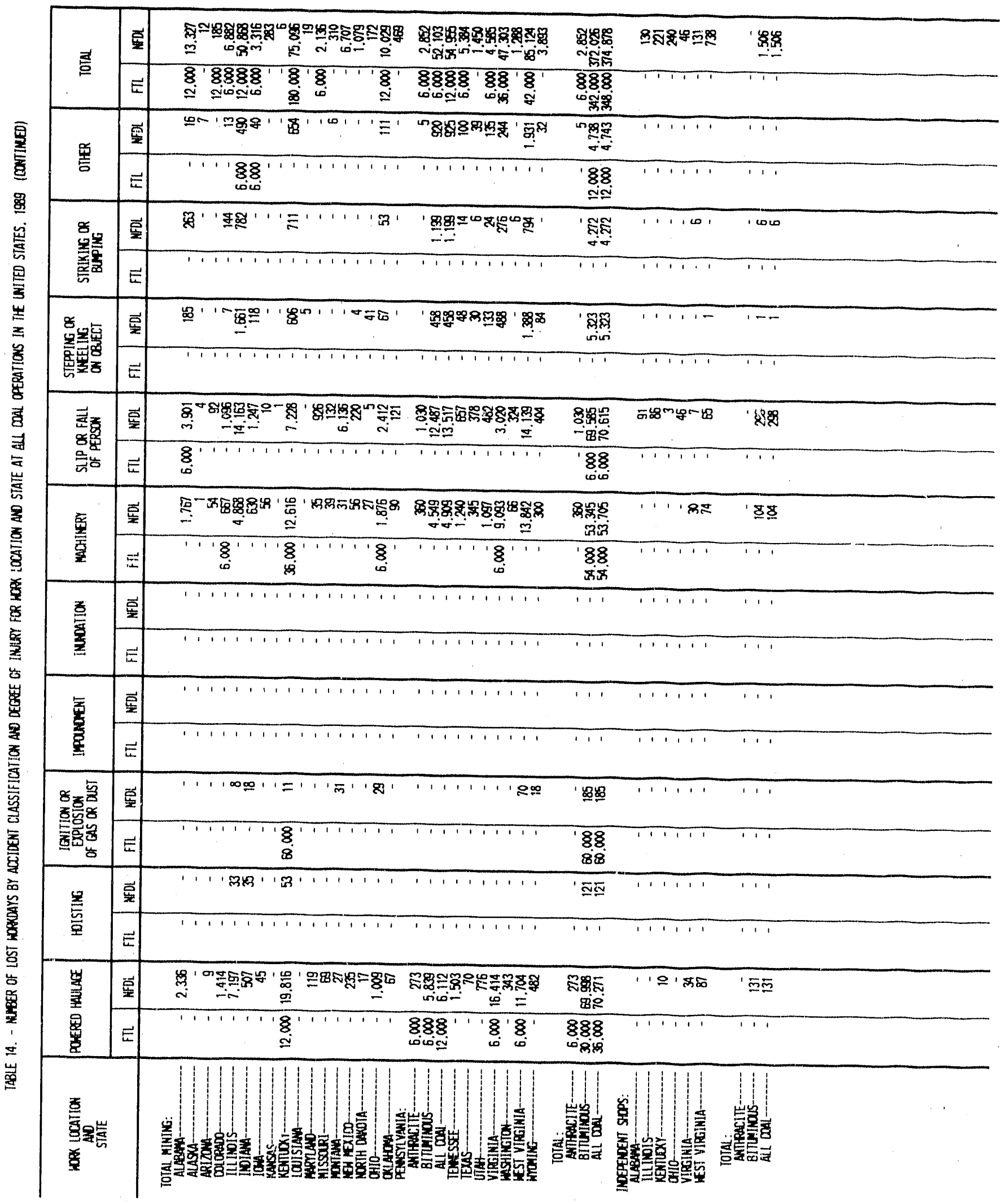




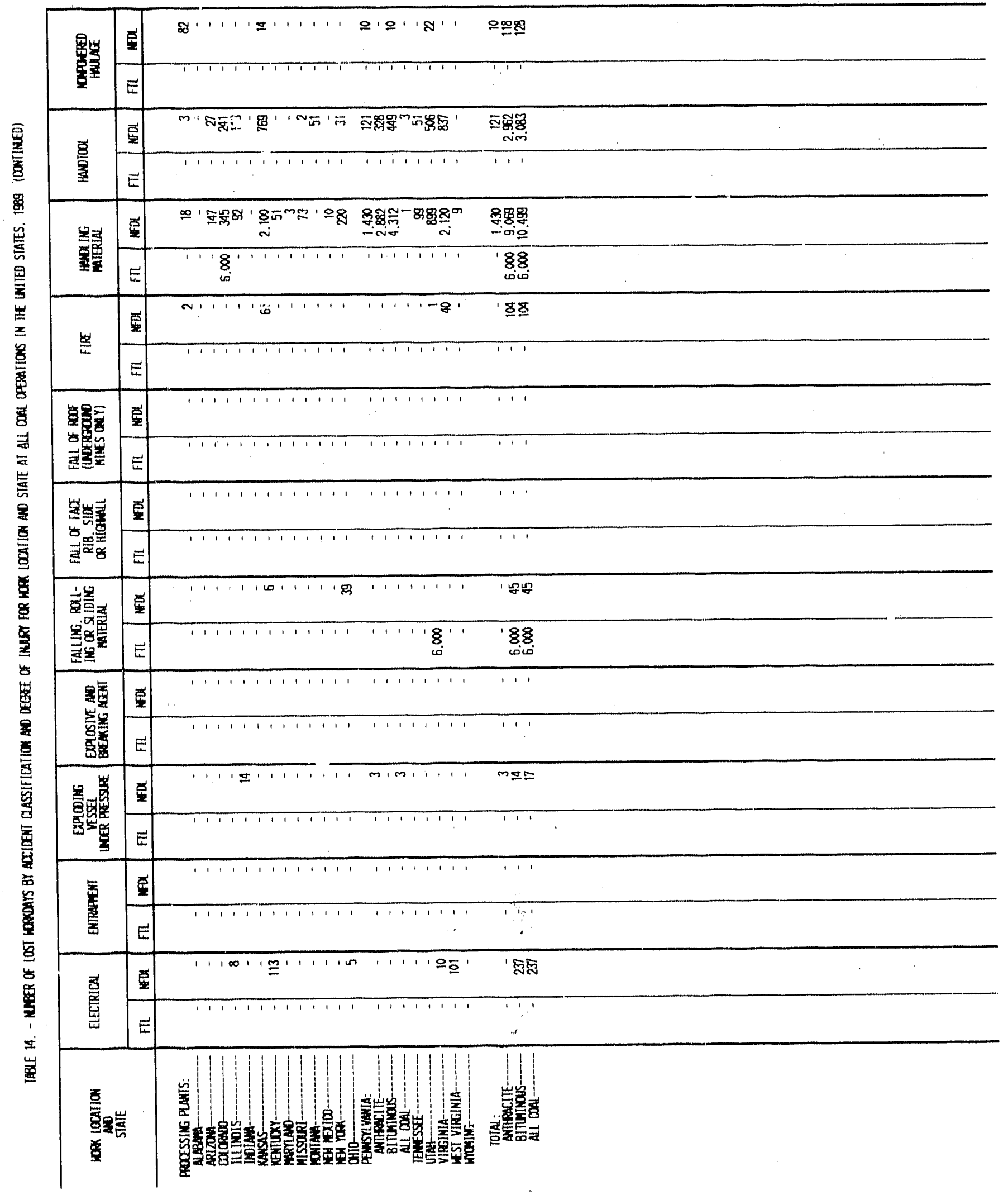




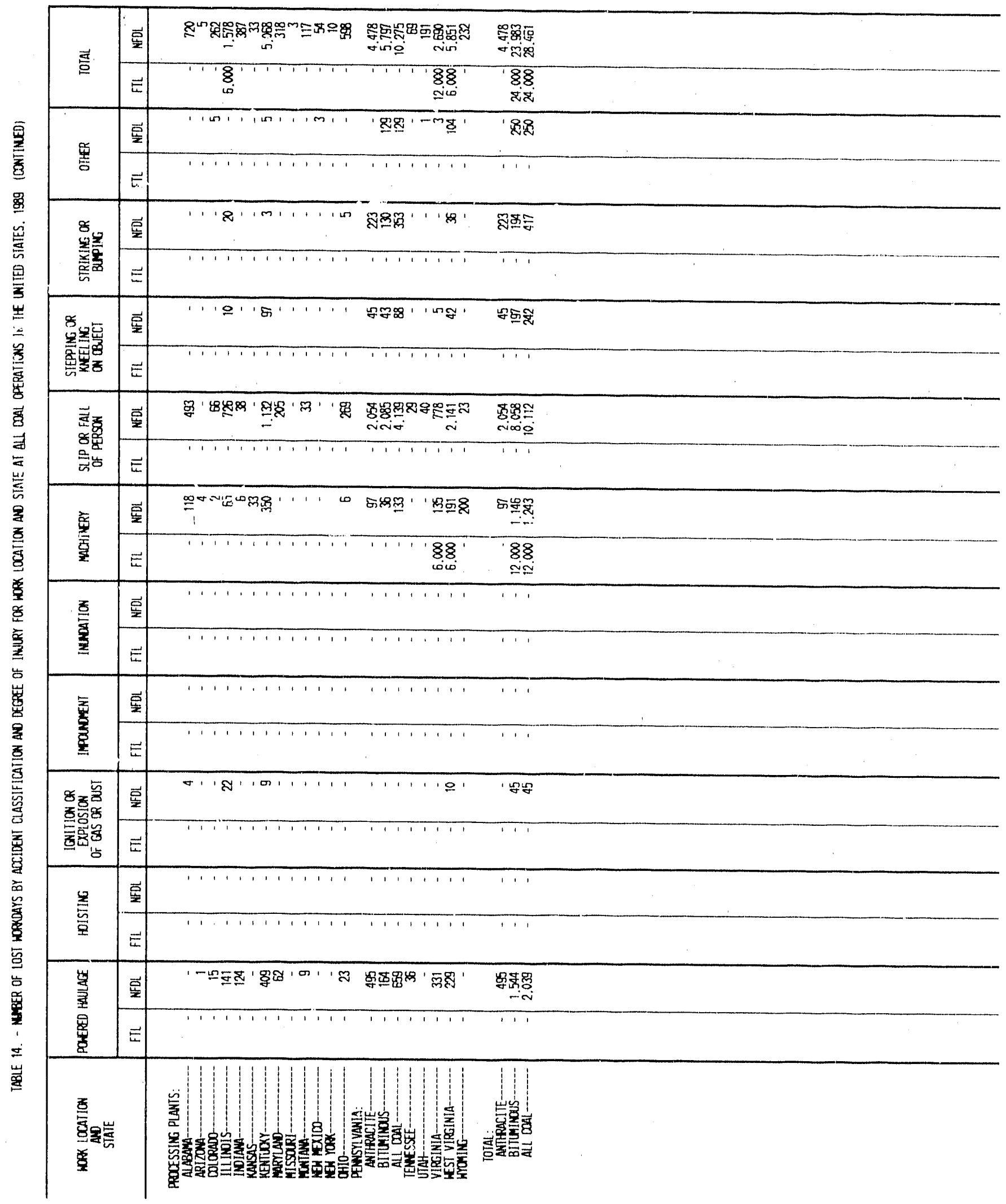




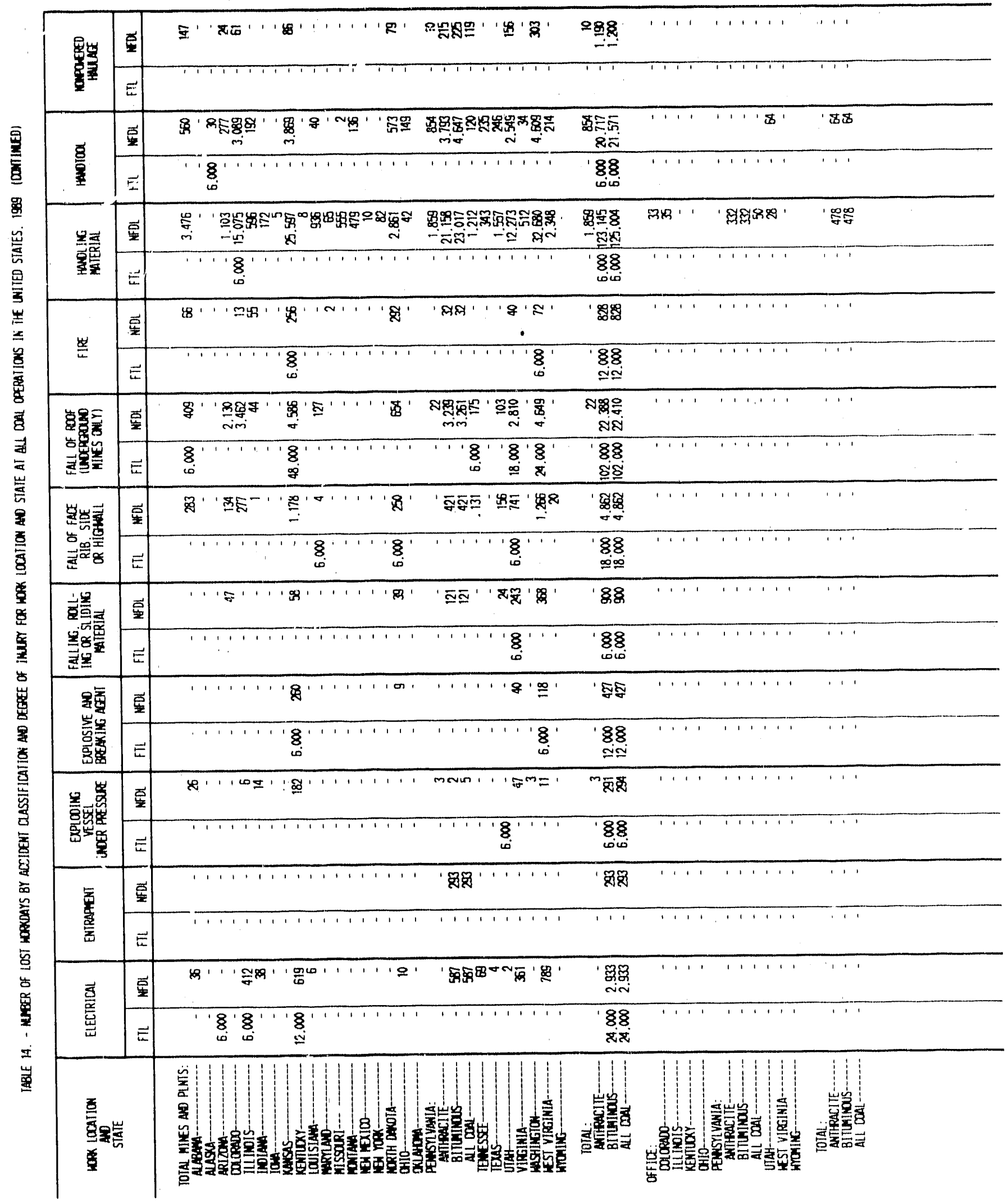




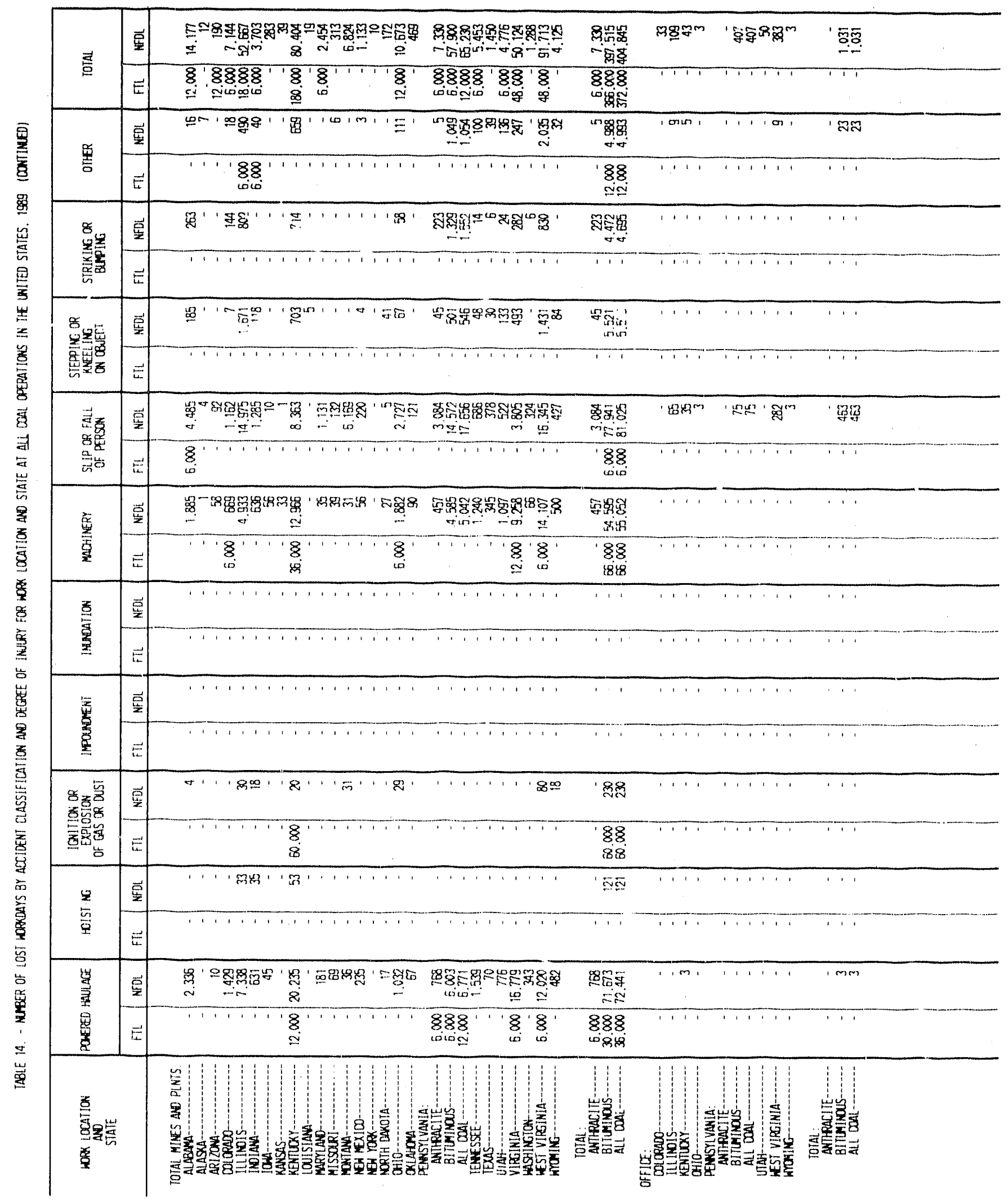




\begin{tabular}{|c|c|c|c|}
\hline \multirow{2}{*}{ 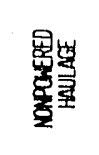 } & 豆 & 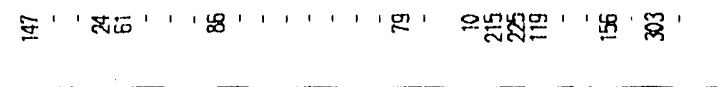 & 으요뭉 \\
\hline & $E$ & 111111 & 111 \\
\hline \multirow{2}{*}{ 吾 } & 高 & 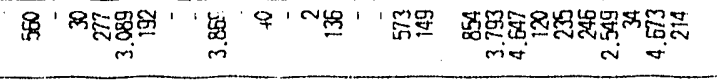 & 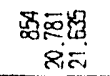 \\
\hline & 란 & 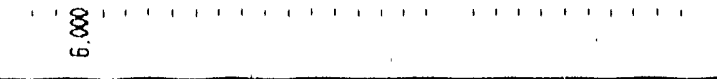 & ' \\
\hline \multirow{2}{*}{ 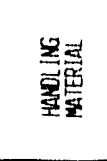 } & 章 & 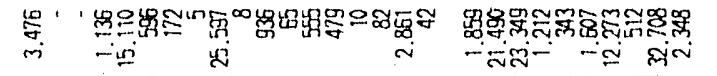 & 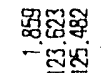 \\
\hline & $E$ & 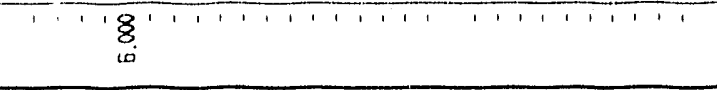 & 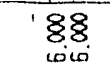 \\
\hline \multirow{2}{*}{ 些 } & 妾 & 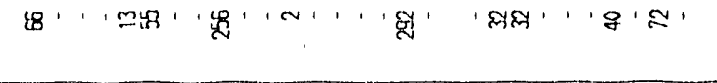 & 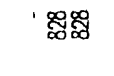 \\
\hline & $\overrightarrow{\mathrm{E}}$ & $1+1+1+1+\underbrace{8}_{6}$ & ن \\
\hline \multirow{2}{*}{ 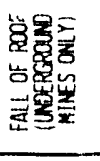 } & 豆 & 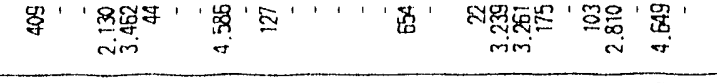 & 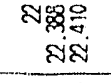 \\
\hline & $\vec{E}$ & $\begin{array}{l}\sum_{i 0}^{\prime} \\
\end{array}$ & $\begin{array}{l}88 \\
\text { s.8 } \\
\text { sid } \\
\end{array}$ \\
\hline \multirow{2}{*}{ 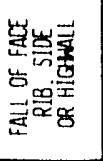 } & 妾 & 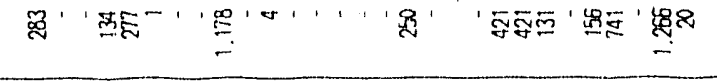 & : :্ঞ: \\
\hline & $\vec{E}$ & $\begin{array}{l}-8 \\
0 \\
0\end{array}$ & 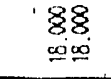 \\
\hline \multirow{2}{*}{ 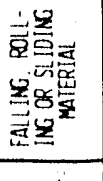 } & $\vec{a}$ & 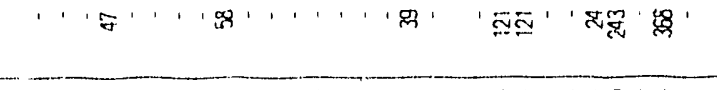 & ৪্ঞ \\
\hline & $\overrightarrow{2}$ & 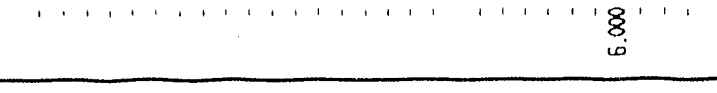 & 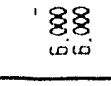 \\
\hline \multirow{2}{*}{ 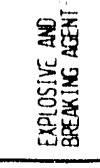 } & 矛 & 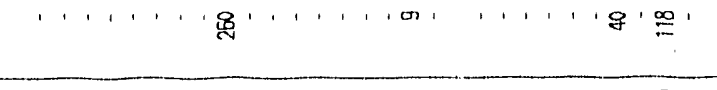 & ร్ูำ \\
\hline & $\ddot{E}$ & $+\underset{10}{8}+\cdots+1+1$ & ن \\
\hline \multirow{2}{*}{ 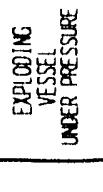 } & 可 & 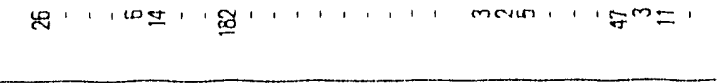 & m市莣 \\
\hline & $E$ & 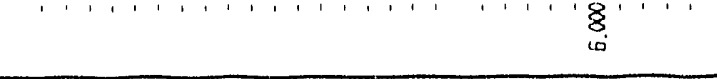 & $\begin{array}{l}\begin{array}{l}88 \\
\text { cio } \\
\text { cio }\end{array} \\
\end{array}$ \\
\hline \multirow{2}{*}{ 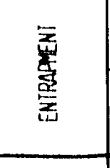 } & 总 & 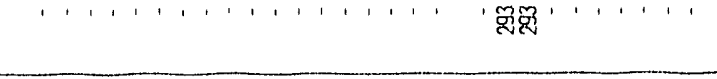 & ' 贸吕 \\
\hline & 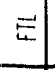 & 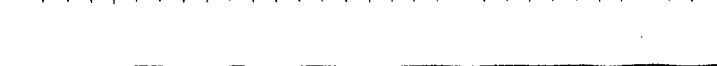 & \\
\hline \multirow{2}{*}{ 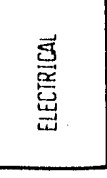 } & 豆 & 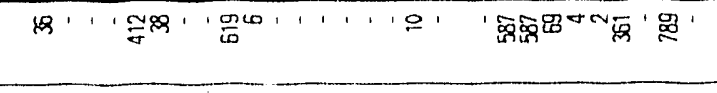 & 兹器 \\
\hline & $\vec{b}$ & 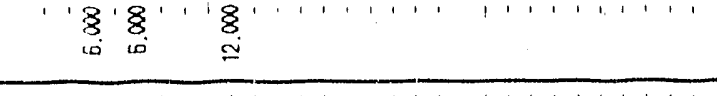 & $\begin{array}{l}88 \\
98+1 \\
\end{array}$ \\
\hline 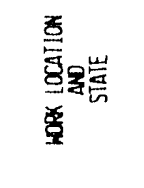 & & 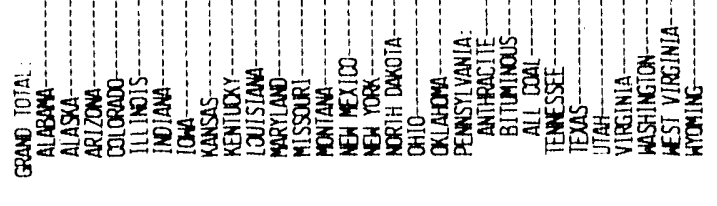 & 峞岁 \\
\hline
\end{tabular}




\begin{tabular}{|c|c|c|c|}
\hline \multirow{2}{*}{$\overrightarrow{5}$} & $\overrightarrow{\underline{Q}}$ & 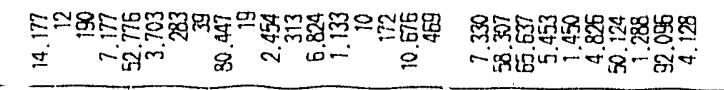 & 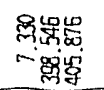 \\
\hline & $\vec{E}$ & 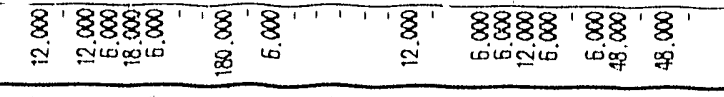 & 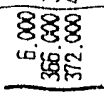 \\
\hline \multirow{2}{*}{ 誉 } & 㝘 & 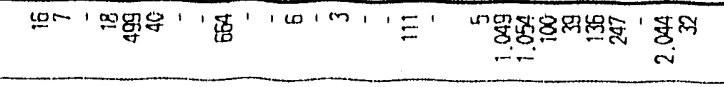 & 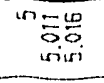 \\
\hline & $E$ & 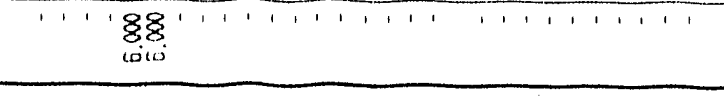 & 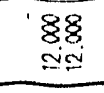 \\
\hline \multirow{2}{*}{ 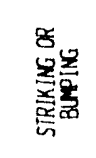 } & 产 & 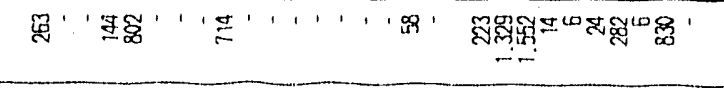 & 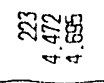 \\
\hline & $E$ & 1111,1111111 & 1.1 \\
\hline \multirow{2}{*}{ 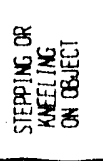 } & $\underline{\underline{\underline{n}}}$ & 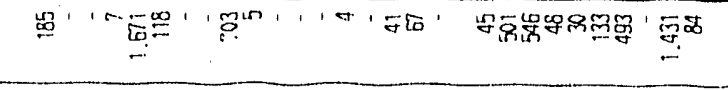 & 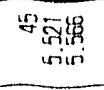 \\
\hline & $\vec{E}$ & . & ' \\
\hline \multirow{2}{*}{ 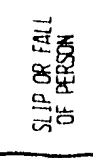 } & 豆 & 参 & 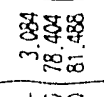 \\
\hline & $E^{\prime \prime}$ & $\begin{array}{l}\boldsymbol{g}_{0} \\
\mathbf{0} \\
\end{array}$ & 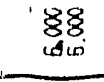 \\
\hline \multirow{2}{*}{ 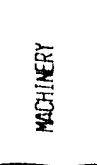 } & 訾 & 将- & 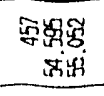 \\
\hline & $\vec{\omega}$ & $\begin{array}{c}\sum_{0}^{\prime} \\
\end{array}$ & $\begin{array}{l}88 \\
88 \\
8818 \\
\end{array}$ \\
\hline \multirow{2}{*}{ 㤣 } & 产 & ' ' & ' \\
\hline & $\vec{E}$ & ' & ' \\
\hline \multirow{2}{*}{ 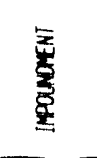 } & 旁 & 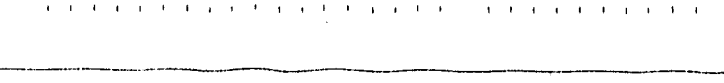 & 1,1 \\
\hline & $\vec{\Delta}$ & $1, \ldots+1, \ldots$ & $\cdots$ \\
\hline \multirow{2}{*}{ 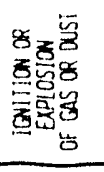 } & $\overrightarrow{0}$ & 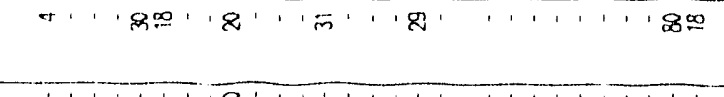 & 多名 \\
\hline & $=$ & $1+1+1,1$ & $\begin{array}{l}88 \\
88 \\
88 \\
\end{array}$ \\
\hline \multirow{2}{*}{$\frac{\text { 몬 }}{\frac{5}{5}}$} & 总 & m吕 ' & ' \\
\hline & $E$ & ' & ' \\
\hline \multirow{2}{*}{ 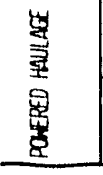 } & 要 & 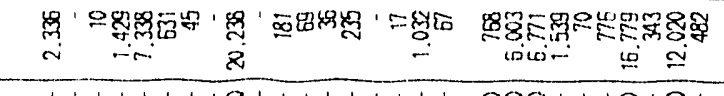 & 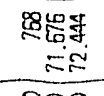 \\
\hline & $\vec{E}$ & 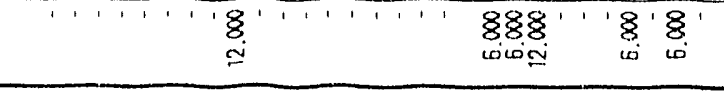 & $\begin{array}{r}888 \\
\text { cosis } \\
\end{array}$ \\
\hline 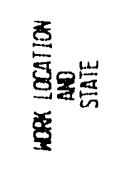 & & 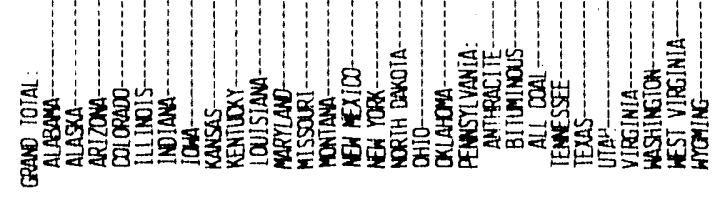 & 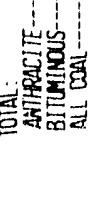 \\
\hline
\end{tabular}




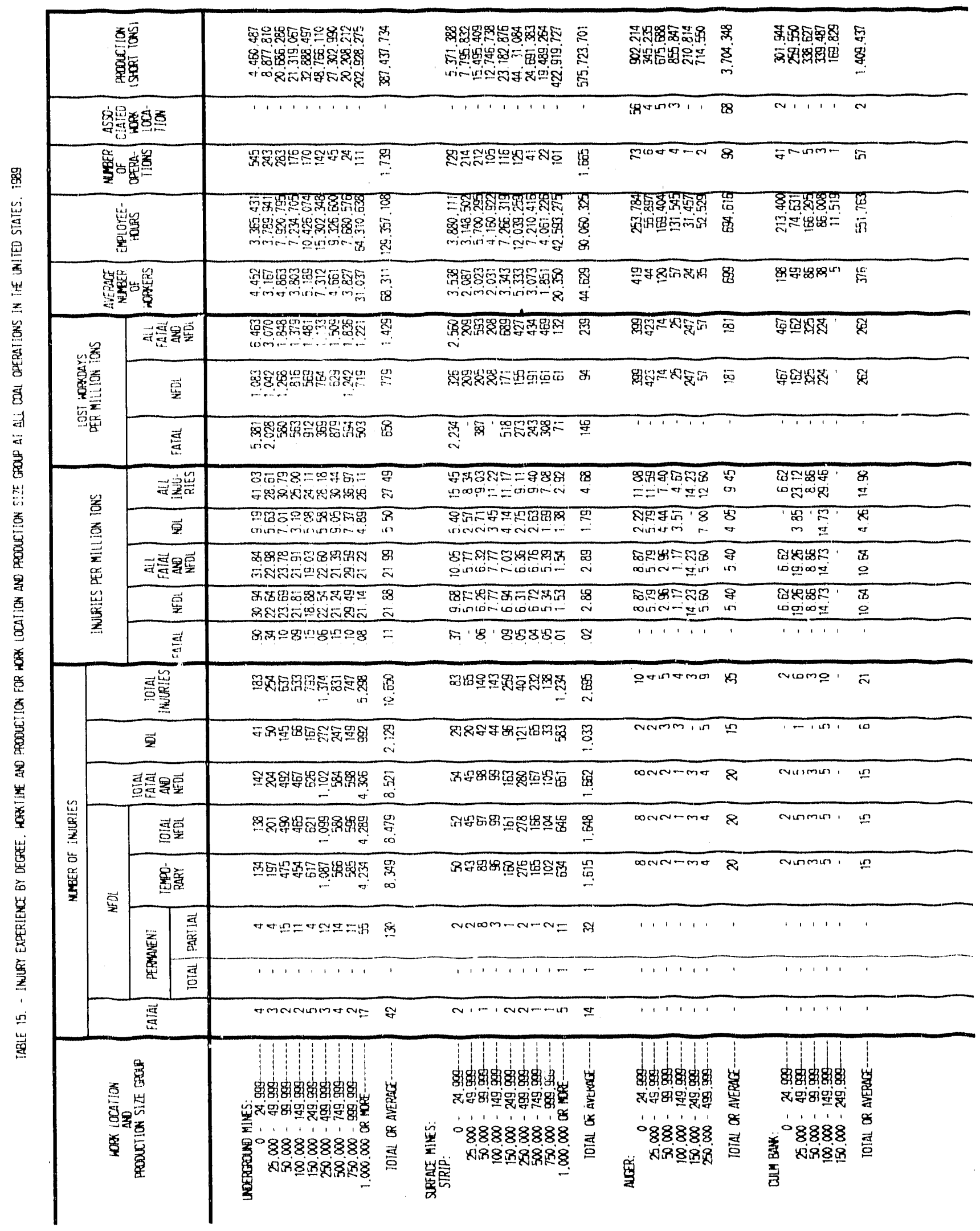




\begin{tabular}{|c|c|c|c|c|c|c|c|c|c|c|c|}
\hline \multicolumn{3}{|c|}{ 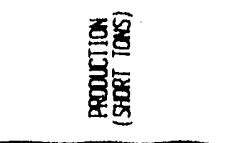 } & 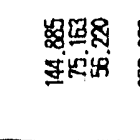 & & 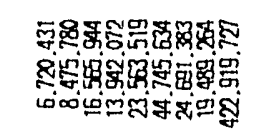 & 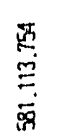 & 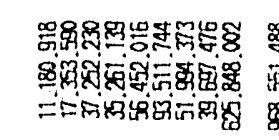 & & & & 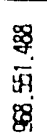 \\
\hline \multicolumn{3}{|c|}{ 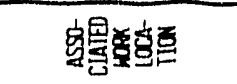 } & 111 & 1 & gotum 1111 & 2 & $\operatorname{sotin} 11,119$ & 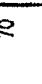 & 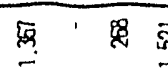 & $\bar{s}$ & 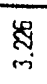 \\
\hline \multicolumn{3}{|c|}{ 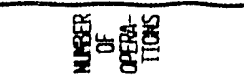 } & ম্ver & 40 & 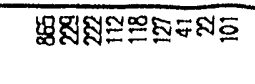 & $\bar{్}$ & 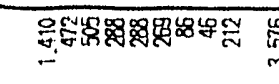 & & $\bar{y}$ bi & श्र & 尊 \\
\hline \multicolumn{3}{|c|}{ 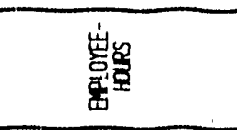 } & 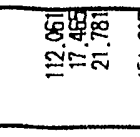 & & 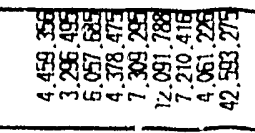 & \begin{tabular}{l}
\multicolumn{5}{c}{} \\
og \\
g. \\
on \\
\end{tabular} & 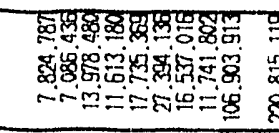 & 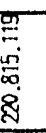 & 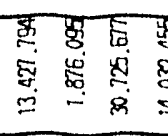 & 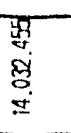 & 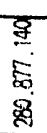 \\
\hline \multicolumn{3}{|c|}{ 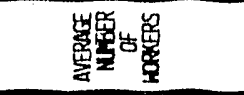 } & $\infty=0$ & $\stackrel{8}{9}$ & 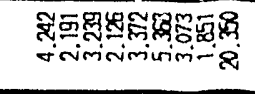 & 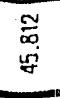 & 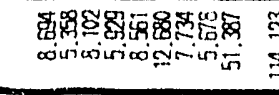 & 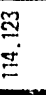 & 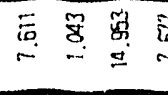 & 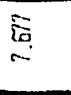 & $\begin{array}{l}\text { 京 } \\
\text { 垔 } \\
\end{array}$ \\
\hline \multirow{3}{*}{ 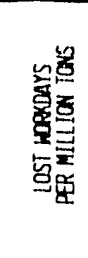 } & 局 & 实要 & & & 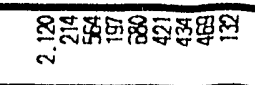 & gृ & 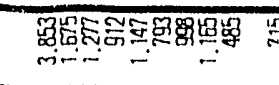 & & & & \\
\hline & & 茜 & ' ' & ' & 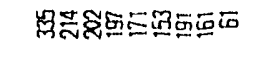 & 尔 & 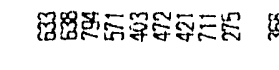 & 禺 & ' ' ' ' & ' & t \\
\hline & & 륜 & 1.1 & 1 & 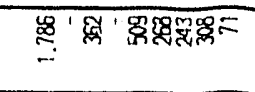 & 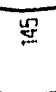 & 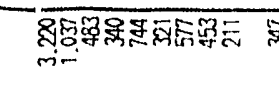 & 突 & & & \\
\hline \multirow{5}{*}{ 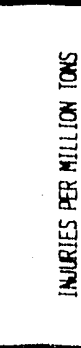 } & \multirow{5}{*}{-} & 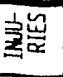 & '兽' & $\underset{m}{q g}$ & 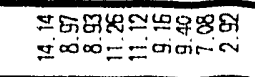 & $\underset{t}{g}$ & 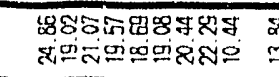 & s. & & & \\
\hline & & 吾 & 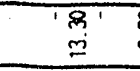 & $\underset{m}{\mathbb{m}}$ & 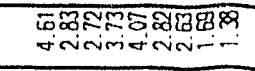 & $\mathscr{- g}$ & 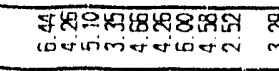 & D্ & & & \\
\hline & & 国要 & 111 & ' & 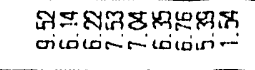 & \& & 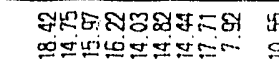 & 幽 & 7 & . & 1 \\
\hline & & 家 & 1.1 & $T$ & 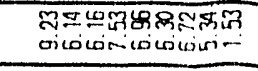 & $\begin{array}{l}8 \\
i\end{array}$ & 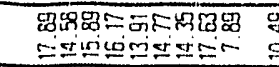 & $\begin{array}{l}\text { gn } \\
\underline{g}\end{array}$ & & $T$ & \\
\hline & & 蛋 & 1, & ' & ' 14.88545 & 5 & $x=94898988$ & 8. & ' & . & \\
\hline \multirow{8}{*}{ 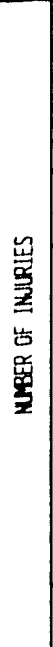 } & \multicolumn{2}{|c|}{ 䞤裳 } & $1-1$ & - & 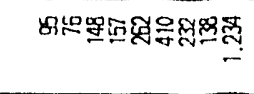 & 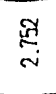 & 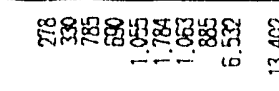 & $\underset{\mathfrak{g}}{\stackrel{q}{m}}$ & 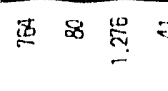 & $\bar{r}$ & 息 \\
\hline & \multicolumn{2}{|l|}{$\underline{\underline{T}}$} & $1-1$ & - & 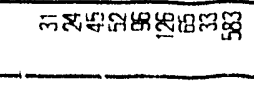 & 㧝 & 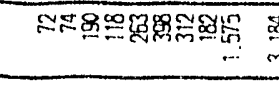 & 苾 & 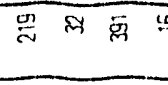 & $\underline{10}$ & 芦 \\
\hline & \multicolumn{2}{|c|}{ 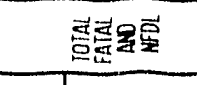 } & $\cdots$ & ' & 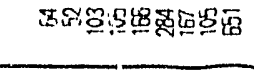 & 舫 & 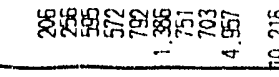 & 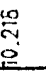 & 莒早最 & $x$ & $\ddot{v}$ \\
\hline & \multicolumn{2}{|c|}{ 彭戛 } & $\cdots$ & ' & 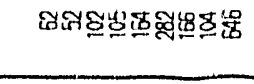 & 孚 & 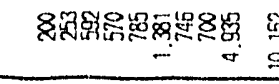 & 㴔 & 第票要 & W & $\stackrel{9: 9.9}{=}$ \\
\hline & \multicolumn{2}{|c|}{ 䔡憘 } & 11. & ' & 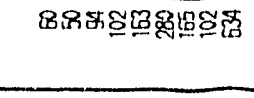 & 8 & 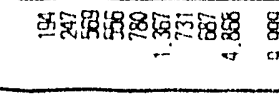 & 思 & 悉 98 & $\bar{x}$ & 要 \\
\hline & 蛋 & \begin{tabular}{l}
\multirow{3}{*}{} \\
音 \\
\end{tabular} & $\cdots$ & ' & Nonom-N-CVE & હ્ભ & 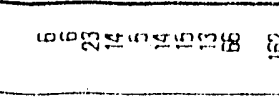 & 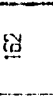 & 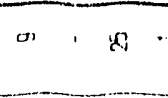 & $\cdots$ & $\underline{\underline{2}}$ \\
\hline & & 홀 & $\cdots$ & i & $\cdots, 1$, & - & $\ldots \ldots \ldots$ & - & 1. & ! & $\cdots$ \\
\hline & \multicolumn{2}{|l|}{ 䃾 } & $\cdots$ & ' & wi- I eventum & $\underline{\underline{x}}$ & commonnurnmey if & $\mathbb{R}$ & 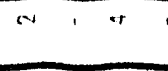 & ' & 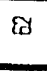 \\
\hline \multicolumn{3}{|c|}{ 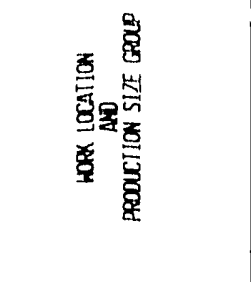 } & 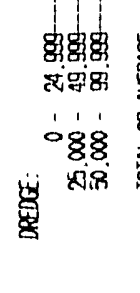 & 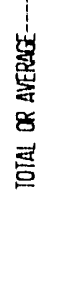 & 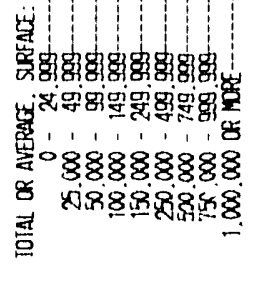 & 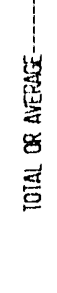 & 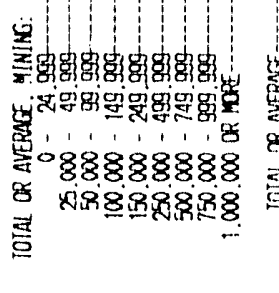 & 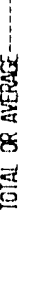 & 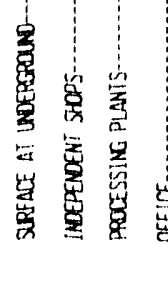 & 焉 & 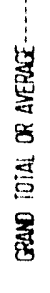 \\
\hline
\end{tabular}




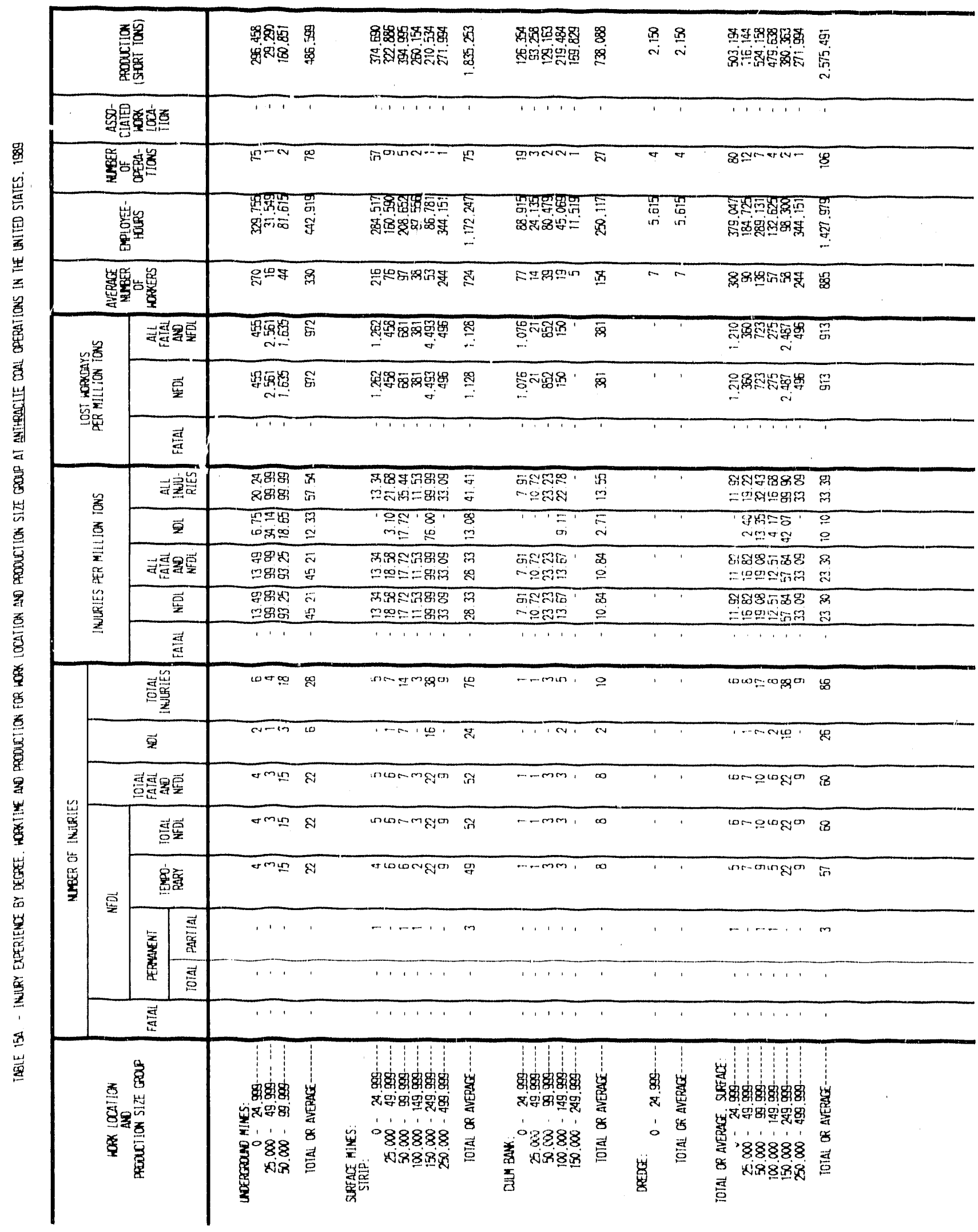




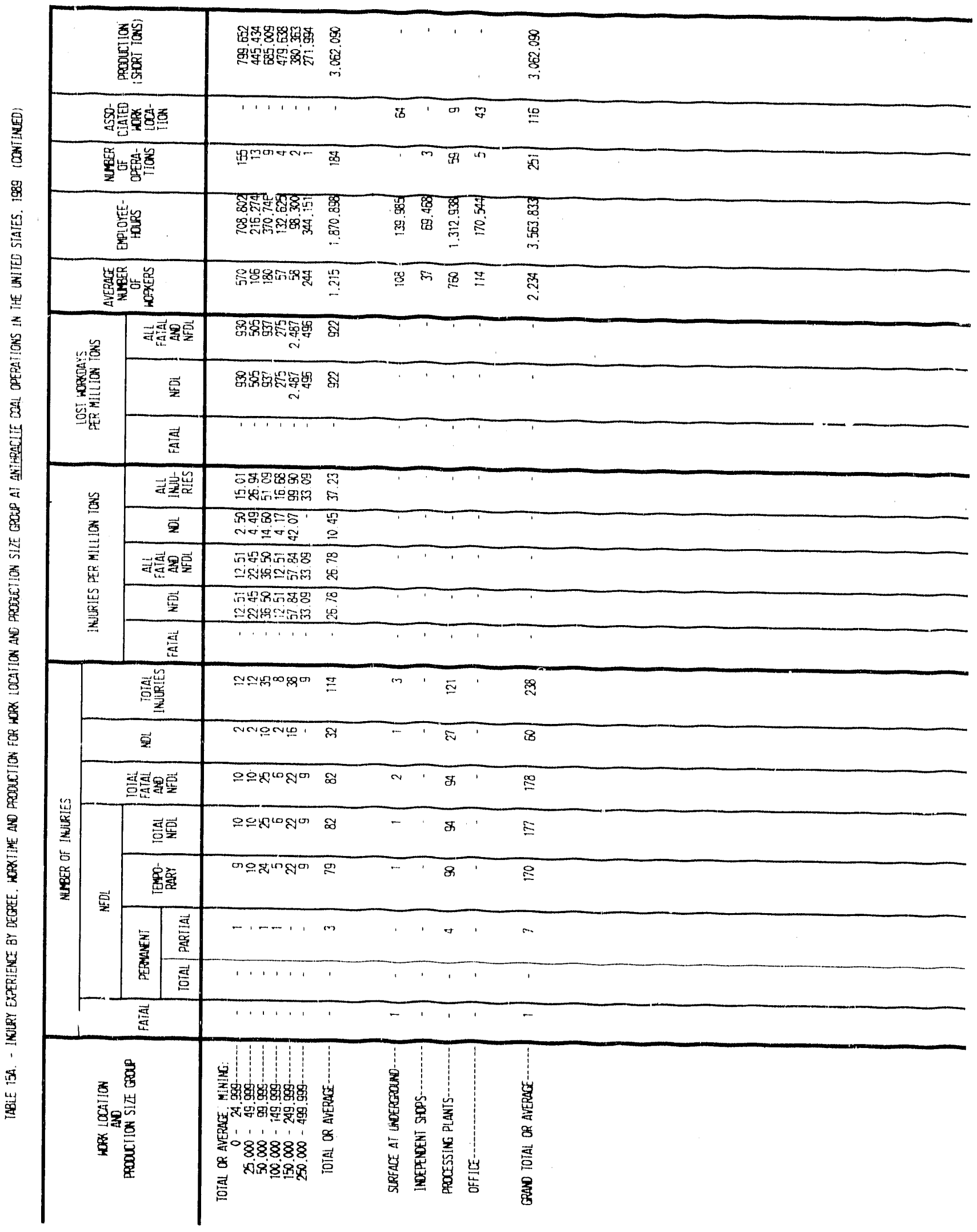




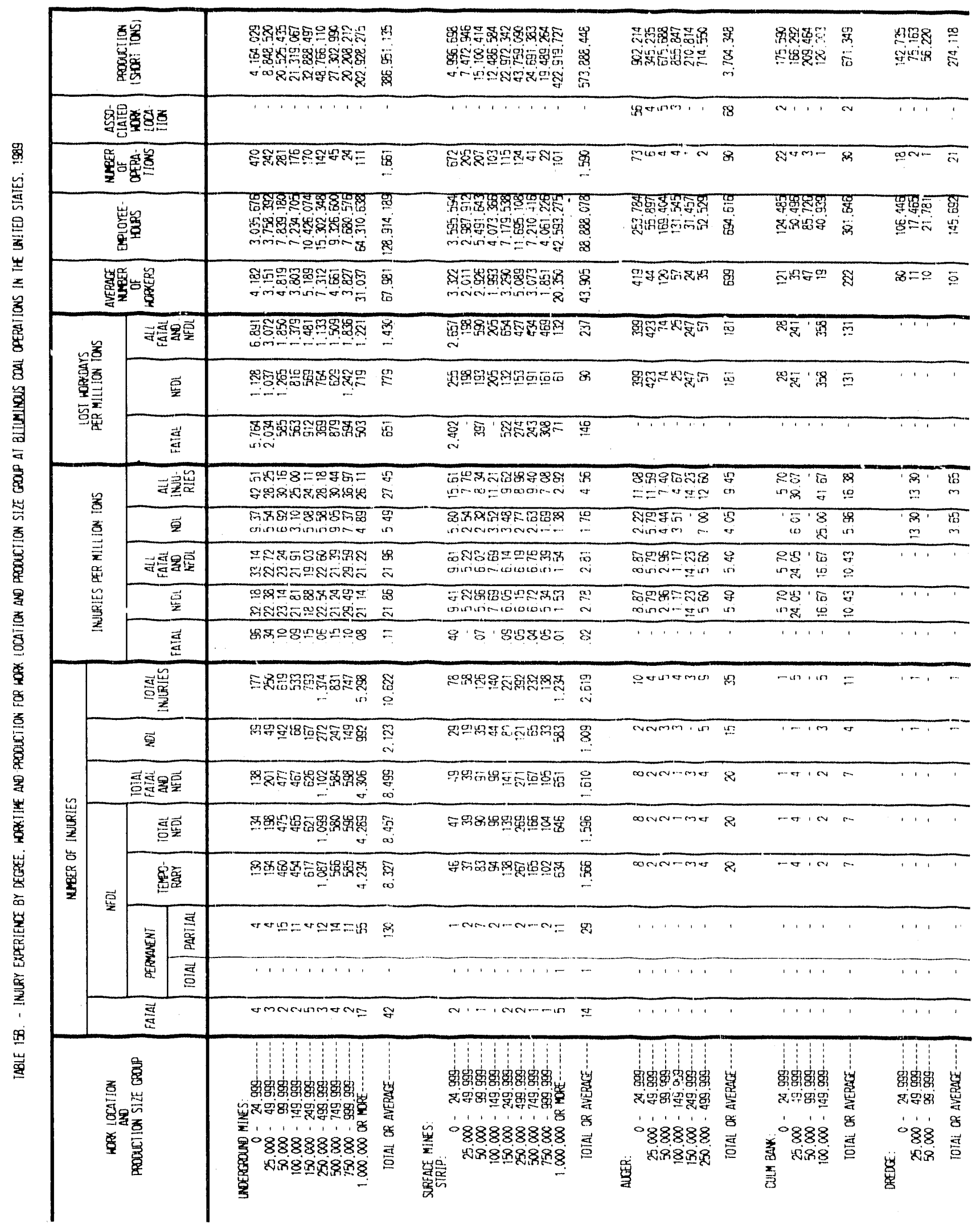




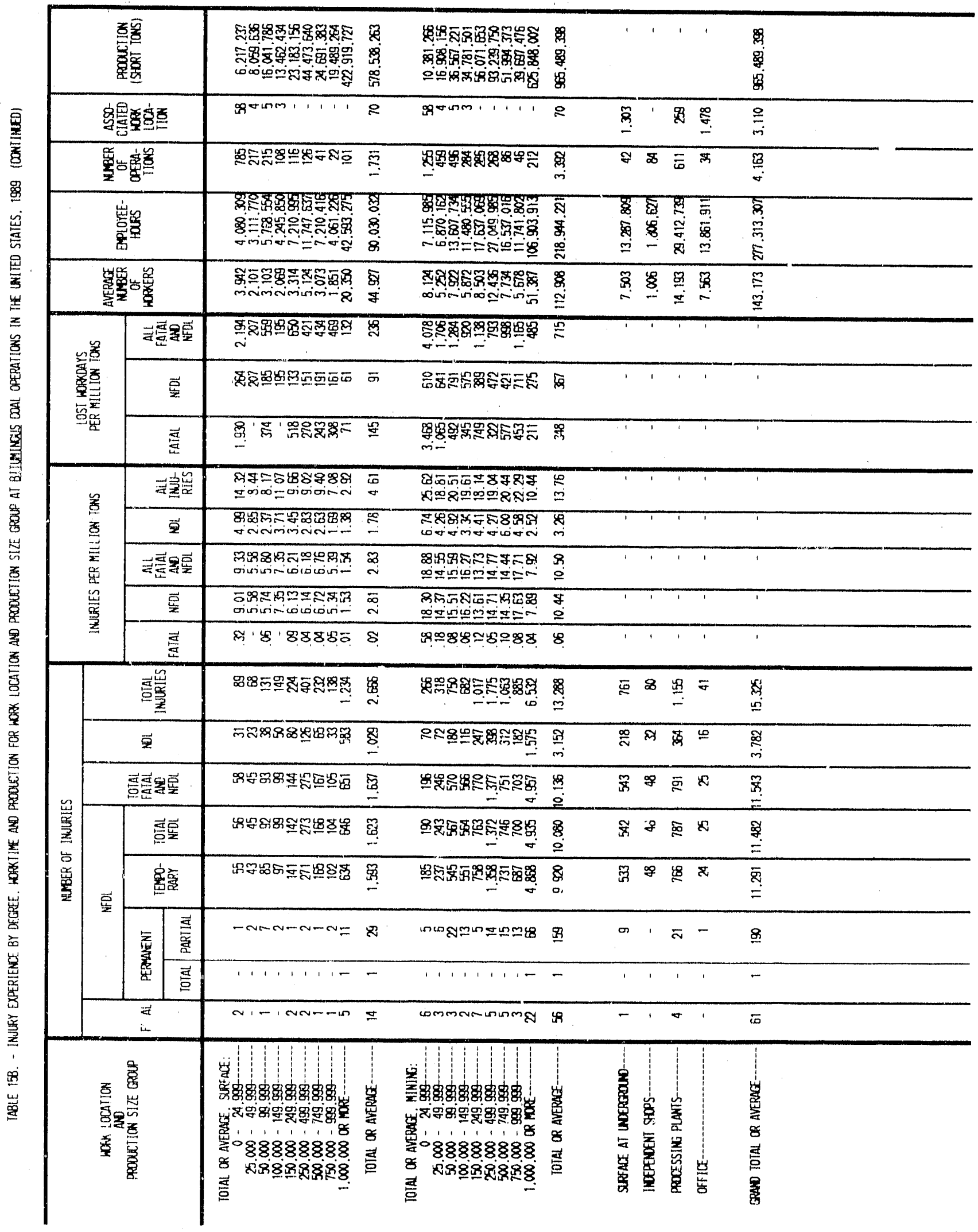




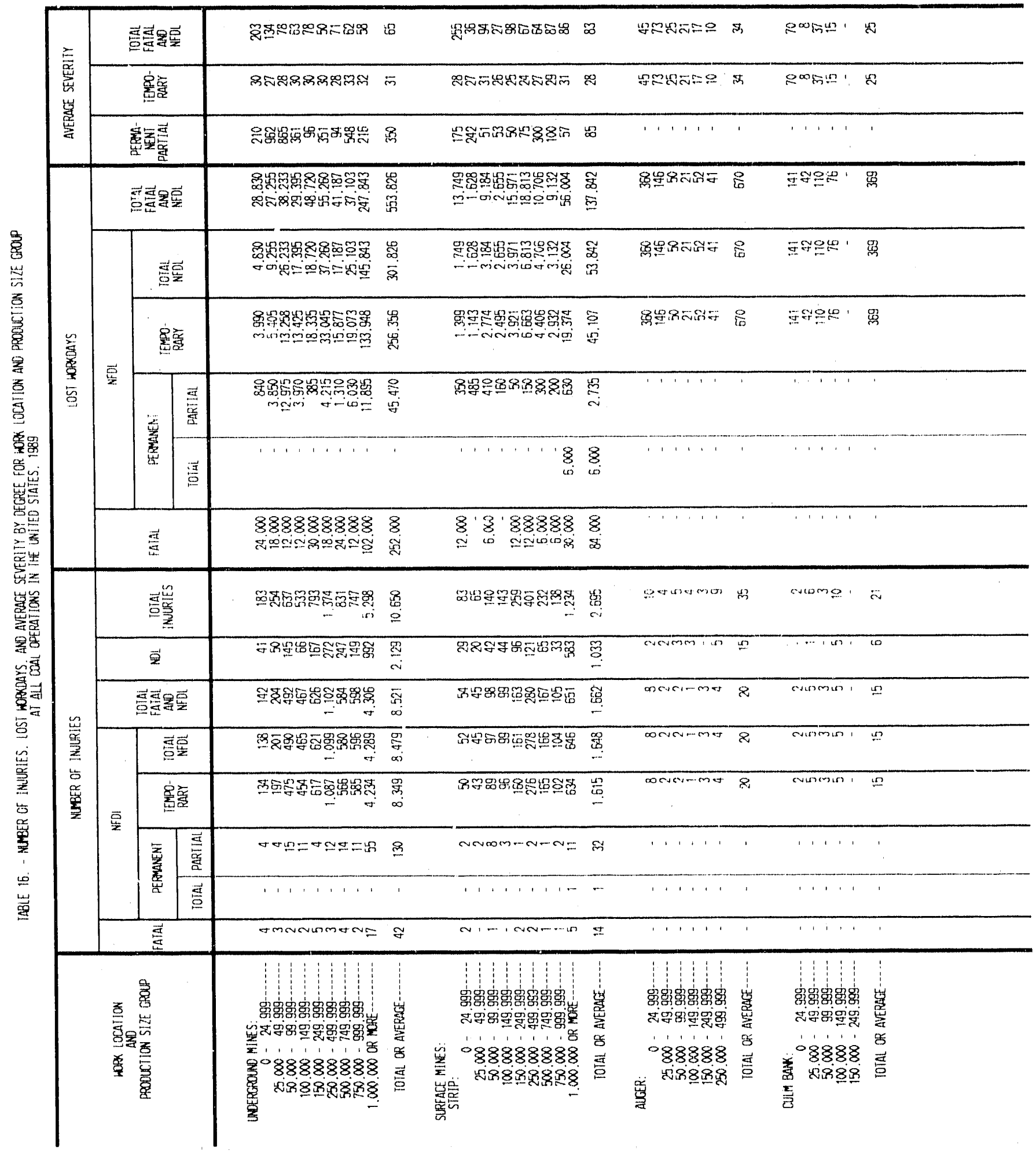




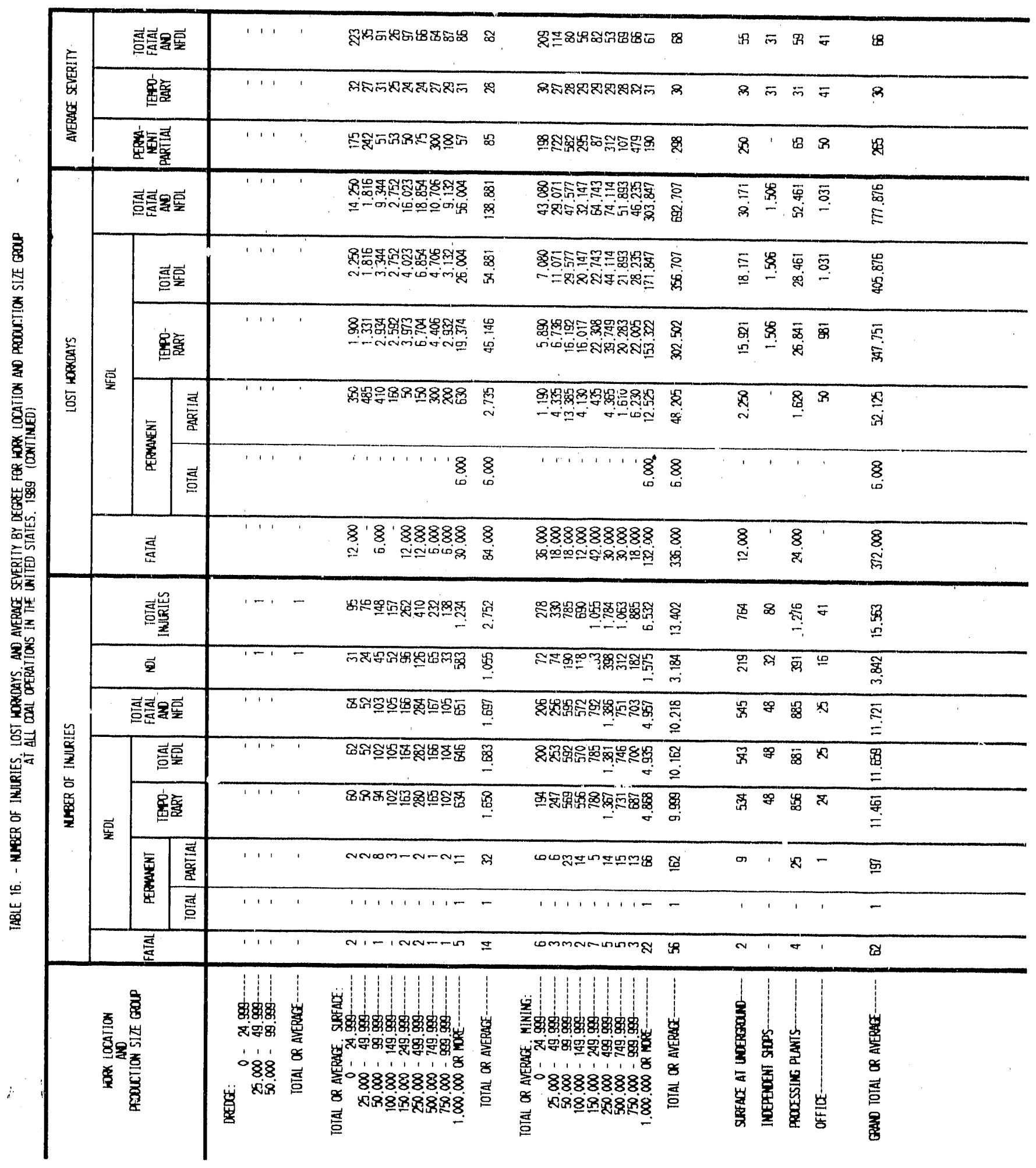




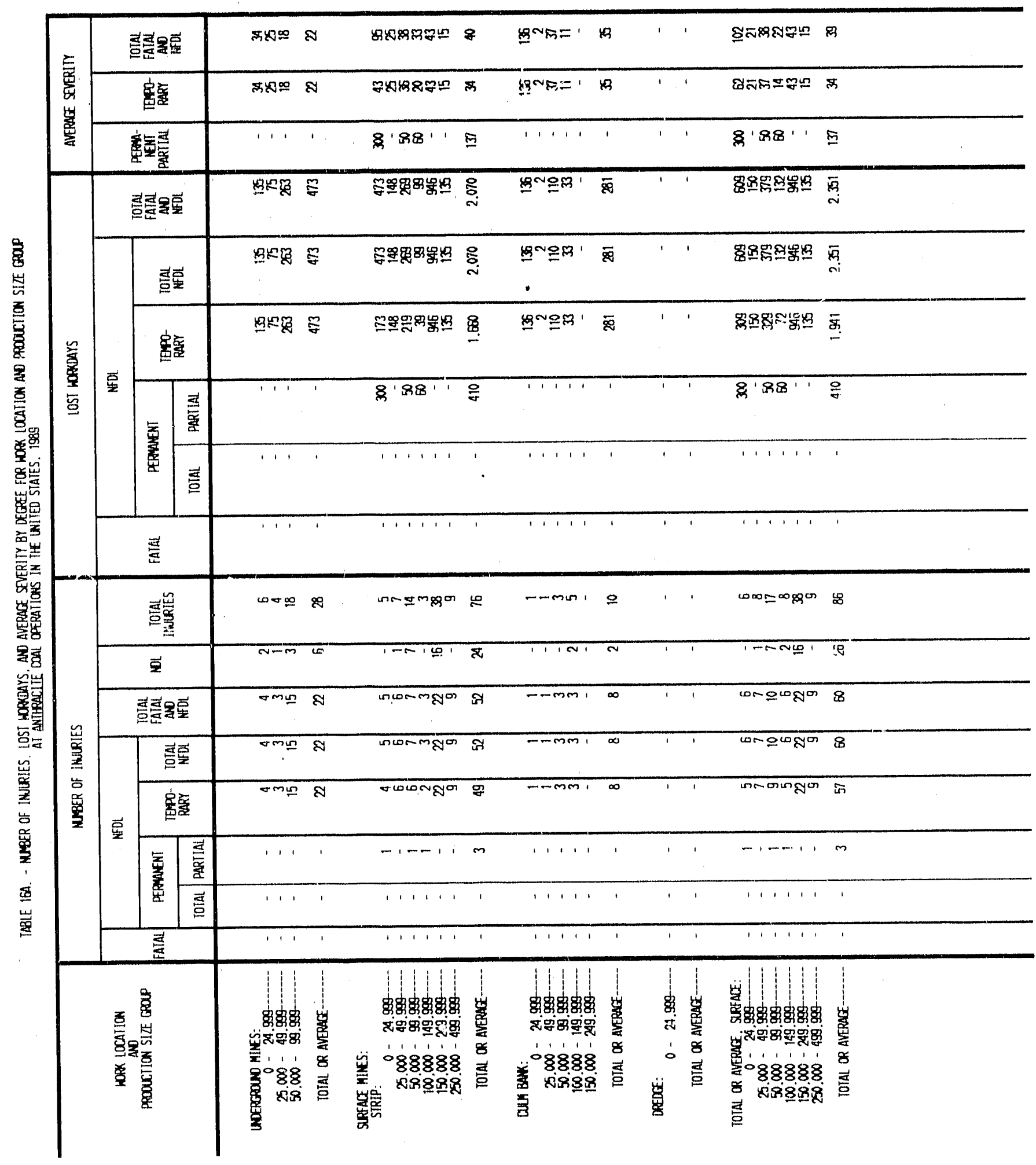




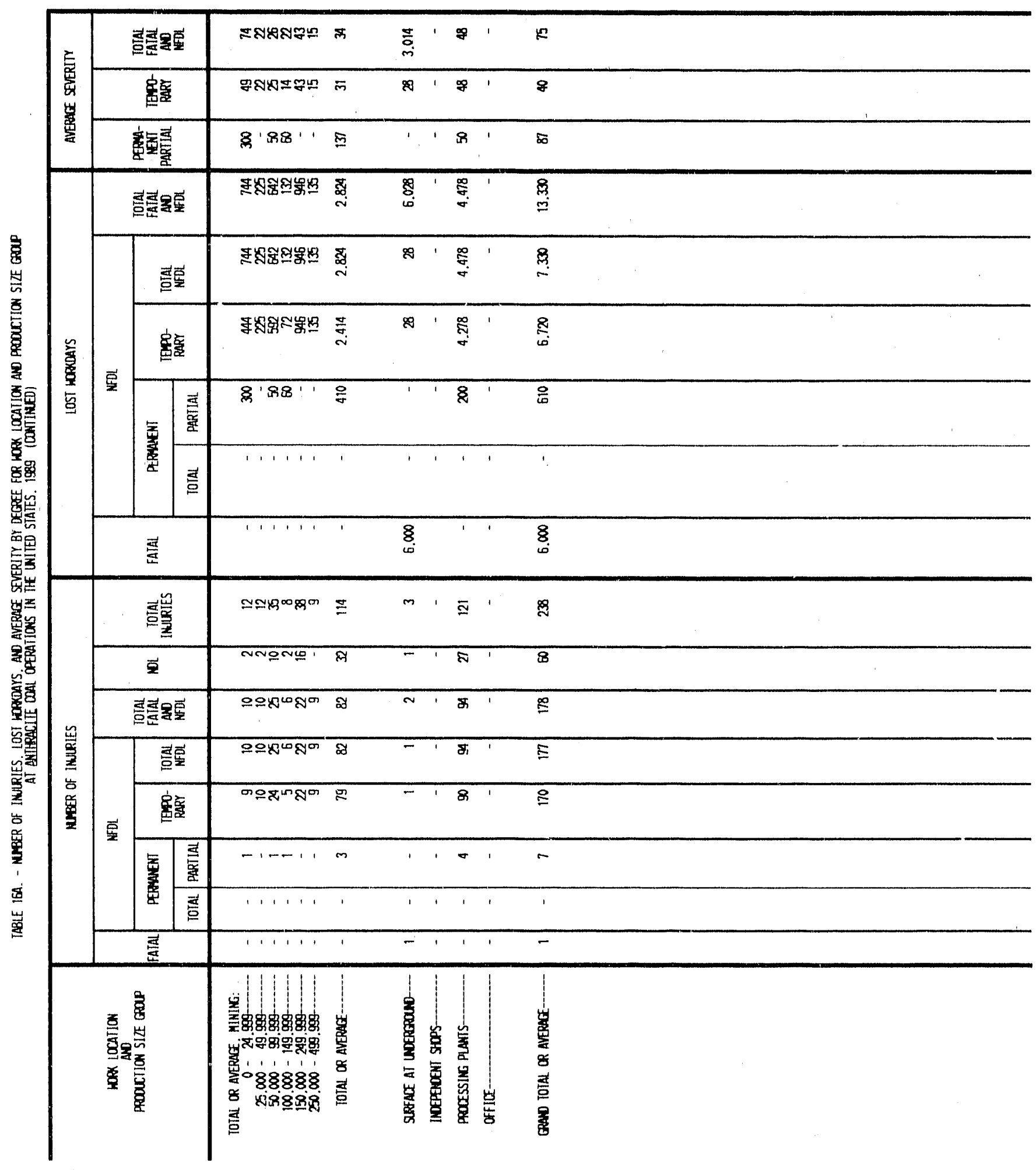




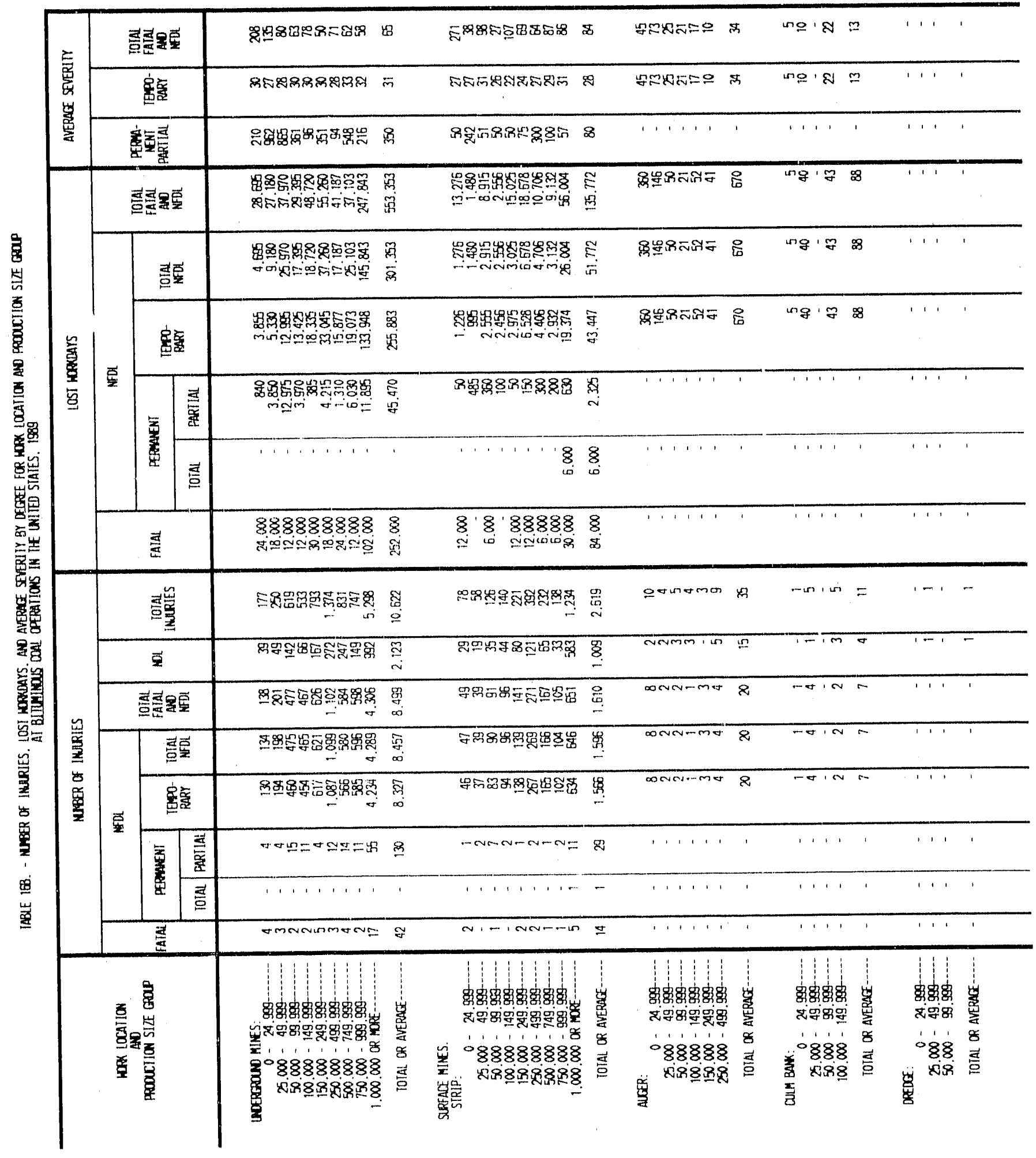




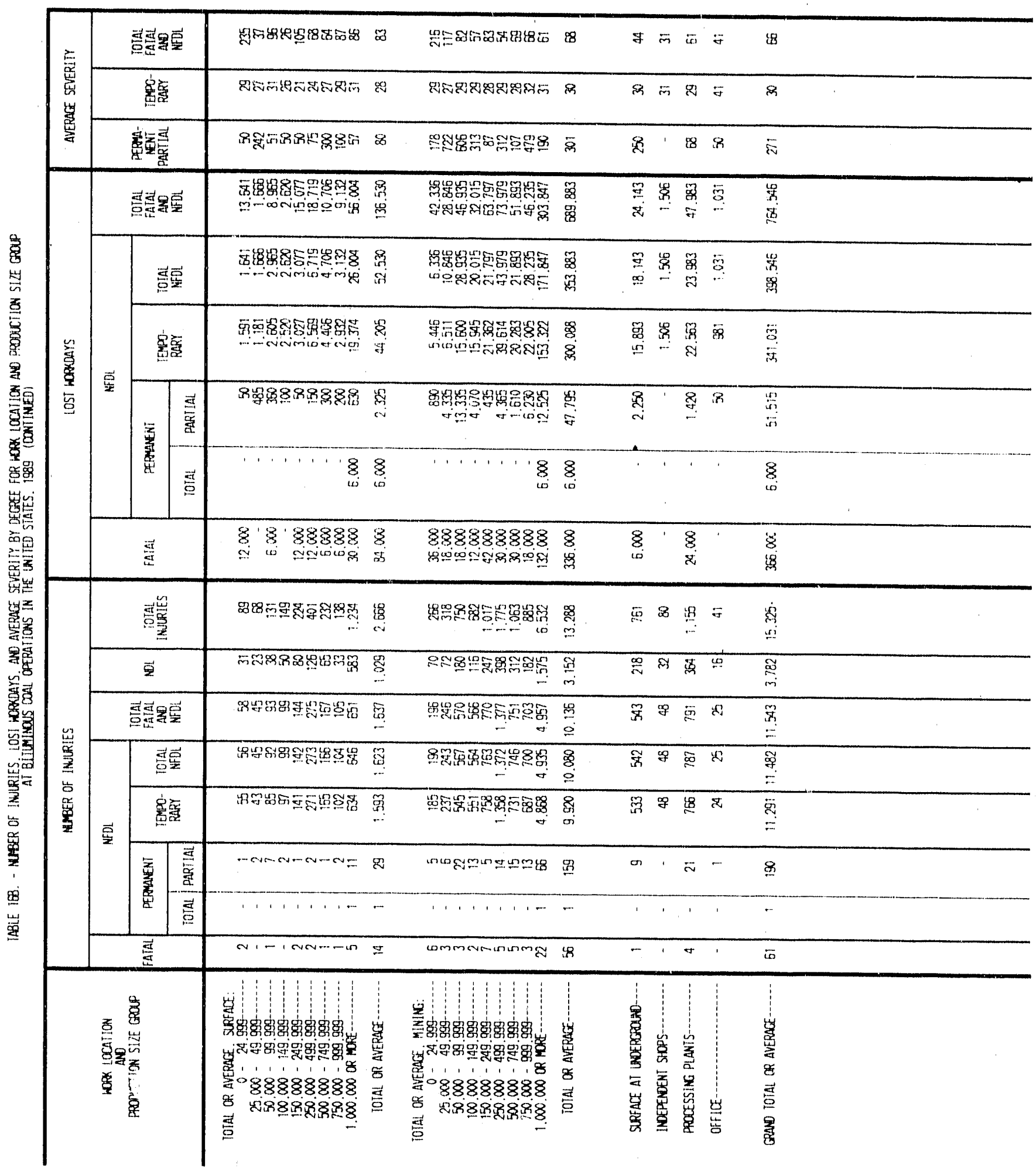




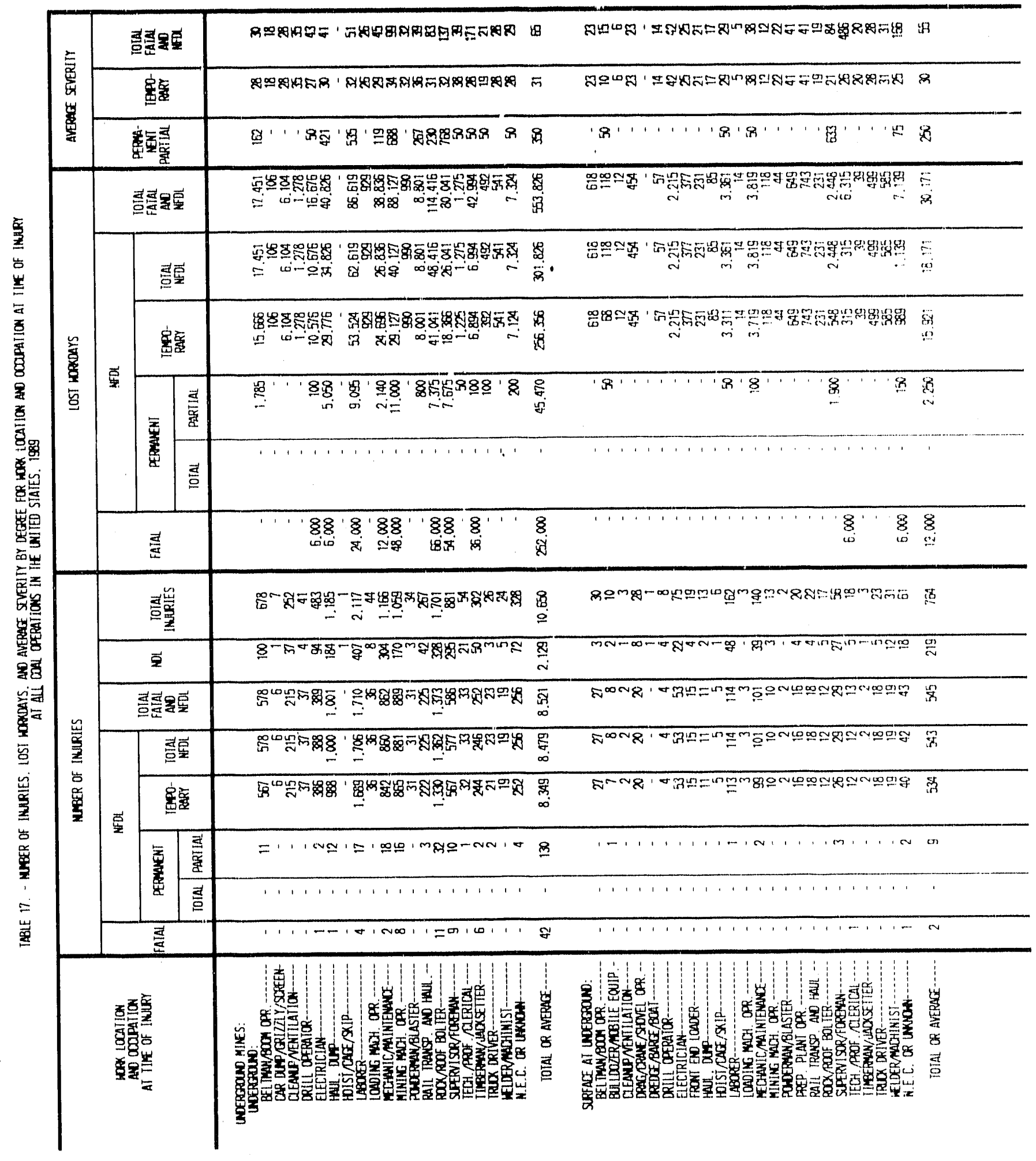




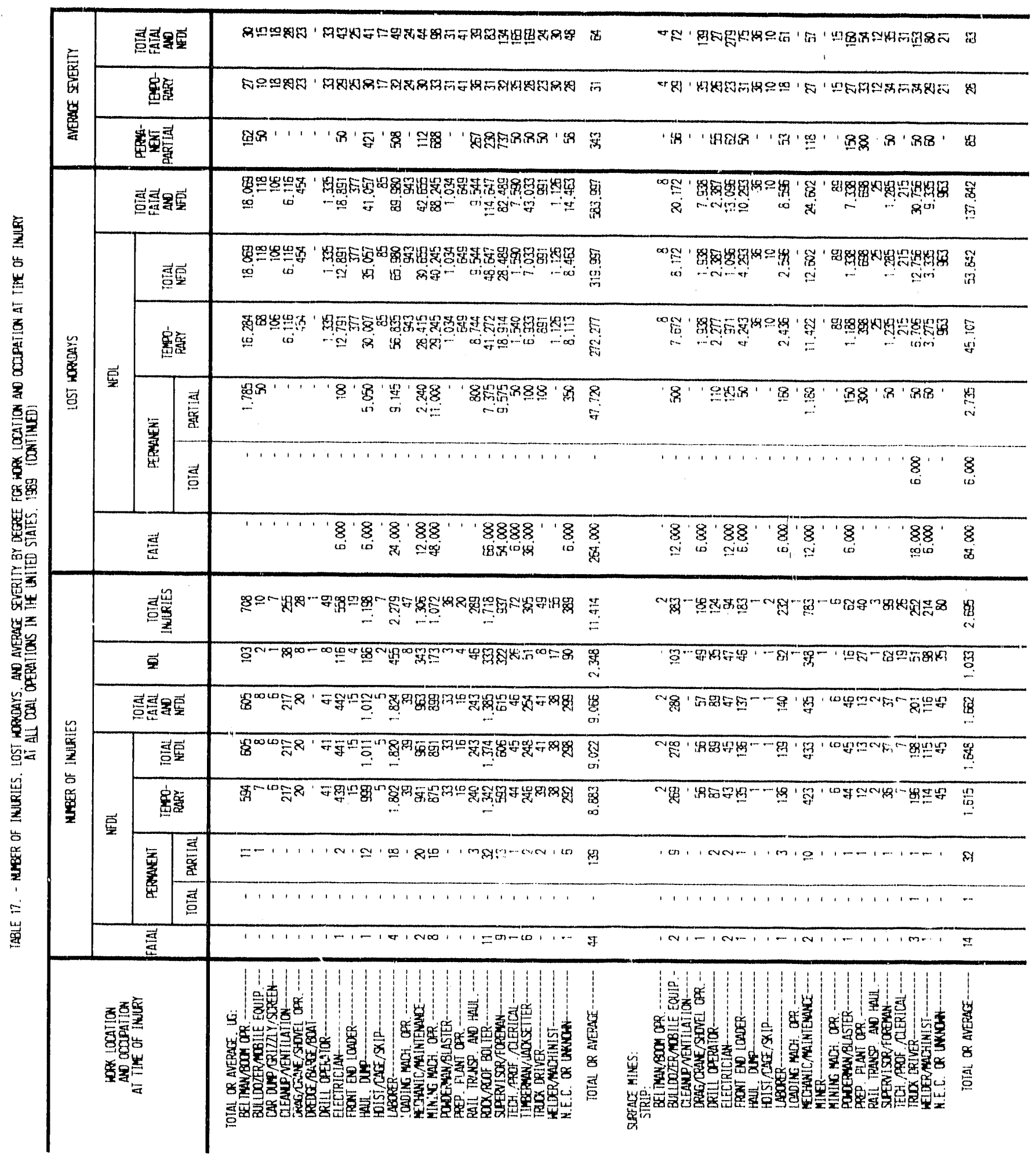




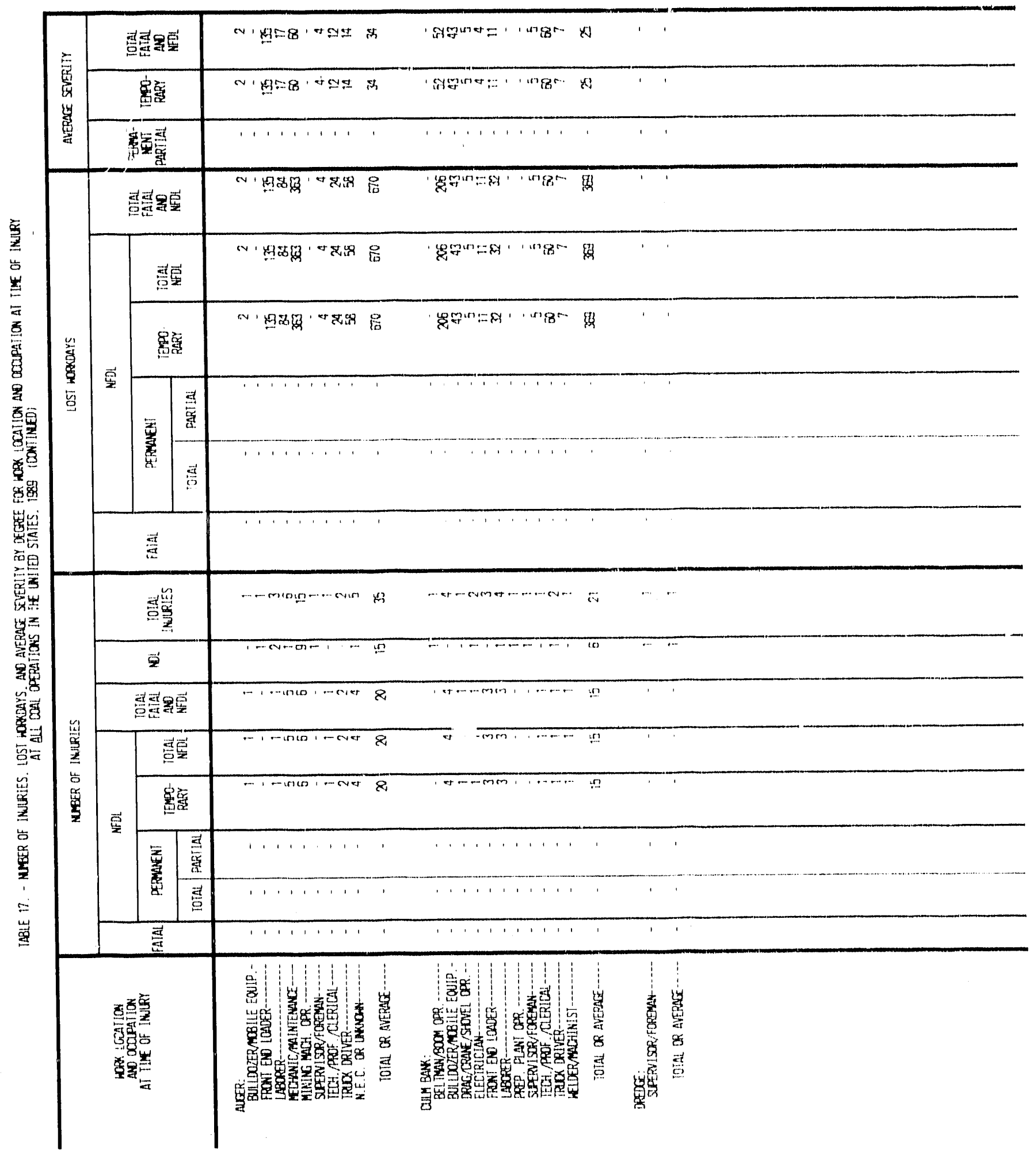




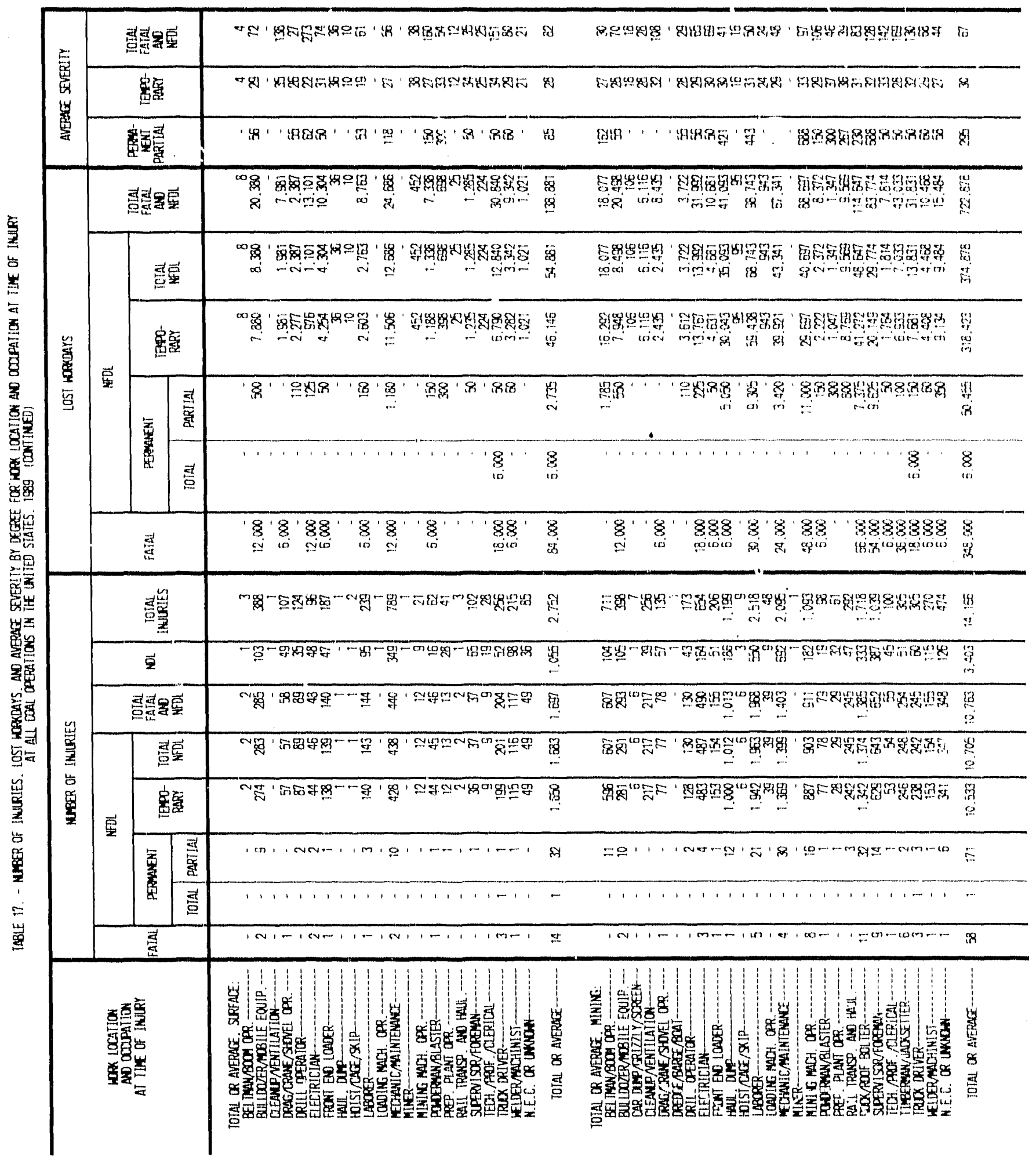




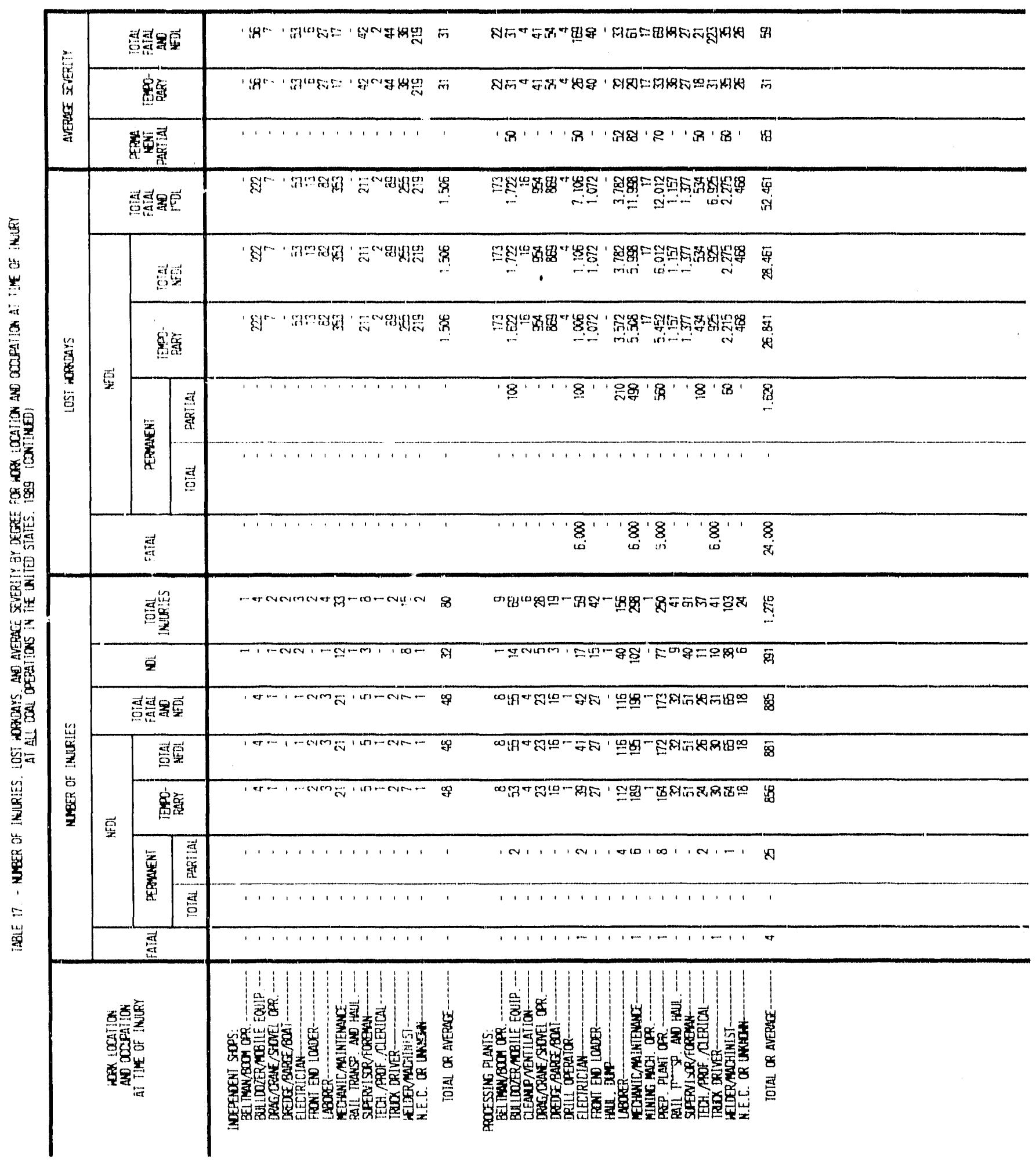




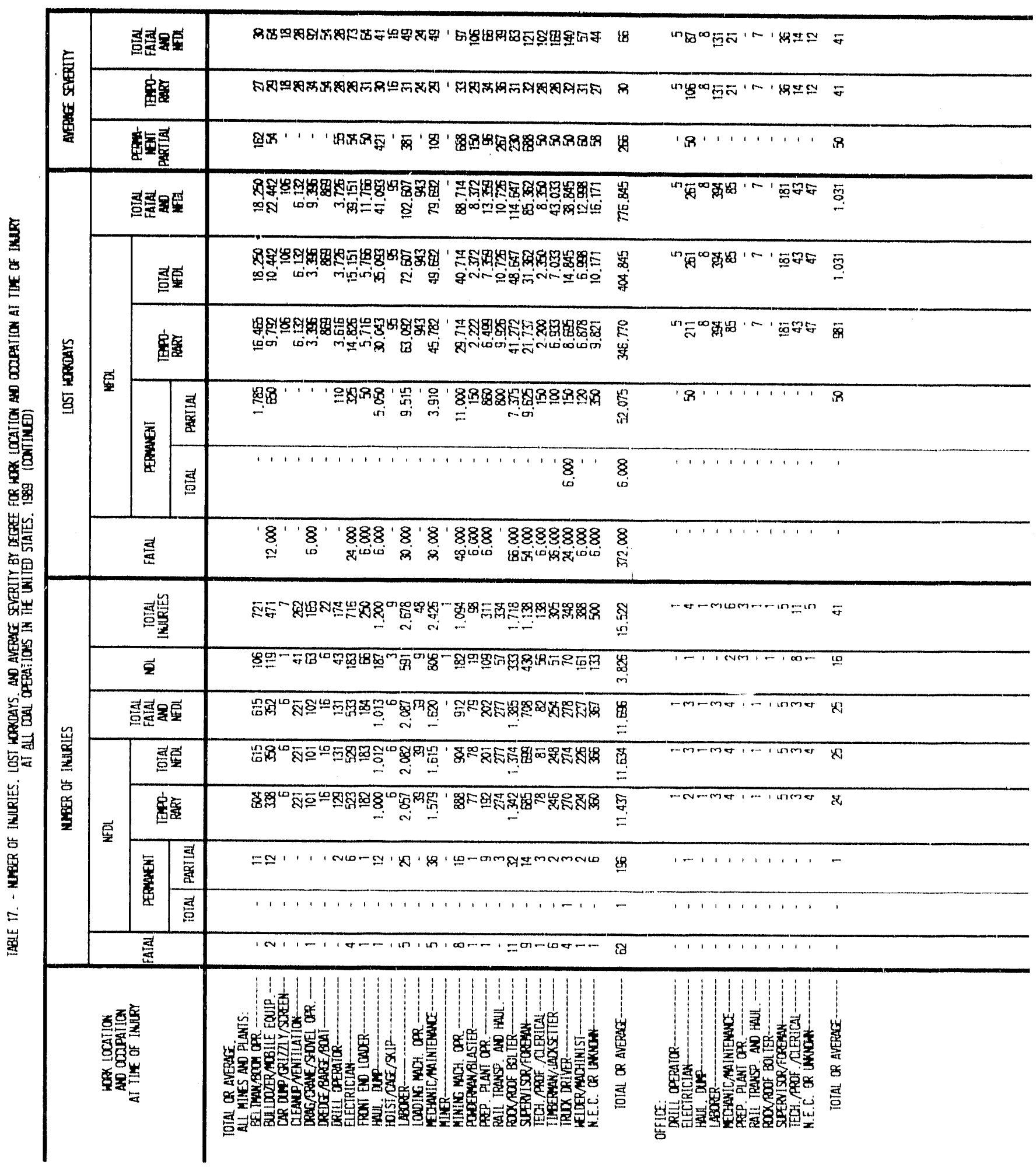




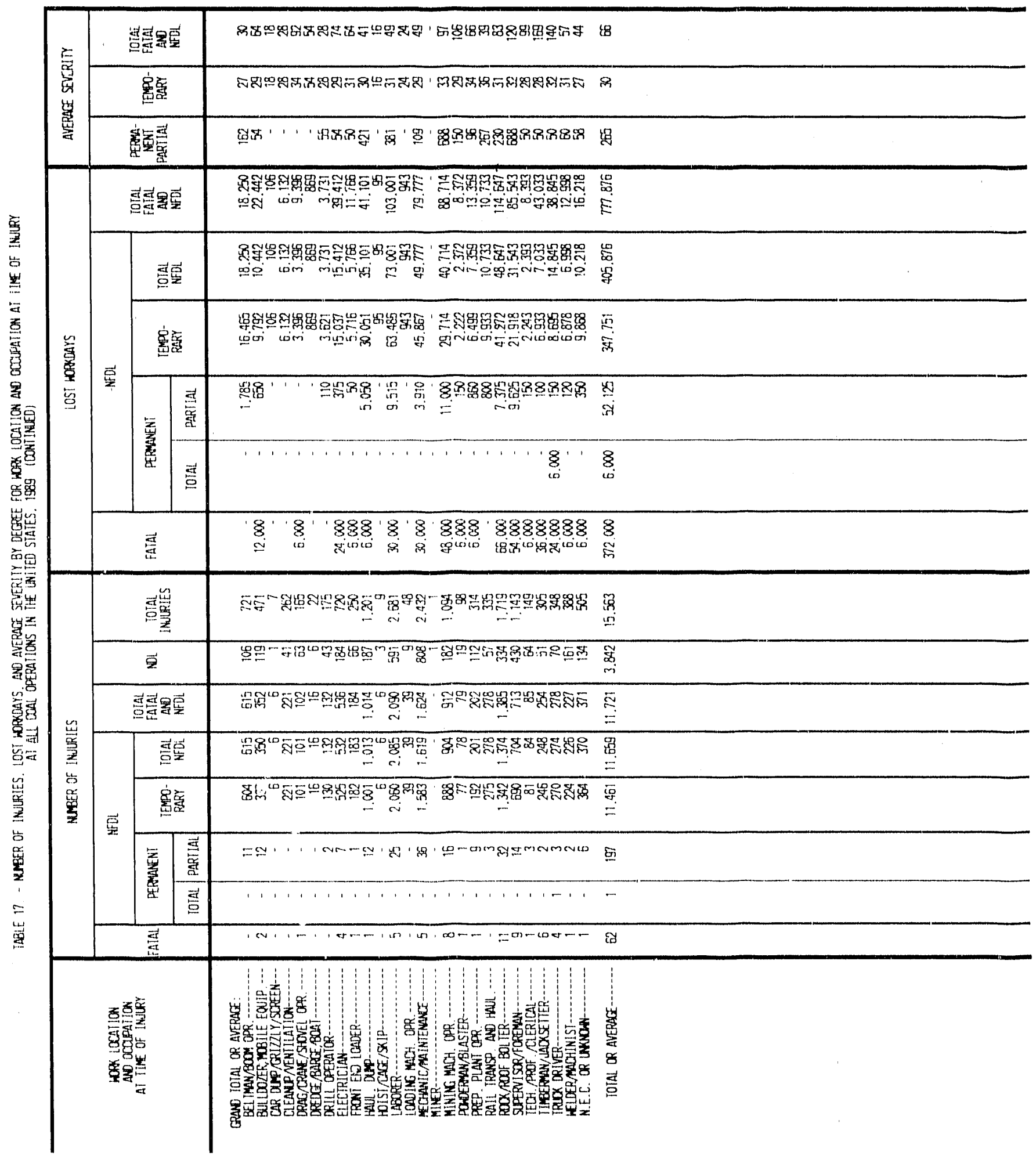




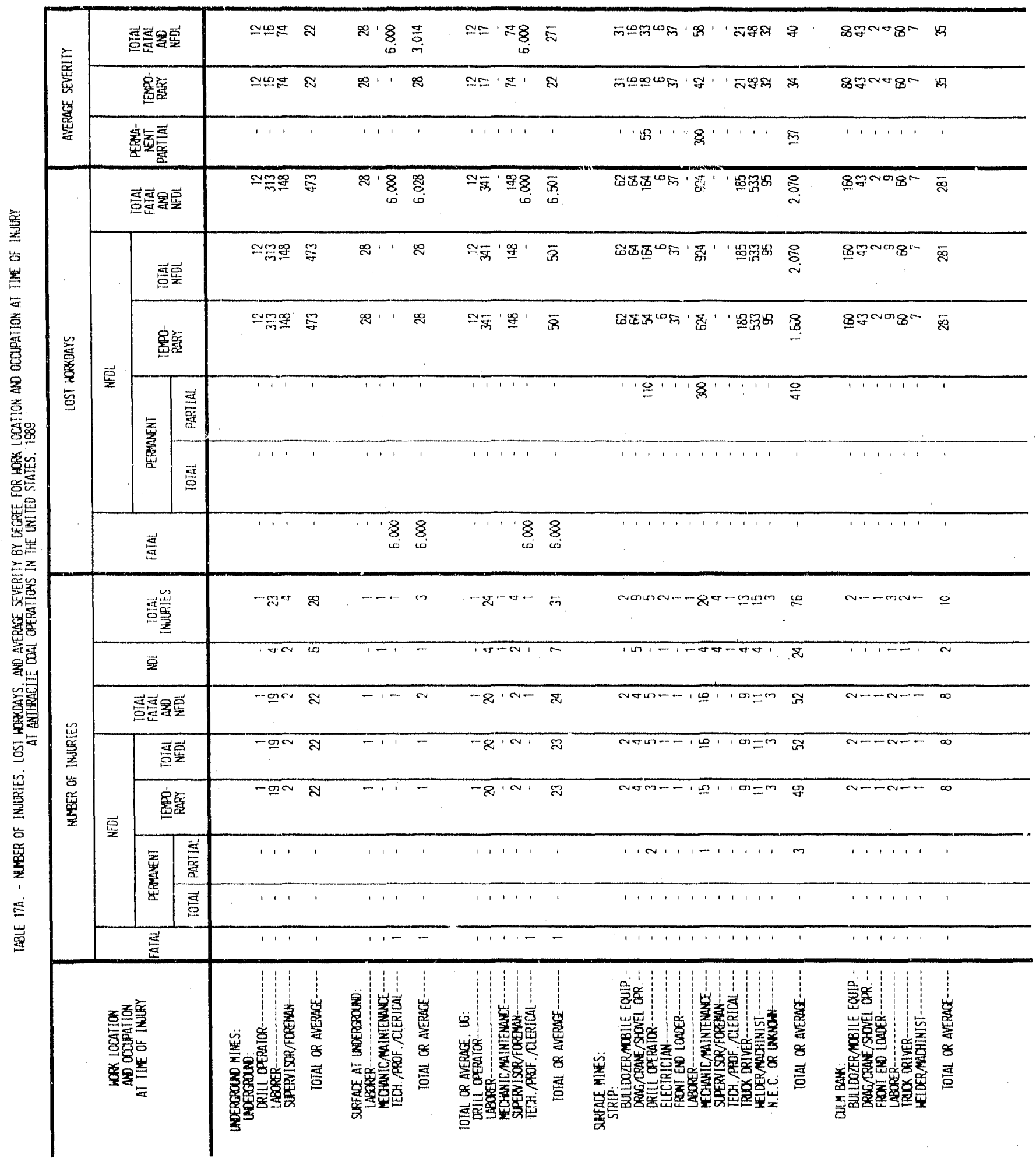




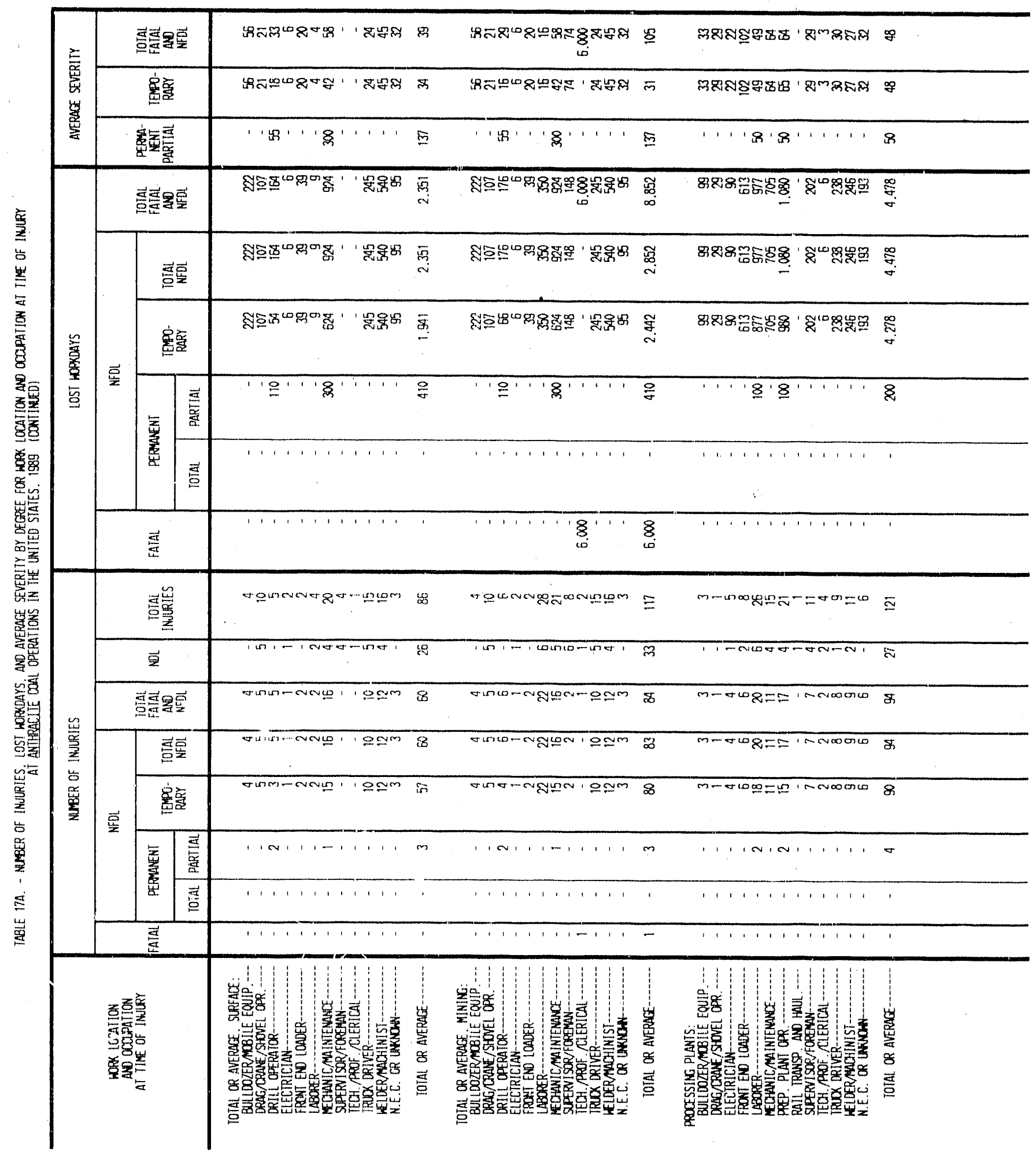




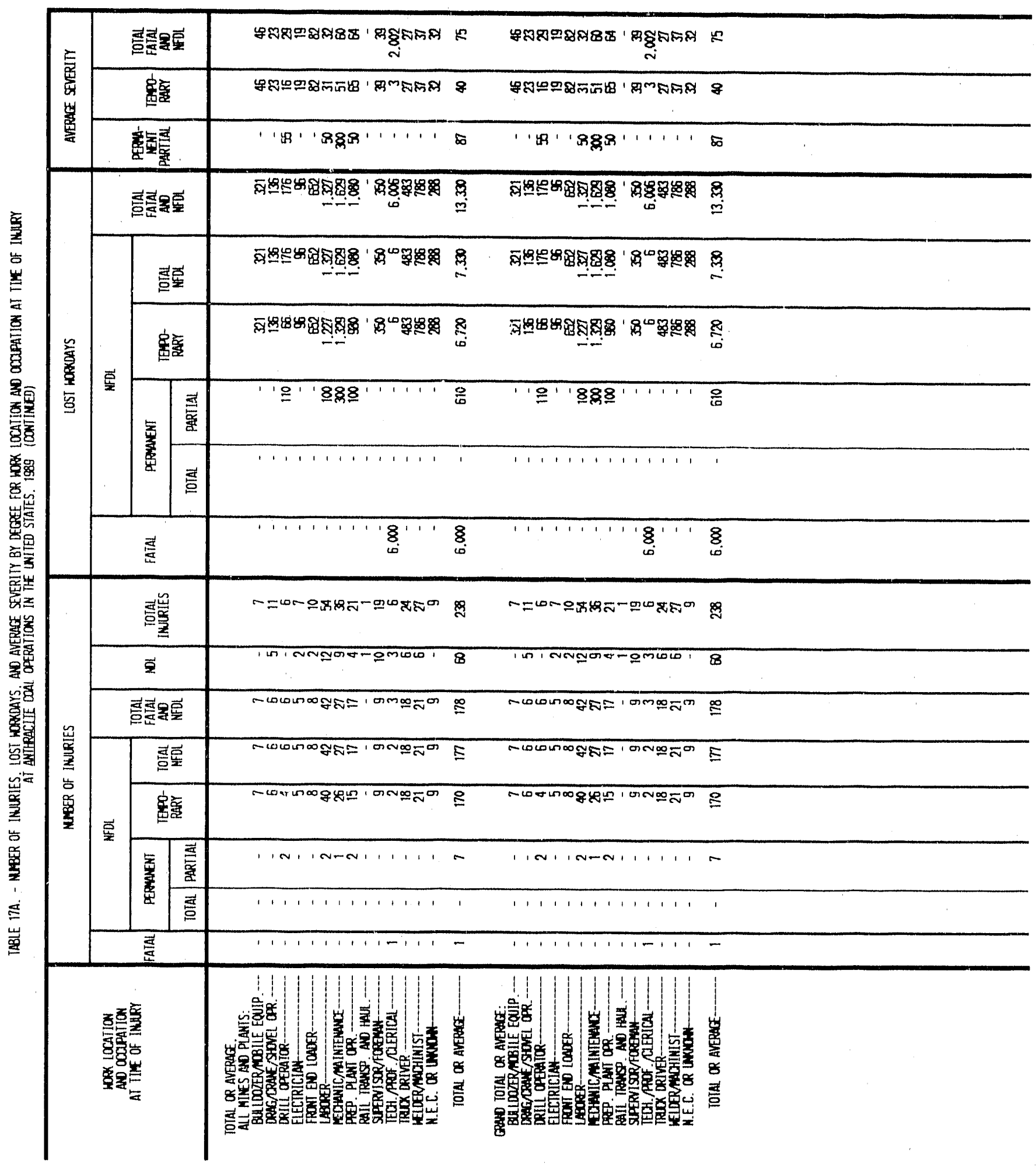




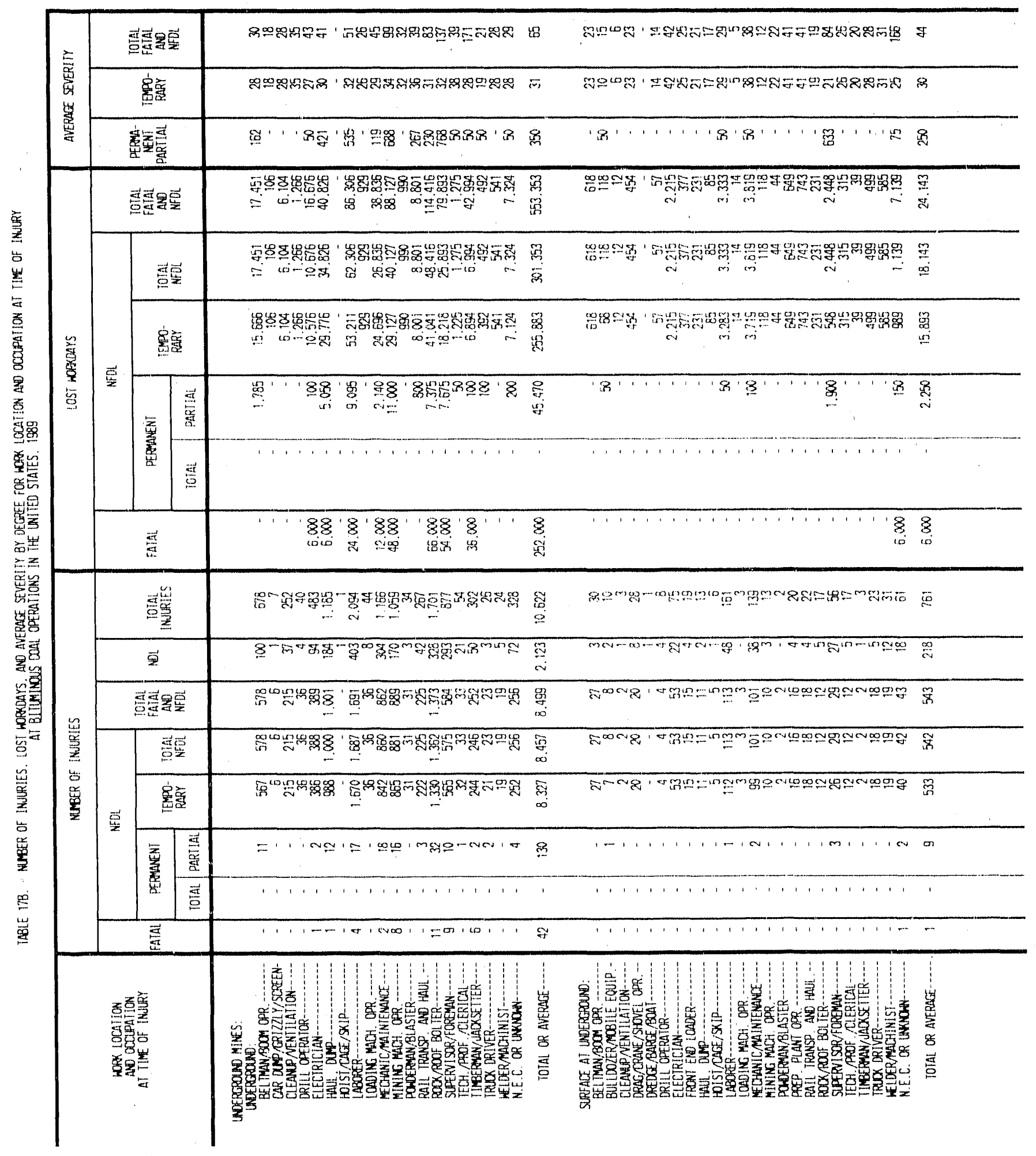




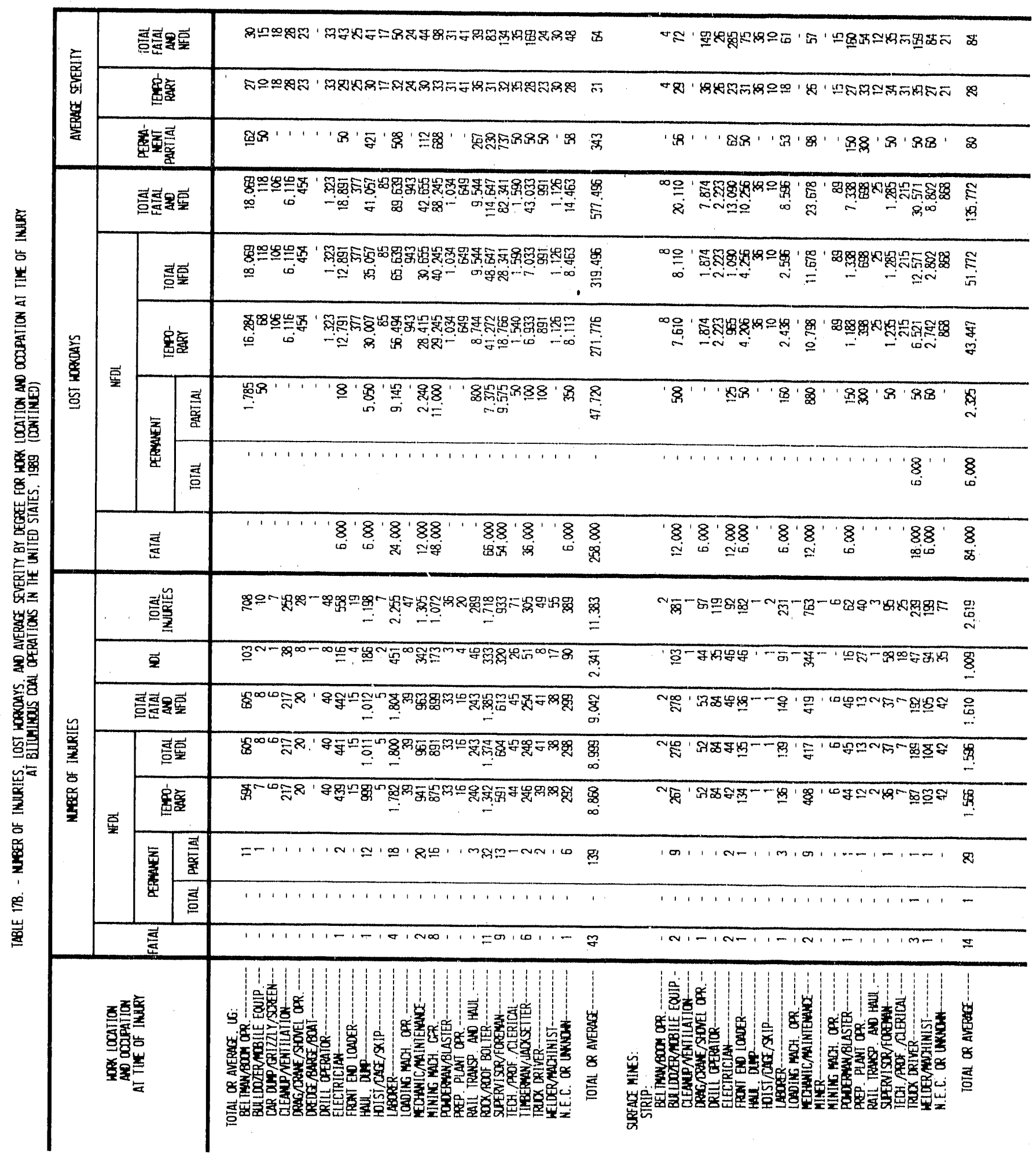




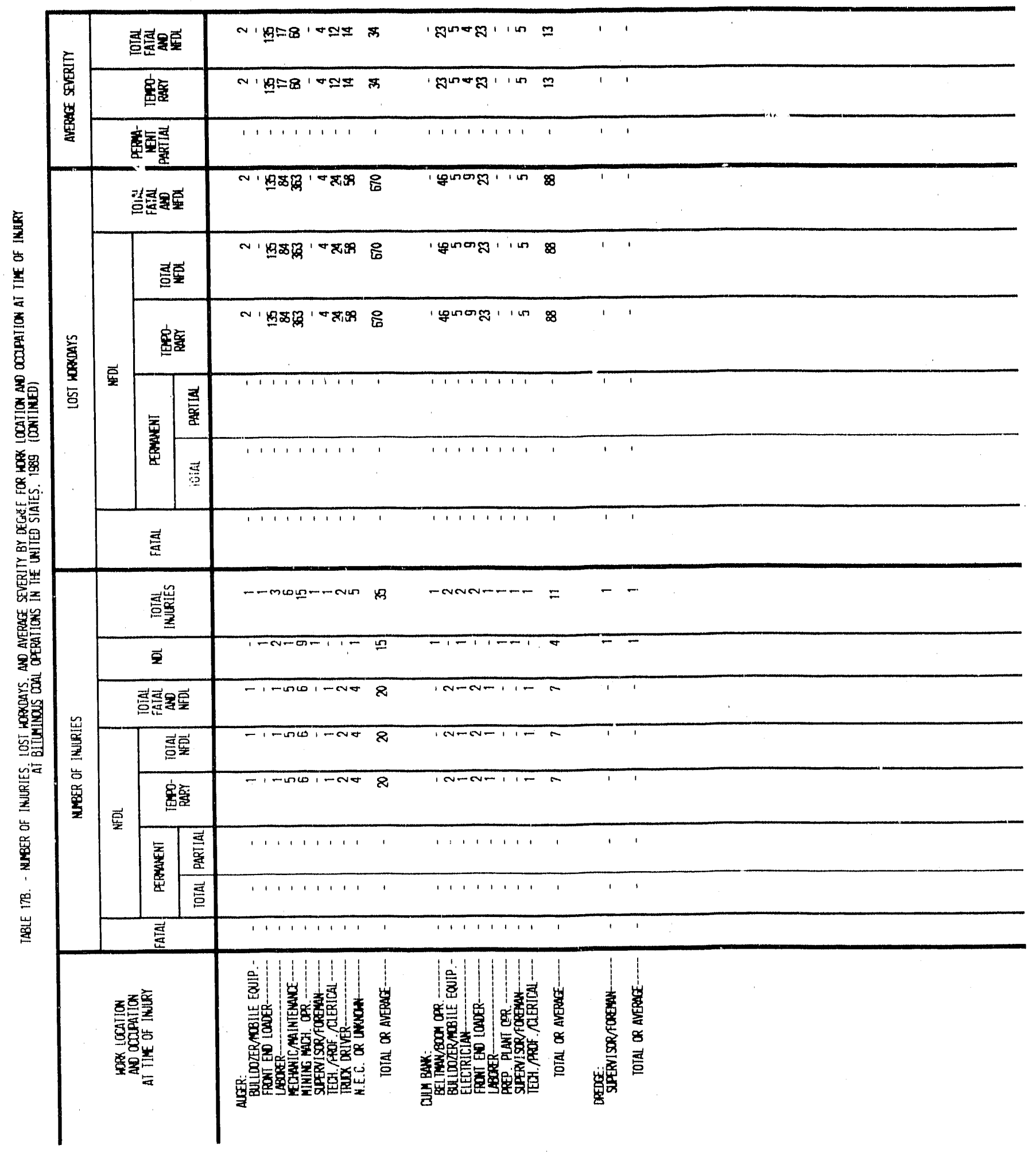




\begin{tabular}{|c|c|c|c|c|c|c|}
\hline \multirow{3}{*}{ 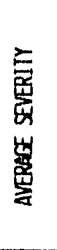 } & \multicolumn{2}{|c|}{ 쳥편울 } & 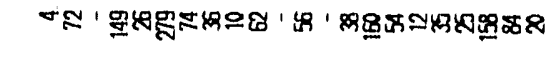 & $\ddot{\infty}$ & 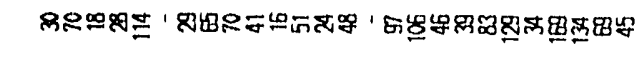 & 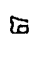 \\
\hline & \multicolumn{2}{|c|}{ 䋗 } & 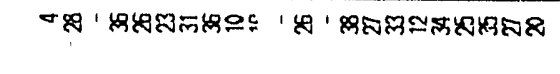 & 8 & 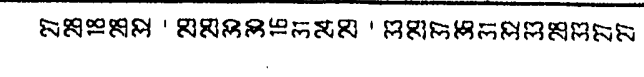 & 8 \\
\hline & \multicolumn{2}{|l|}{ 害率言 } & 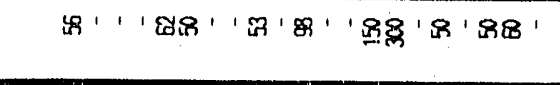 & 8 & 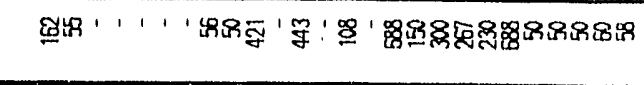 & $\mathscr{\&}$ \\
\hline \multirow{6}{*}{ 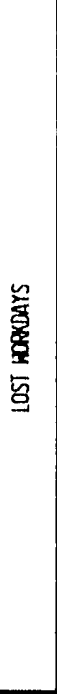 } & \multicolumn{2}{|c|}{ 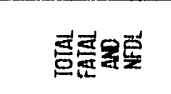 } & 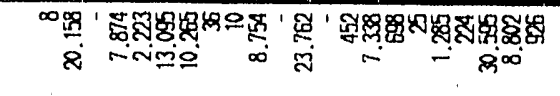 & 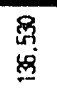 & 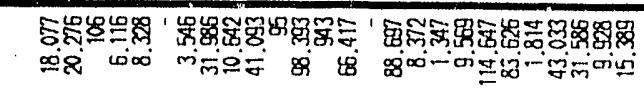 & 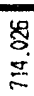 \\
\hline & \multicolumn{2}{|c|}{ 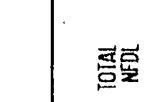 } & 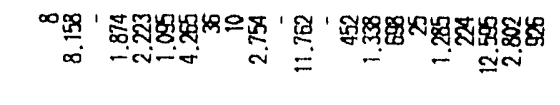 & 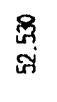 & 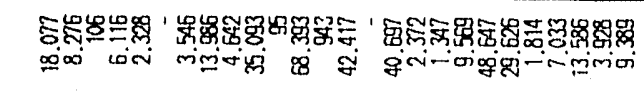 & 峁 \\
\hline & \multicolumn{2}{|c|}{ 旁寒 } & 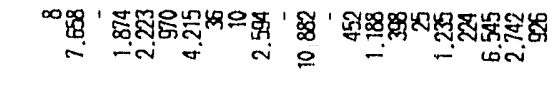 & 岁 & 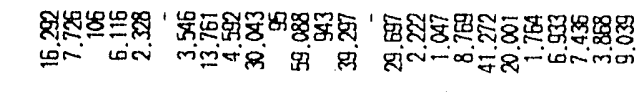 & $\begin{array}{l}\vec{g} \\
\stackrel{\text { wi }}{\mathrm{m}}\end{array}$ \\
\hline & \multirow{2}{*}{ 豆 } & 焉 & 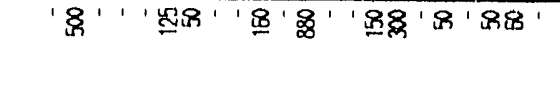 & $\stackrel{\text { id }}{\sim}$ & 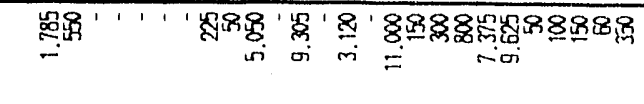 & $\begin{array}{l}\text { 荌 } \\
8 \\
8\end{array}$ \\
\hline & & 홍 & $1,1, \underbrace{\prime}]^{\prime}$ & $\underset{\infty}{8}$ & $11,1,1,1,1,1$ & 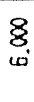 \\
\hline & \multicolumn{2}{|l|}{ 茫 } & 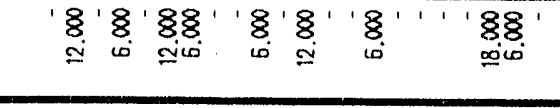 & $\begin{array}{l}8 \\
8 \\
\$\end{array}$ & 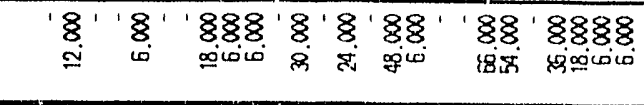 & $\begin{array}{l}8 \\
\stackrel{8}{1} \\
\text { \% }\end{array}$ \\
\hline \multirow{8}{*}{ 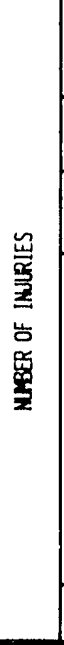 } & \multicolumn{2}{|c|}{ 㺼总宸 } & 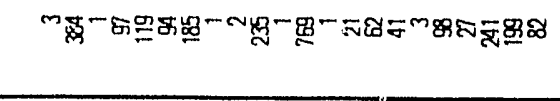 & $\underset{\mathrm{d}}{\mathrm{i}}$ & 三舟 & $\begin{array}{l}\text { gi } \\
\dot{v}\end{array}$ \\
\hline & \multicolumn{2}{|l|}{$\mathbf{s}$} & 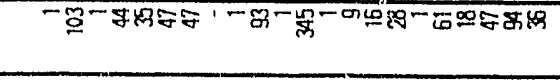 & $\stackrel{5}{-}$ & 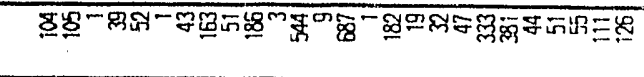 & 용 \\
\hline & \multicolumn{2}{|c|}{ 형련룰 } & 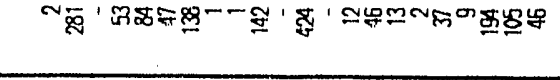 & $\stackrel{\underline{\underline{e}}}{-}$ & 家罗 & 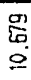 \\
\hline & \multicolumn{2}{|c|}{ 趈要 } & 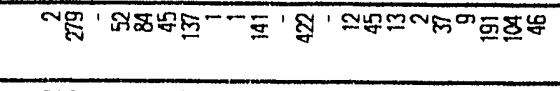 & ๕్g & 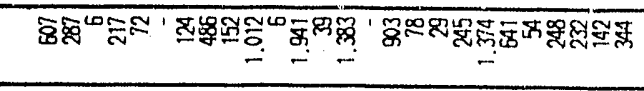 & $\underset{0}{10}$ \\
\hline & \multicolumn{2}{|c|}{ 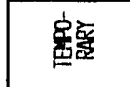 } & 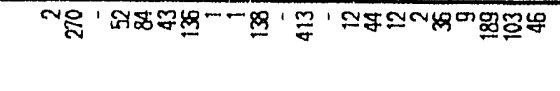 & 蛋 & 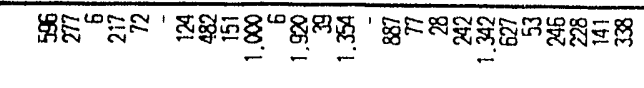 & 氶 \\
\hline & & 혈 & 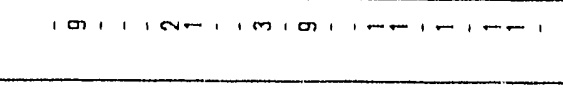 & $g$ & 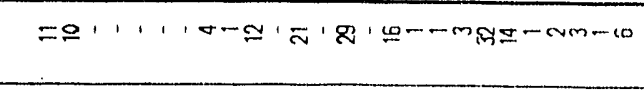 & $\stackrel{8}{0}$ \\
\hline & & 홍 & $111,1,1,1,1$ & - & . & - \\
\hline & \multicolumn{2}{|l|}{ 表 } & $1 \mathrm{~m}-1$ & \pm & & \\
\hline & \multicolumn{2}{|l|}{ 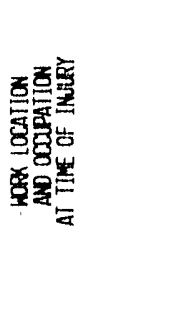 } & 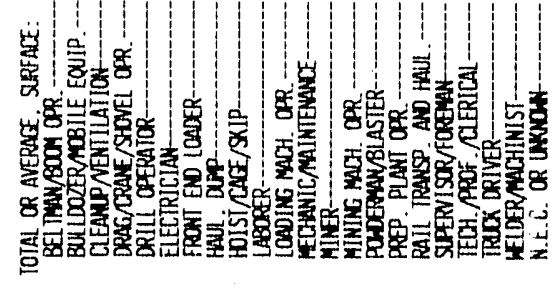 & 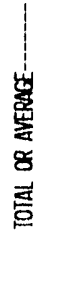 & 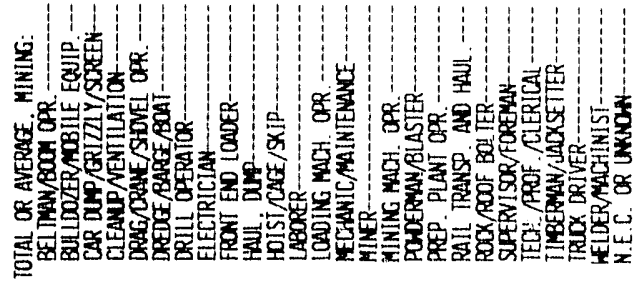 & 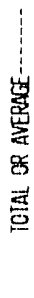 \\
\hline
\end{tabular}




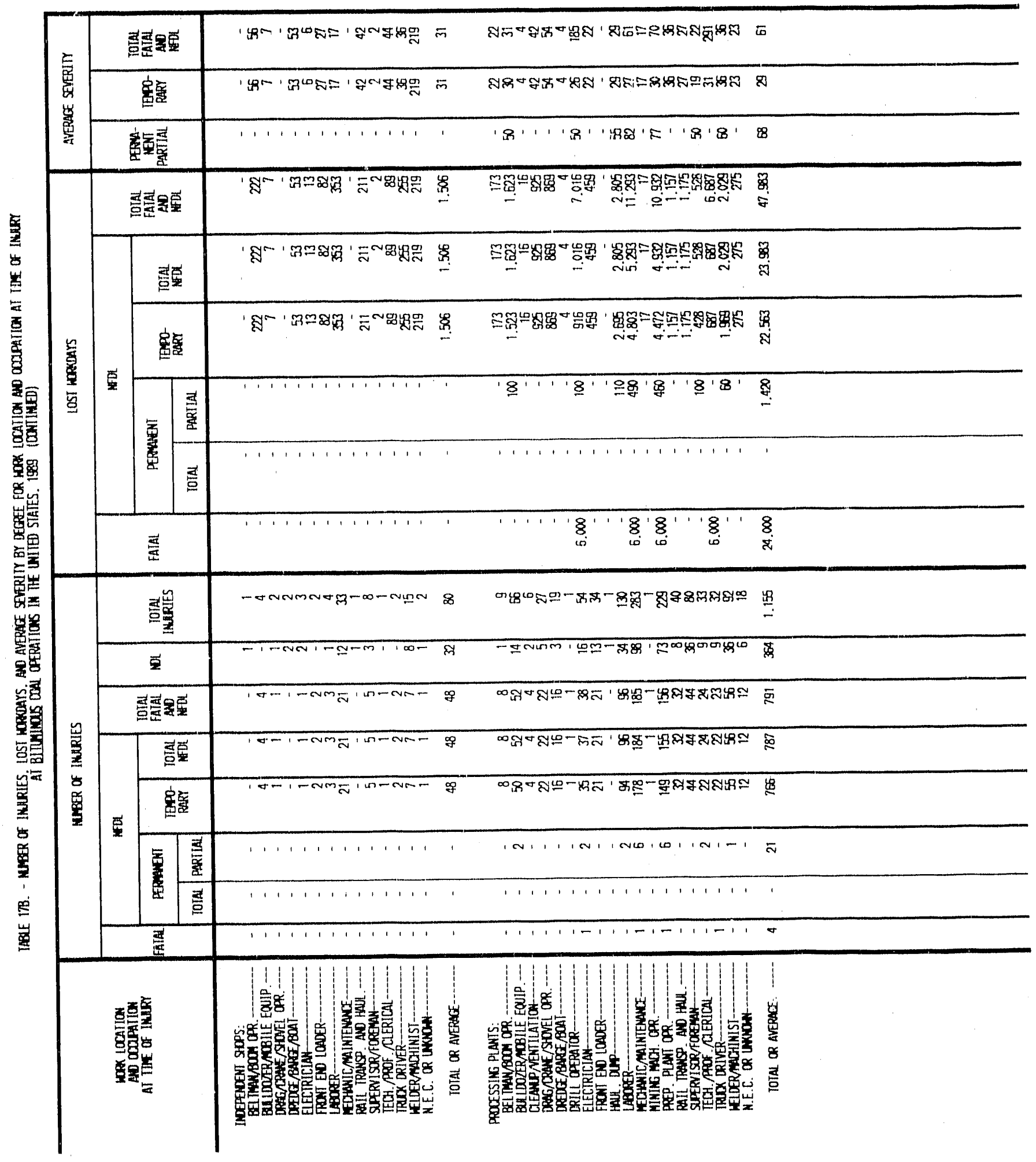




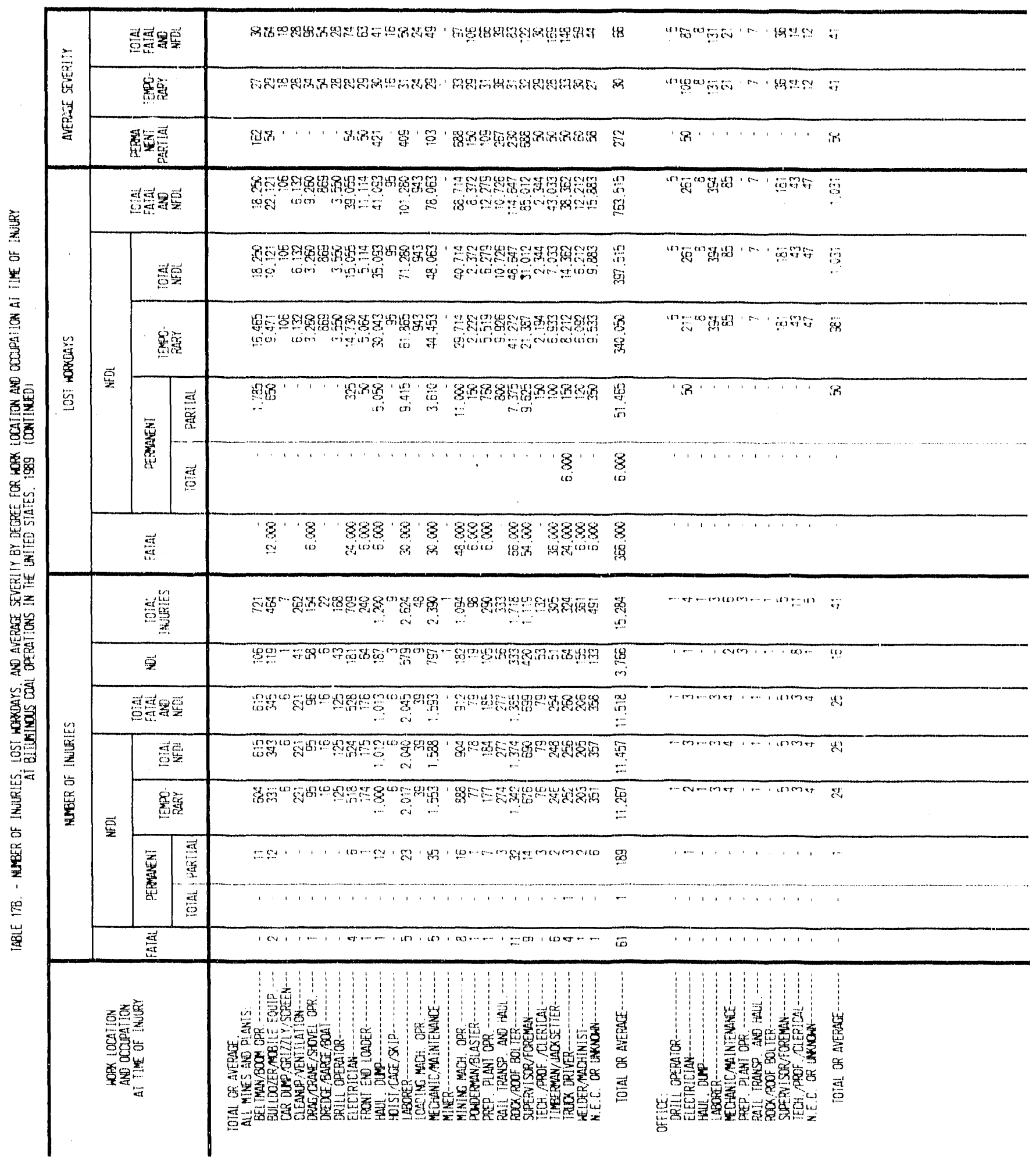




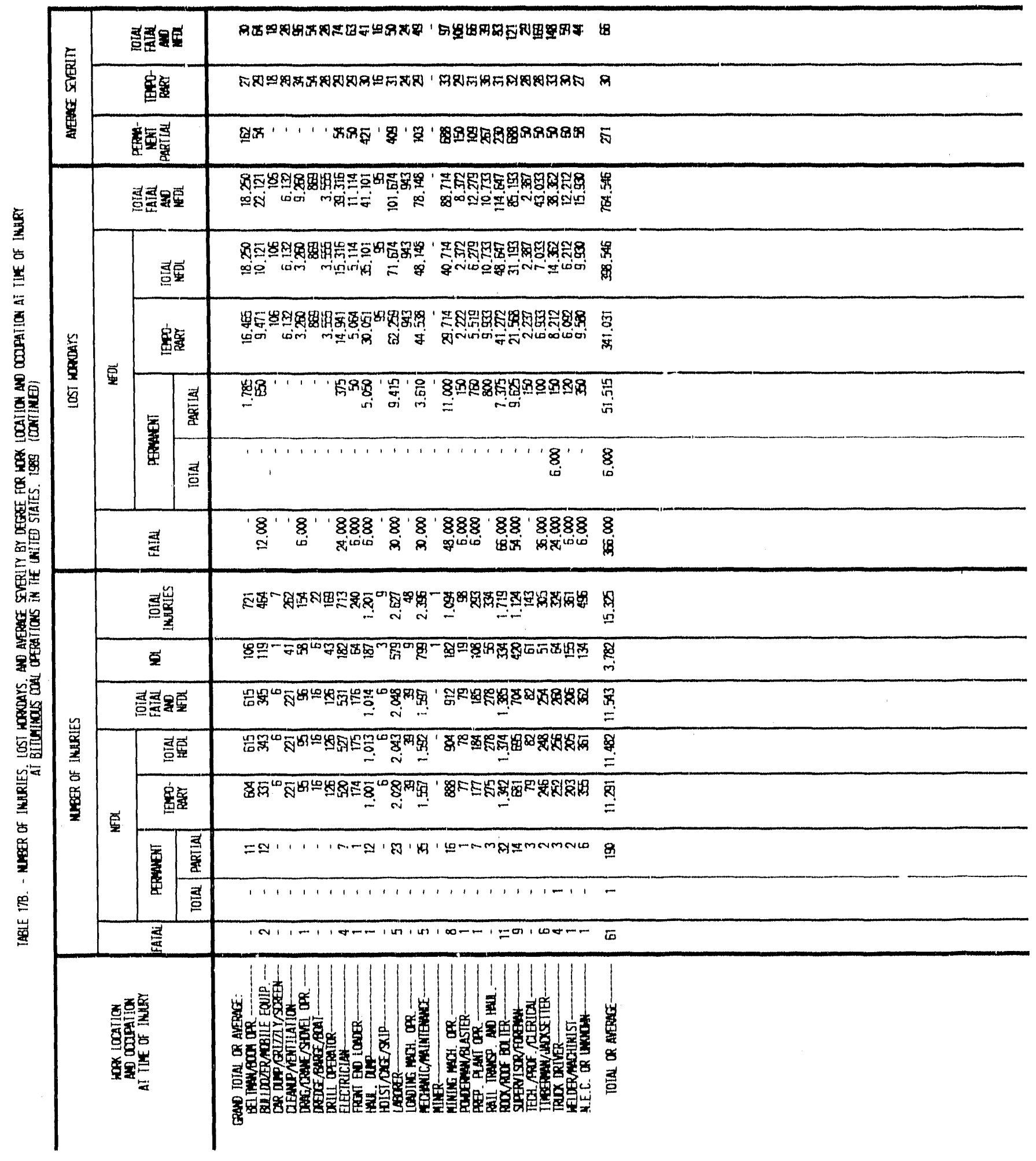




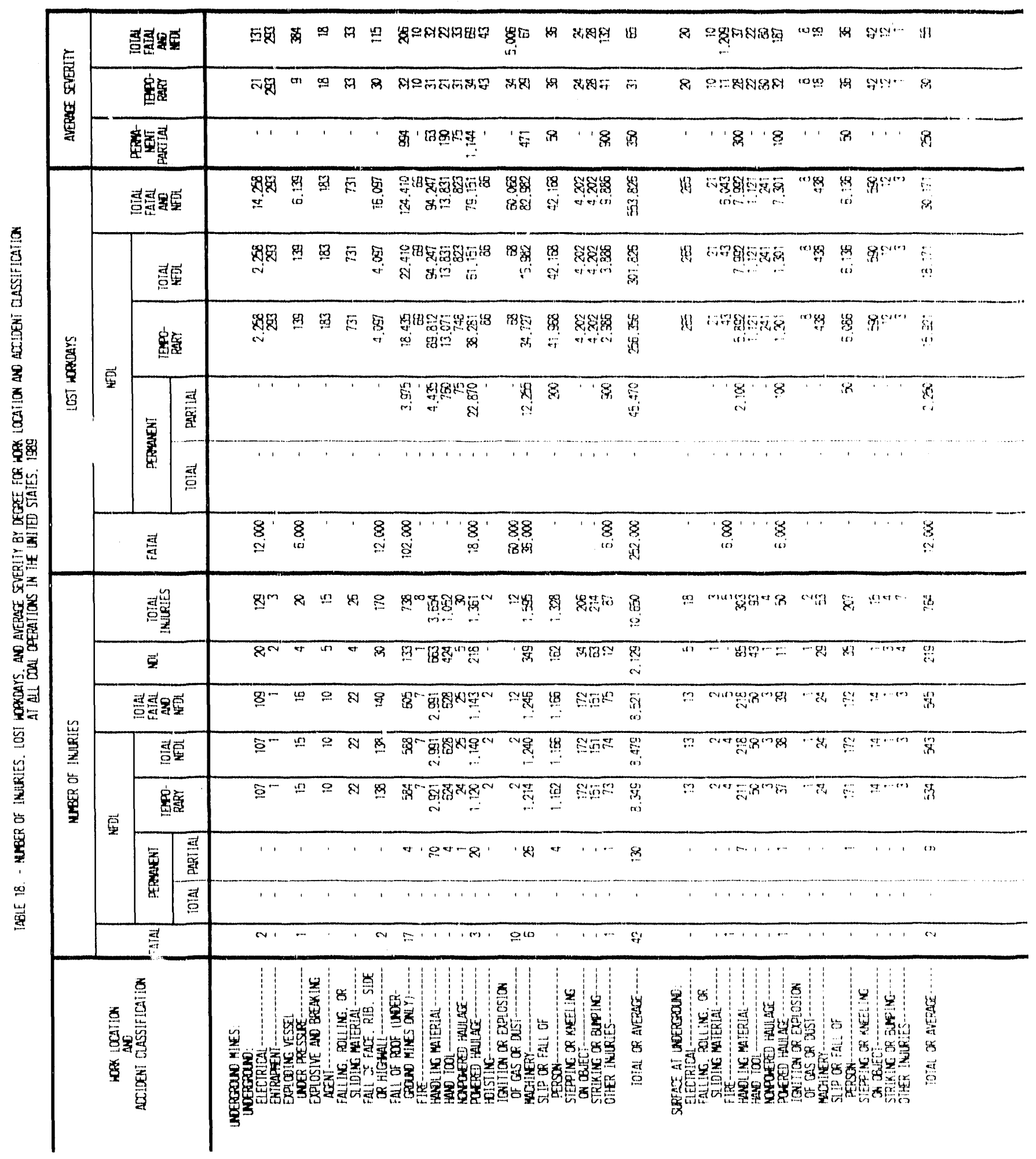




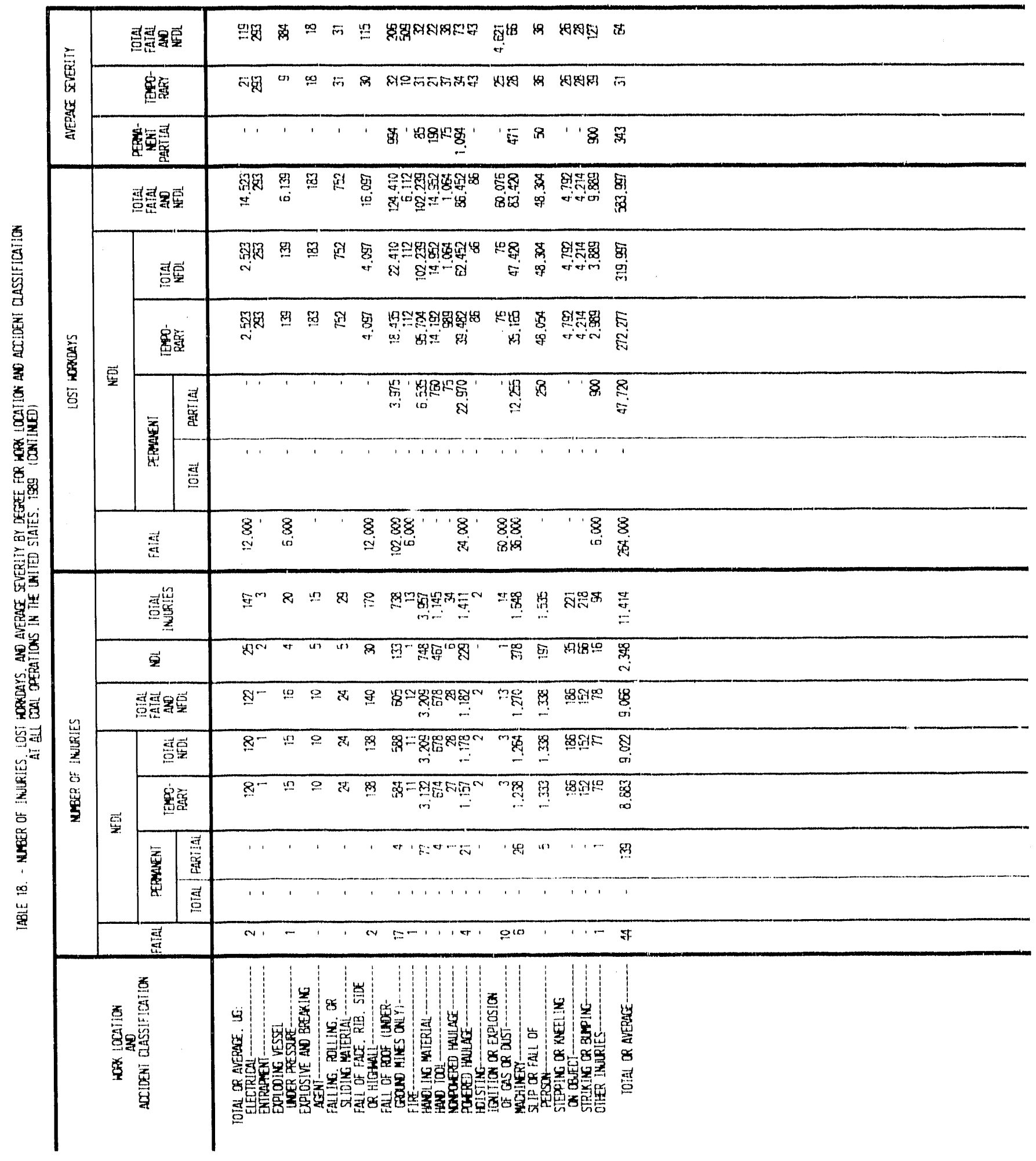




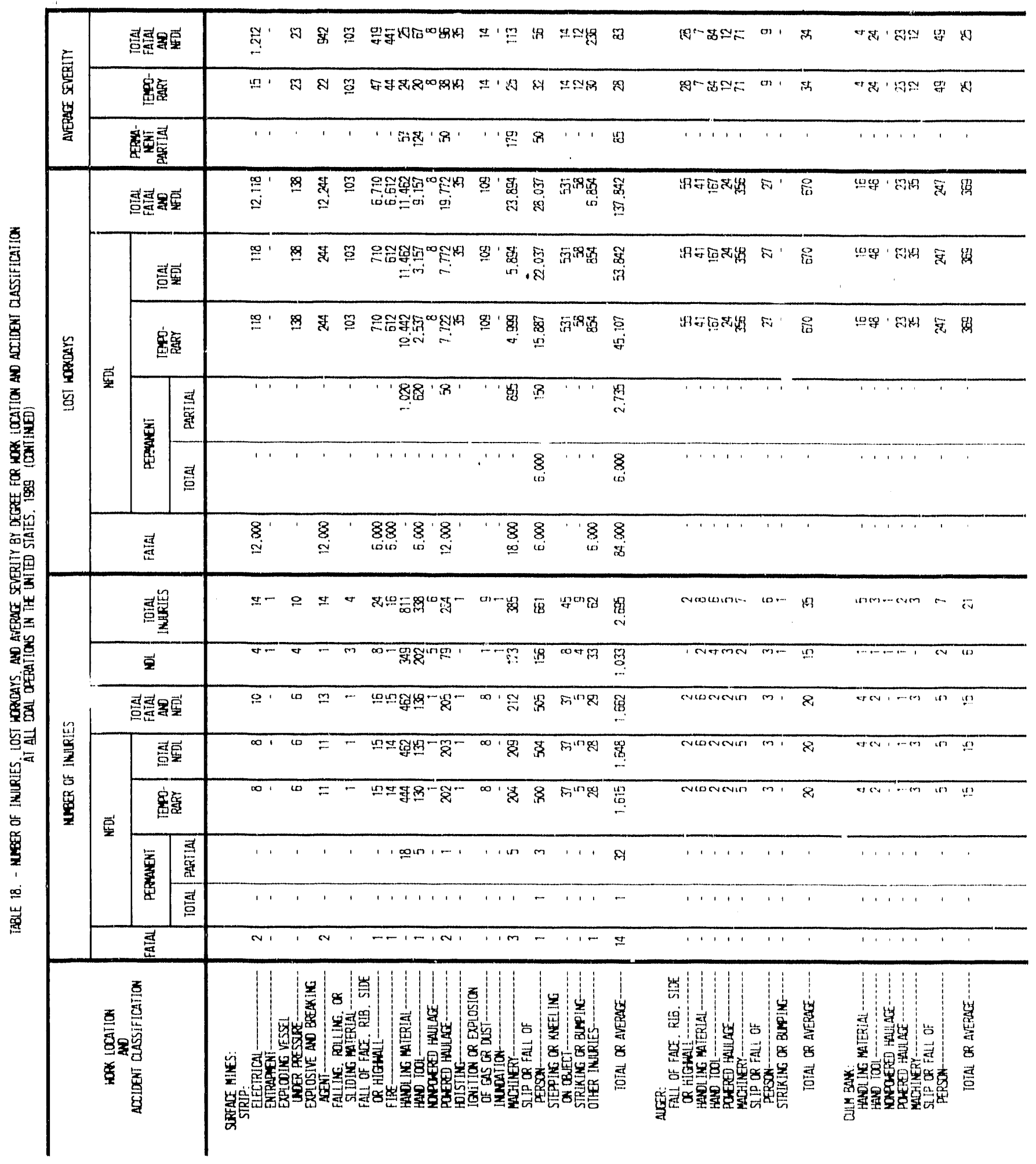




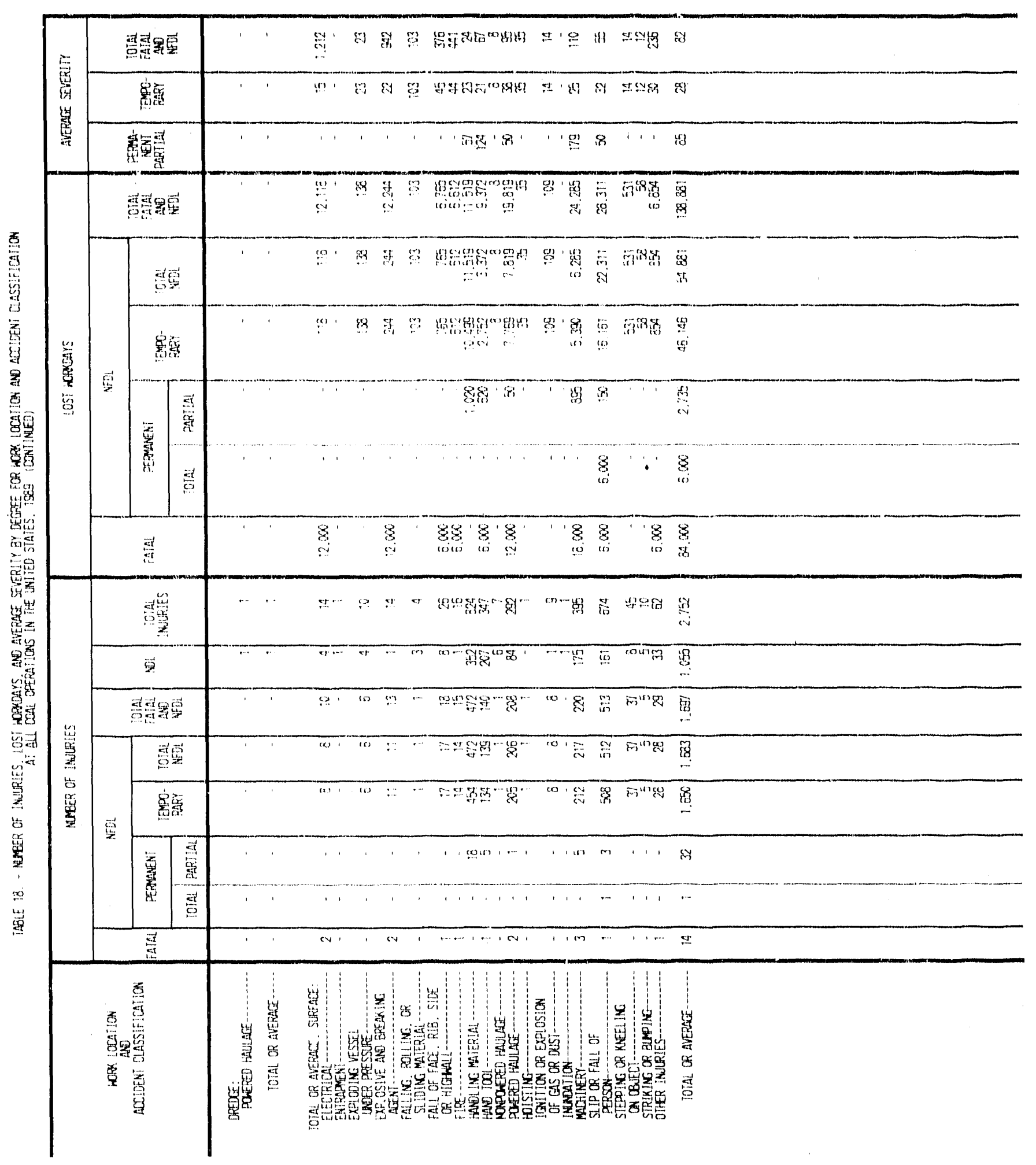




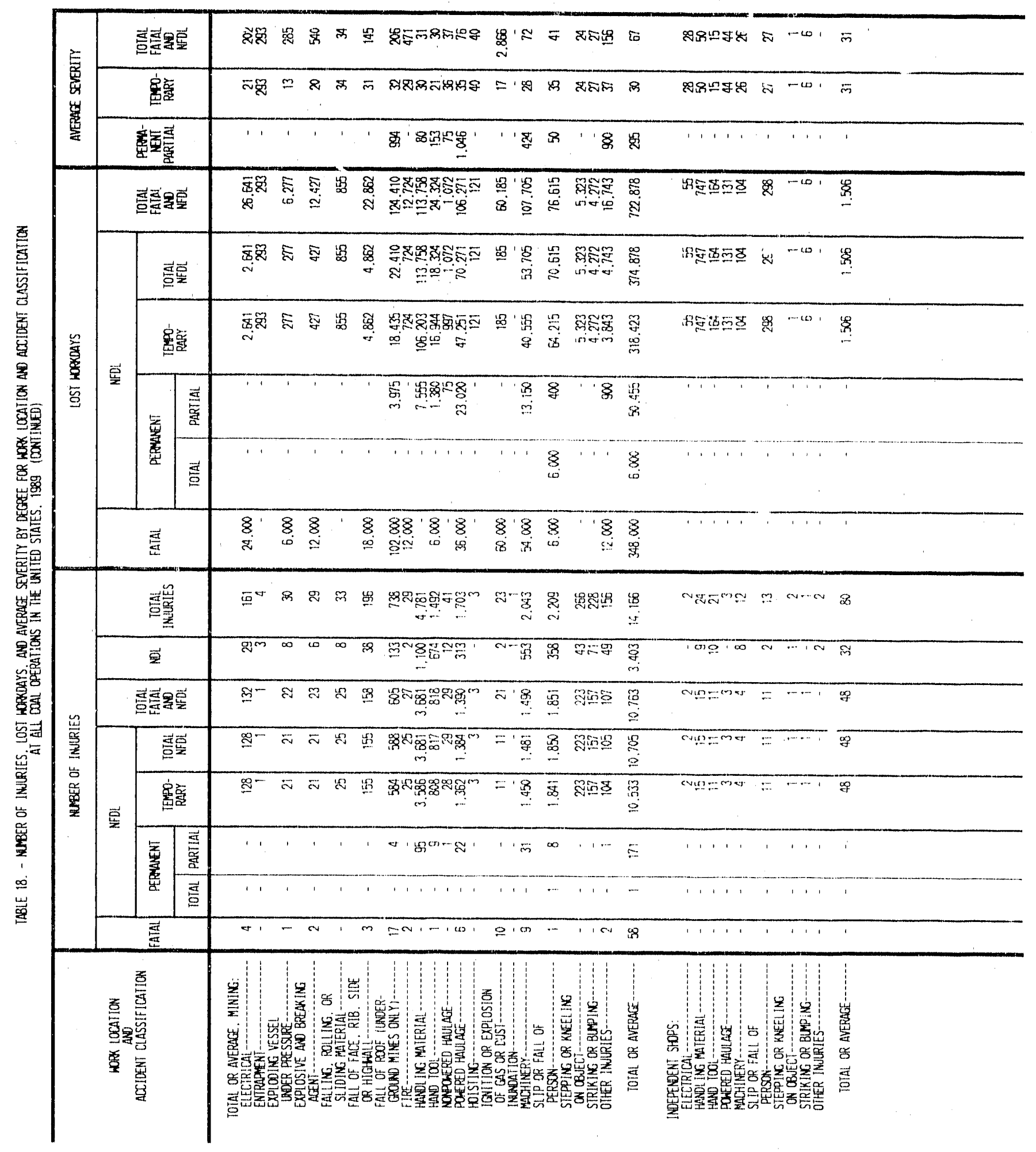




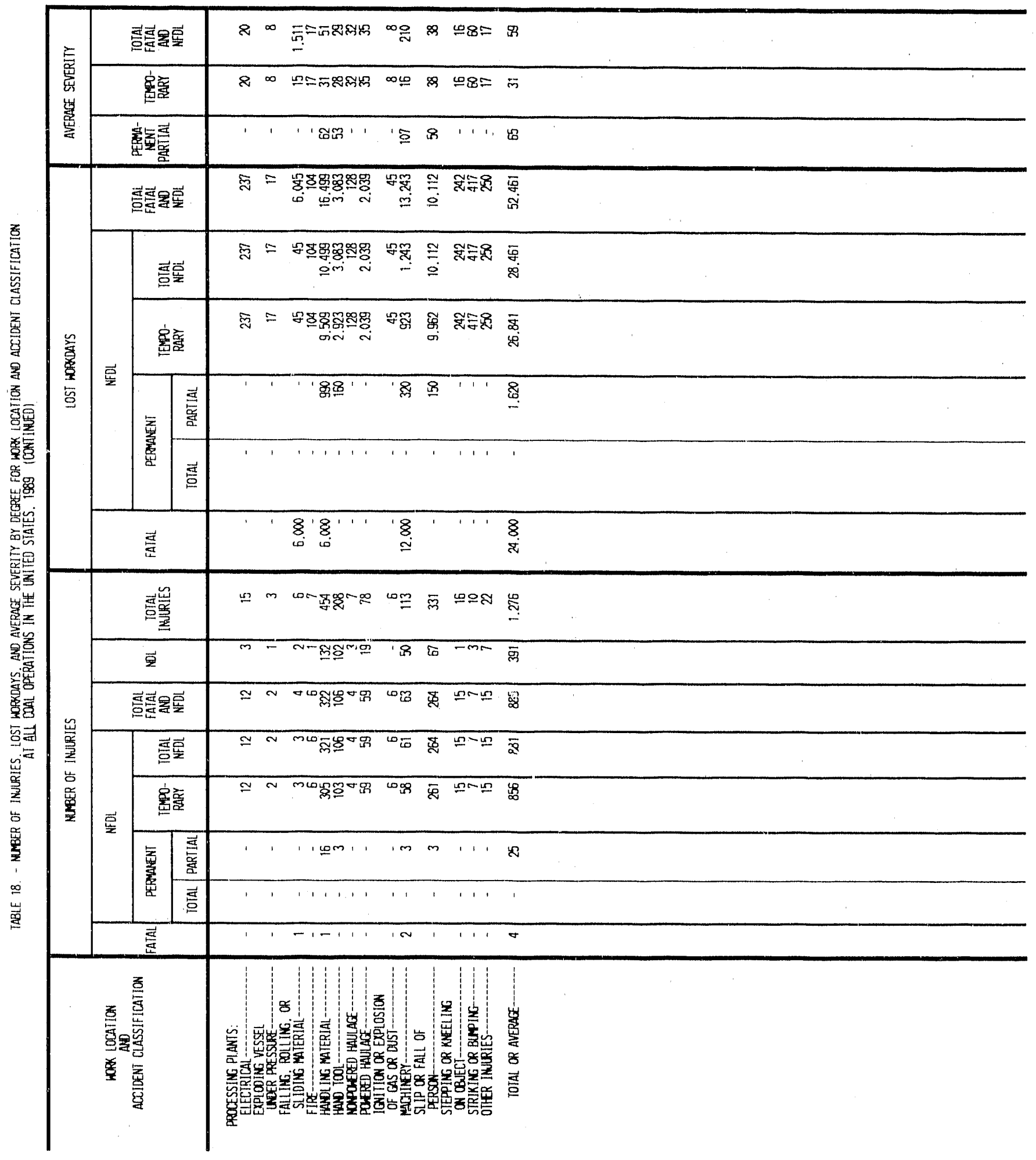




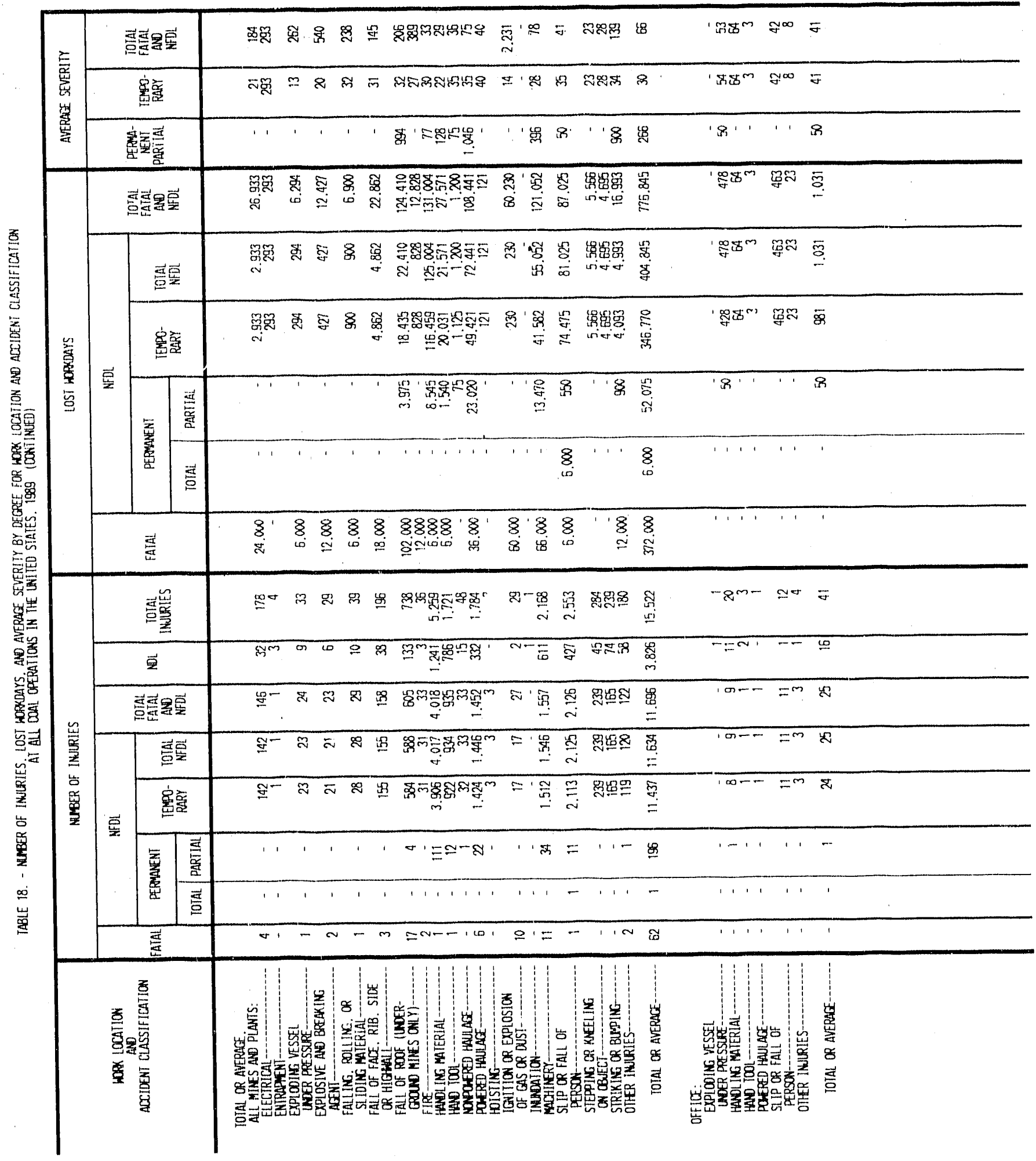




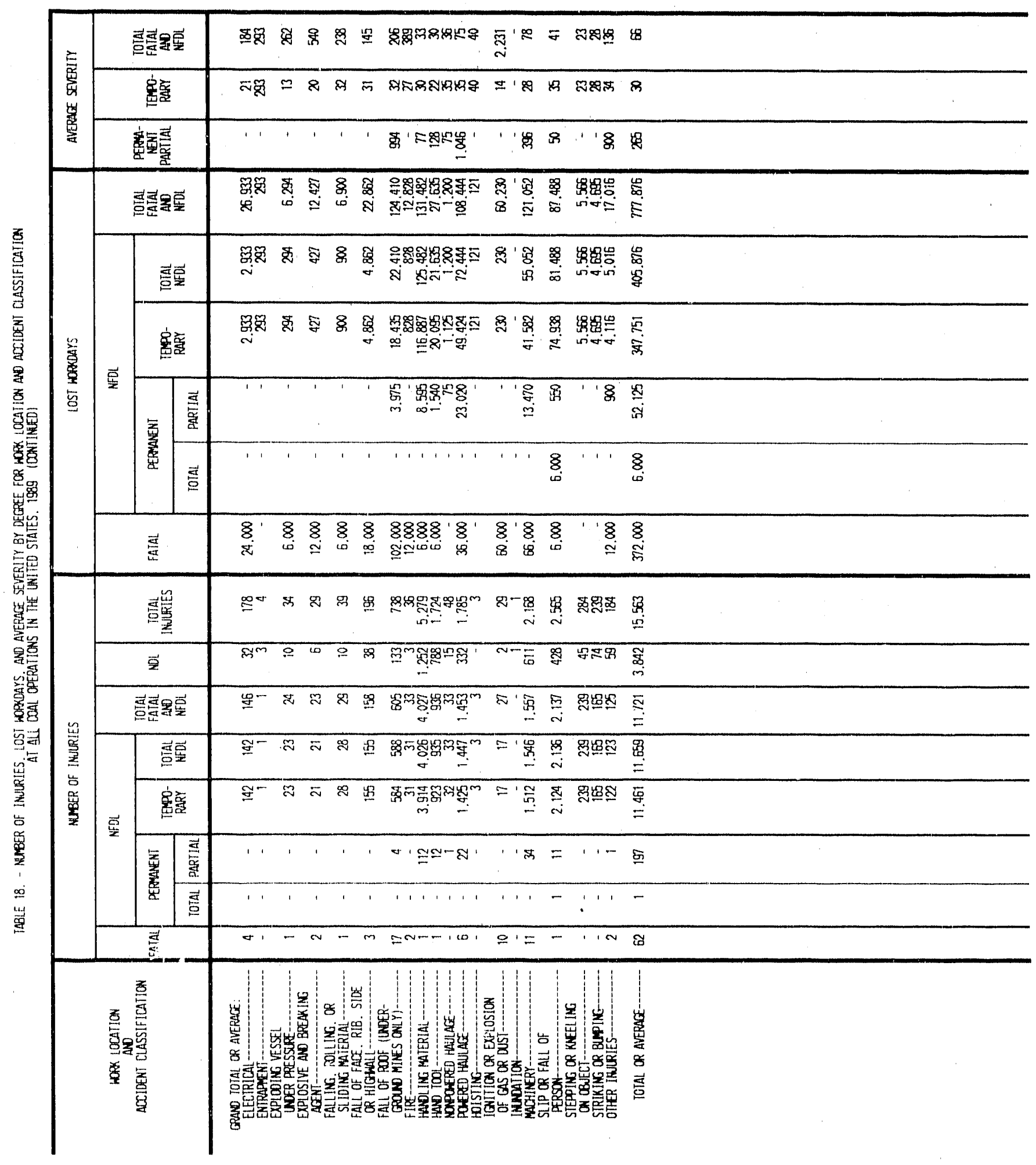




\begin{tabular}{|c|c|c|c|c|c|c|c|c|c|c|}
\hline \multirow{3}{*}{ 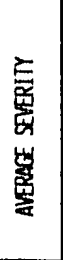 } & \multicolumn{2}{|c|}{ 졍현월 } & $r \pm=48 \%=$ & ส & $\underset{6}{8}$ & & $m=98$ & $\overline{\text { ธ }}$ & 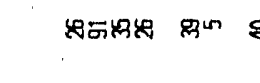 & 8 \\
\hline & \multicolumn{2}{|c|}{ 衰寒 } & $-y=489$ & $\mathfrak{A}$ & 119 & 89 & $\therefore=498 \quad \infty$ & $\mathbb{A}$ & 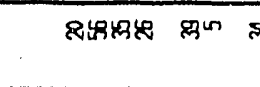 & sh \\
\hline & \multicolumn{2}{|c|}{ 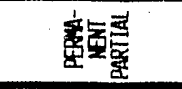 } & 111 & ' & 111 & 1 & 1111 & 1 & सम् & פ \\
\hline \multirow{6}{*}{ 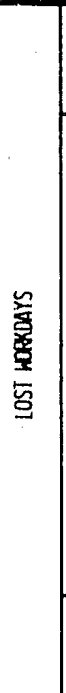 } & \multicolumn{2}{|c|}{ 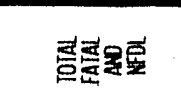 } & 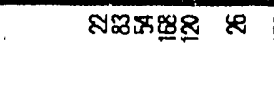 & 8 & $\underset{6}{8}$ & क्ष & 재재요 & 疍 & 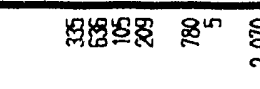 & 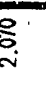 \\
\hline & \multicolumn{2}{|c|}{ 营莫 } & 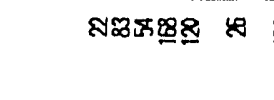 & 等 & 1129 & $\approx$ & 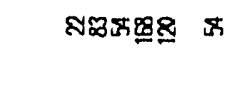 & $\overline{8}$ & 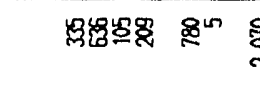 & 号 \\
\hline & \multicolumn{2}{|c|}{ 宦容 } & 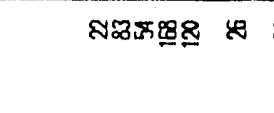 & $\frac{2}{8}$ & 1 1 & \& & รஐங⿱ & $\bar{\Omega}$ & 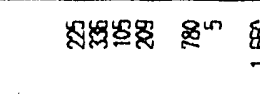 & 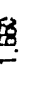 \\
\hline & \multirow{2}{*}{ 垔 } & $\begin{array}{l}\text { 호 } \\
\text { 妾 }\end{array}$ & 111111 & 1 & 11 & 1 & 11111 & ! & 용's'1's & $\frac{0}{7}$ \\
\hline & & 퐇 & 11111.1 & ' & 111 & 1 & 11111 & 1 & 111,11 & 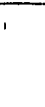 \\
\hline & \multicolumn{2}{|l|}{ 莣 } & 11111 & ' & ' & 8 & ' ' ' & $\underset{6}{8}$ & 1.11 & T \\
\hline \multirow{8}{*}{ 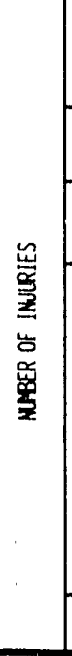 } & \multicolumn{2}{|c|}{ 층 } & m̃oremo m & $\mathscr{q}$ & -- & m & mower & $\bar{m}$ & $x=m a n$ & 电 \\
\hline & \multicolumn{2}{|l|}{$\overline{2}$} & $1 m-1-$ & $\omega$ & -1 & $=$ & $\overline{\sigma-1}-$ & $\pi$ & श्र1-6न & $\bar{Z}$ \\
\hline & \multicolumn{2}{|c|}{ 형현현물 } & mownm $\sim$ & สู & $1-$ & $\bar{n}$ & mointom $m$ & $\pi$ & mamos \&- & 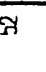 \\
\hline & \multicolumn{2}{|c|}{ 흠훌 } & morimm $\sim$ & ม & $1-$ & $=$ & mounmm $\mathrm{m}$ & m & mamos \& & \% \\
\hline & \multicolumn{2}{|c|}{ 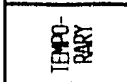 } & morsmm N & ร & $11-$ & $=$ & mळinmm $\mathrm{m}$ & $\approx$ & $=$ wom $8^{-}$ & $g$ \\
\hline & 婹 & 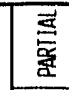 & 1,1, & , & 1 & , & 111, & . & $n-1,1,0$ & $m$ \\
\hline & & 홍 & $1,1,1$ & ' & 1 . & 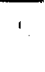 & 11111 & ' & 11 & , \\
\hline & \multicolumn{2}{|l|}{ 存 } & $1.1,1$ & 1 & $1-1$ & $=$ & $111-11$ & - & 11 & 1 \\
\hline & \multicolumn{2}{|l|}{ 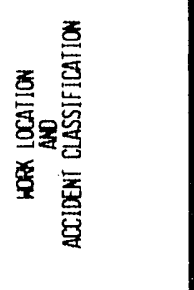 } & 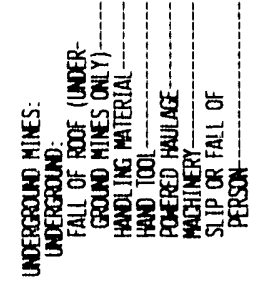 & 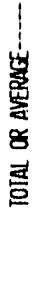 & 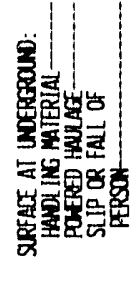 & 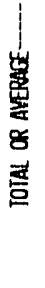 & 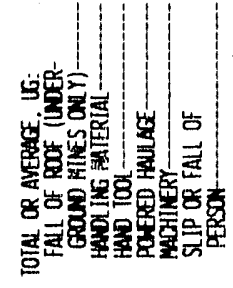 & 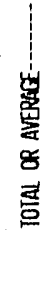 & 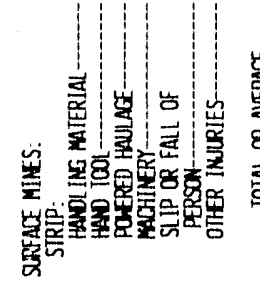 & 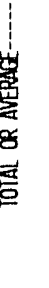 \\
\hline
\end{tabular}




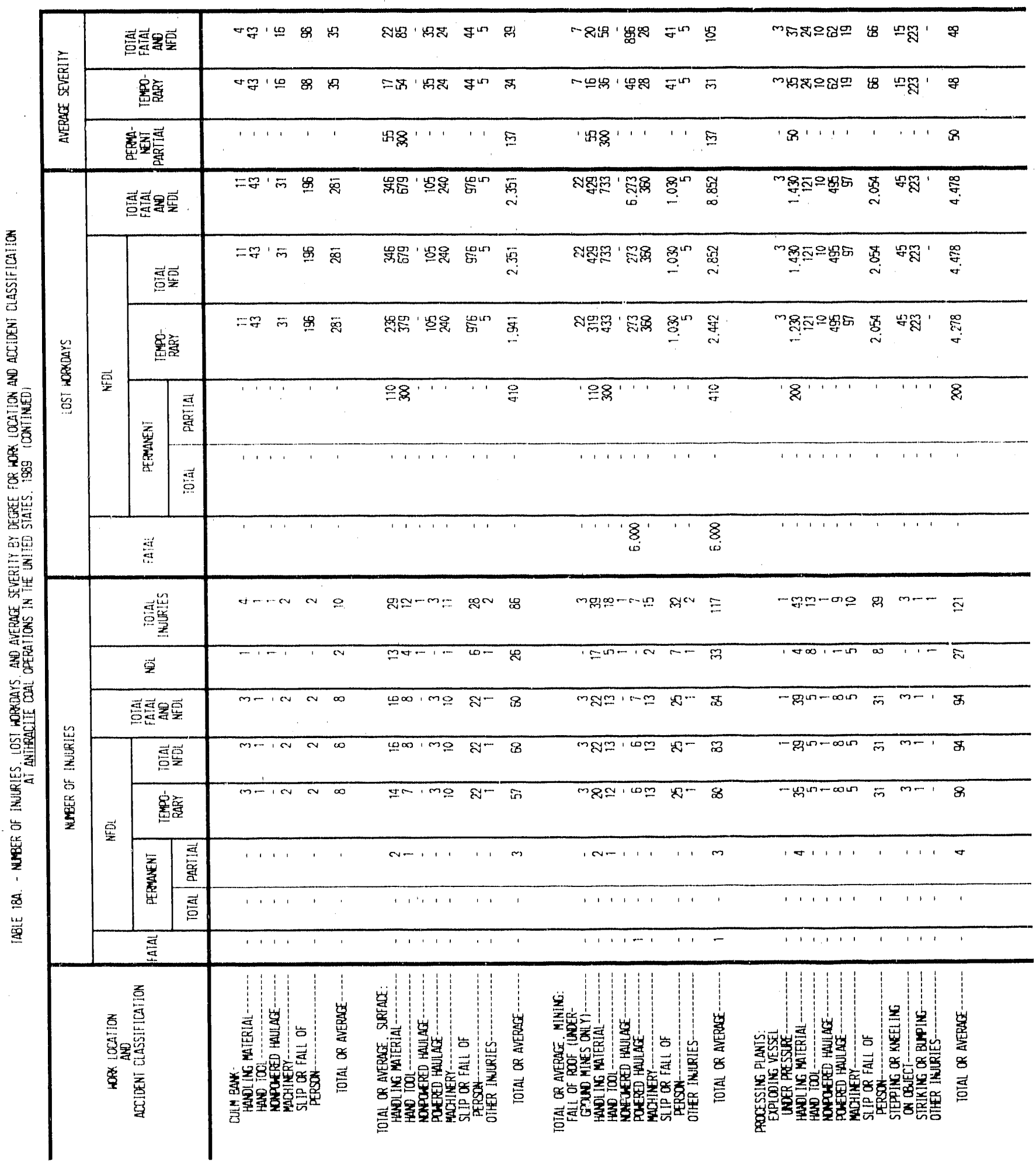




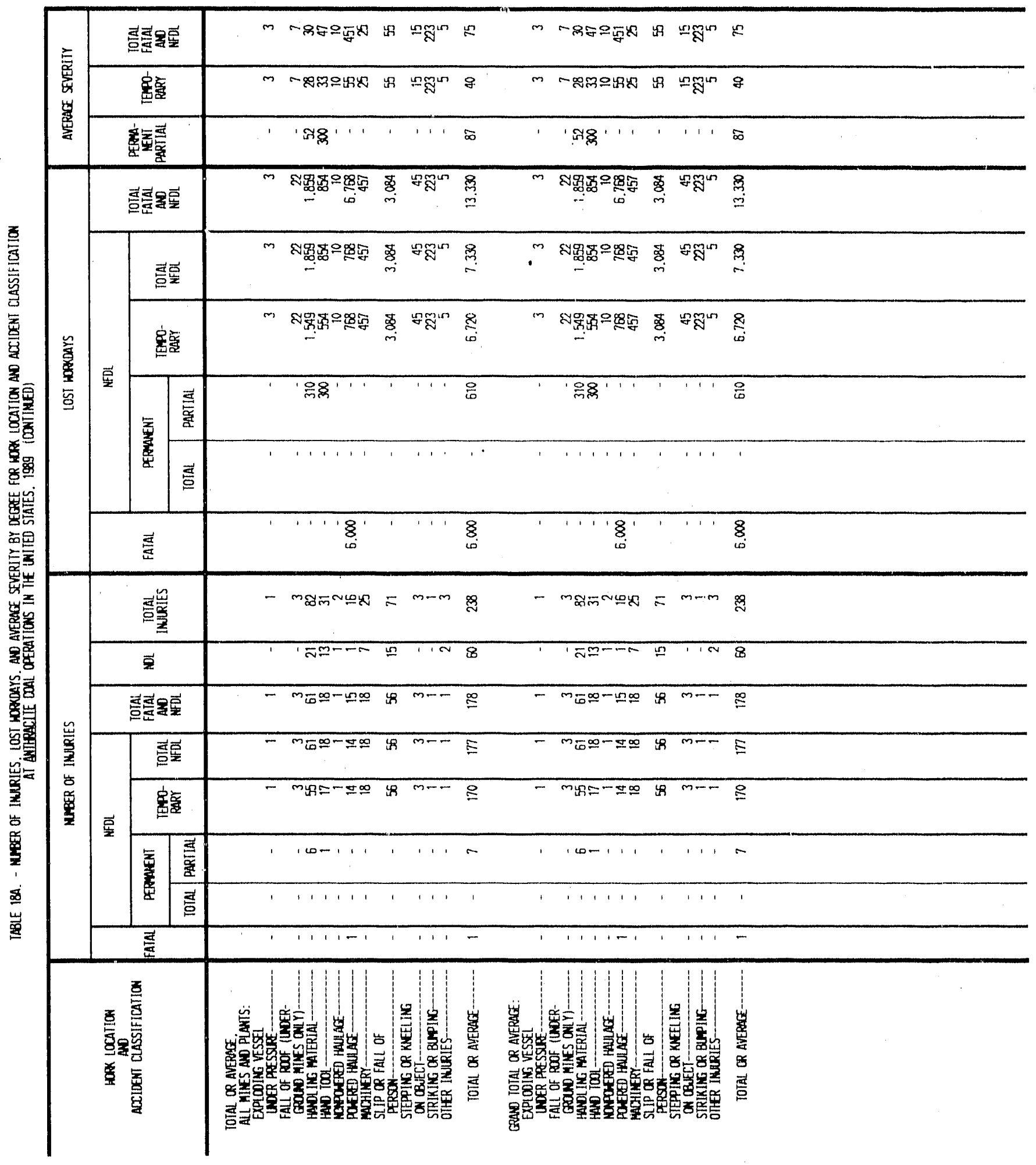




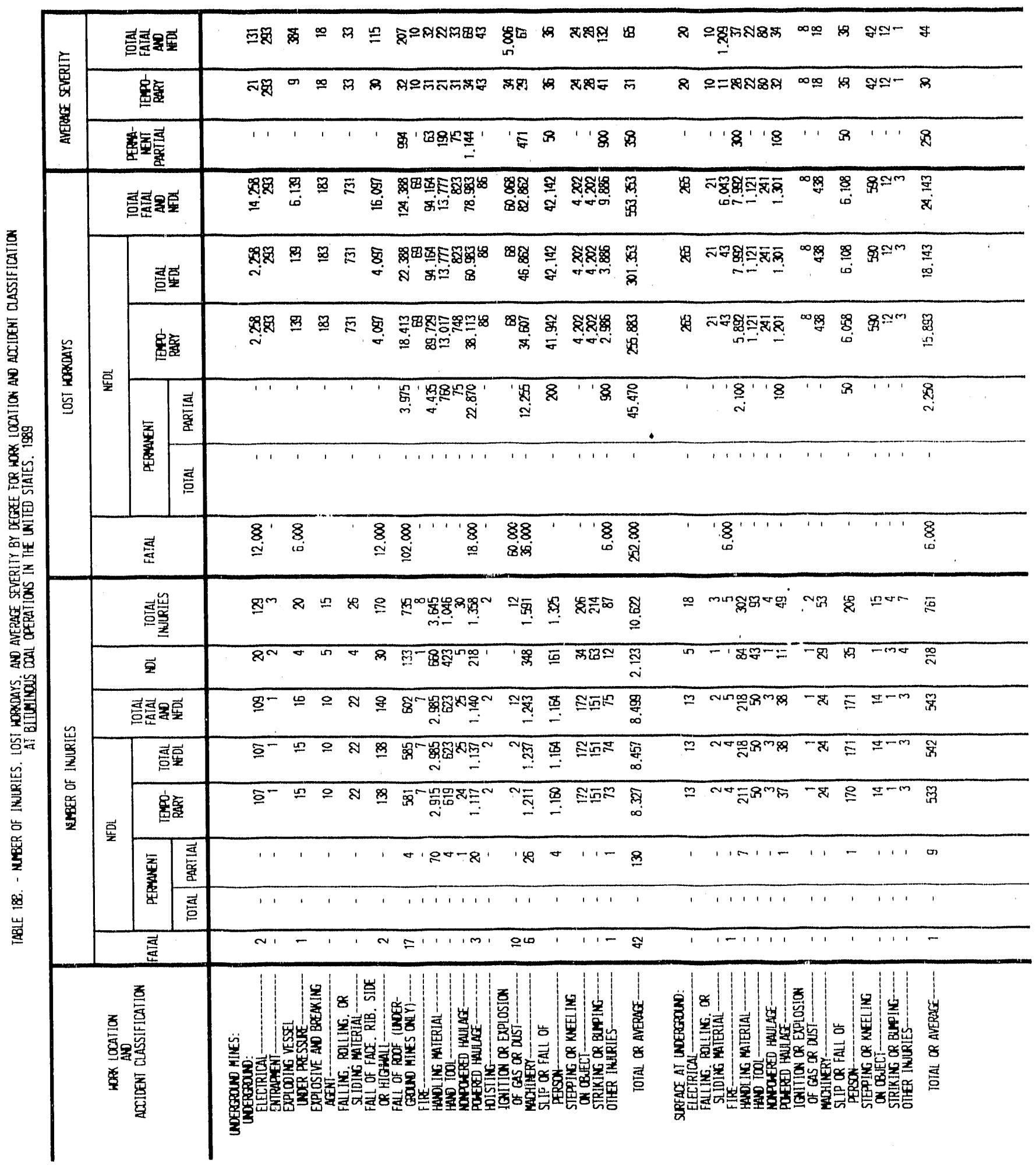




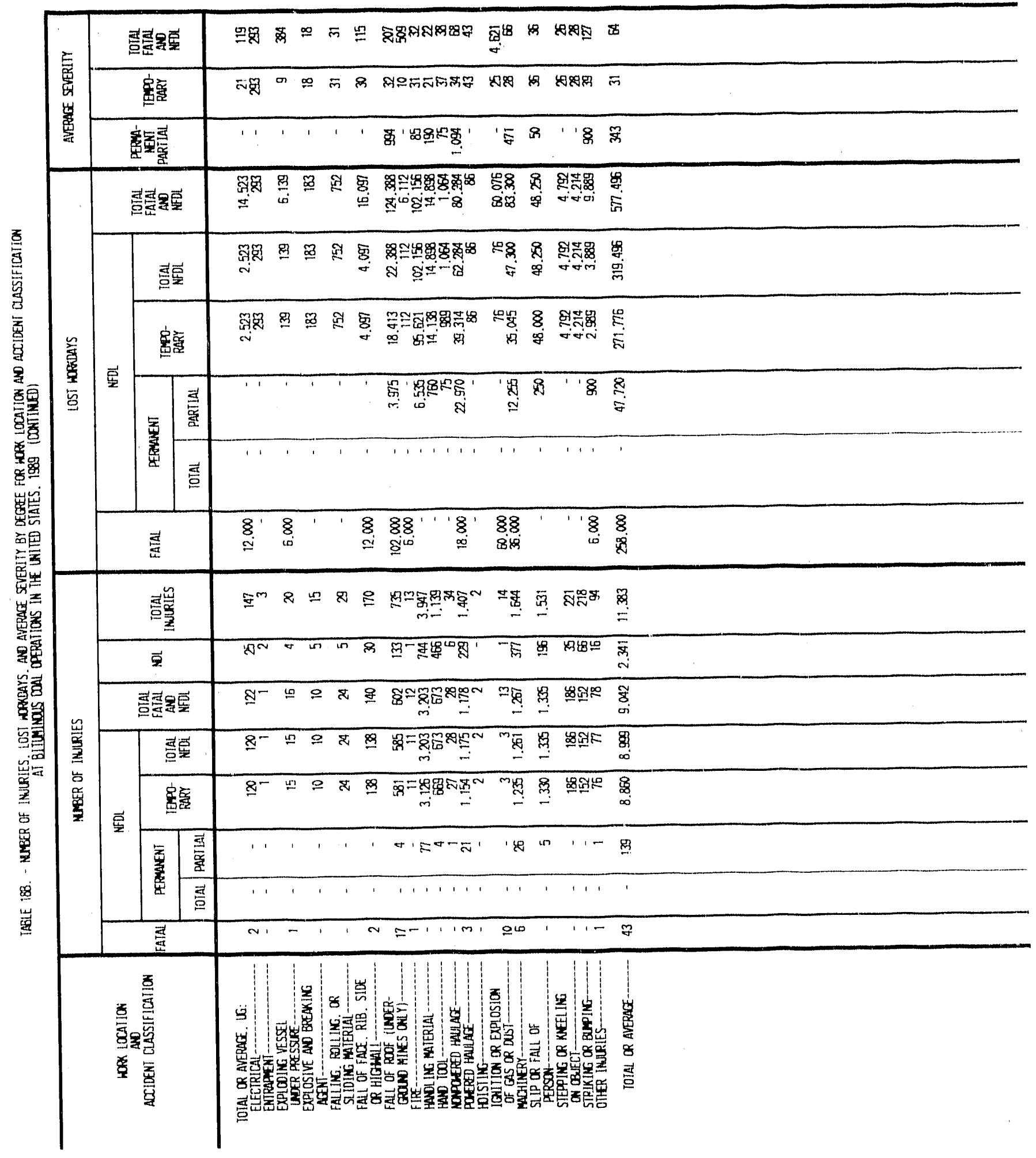




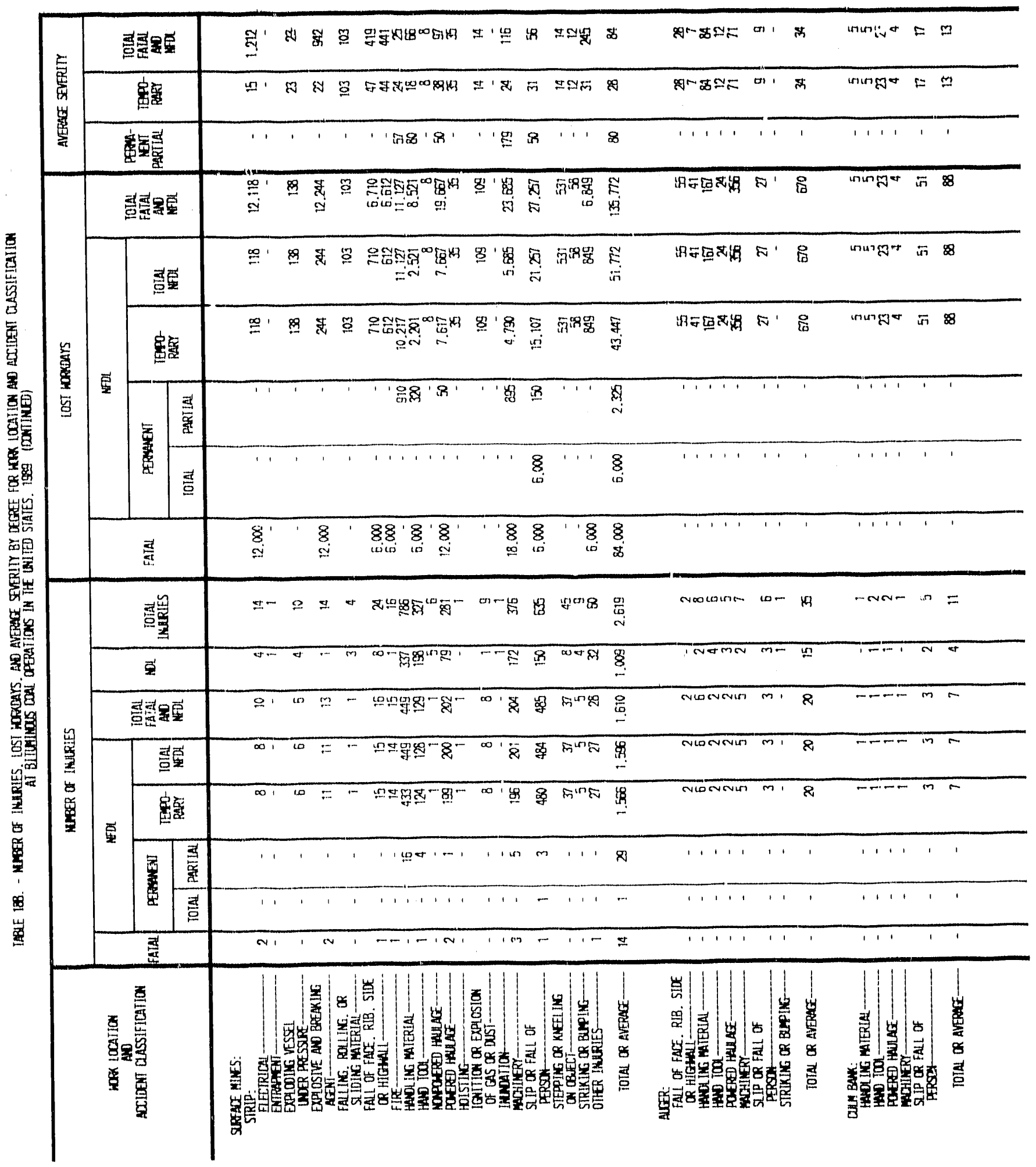




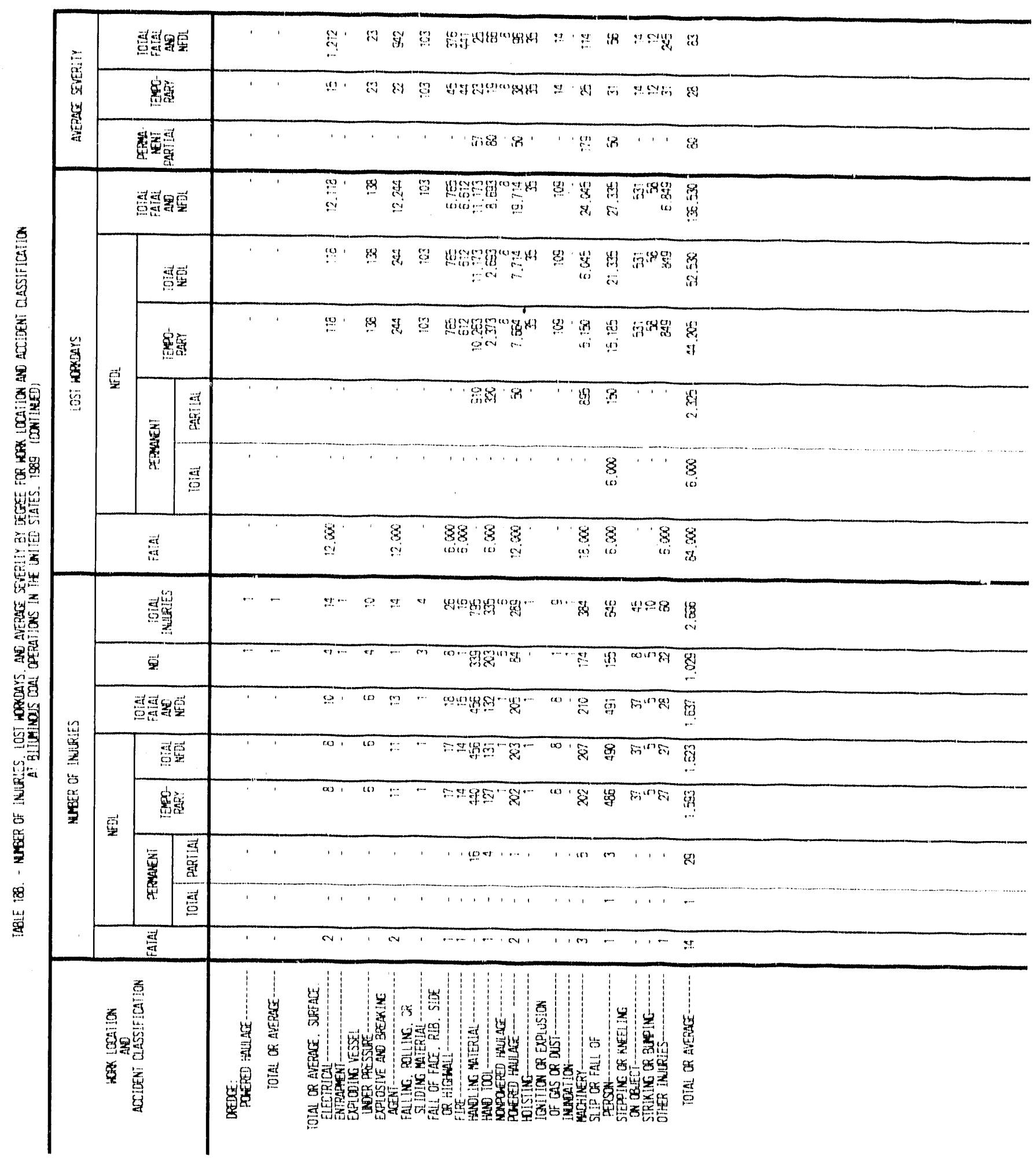




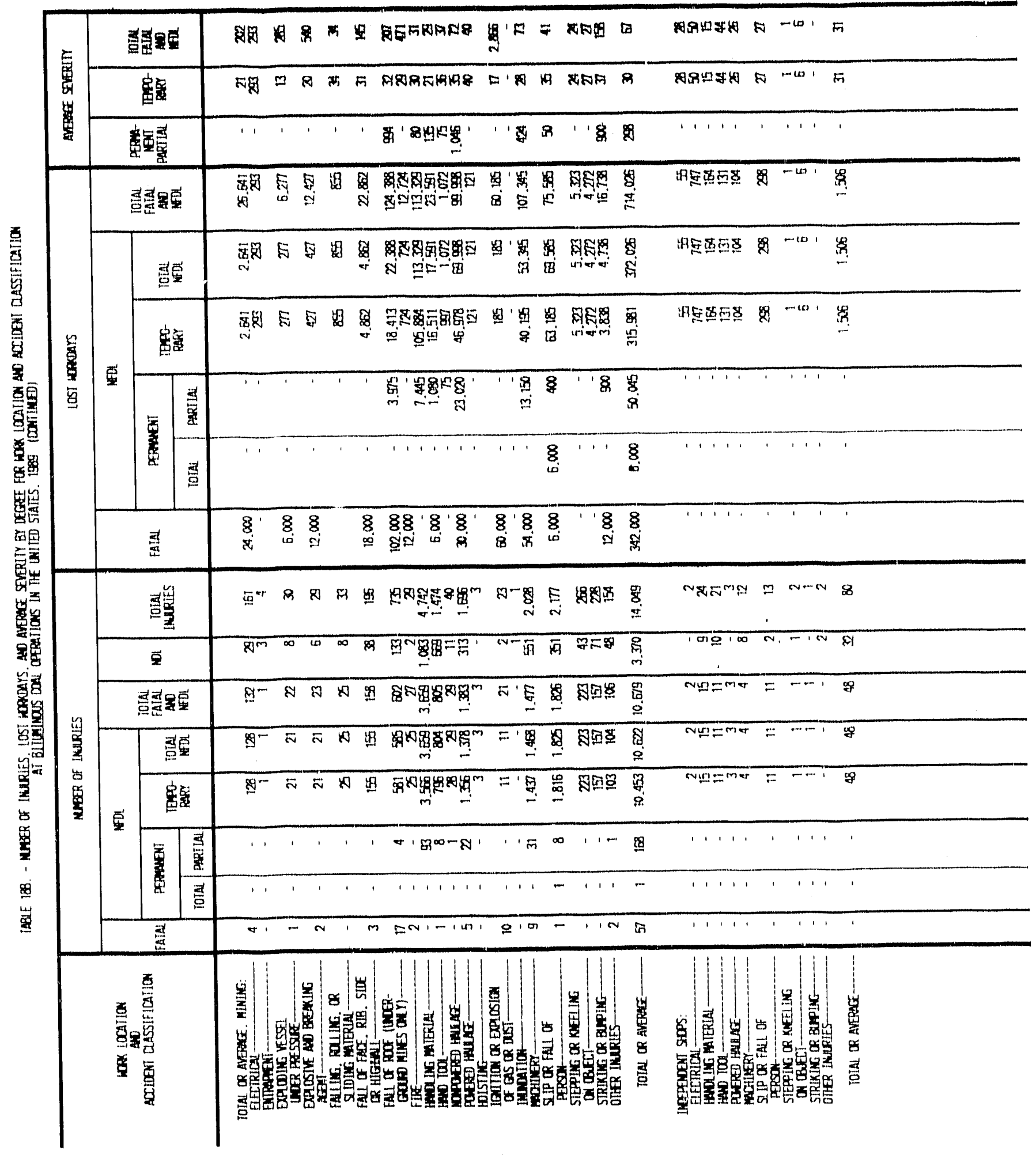




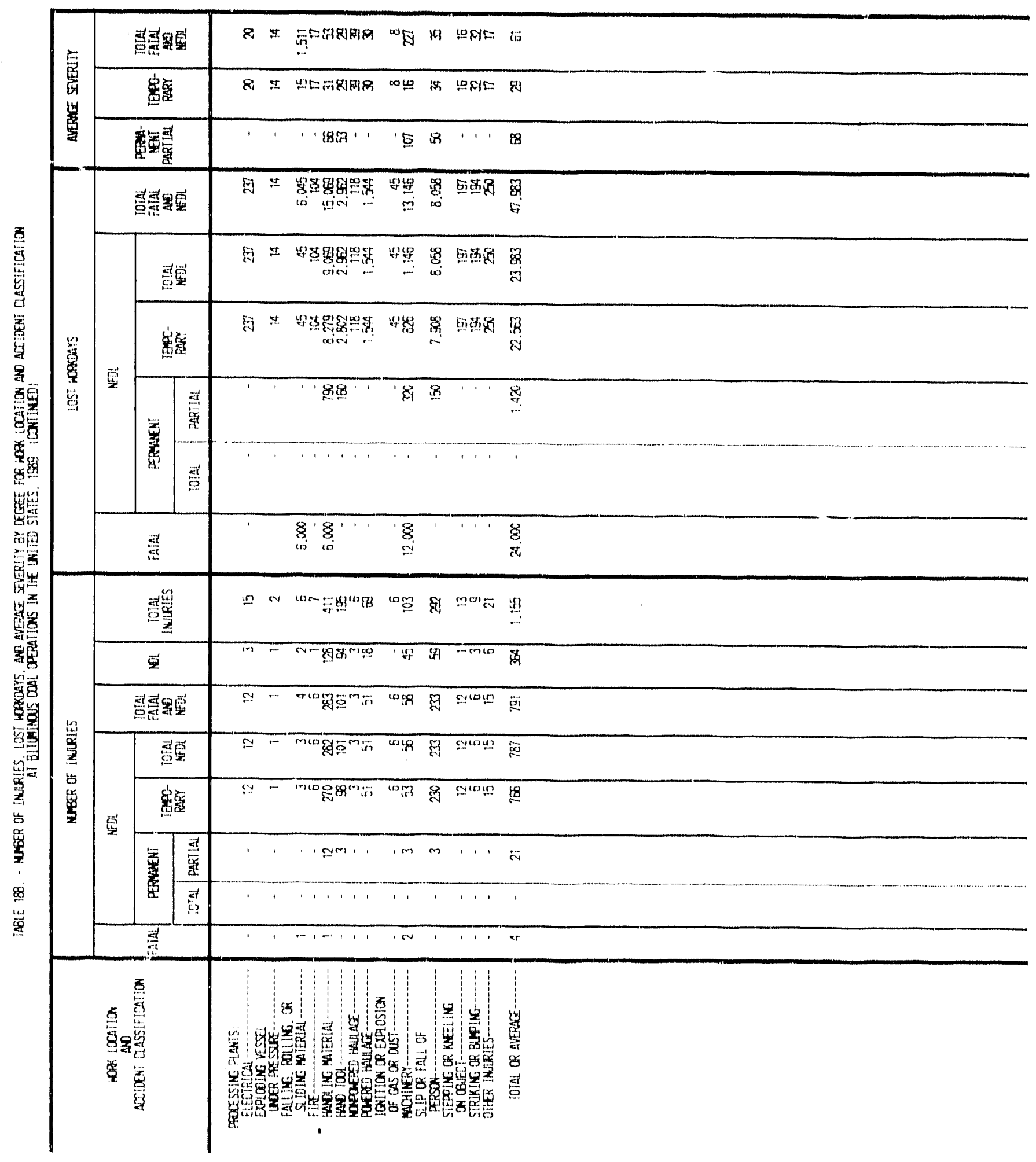




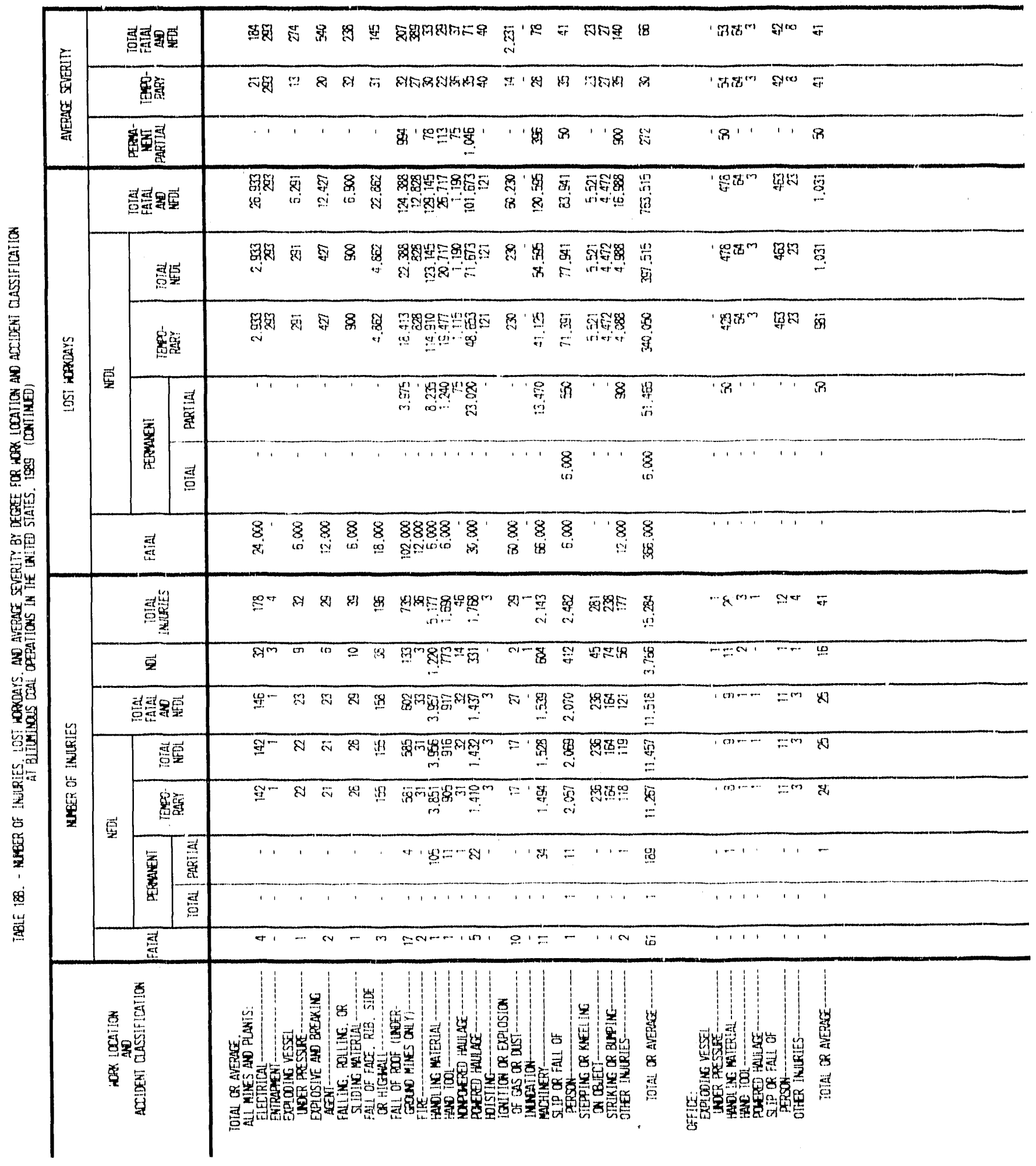




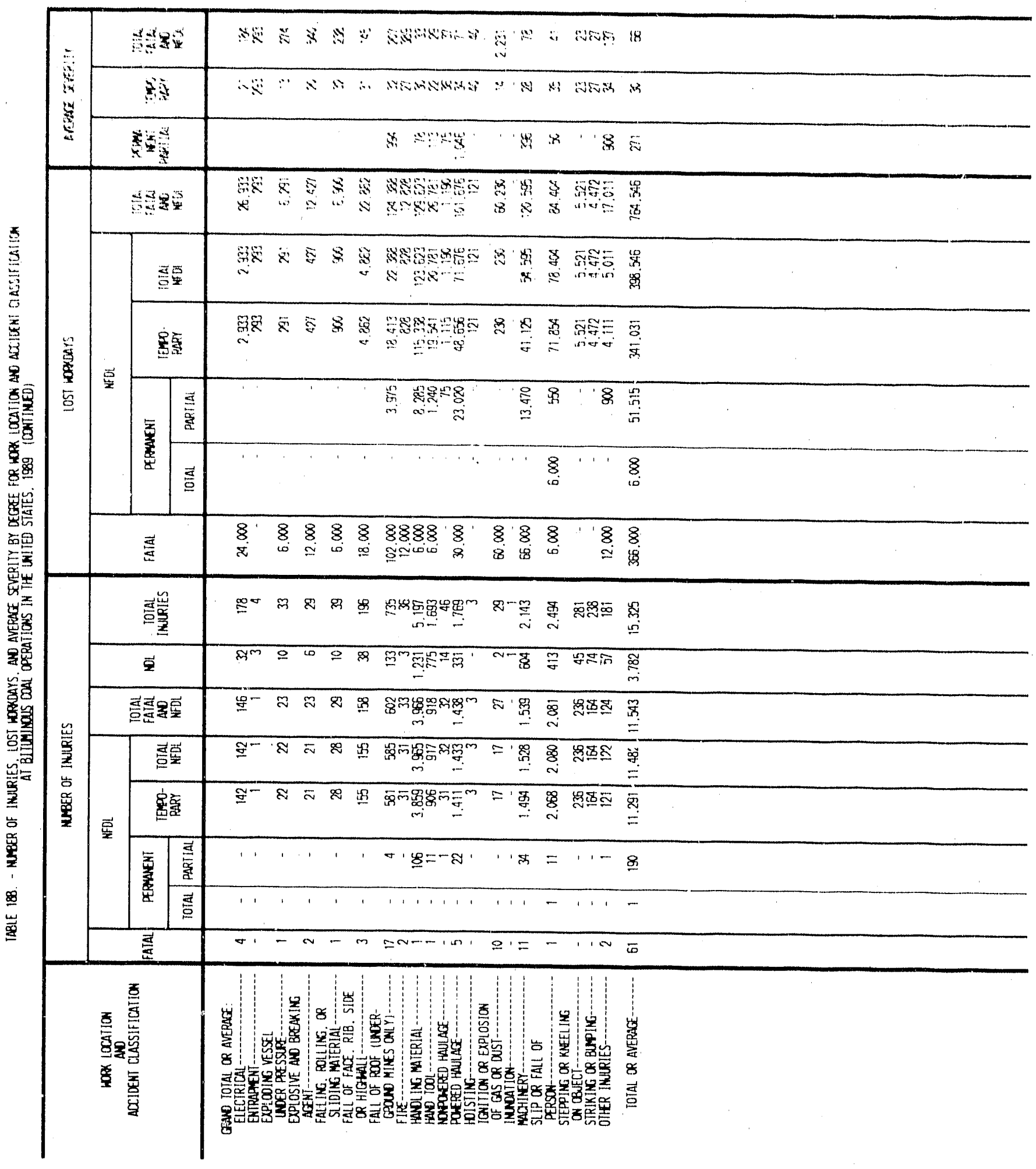




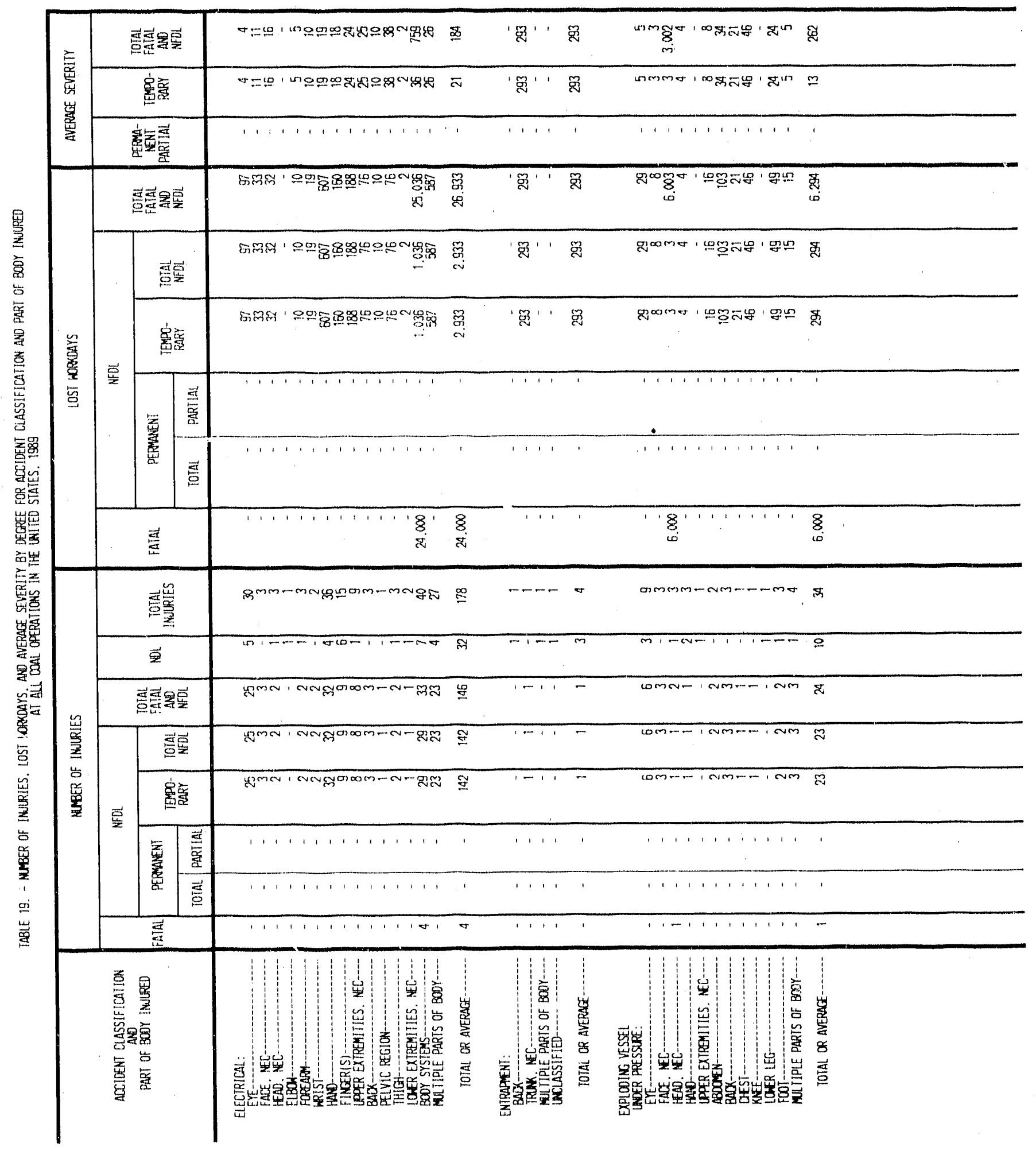




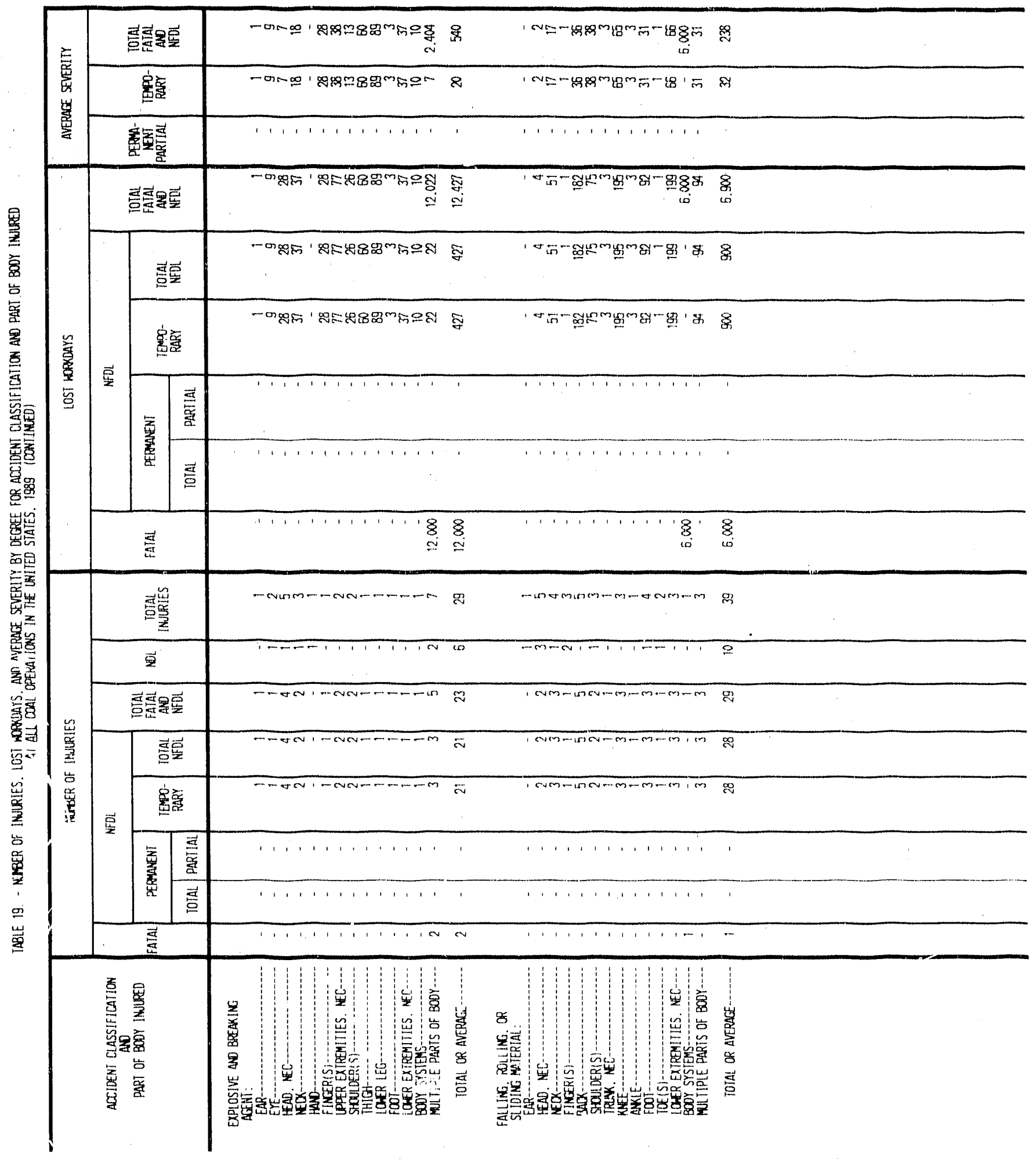




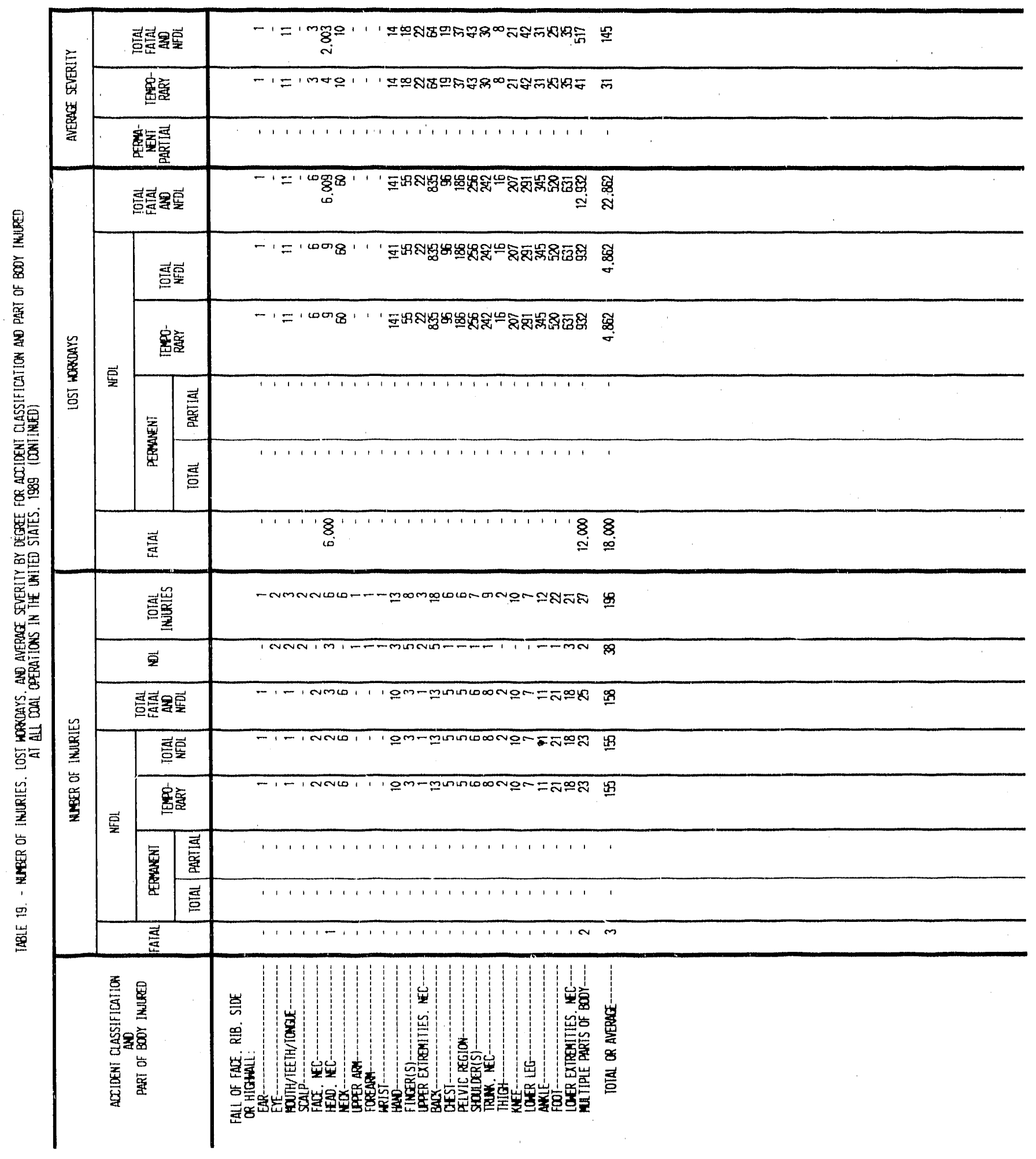




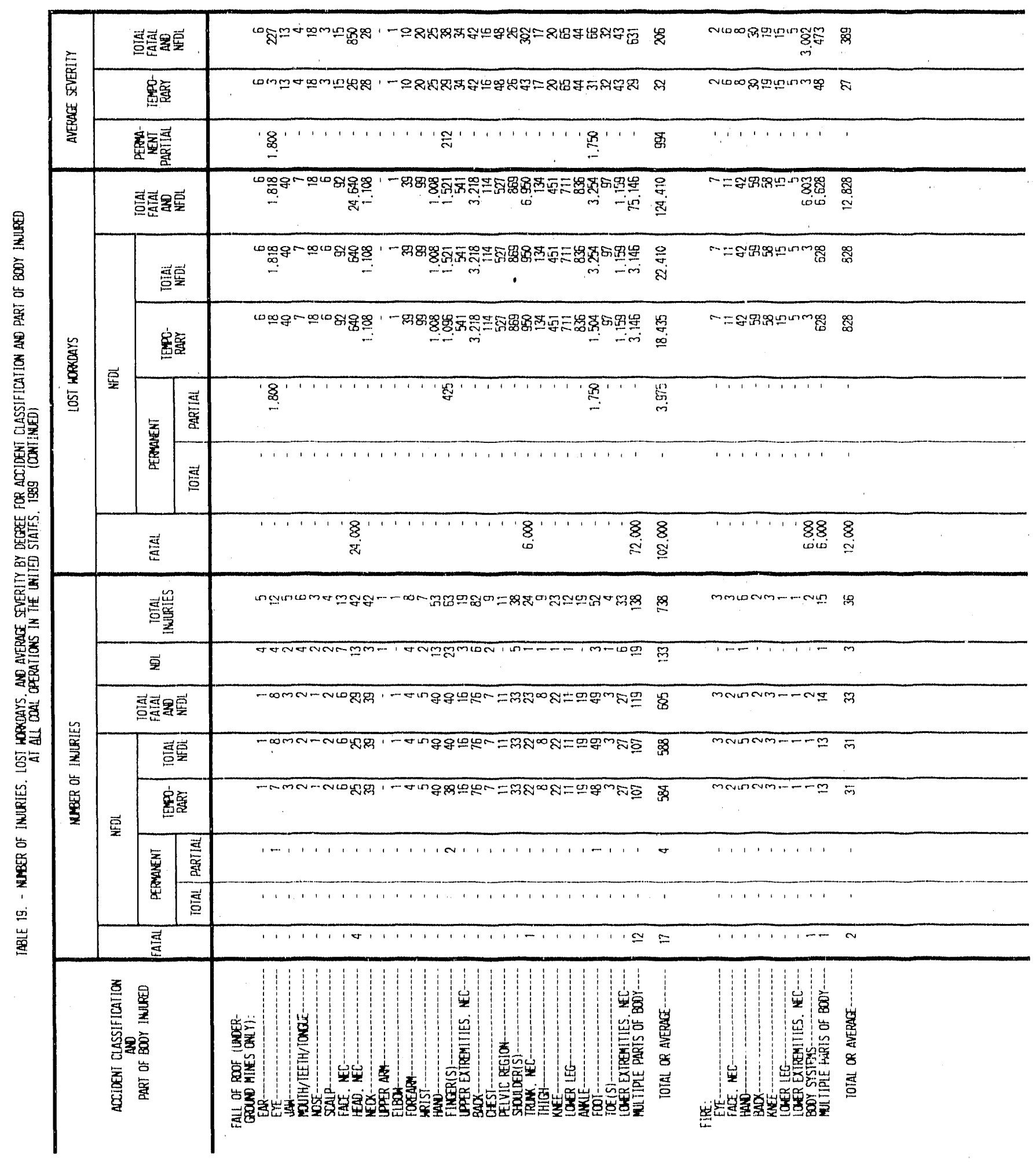




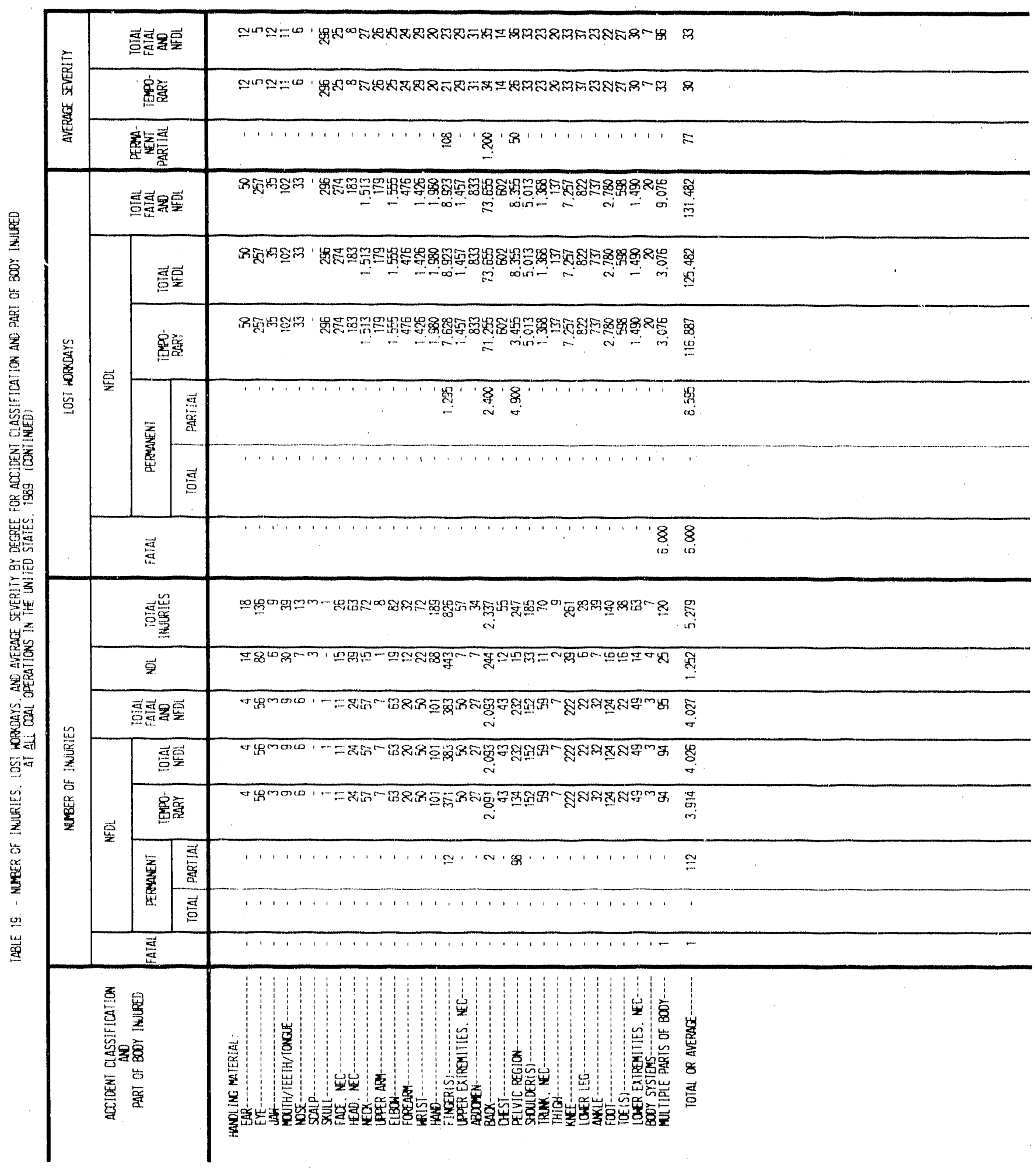




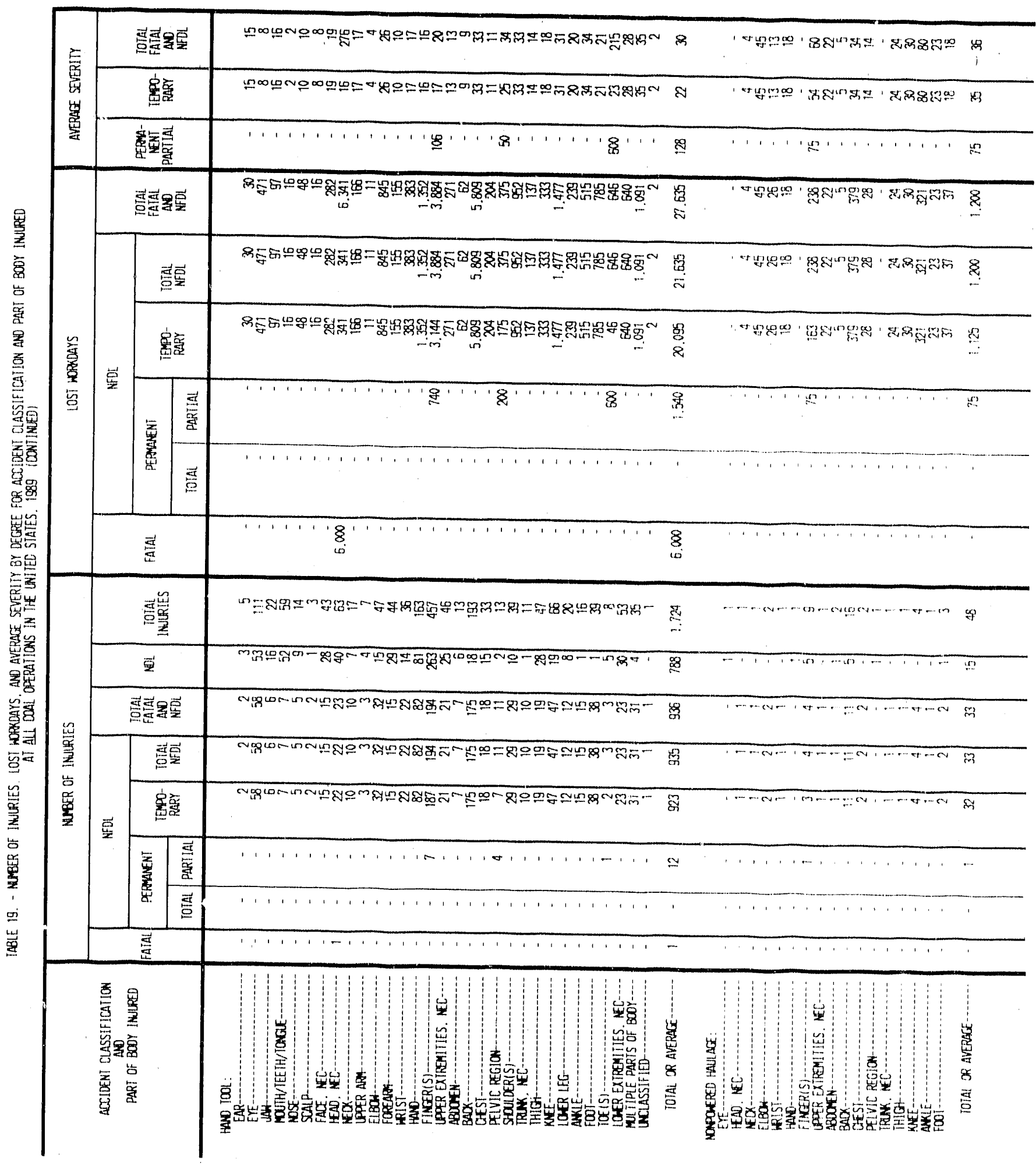




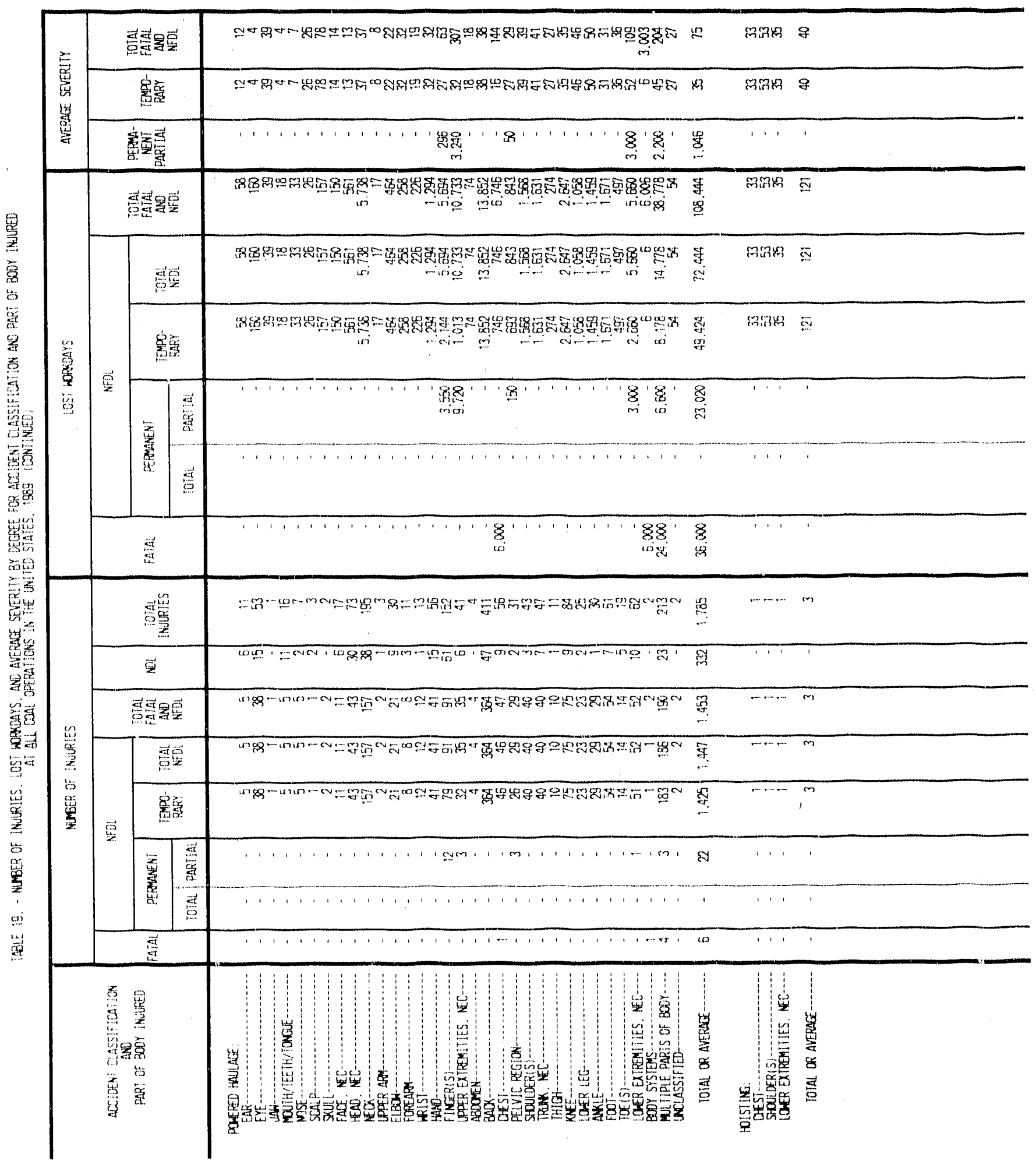




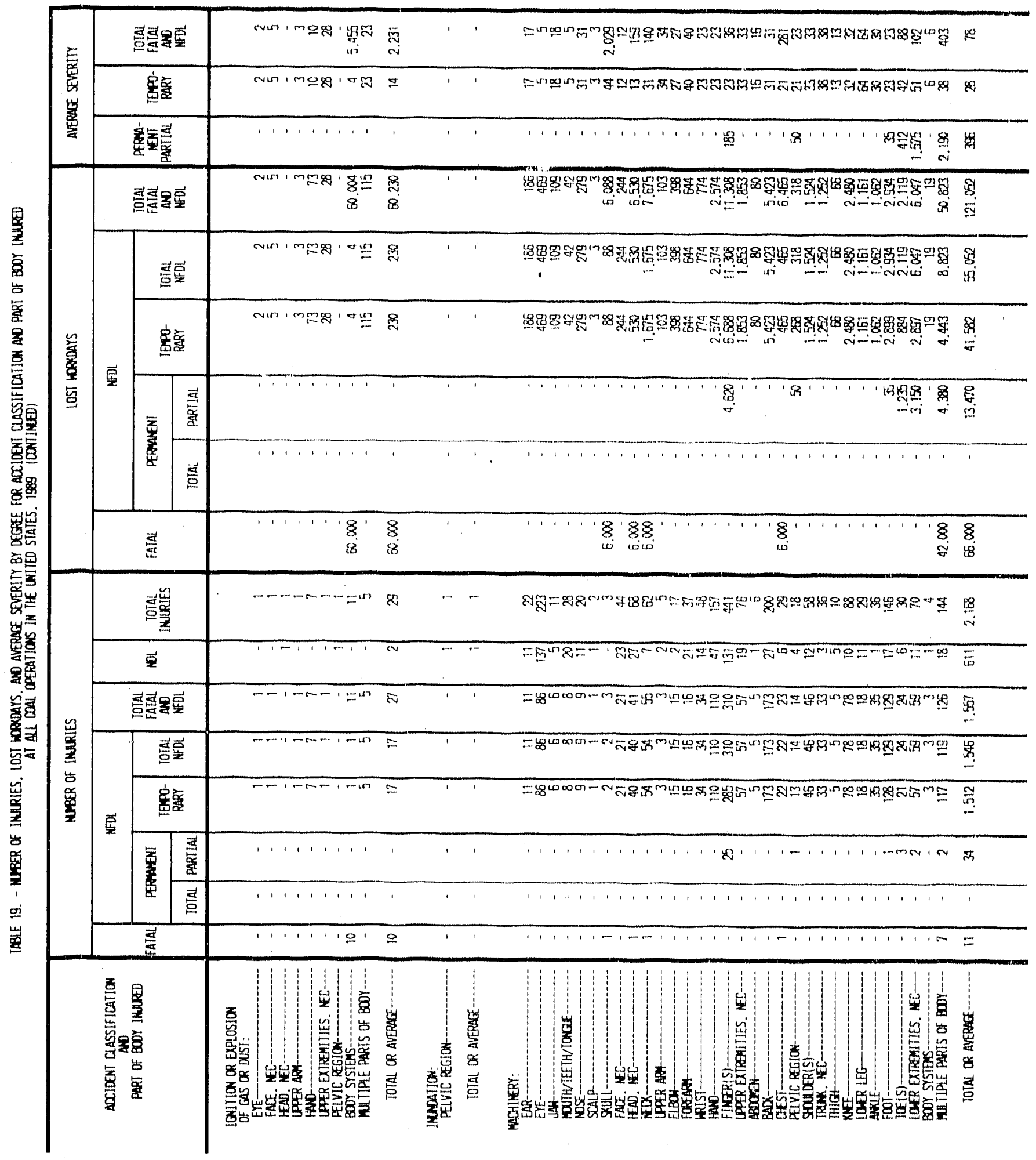




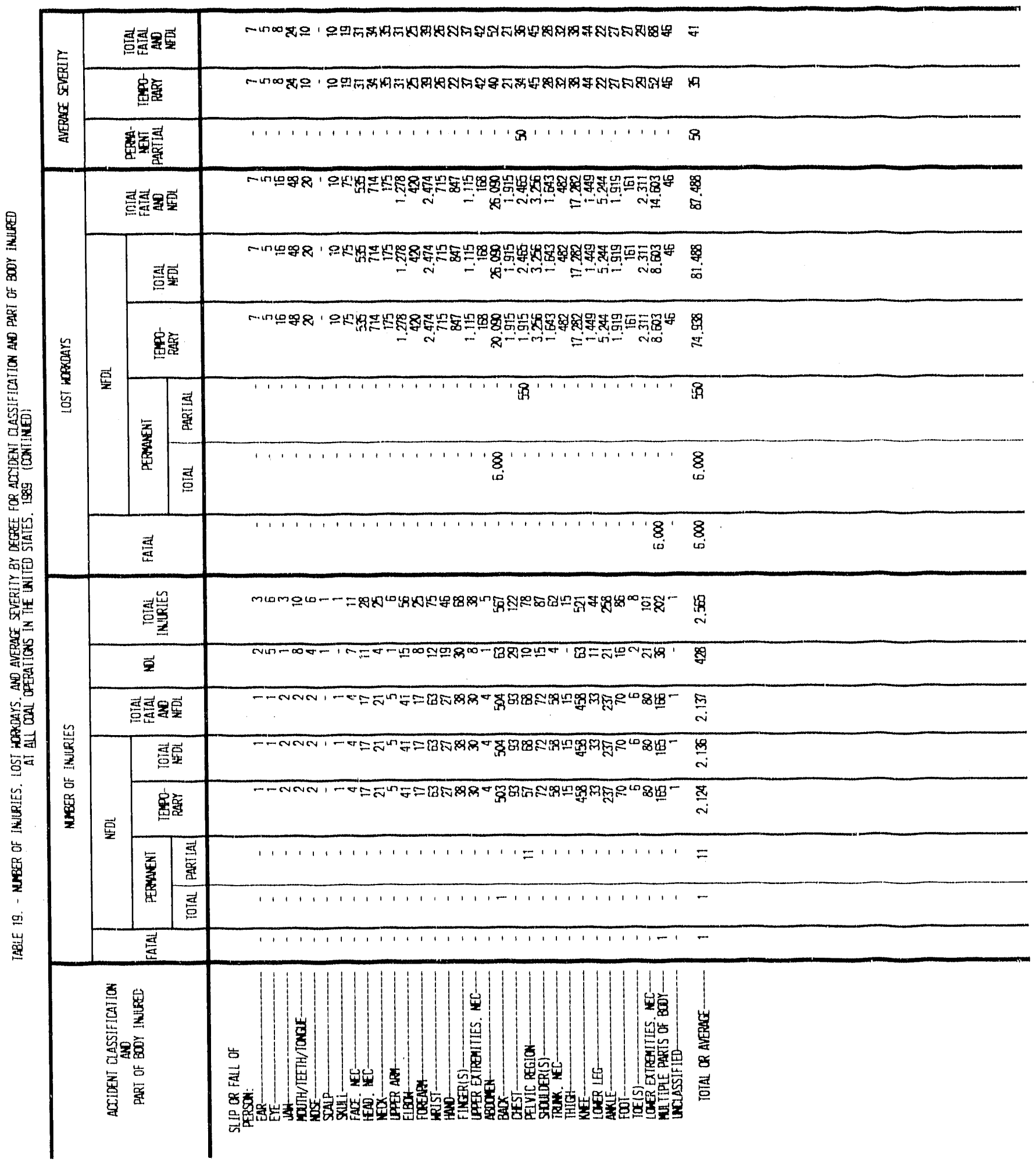




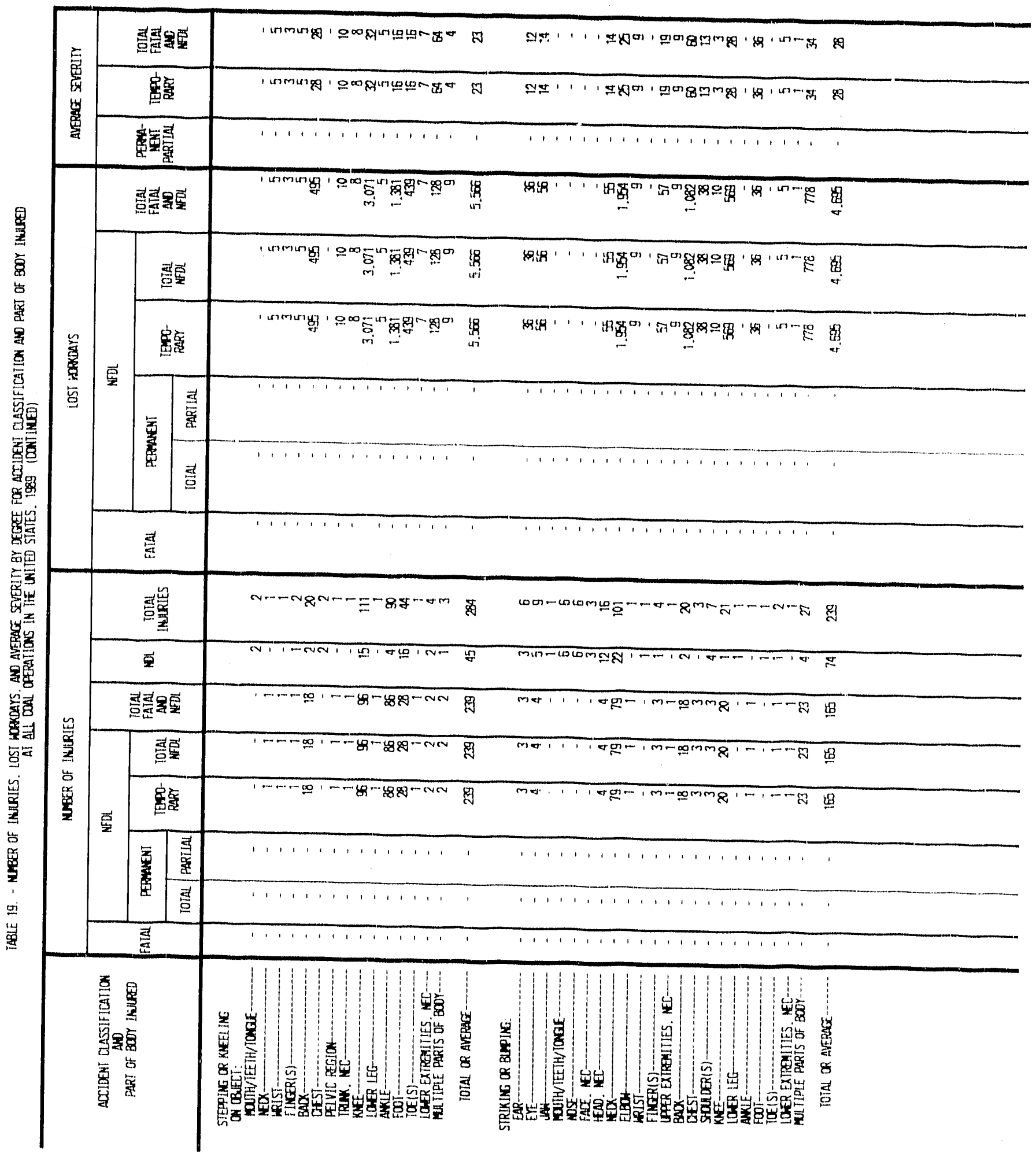




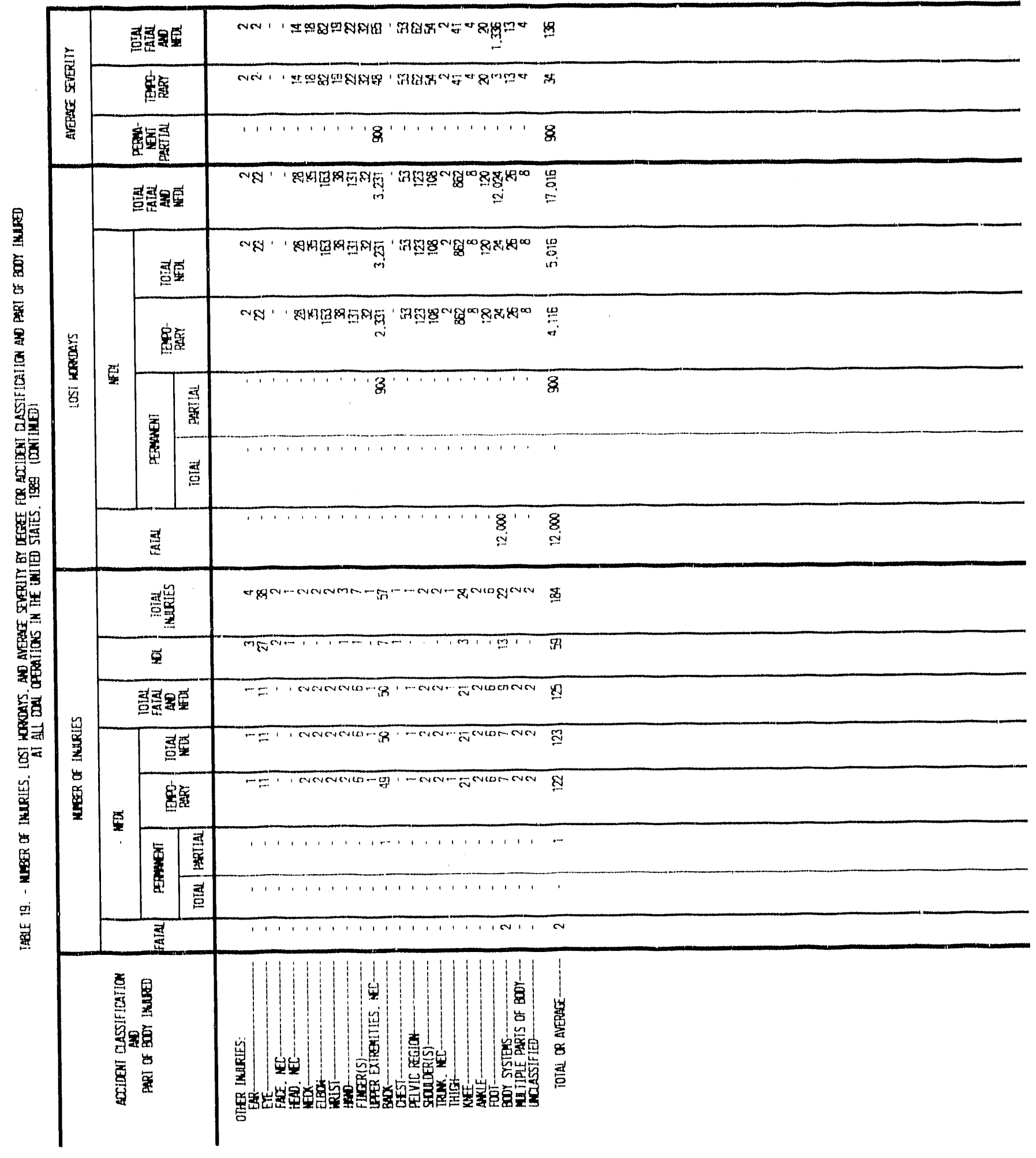




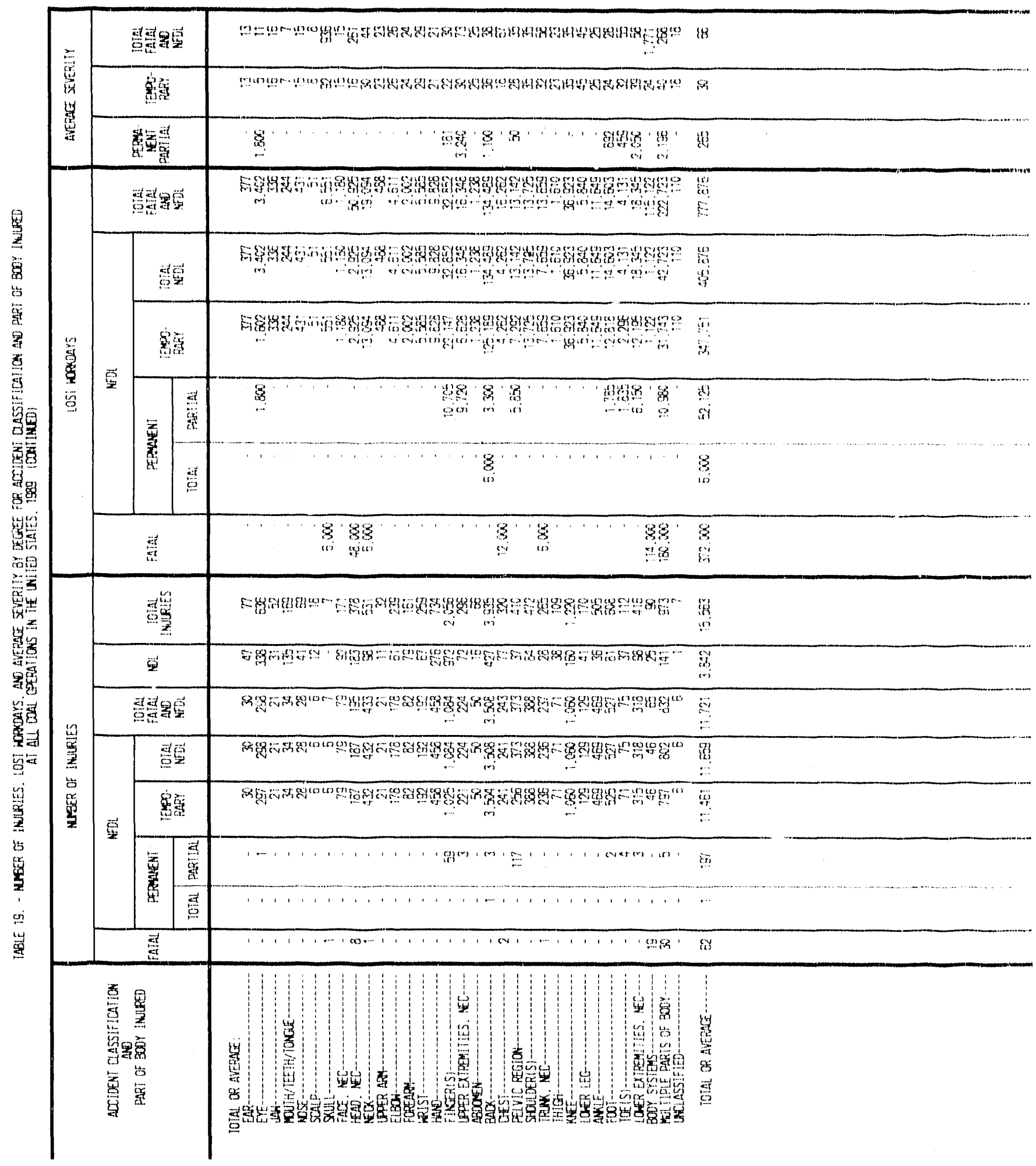




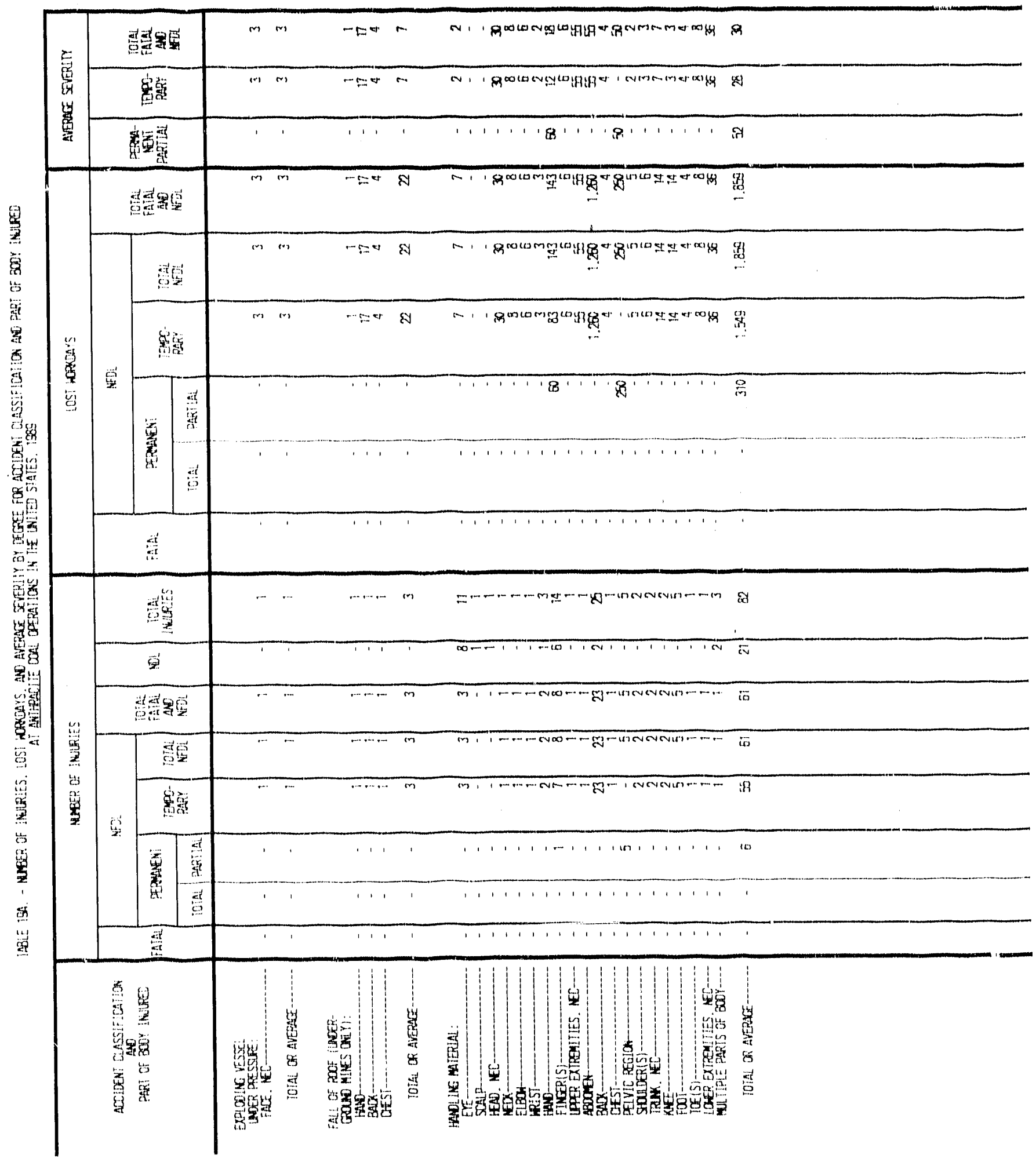




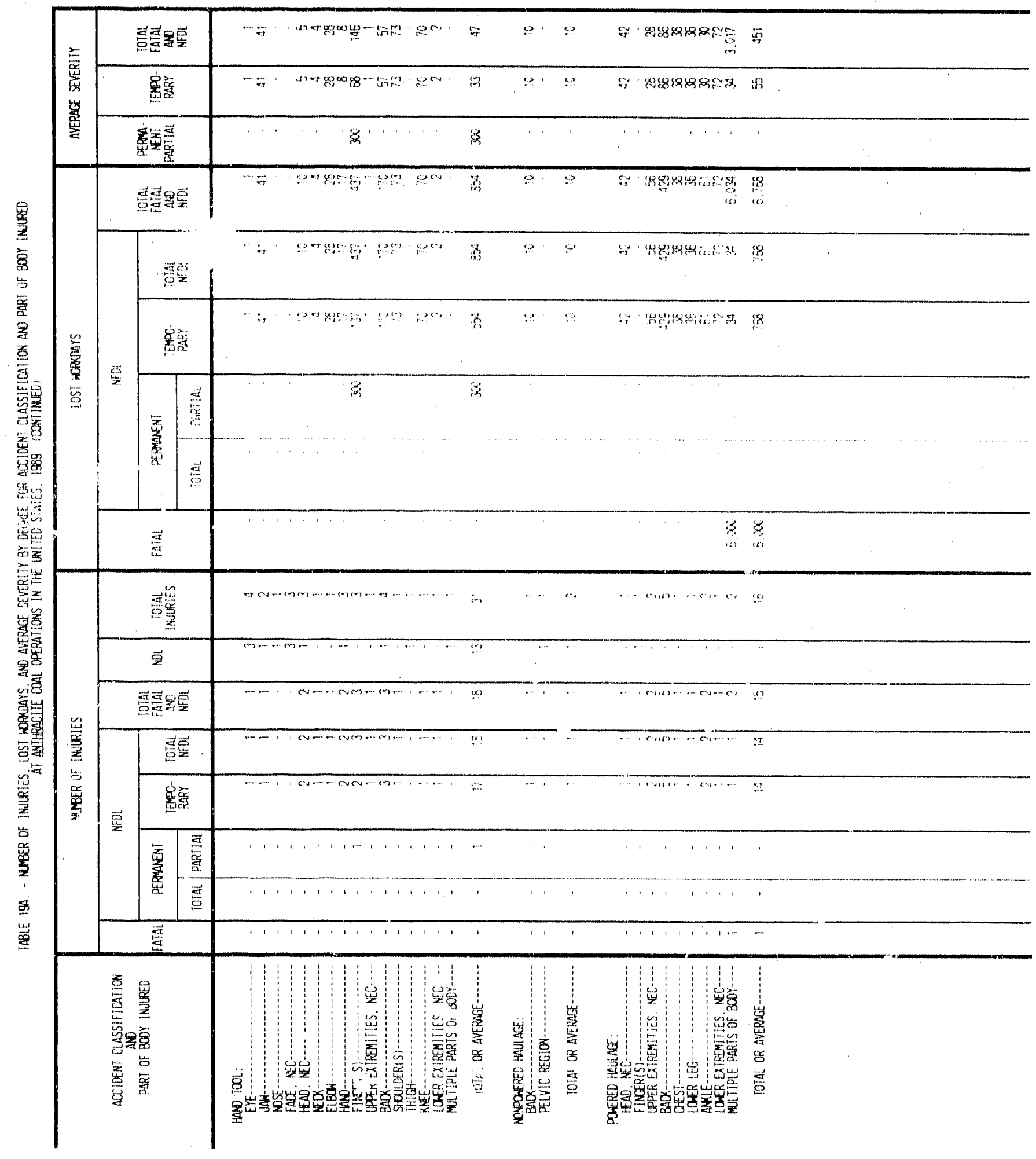




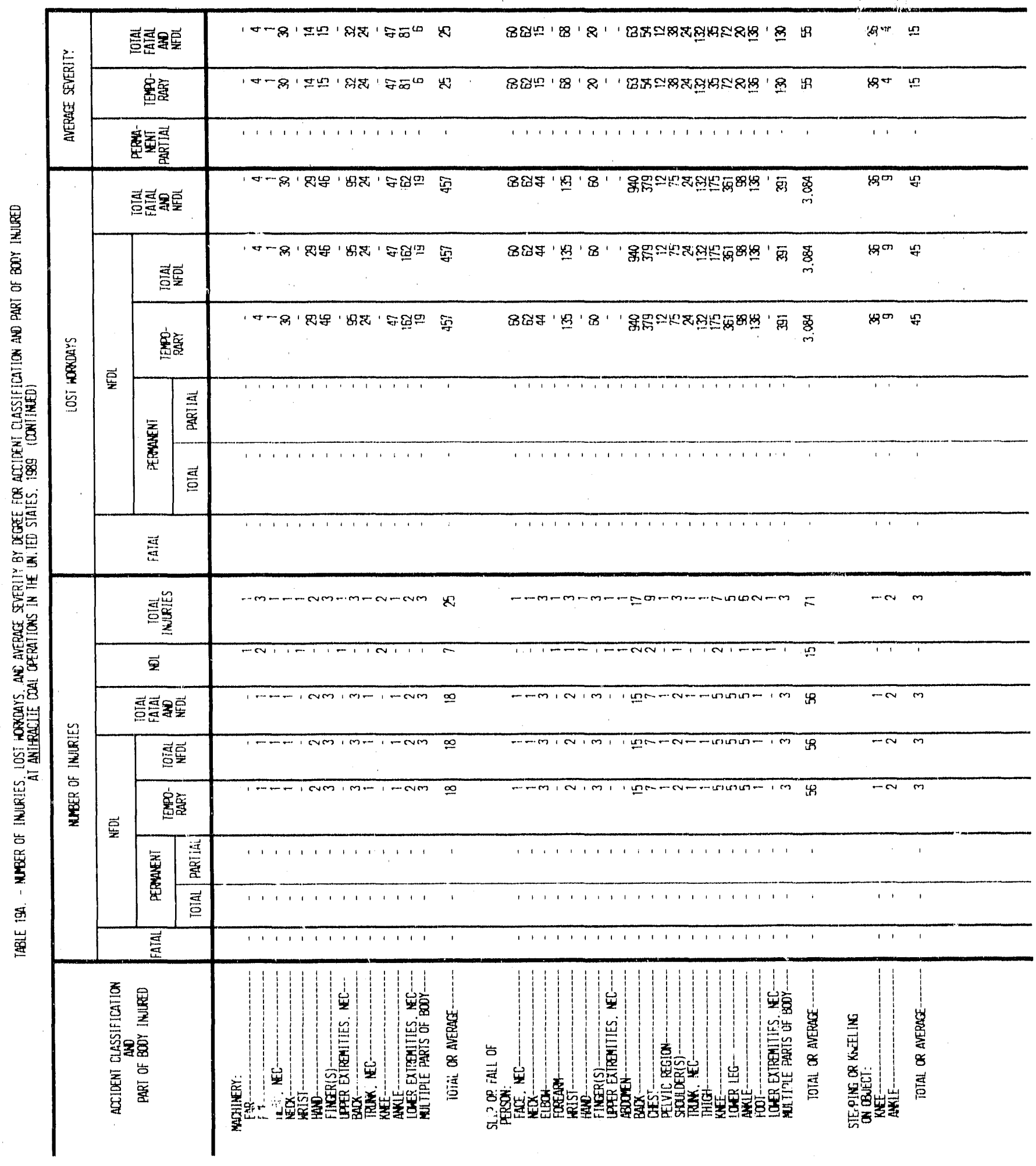




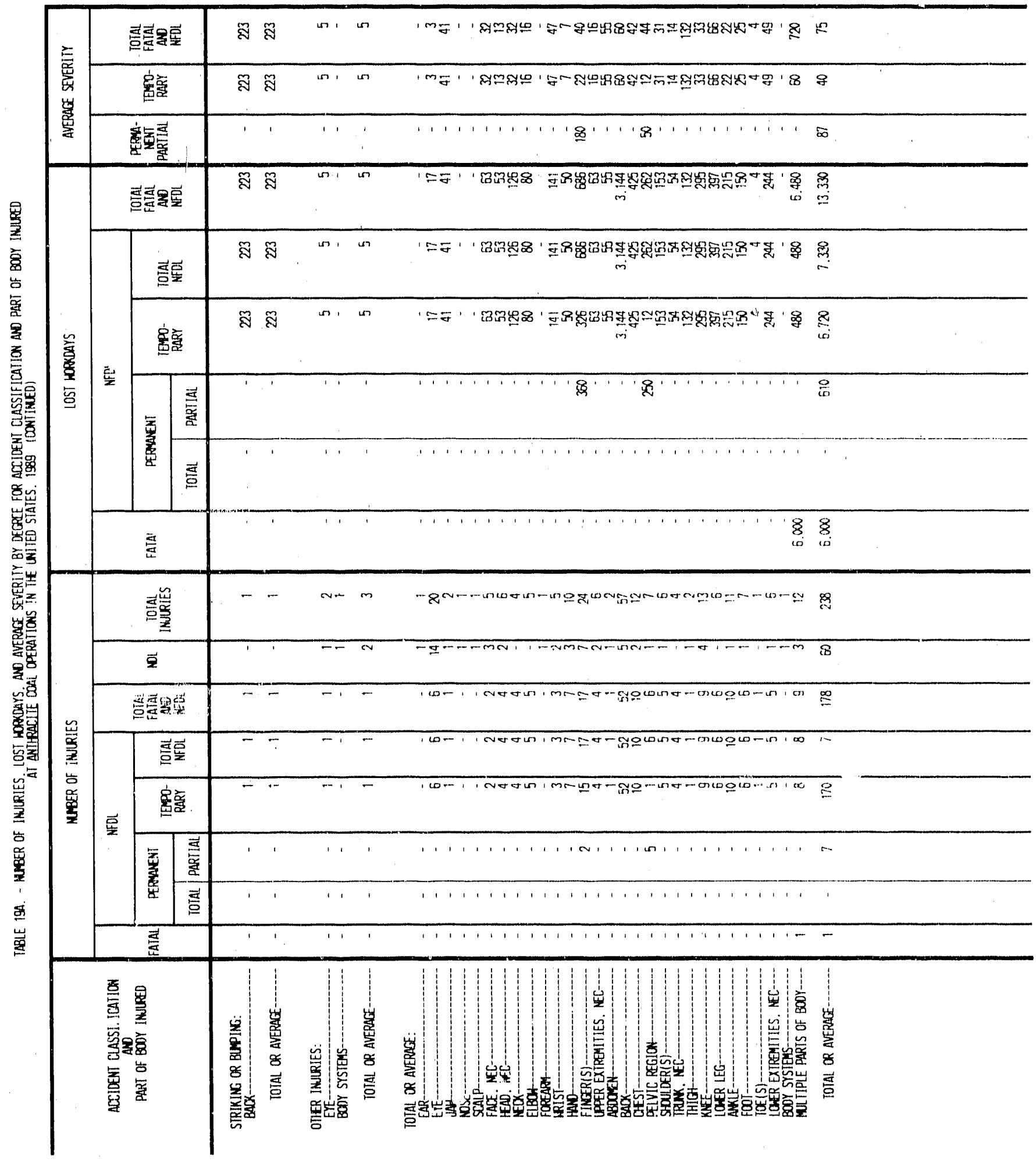




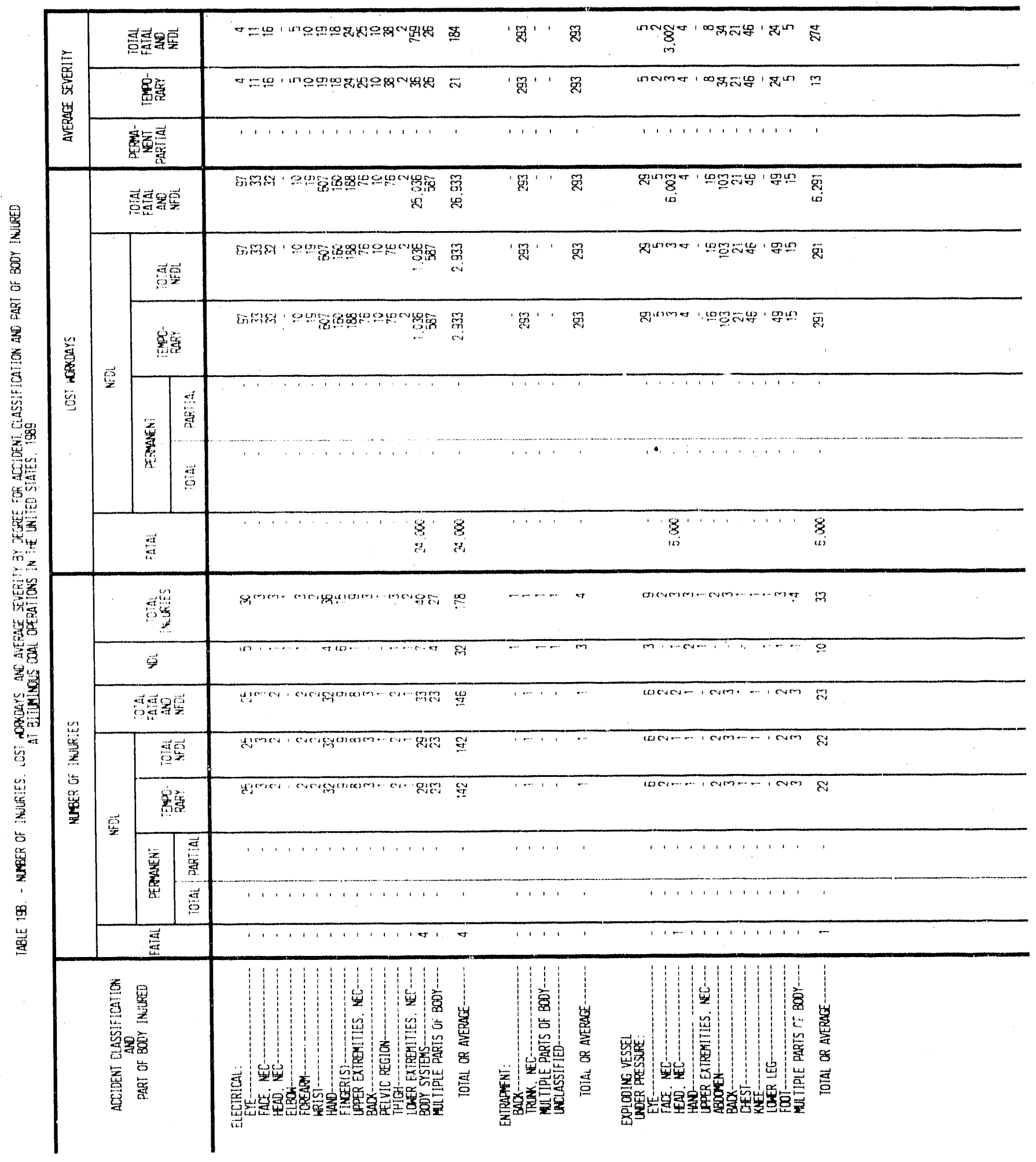




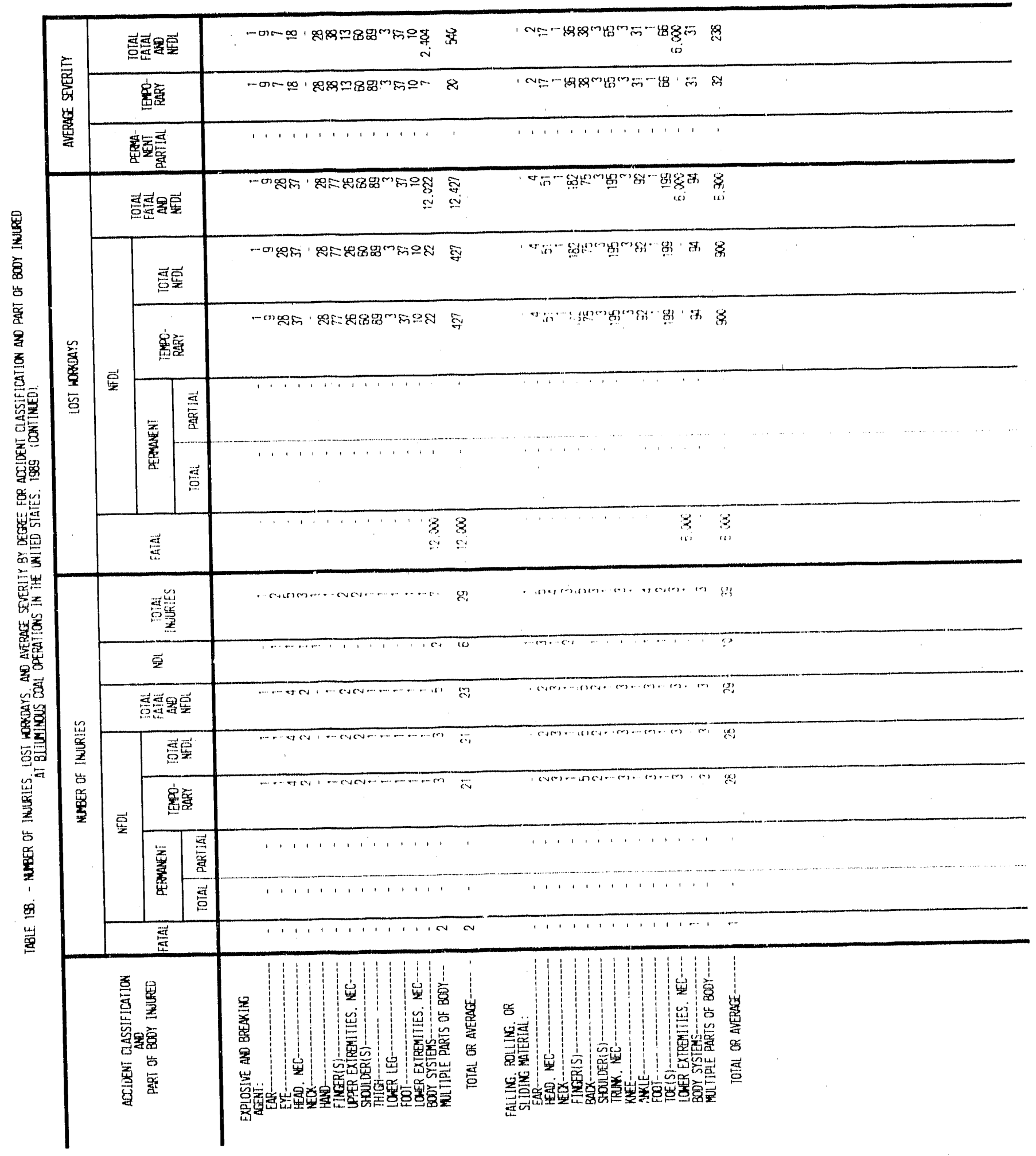




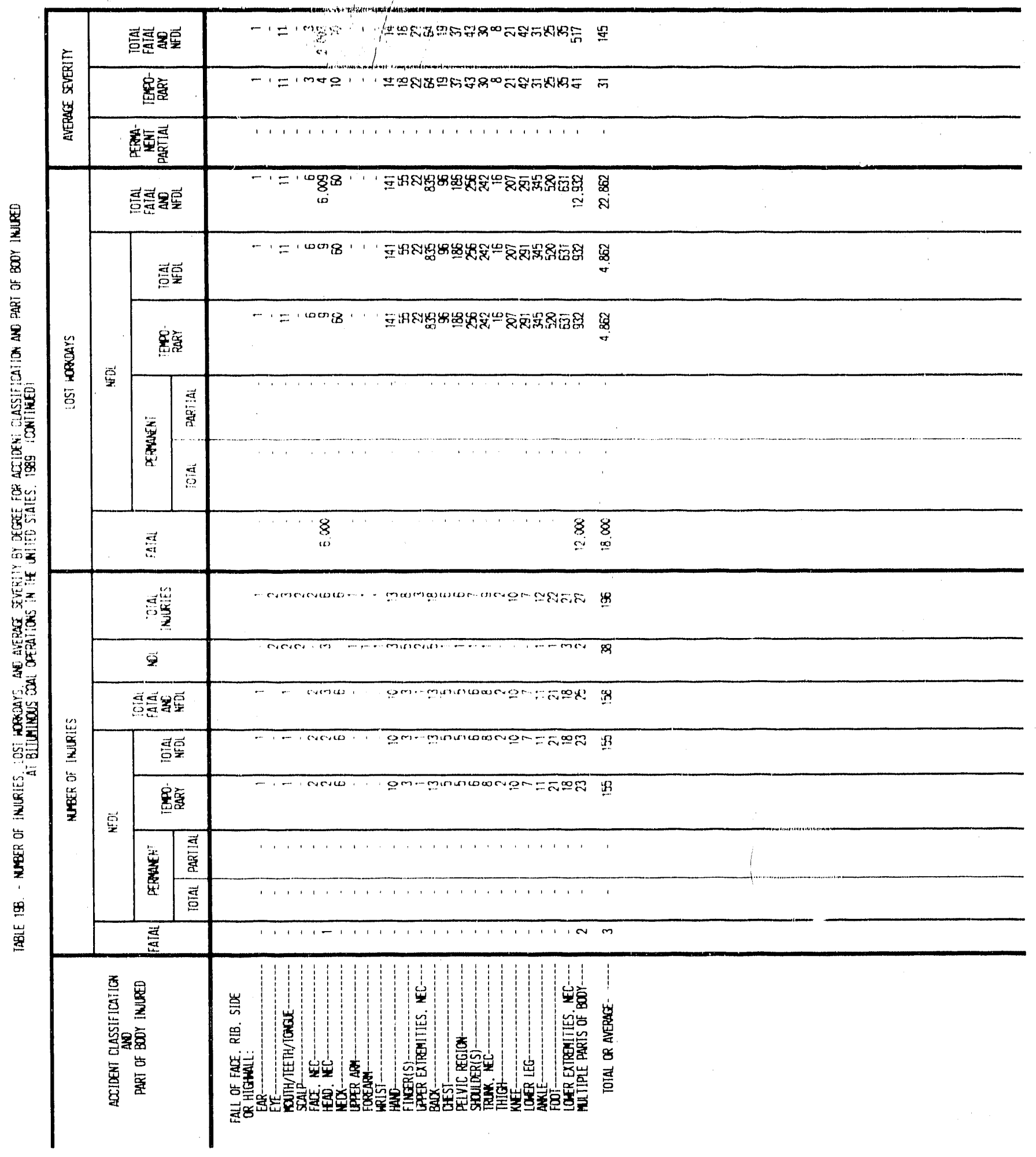




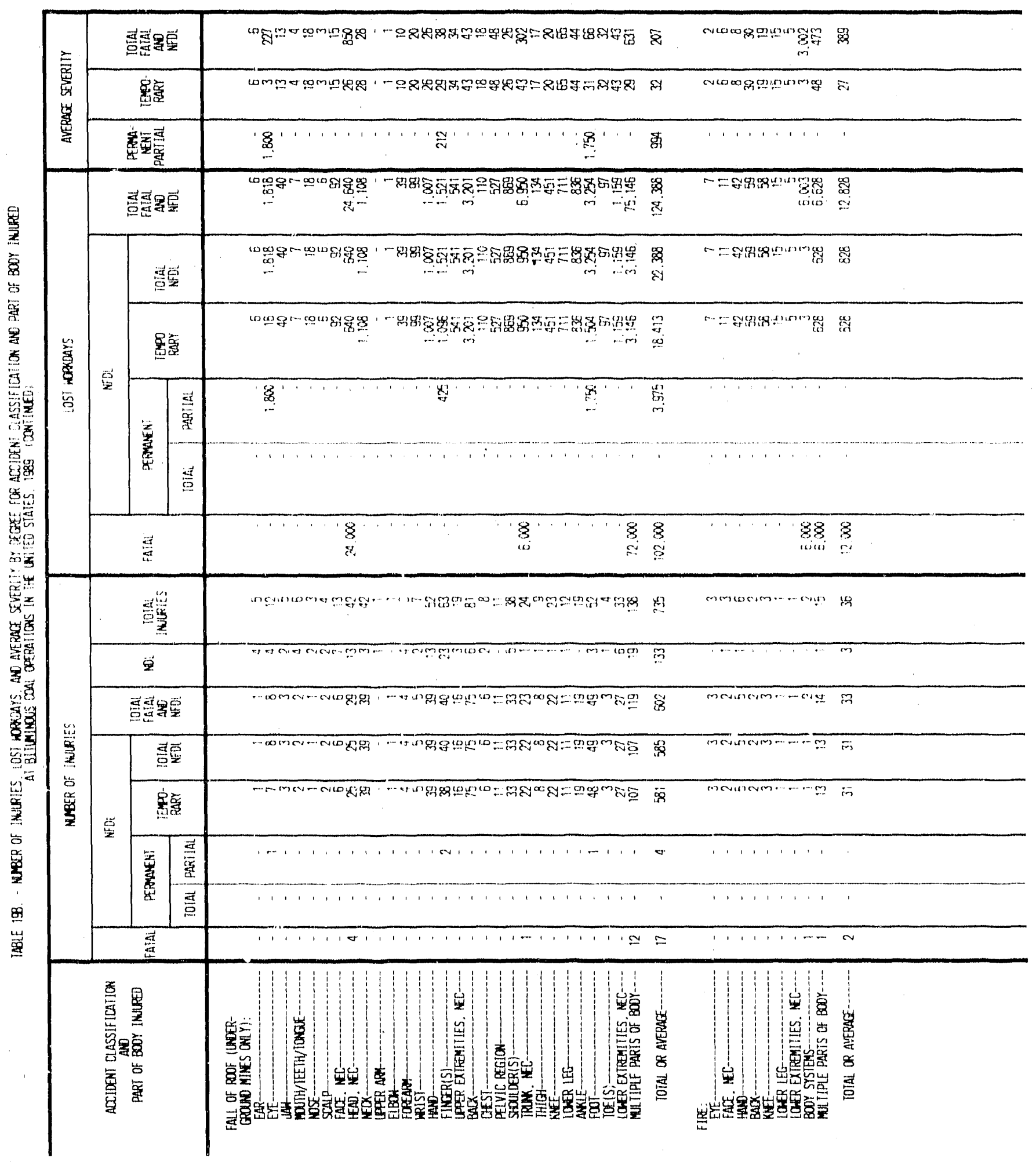




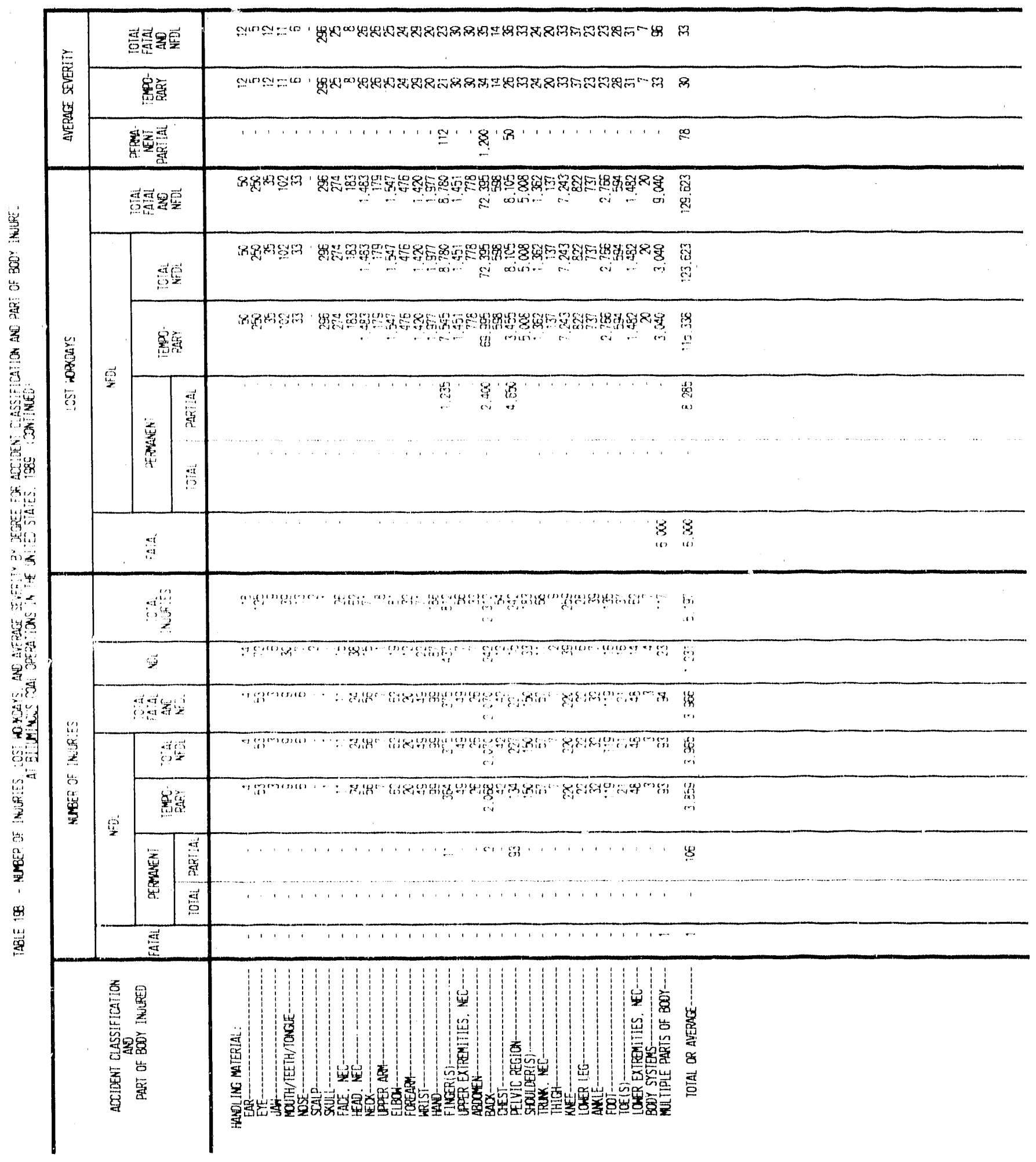




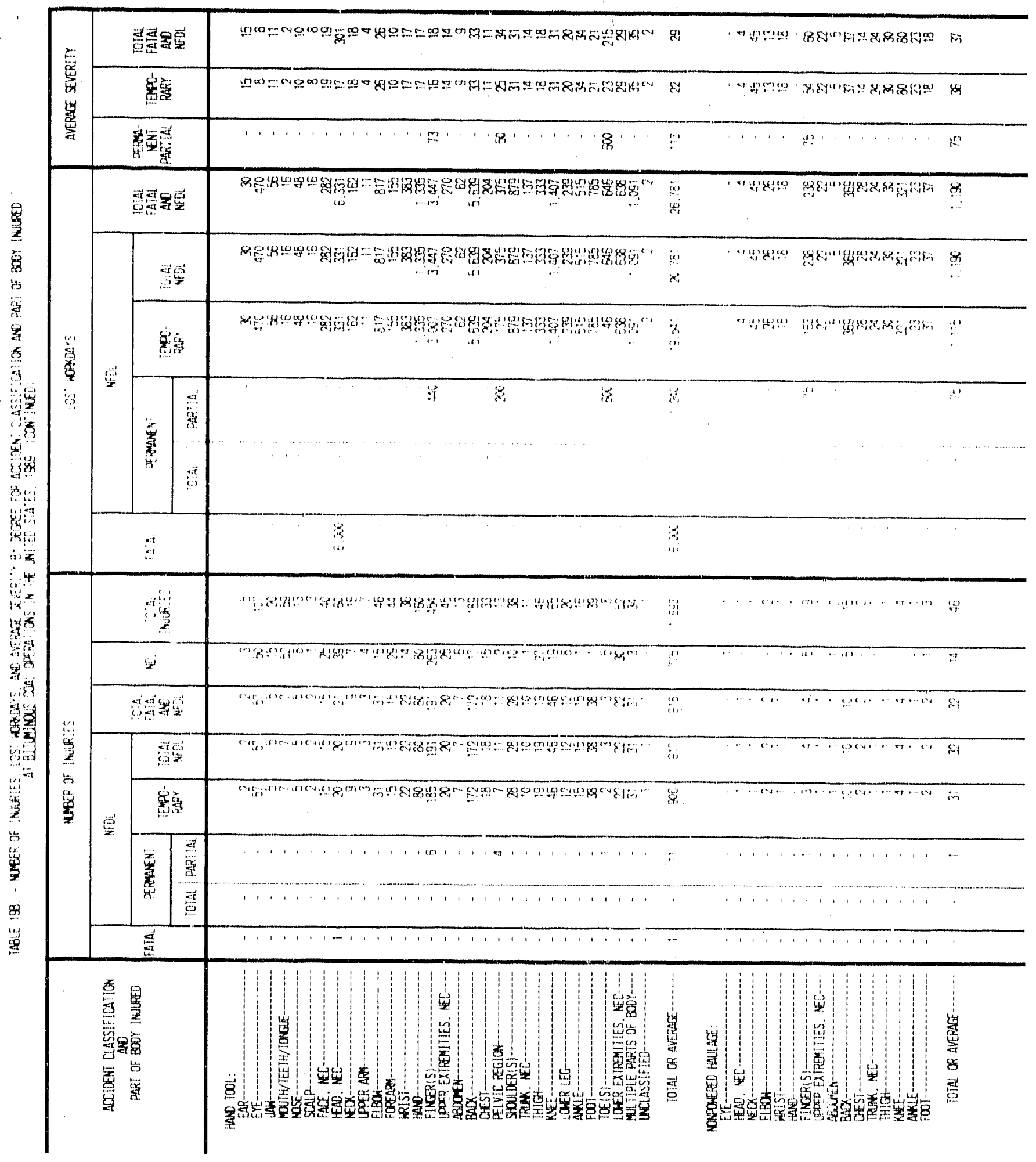




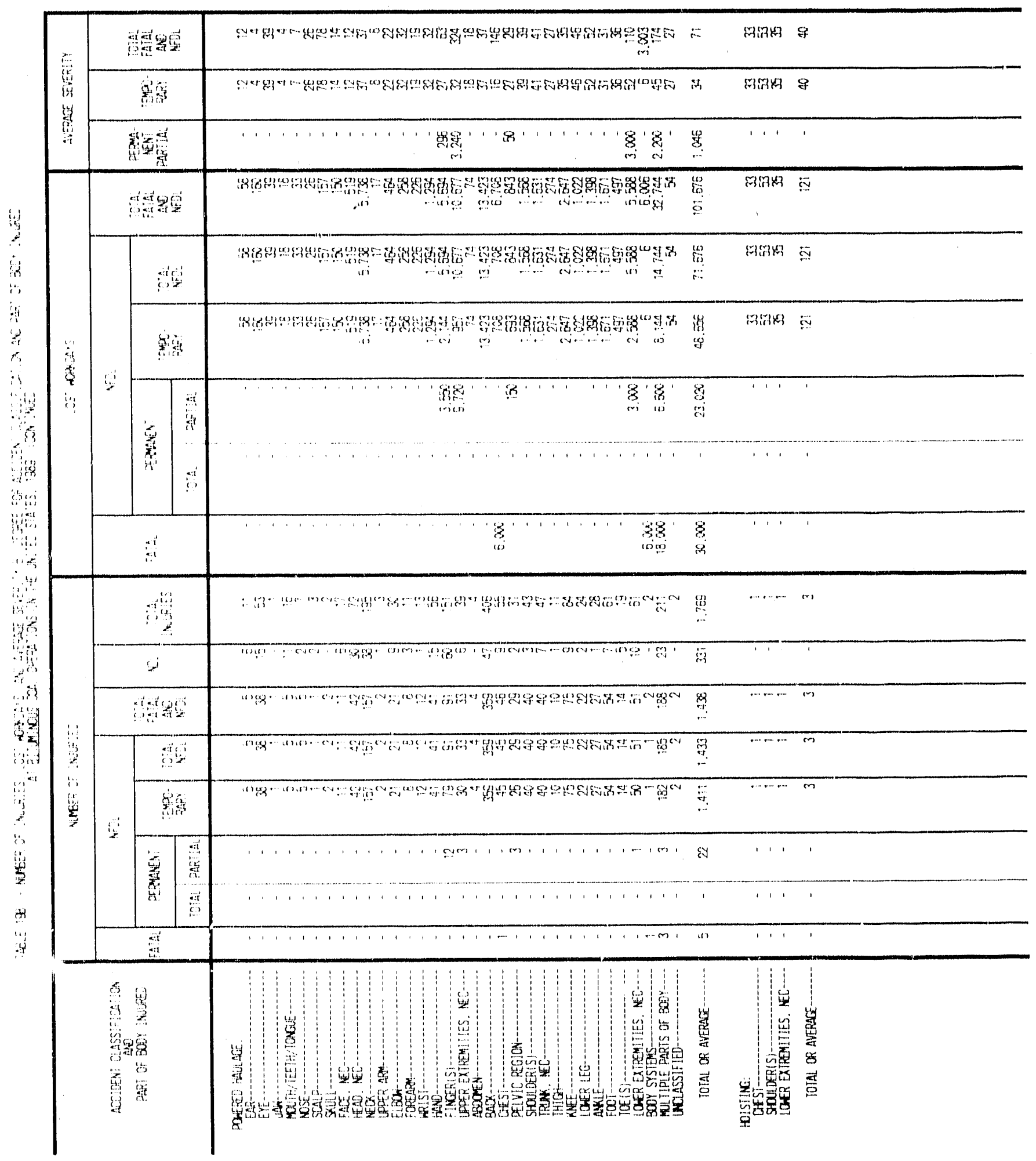




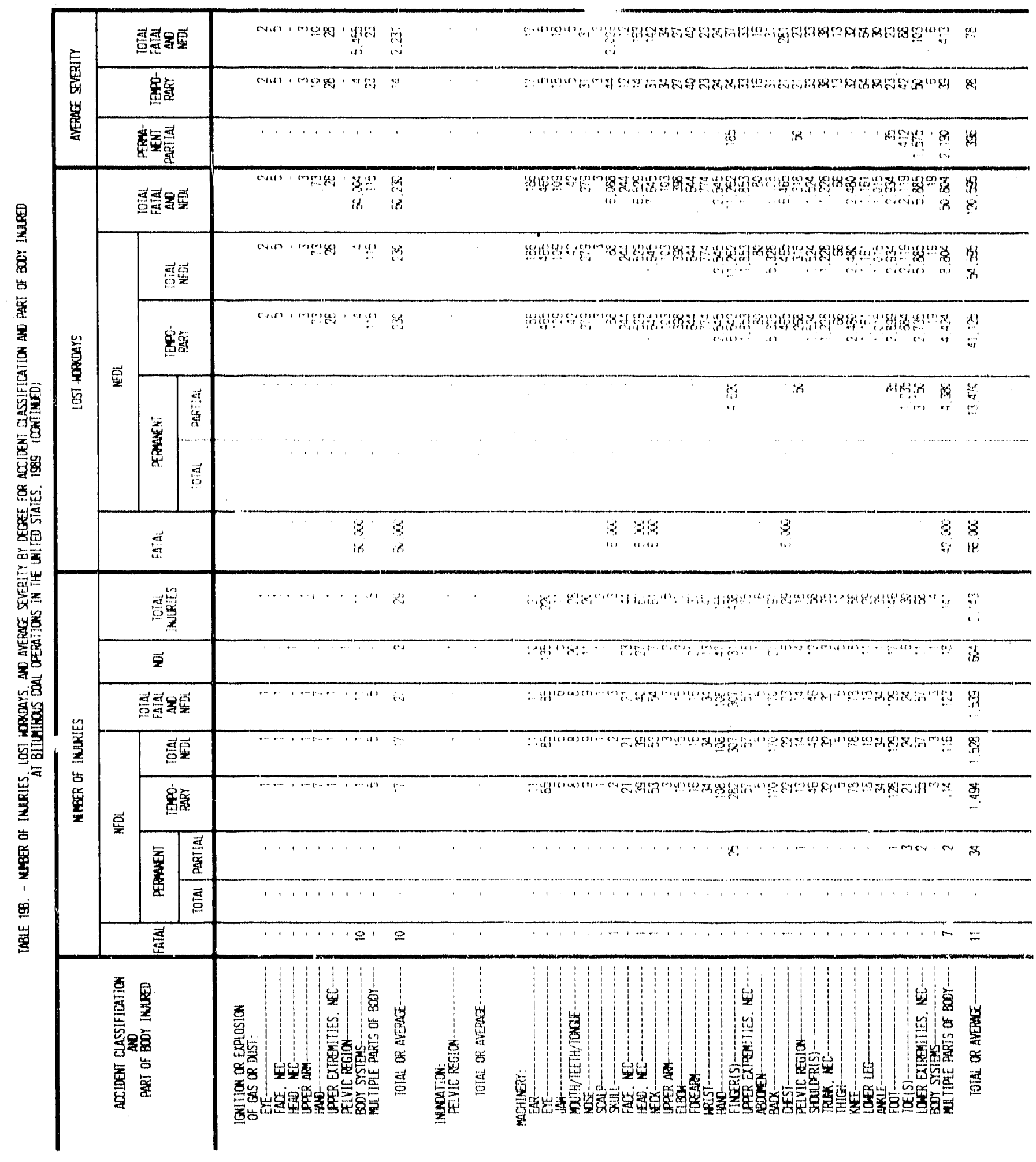




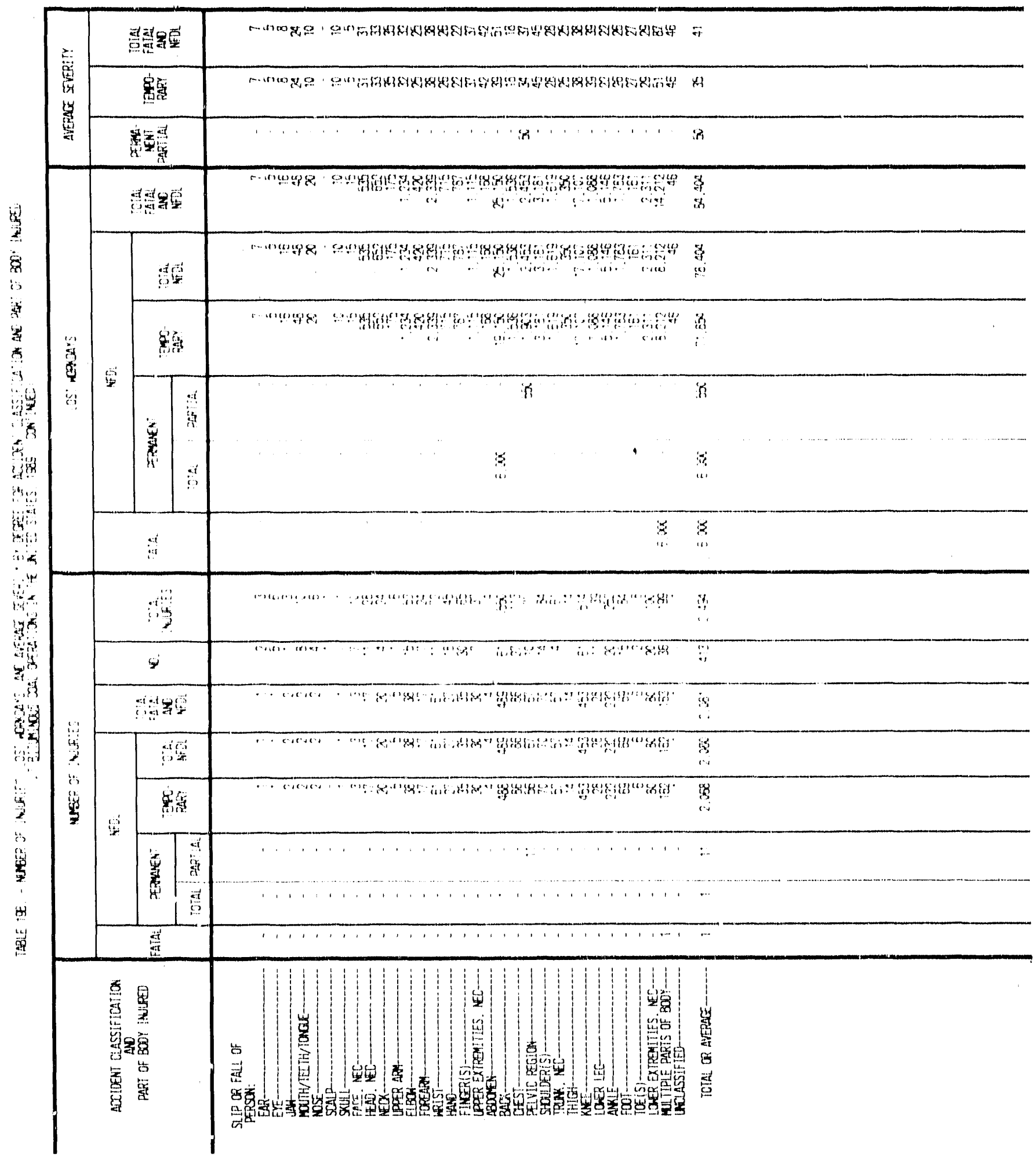




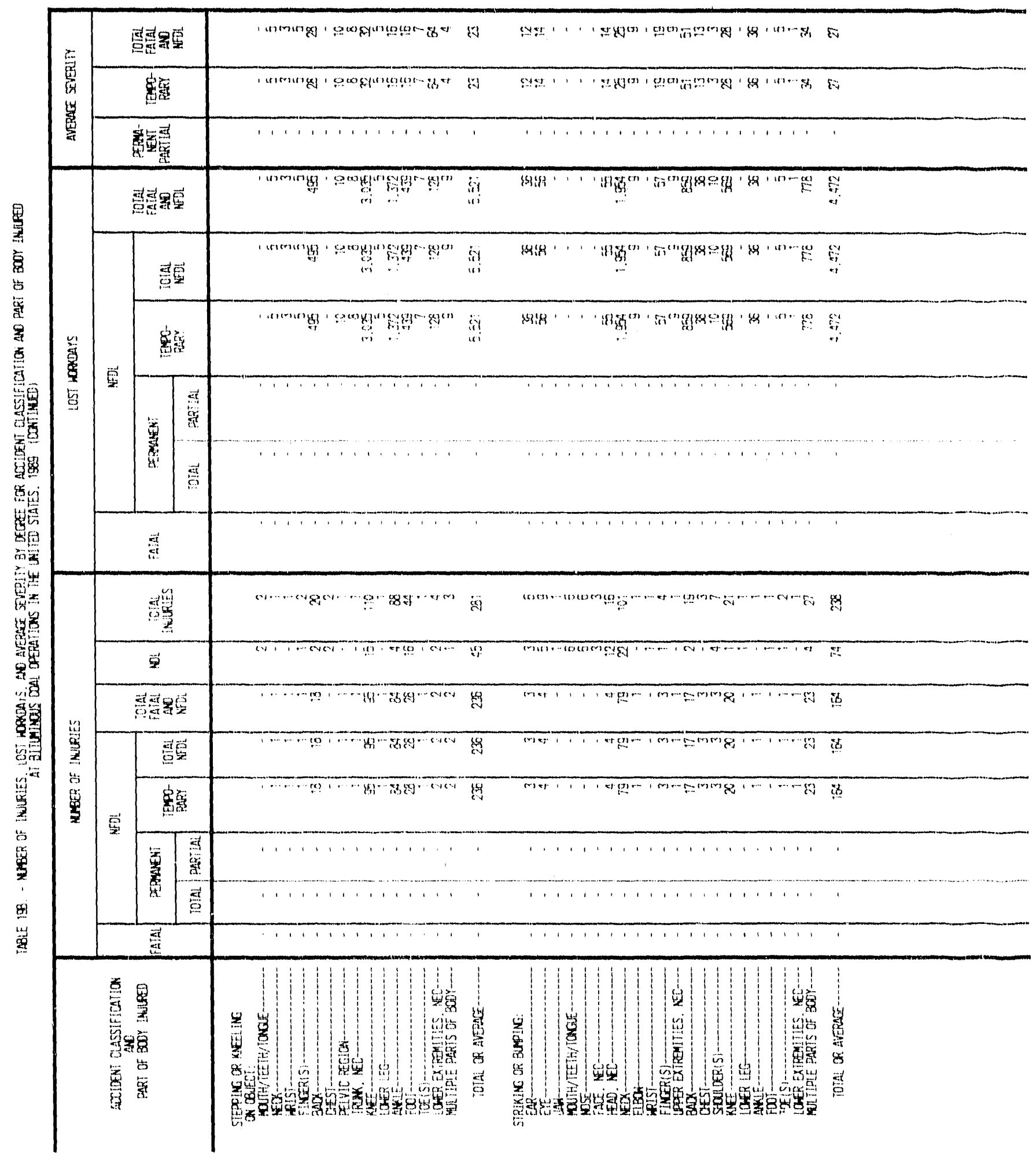




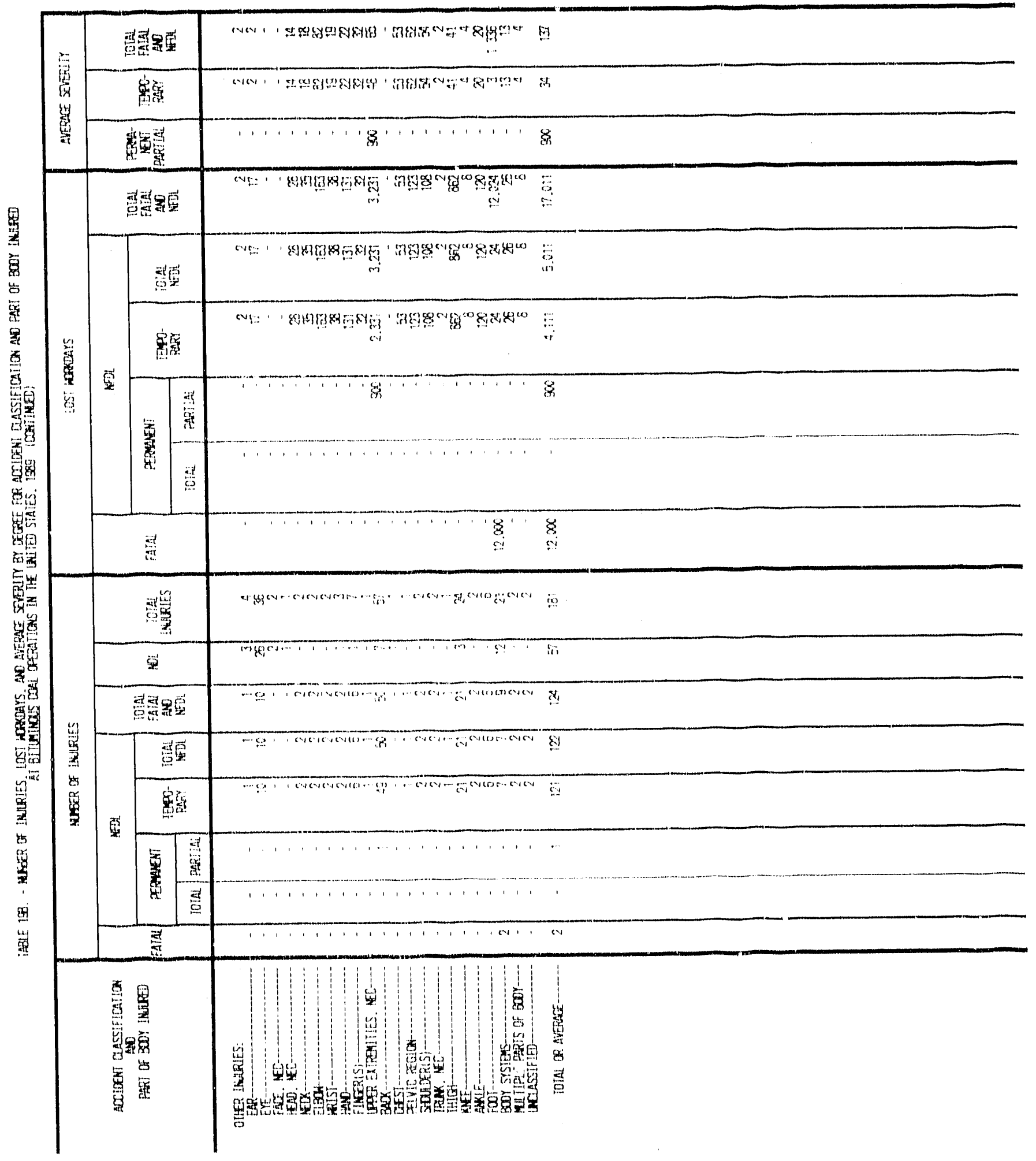




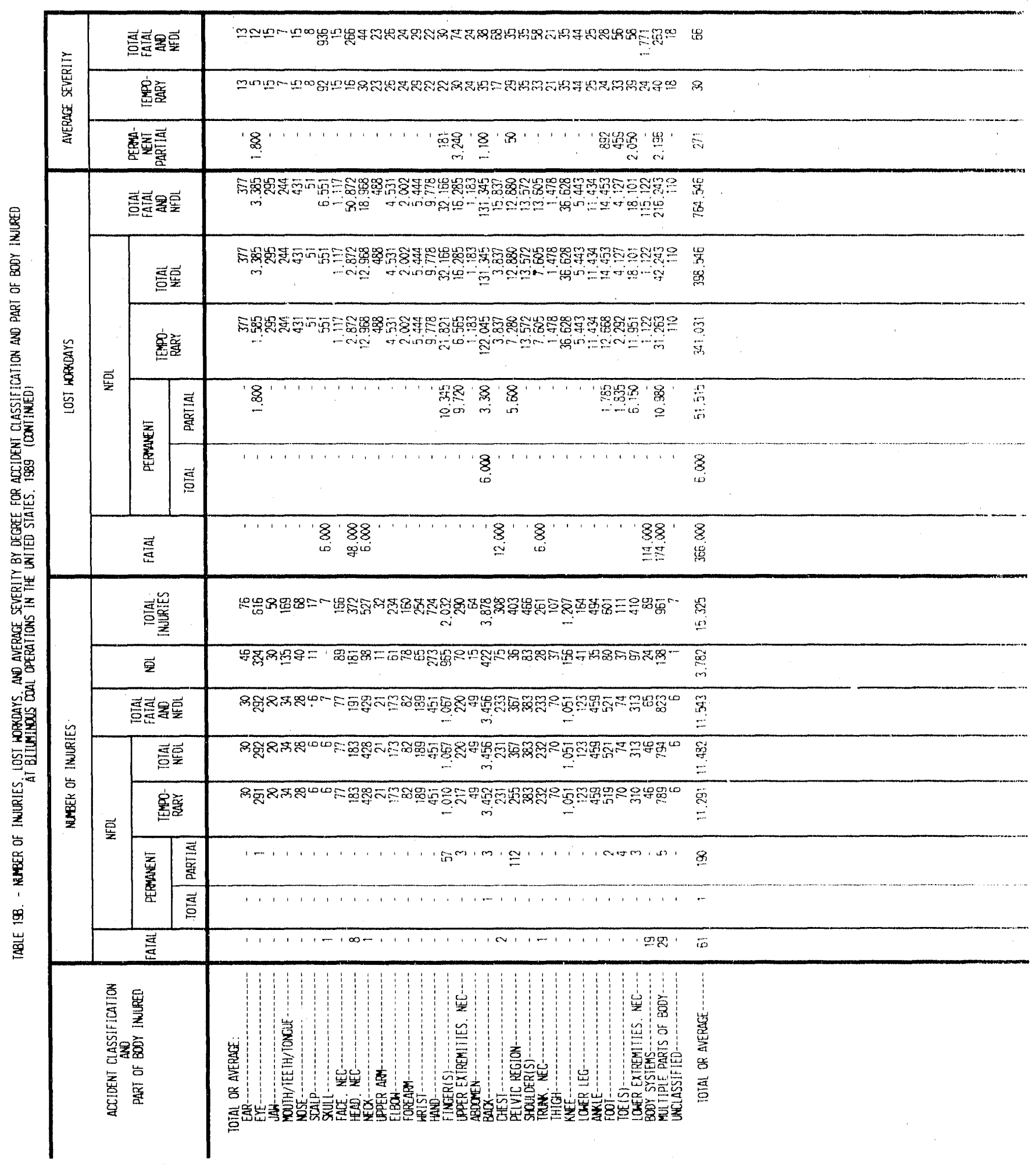




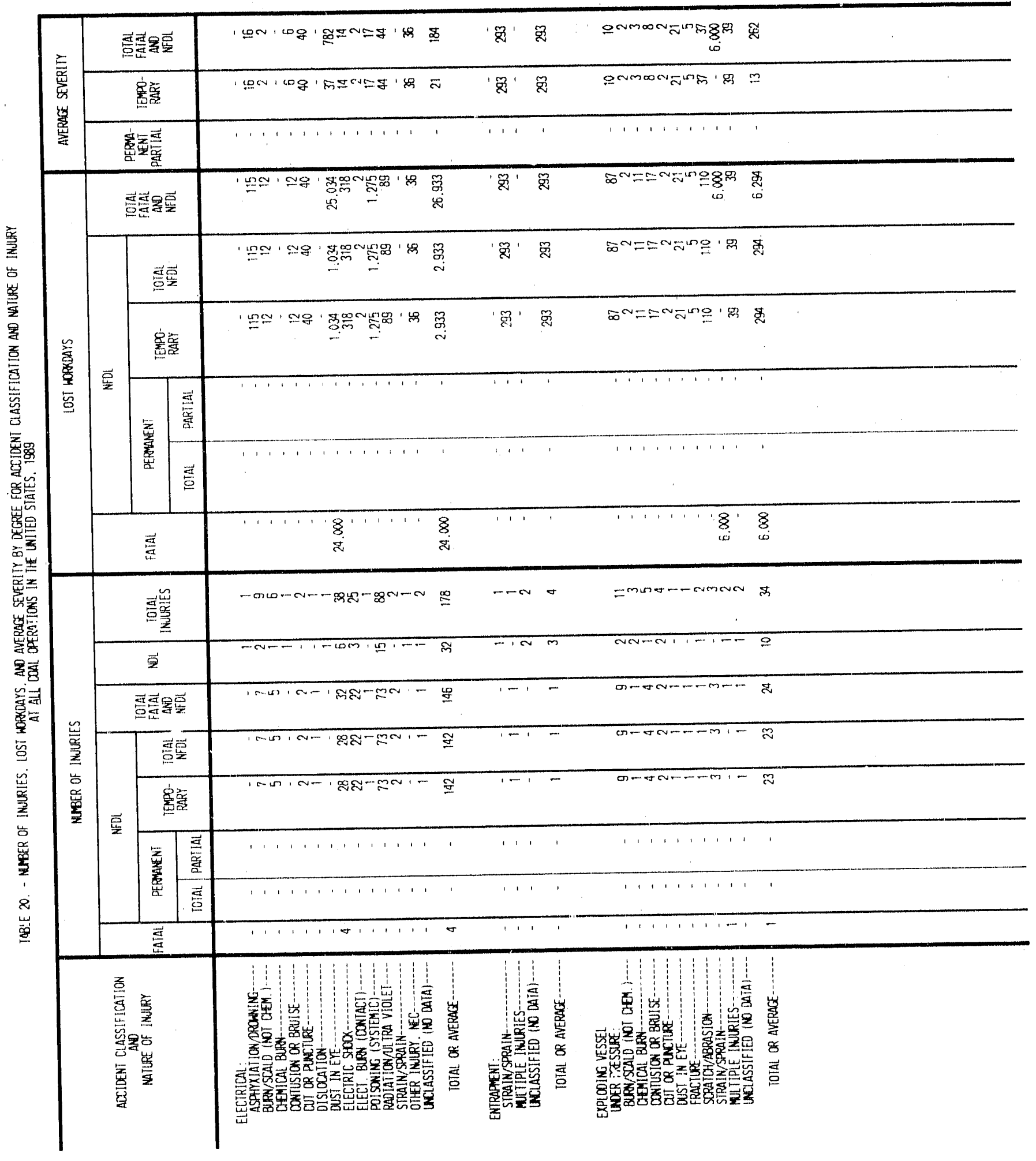




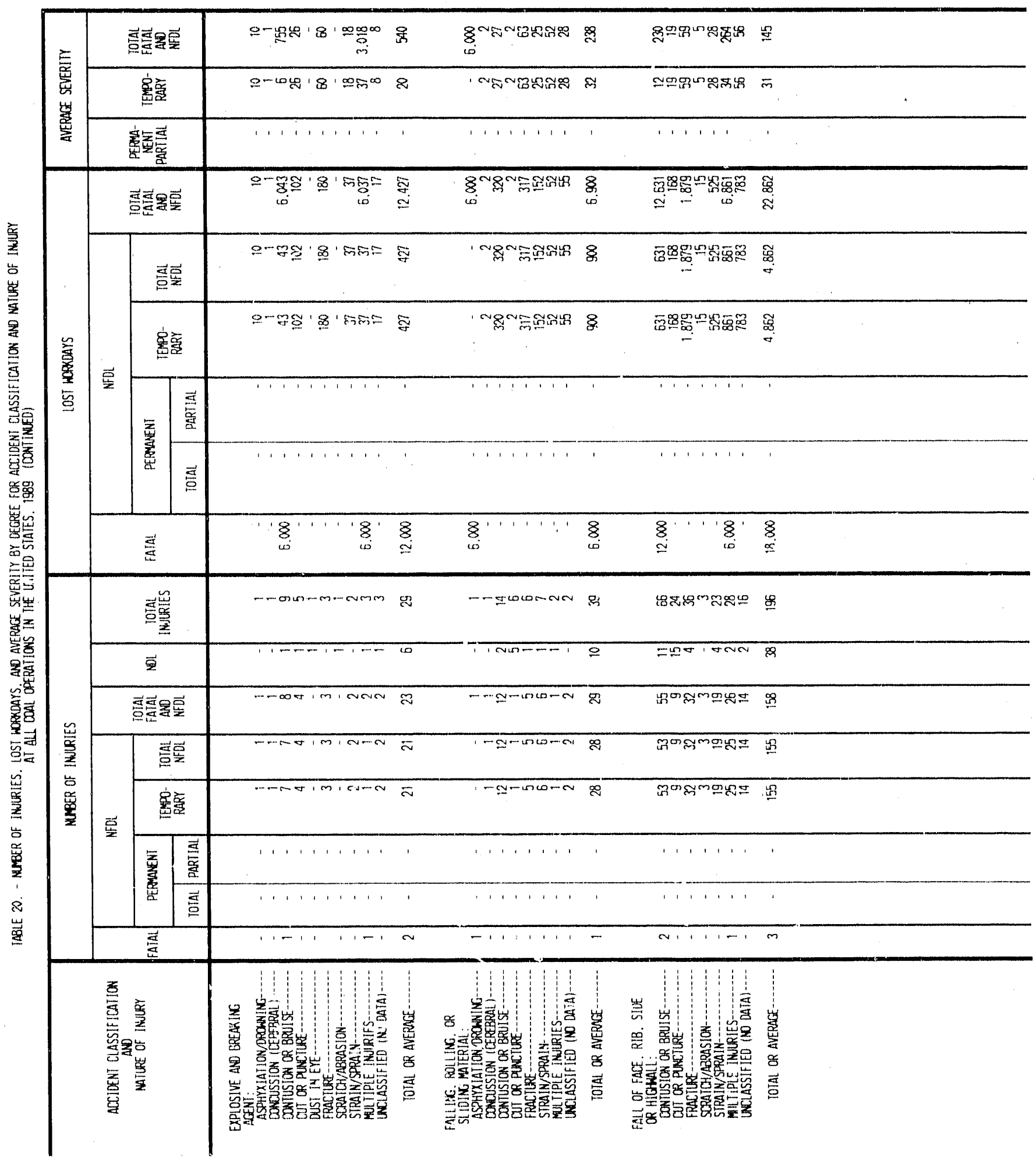




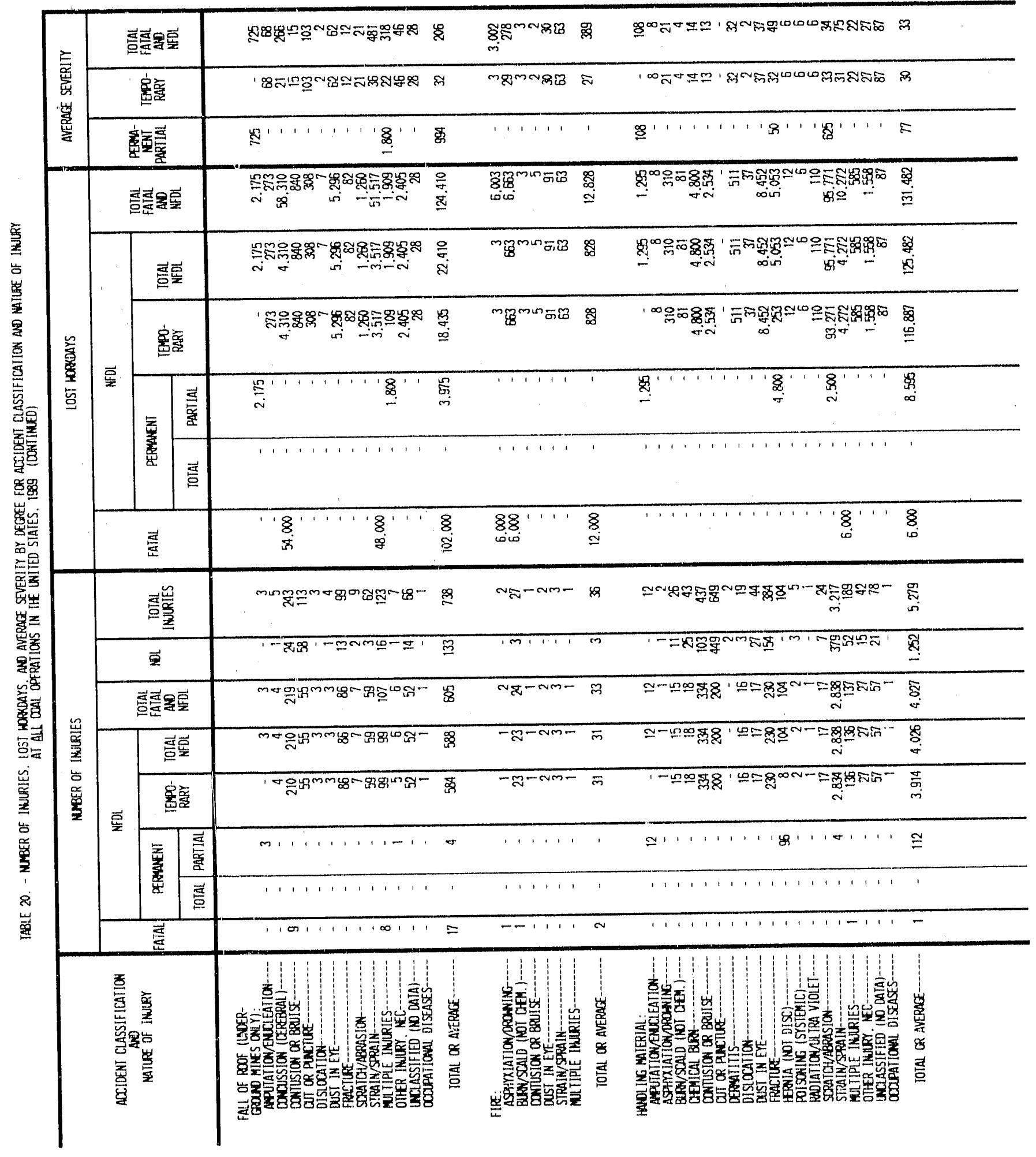




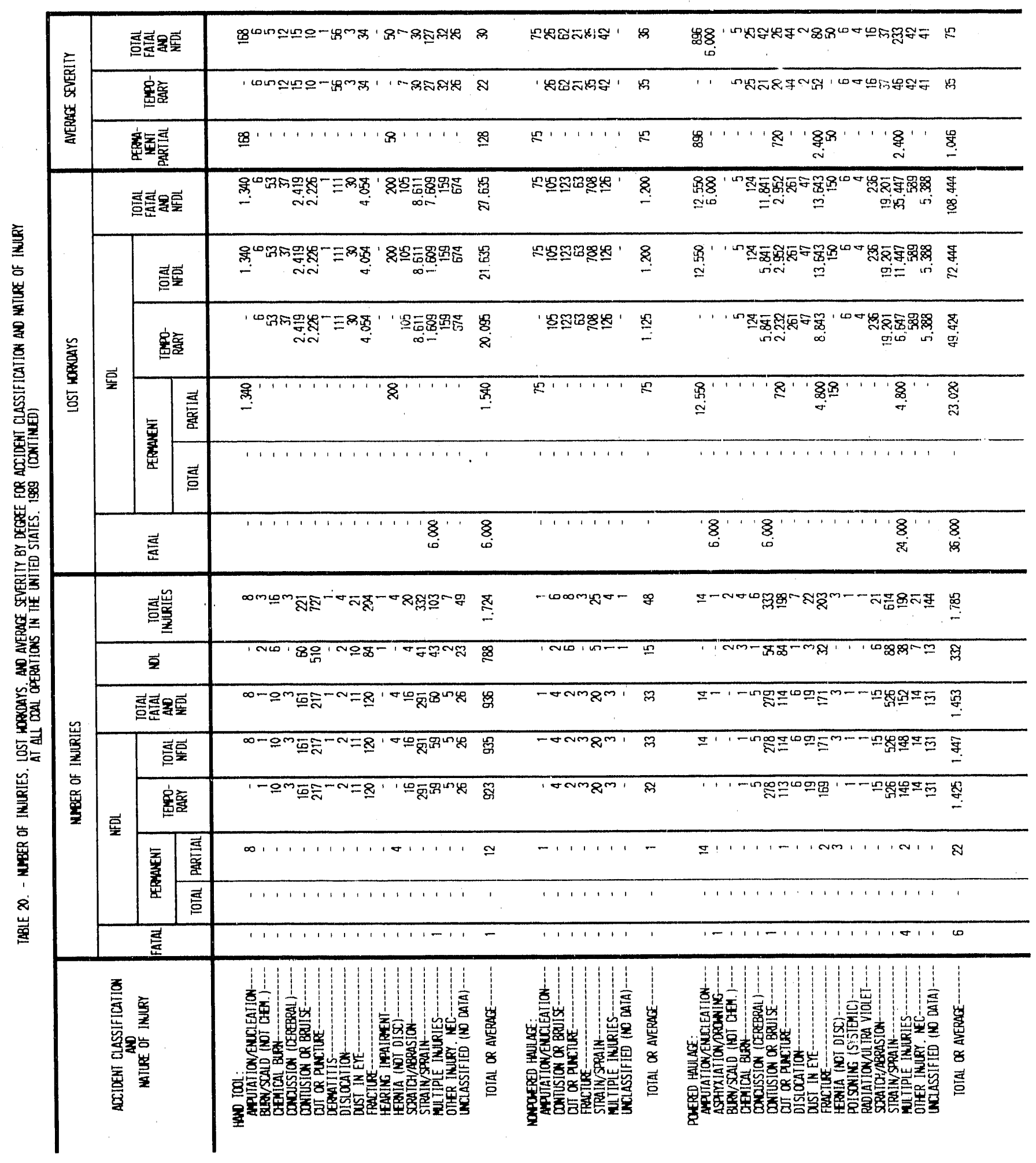




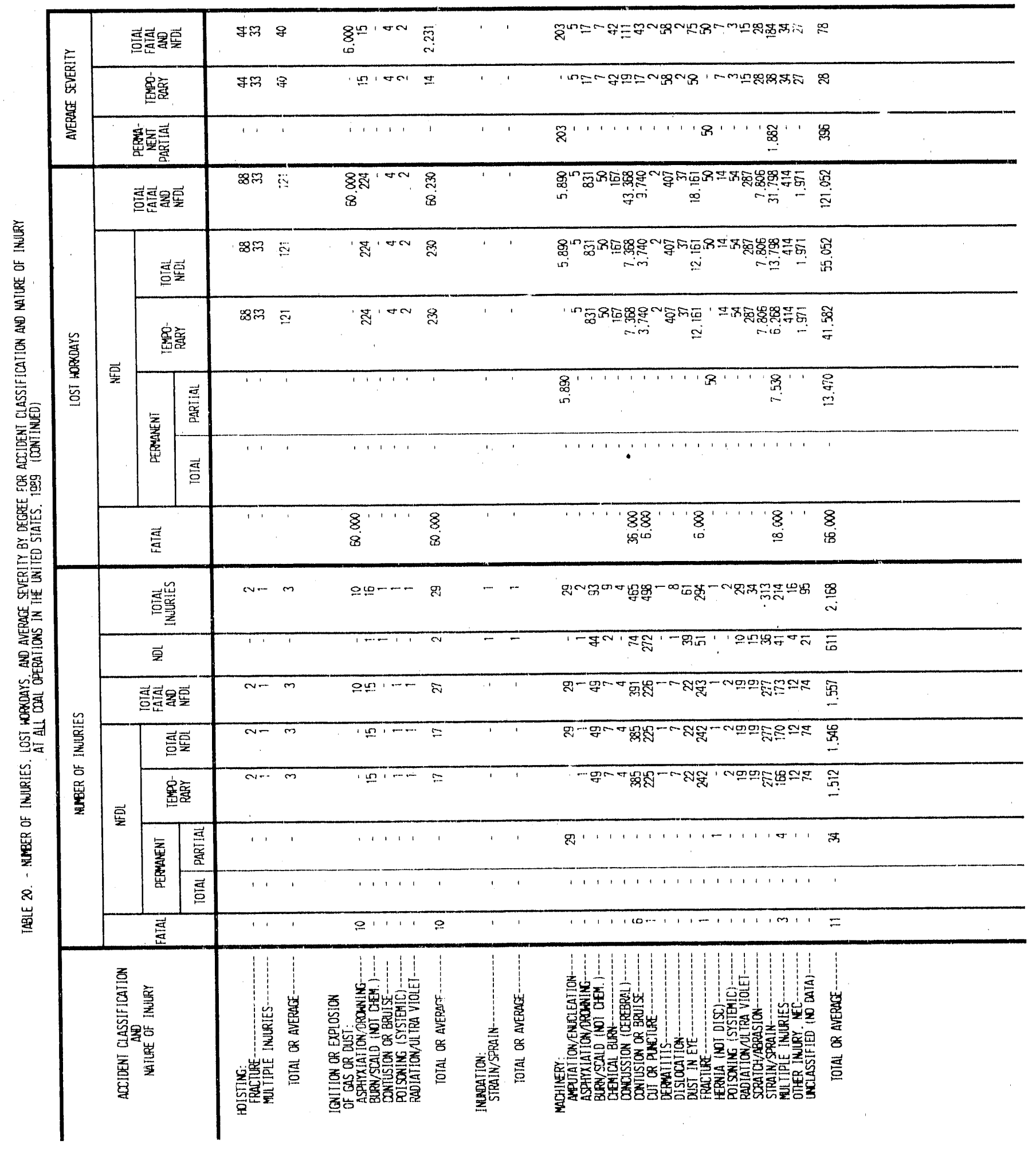




\begin{tabular}{|c|c|c|c|c|c|c|c|}
\hline \multirow{3}{*}{ 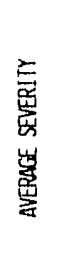 } & \multicolumn{2}{|c|}{ 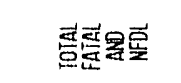 } & 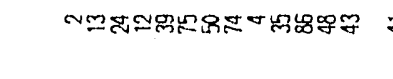 & $\bar{\xi}$ & - & $z_{i}=r=-\operatorname{sig} \bar{m}$ x & \\
\hline & \multicolumn{2}{|c|}{ 害䇾 } & 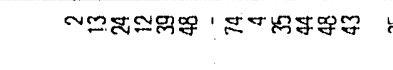 & 要 & 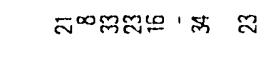 & 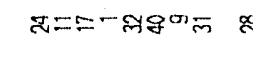 & \\
\hline & \multicolumn{2}{|c|}{ 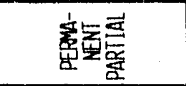 } & 11118 & 8 & 11 & $\cdots$ & ' \\
\hline \multirow{6}{*}{ 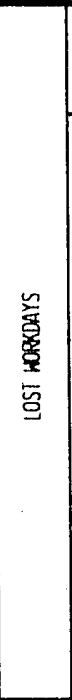 } & \multicolumn{2}{|c|}{ 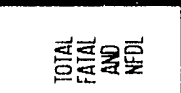 } & 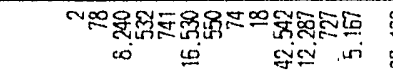 & \begin{tabular}{c}
$\substack{a \\
\hdashline \\
\infty \\
\infty}$ \\
$\infty$
\end{tabular} & 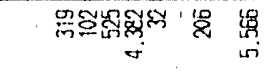 & 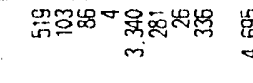 & ?ר: \\
\hline & \multirow{4}{*}{ 高 } & 高垩 & 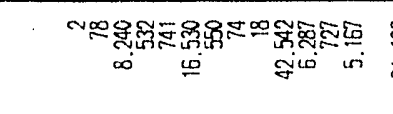 & $\frac{\mathscr{m}}{\infty}$ & 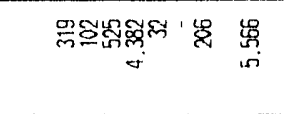 & 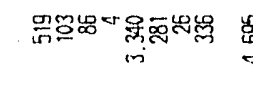 & 虽 \\
\hline & & 容筟 & 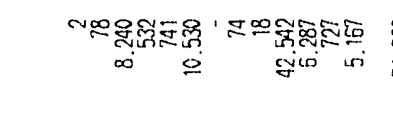 & 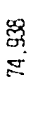 & 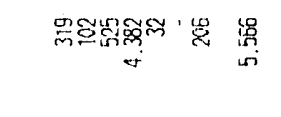 & 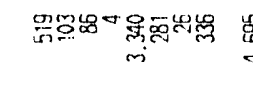 & : \\
\hline & & \multirow{2}{*}{ 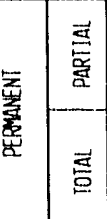 } & 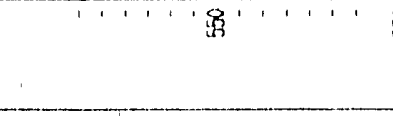 & 䒺 & 1 & & \\
\hline & & & ' ' ' & $\underset{c}{8}$ & 1 & $\cdots$ & . \\
\hline & \multicolumn{2}{|c|}{ 咅 } & ' ' n ' & $\underset{10}{8}$ & ' ' ' & ' 11.1 & \\
\hline \multirow{8}{*}{ 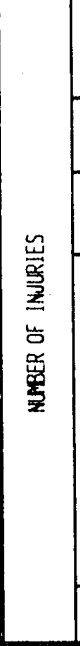 } & \multicolumn{2}{|c|}{ 琴璦 } & 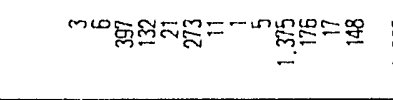 & 㟔 & gू- & 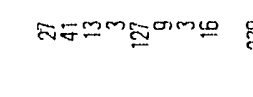 & gg \\
\hline & \multicolumn{2}{|r|}{ 至 } & 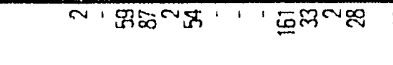 & 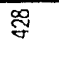 & 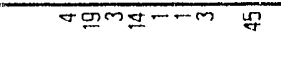 & 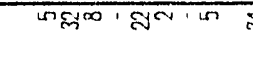 & I \\
\hline & \multicolumn{2}{|c|}{ 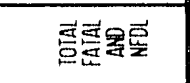 } & - & $\stackrel{\bar{g}}{i}$ & 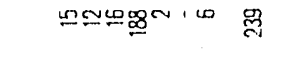 & 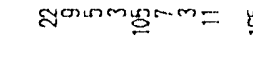 & $\stackrel{9}{9}$ \\
\hline & \multicolumn{2}{|r|}{ 吾要 } & - & $\stackrel{\underline{m}}{i}$ & 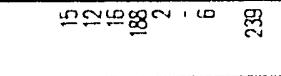 & gormm & 照 \\
\hline & \multirow{3}{*}{ 豆 } & 裹害 & 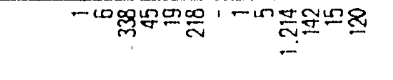 & $\underset{d}{i}$ & 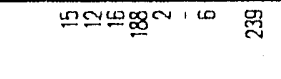 & 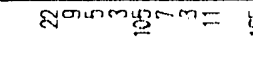 & 㽞 \\
\hline & & \multirow{2}{*}{ 氞 } & & $=$ & ' & & ' \\
\hline & & & ' ' & - & $\cdots$ & ' & ' \\
\hline & \multicolumn{2}{|r|}{ 宸 } & , & - & 11 & 1. & . \\
\hline & \multicolumn{2}{|c|}{ 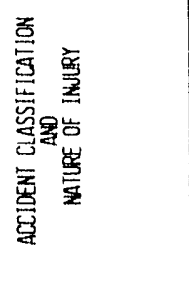 } & 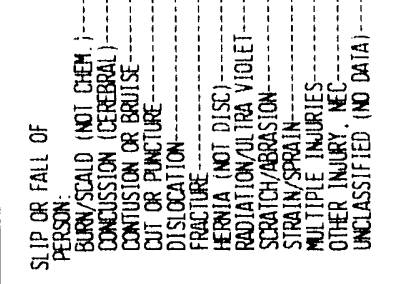 & 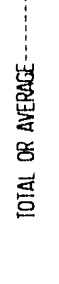 & 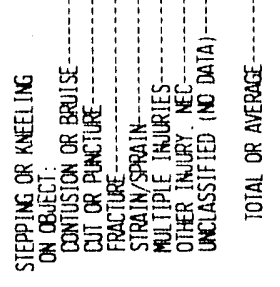 & 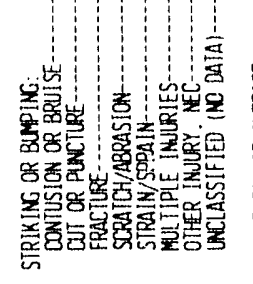 & 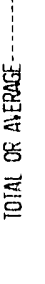 \\
\hline
\end{tabular}




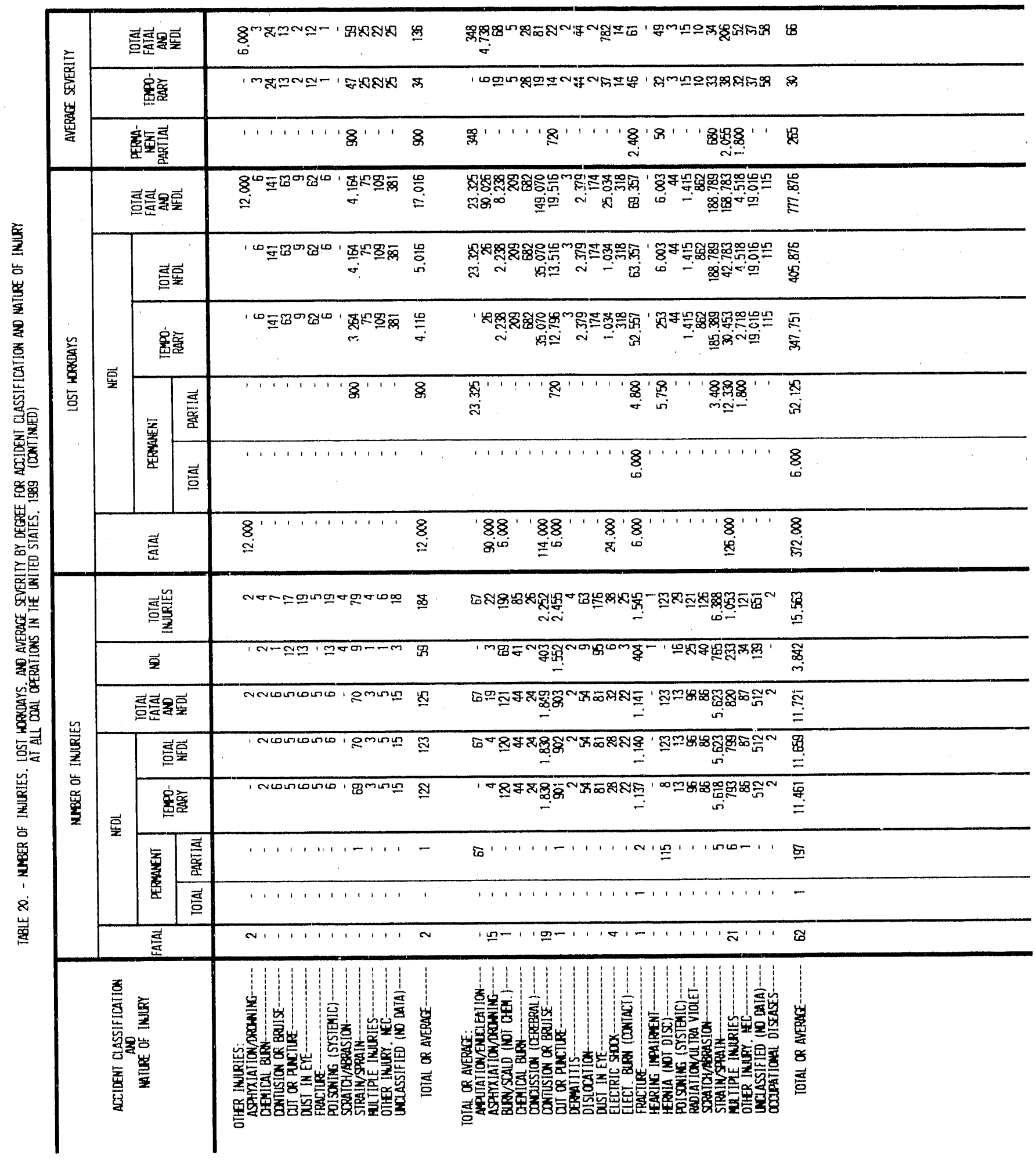




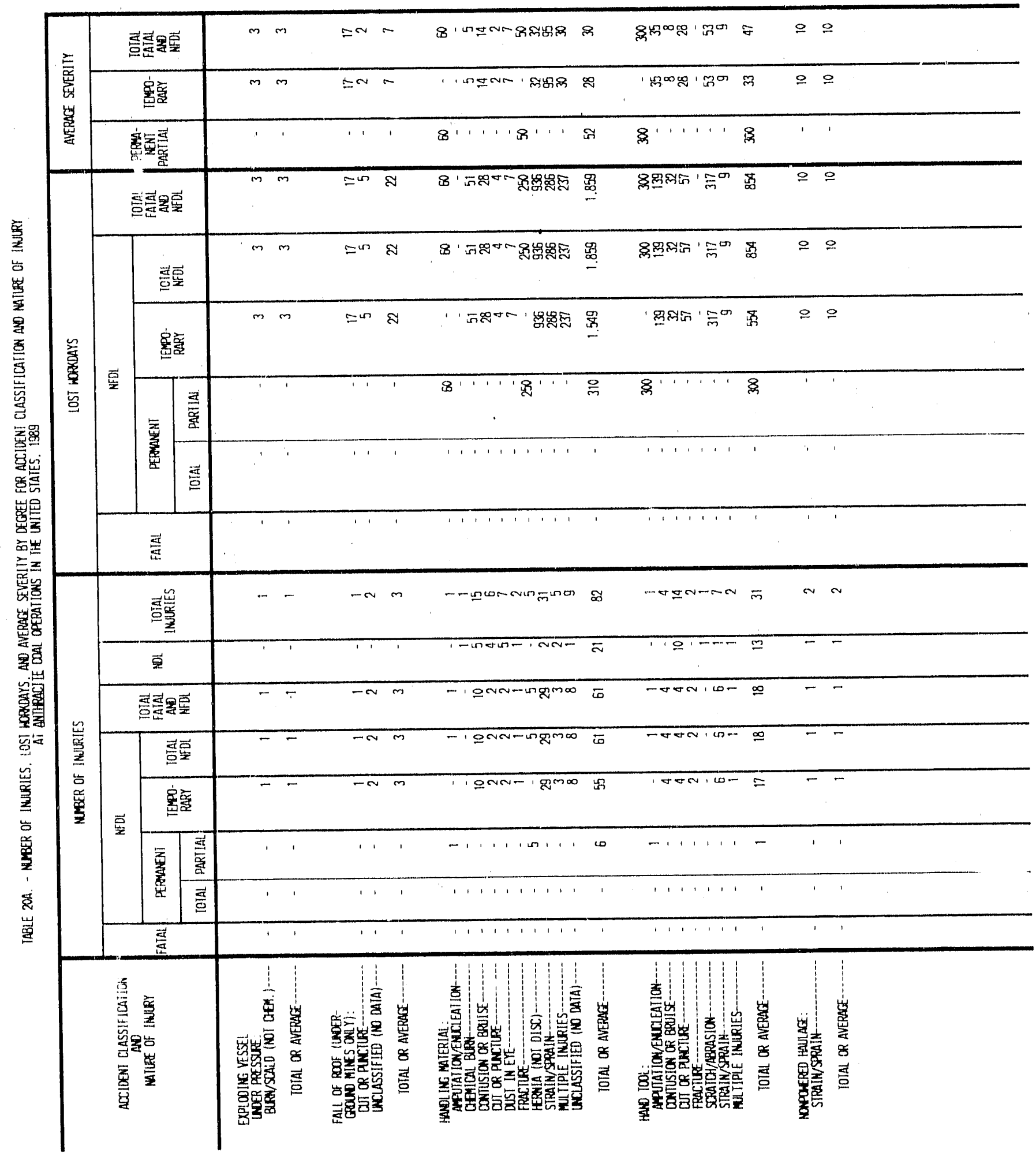




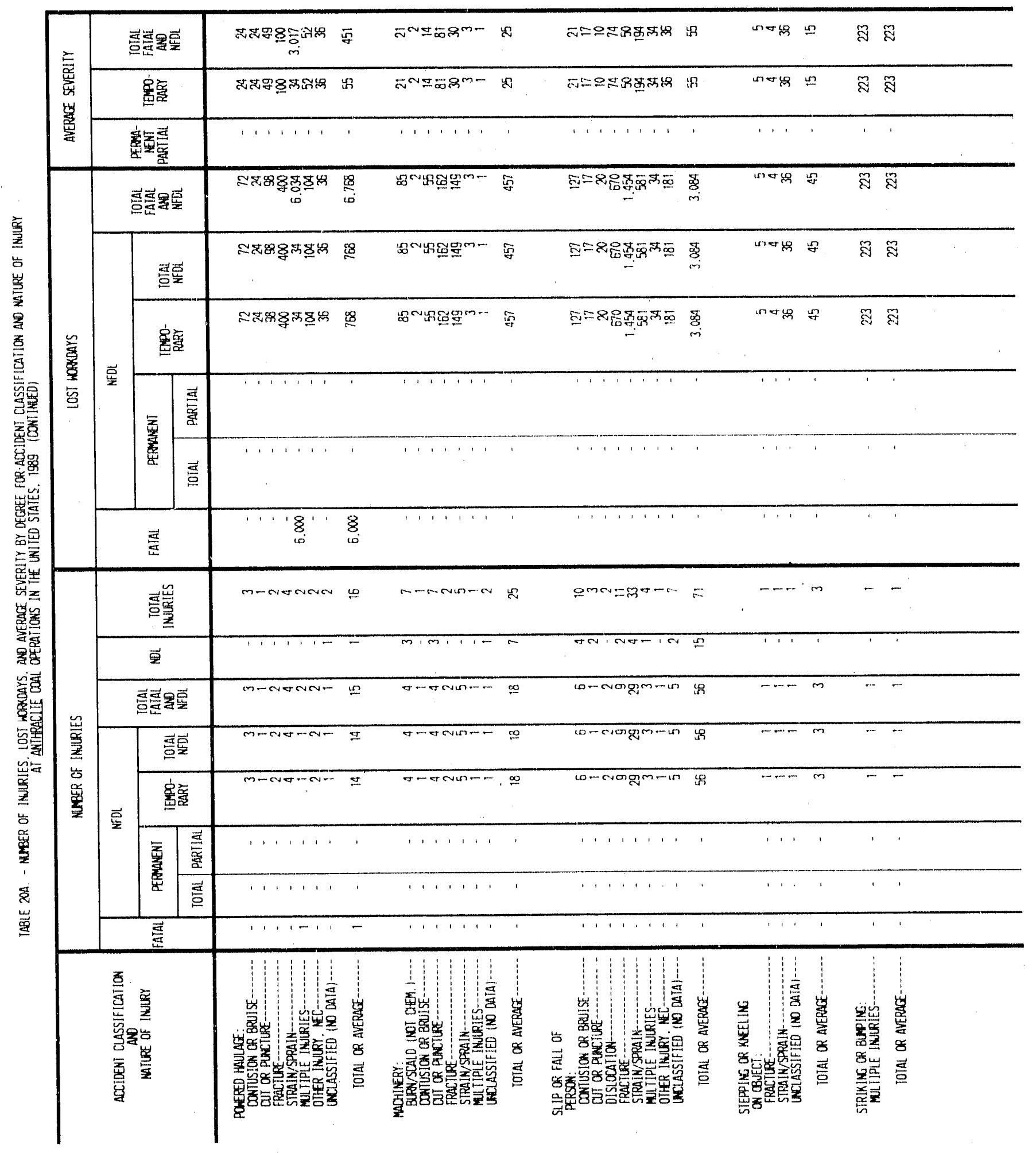




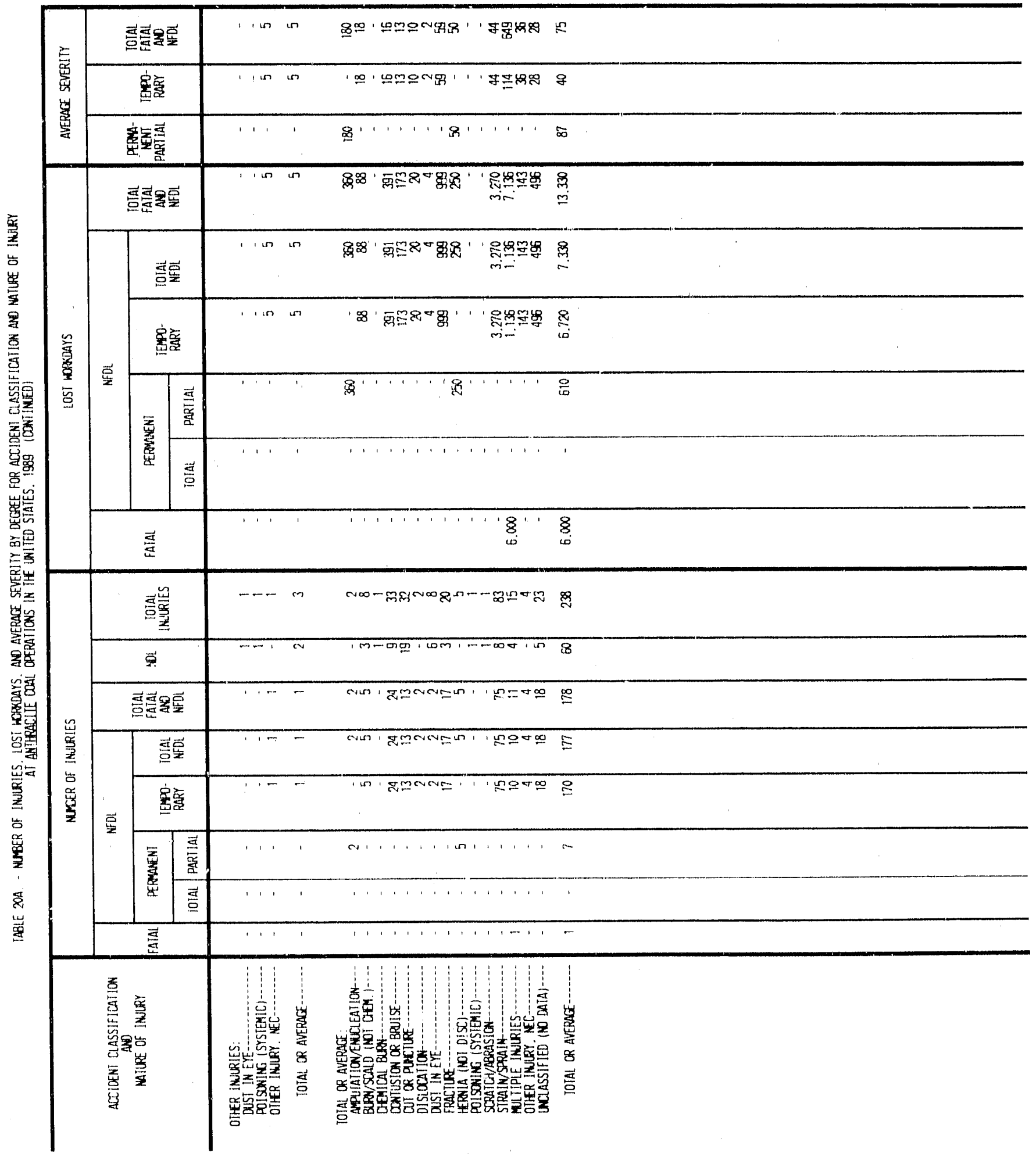




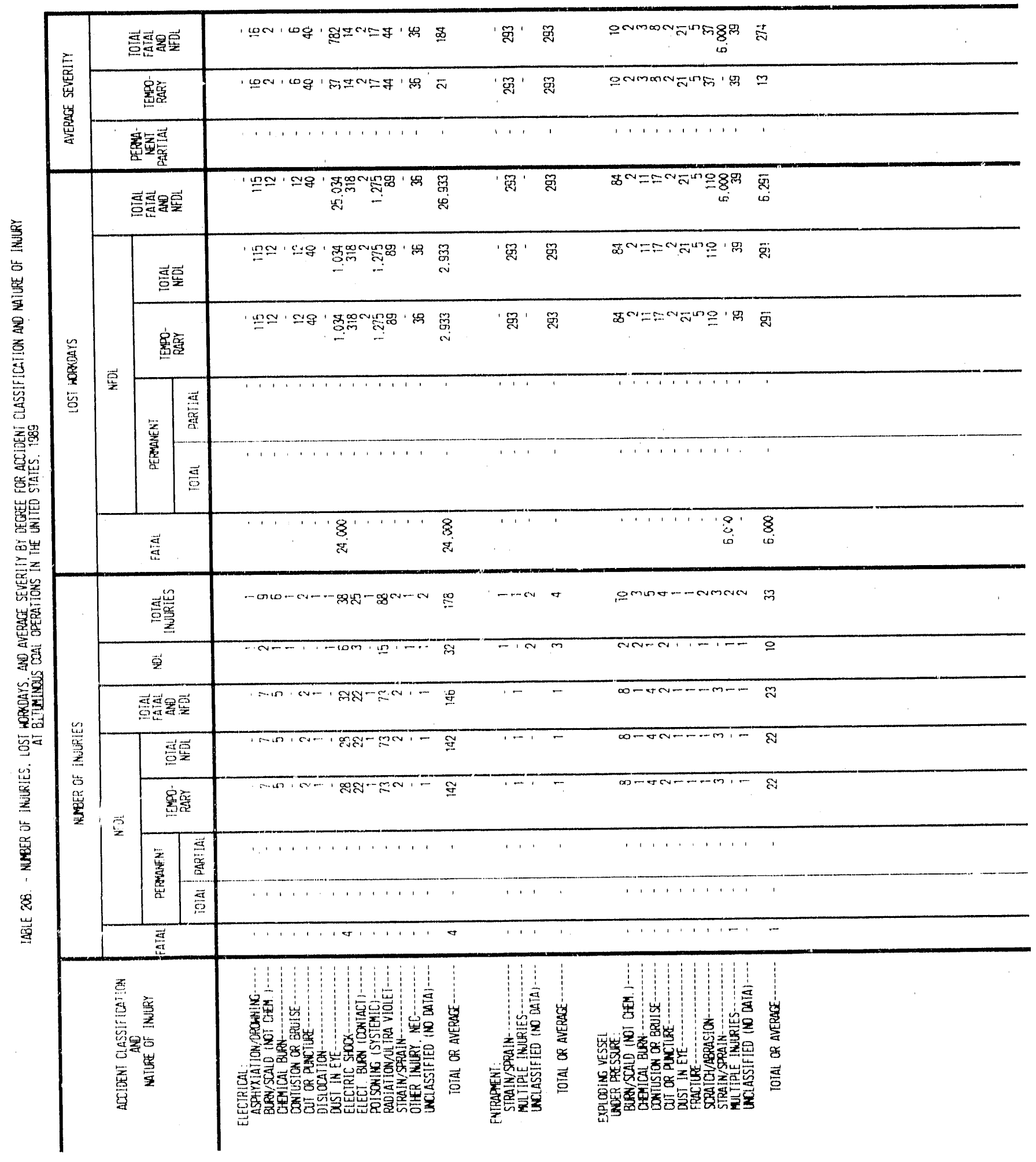




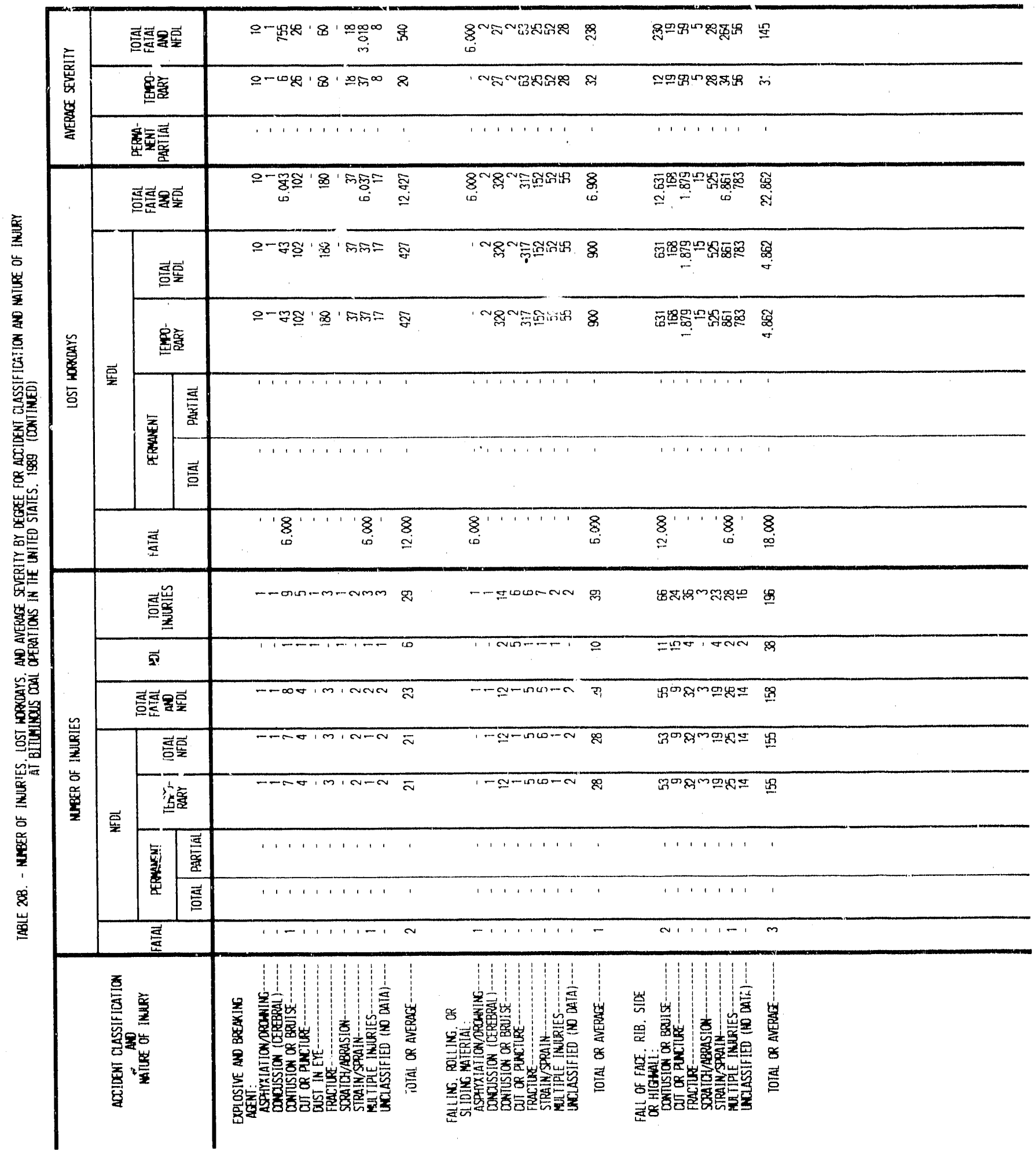




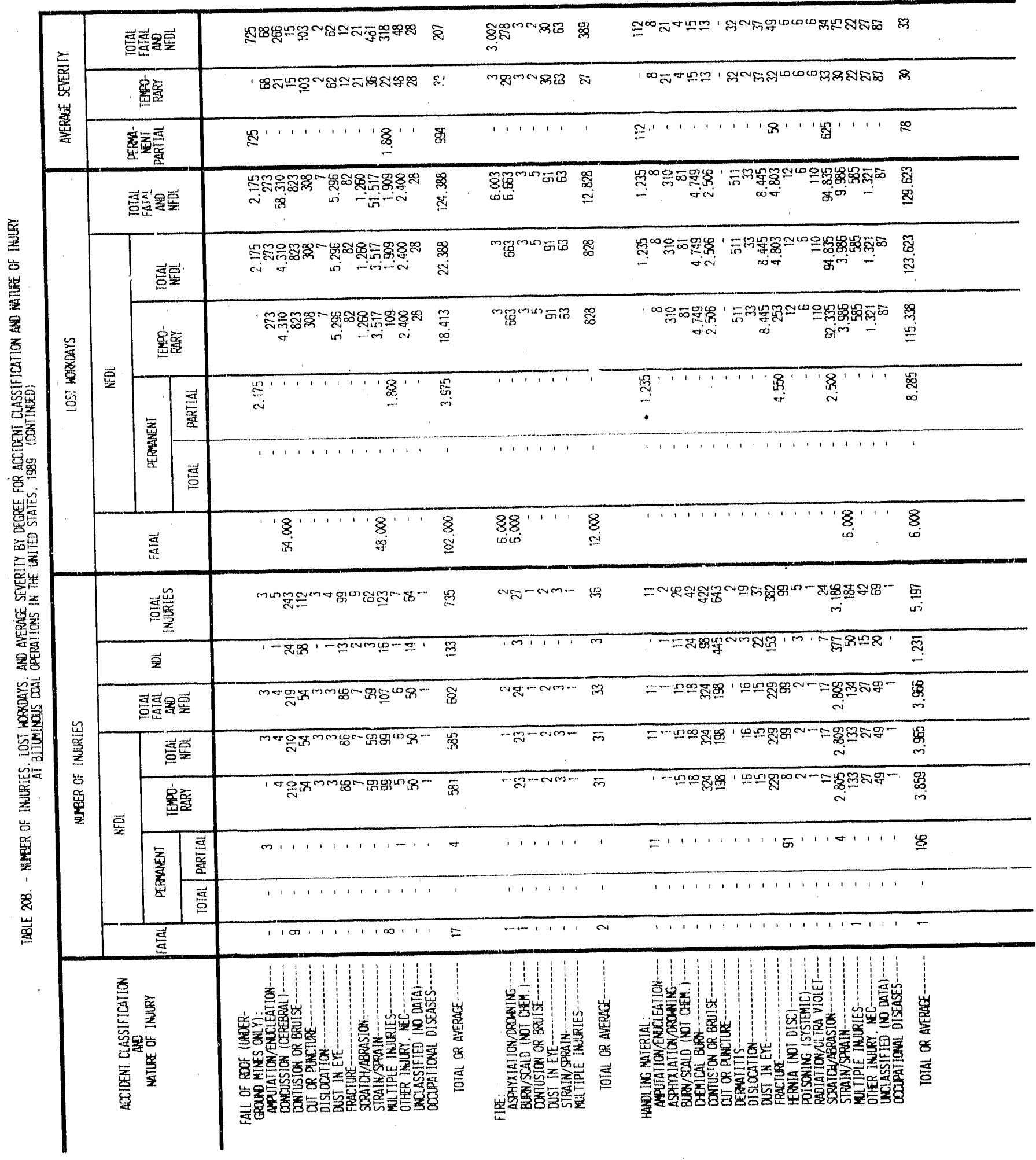




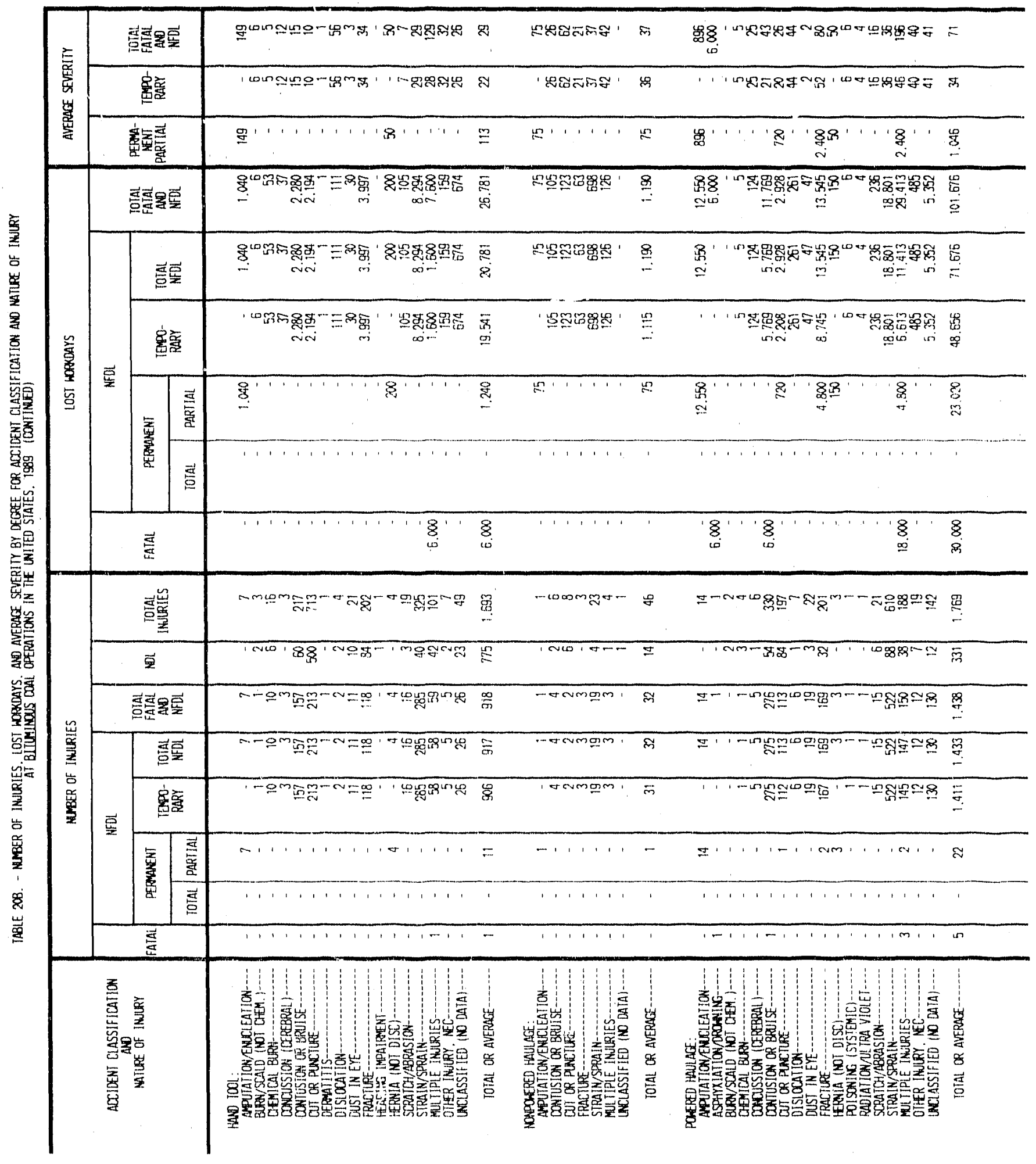




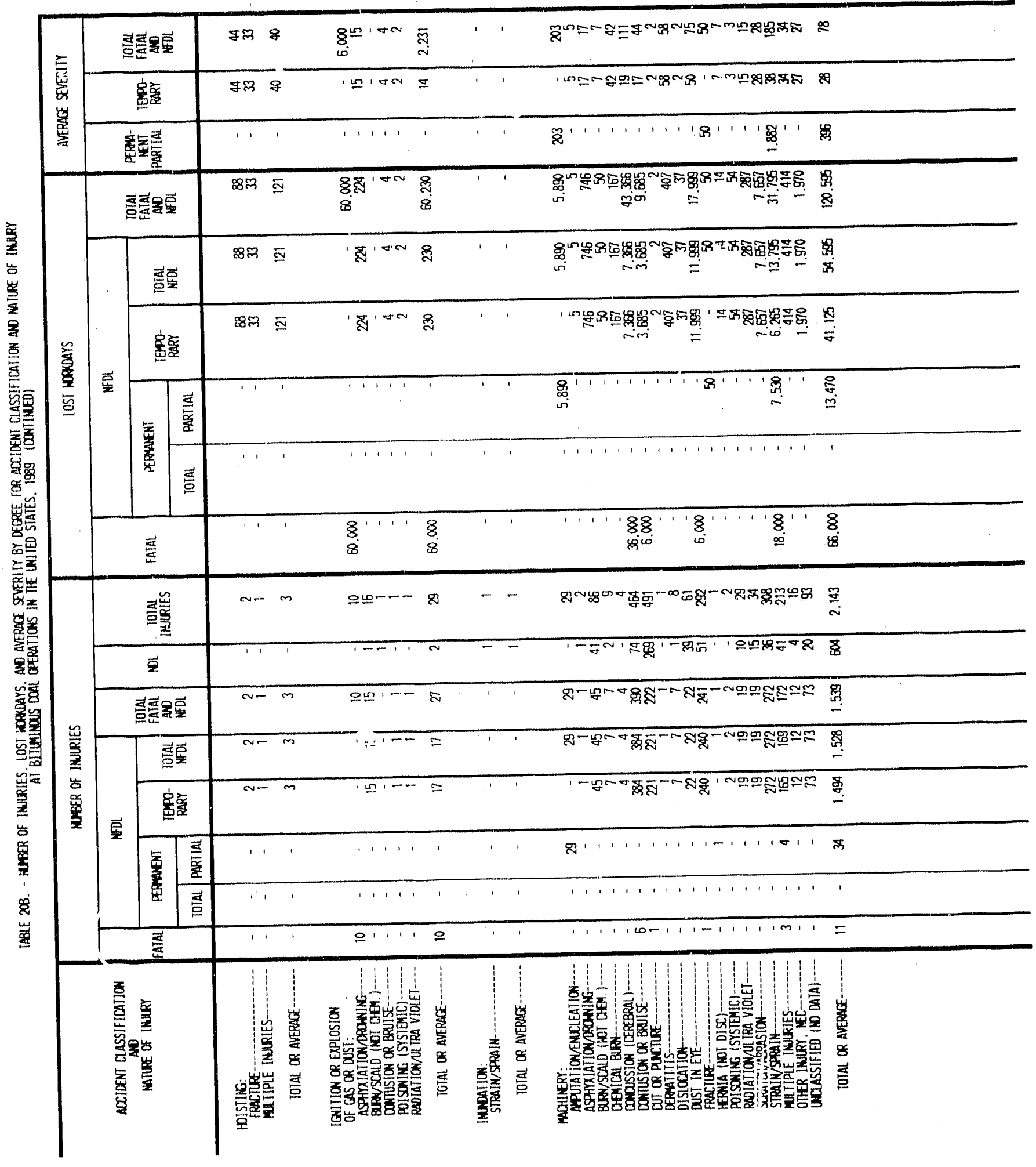




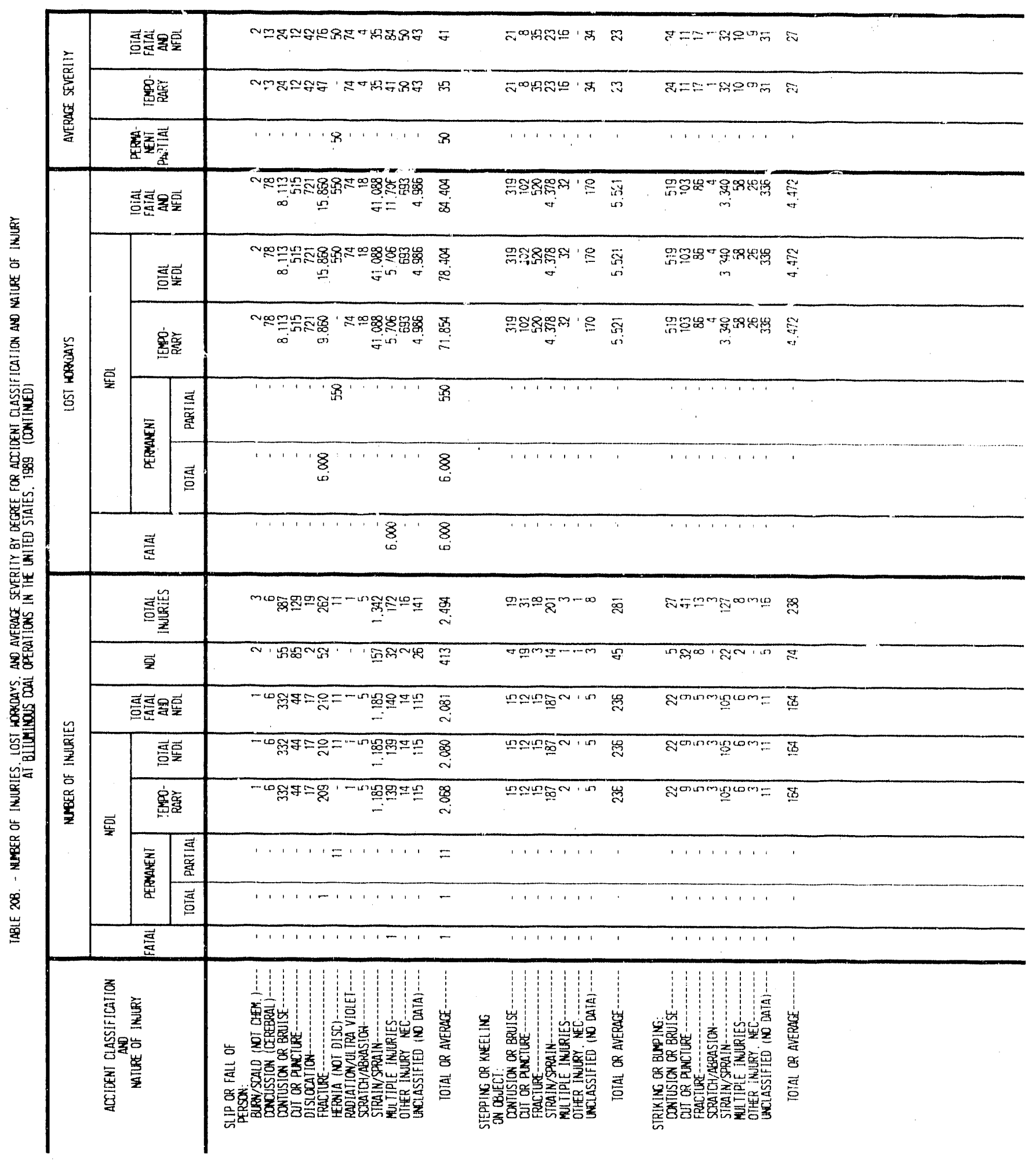




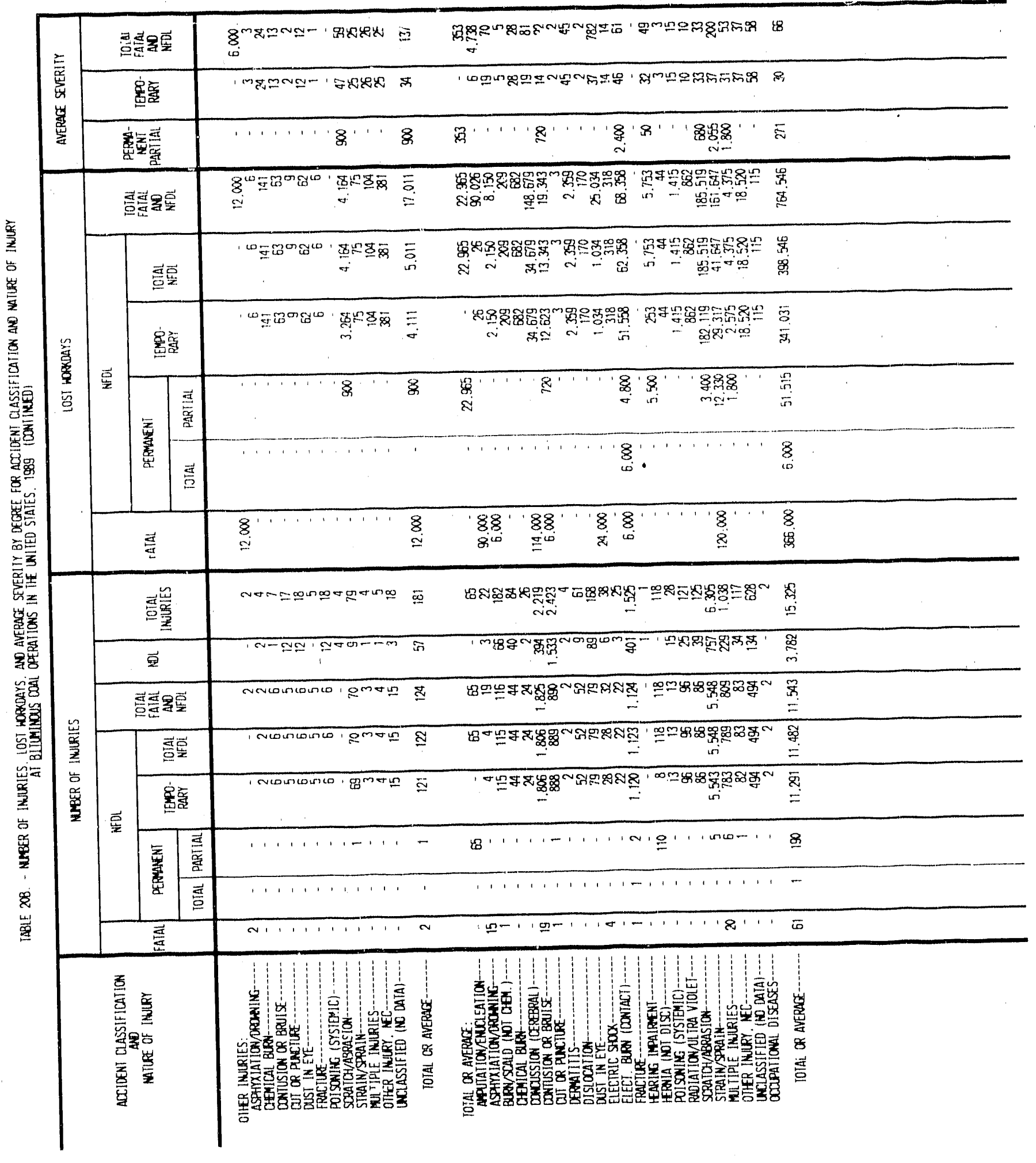




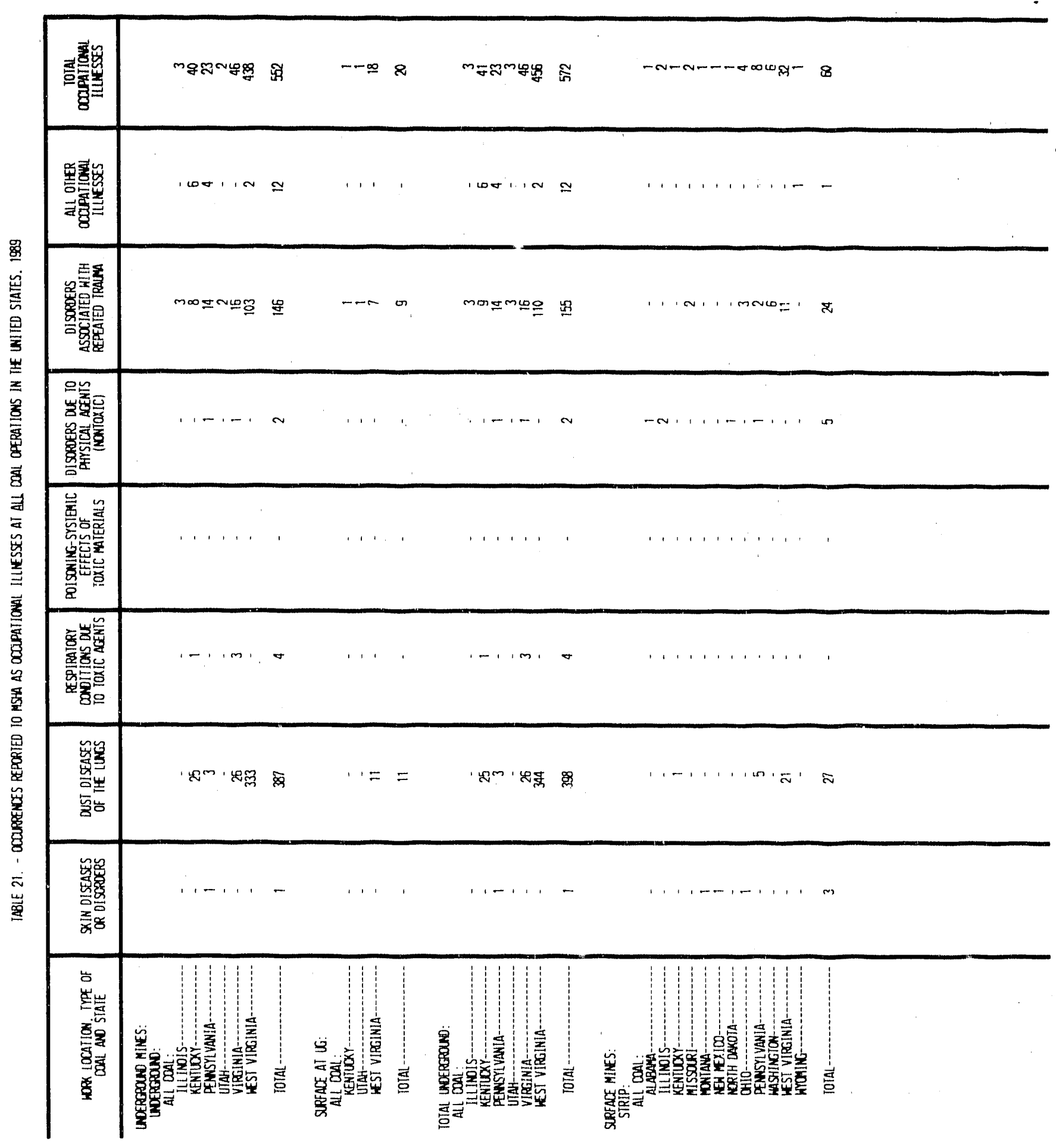




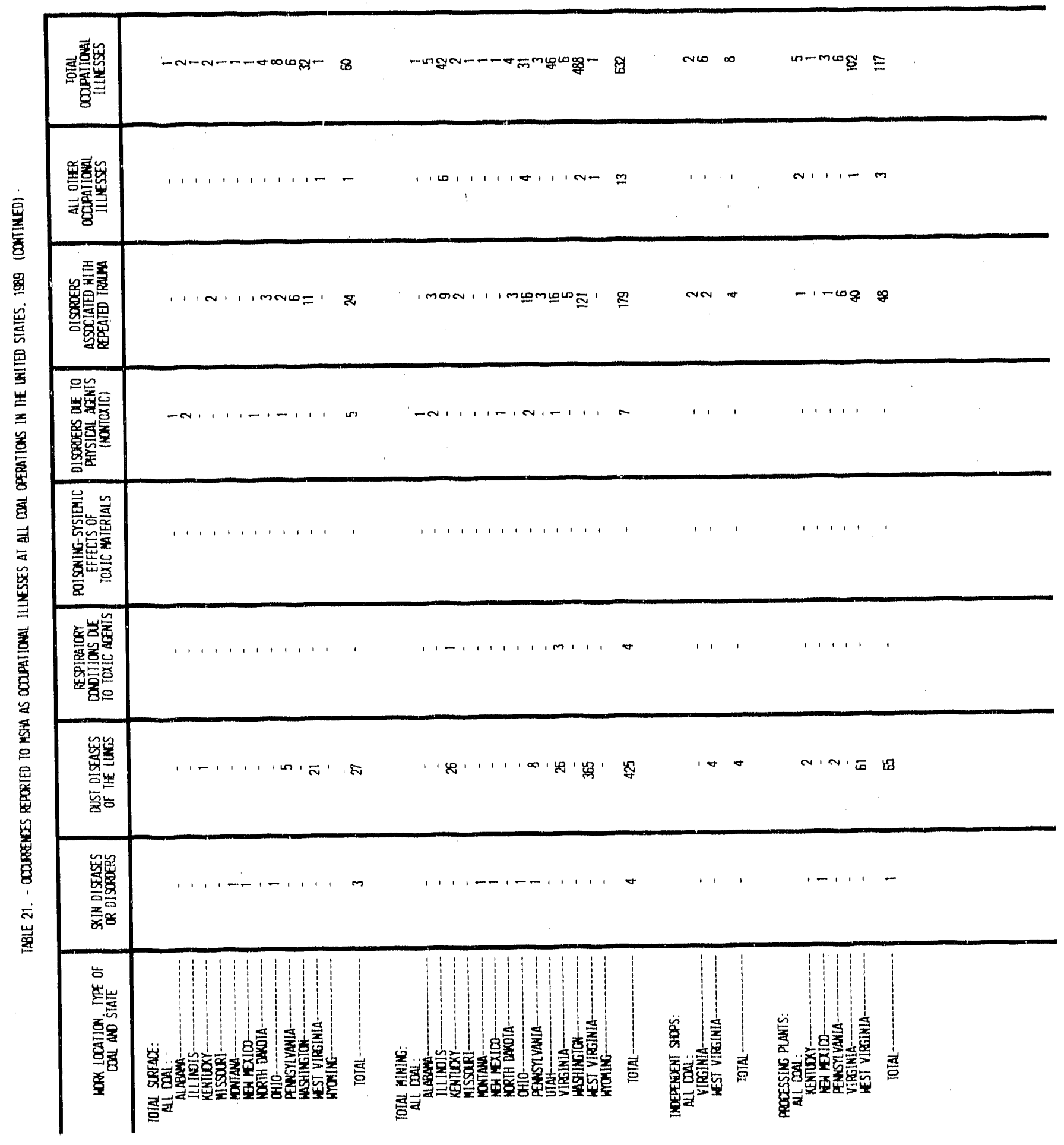




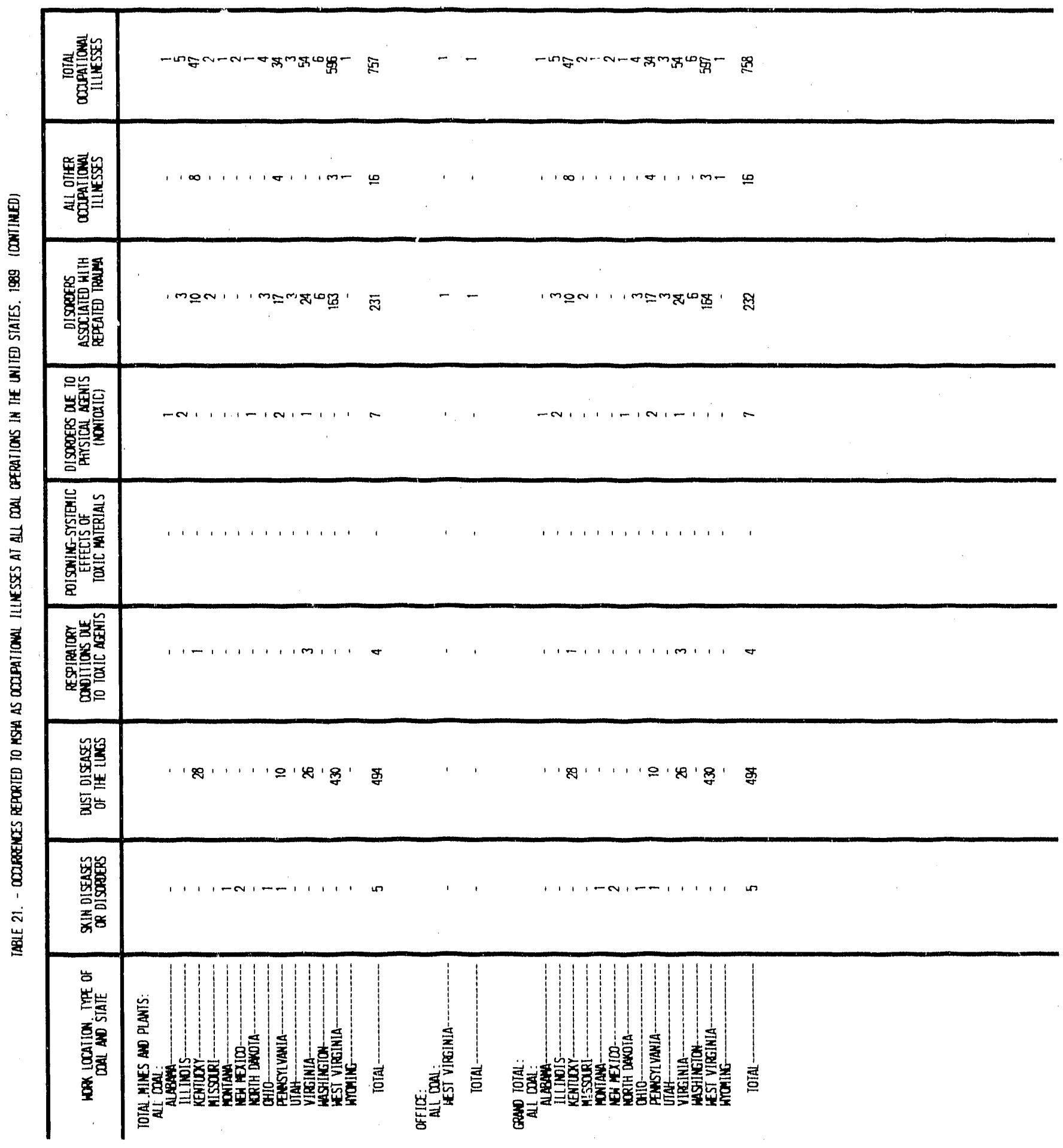




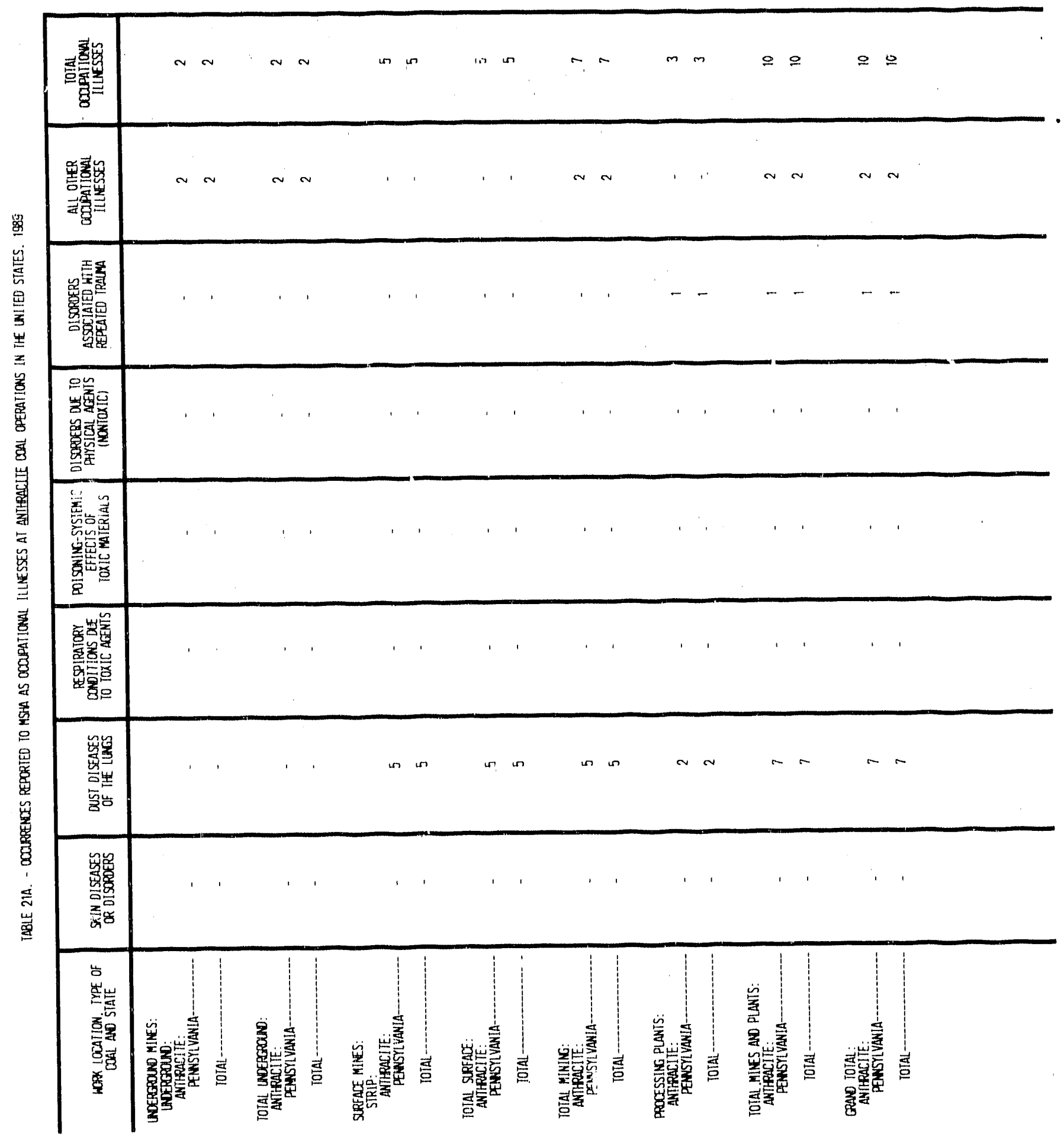




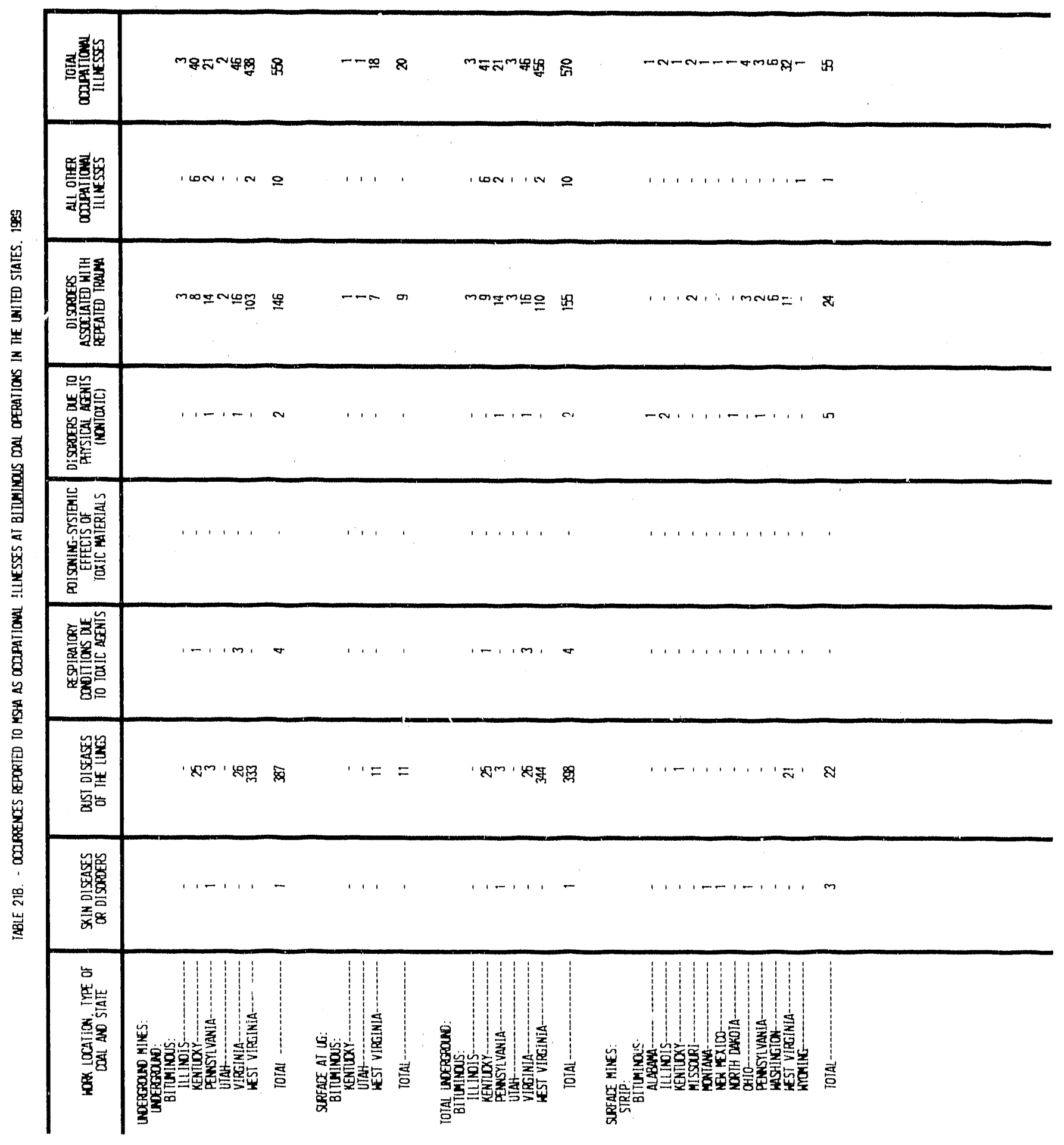




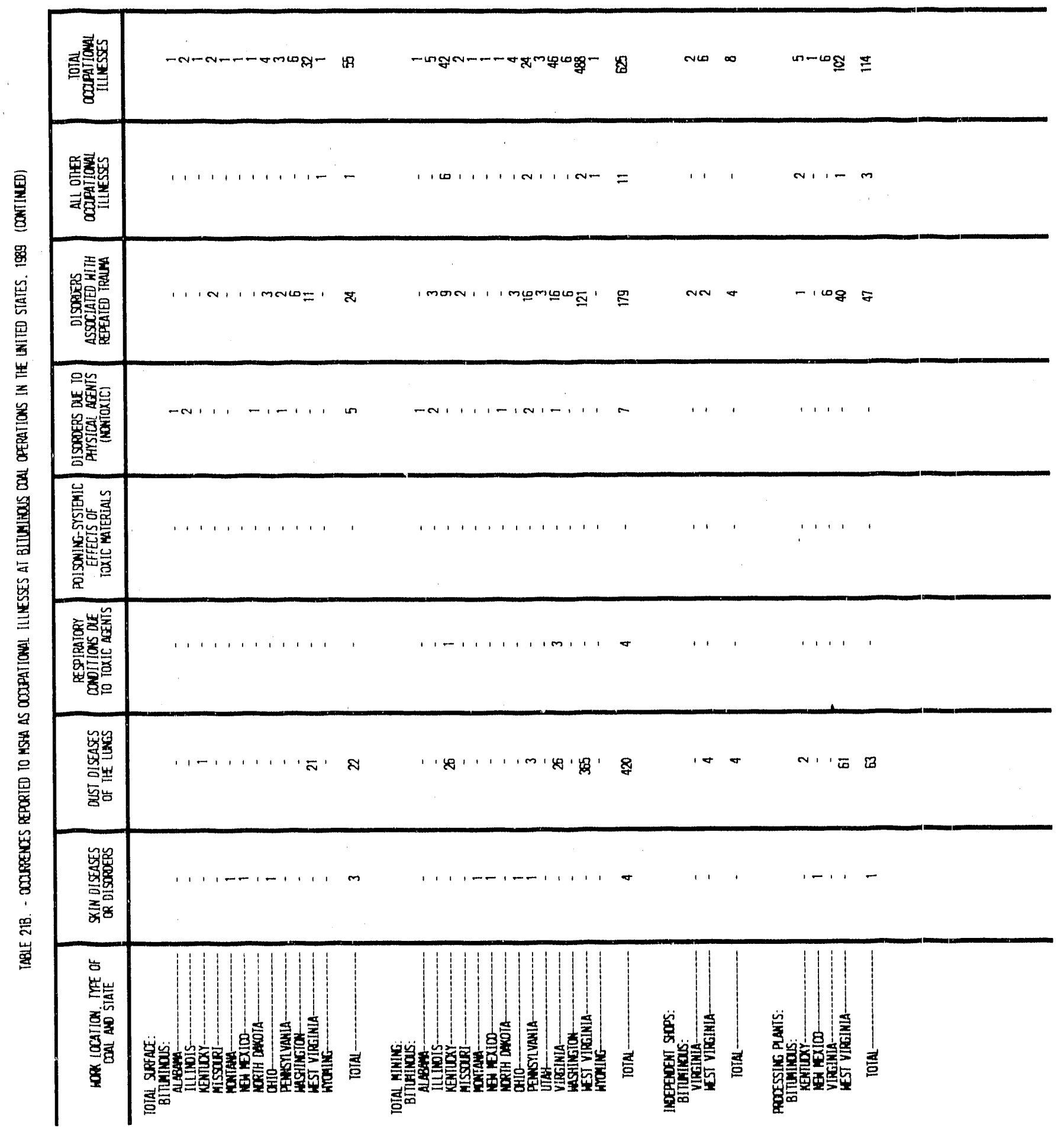




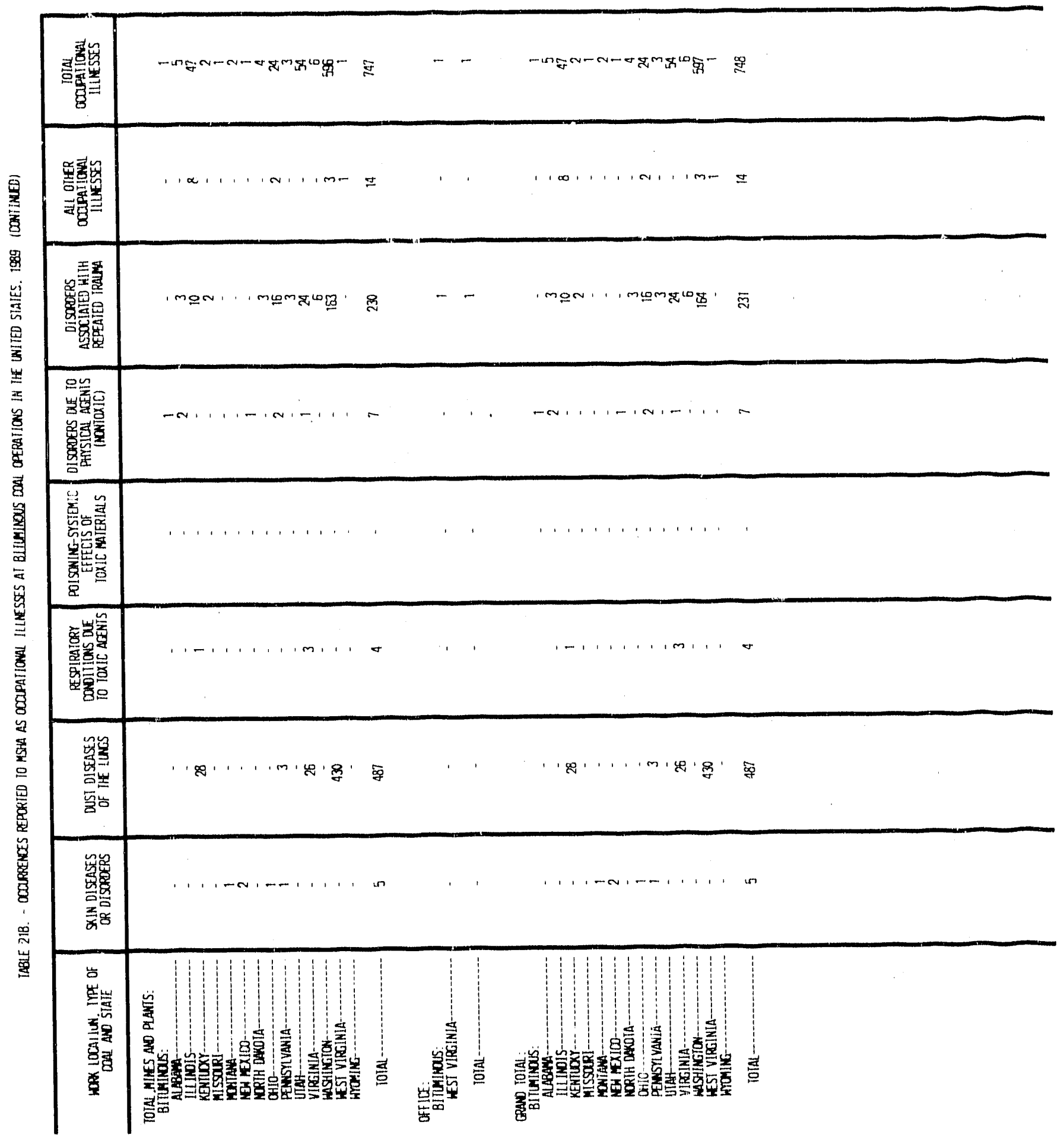




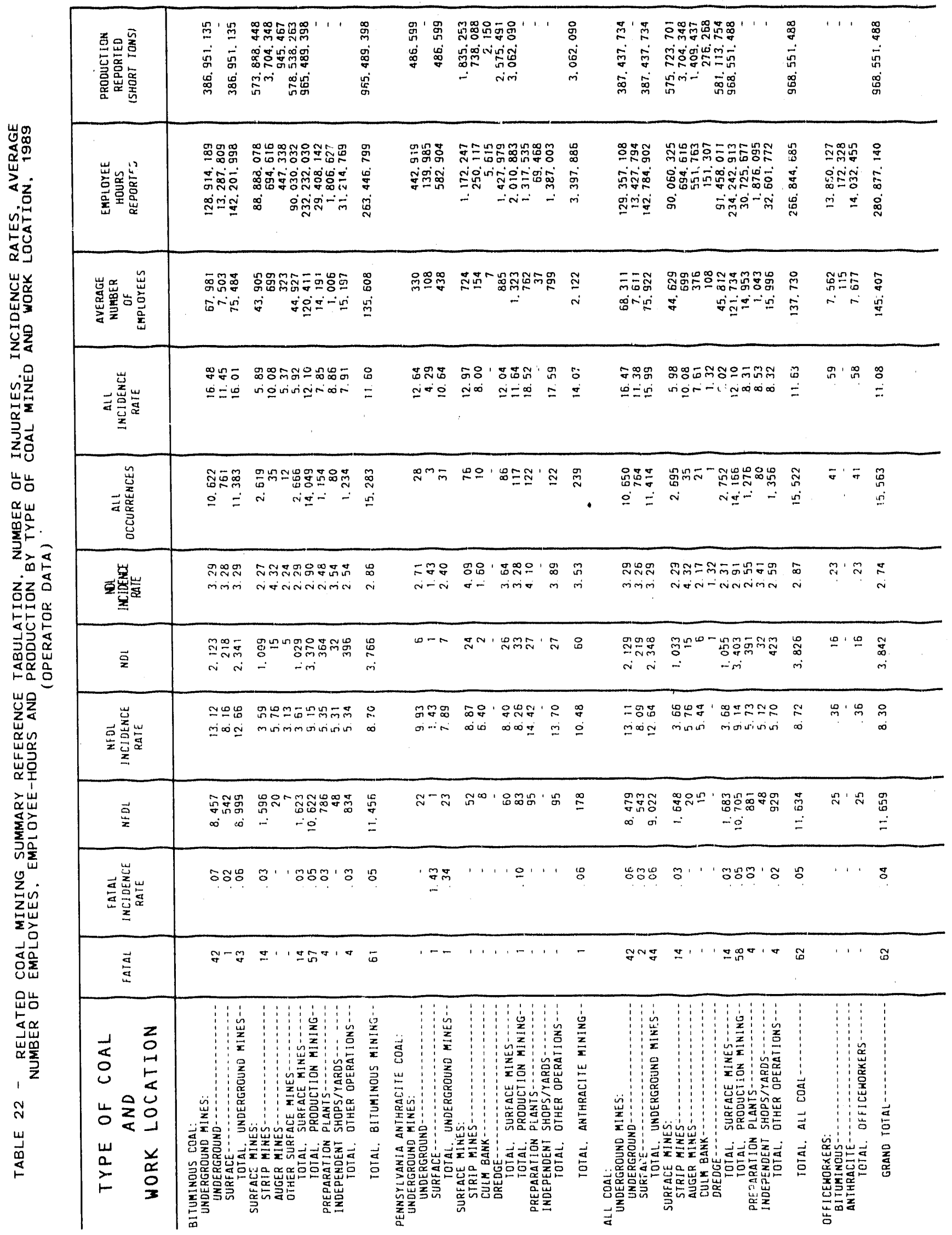




\begin{tabular}{|c|c|c|c|c|c|c|c|c|}
\hline 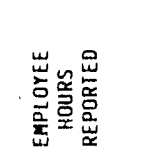 & 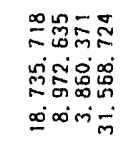 & 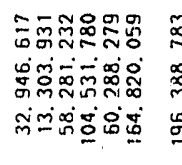 & 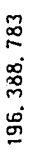 & 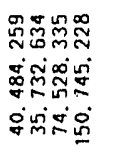 & 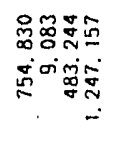 & 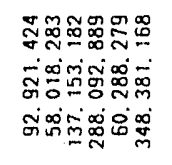 & 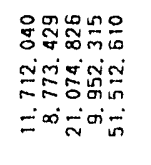 & 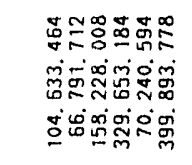 \\
\hline 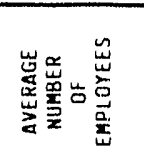 & 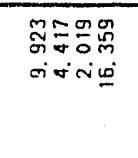 & 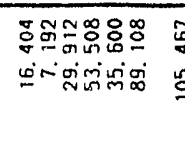 & 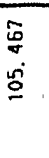 & 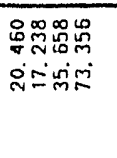 & 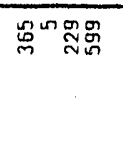 & 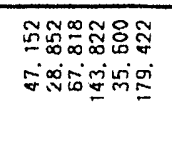 & 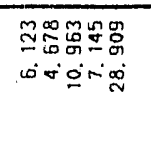 & 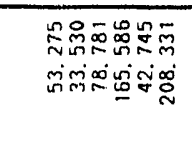 \\
\hline 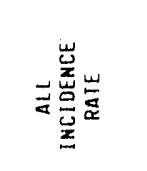 & 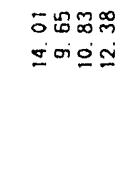 & 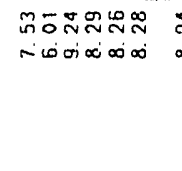 & $\begin{array}{l} \\
\text { a } \\
\infty\end{array}$ & 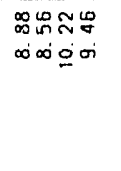 & 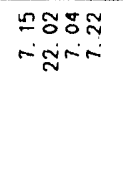 & 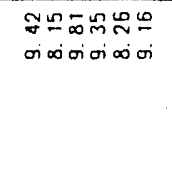 & 밈슈요 & 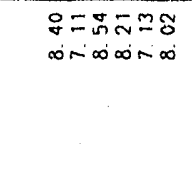 \\
\hline 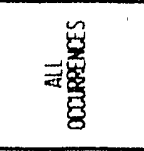 & 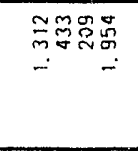 & 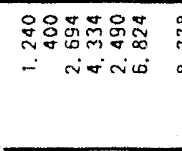 & $\begin{array}{l}\infty \\
\stackrel{\infty}{\infty} \\
\infty\end{array}$ & 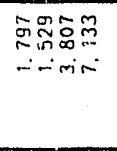 & $\bar{a}^{-5}$ & 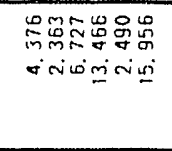 & $\overline{2 M \infty} \approx \mathbb{R}$ & 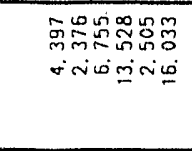 \\
\hline 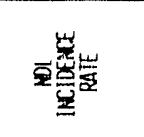 & 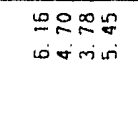 & 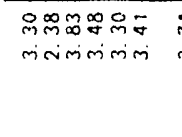 & $\therefore$ & 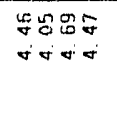 & 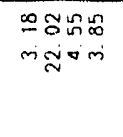 & 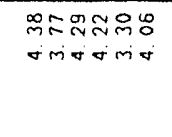 & $\because \bar{\Omega} \pm \simeq \omega$ & 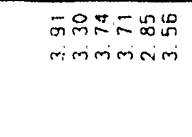 \\
\hline 荃 & 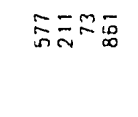 & 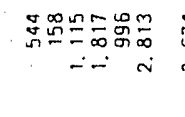 & $\begin{array}{c}\frac{y}{6} \\
\dot{m} \\
\dot{m}\end{array}$ & 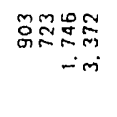 & 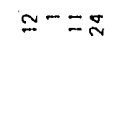 & 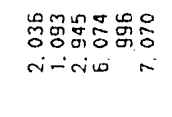 & 음ㅁ요요 & 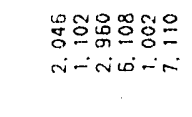 \\
\hline 崖. & 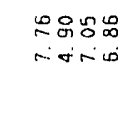 & 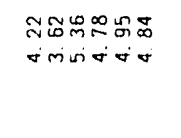 & 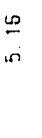 & 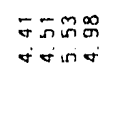 & 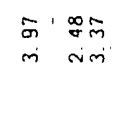 & 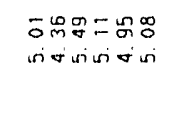 & g g g요요 & 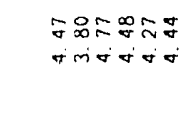 \\
\hline $\overrightarrow{\underline{a}}$ & 추줌ำ & 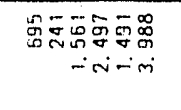 & $\begin{array}{l}\overline{0} \\
\text { si }\end{array}$ & 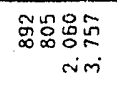 & $\simeq 10 \bar{N}$ & 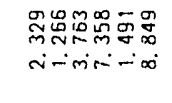 & $=\sigma \underline{m} \bar{m}$ & 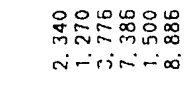 \\
\hline 崖 & 용요. & 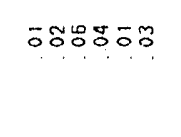 & m & $\overline{0} \overline{0} 0 \overline{0}$ & & ธธ్๐ & & รธํㅇ융ํ \\
\hline$\vec{a}$ & $\infty N \cdot$. & $-\cdots \infty$ & $m$ & $h-d$ & & $=\sigma \Phi \Phi m \tilde{m}$ & & 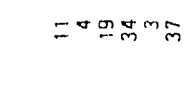 \\
\hline 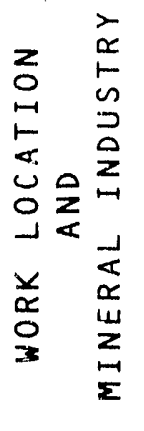 & 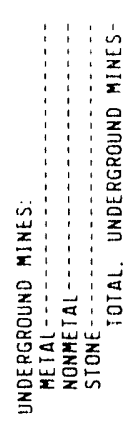 & 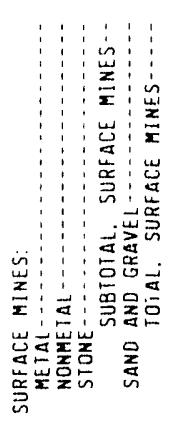 & 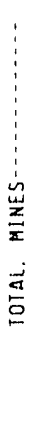 & 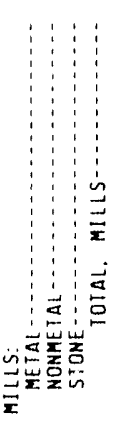 & 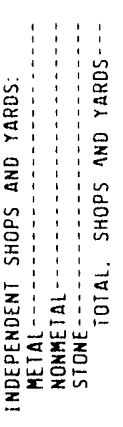 & 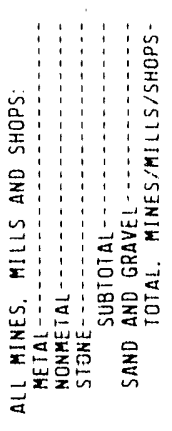 & 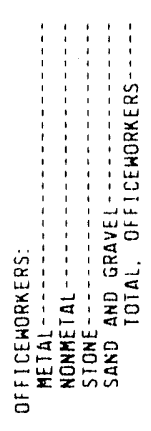 & 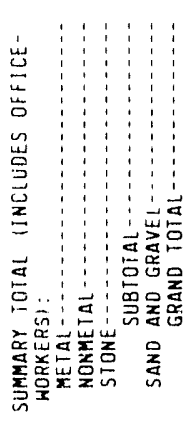 \\
\hline
\end{tabular}


CONTRACTOR DATA 
The following reference guide facilitates locating particular data elements contained in the annual statistical tables for contractors. The number appearing in each cell indicates the table number in which that particular element may be found. All elements listed under the stub headings appear as vertical headings in the tables. Elements 1 isted under colimn headings appear as horizontal headings. The only exception to this is table 1 , in which the headings bave been reversed.

To use this guide, read down or across the elements listed in both the stub and colum headings to determine which combinations are of interest. For example, lost workdays by work location appear in six different tables, numbers $5,7,9,12,13$, and 14 . However, only one of these, table 9 , provides a further division by nature of injury as well. The occupation of the injured is found in table 13 and, as seen in the colum headings, provides the number of injuries, lost workdays, and average severity.

Although injuries and illnesses occurring to contractor employees are identifiable as to state in which they occurred or conmodity mined, matching exposure hours are nat available. Incidence rates for such employees are possible only at the national level for the various subunits and only for the coal industry as a whole and the noncoal industry as a whole. Consequently, certain tables have been entirely eliminated from the contractor portion of this report. The remaining tables are numbered to correspond with the comparable table in the operator portion of this report. 
R.EFERENCE GUIDE TO COAL STATISTICAL TABLES (CONTRACTOR DATA)

\begin{tabular}{|c|c|c|c|c|c|c|c|c|c|c|c|c|}
\hline 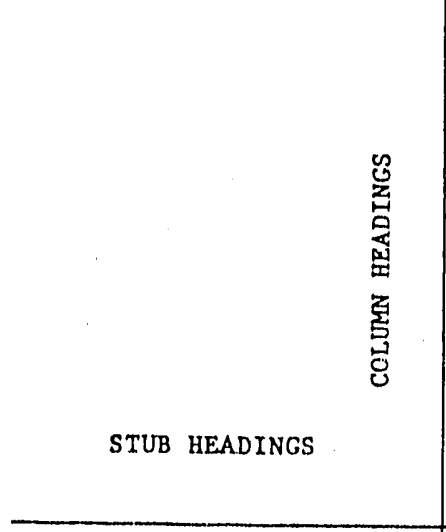 & 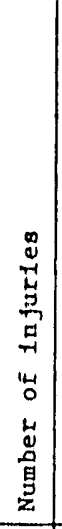 & 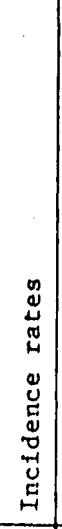 & 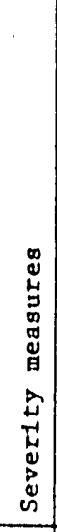 & 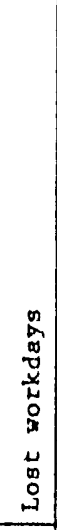 & 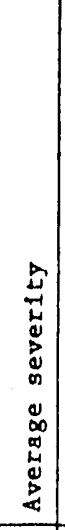 & 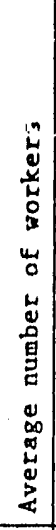 & 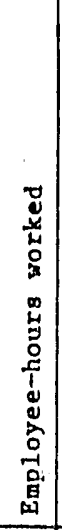 & 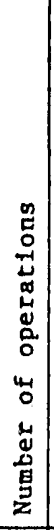 & 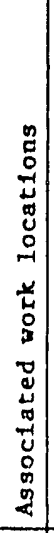 & 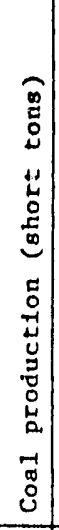 & 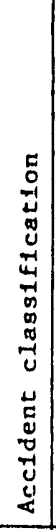 & 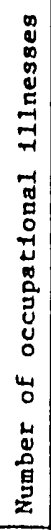 \\
\hline $\begin{array}{l}\text { Prior year data } \\
\text { w1th and without off } 1 \mathrm{ce}^{1 /}\end{array}$ & 1 & 1 & 1 & & 1 & 1 & 1 & 1 & 1 & 1 & & \\
\hline \multirow{15}{*}{ Work location } & 2 & 2 & 2 & & & 2 & 2 & 2 & 2 & 2 & & \\
\hline & 3 & & & 3 & 3 & & & & & & & \\
\hline & 4 & 4 & 4 & & & 4 & 4 & 4 & 4 & द) & & \\
\hline & 5 & & & 5 & 5 & & & & & & & \\
\hline & 7 & & & 9 & 7 & & & & 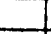 & & & \\
\hline & 8 & 8 & 8 & & & & & & 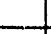 & & & \\
\hline & 9 & & & 9 & 9 & & & & & & & \\
\hline & 10 & 10 & 10 & & & & & & & & & \\
\hline & $\frac{11}{13}$ & & & 11 & 11 & & & & & & 13 & \\
\hline & & & & 14 & & & & & & & 14 & \\
\hline & 17 & & & 17 & 1) & & & & & & & 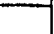 \\
\hline & 1.8 & & & 18 & 18 & & & & & & & \\
\hline & & & & & & & & & & & & 21 \\
\hline & 22 & 22 & & & & 22 & 22 & & & 22 & & \\
\hline & 23 & 23 & & & & 23 & 23 & & & & & \\
\hline \multirow{2}{*}{ Employment size group } & 4 & 4 & 4 & & & 4 & 4 & 4 & 4 & 4 & & \\
\hline & 5 & & & 5 & 5 & & & & & & & \\
\hline \multirow{3}{*}{ State } & 7 & & & 7 & 7. & & & & & & & \\
\hline & 13 & & & & & & & & & & 13 & \\
\hline & & & & 14 & & & & & & & 14 & \\
\hline \multirow{4}{*}{ Part of body infured } & 87 & 81 & & & & & & & & & & 21 \\
\hline & 9 & 0 & & 9 & 9 & & & & & & & \\
\hline & 12 & & & 12 & 12 & & & & & & & \\
\hline & 19 & & & 19 & 19 & & & & & & & \\
\hline \multirow{4}{*}{ Nature of Infury } & 10 & 10 & 10 & & & & & & & & & \\
\hline & 11 & & & 11 & 11 & & & & & & & \\
\hline & 12 & & & 12 & 12 & & & & & & & \\
\hline & 20 & & & 20 & 20 & & & & & & & \\
\hline $\begin{array}{l}\text { Occupation at time of } \\
\text { Infury }\end{array}$ & 17 & & & 17 & 17 & & & & & & & \\
\hline \multirow{3}{*}{ Accldent classification } & 18 & & & 18 & 18 & & & & & & & \\
\hline & 19 & & & 19 & 19 & & & & & & & \\
\hline & 20 & & & 20 & 20 & & & & & & & \\
\hline Type of coal & 22 & 22 & & & & 22 & 22 & & & 22 & & \\
\hline Mineral Induatry & 23 & 23 & & & & 23 & 23 & & & & & \\
\hline
\end{tabular}

$1 /$ For table 1 only, the headings have been reversed to oloplify this guide; that 1s, the data elements indicated in the column headinga for thlo table actually appear a vertical headings. 


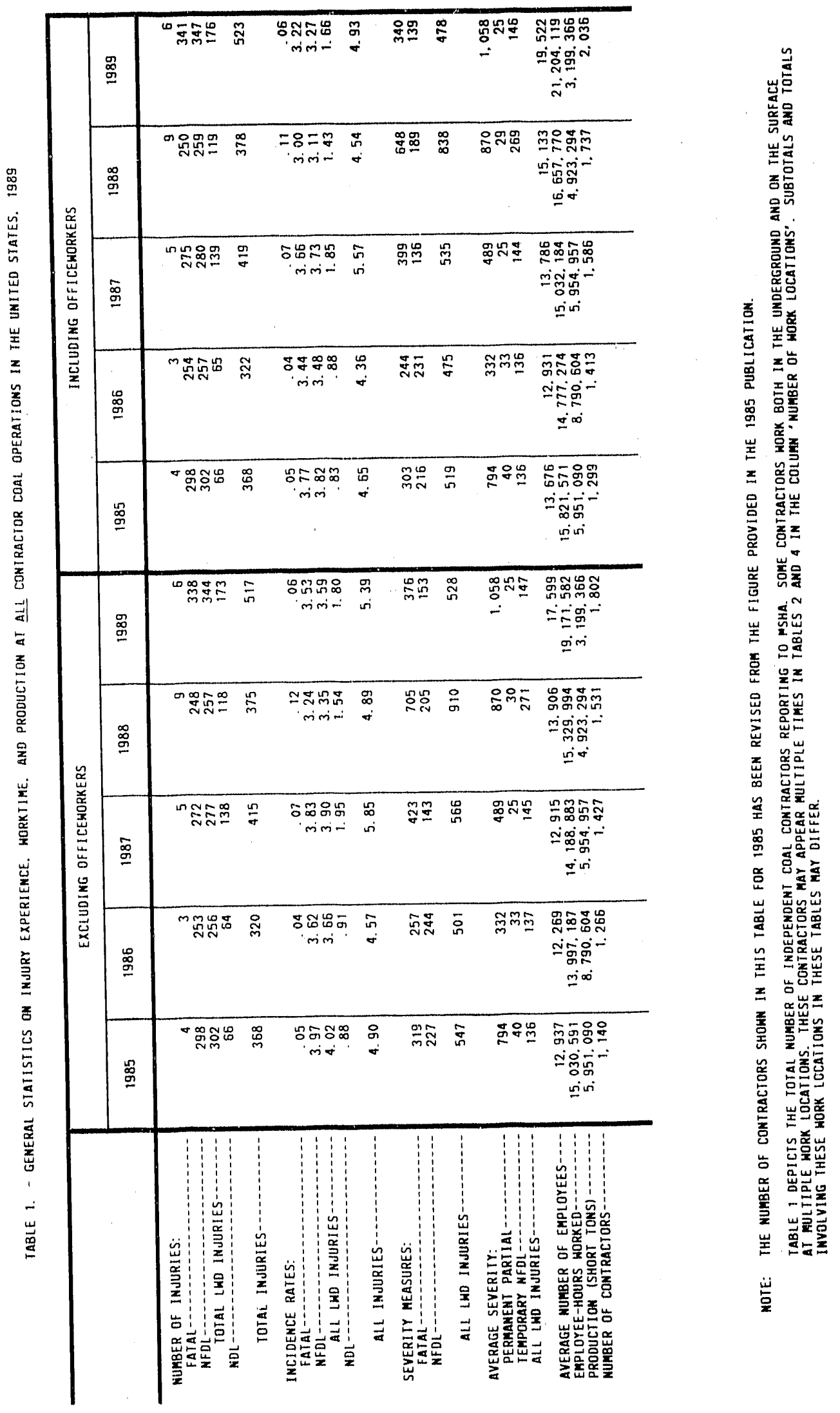




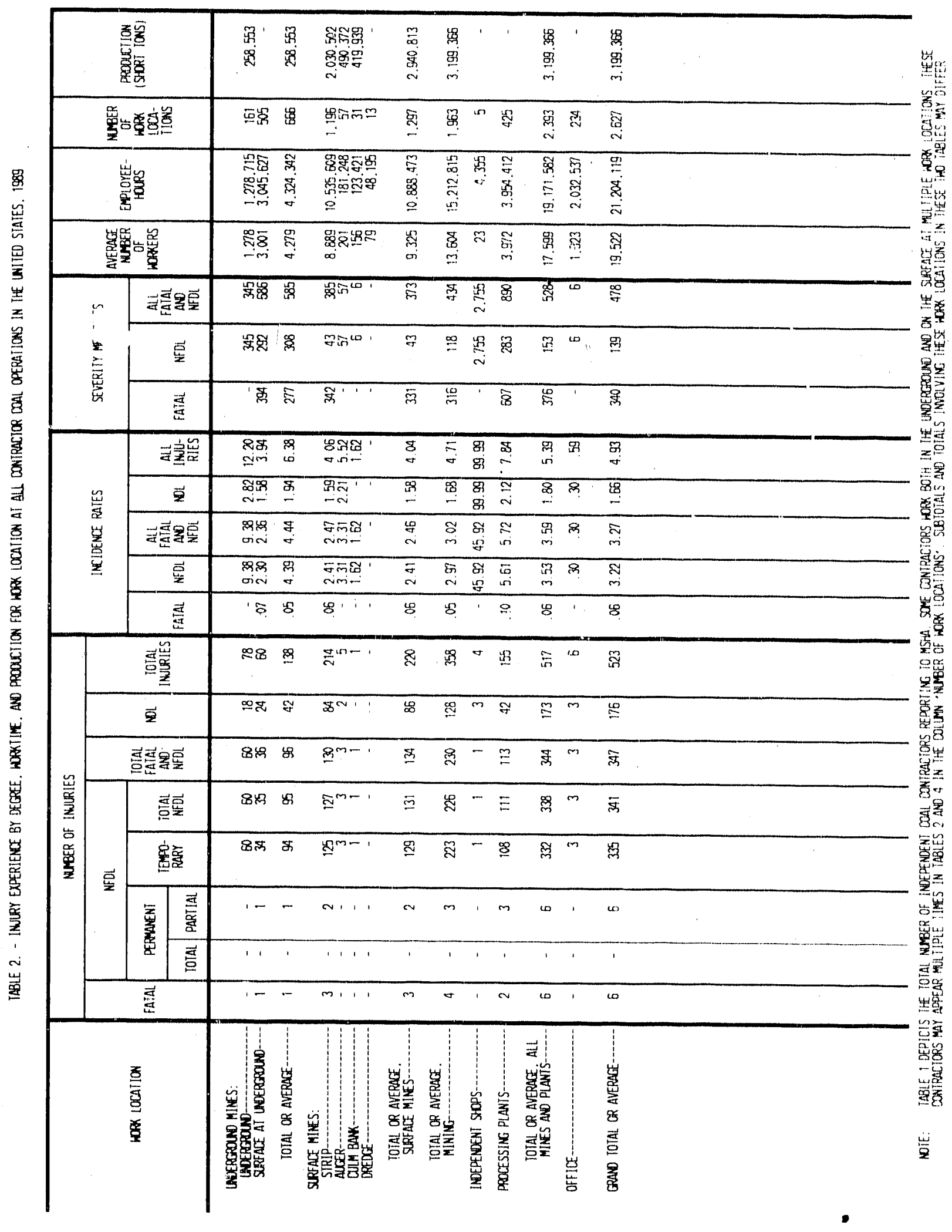




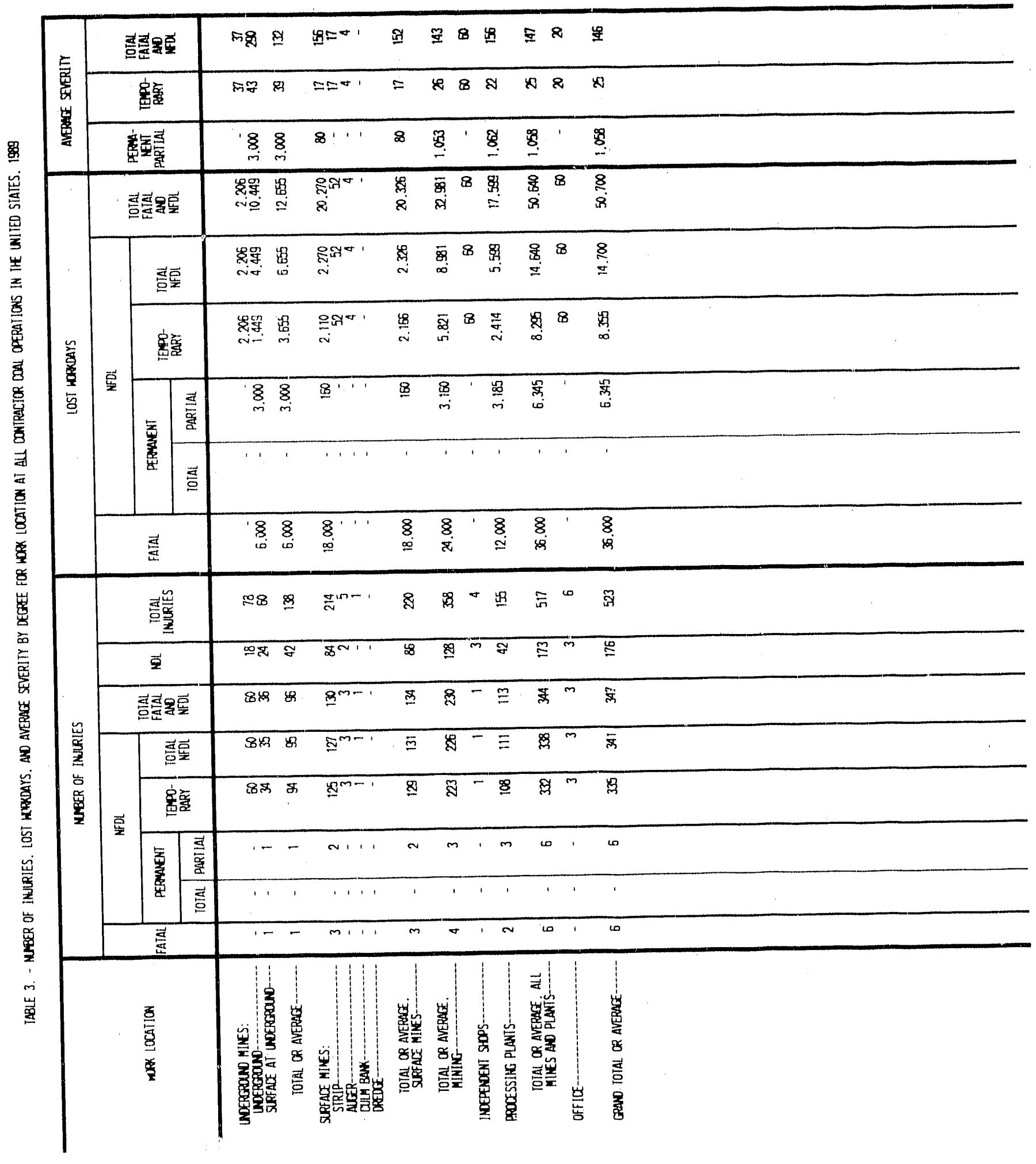




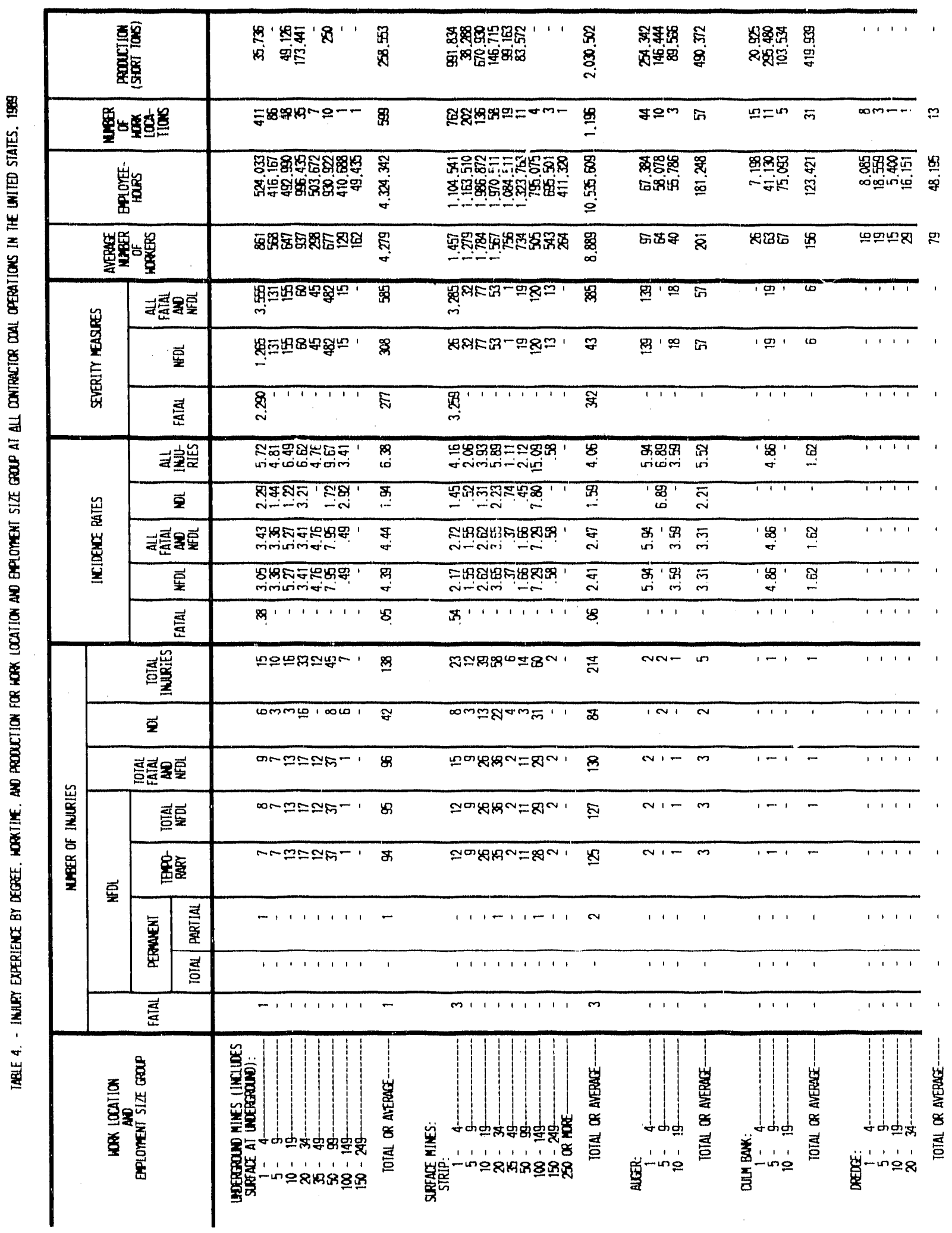




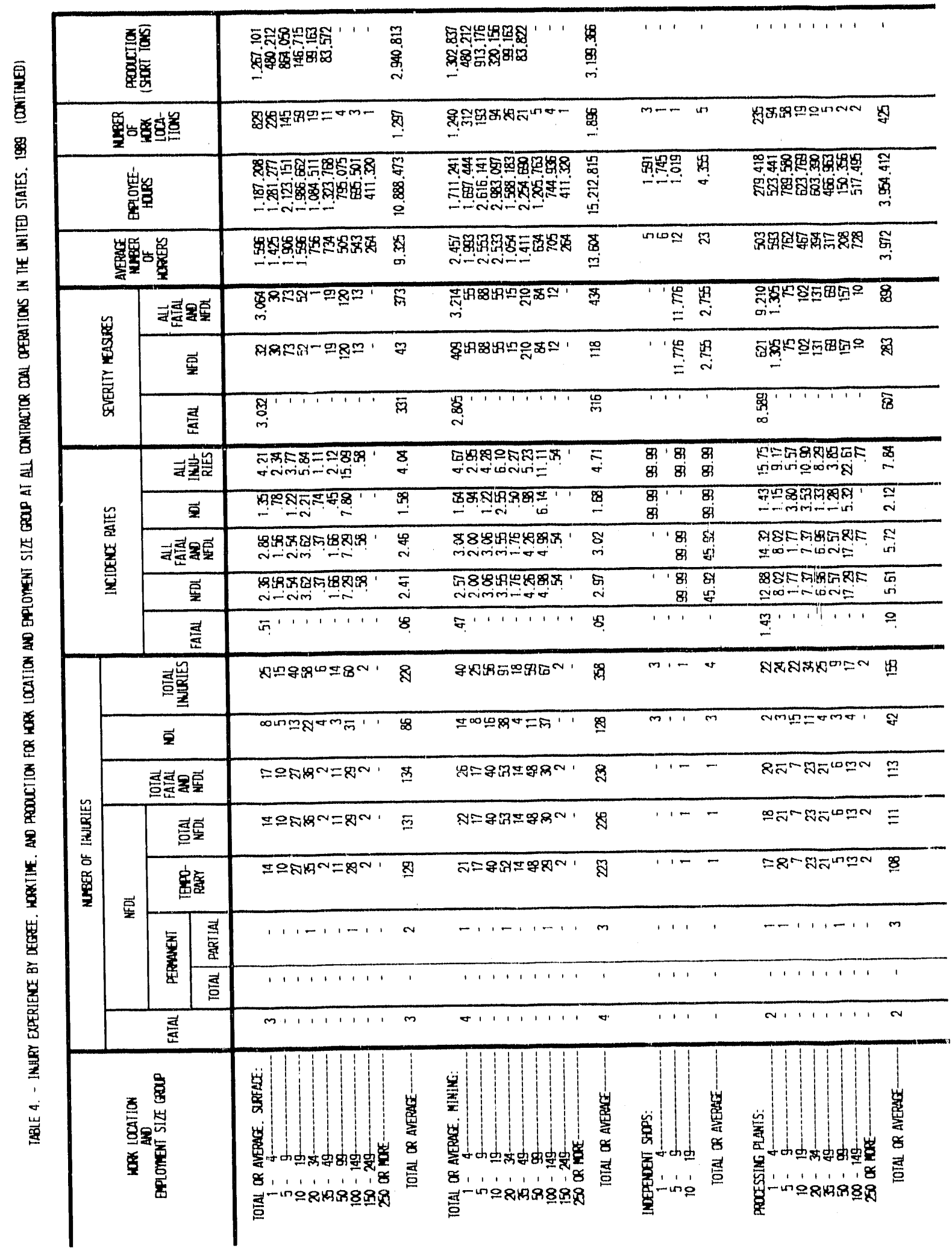




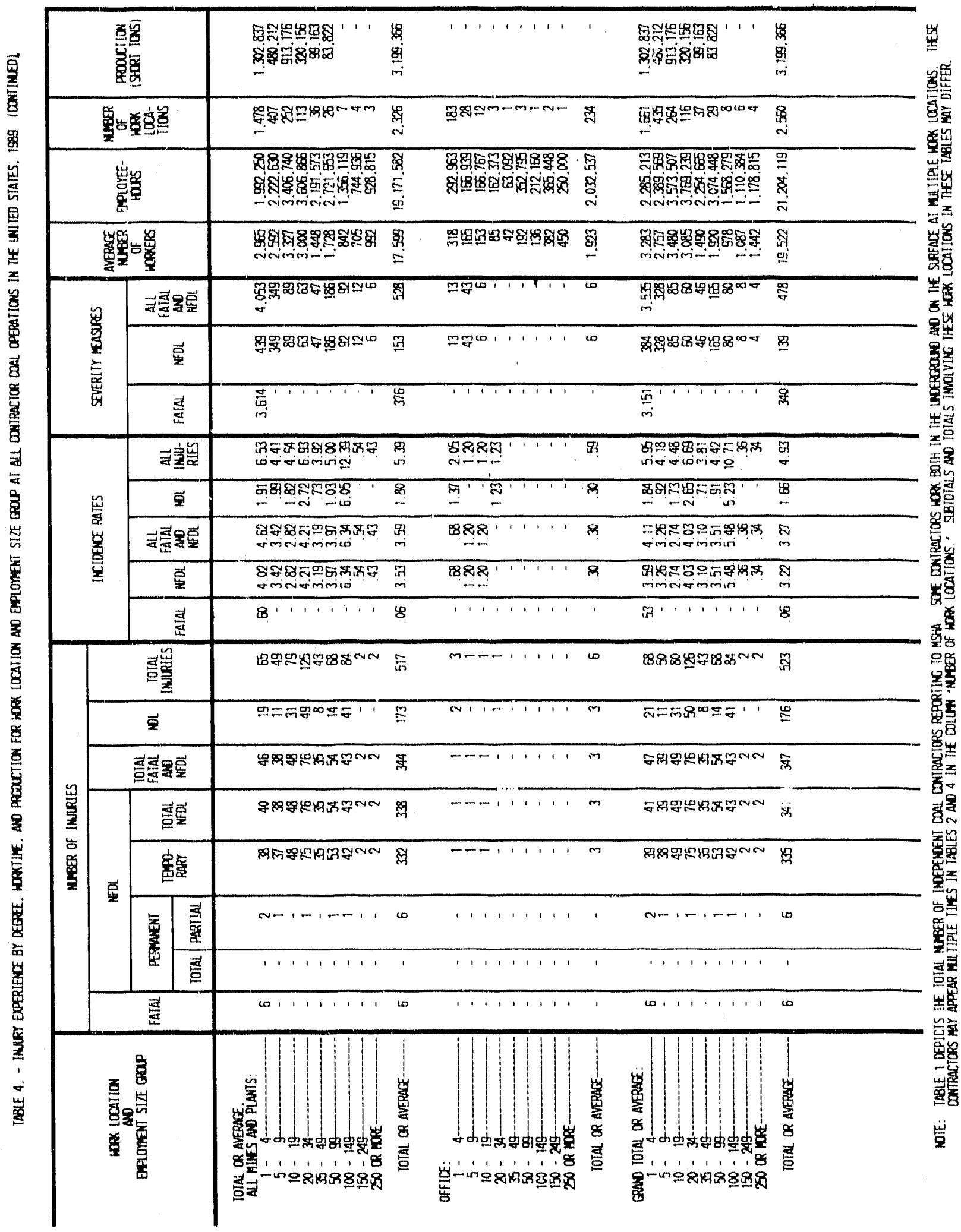




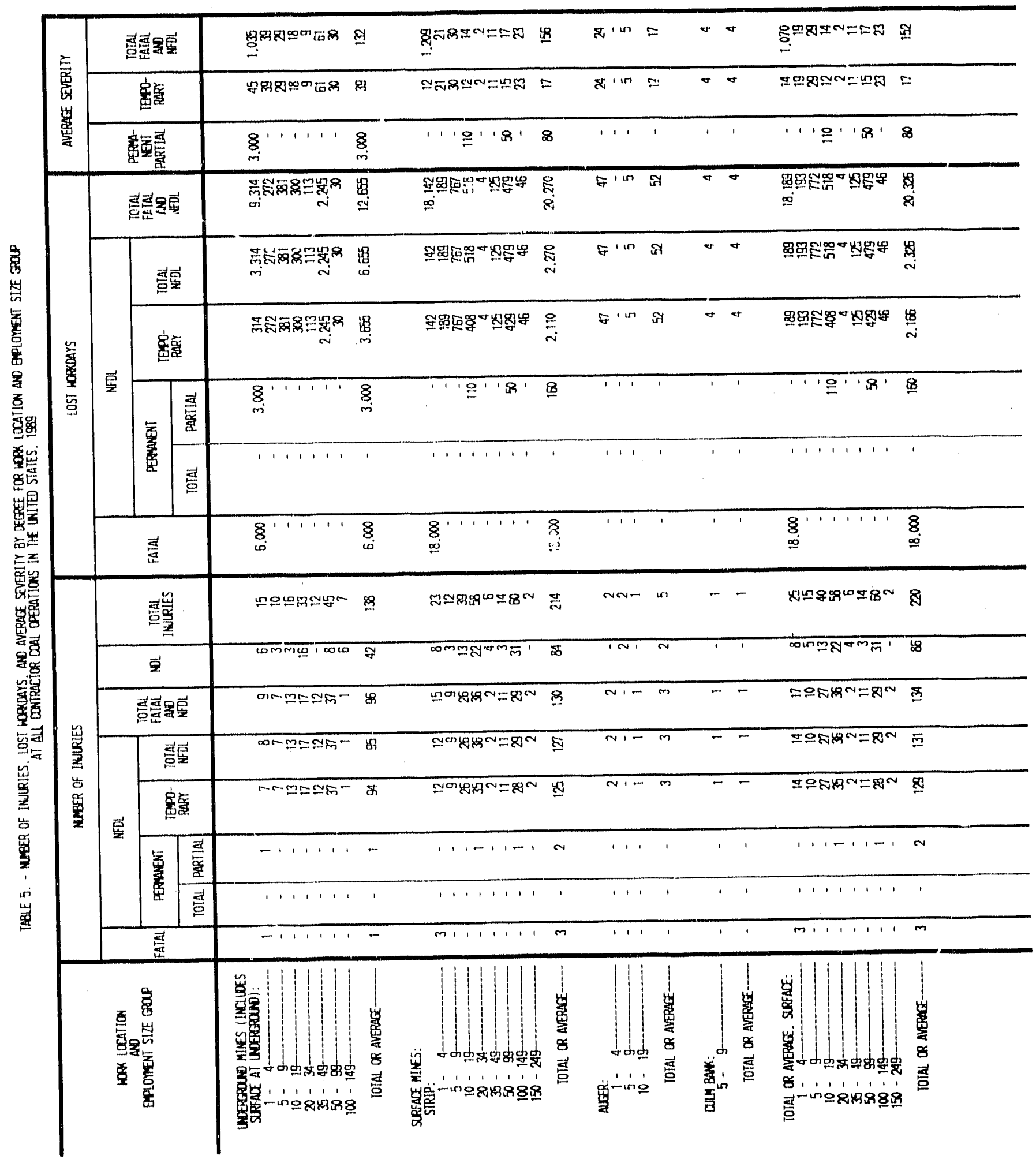




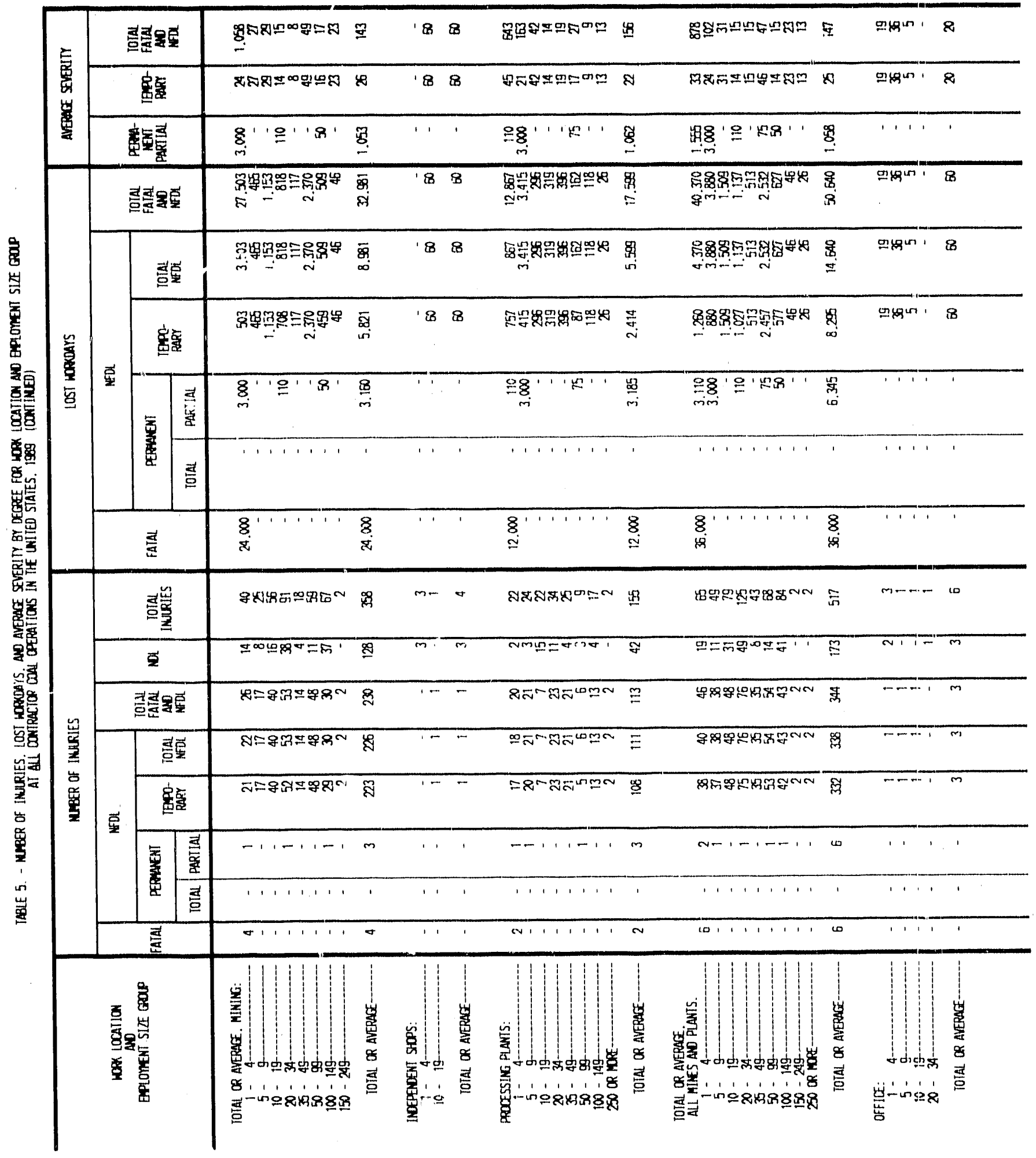




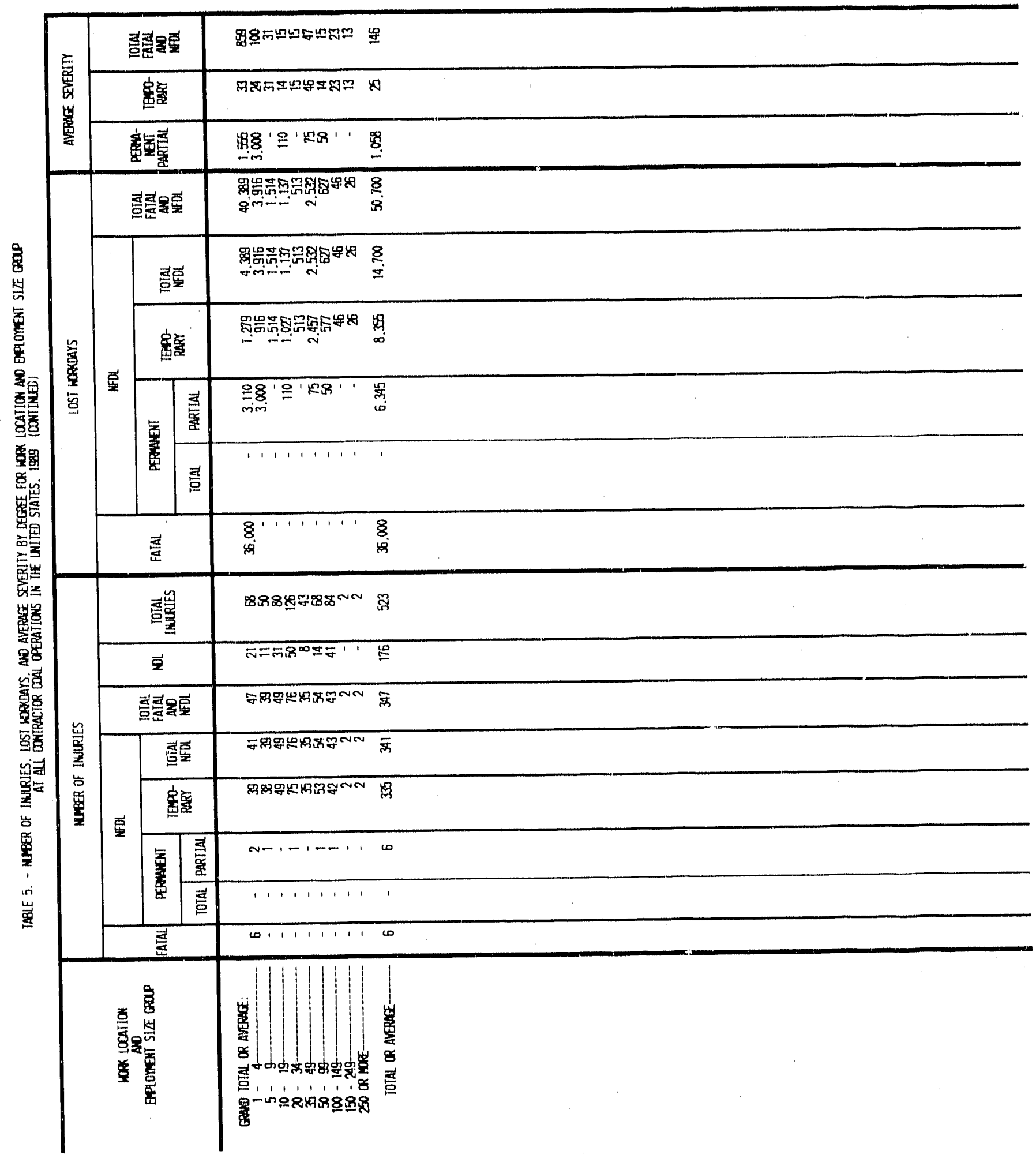




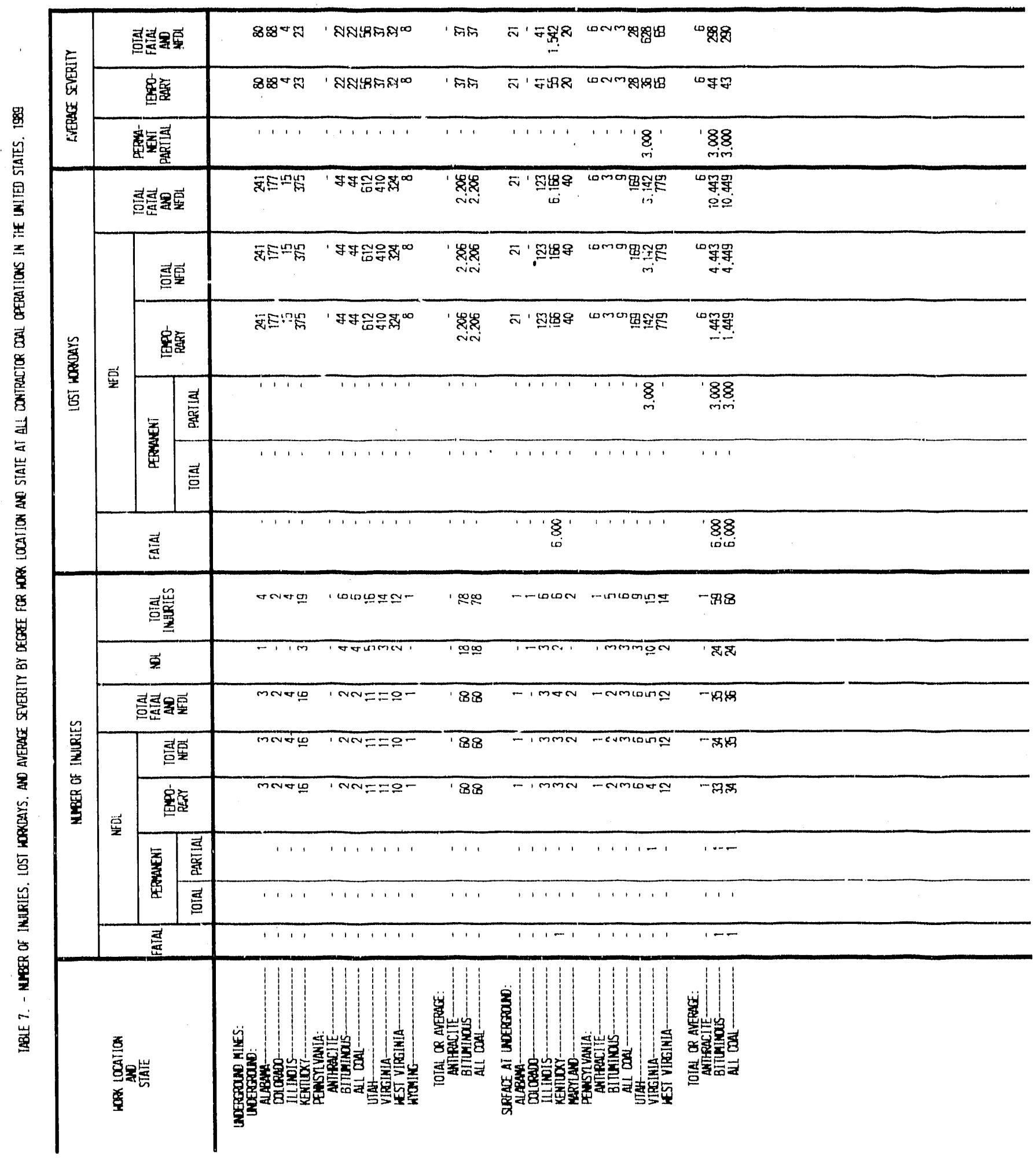




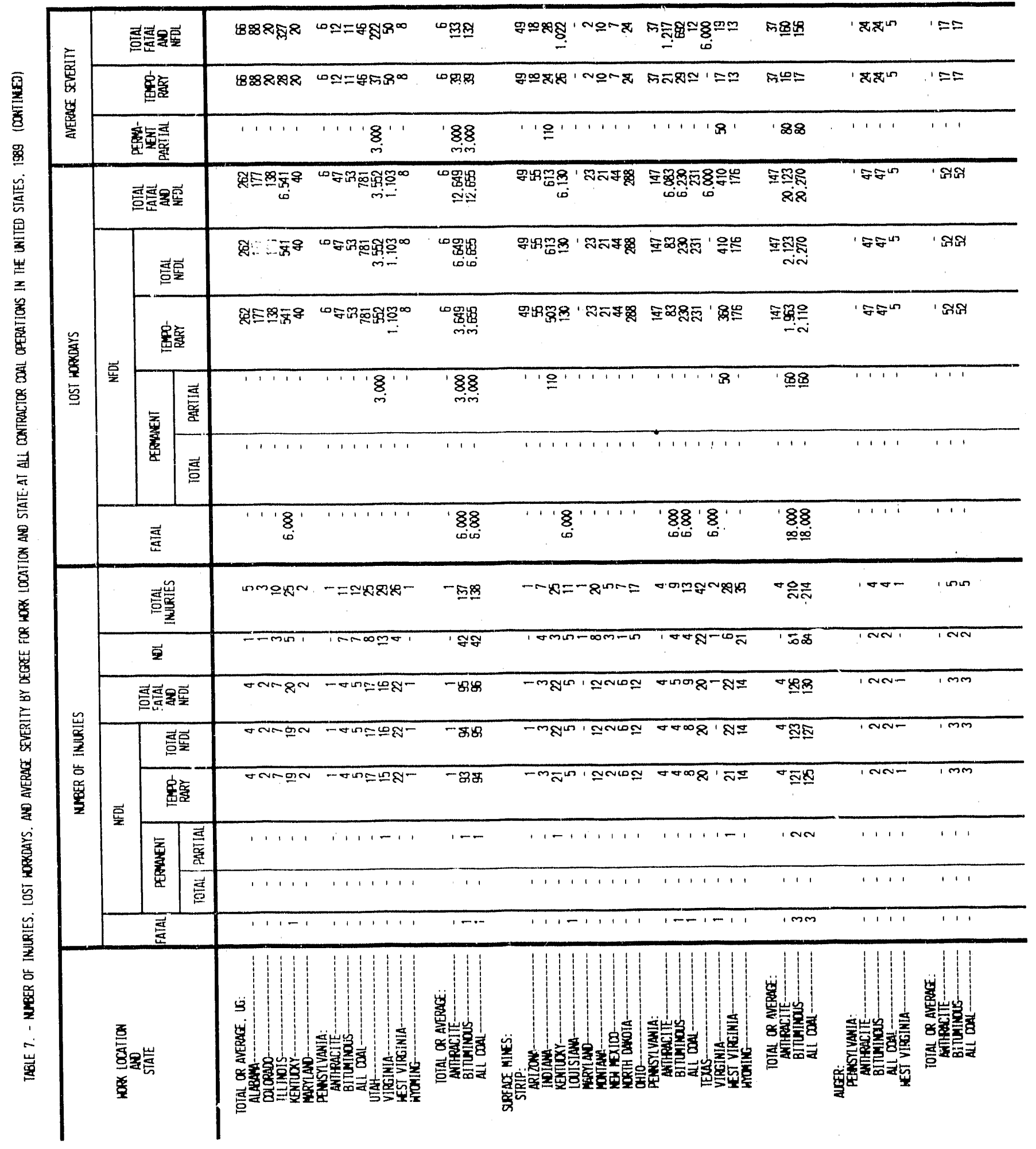




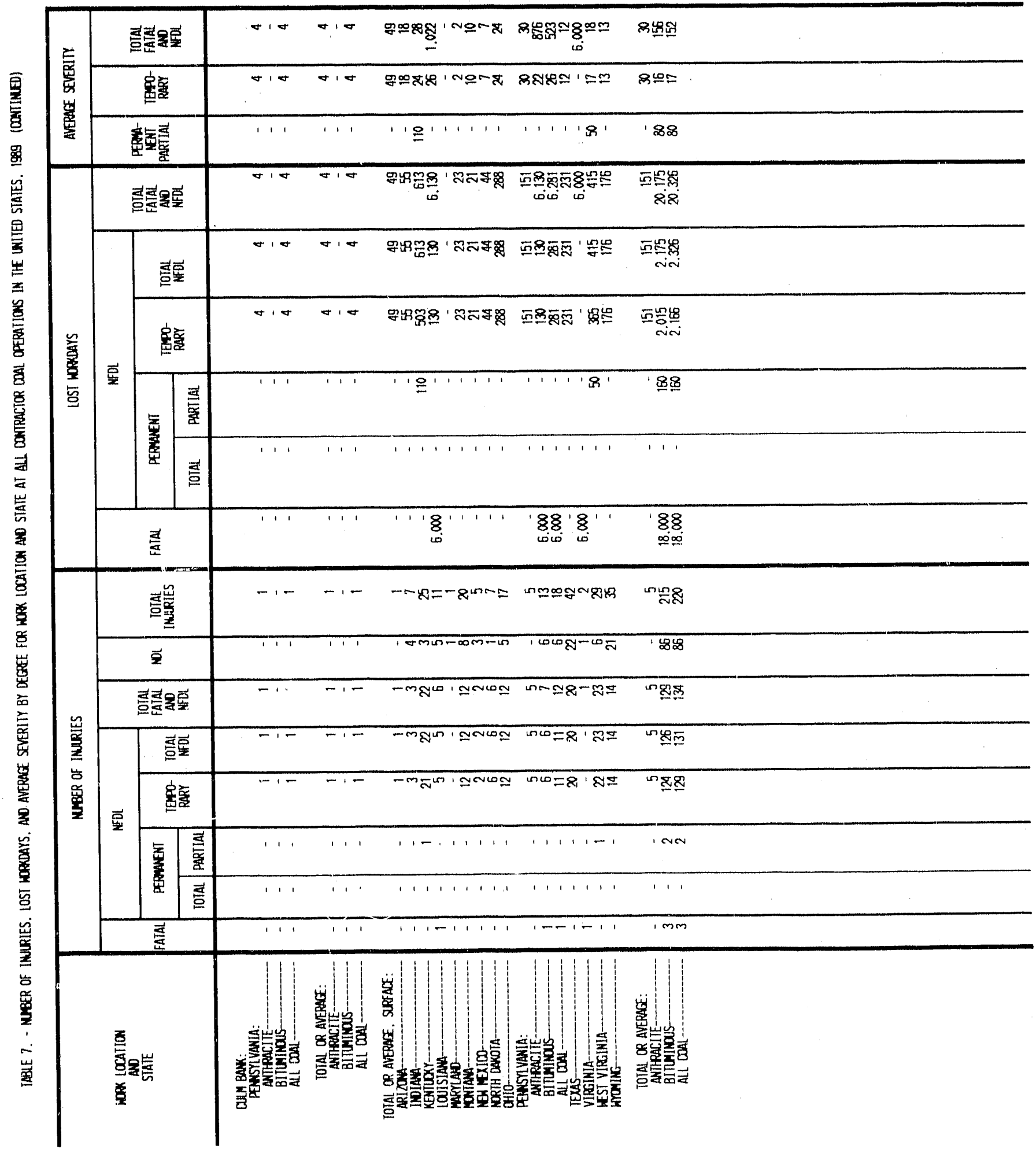




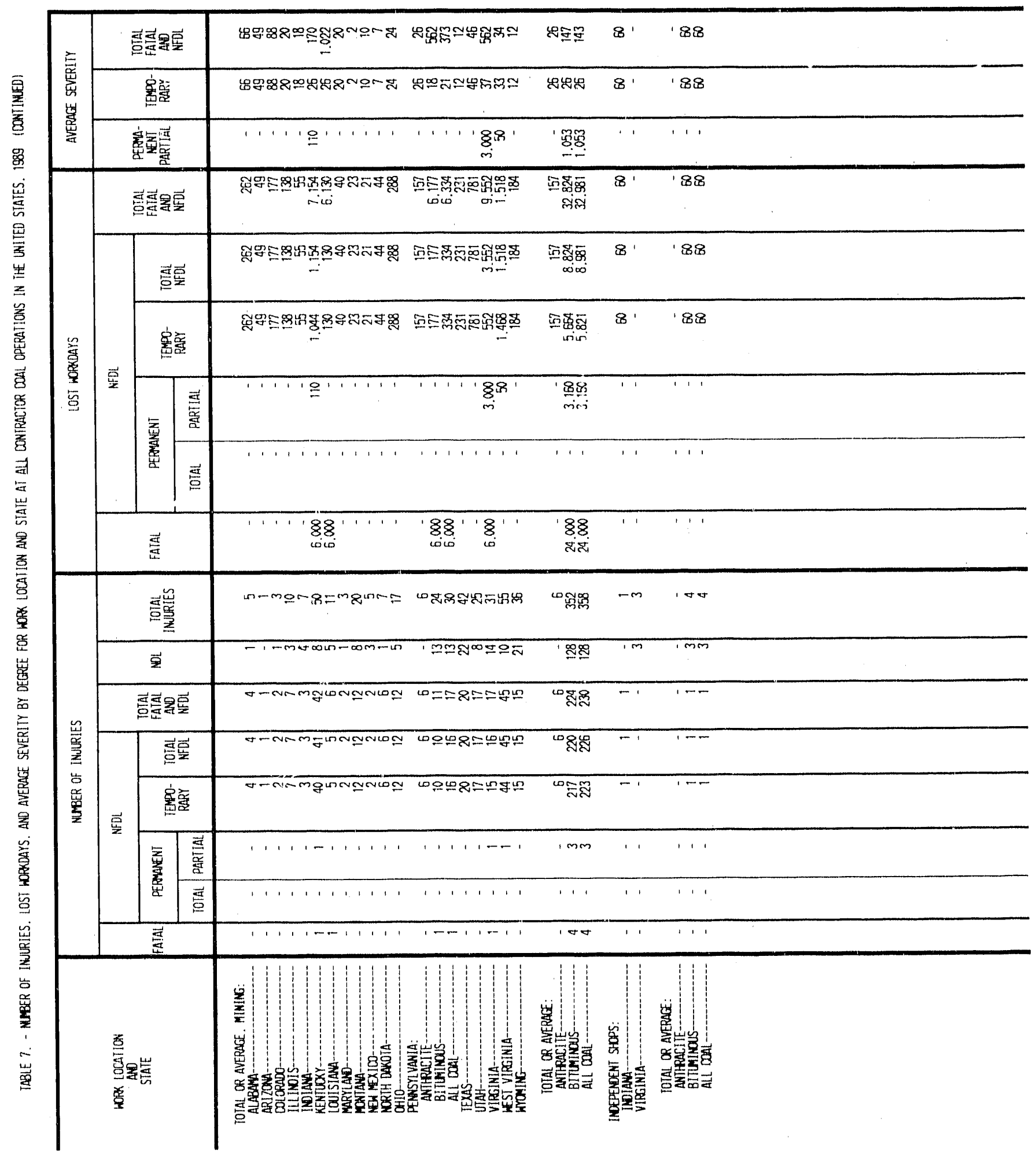




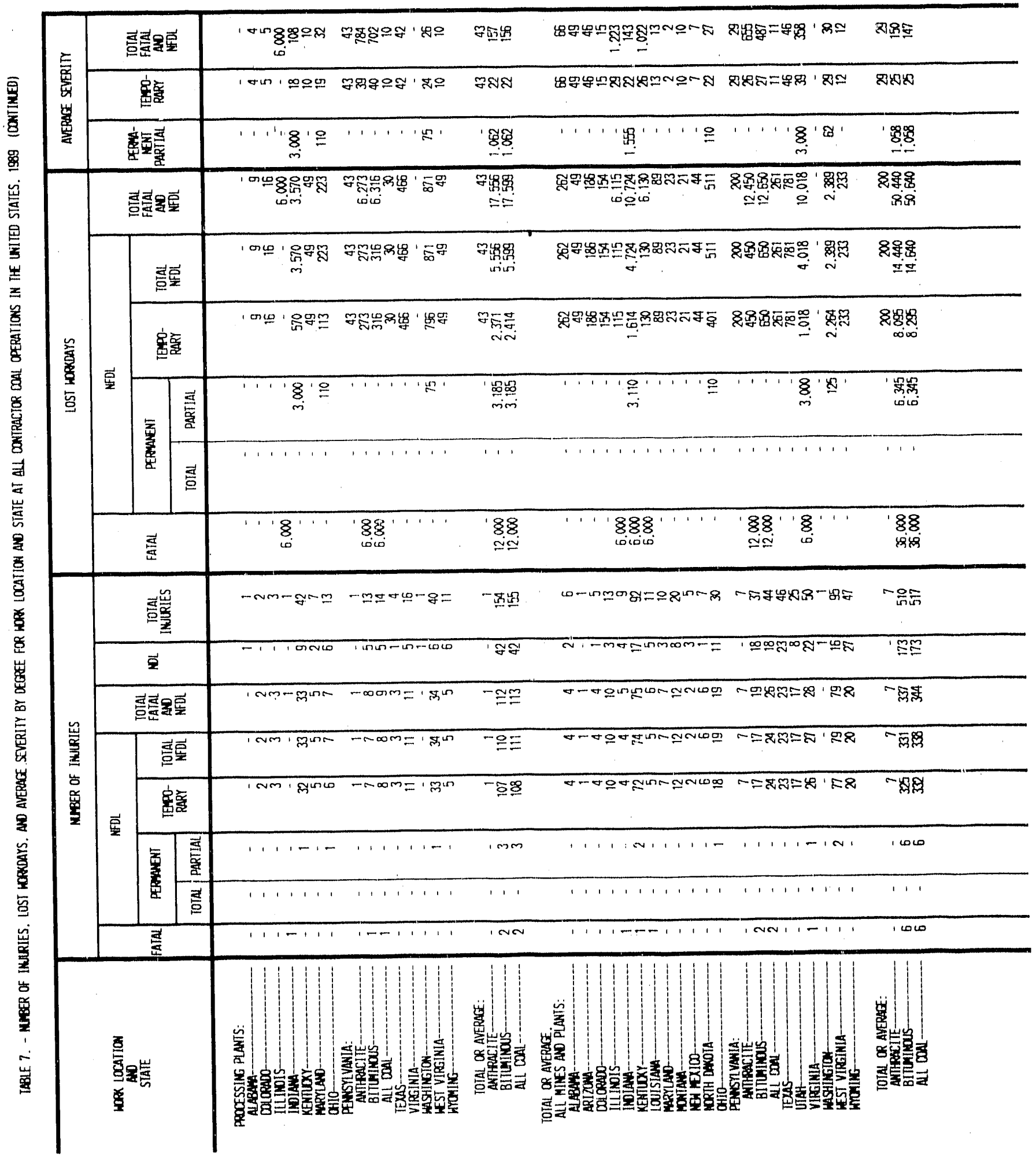




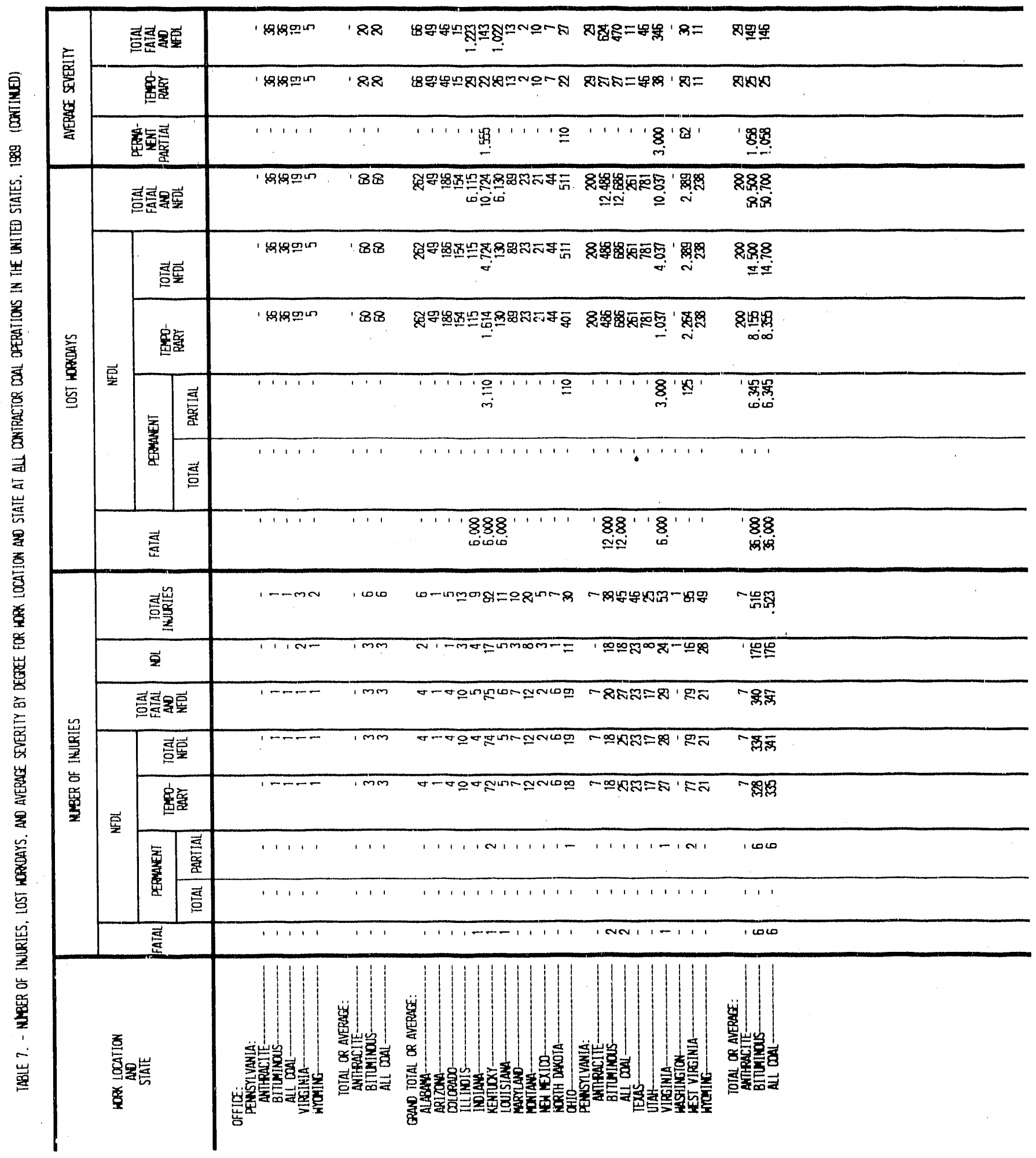




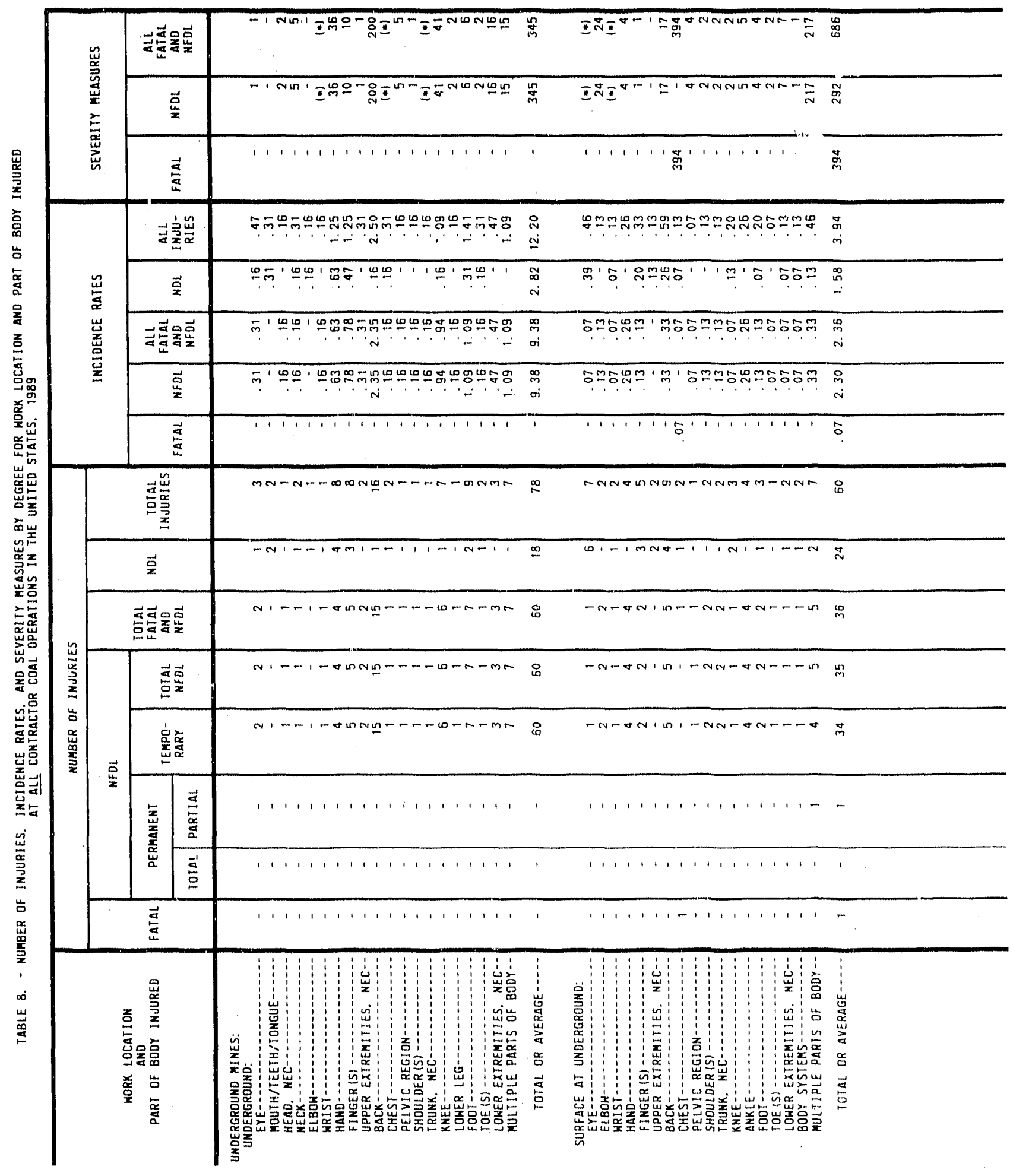




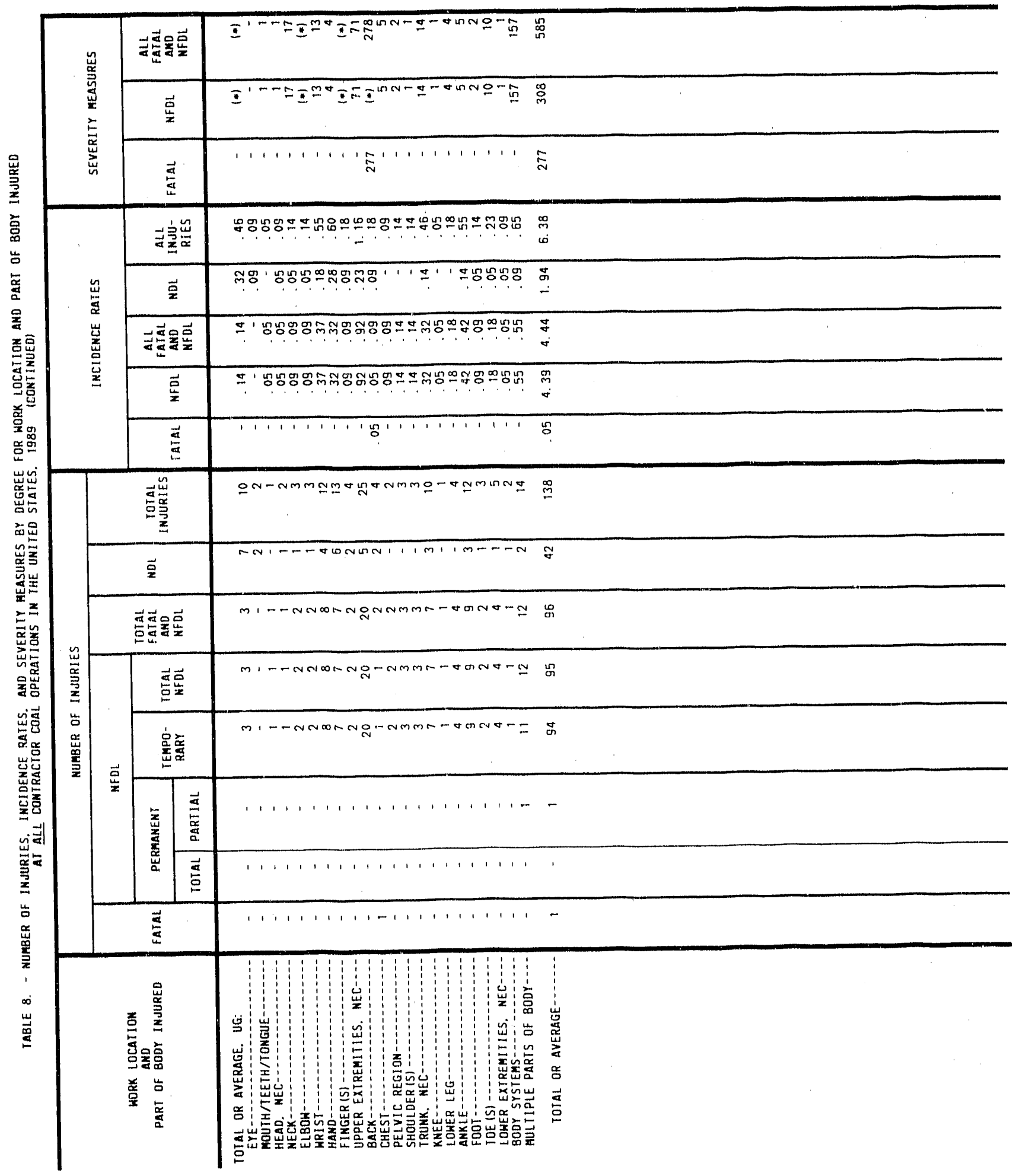




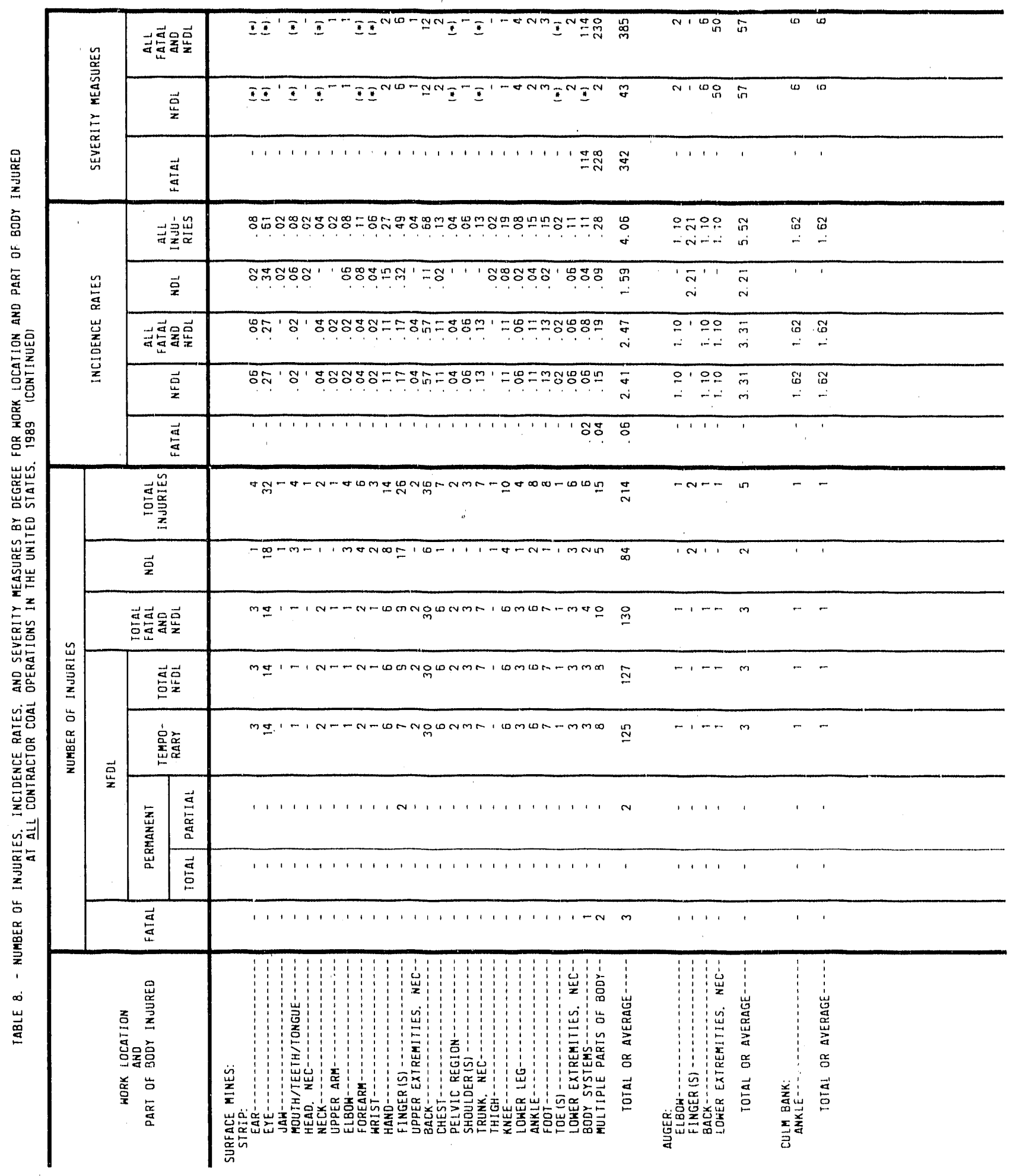




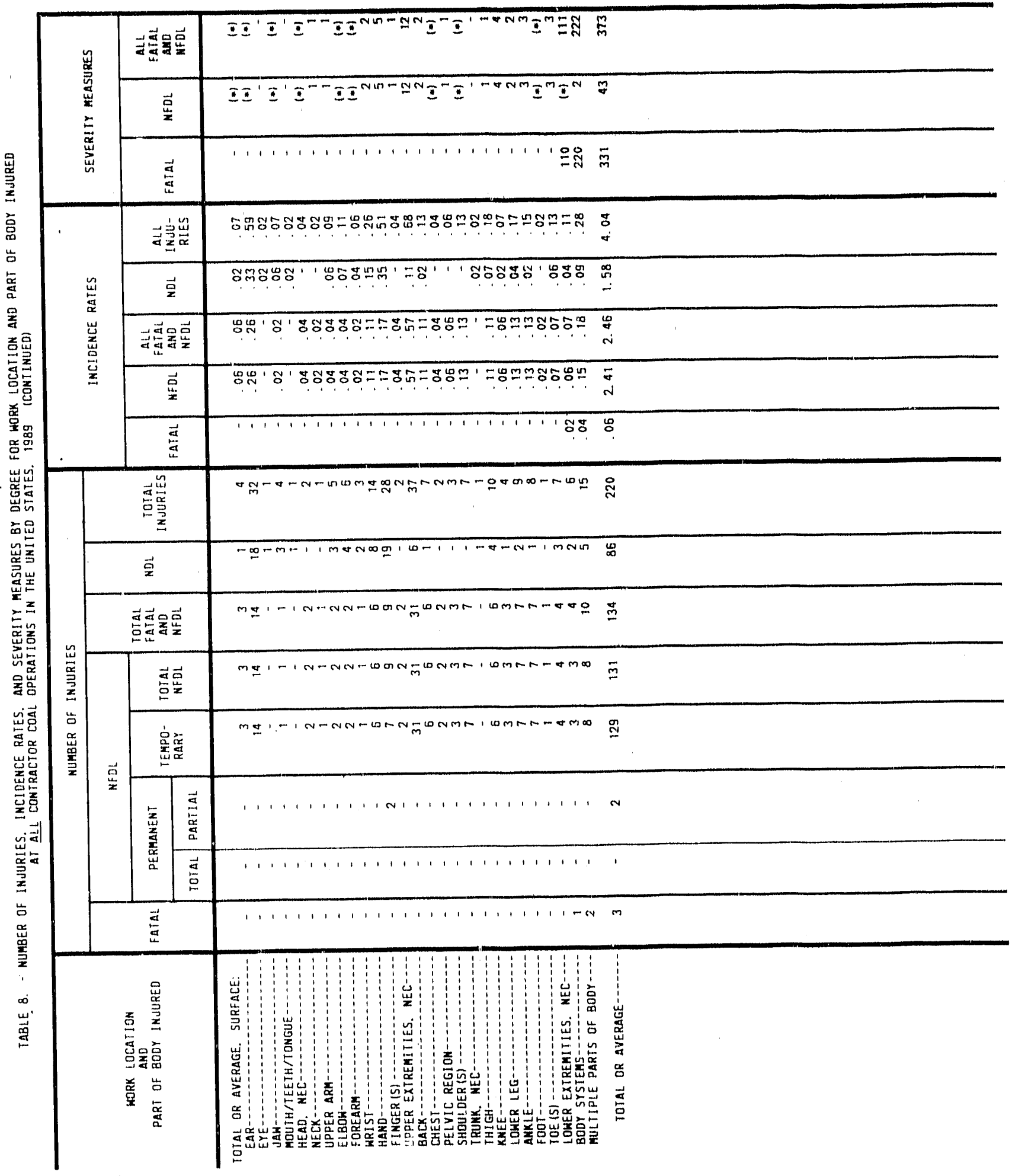




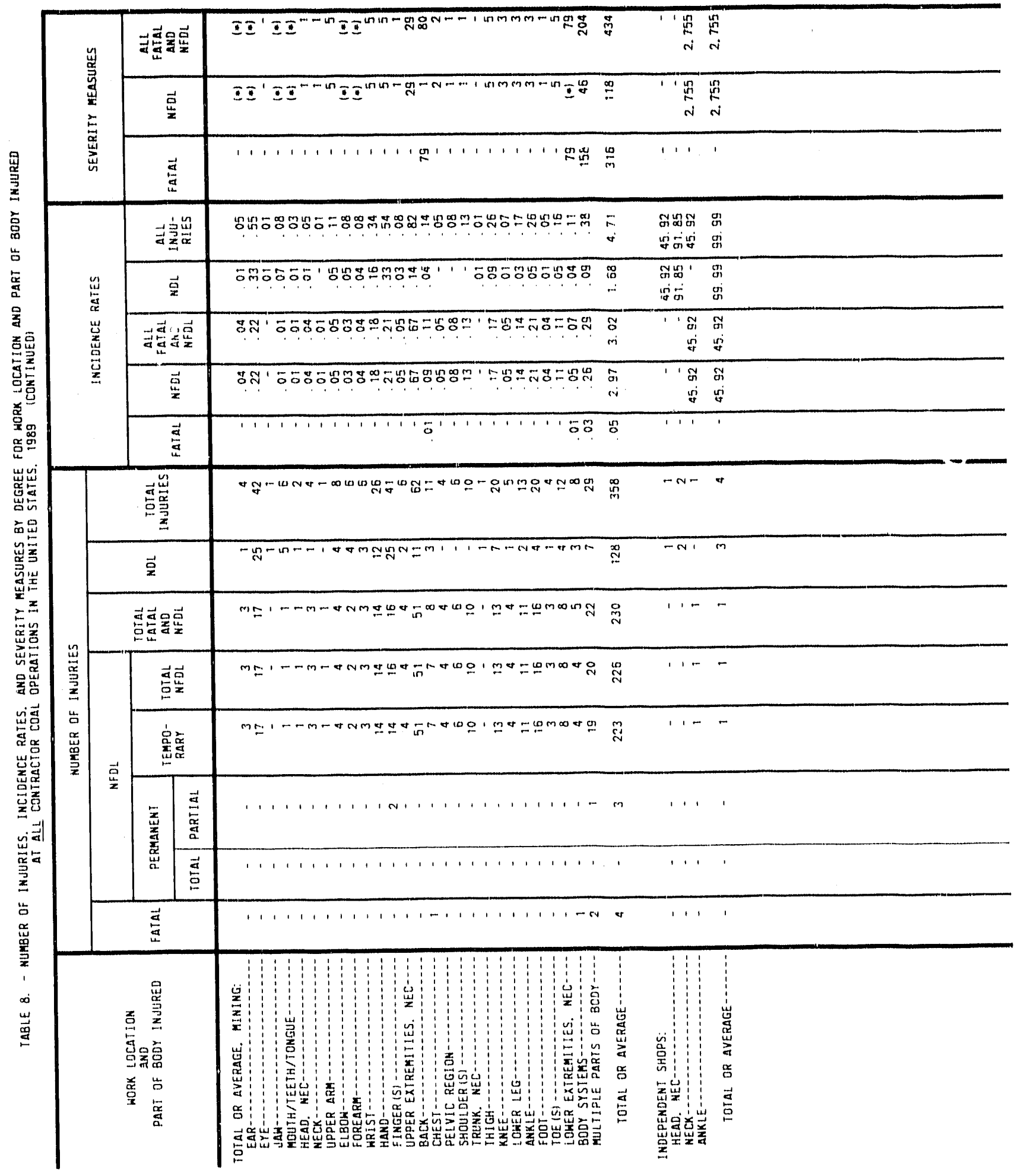




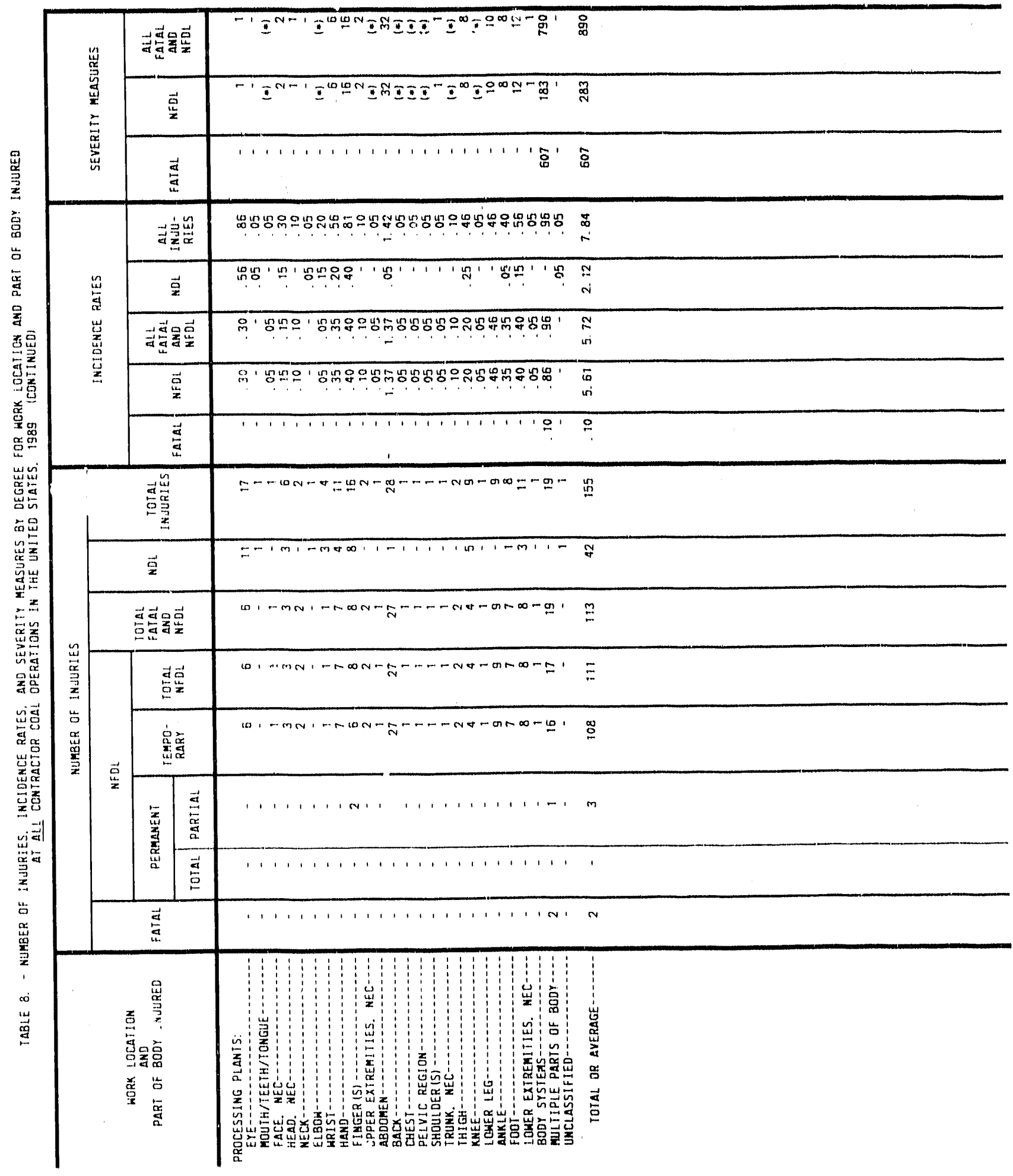




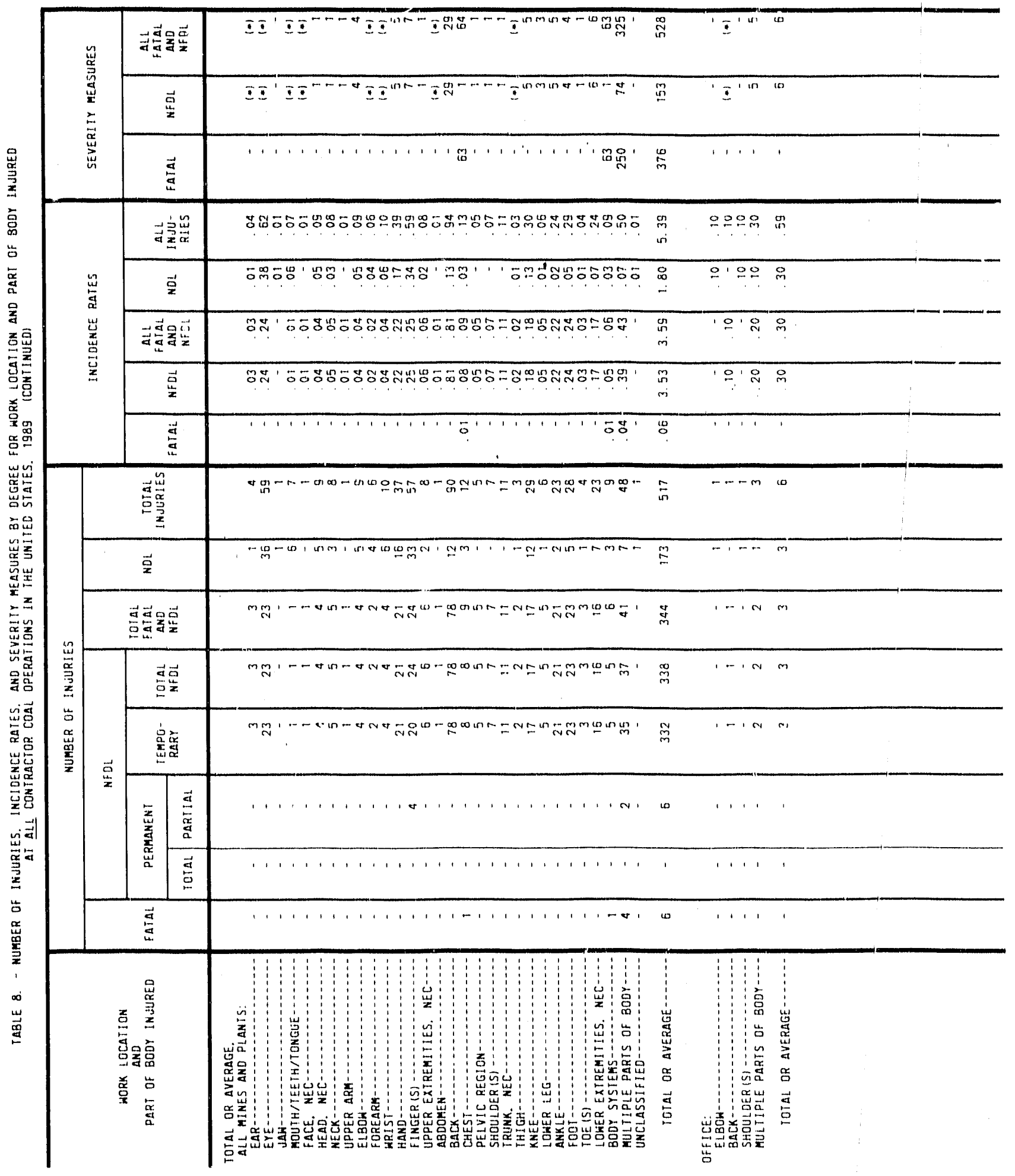




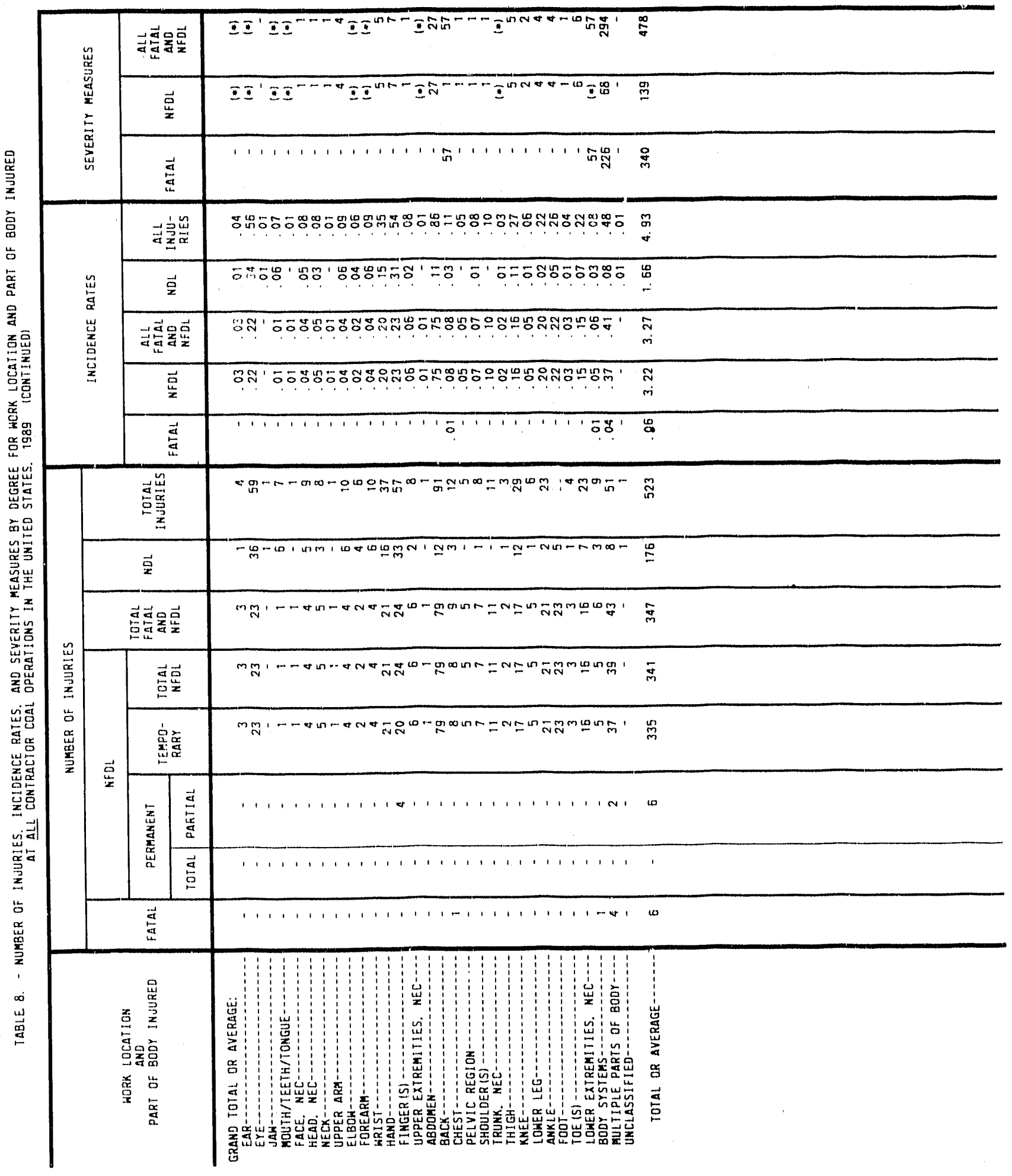




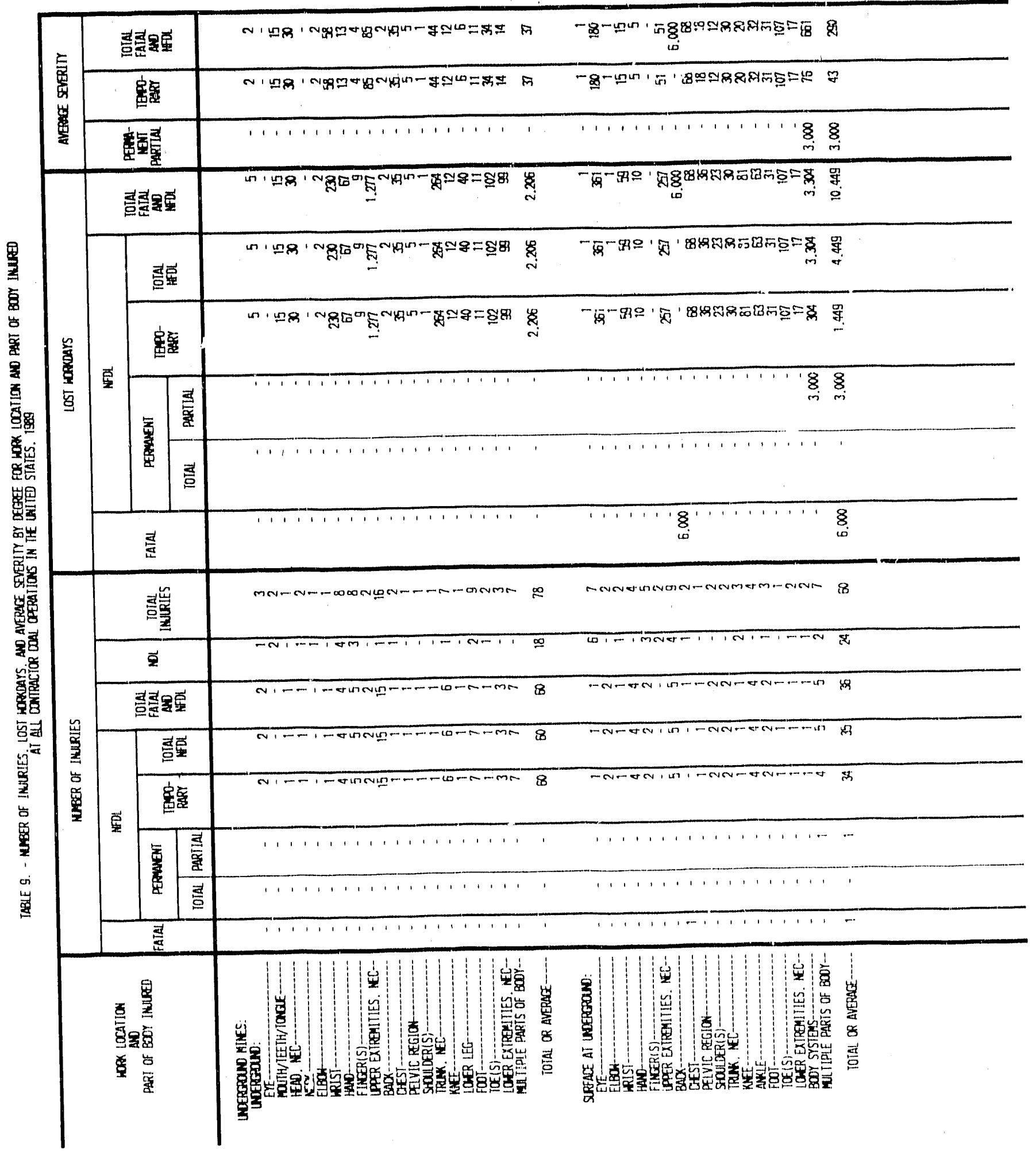




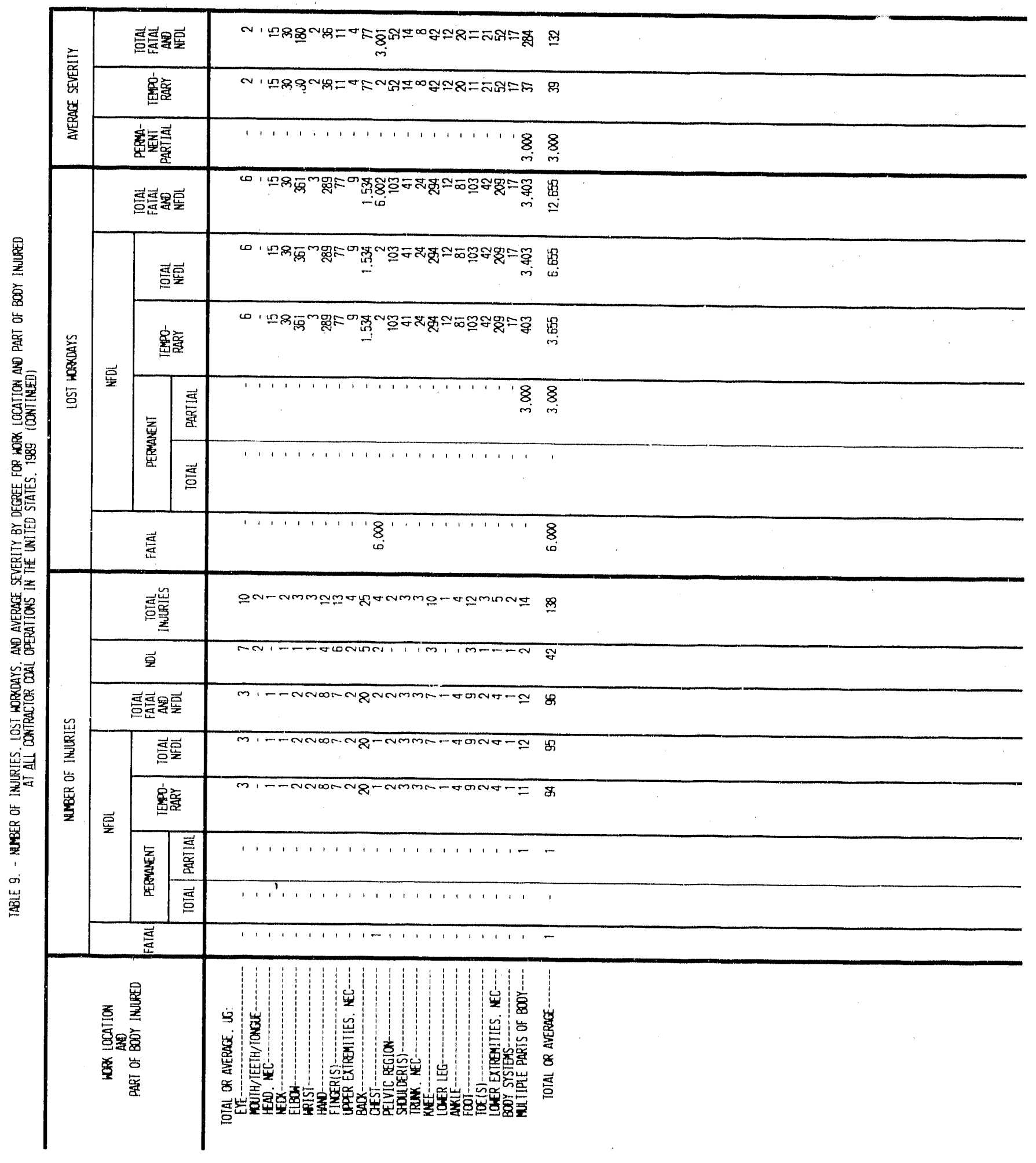




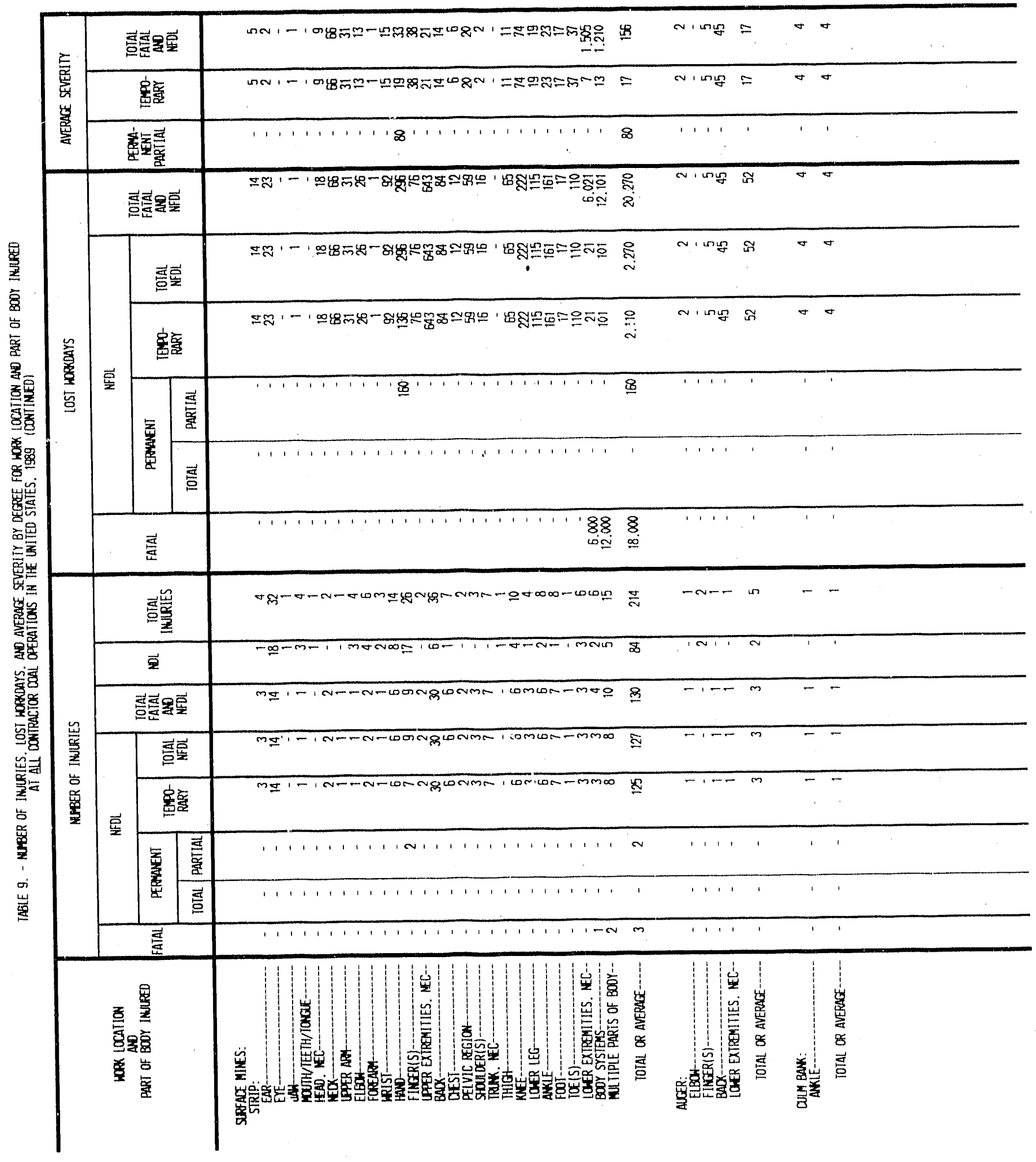




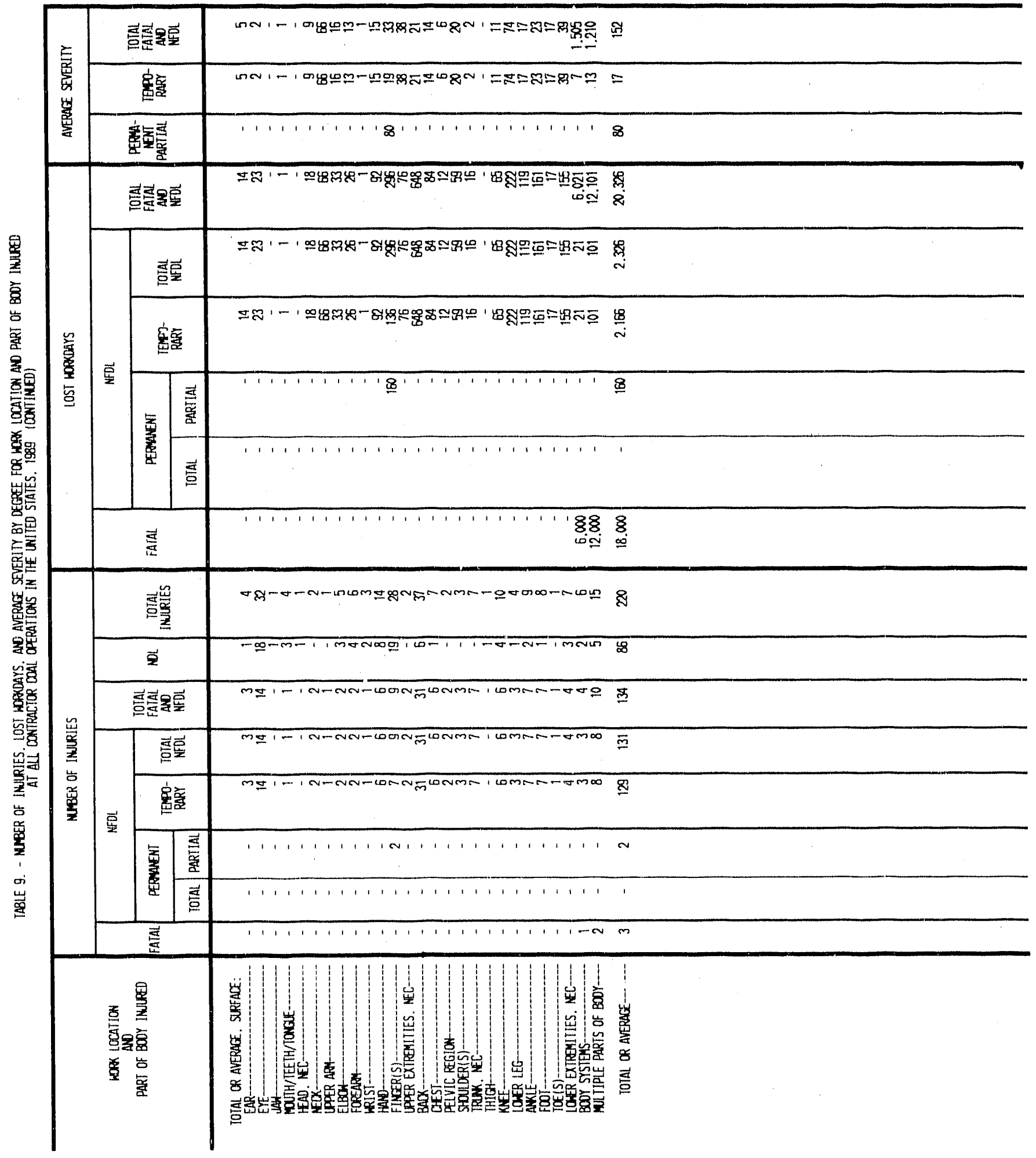




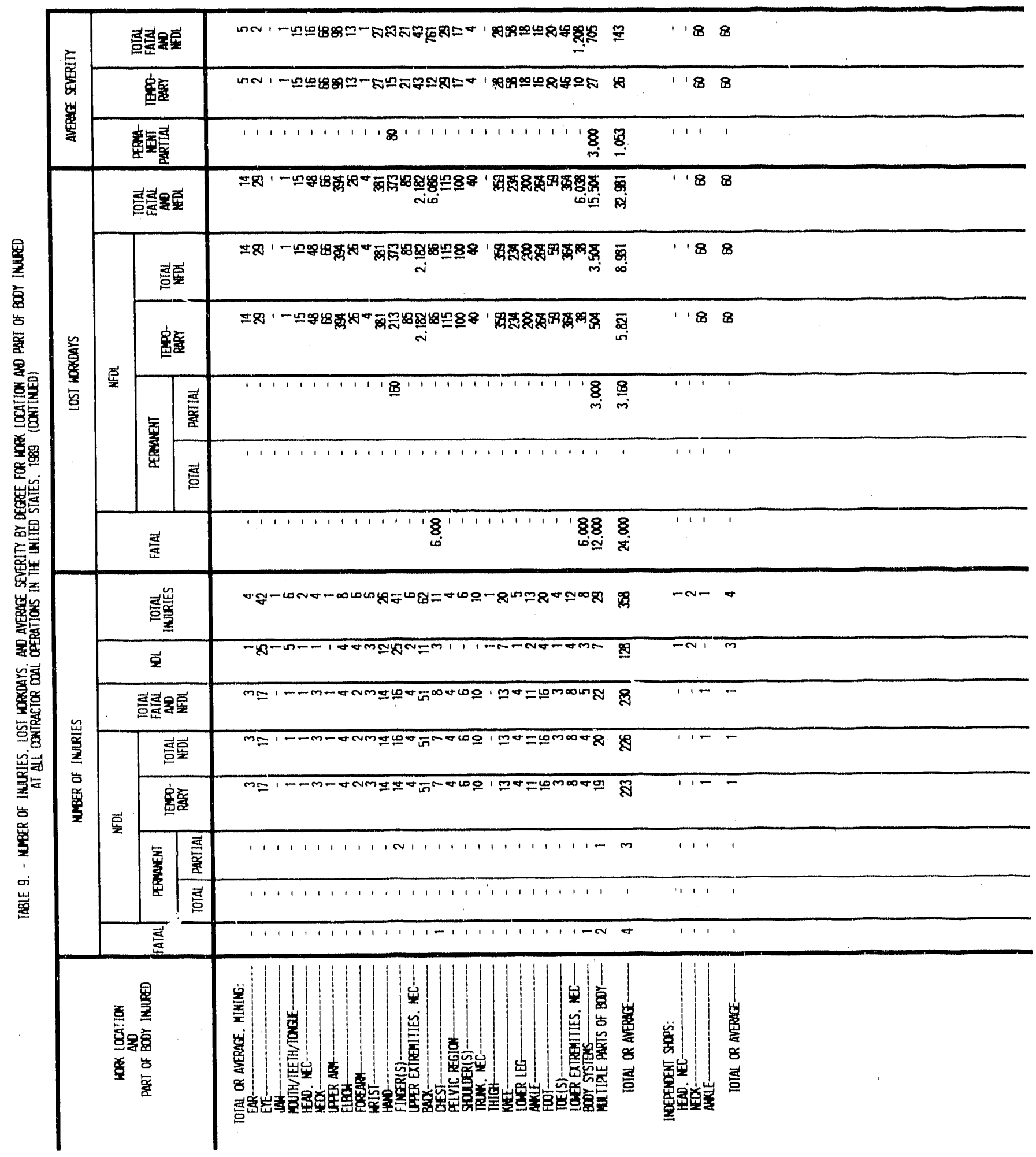




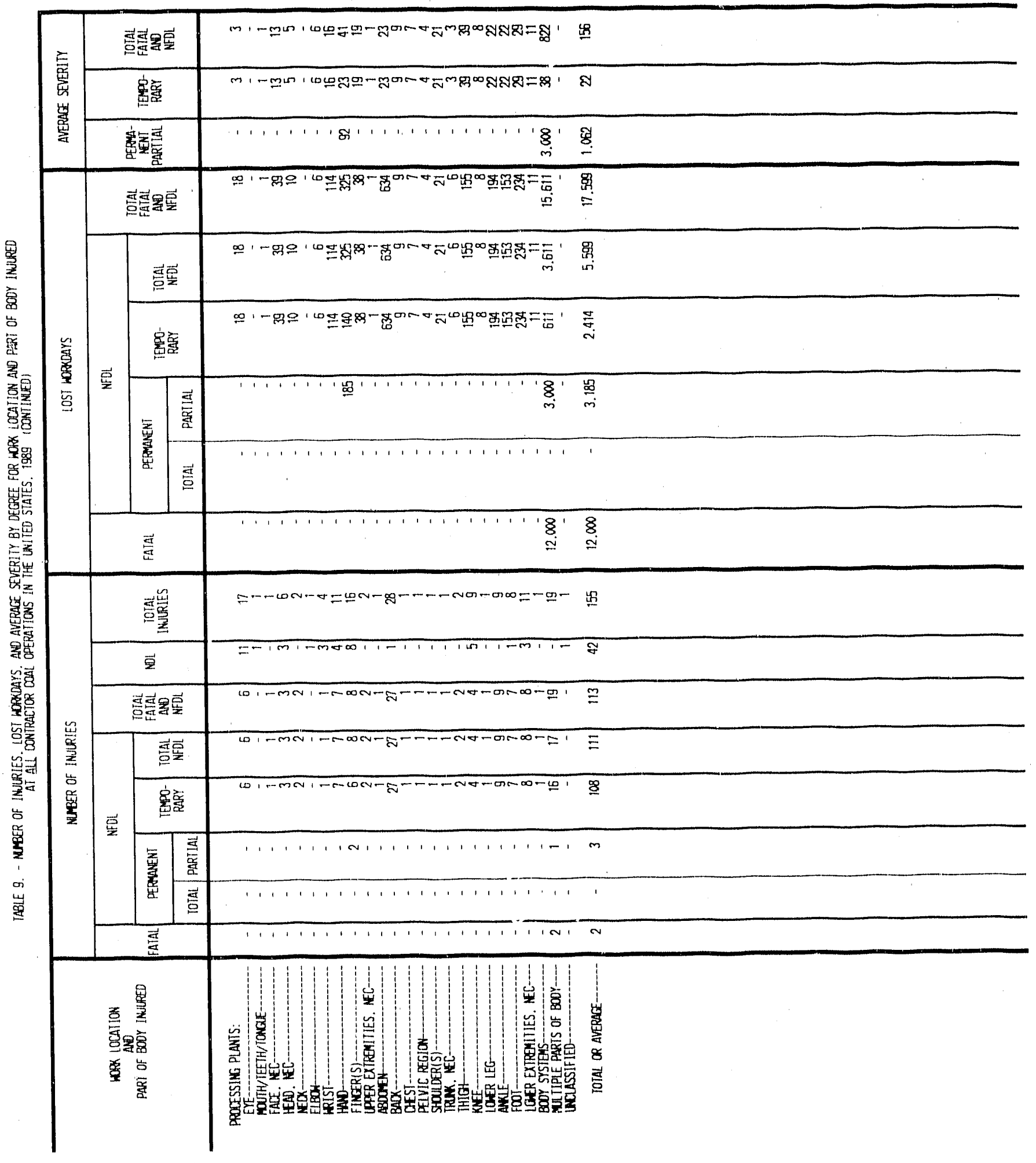




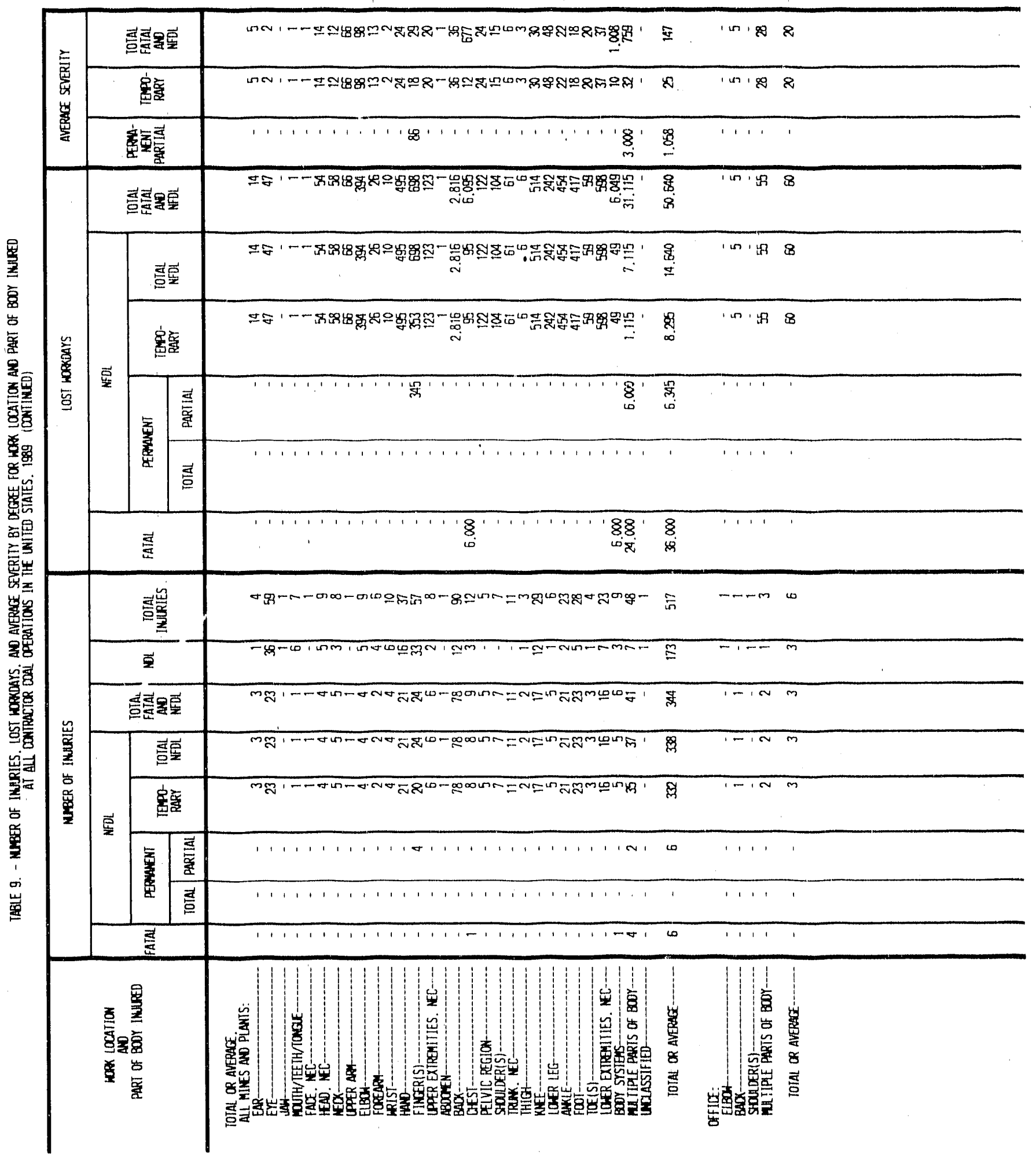




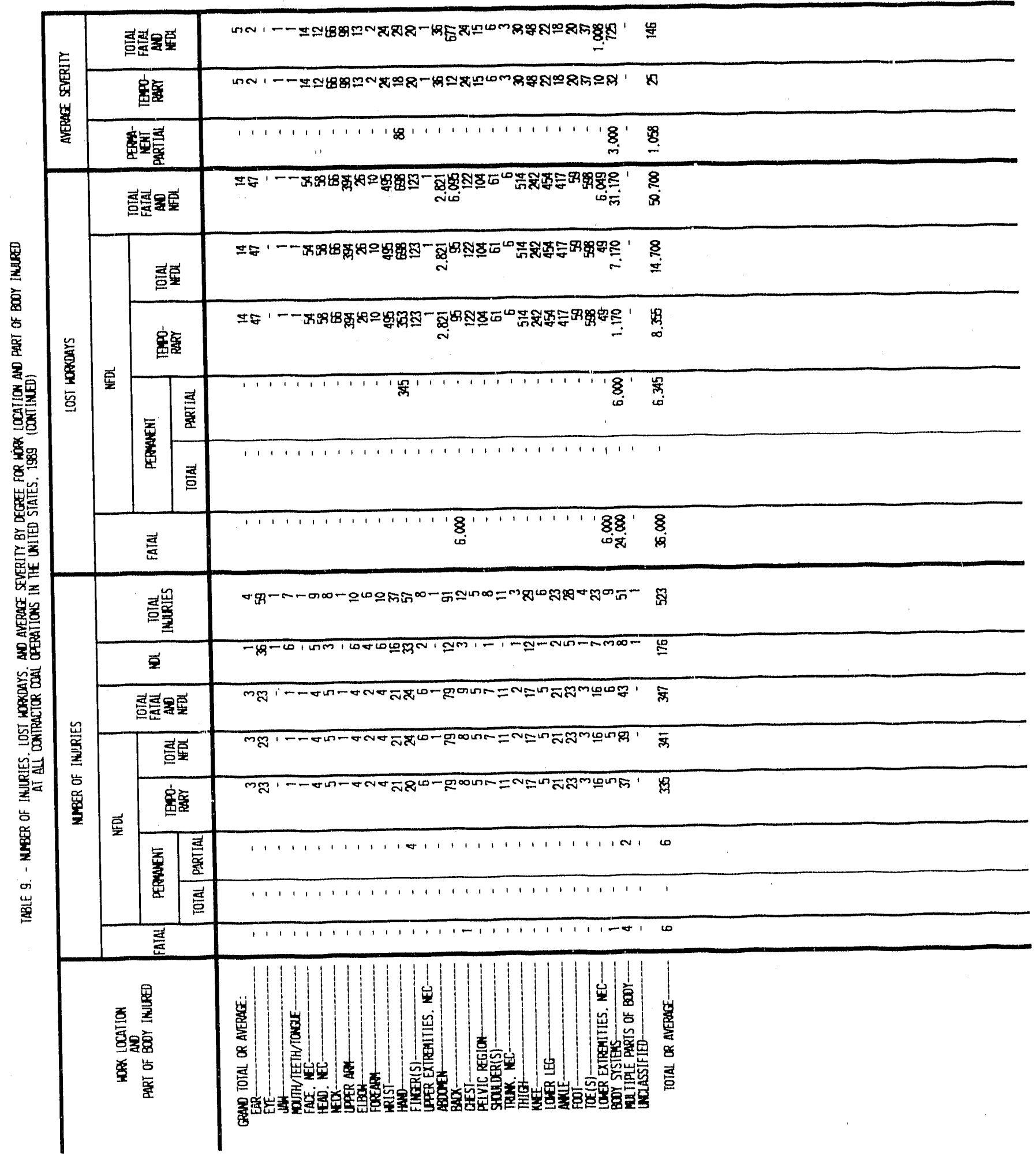




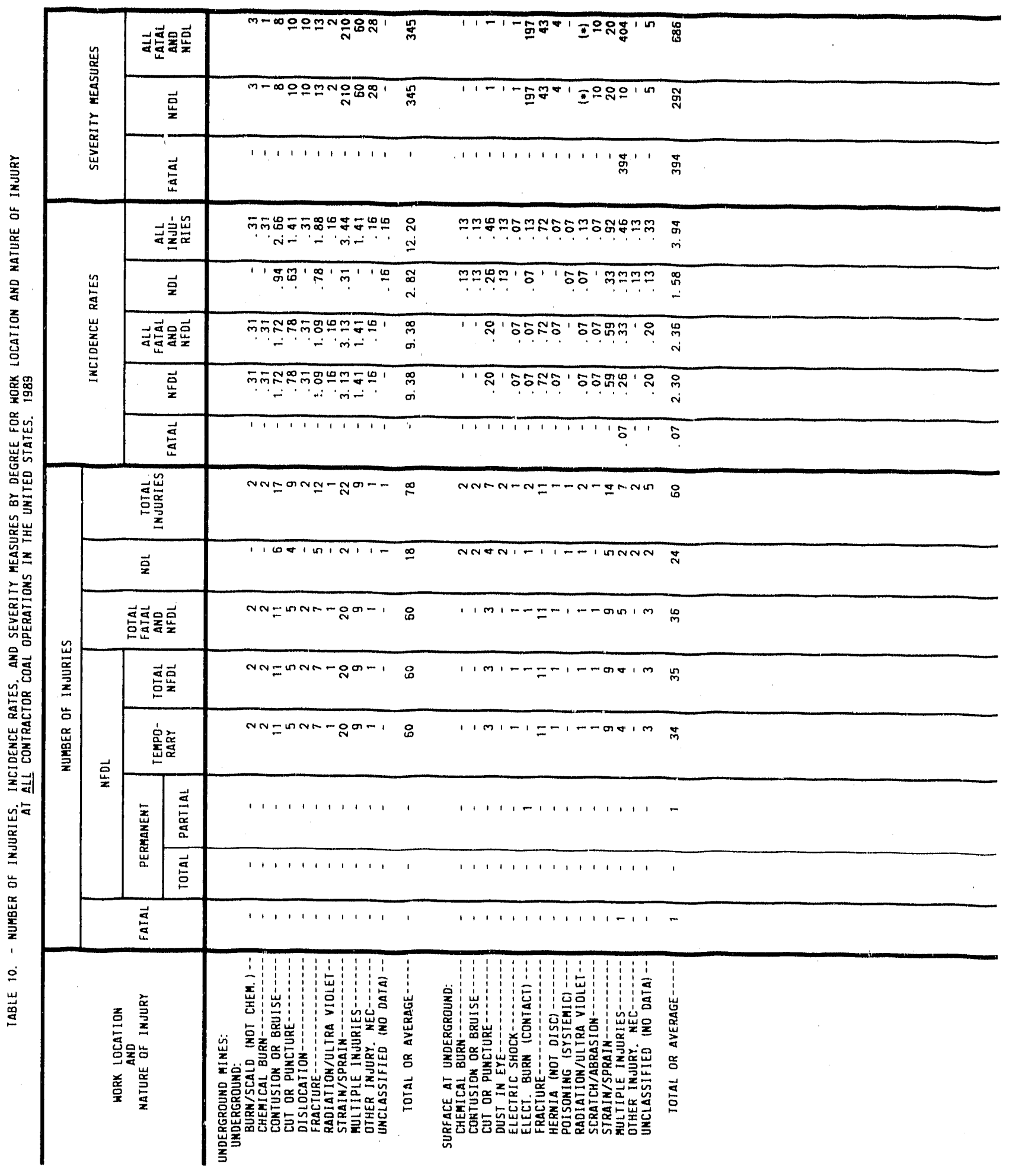




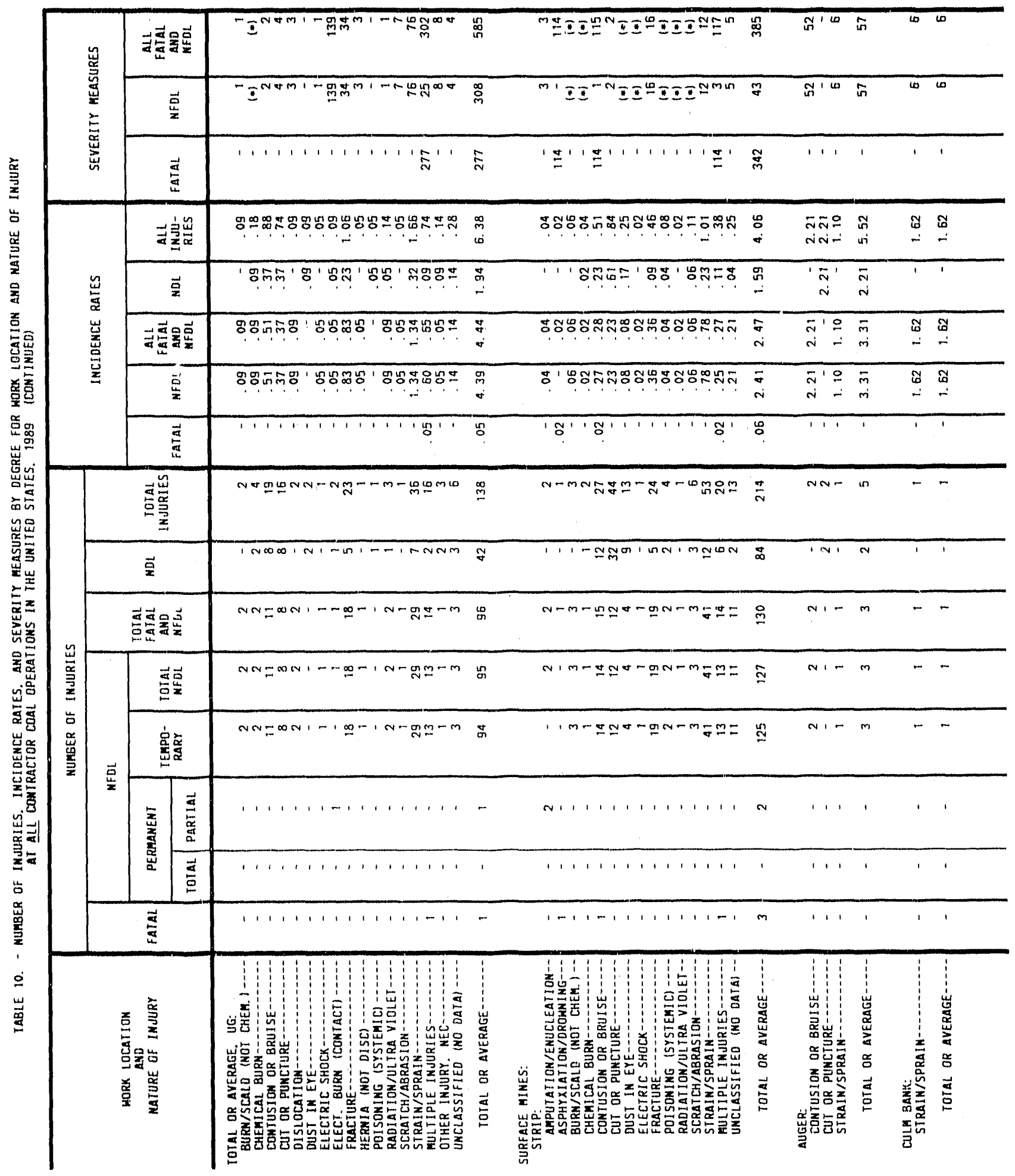




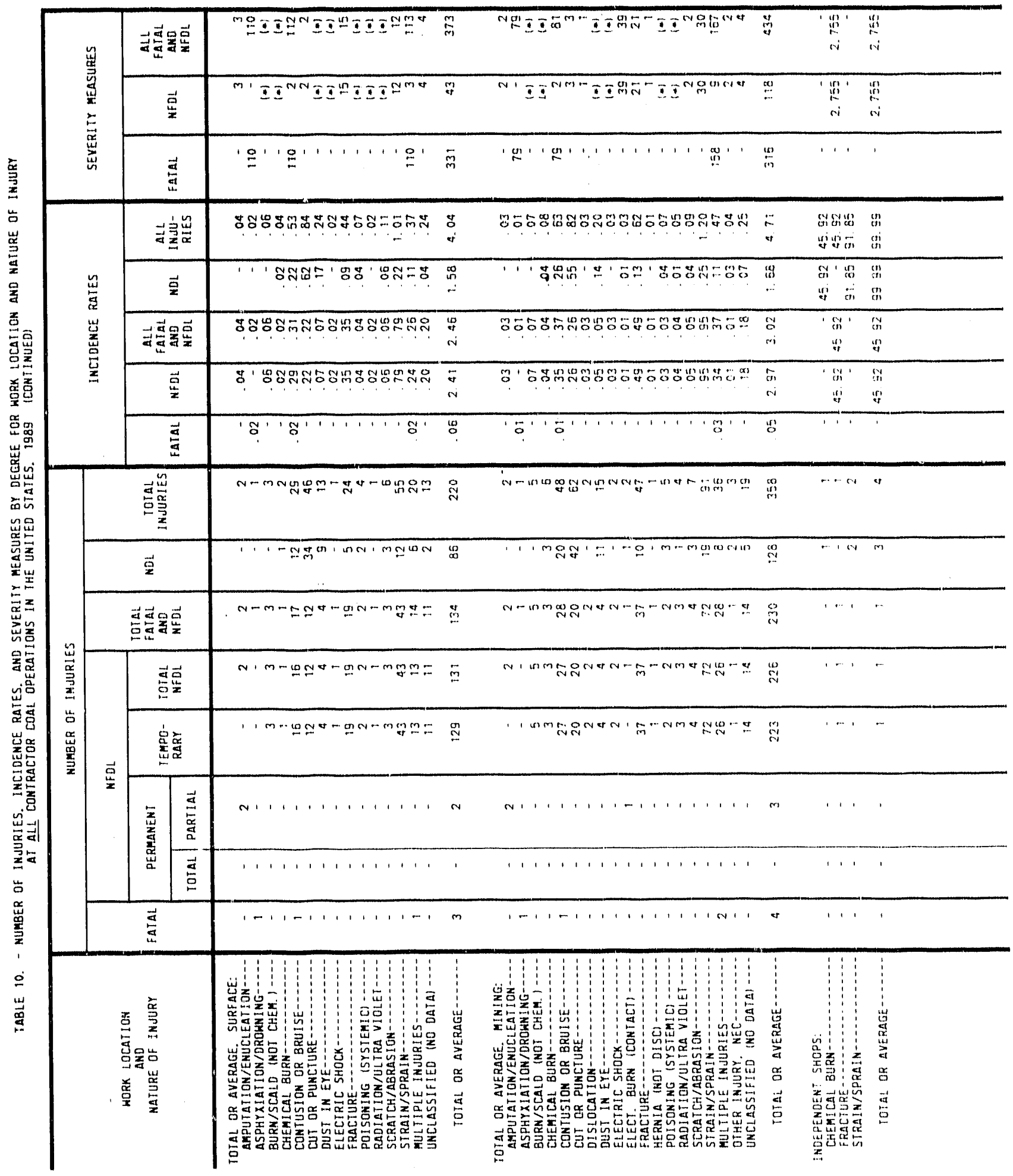




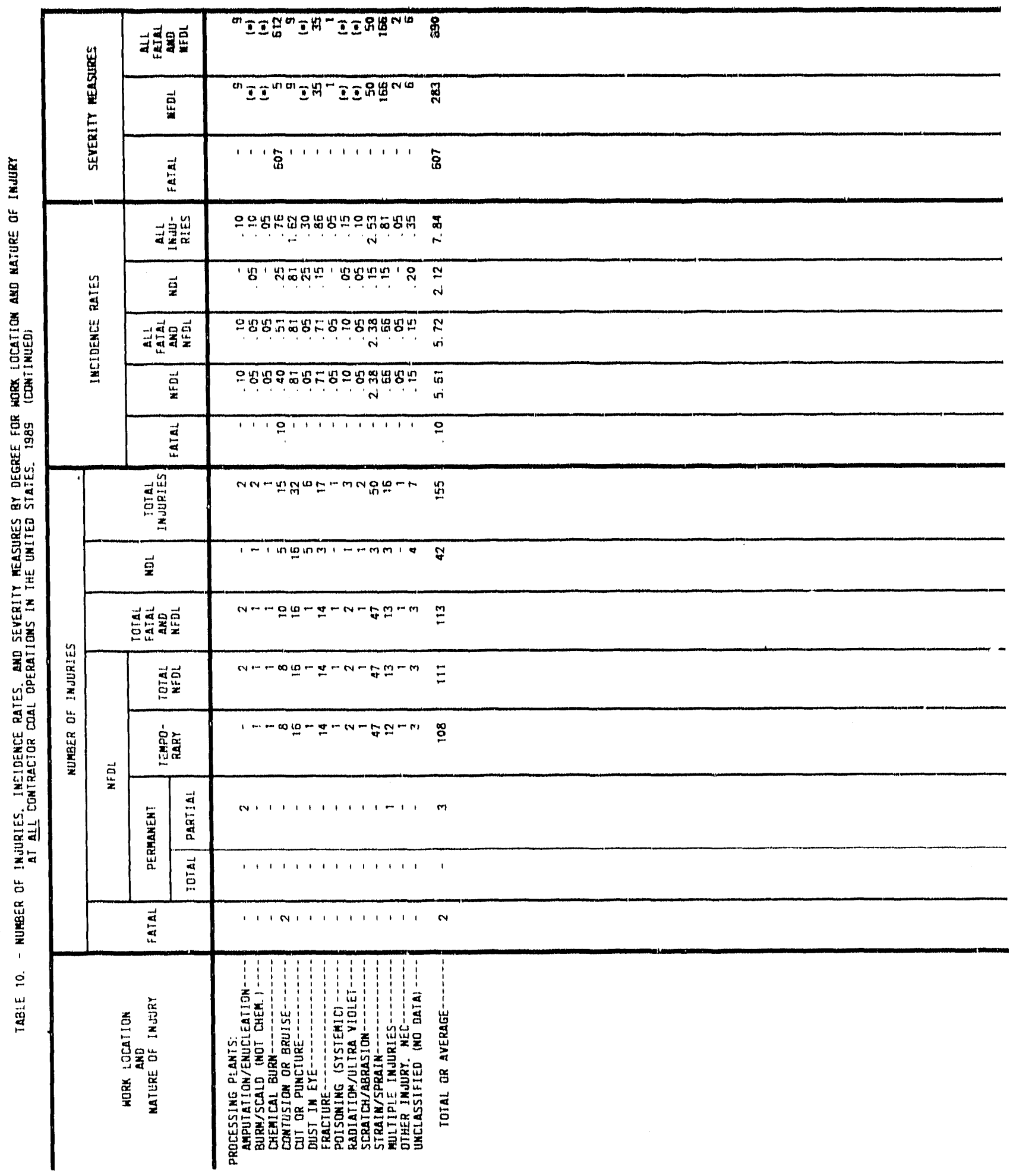




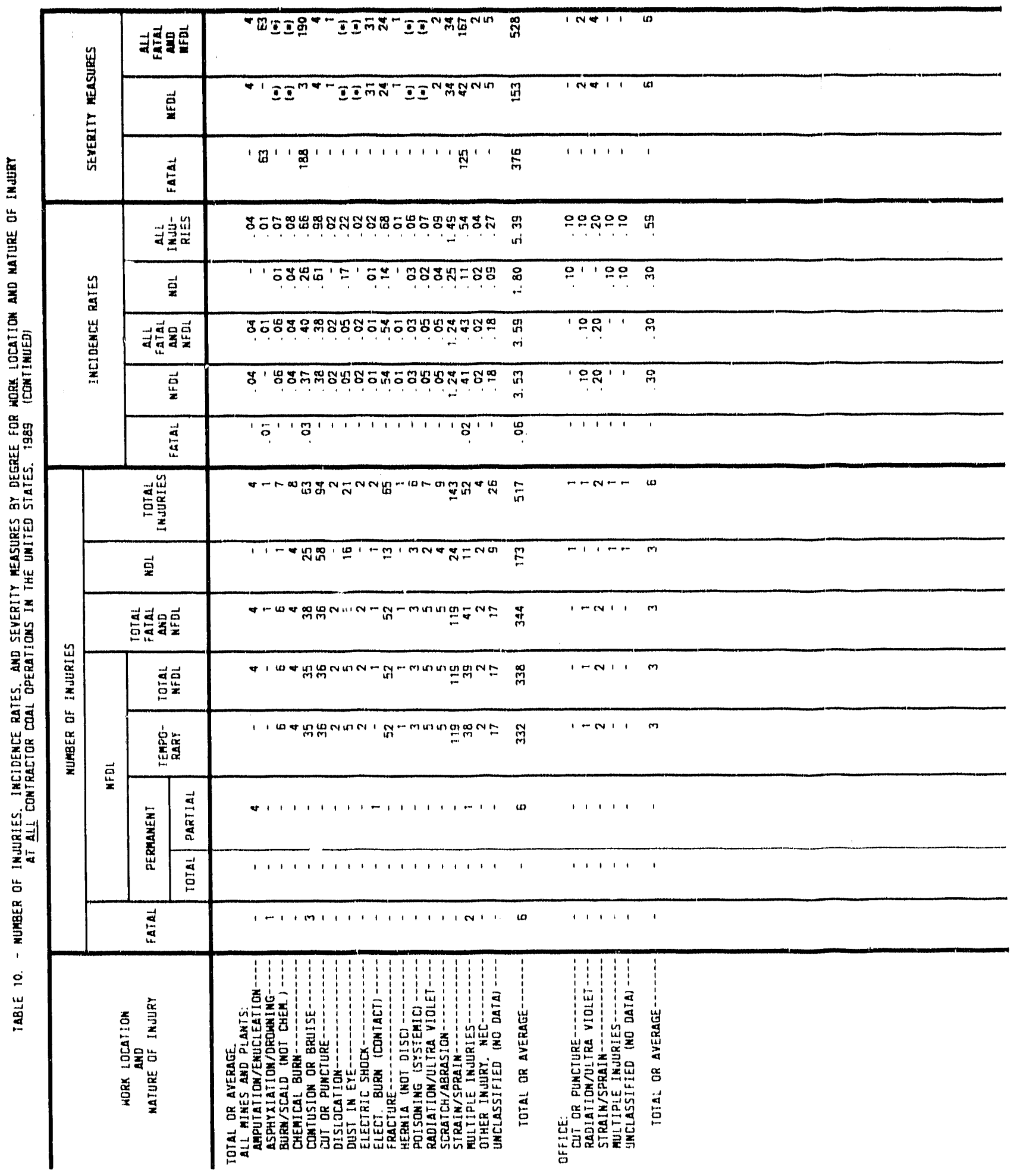




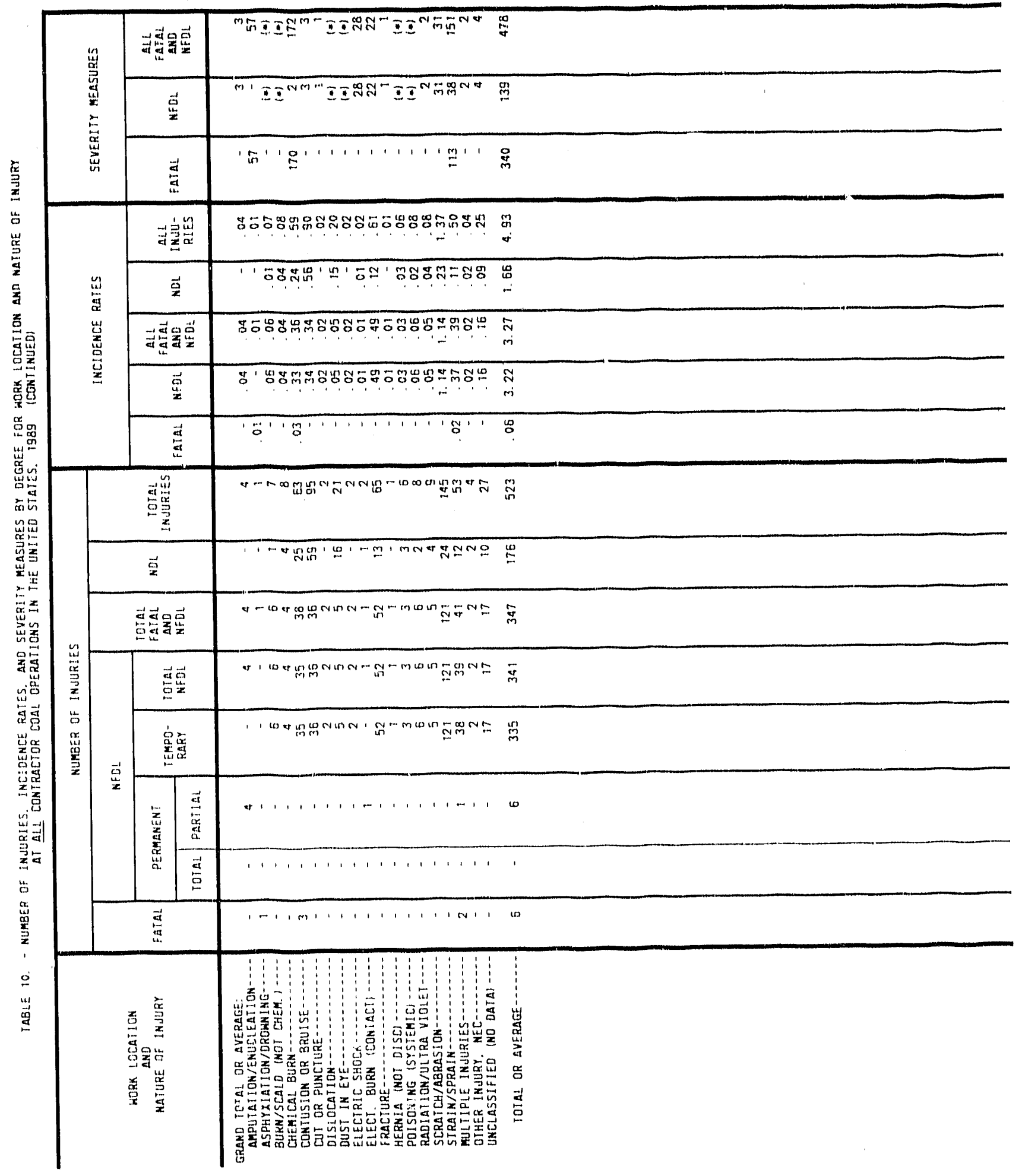




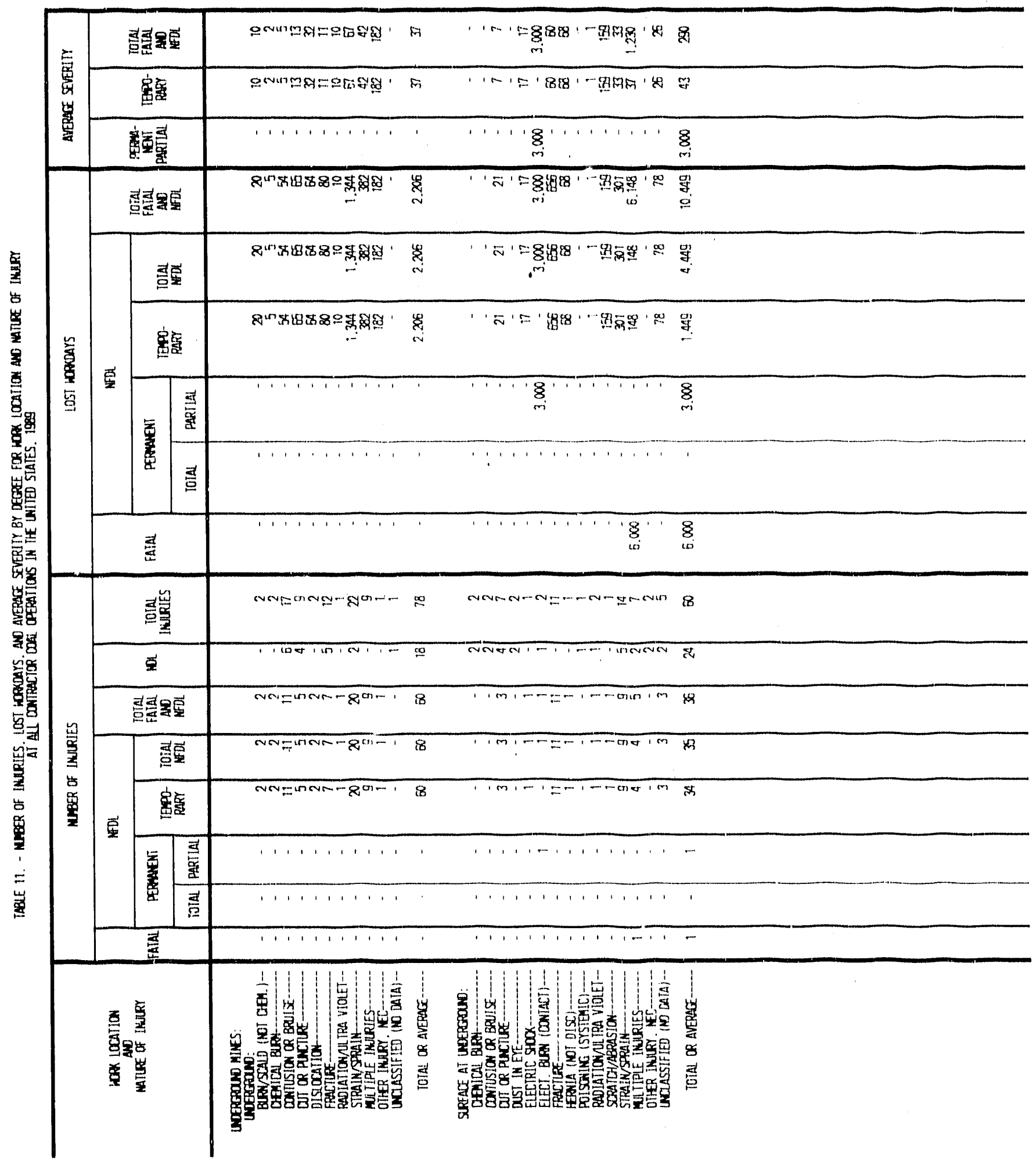




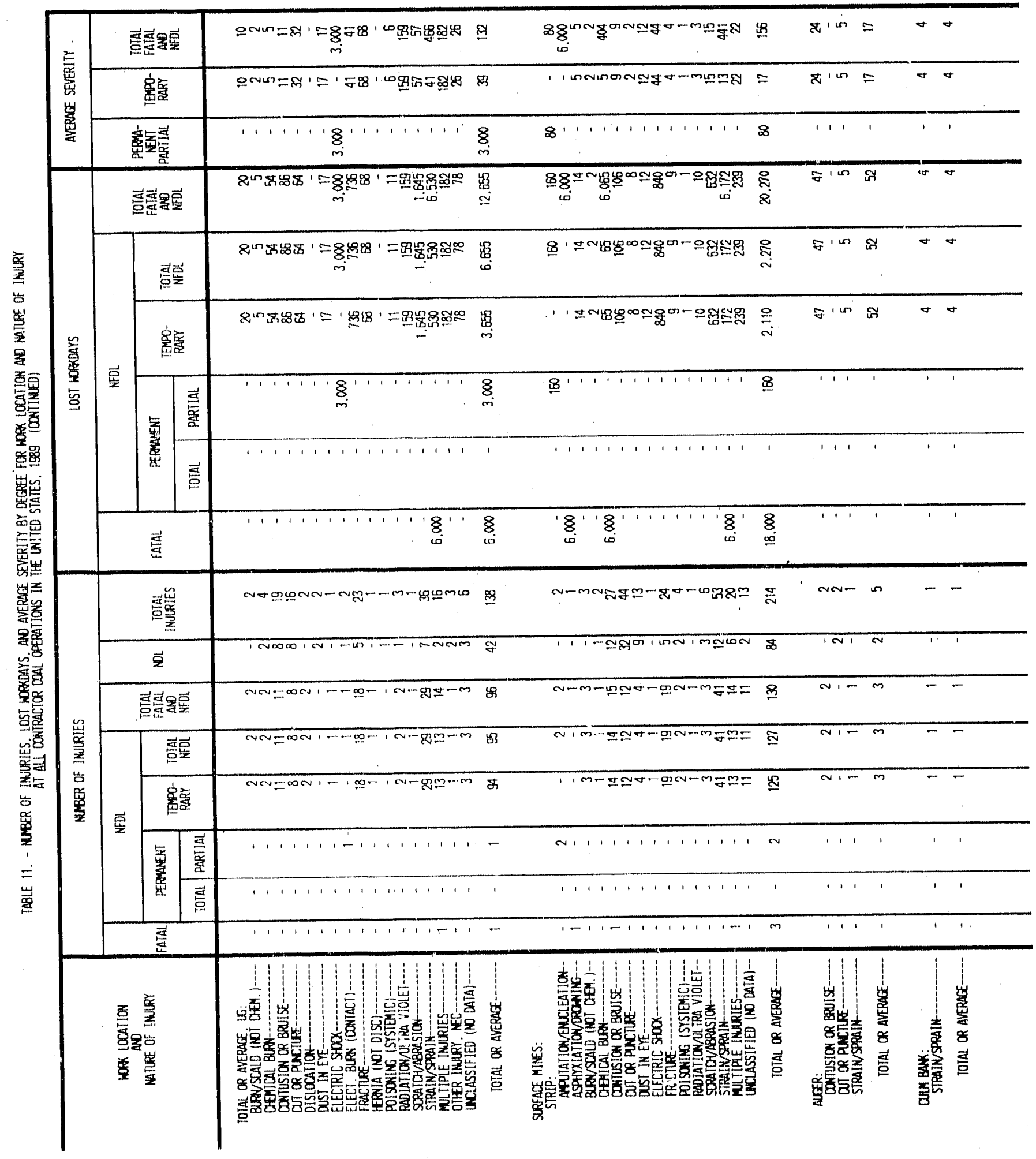




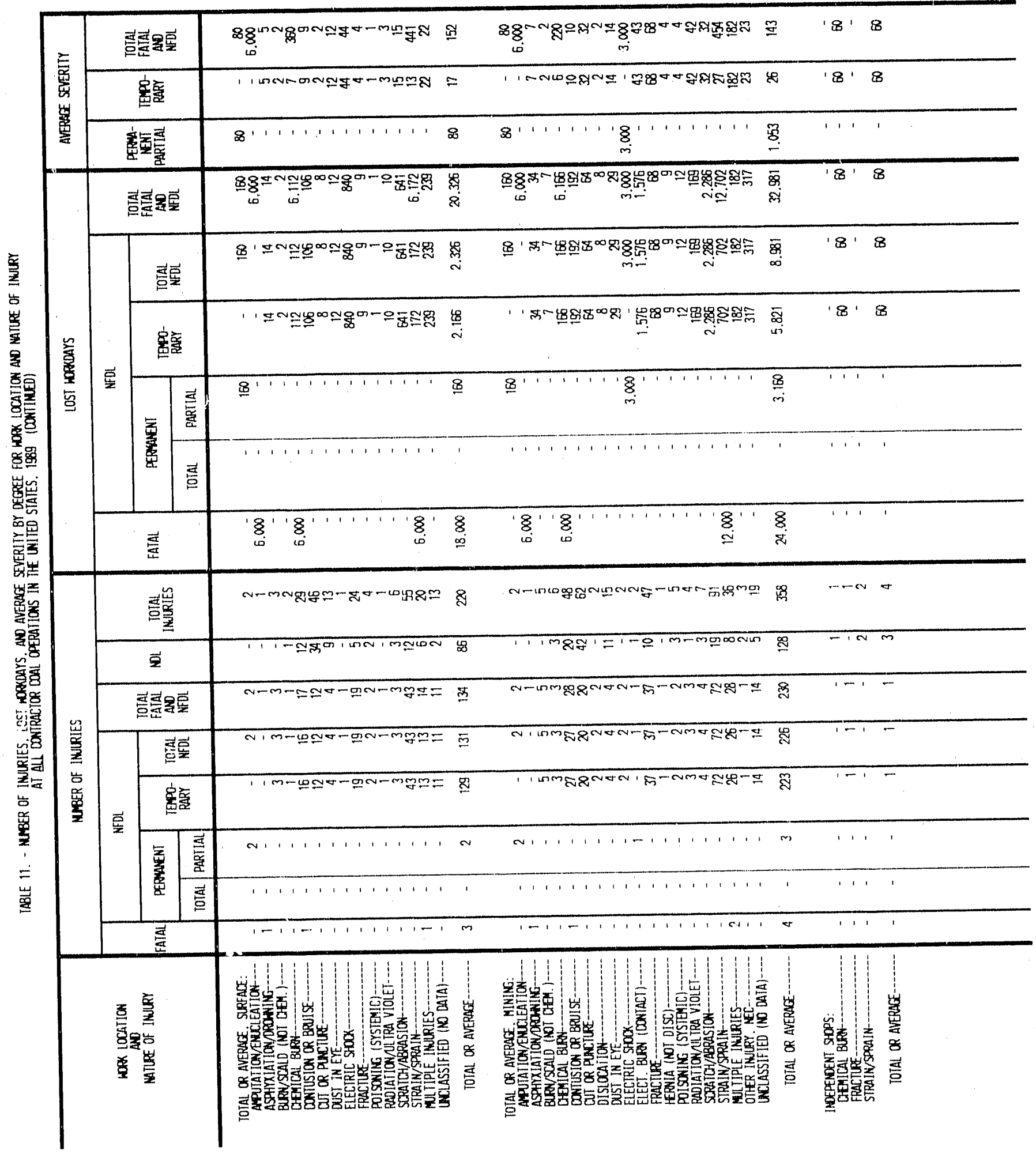




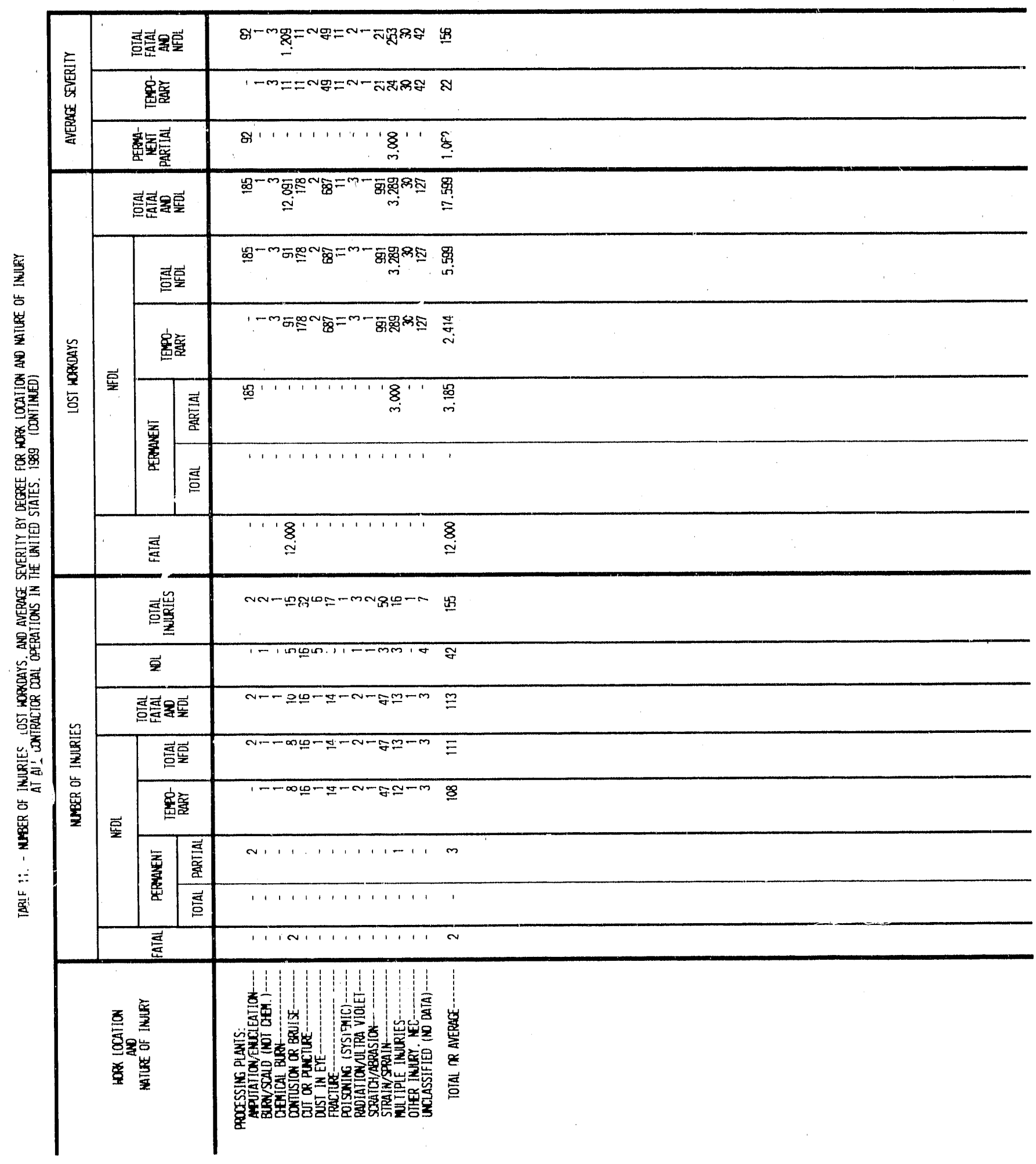




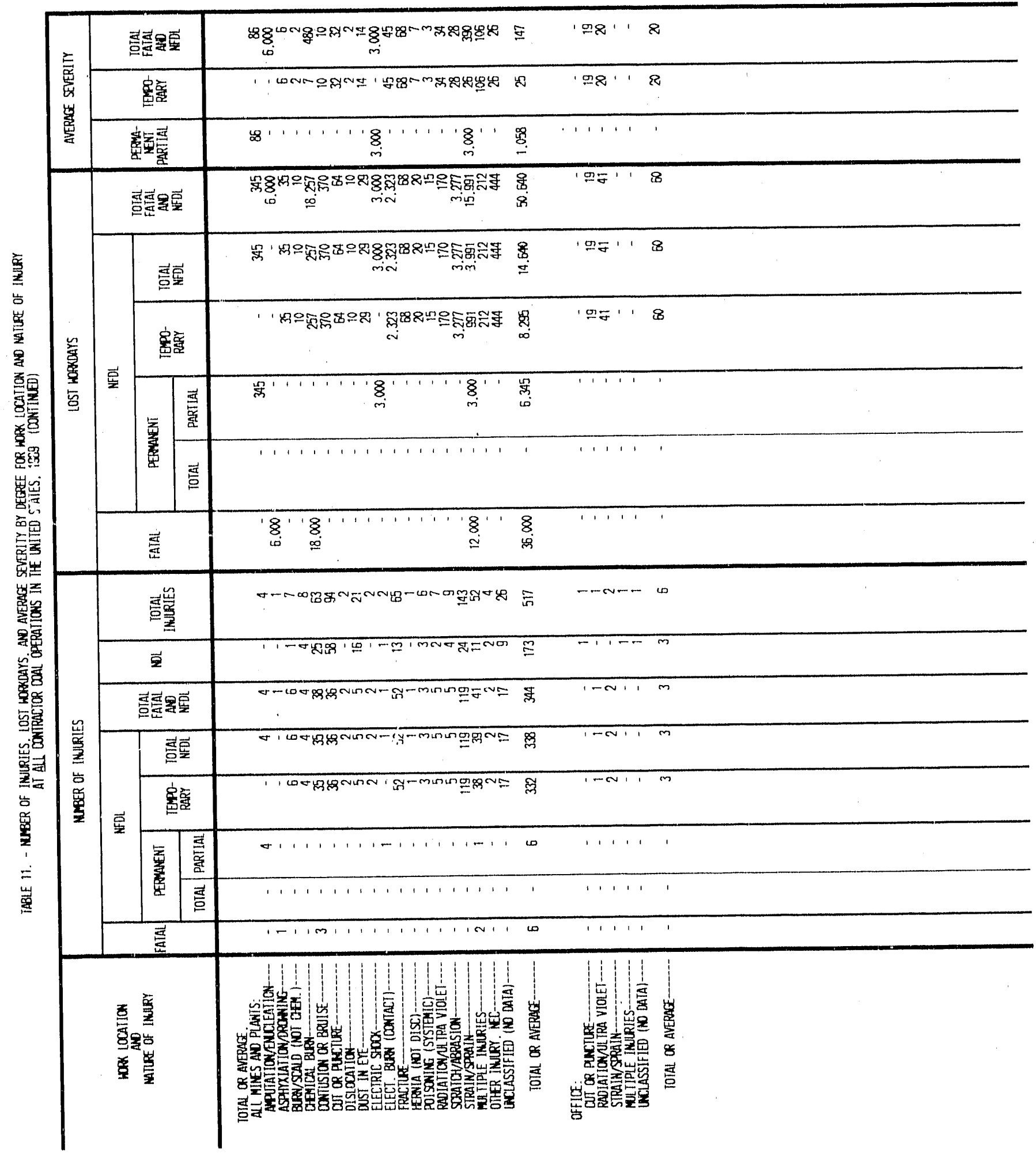




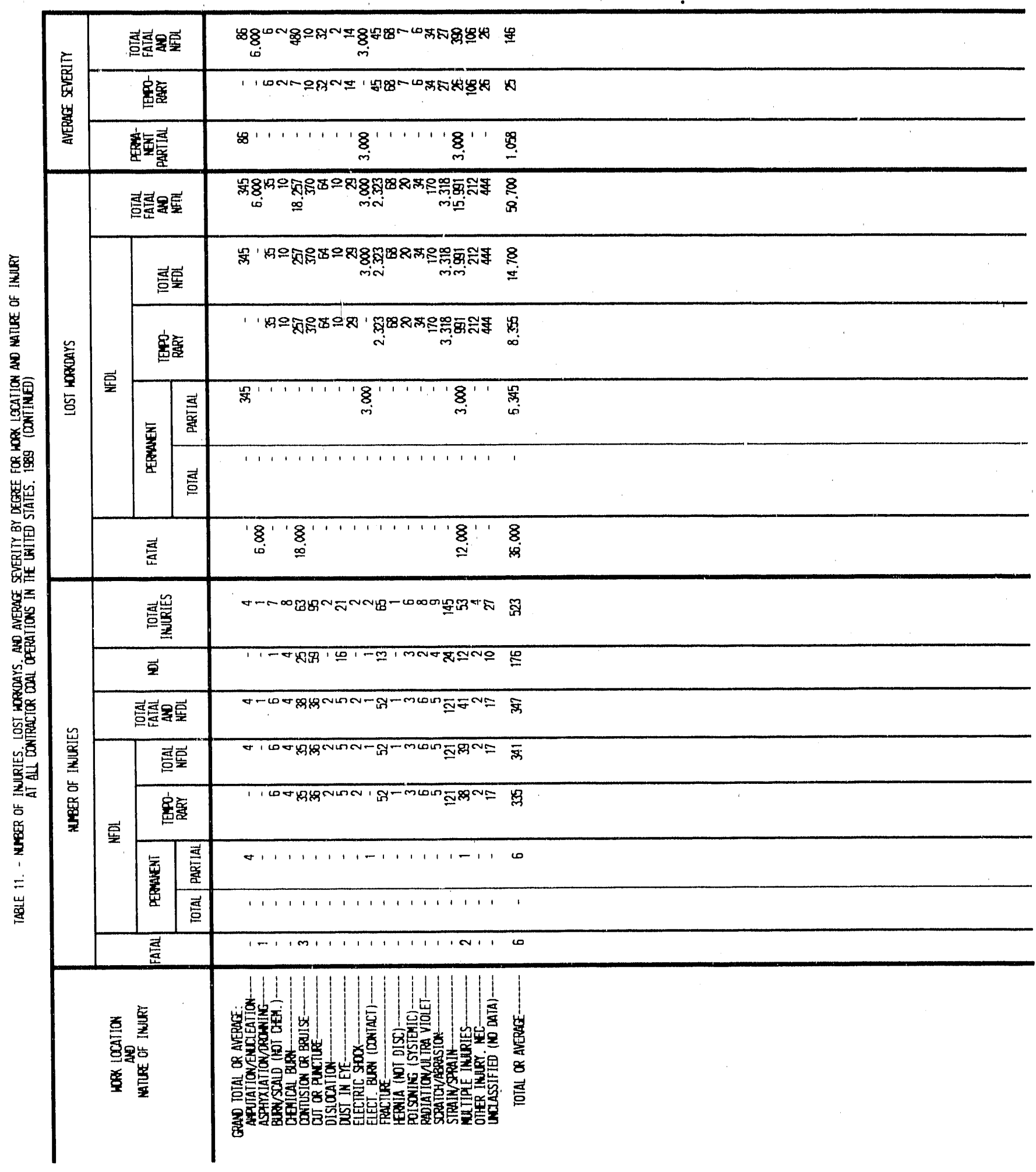




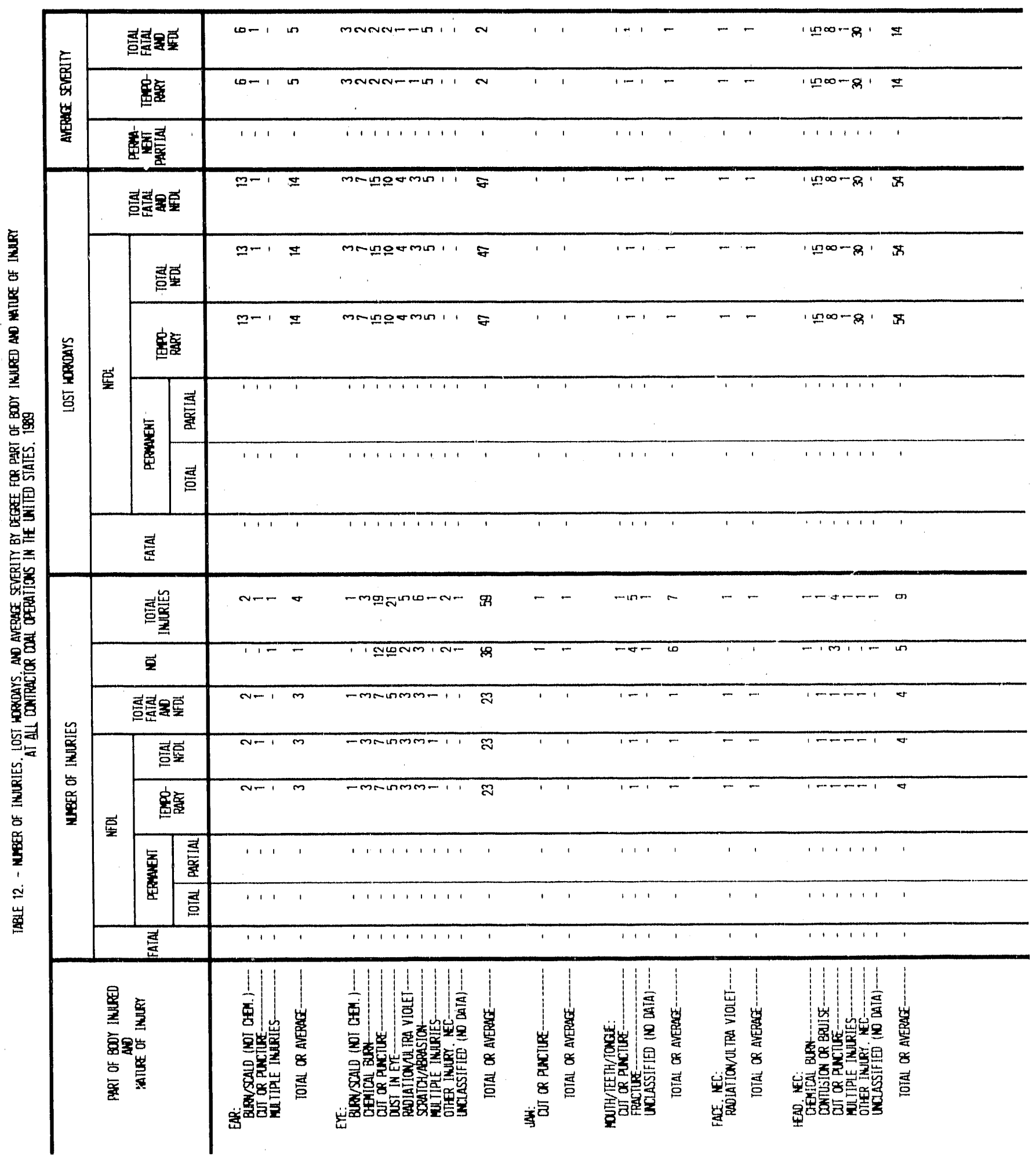




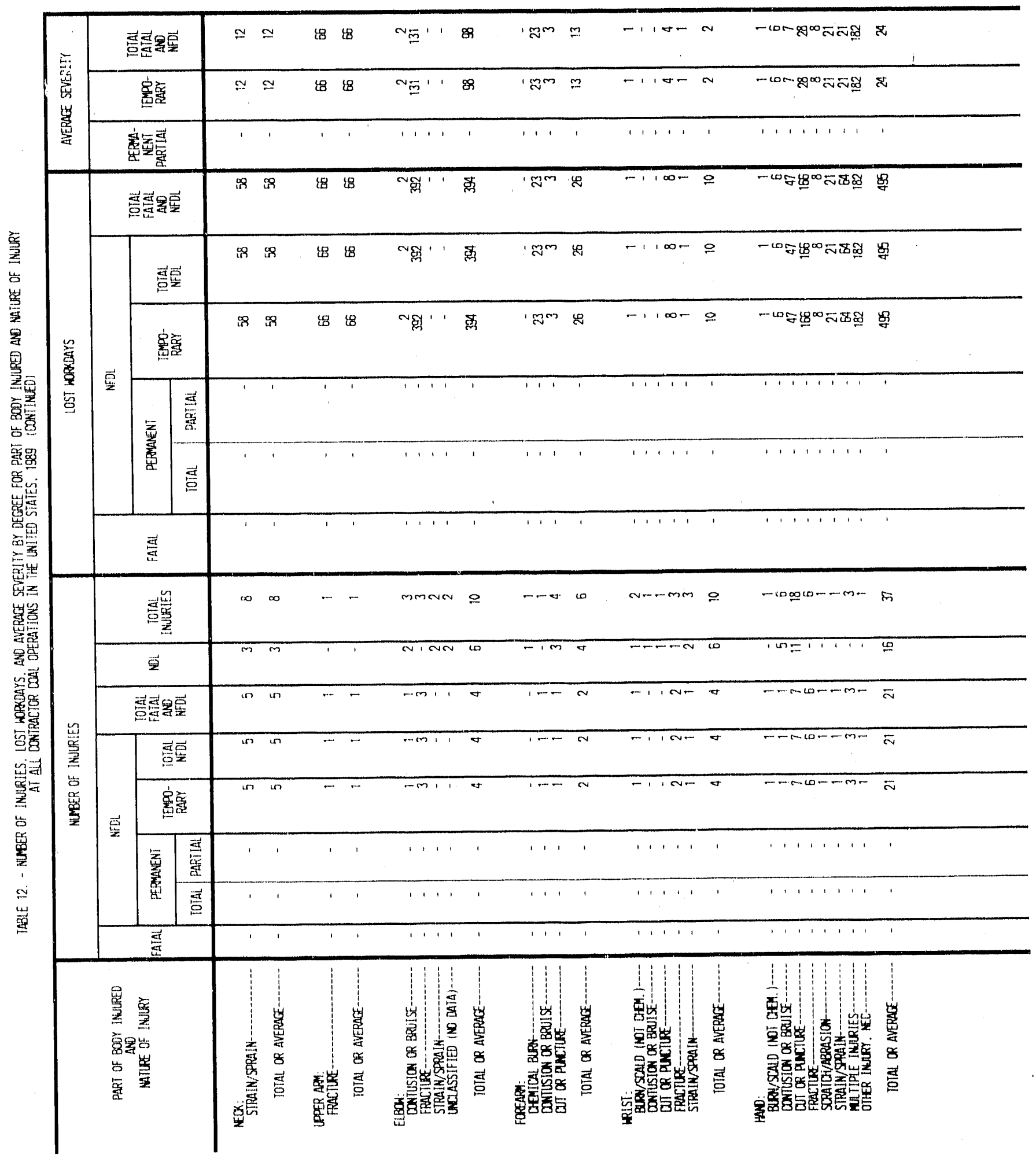




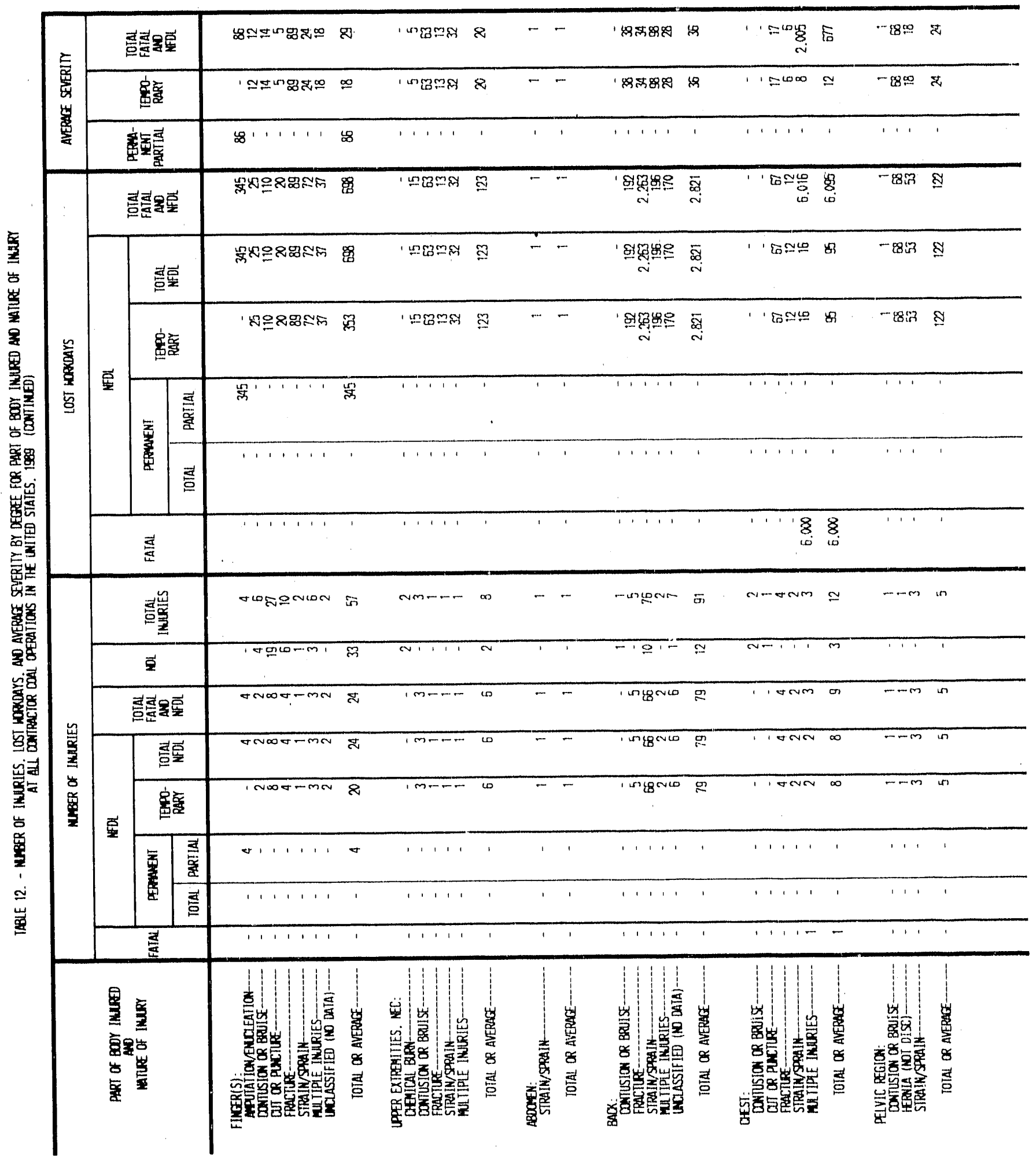




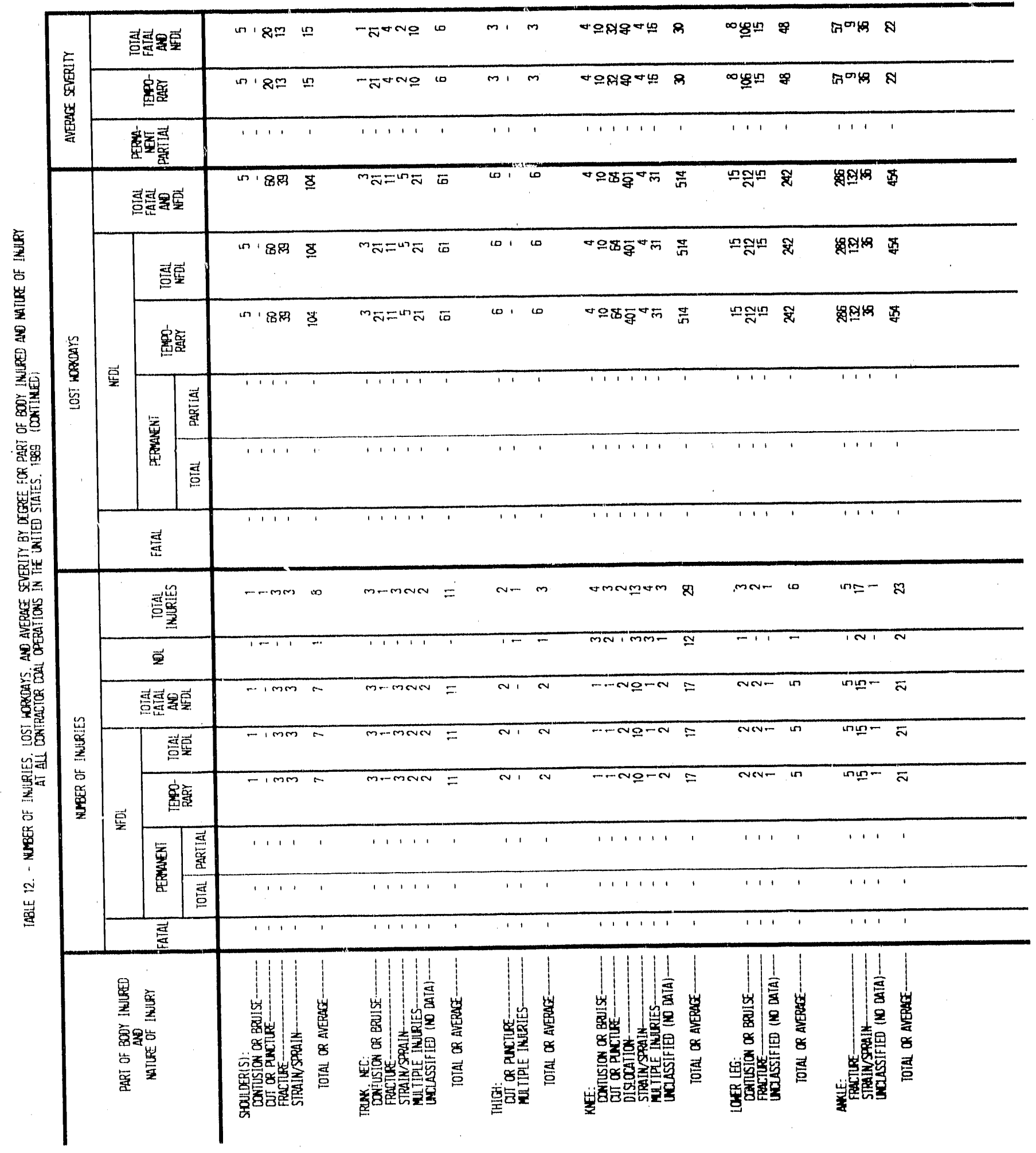




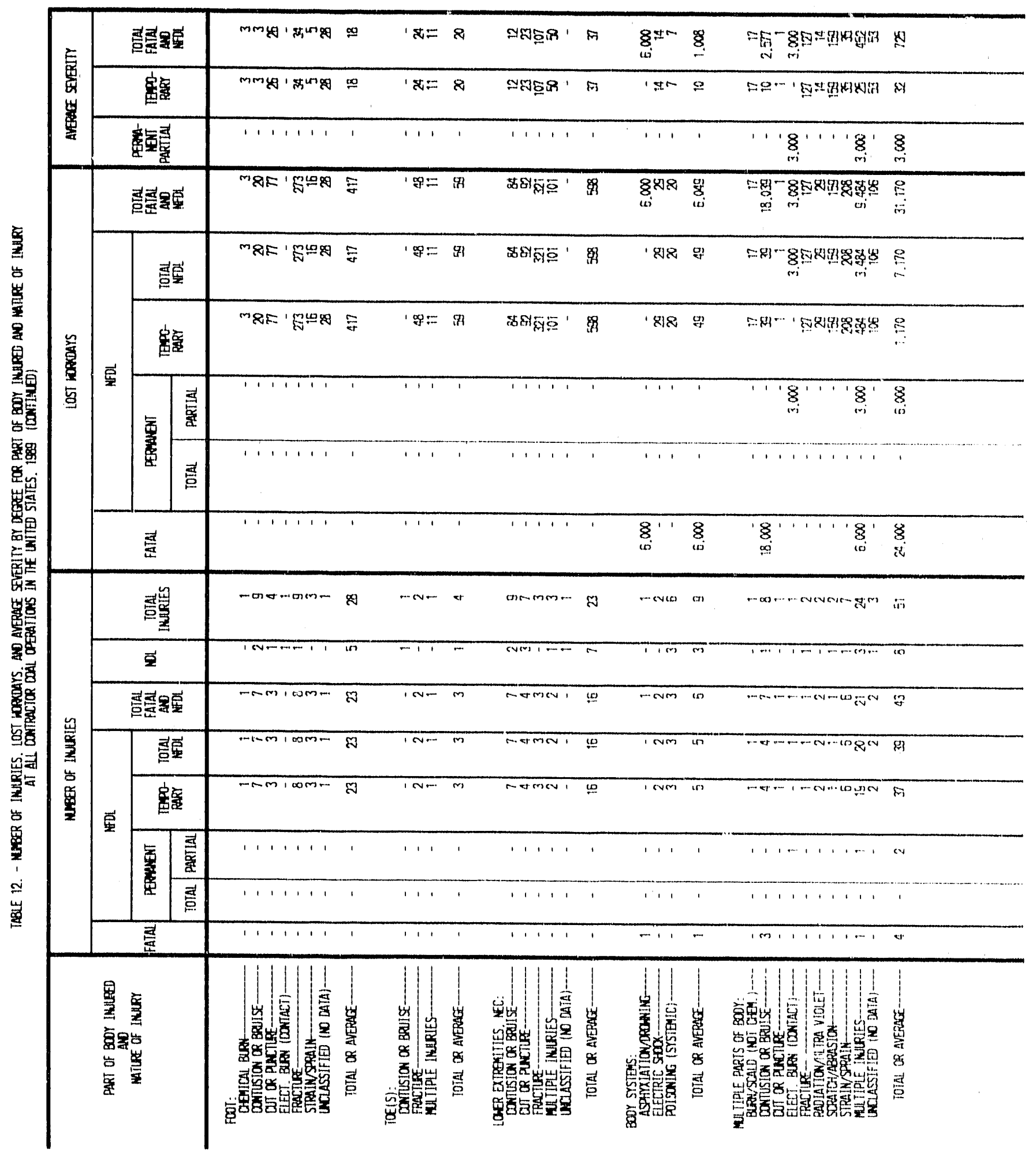




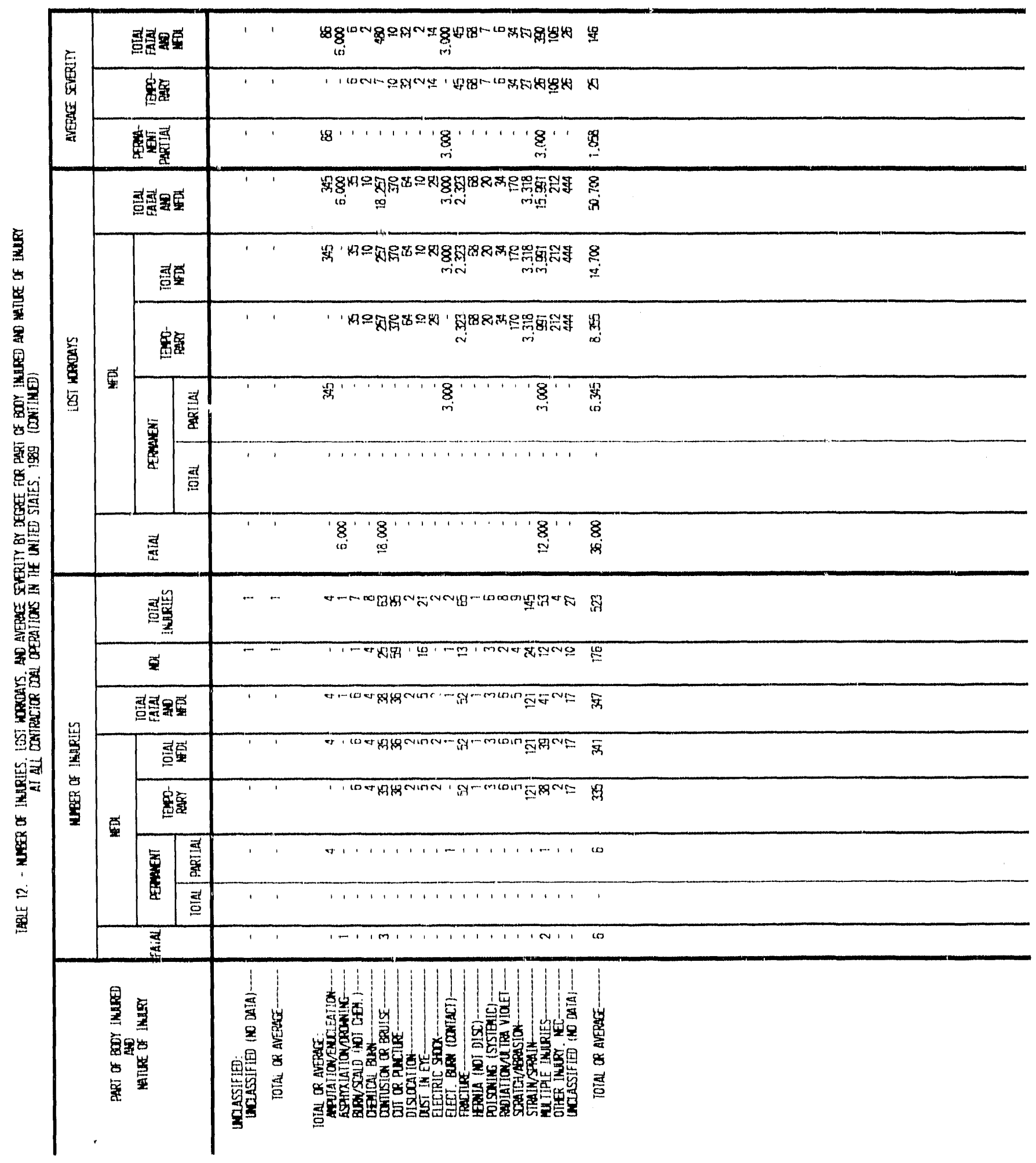




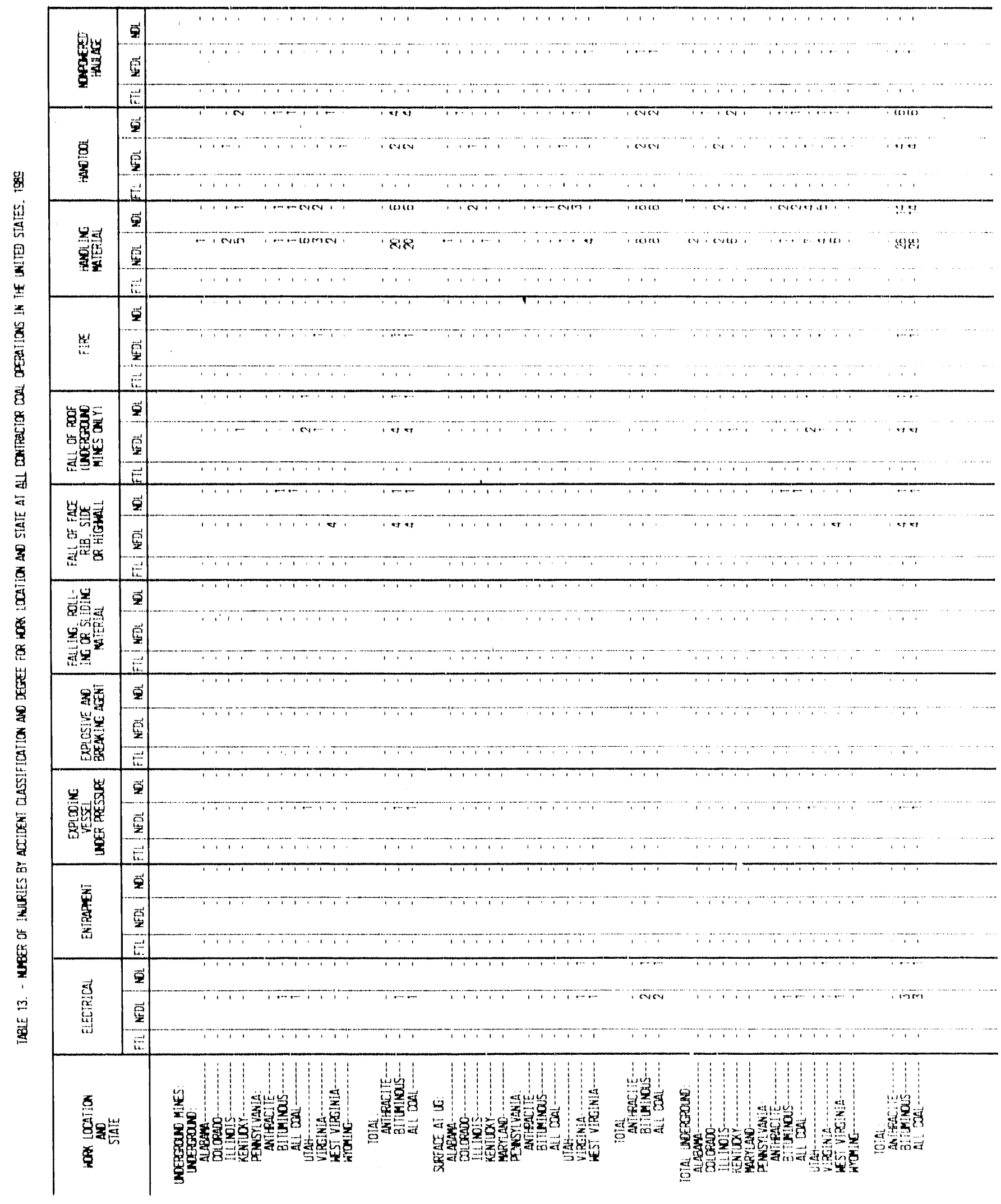




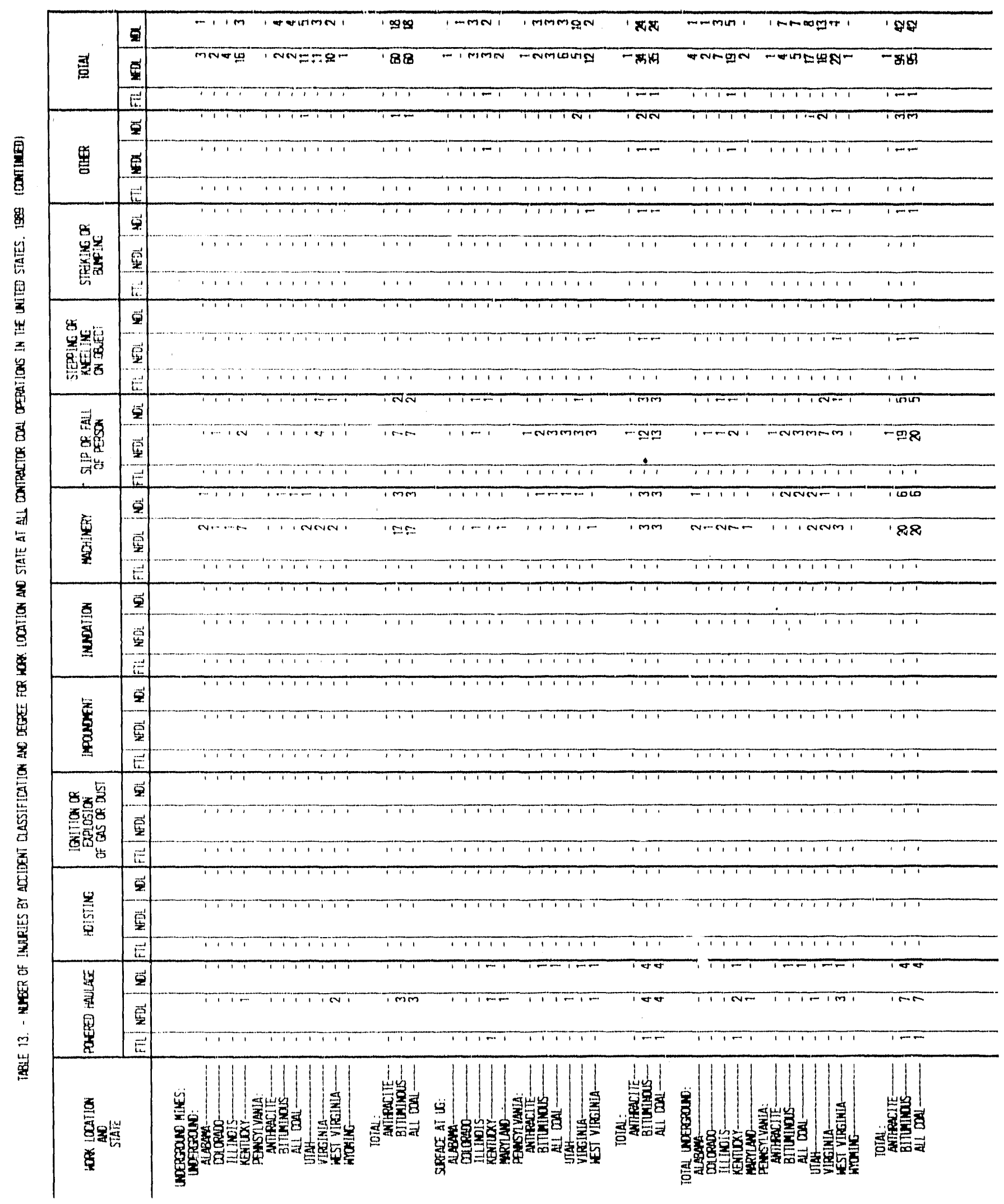




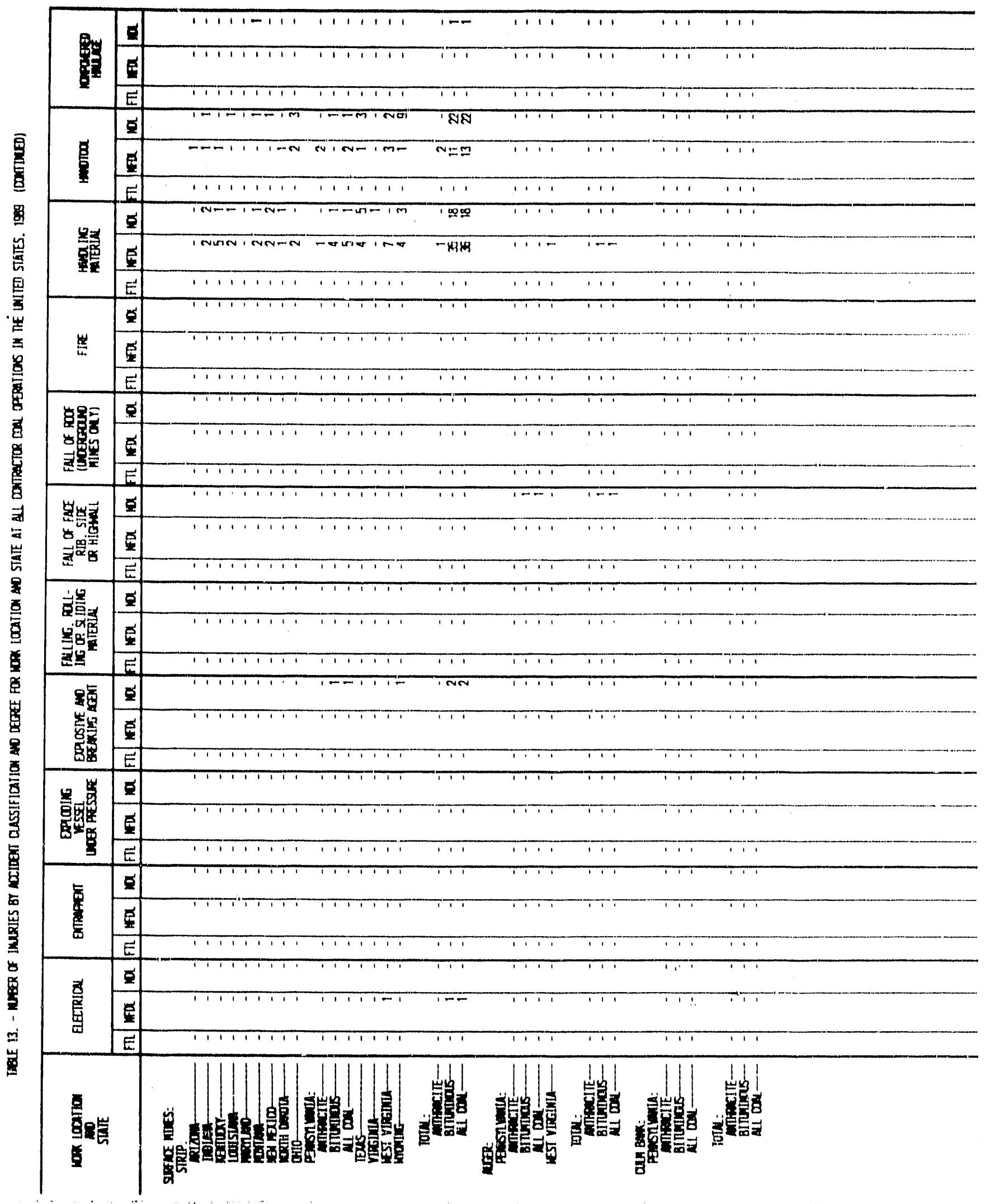




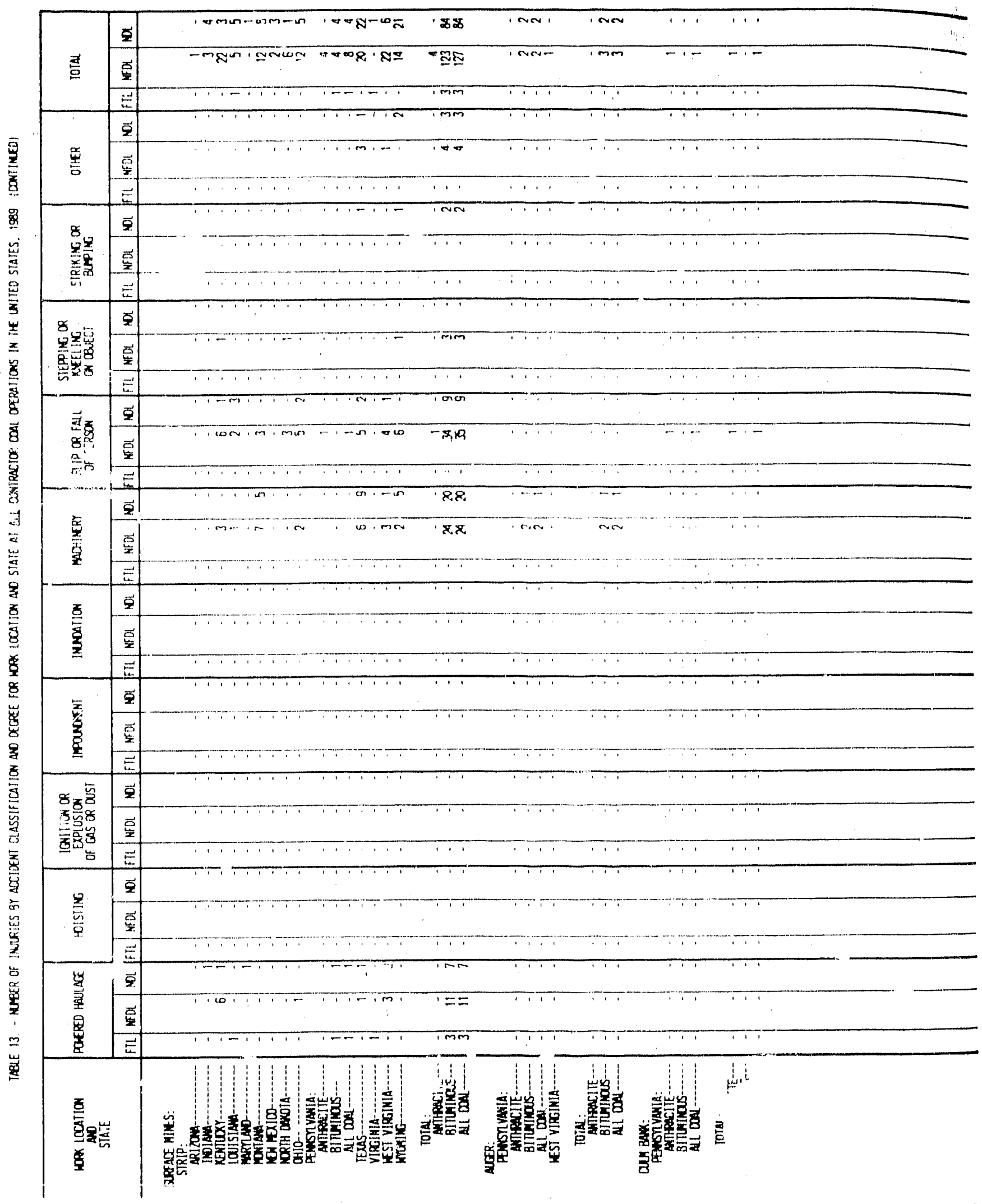




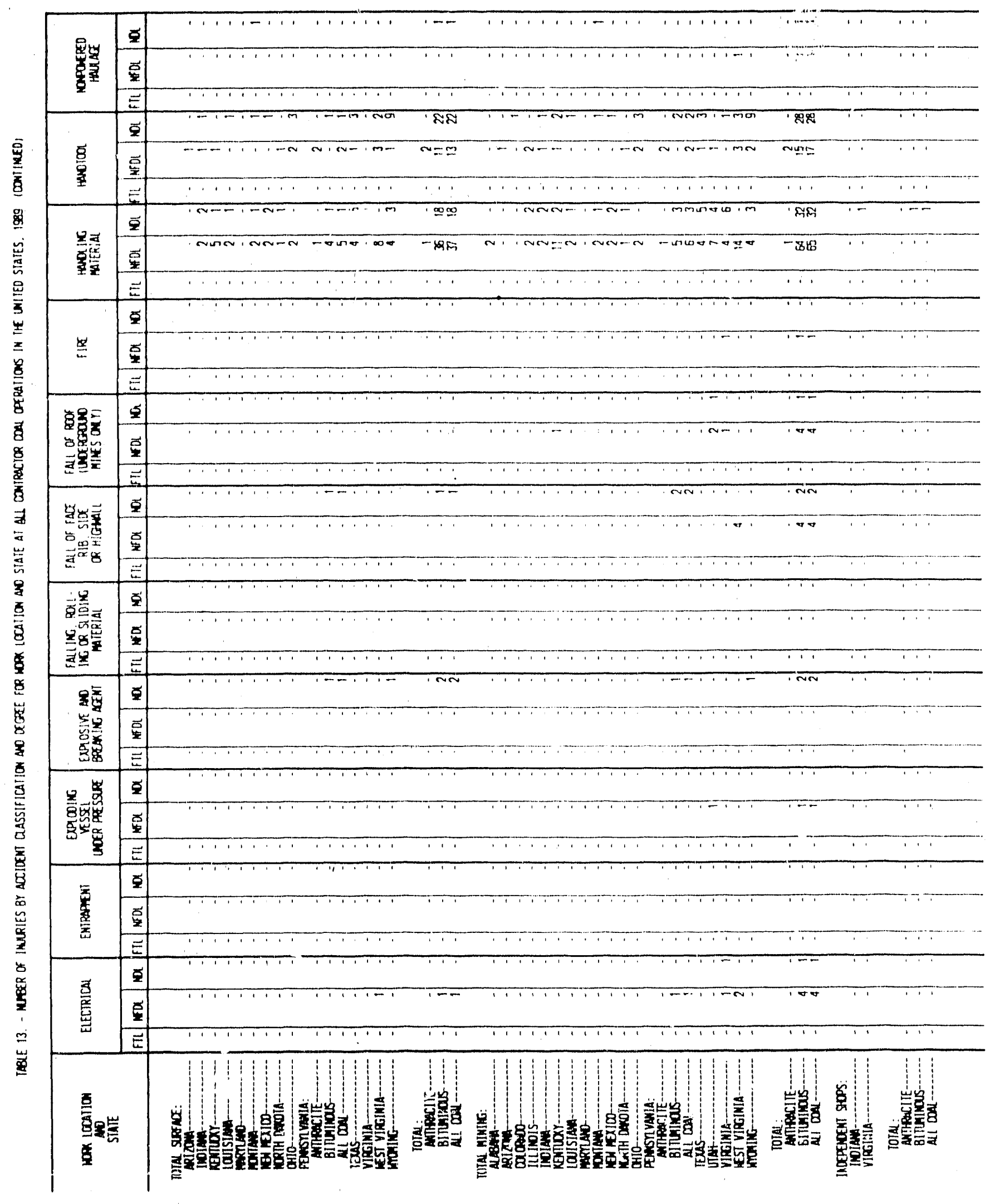




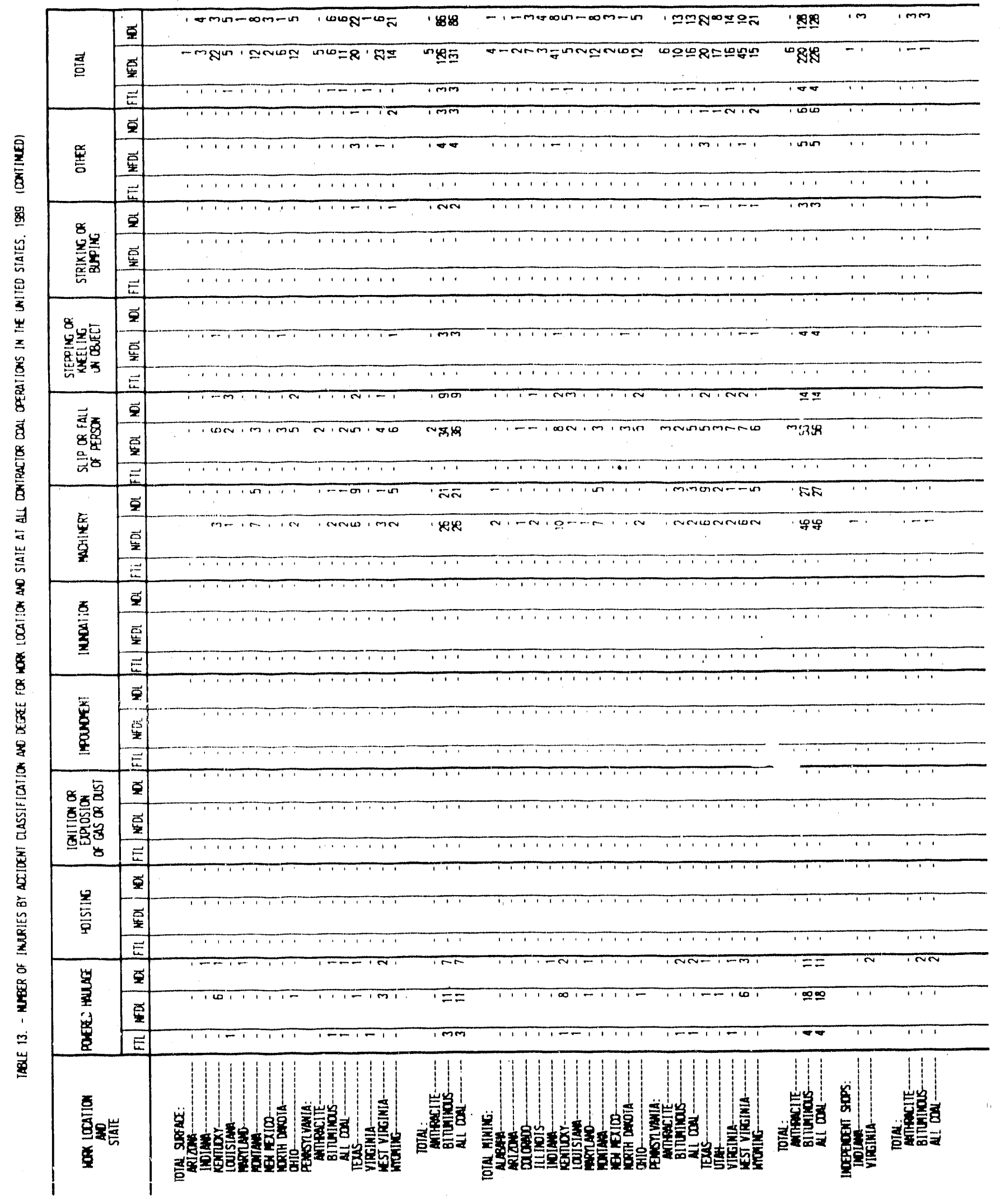




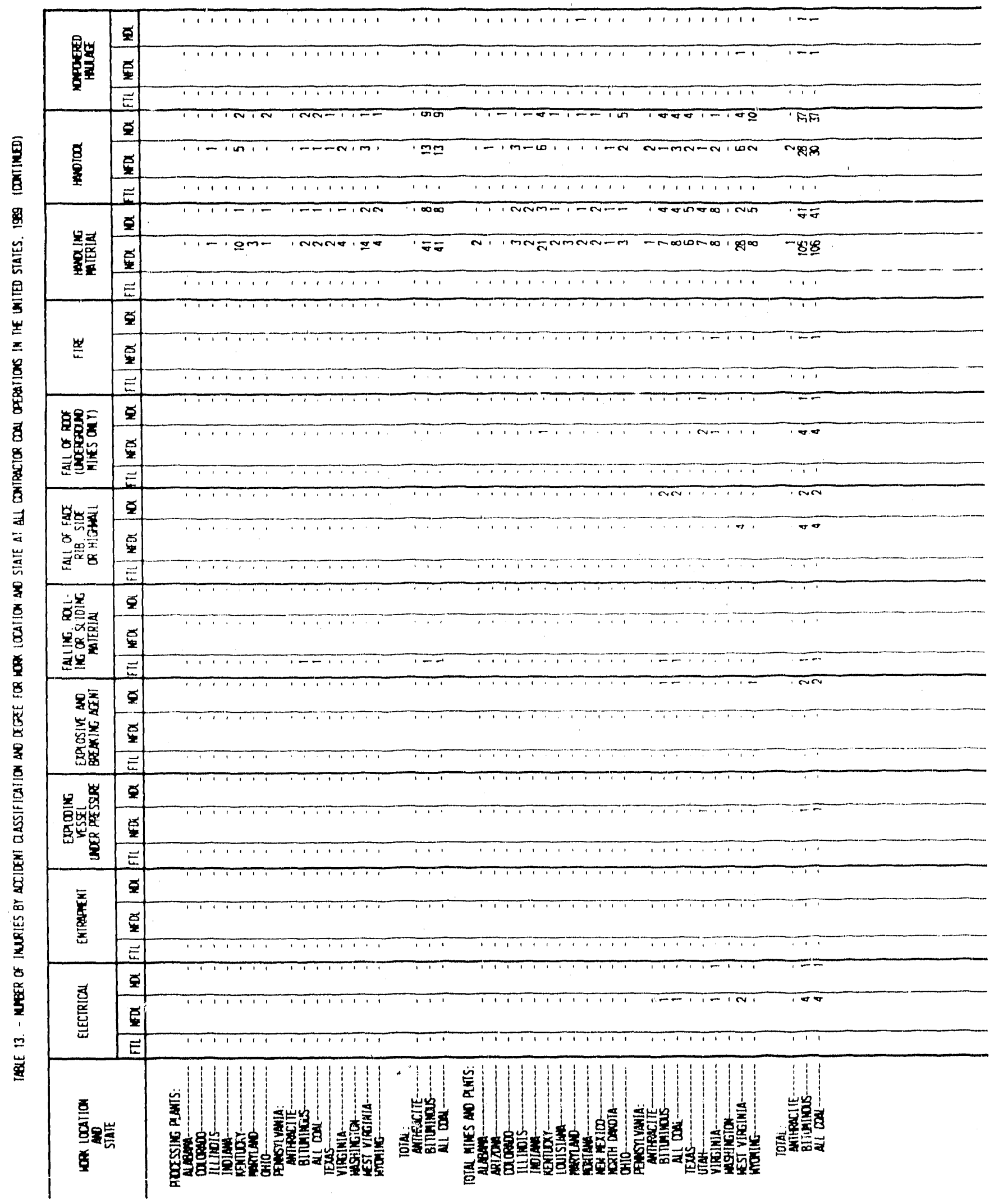




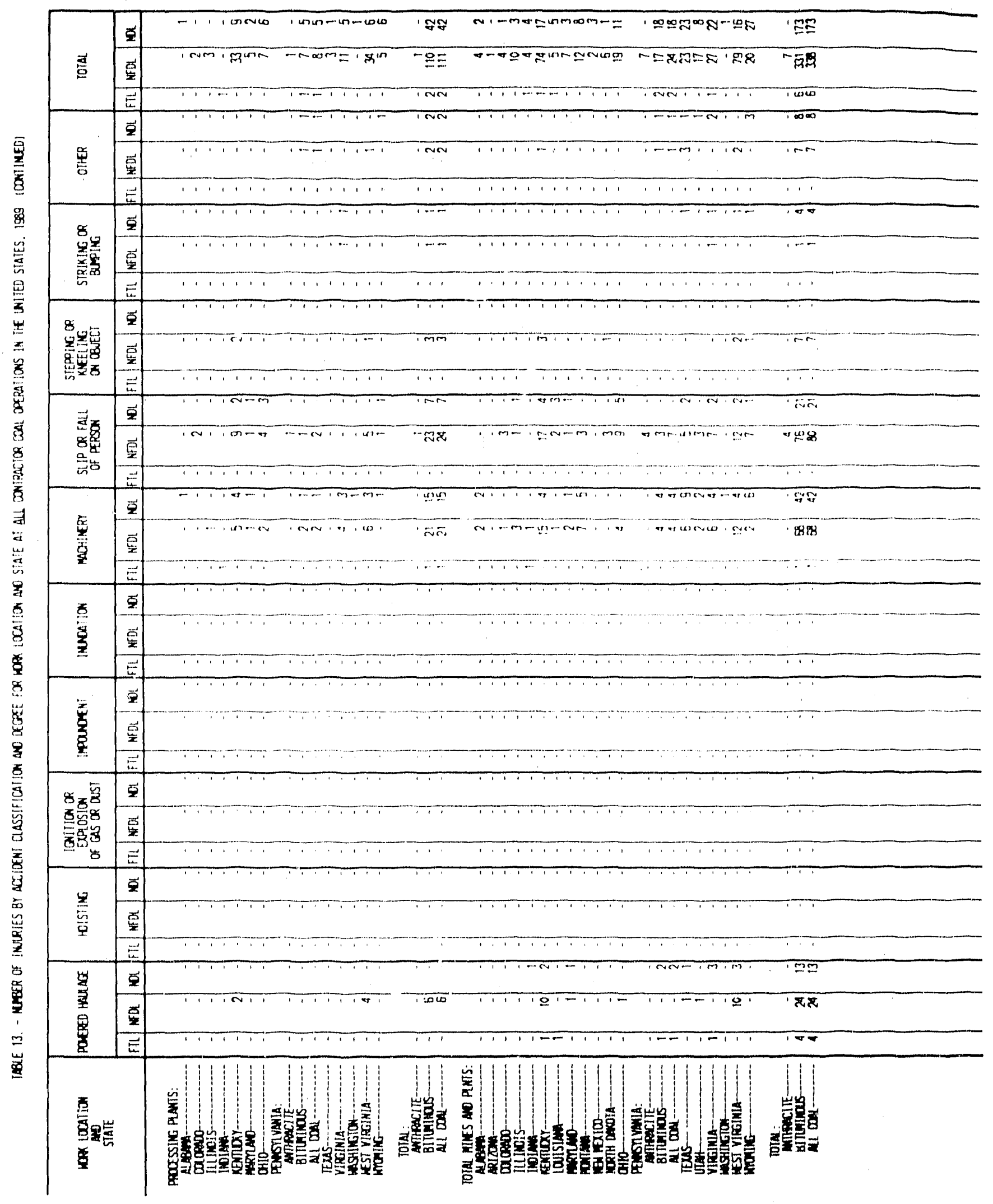




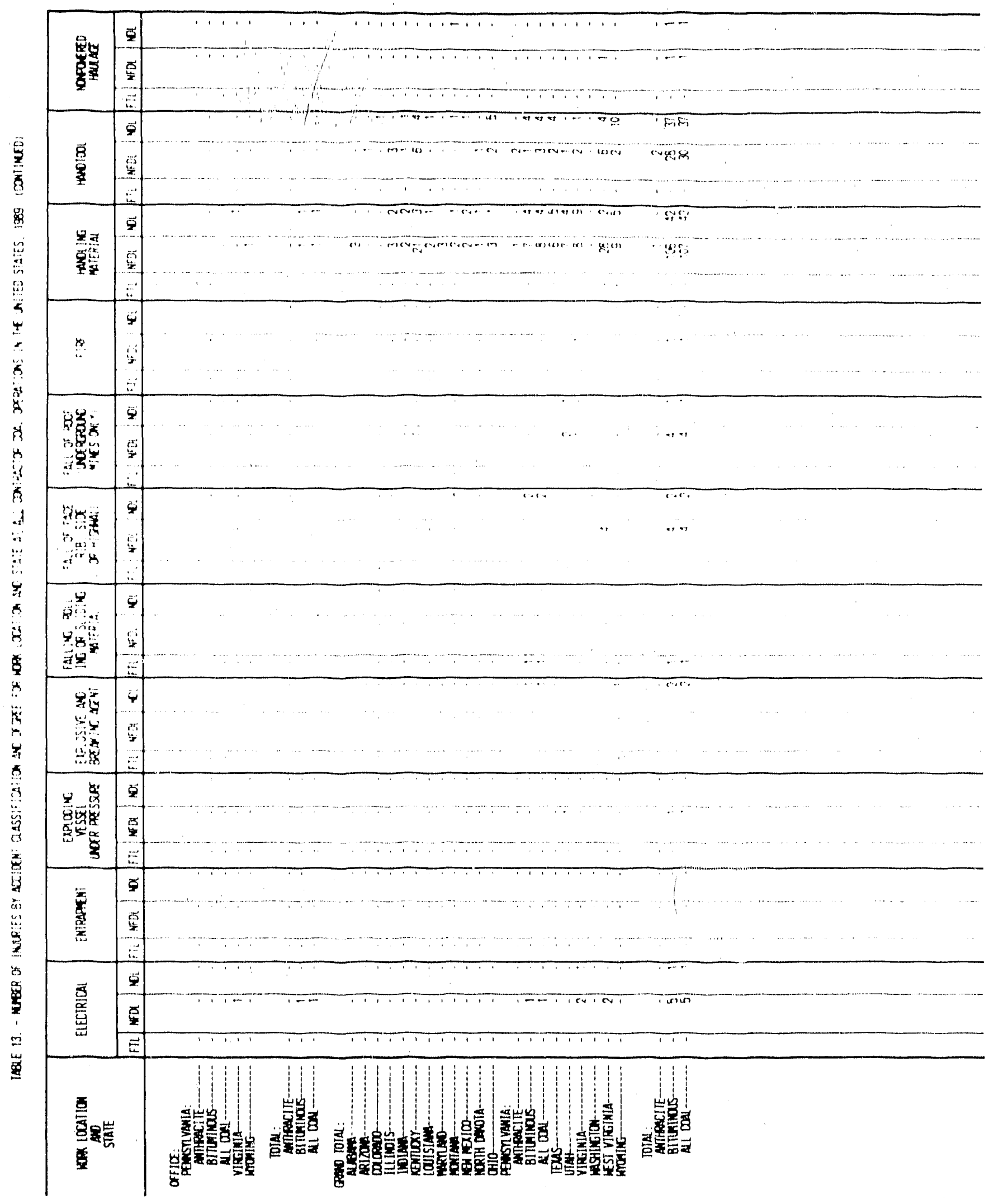




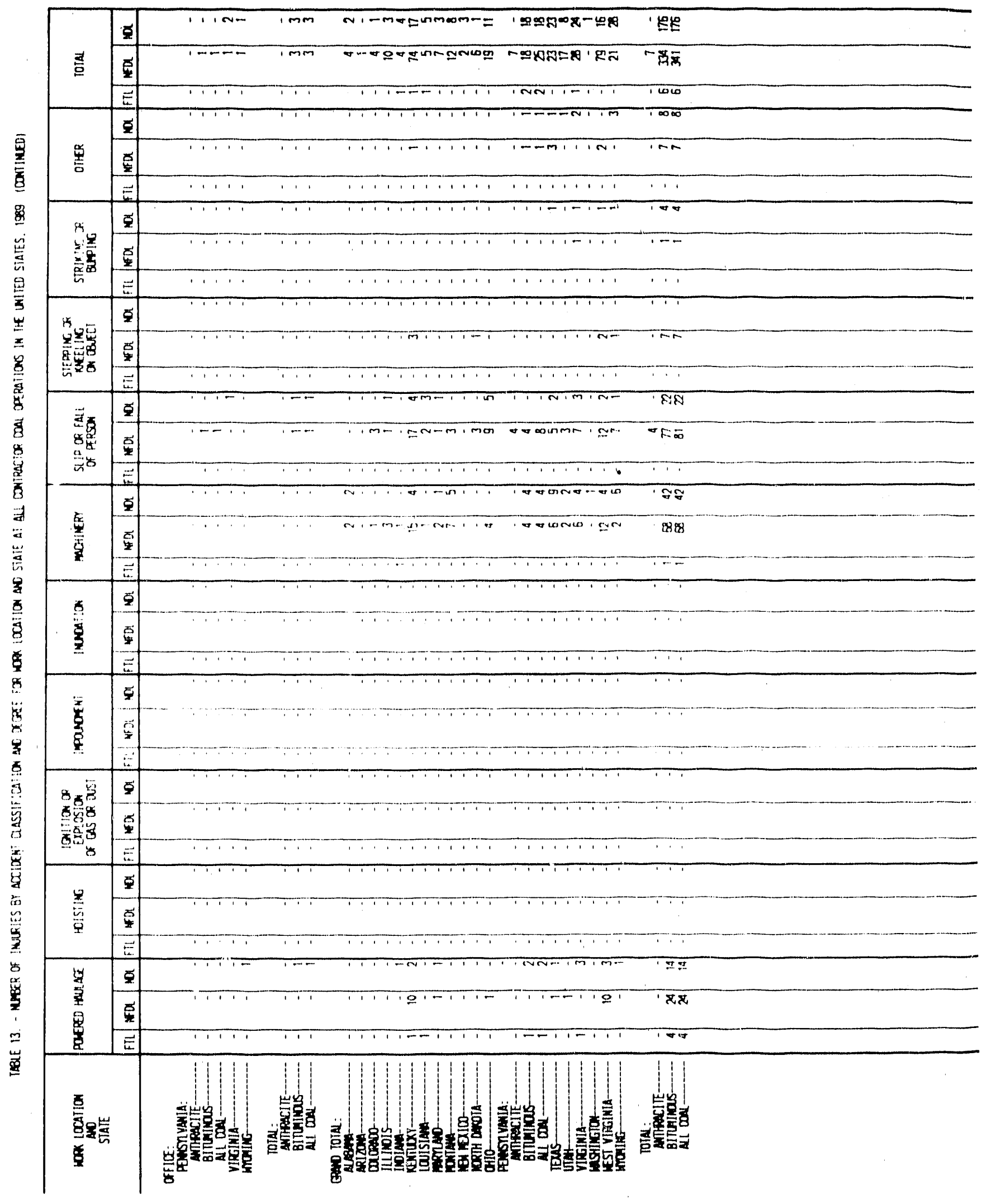




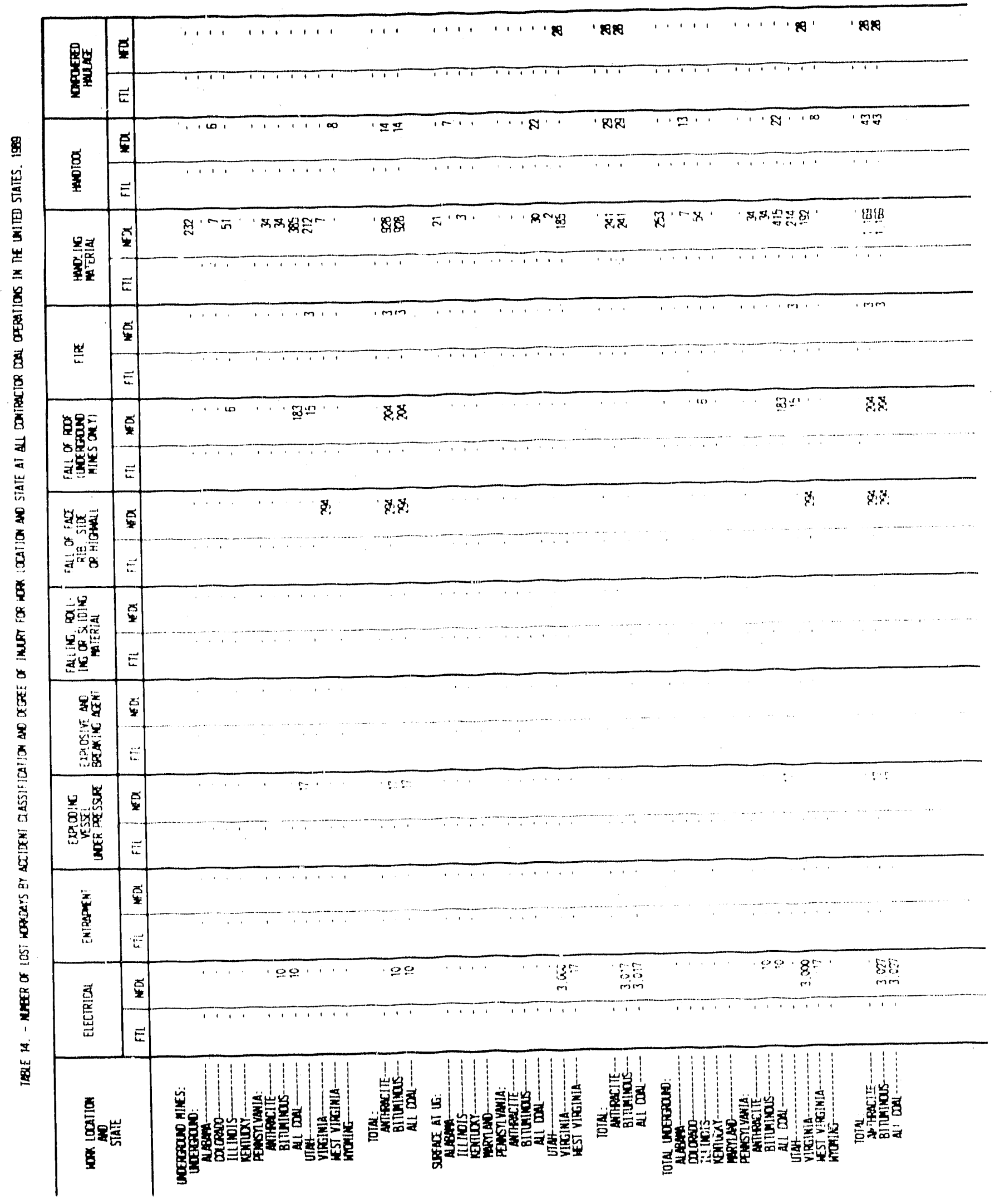




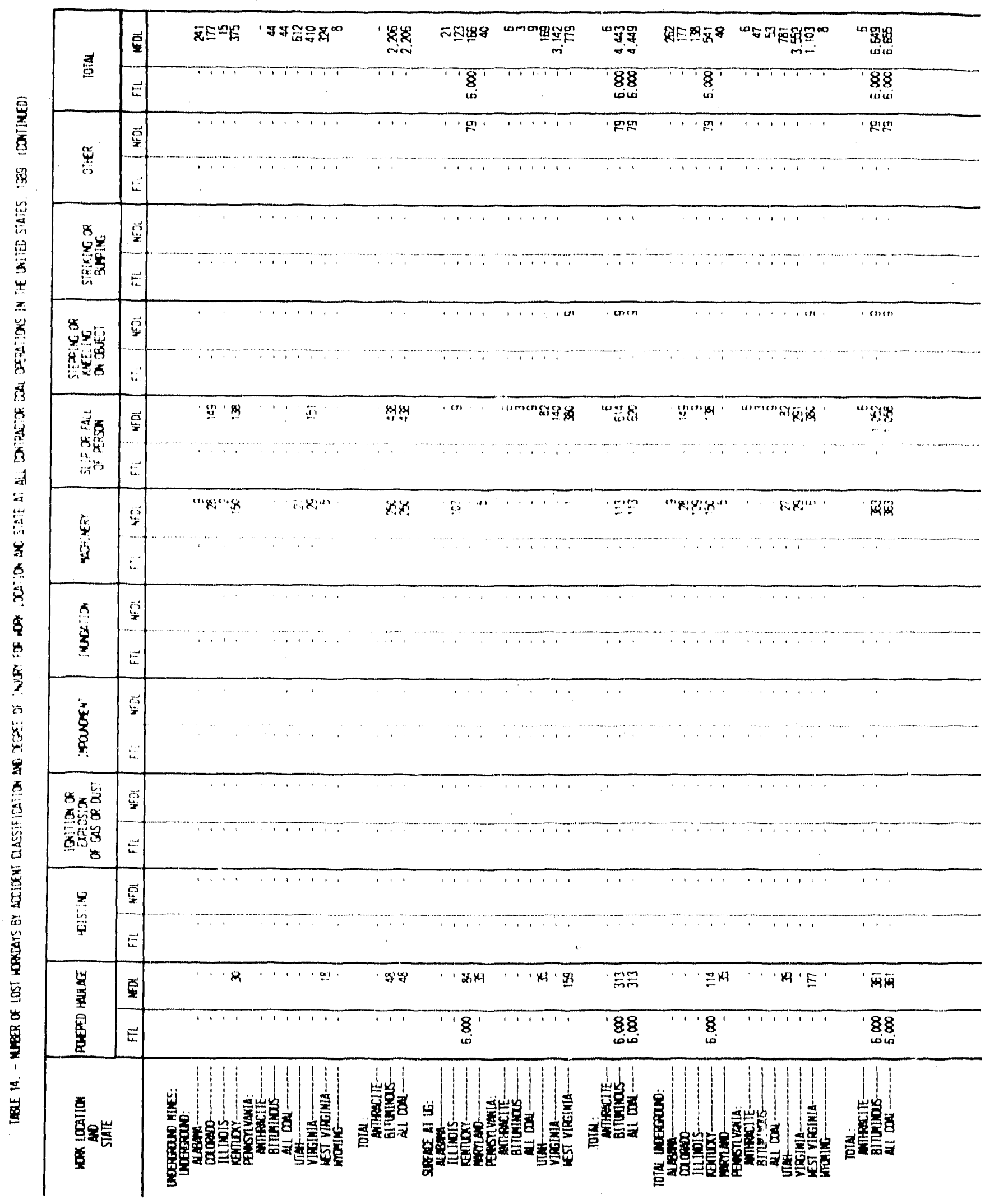




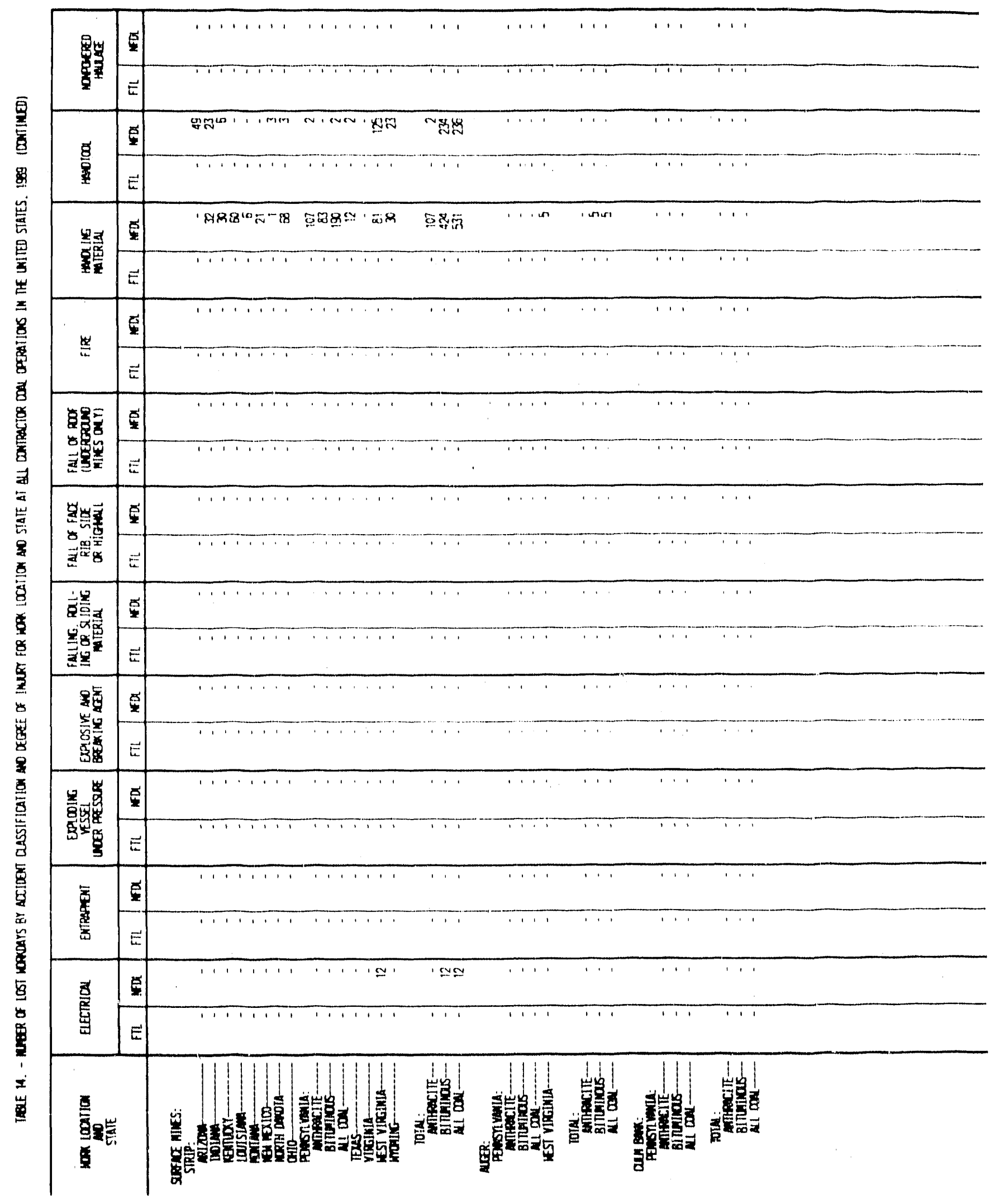




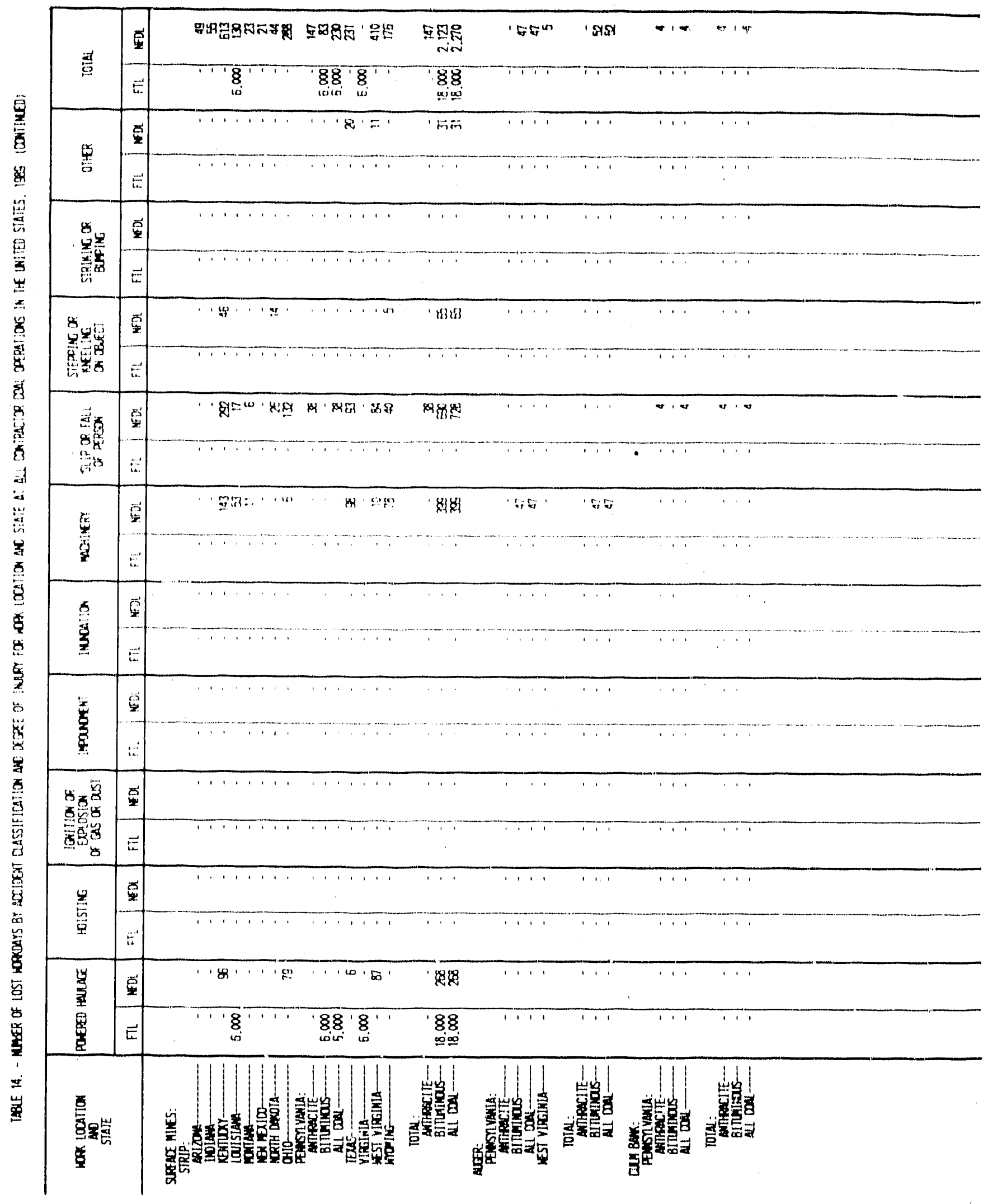




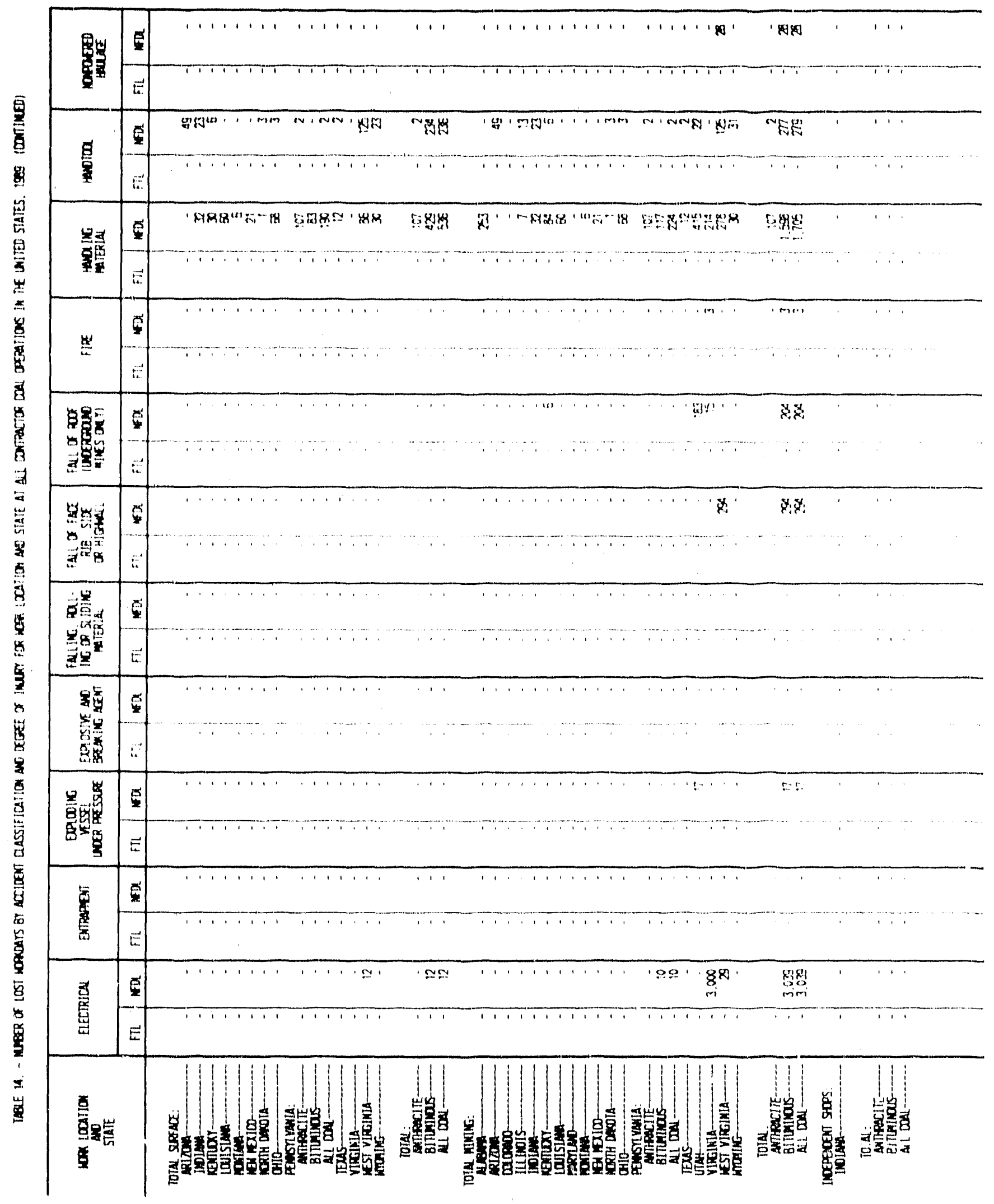




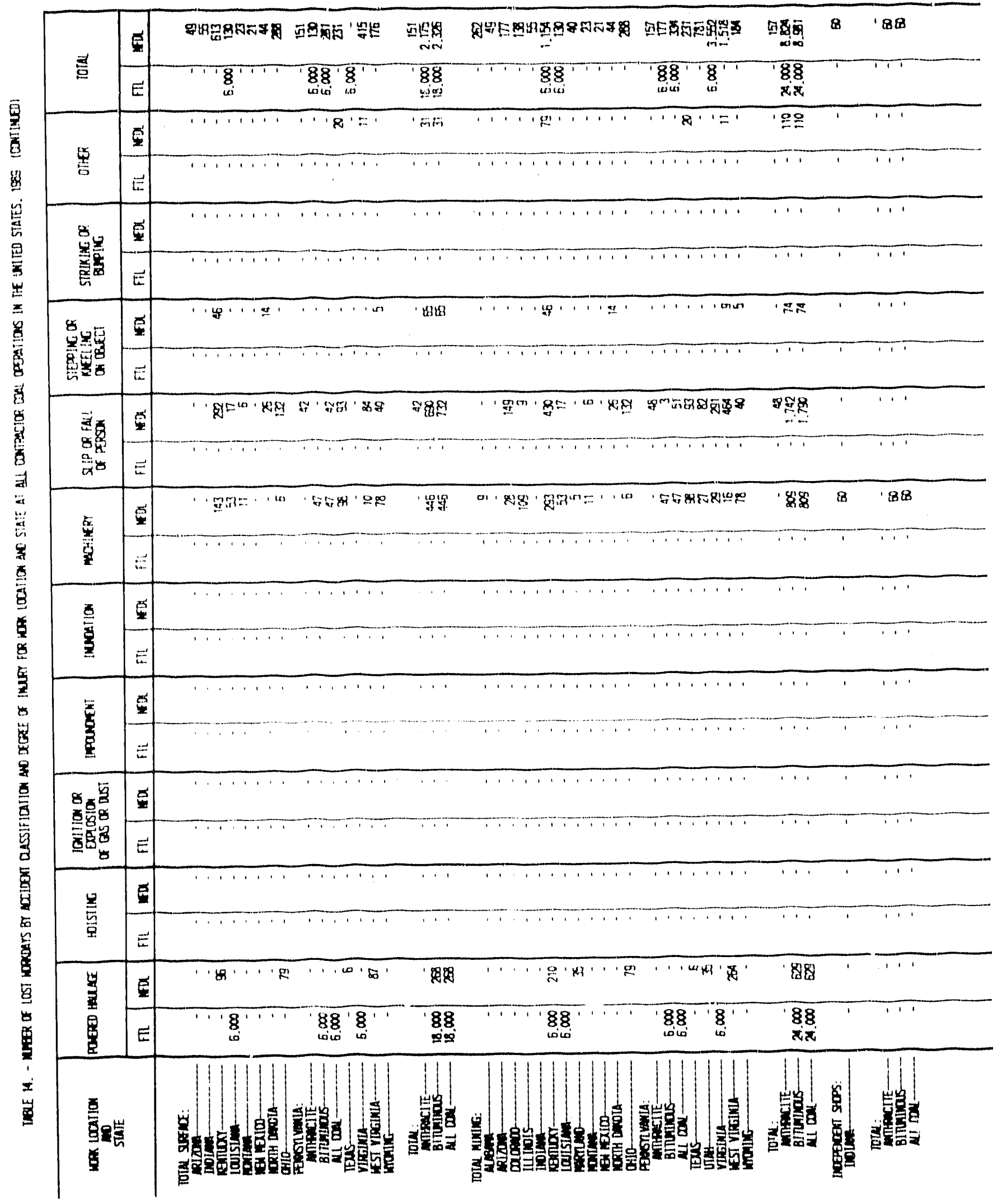




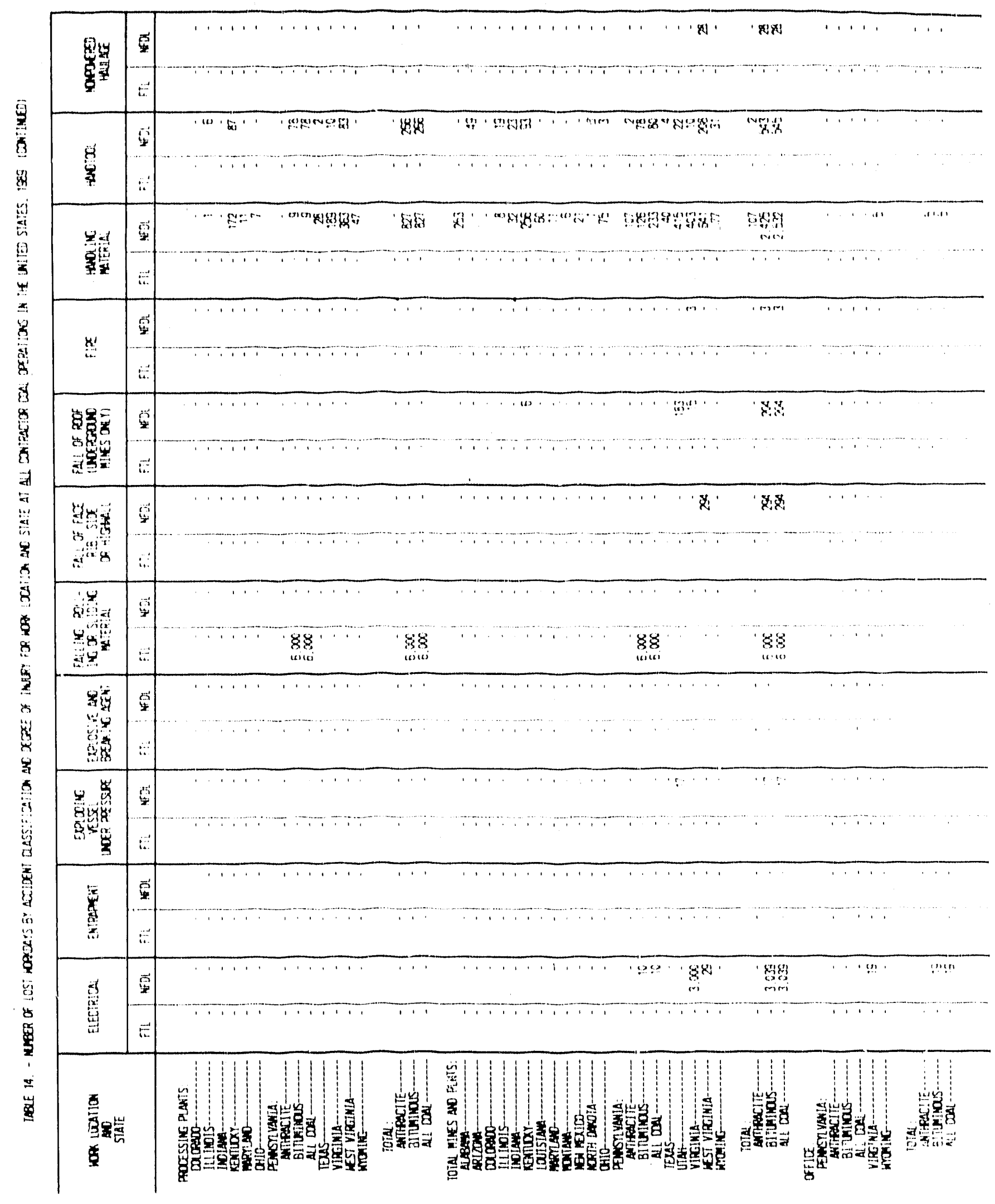




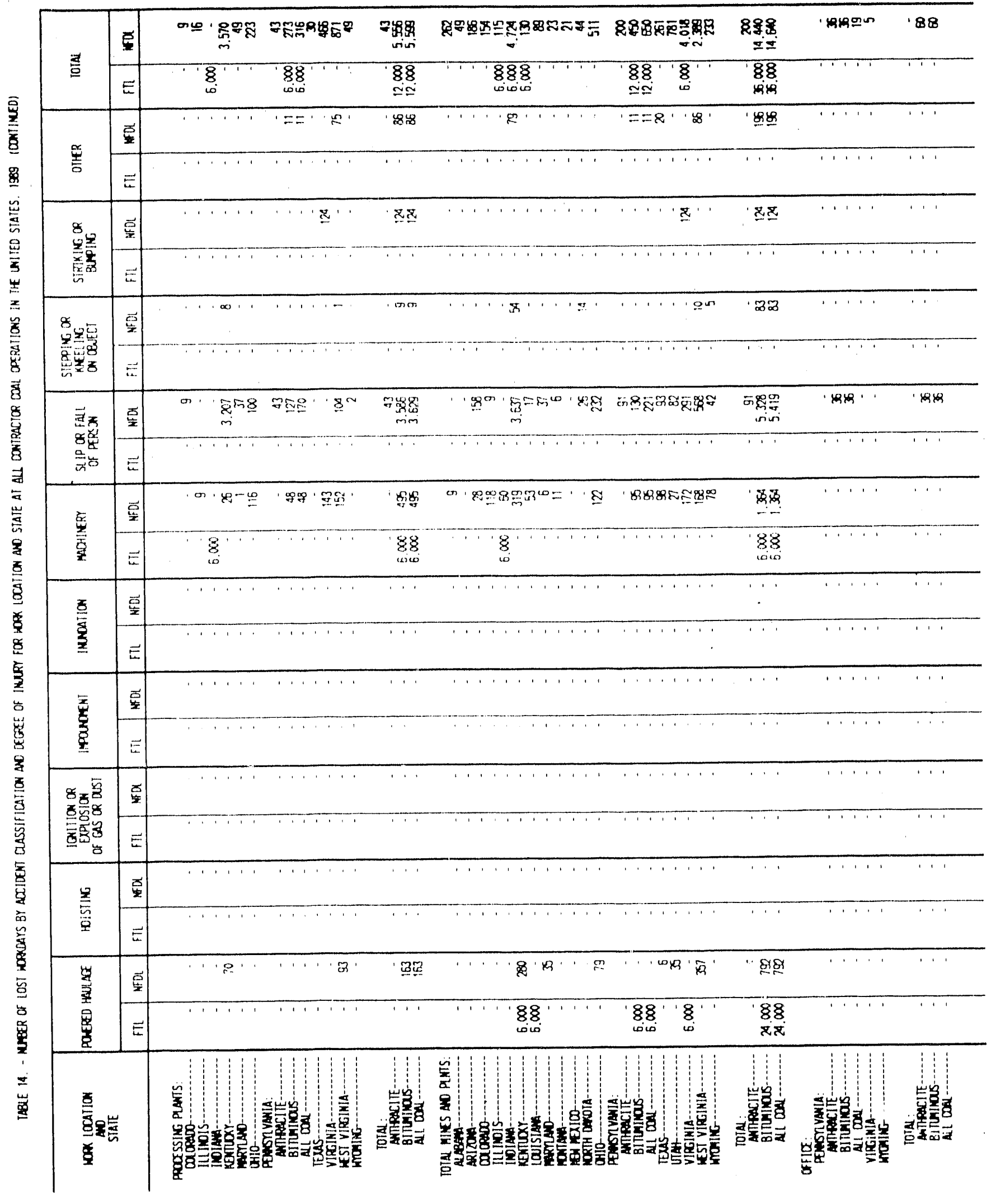




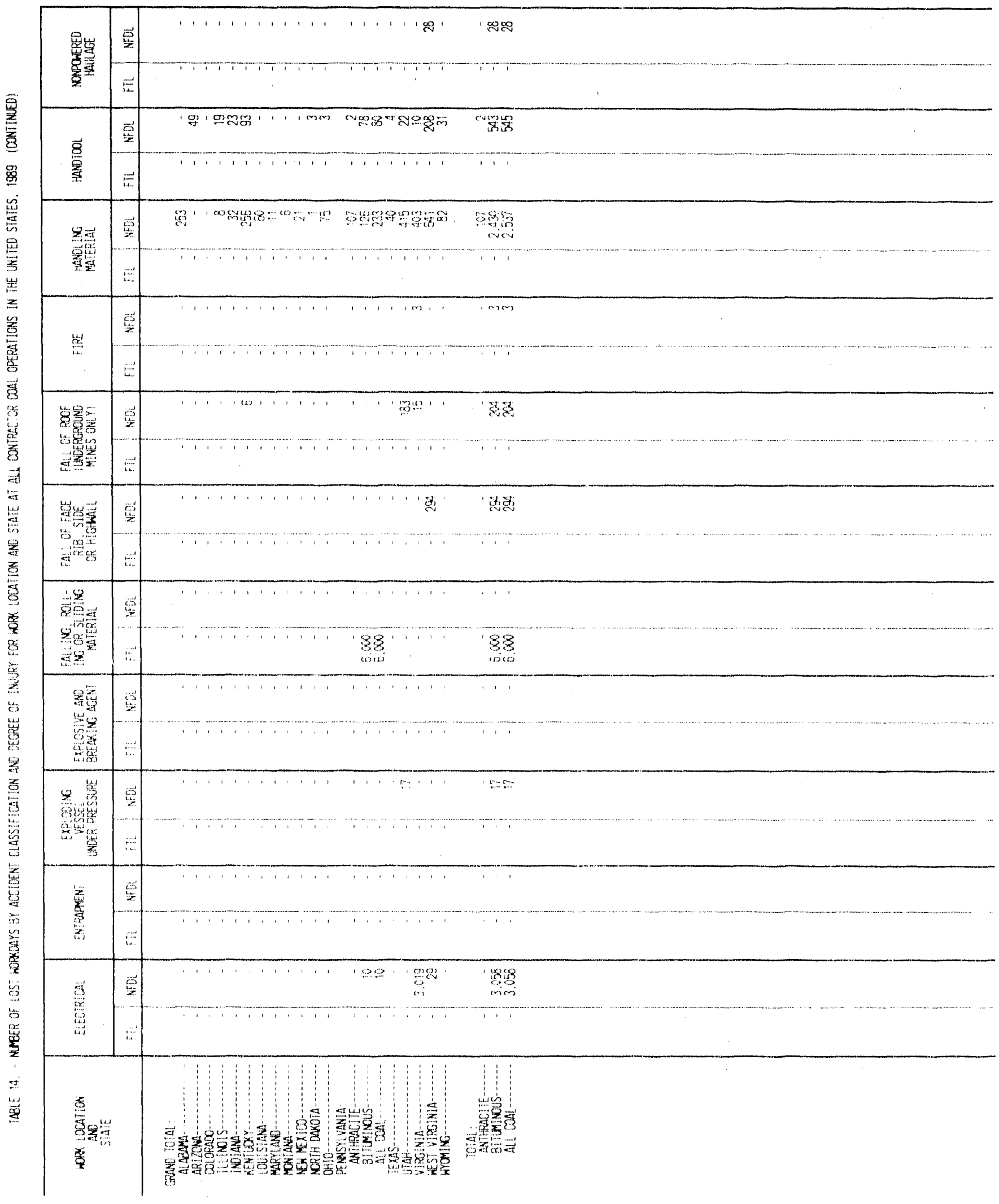




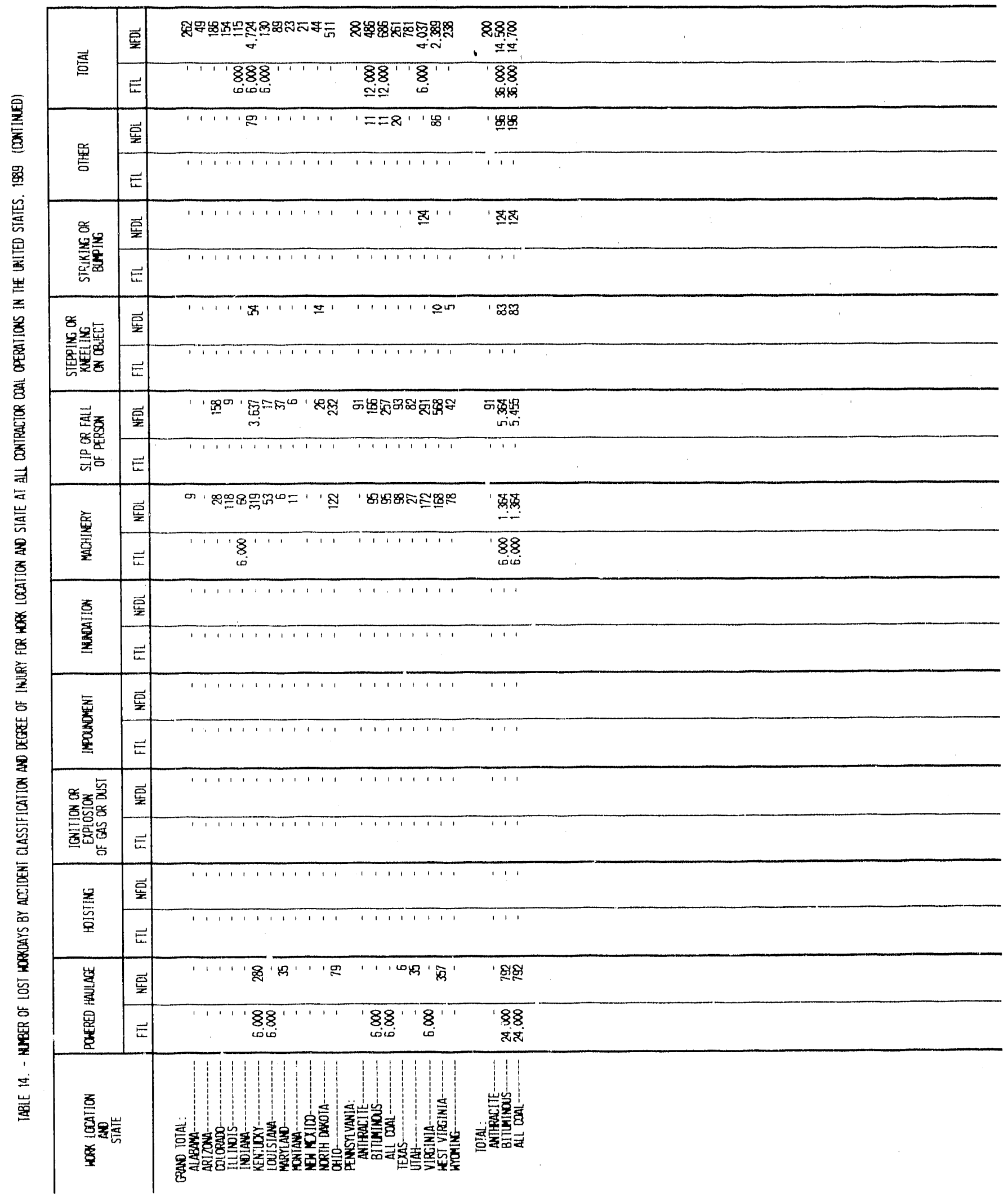




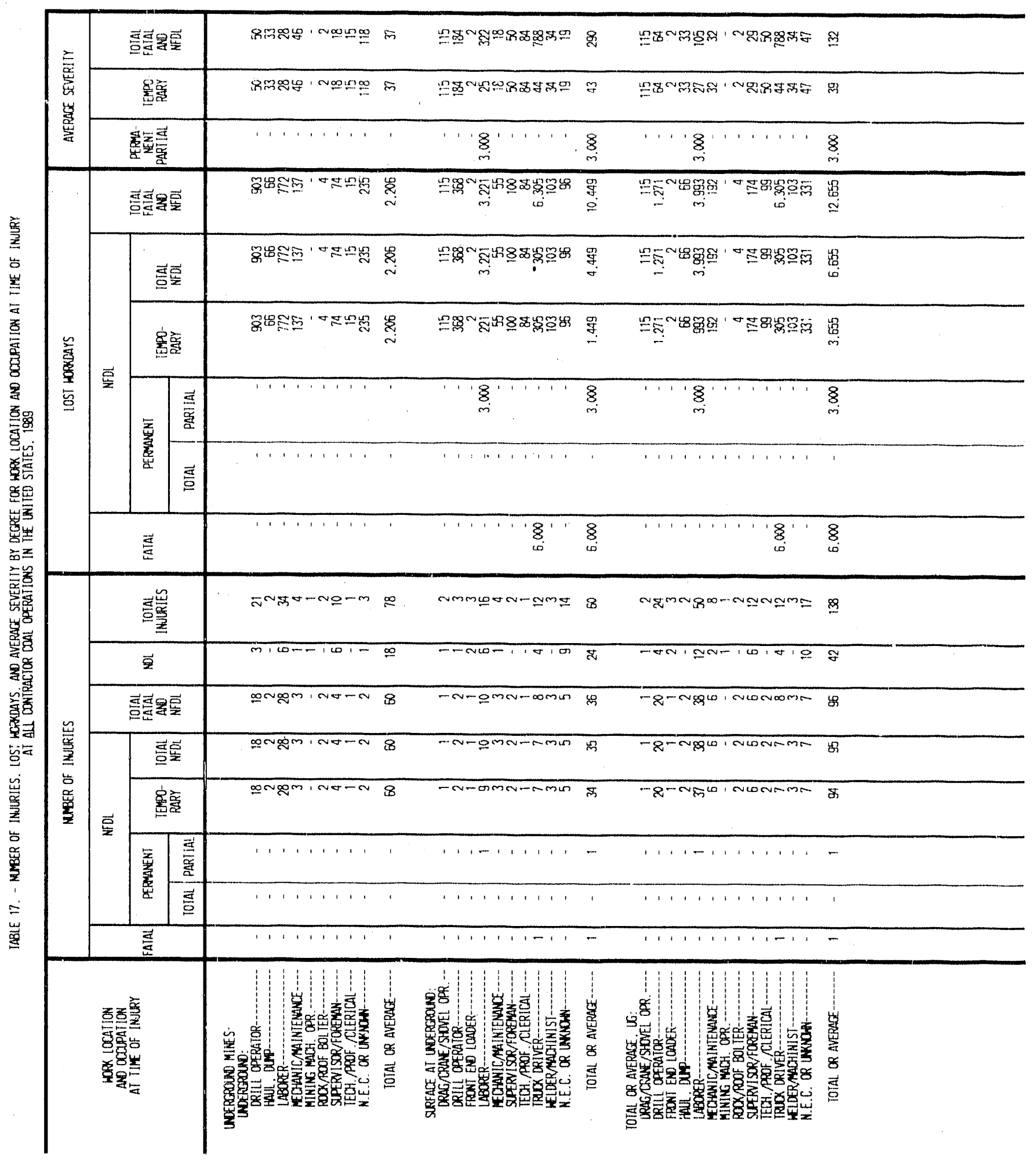




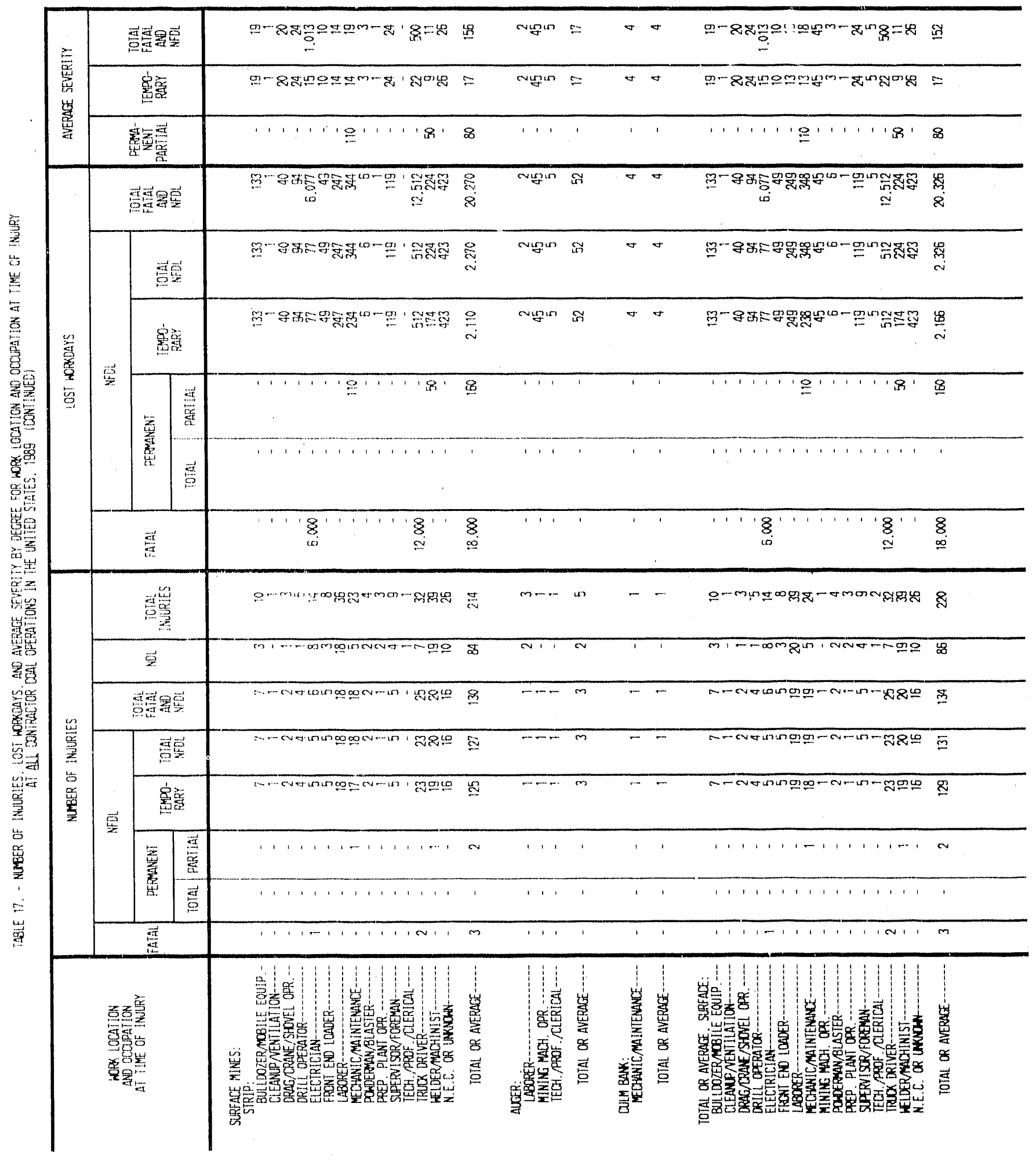




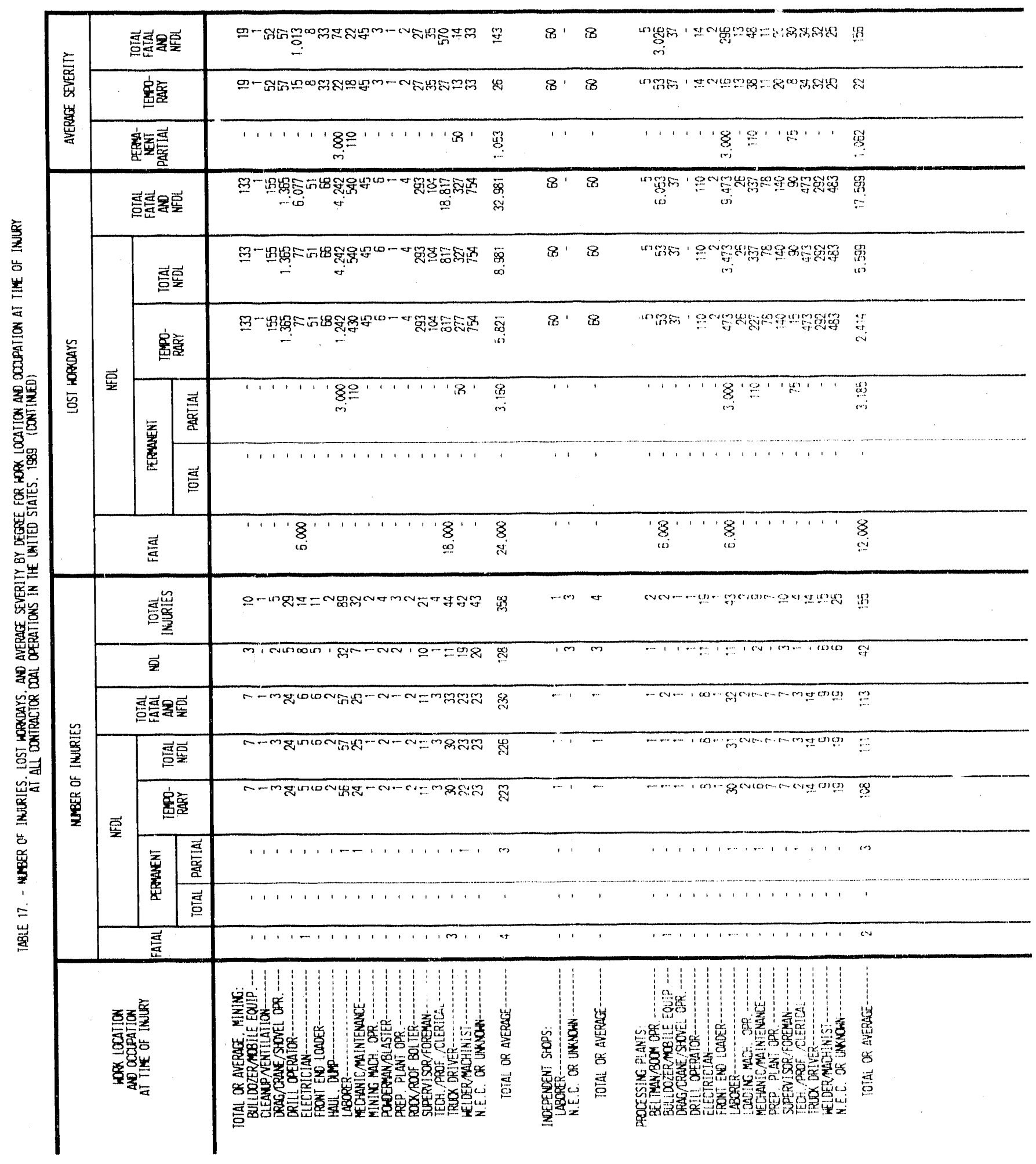




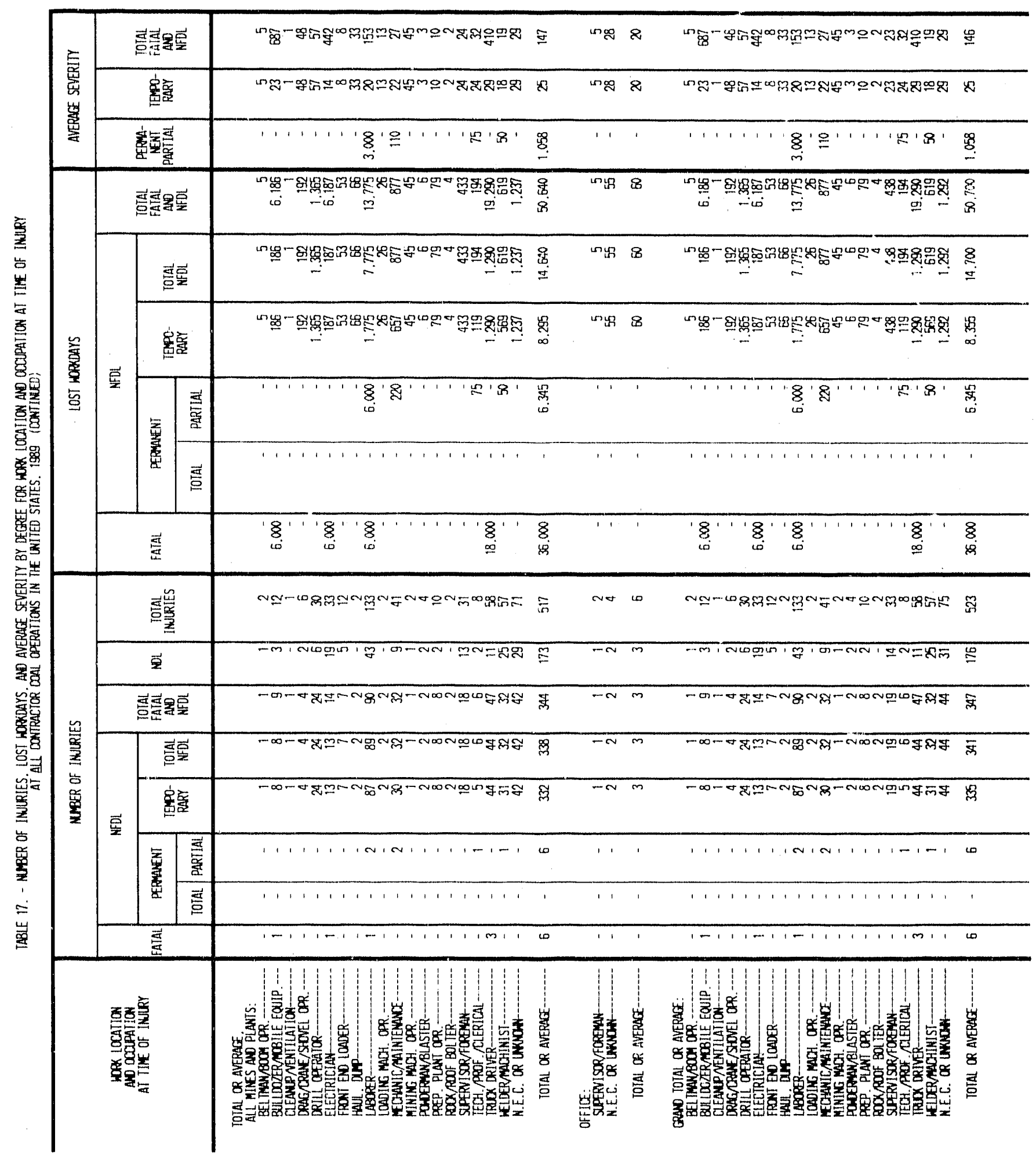




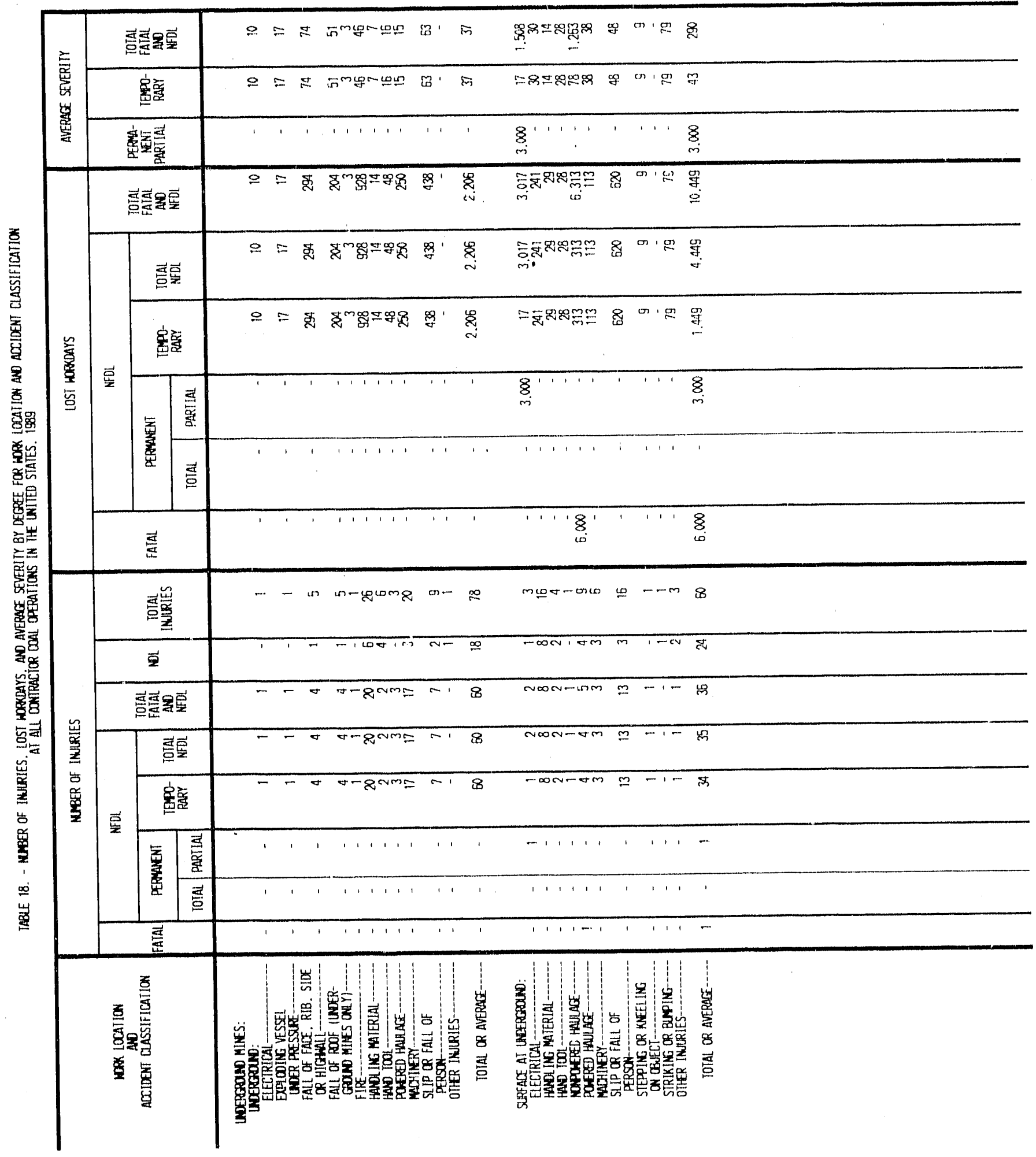




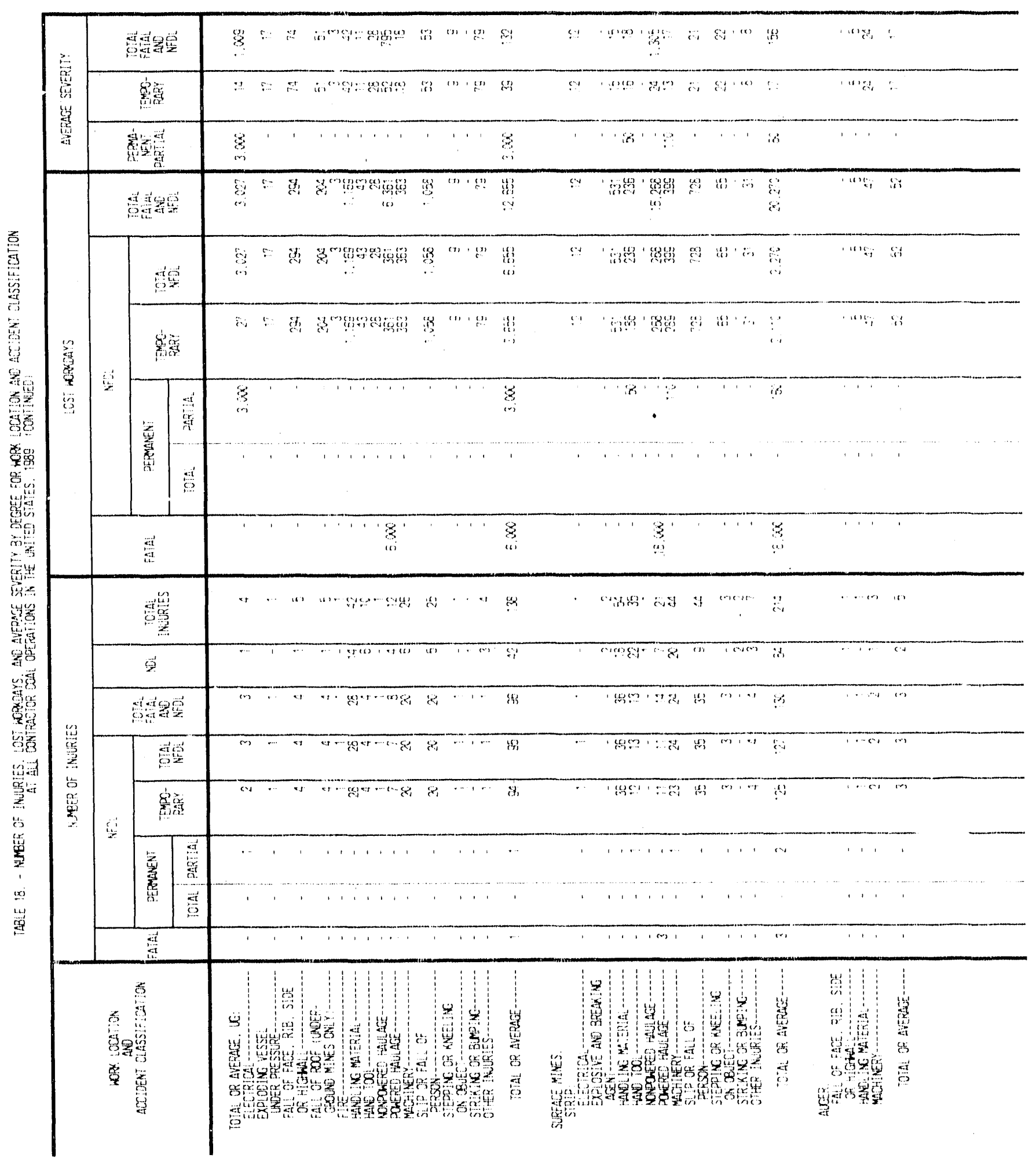




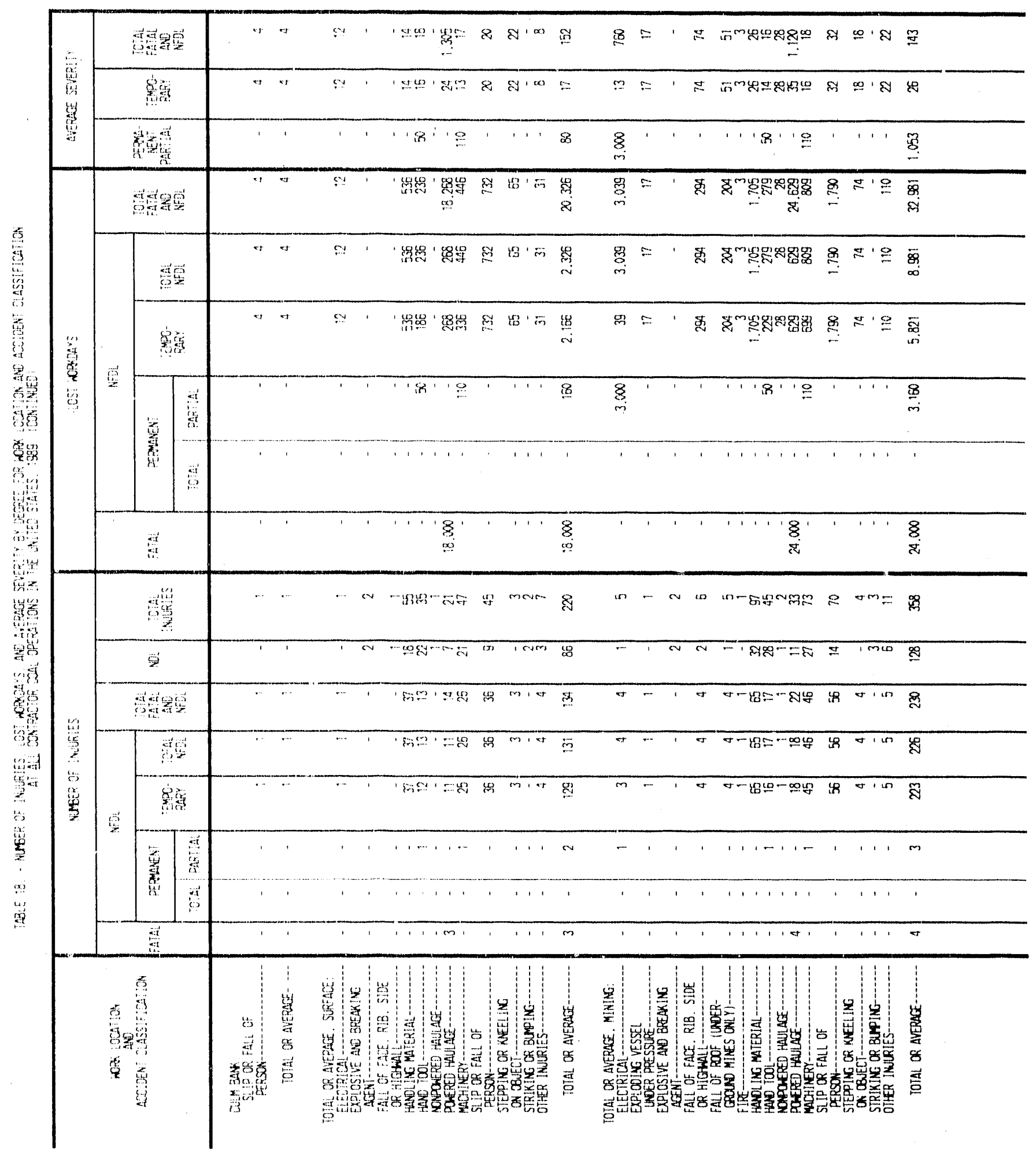




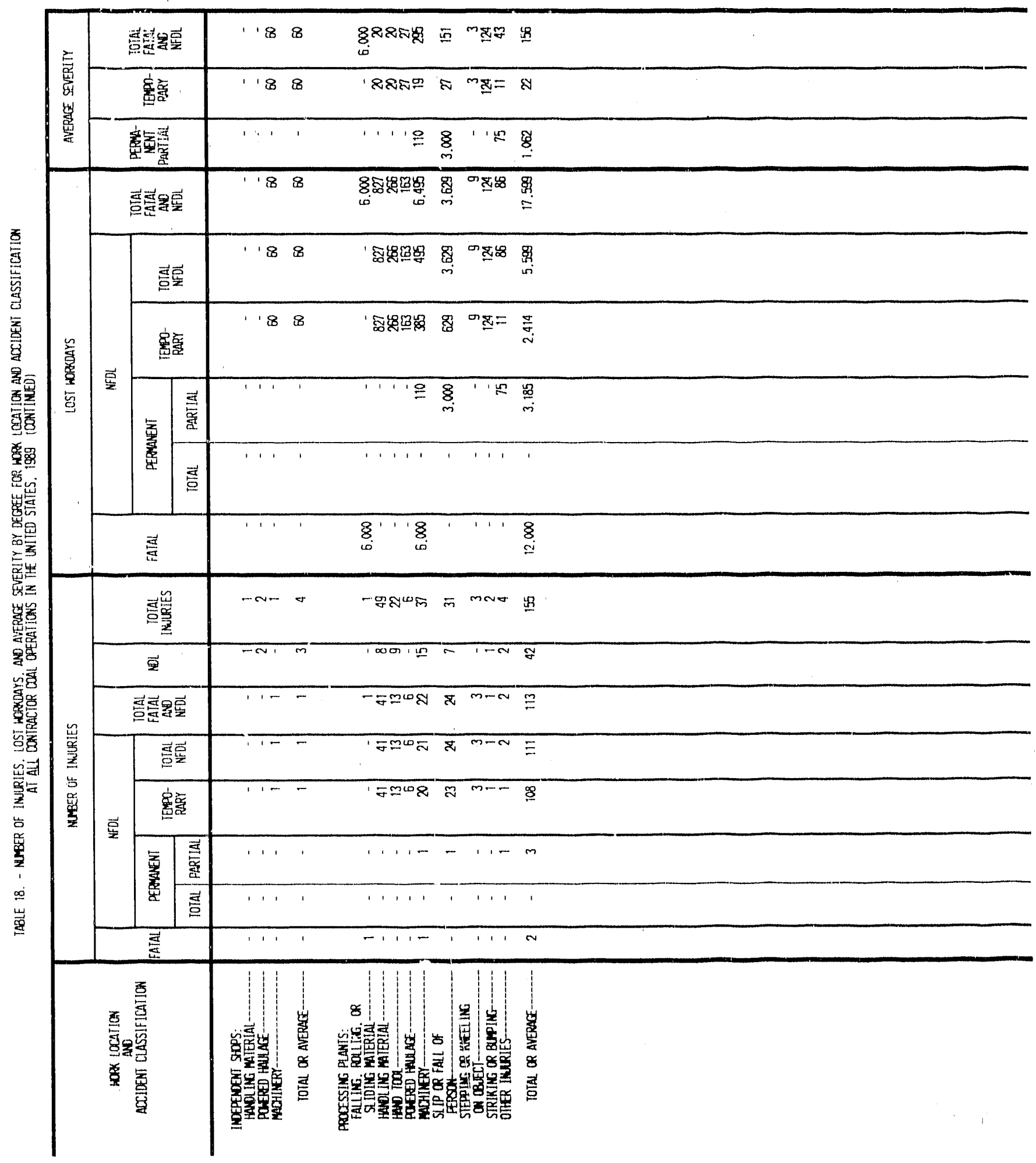




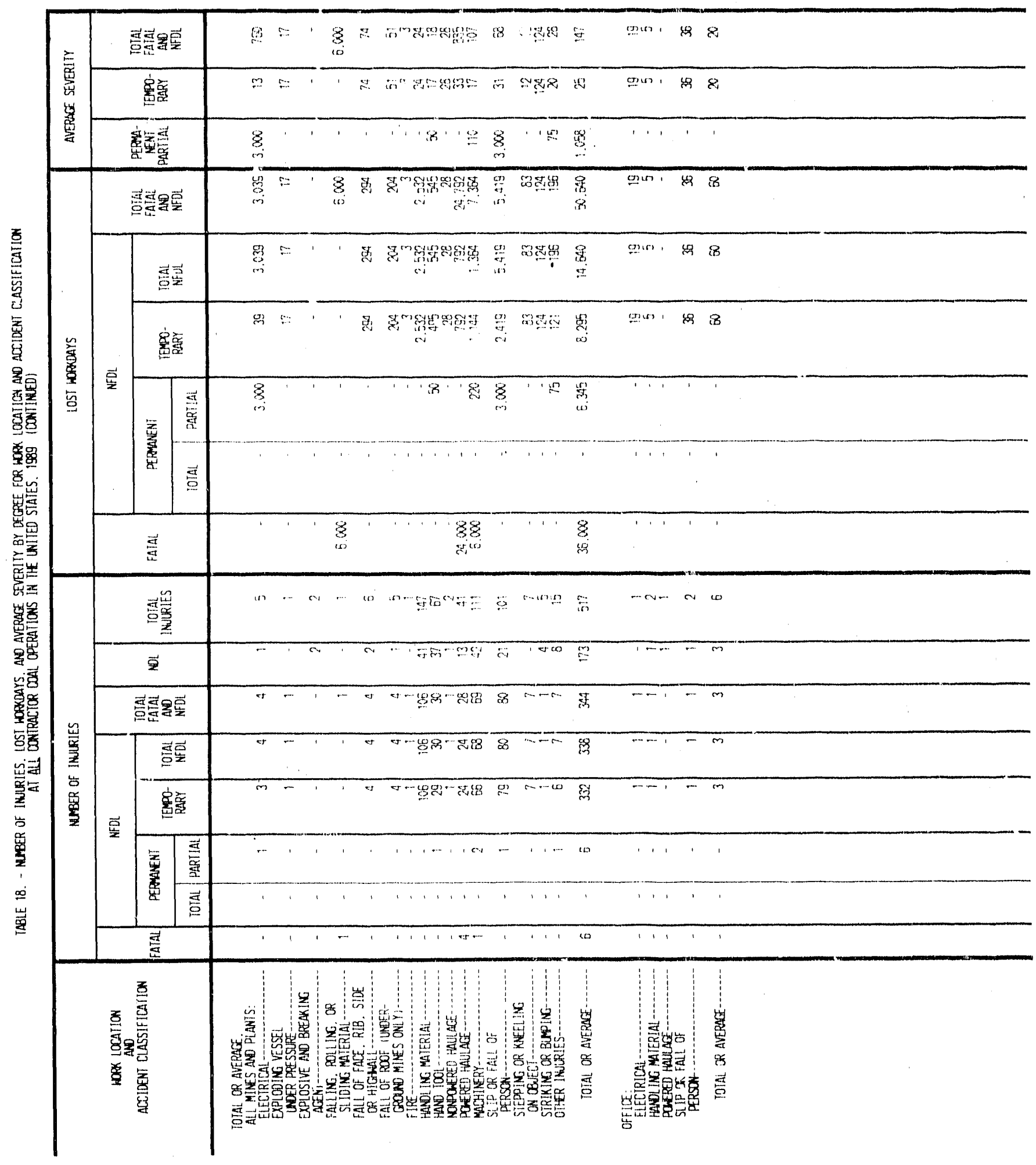




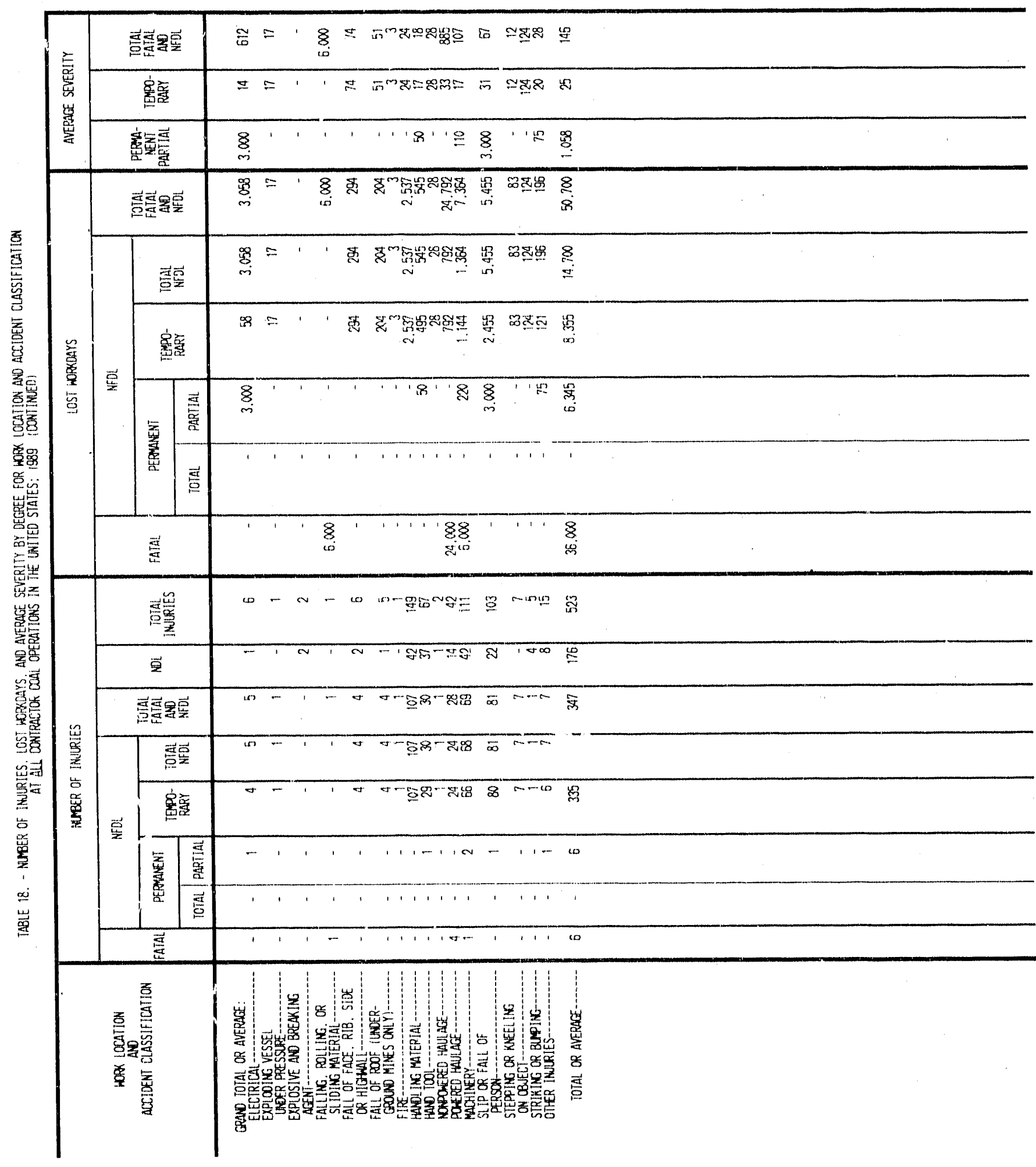




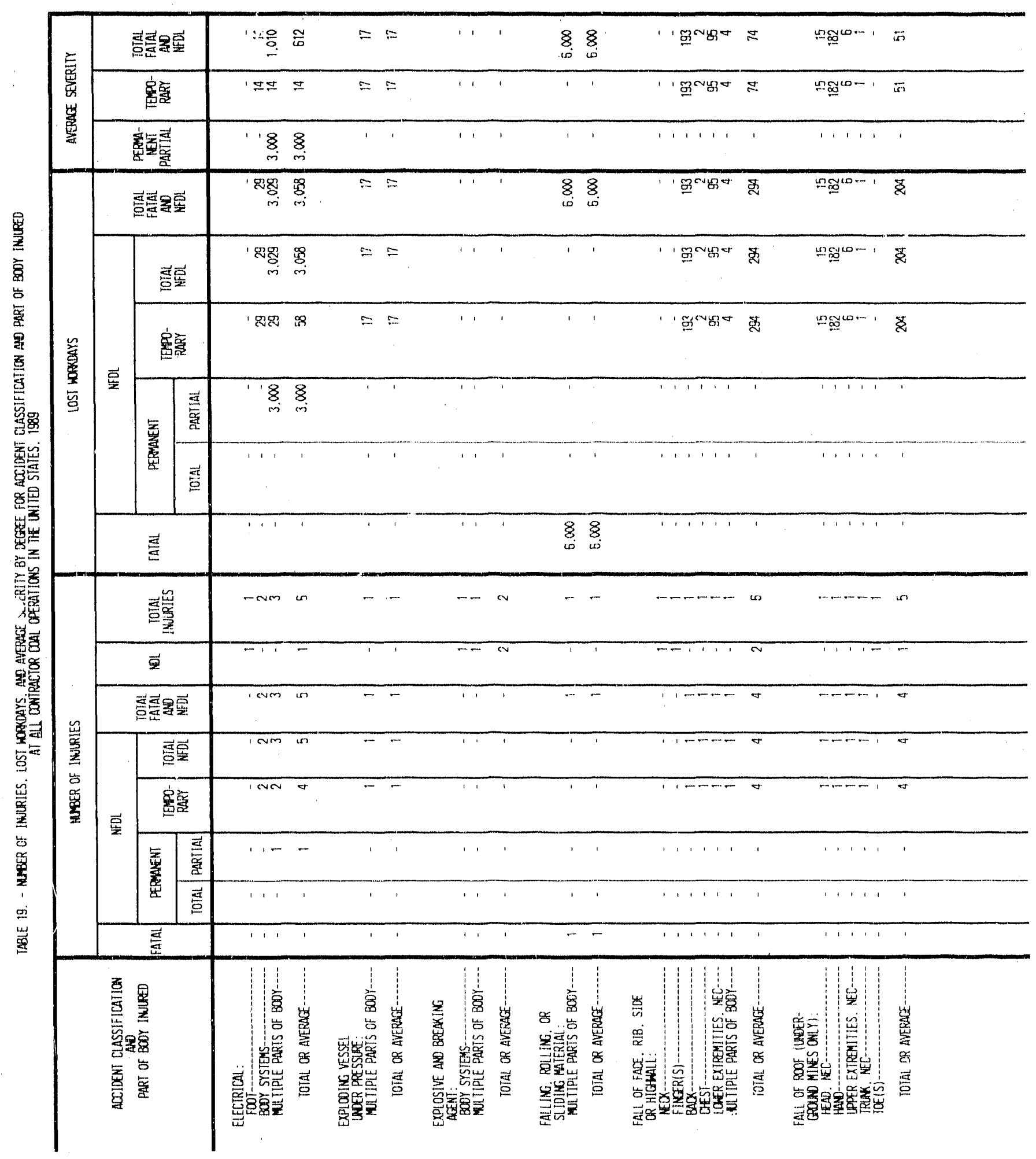




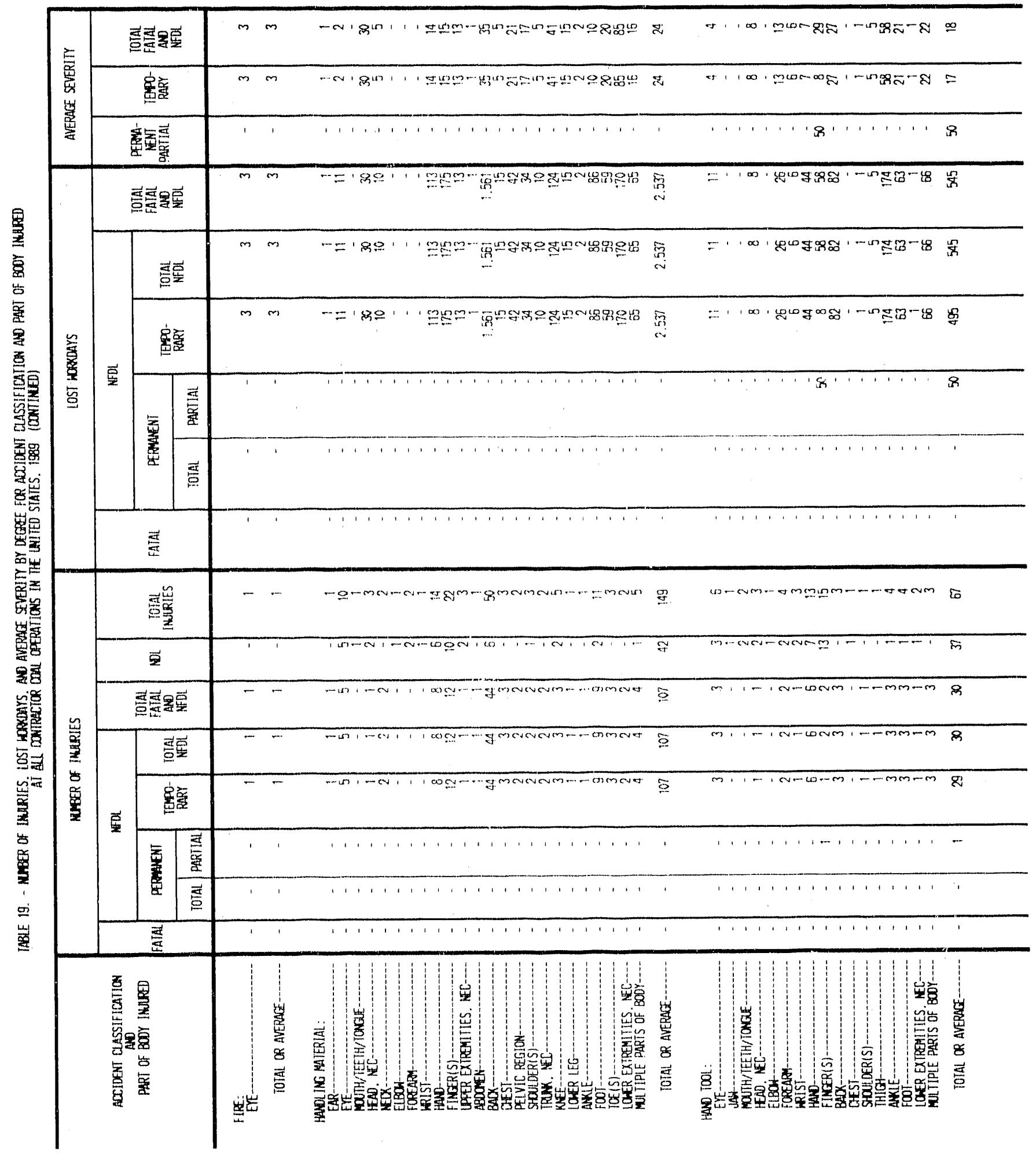




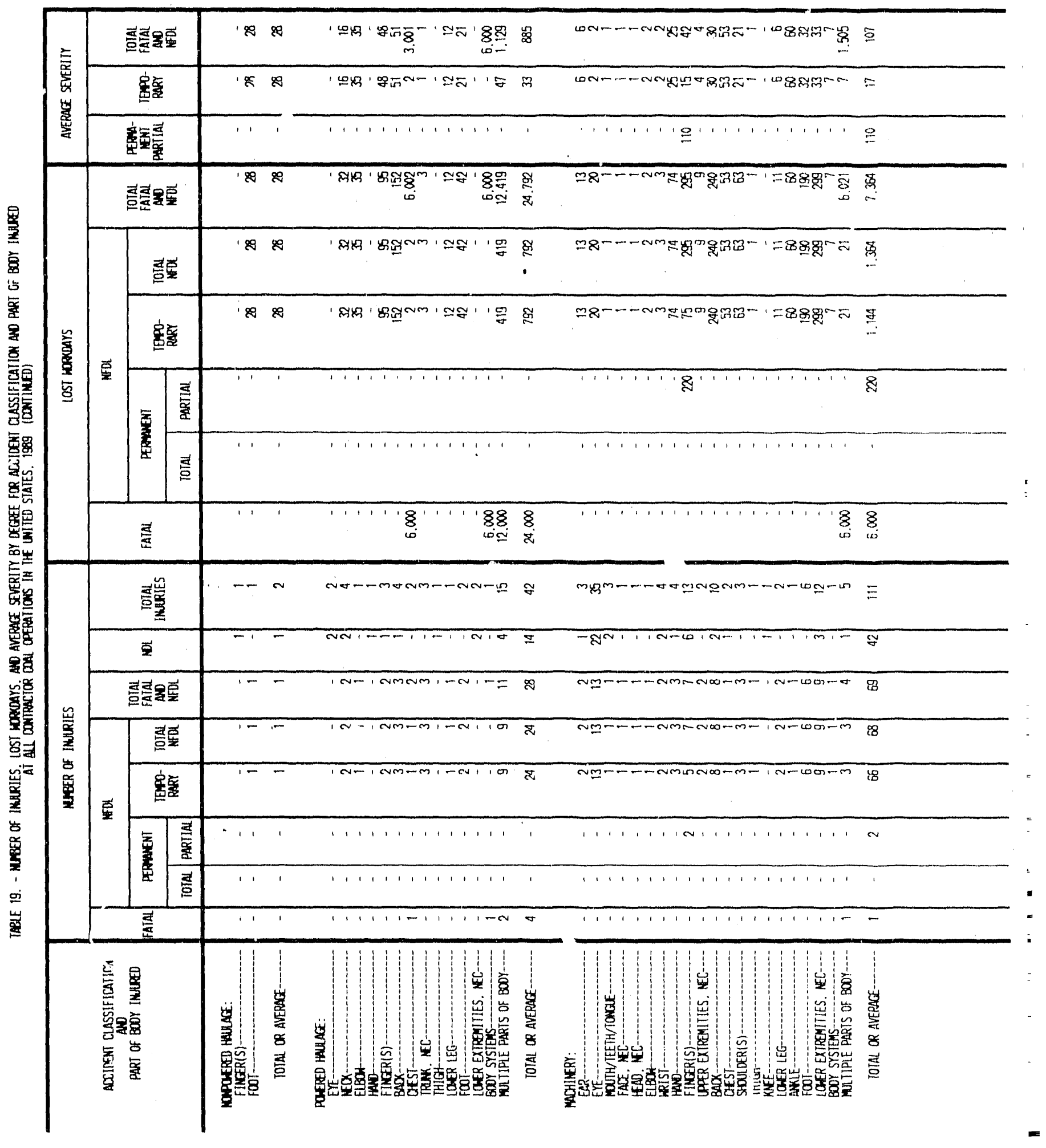




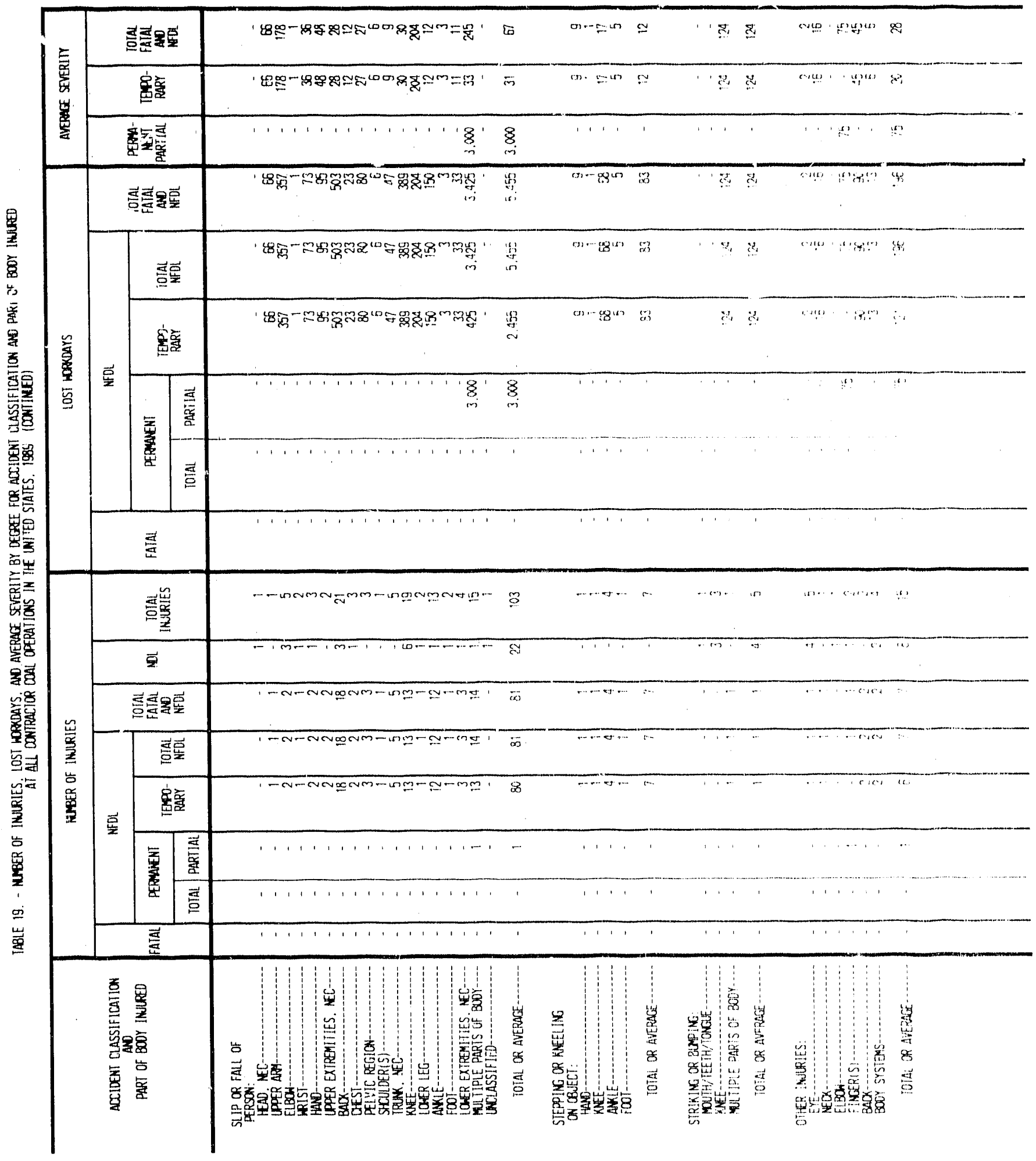




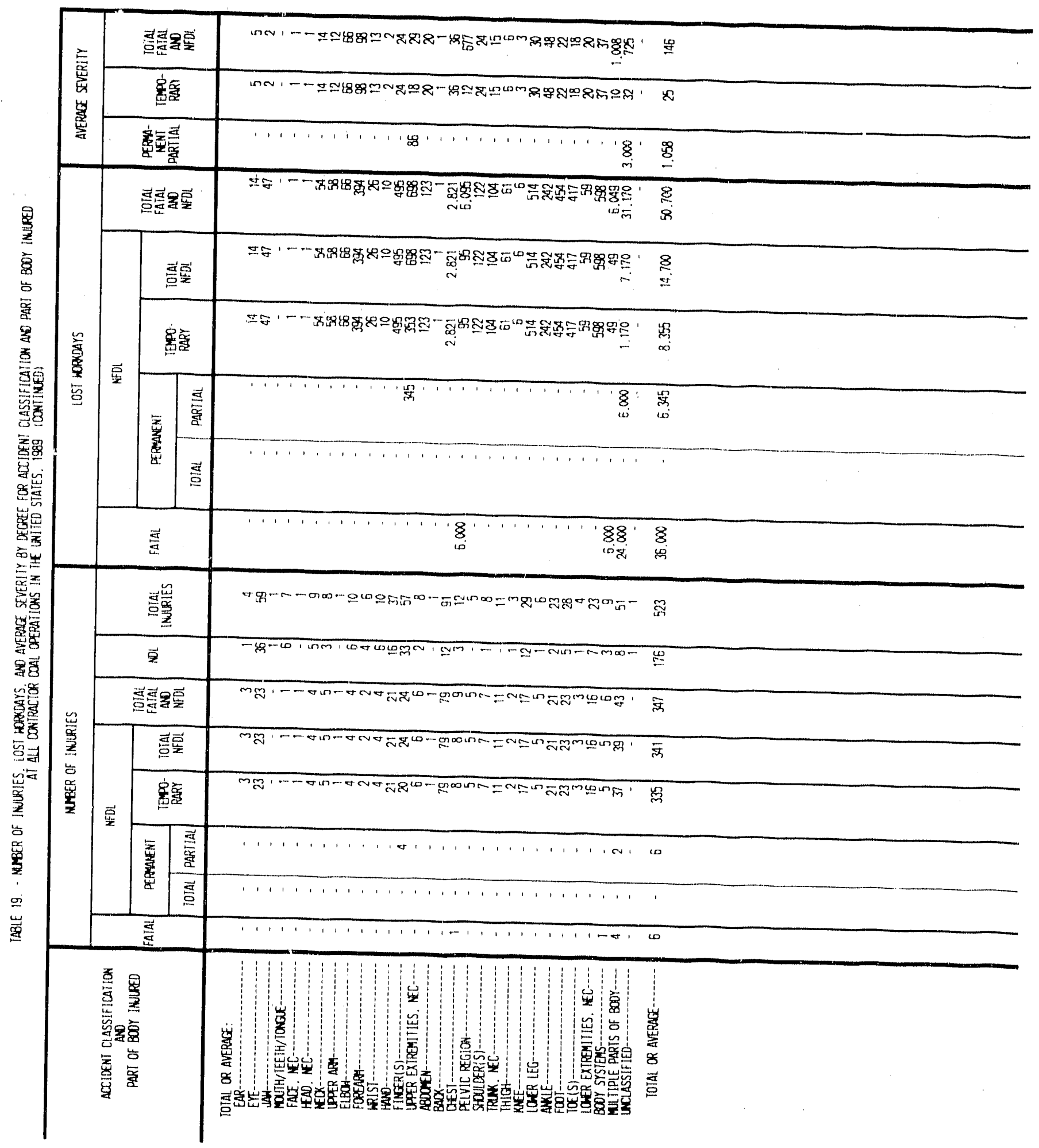




\begin{tabular}{|c|c|c|c|c|c|c|c|c|c|c|c|c|c|c|c|}
\hline \multirow{3}{*}{ 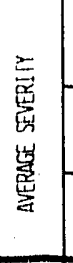 } & \multicolumn{2}{|c|}{ 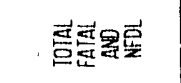 } & 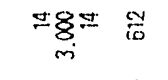 & $=$ & & $\therefore$ & & $\underset{10}{8}$ & & W E & & $m_{0}$ & & & $m$ \\
\hline & \multicolumn{2}{|l|}{ 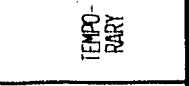 } & $\pm 1 \pm \quad \pm$ & $=$ & $=$ & 11 & 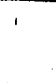 & ' & ' & 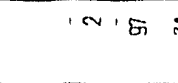 & \pm & - & $\pi$ & $m$ & $m$ \\
\hline & \multicolumn{2}{|c|}{ 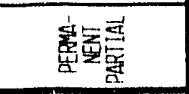 } & 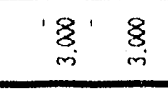 & ' & ' & $\cdots$ & ' & ' & ' & ' & ' & ' & ' & ' & ' \\
\hline \multirow{6}{*}{ 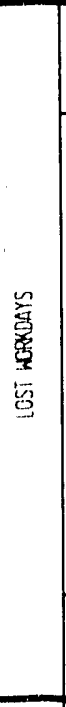 } & \multicolumn{2}{|c|}{ 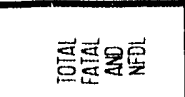 } & 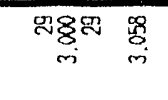 & $=$ & $=$ & 11 & 1 & $\begin{array}{c}8 \\
10\end{array}$ & $\underset{10}{8}$ & N'思 & 总 & \&ळ్ & \& & $m$ & $\bar{m}$ \\
\hline & \multicolumn{2}{|c|}{ 흥를 } & 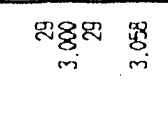 & $=$ & $=$ & 11 & & ' & . & in'g & 盯 & 정 & $z$ & $m$ & $m$ \\
\hline & \multicolumn{2}{|c|}{ 金㟥 } & 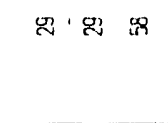 & $\because$ & $=$ & 11 & ' & ' & . & $\cdots$ & 幽 & สฺ্ & 茫 & m & $m$ \\
\hline & \multirow[t]{2}{*}{ 昱 } & 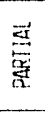 & '政 & i & ' & 11 & & . & ' & $\cdots$ & 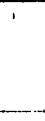 & 1. & i & T & ? \\
\hline & & 袩 & '.' & ' & ' & $\cdots$ & & & & $\ldots$ & & ' & . & ' & $\cdot$ \\
\hline & \multicolumn{2}{|l|}{ 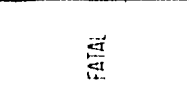 } & ' & ' & ' & i & & $\underset{B}{3}$ & $\underset{i n}{8}$ & & & & & & \\
\hline \multirow{8}{*}{ 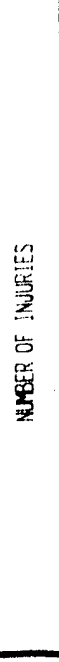 } & \multicolumn{2}{|c|}{ 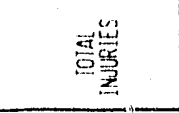 } & cores is & - & - & $\cdots \cdot$ & $\sim$ & - & - & $-\ldots \ldots$ & $\infty$ & $\pi-$ & w & -. & - \\
\hline & \multicolumn{2}{|l|}{$\ddot{z}$} & 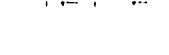 & ' & ' & $\cdots$ & $a$ & ' & & $1-$ & n & $\cdots$ & & 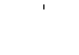 & \\
\hline & \multicolumn{2}{|c|}{ 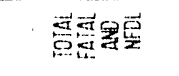 } & 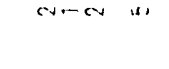 & - & $\cdots$ & $\cdots$ & ' & $\cdots$ & $\cdots$ & $1-m$ & $\sigma$ & $m-$ & 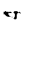 & $\cdots$ & - \\
\hline & \multicolumn{2}{|c|}{ 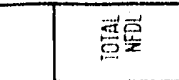 } & $\operatorname{coch}(5)$ & $\cdots$ & $\cdots$ & '. & & ' & & $1 \cdots 1 \mathrm{~m}$ & + & m. & $\sigma$ & $-\cdots$ & - \\
\hline & \multicolumn{2}{|c|}{ 䔡管 } & $\alpha+\alpha \sigma$ & - & - & 1.1 & & & 1 & $1 \cdots, m$ & $\pi$ & $m$ & $\sigma$ & - & - \\
\hline & & 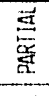 & $1-$ & & , & $\therefore$ & & . & ' & . & . & '. & & 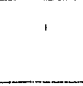 & ' \\
\hline & & 吾 & $\cdots$, & ' & ' & ' ' & & . & & 1 & ' & & & & \\
\hline & \multicolumn{2}{|l|}{ 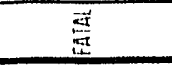 } & ' & . & 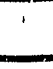 & ' & & $\cdots$ & $-\cdots$ & $\cdots$ & 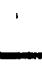 & 11 & ' & 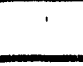 & ' \\
\hline & \multicolumn{2}{|l|}{ 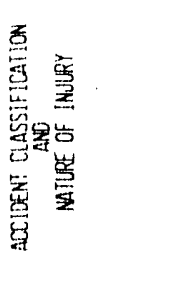 } & 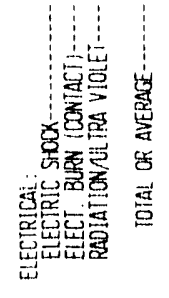 & 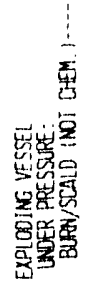 & 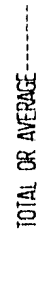 & 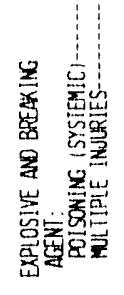 & 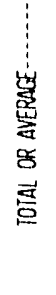 & 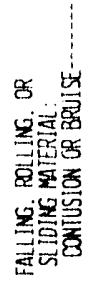 & 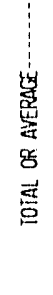 & 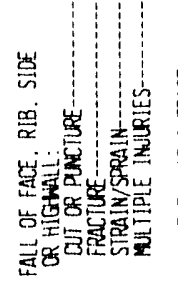 & 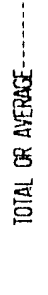 & 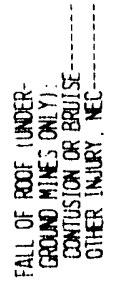 & 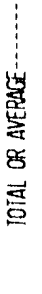 & 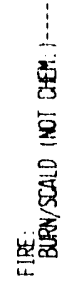 & 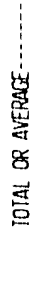 \\
\hline
\end{tabular}




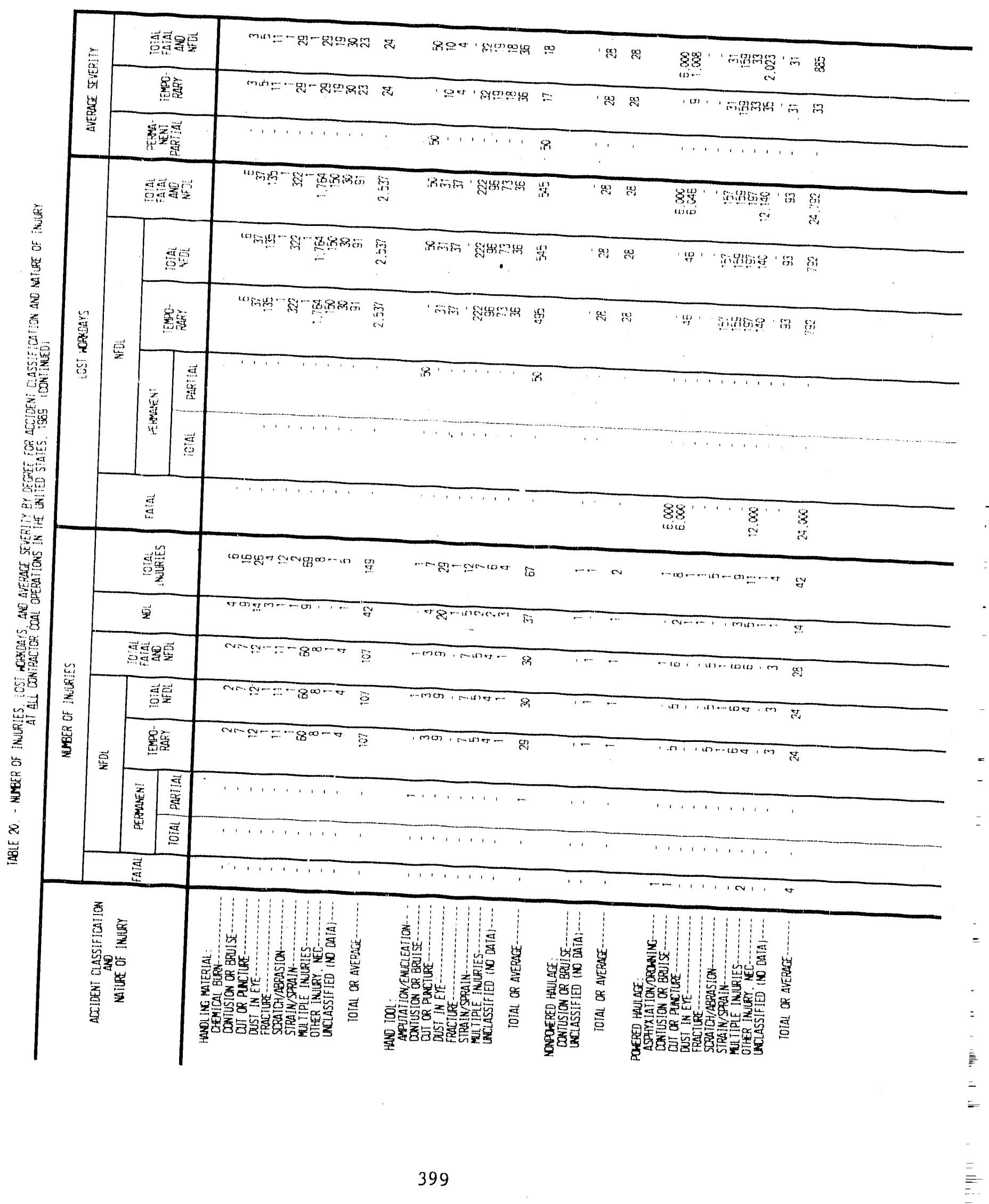




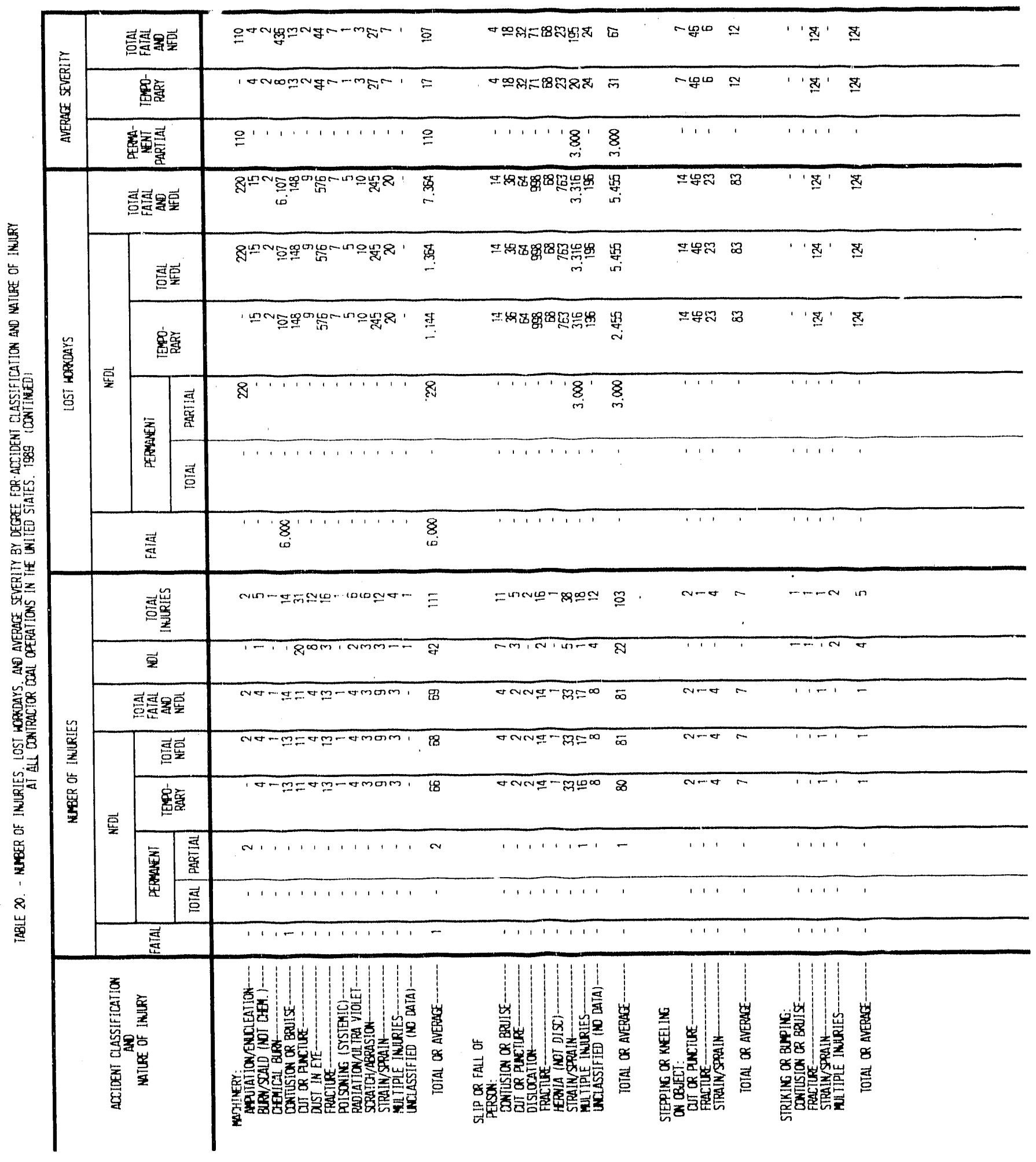




\begin{tabular}{|c|c|c|c|c|c|}
\hline \multirow{3}{*}{ 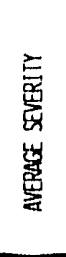 } & \multicolumn{2}{|c|}{ 형현울롤 } & now $1 \cos ^{\prime}$ & 188 & \\
\hline & \multicolumn{2}{|c|}{ 产愛 } & 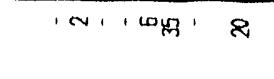 & 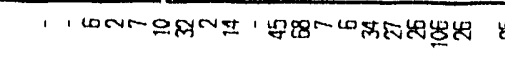 & 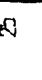 \\
\hline & \multicolumn{2}{|c|}{ 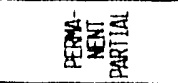 } & $421,1, k$ & 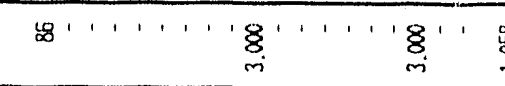 & s. \\
\hline \multirow{6}{*}{ 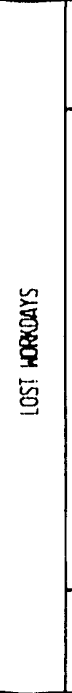 } & \multicolumn{2}{|c|}{ 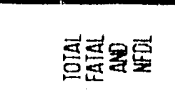 } & WN ' 9919 & 嵌8 & $\frac{8}{8}$ \\
\hline & \multirow{4}{*}{ 㬅 } & 吾要 & 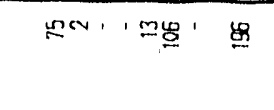 & 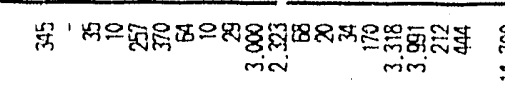 & $\begin{array}{l}8 \\
\square \\
\square\end{array}$ \\
\hline & & 象蜜 & 'N'罗 & 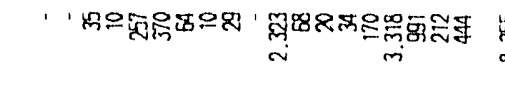 & 崔 \\
\hline & & & $m+1,19$ & 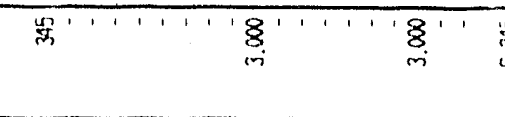 & o. \\
\hline & & & $\cdots+\cdots$ & $\cdots,{ }^{\prime}$ & , \\
\hline & \multicolumn{2}{|l|}{ 窟 } & $1,1,1$ & 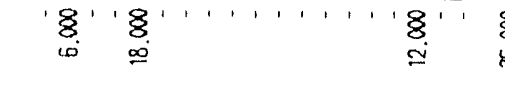 & $\begin{array}{l}8 \\
\text { s }\end{array}$ \\
\hline \multirow{8}{*}{ 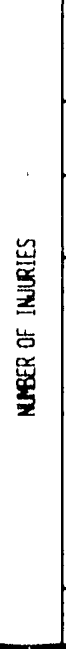 } & \multicolumn{2}{|c|}{ 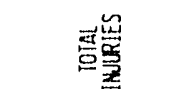 } & $-m-a+r$ & 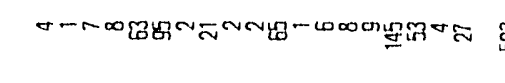 & $\dddot{\sim}$ \\
\hline & \multicolumn{2}{|l|}{$\bar{q}$} & $\infty$ & : : & 10 \\
\hline & \multicolumn{2}{|c|}{ 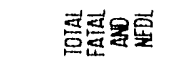 } & $-\infty, 1 \mathrm{Nm}+\mathrm{N}$ & 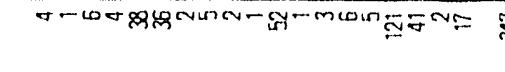 & कि \\
\hline & \multicolumn{2}{|c|}{ 氞哥 } & $--1 . \mathrm{erm} 1-$ & 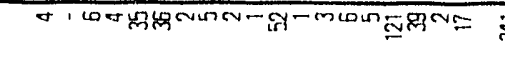 & $\overline{\bar{m}}$ \\
\hline & \multicolumn{2}{|c|}{ 蔤言 } & $1-1.1 \mathrm{Nm} 1 \mathrm{t}$ & 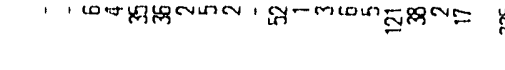 & 帅 \\
\hline & 蒵 & 焉 & $-1,1, \ldots$ & $\sigma, \ldots, 1, \ldots, 1, \ldots, 1$, & $\infty$ \\
\hline & & 总 & $\cdots+1,1$, & .', ' & \\
\hline & \multicolumn{2}{|l|}{$\overline{\frac{\vec{d}}{E}}$} & 11 & 11021 & 10 \\
\hline & \multicolumn{2}{|l|}{ 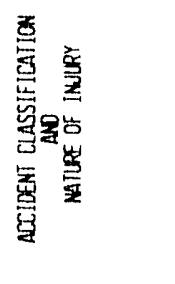 } & 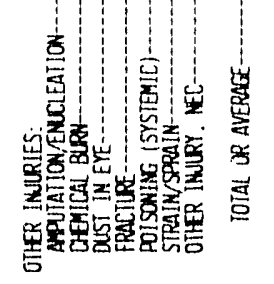 & 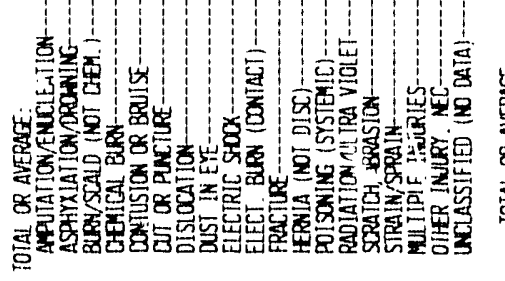 & 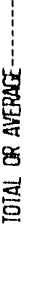 \\
\hline
\end{tabular}




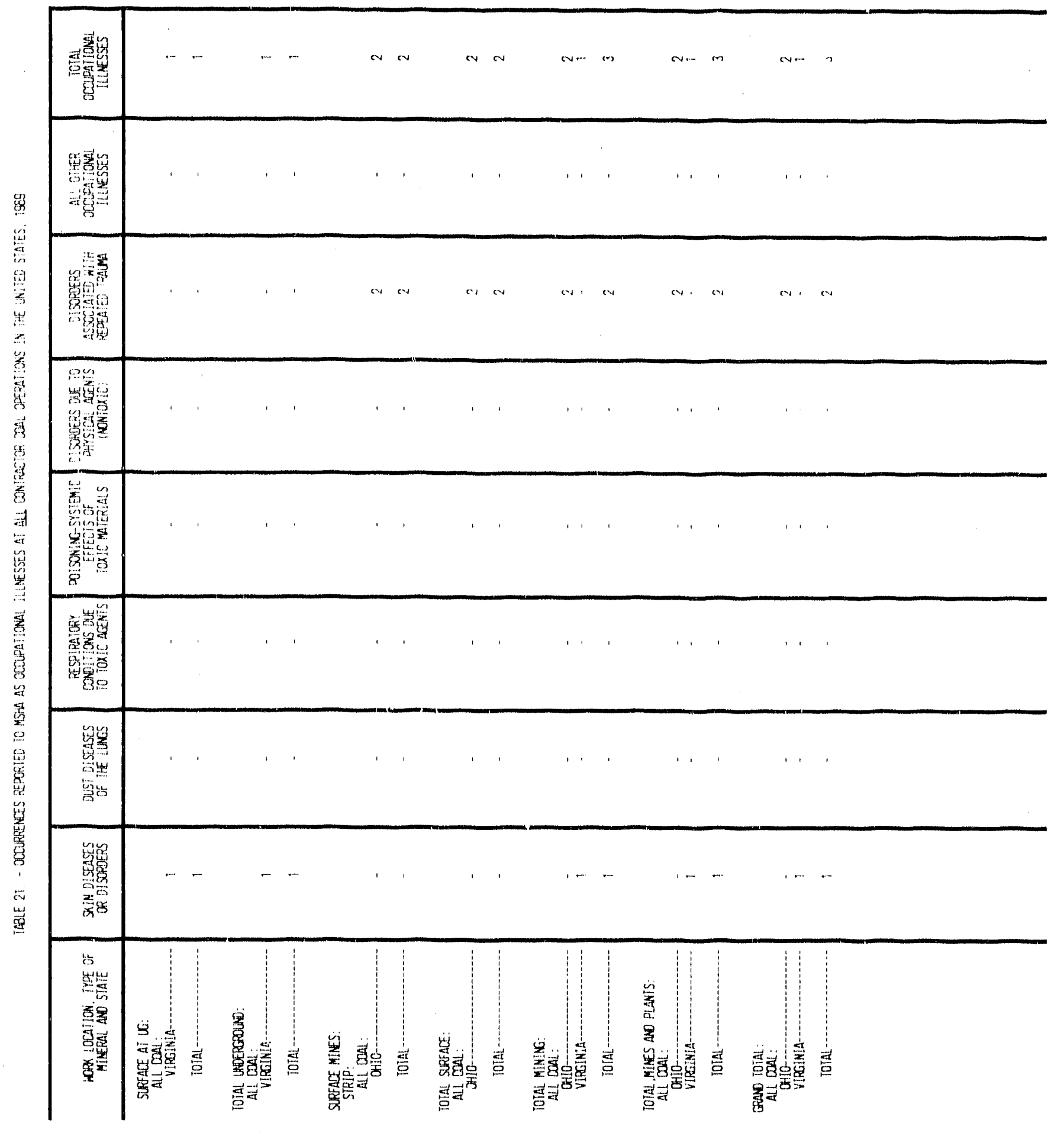




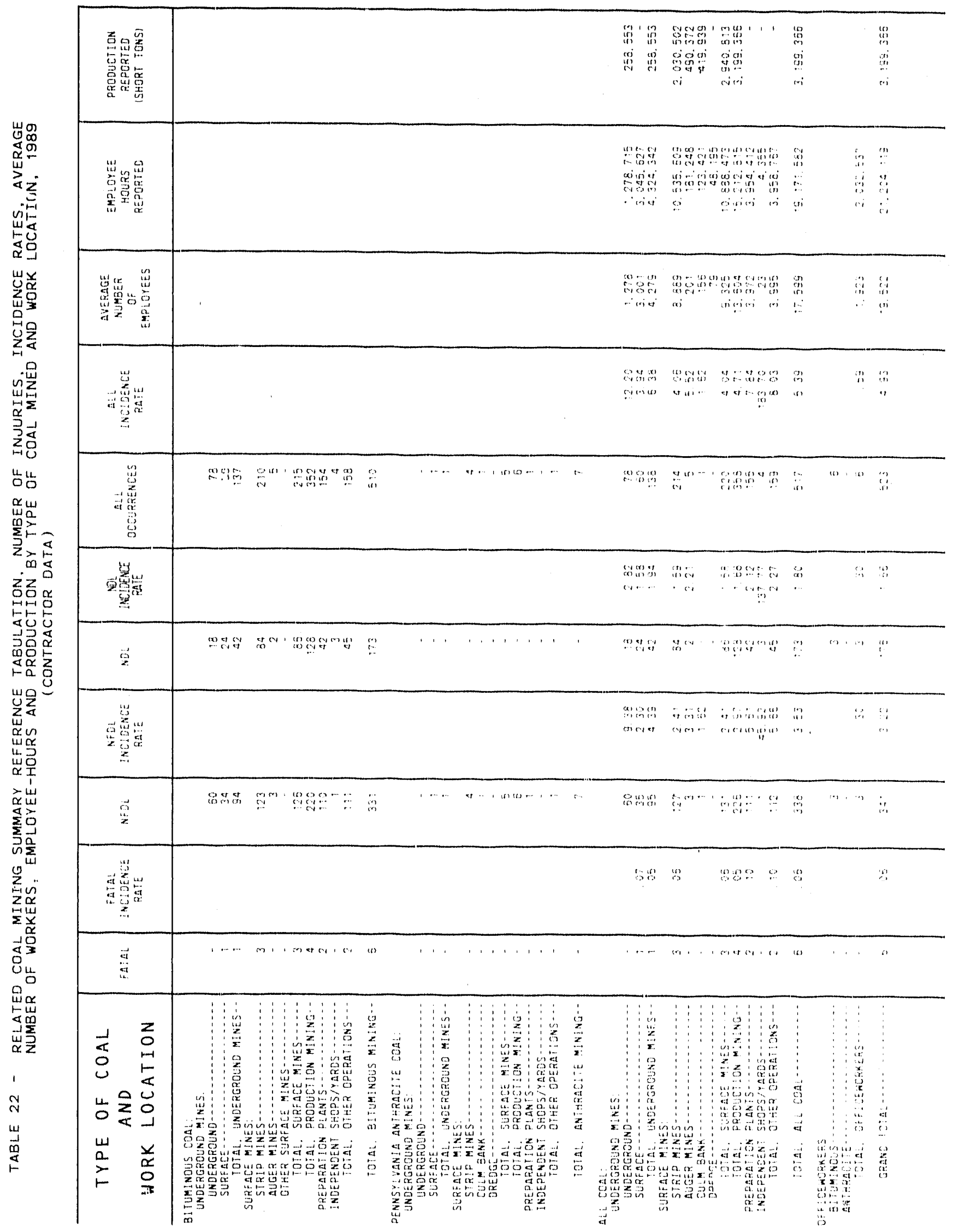




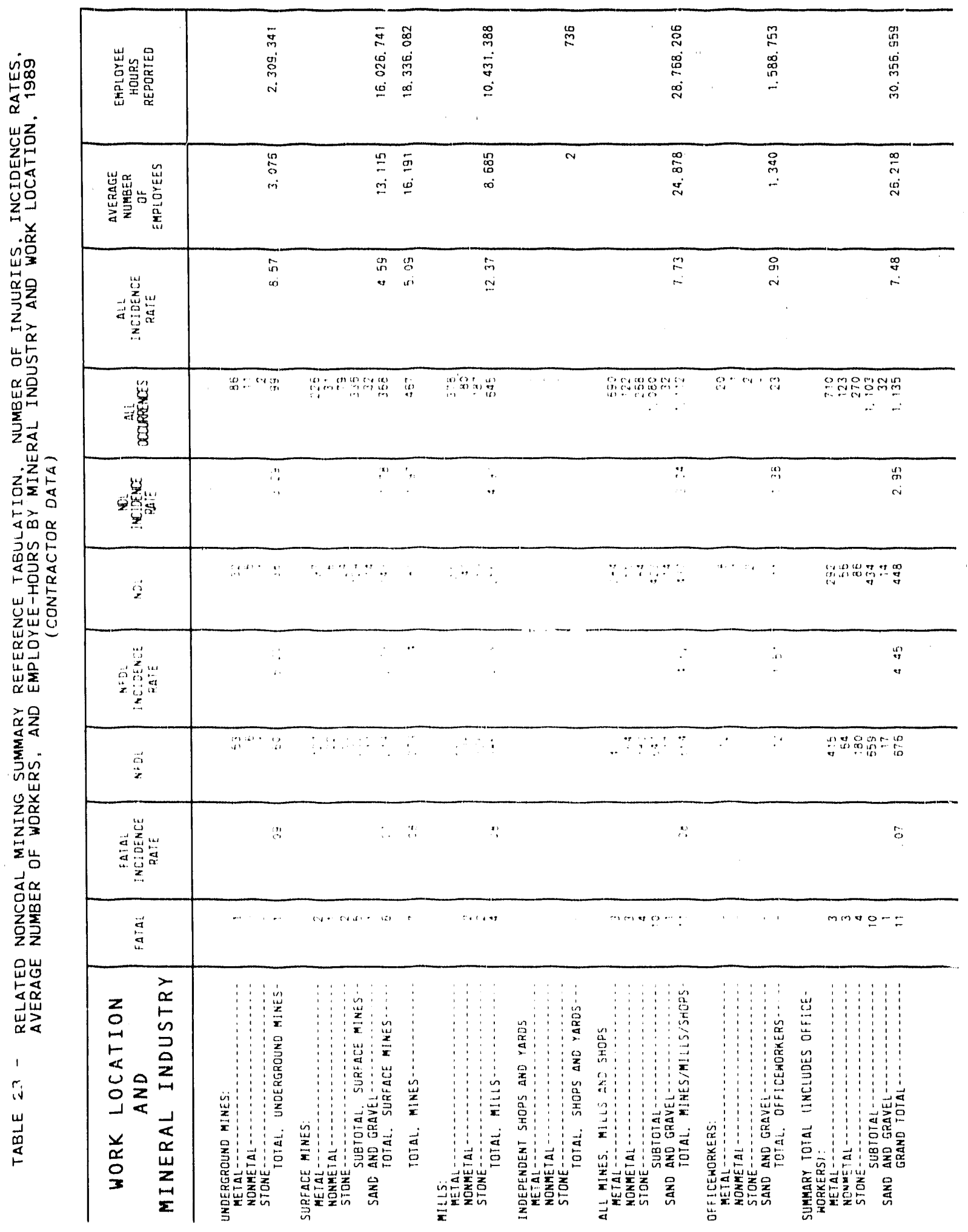



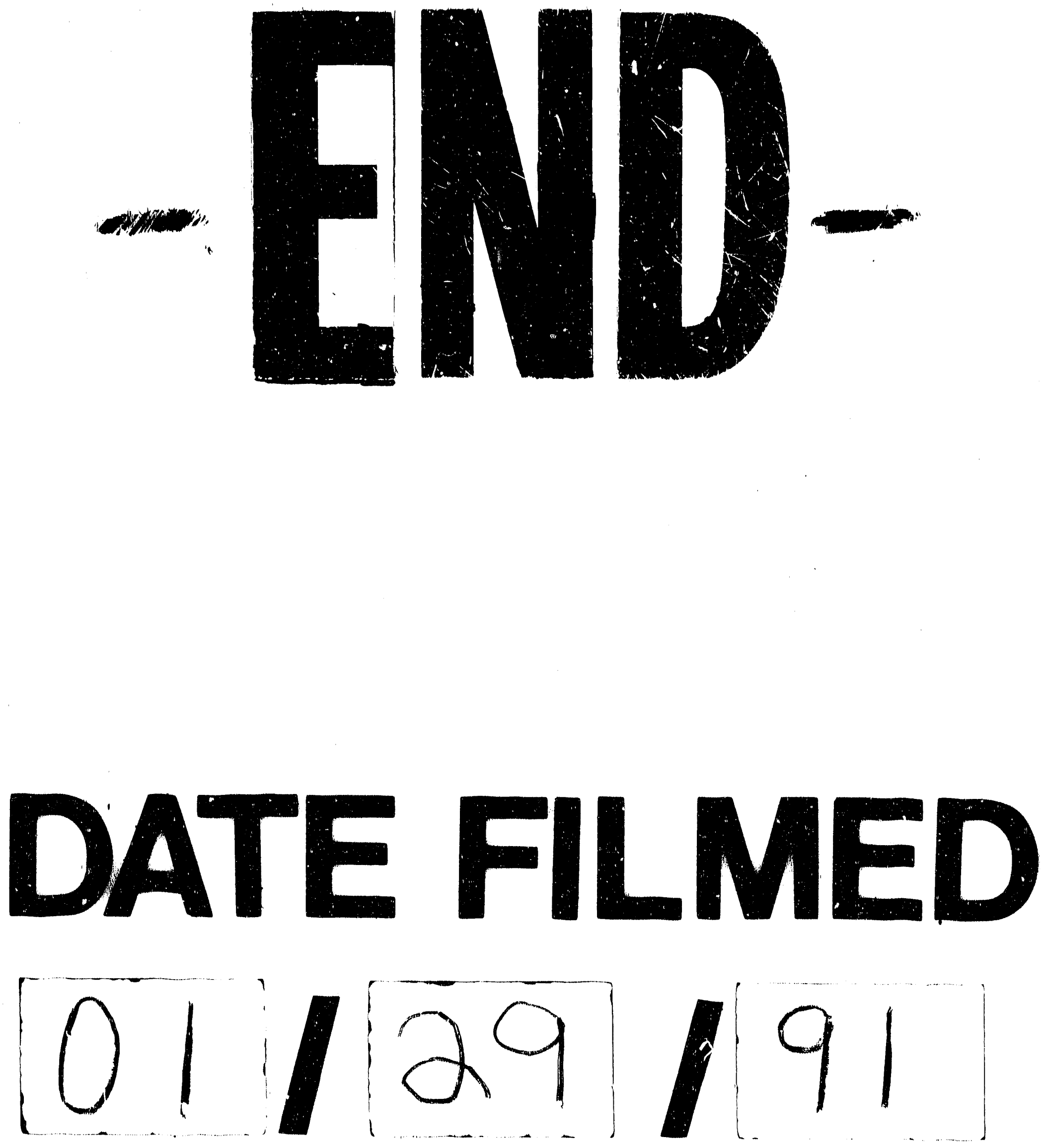